\title{
EAP 2019 Congress and Master Course
}

\section{Oral Presentations}

735

Adolescent Medicine

Educational Attainment, Employment Status and Personal Autonomy of Young Adults with Neurodevelopmental Disorders Zakhar Shchomak ${ }^{1}$, Cláudia Limal ${ }^{1}$ Sara Pereira ${ }^{1}$, Manuela Baptistal ${ }^{T}$ Department of Pediatrics, Centro Hospitalar Universitário Lisboa Norte, Portugal

Background: Neurodevelopmental disorders are characterized by developmental deficits that produce impairments of personal, social, academic or occupational functioning.

Objective: To access the educational attainment, employment status and personal autonomy among adolescents aged 17-20 years followed in the neurodevelopment clinic of a tertiary hospital.

Methods: A telephonic survey was elaborated and conducted to assess educational level, employment status, professional internship enrolment, parental sensation of personal autonomy and other features like getting a bank account or a driving licence. Patients born in 1999 and 2000 followed in our centre from 2009 to 2018 were included. Clinical information comprising diagnosis, comorbidities and age at the first and the last appointments were retrieved from personal records.

Results: Fifty-two of 83 eligible patients $(63 \%)$ responded to the survey; $69 \%$ were boys. The results were analysed by three major diagnosis groups i.e. Autism Spectrum Disorder (ASD) $(\mathrm{n}=10)$, Intellectual Disability (ID) $(n=13)$ and Attention-Deficit/Hyperactivity Disorder (ADHD) $(n=21)$. Concerning educational attainment, the majority were attending high school or higher levels of education (ASD 100\%, ID 54\% and ADHD 76\%). Regarding employment the majority were enrolled in a professional internship or were employed (ASD 40\%, ID 46\% and ADHD 52\%), while the rest were living at home under parental care or attending occupational centre activities. Parental sensation of personal autonomy was achieved in $60 \%$ of ASD, $54 \%$ of ID and $100 \%$ of ADHD patients. The number of adolescents obtaining a driving licence (ASD 40\%, ID 15\% and ADHD 85\%) and owning/managing a bank account (ASD 40\%/0\%, ID 38\%/15\% and $\mathrm{ADHD} 90 \% / 40 \%$ ) were heterogenous among the groups.

Conclusion: Educational levels, employment possibilities and personal autonomy have been increasing among patients with neurodevelopmental disorders, however, vary significantly and depend on disorder itself and its severity. A better guidance is needed to support these adolescents in the transition to adulthood.

\section{4}

\section{Adolescent Medicine}

Characteristics of a Decade of Alcohol Intoxications in Adolescents in Pediatric Departments in Dutch Hospitals

Nicolaas van der Lelij ${ }^{1}$, Loes de Veld ${ }^{l}$, Joris van Hoof $f^{2}$

${ }^{T}$ Pediatric department, Reinier de Graaf Gasthuis, Netherlands

${ }^{2}$ Behavioral Sciences Faculty, University of Twente, Netherlands
Aim: Admission of adolescents with an alcohol intoxication has become a health concern in current pediatrics. Over the years 2007 till 2016 we conducted a longitudinal study by monitoring the intake and treatment of 5,893 adolescents in all Dutch Pediatric Departments.

Methods: from 2007 till 2016 data were collected on all adolescents (aged younger than 18 and with a positive BAC), treated in all Pediatric Departments in The Netherlands. The Dutch Pediatric Surveillance System (NSCK) consists of a system in which all pediatricians report all admitted adolescents by completing a questionnaire, making use of a patient interview.

Results: 5,893 adolescents were treated in totally, most of them $(4,678$; $88 \%$ ) related to severe alcohol intoxication; mean age was 15.4 years, and $48 \%$ were girls. BAC level increased during this period (1.82 in 2007 and 2.01 in 2016), and reduced consciousness lasted from 2.24 hours in 2007 till 3.12 hours in 2016. Of the adolescents with alcohol intoxication, $11.4 \%$ had simultaneous drug usage. The attitude of the parents changed during the years: in 2011 (first year of registration) $68 \%$ of the parents gave permission to their child to drink alcohol, in 2016 this decreased to only $19 \%$.

Conclusions: The last decades, alcohol intoxication among youngsters has become an important aspect in Pediatrics. The Dutch Surveillance System was used to conduct a longitudinal analysis on alcohol intoxication characteristics, medical treatment and effects of interventions in this group.

496

Allergy \& Immunology

Assessment of Parent, Adolescent and Family Cognitive and Behavioral Dimensions Associated with Adherence to Treatment and Asthma Control

Luisa Barros ${ }^{l}$, Margarida Custódio dos Santos ${ }^{1,2}$, Ana Rita Oliveira ${ }^{l}$, $\overline{\text { Raquel Nunes }}^{1}$, Margarida Bonança ${ }^{3}$, Elsa Pereira $^{3}$, Ana Margarida Neves $^{3}$

${ }^{1}$ Faculdade de Psicologia, Universidade de Lisboa, Portugal

${ }^{2}$ Psicologia, Escola Superior de Tecnologia da Saúde de Lisboa, Portugal

${ }^{3}$ Consulta de Asmologia, Centro Hospitalar Universitário Lisboa Norte, Portugal

Background: Pediatric asthma management involves the development of a treatment plan and its implementation by the patient and the family. Adherence levels tend to be especially problematic during adolescence. Individual and family psychological dimensions contribute to a more comprehensive understanding of asthma management and control.

Objective: The aim of this study was to explore the relation between parents and adolescents with asthma, related knowledge and beliefs, family asthma management, and adherence to treatment and asthma control. Methods: Sample of 112 adolescents with asthma, 11-18 years old and their caregiver filled measures of Asthma Knowledge and Beliefs about Asthma Medication. Physicians rated the asthma control and nurses rated adherence. A sub sample of 32 caregiver and adolescent dyads completed the Family Asthma Management System Scale (FAMSS). 
Results: Show that both parents and adolescents have a reasonable knowledge about asthma, with wrong answers in similar domains, and overall positive beliefs regarding medication. Parents and adolescents knowledge about asthma were associated with asthma control, and with adherence for girls, but not for boys. Adherence was significantly associated with asthma control and with lower concerns about the secondary effects of medication. The overall family management and several dimensions of the FAMSS measure were positively associated with better adherence to treatment and asthma control.

Conclusion: FAMSS was a useful tool to identify domains of worse adaptation in families with lower adherence to treatment Understanding parents and adolescents asthma related knowledge and beliefs, and how the family organizes herself to manage the disease, may contribute to a more tailored educational intervention to increase adolescents levels of adherence and asthma control.

\section{7}

\section{Allergy \& Immunology}

Visceral Leishmaniasis in a Patient with Hyper-IgM Syndrome Secondary to PIK3CD Mutation: A Case Report

Mariano Silva Hernández ${ }^{1}$, Virginia Santana Rojo ${ }^{1}$, Serafin Castellano Dámaso $^{1}$, María Bravo-García-Morato ${ }^{2}$, Rebeca Rodríguez Pena ${ }^{2}$, María Alba-Domínguez, ${ }^{2}$, Fernando Baquero Artigao ${ }^{3}$, Eduardo LópezGranados $^{2}$, Francisco Javier Aracil Santos ${ }^{3}$

${ }^{1}$ Department of Paediatrics, Hospital Universitario La Paz, Spain

${ }^{2}$ Department of Immunology, Hospital Universitario La Paz, Spain

${ }^{3}$ Department of General Paediatrics and Paediatric Infectious Diseases, Hospital Universitario La Paz, Spain

Background: Visceral leishmaniasis is rare in patients with primary immunodeficiencies, especially, in patients with hypogammaglobulinemia. Recently, gain of function in PI3CKD have been related to a hyper-IgM syndrome, characterised by autoimmunity and lymphoproliferative disease, apart from hypogammaglobulinemia and predisposition to infections. As PI3CKD is part of the mTOR pathway, its treatment is based on blocking of different steps of it, with drugs such as sirolimus.

We present a case of visceral leishmaniasis in a 14-year-old boy with hyper-IgM syndrome secondary to PIK3CD mutation.

Case Presentation: Our patient is a 14-year-old boy with hyper-IgM syndrome secondary to PIK3CD mutation treated with immunoglobulin, sirolimus, cotrimoxazole, pantoprazole and calcium.

He had developed previous outbreaks of lymphoproliferative disease, consisting mainly of enlarged lymph nodes and tonsils, but they were controlled with sirolimus.

He referred a 6-week history of daily fever associated to headache and pain of his lymphadenopathies, more intense after the administration of immunoglobulin. On physical examination, he was pale and his lymphadenopathies were as usual, but slight splenomegaly was present. Laboratory tests showed a progressive pancytopenia (minimum values: haemoglobin $5 \mathrm{~g} / \mathrm{dL}, \mathrm{WBC}$ $1120 / \mu \mathrm{L}$, neutrophils $610 / \mu \mathrm{L}$, lymphocytes $290 / \mu \mathrm{L}$, platelets $95000 / \mu \mathrm{L}$ ). Sirolimus blood levels were higher than previous controls, without dosage changes nor renal disturbances. Sirolimus was discontinued, but there was not improvement of the symptoms and pancytopenia. Studies performed because of the pancytopenia were negative, except for positive PCR for Leishmania infantum in blood. PCR for Leishmania infantum was also positive in the bone marrow biopsy, although amastigotes were not visualized. Patient was treated with liposomal amphotericin B for 10 days, followed by additional doses at days 14 and 21. Progressively, fever, splenomegaly and analytic disturbances disappeared. After recovery, he received monthly suppressive therapy with liposomal amphotericin B for 6 months.

Conclusions: Visceral leishmaniasis should be considered as a possible cause of lasting fever in patients with immunodeficiencies, regardless of the type of immunodeficiency. Mainly, when fever is associated with pancytopenia and splenomegaly.
832

\section{Critical / Emergency Care}

Long-stay Patients in the Pediatric Intensive Care Unit Joana Caldeira Santos ${ }^{1}$, Francisca Martins ${ }^{2}$, Marta Grilo ${ }^{3}$, Augusto Ribeiro

${ }^{1}$ Department of Pediatrics, Centro Hospitalar Vila Nova de Gaia/Espinho, Portugal

${ }^{2}$ Department of Pediatrics, Unidade Local de Saúde do Alto Minho, Portugal ${ }^{3}$ Pediatric Intensive Care Unit, Centro Hospitalar de São João, Portugal

Background: Long-stay patients (LSP) in pediatric intensive care unit (PICU) are associated with high healthcare resource utilization.

Objective: To identify factors associated with long hospitalization in PICU. Methods: Retrospective cohort study of all pediatric patients admitted in a PICU of Northern Portugal in 2017 and 2018. LSP were defined as patients having a length-of-stay greater than the $90^{\text {th }}$ percentile ( $\geq 14$ days).

Results: A total of 626 patients were admitted, $10.7 \%(n=67)$ were LSP, with a mean age of $7.2 \pm 6.5$ years and $59.7 \%(n=40)$ were male. Using univariate binary logistic models, we determine a significant association between admission from another intrahospital department $(\mathrm{OR}=0.35,95 \%$ confidence interval [CI 0.21-0.59; p0.001), emergency admission ( $\mathrm{OR}=4.04,95 \%$ CI 2.29-7.13; p0.001), nonsurgical admission (OR=2.67, 95\% CI 1.59-4.45; p0.001), neurological disorder admitting diagnosis $(\mathrm{OR}=2.89,95 \% \mathrm{CI} 1.35-6.18$; $\mathrm{p}=0.006)$, cardiovascular condition admitting diagnosis $(\mathrm{OR}=2.85,95 \%$ CI 1.17-6.94; $\mathrm{p}=0.021)$, trauma related admission $(\mathrm{OR}=4.30,95 \%$ CI $2.24-8.25 ; \mathrm{p} 0.001)$ and the length-of-stay. Additionally, it was tested the association between the previously independent variables and the length-of-stay in a multivariate regression model, retaining only the significant variables. An association was determined between nonsurgical and trauma admissions with longer length-of-stay, with corresponding $\mathrm{OR}_{\mathrm{a}}$ of $4.68,95 \%$ CI 2.54-8.63 and 9.04, 95\% CI 4.2319.33 , respectively. The model classified correctly the observations in $89.3 \%$ of the cases. Model discrimination was assessed using a ROC curve, with corresponding AUROC $=0.72(95 \% \mathrm{CI}=0.65-0.78 ; \mathrm{p} 0.001)$

Conclusion: The clinical profile of LSP includes nonsurgical and trauma patients. A predictive model could help to identify patients with high risk of longer length-of-stay with a potential applicability for internal management regarding quality and cost-saving interventions.

\section{9}

\section{Critical / Emergency Care}

Acute Pain in Pediatric Emergency Settings: Challenges and Solutions

Kristina Ganzijeva $^{1}$, Ieva Kindereviciute ${ }^{1}$, Lina Jankauskaite ${ }^{1,2}$, Algirdas Dagys $^{1,2}$

${ }^{1}$ Medical Academy, Lithuanian University of Health Sciences, Lithuania ${ }^{2}$ Department of Pediatrics, Hospital of Lithuanian University of Health Sciences Kauno klinikos, Lithuania

Introduction: Today, pain is characterized not only as physiological reaction and response to tissue damage, it is recognized as multidisciplinary issue and multi-profile problem. Over the last decades great progress was made in pediatric pain evaluation and pain management. Unfortunately, acute pediatric pain still remains misunderstood, under-diagnosed, and under-treated.

Aims: To investigate accuracy of acute pain assessment and management in Pediatric Emergency Department (PED) in Lithuania University of Health Sciences Hospital Kauno Klinikos (LSMU KK).

Methods: We performed a retrospective card record analysis before (year 2017) and after (year 2018) pediatric pain training course was conducted. In total, 1000 randomly selected outpatient card records were analyzed. All cases were divided into two groups: group A records from 2017, group B - from 2018. Cases were further divided into trauma and nontrauma and subdivided into 4 different age groups. We collected patient age, origin of pain, pain characteristics, pain score and medication. 
Results: We compared 500 pain cases in each group. Group A and B consisted of $154(30.8 \%)$ and $116(23.2 \%)$ traumatic patients respectively. Pain was scored less in group A (420 children (84\%)) comparing to group B (94.4\% of all 500 cases, p0.001). In all age groups of group B pain was assessed more frequently and pain medication was prescribed more often compared to group A ( $\mathrm{p} 0.001$ ). There was a tendency to assess pain more often in non-traumatic patients in group A $(\mathrm{p}=0.054)$. However, pain relief in traumatic patients was less adequate compared to non-traumatic. Conclusion: Pain evaluation differed in both groups. In group B pain was evaluated more frequently and received pain-medication more often than group A. Teenagers are still less likely to receive analgesics than toddlers. Tendency remains to give less painkillers to trauma patients compared to nontraumatic children.

\section{2}

\section{Critical / Emergency Care}

Task-Sharing is Associated with Reduced In-Hospital Paediatric Mortality in Sierra Leonean Hospitals Christopher Hands ${ }^{l}$, Sandra Hands ${ }^{l}$, Emma Bailey ${ }^{l}$, Mannah Janet ${ }^{2}$, Mamasu Conteh ${ }^{2}$, Dennis Marke ${ }^{3}$, Andrew Fryer ${ }^{1}$, James Bunn ${ }^{4}$

${ }^{1}$ Global Team, Royal College of Paediatrics and Child Health, UK

${ }^{2}$ Resus Department, Ola During Children's Hospital, Sierra Leone

${ }^{3}$ Child Health Team, Ministry of Health and Sanitation, Sierra Leone

${ }^{4}$ Child Health, World Health Organisation, Sierra Leone

Background: Following the Ebola epidemic in Sierra Leone, the Ministry of Health and Sanitation (MoHS) initiated measures to regain and extend improvements in healthcare for children. Within a wider strategy to reduce child mortality, UKAID supported MoHS and RCPCH to introduce locally adapted WHO Emergency Triage Assessment and Treatment (ETAT+) protocols to all district hospitals.

Objectives: Analyse operational programme data to identify trends in mortality rates and quality of care.

Methods: In 2017 two resident clinical mentors (one Sierra Leonean, one international) delivered a three-month on-the-job training programme alongside mentorship for nurses, with written and practical examinations (74\% pass rate). Nurses passing the exams were authorised to initiate emergency paediatric treatment and were supported by clinical mentorship for three further months.

Mentors collected treatment data each day on children admitted the previous day and recorded discharge outcomes. These data were analysed to identify adherence to clinical protocols and to calculate mortality rates. In 2018, with intermittent support from RCPCH teams, admission and mortality data collected by hospital M\&E officers were analysed in onemonth blocks over six months.

Results: The 2017 data collection protocol was followed at 11 of 13 hospitals. Mean combined inpatient mortality over the first twelve weeks of the programme was $14 \%$, and for the second twelve weeks was $9.6 \%$. Adherence with clinical protocols improved for all emergency treatments, including appropriate use of oxygen ( $52 \%$ to $93 \%$ ), correct antibiotic prescriptions (39\% to $85 \%$ ), and management of convulsions (54\% to 83\%). In 2018 data collection was undertaken at 12 of 13 hospitals. Combined mortality was $10.4 \%$, and following further training fell to $7.3 \%$ in the second twelve weeks.

Conclusions: This analysis of operational data suggests that sustained reductions in hospital-level child mortality may be achievable in lowresource settings, when a task-sharing approach is combined with onthe-job training and mentorship.

188

\section{Critical / Emergency Care}

Faking it: Evaluating the Effectiveness of Low-Fidelity SimulationBased Paediatric Teaching in a District Hospital
Michael Kershaw ${ }^{1}$, Sobia Bilal ${ }^{1}$

${ }^{T}$ Paediatrics, Harrogate and District NHS Foundation Trust, UK

Background: Simulation-based teaching has become a key part of Paediatric training by allowing the development of the skills and knowledge to manage conditions and emergencies which are infrequently encountered in clinical practice. The current literature of simulation-based teaching focuses on large paediatric units with high-cost training programs, which could be prohibitively expensive for district hospitals.

Objectives: By conducting this prospective observational study we aimed to evaluate the low-fidelity ward-based simulation being conducted in Harrogate District Hospital. We aimed to demonstrate its effectiveness as well as assess participant's attitudes towards this method of teaching.

Methods: Low-fidelity simulation-based teaching sessions were undertaken with a multi-disciplinary team during periods of low ward activity. They used a simple mannequin with expired, or spare, equipment and were run by the Consultant on the ward. Questionnaires were distributed to evaluate the participants attituded towards simulation as well as their confidence levels before and after simulation of Paediatric and Neonatal emergency scenarios. A total of 24 responses were received and included in this study. Results: $100 \%$ of participants agreed, or strongly agreed, they wanted more simulation-based teaching. The average feedback from participants was agreement that these sessions improved their knowledge, communication, leadership, decision-making, and day-to-day practice. There was a statistically significant increase in participant's confidence levels with regards to: preparedness to manage emergencies $(\mathrm{p}=0.001)$, understanding their role $(\mathrm{p}=0.008)$, leadership ability $(\mathrm{p}=0.002)$, escalating to seniors $(\mathrm{p}=0.014)$, practical skills $(\mathrm{p}=0.012)$, communicating effectively $(\mathrm{p}=0.025)$, and ability to recognise unwell children $(\mathrm{p}=0.034)$.

Conclusion: This study demonstrated that low-fidelity simulation undertaken at low cost is an effective and flexible teaching method which is regarded highly by participants. There is a high demand from trainees for more simulation-based teaching and we hope the flexibility and effectiveness demonstrated in this study helps to encourage more widespread use.

\section{5}

\section{Critical / Emergency Care}

Uncertain Result of Abdominal Ultrasound Produced by Undertrained Operator Increase the CT in the Pediatric Emergency Department

So Yeon Kim ${ }^{1}$, Jae Yun Jung ${ }^{1}$, Young Ho Kwak ${ }^{1}$, Joong Wan Park ${ }^{1}$, Hyun $\overline{\text { Jung Chung }}^{1}$, Woo Ri Bae

${ }^{I}$ Department of Emergency Medicine, Pediatric Emergency Seoul National University Hospital, South Korea

Background: Children with abdominal pain are commonly evaluated with ultrasound to reduce radiation exposure in the pediatric emergency department (PED). However, ultrasound is highly operator-dependent, and the results are varied according to the level of the operator. Uncertain result by the undertrained operator may increase the CT, paradoxically.

Objective: To compare the correlation between the level of ultrasound operator and the presence of an uncertain conclusion which leads to additional CT scans.

Methods: This study was a retrospective cohort pilot study conducted at a tertiary academic PED. Patients under 18 years old with abdomen ultrasound from January to March 2017 were eligible, and chronic disorders including malignancy, hepatobiliary disease, and previous abdominal surgery history were excluded. Patients were classified into 'Specialist' group (SG) operated ultrasound by radiology specialist, and 'Resident' group (RG) by radiology resident. In our institution, radiology specialist operated ultrasound during the working day (Monday to Friday, 9 AM to $6 \mathrm{PM}$, except holiday), and after working hours, radiology resident operated. We compared the rate of uncertain ultrasound conclusions and additional CT between two groups using Chi-square or Fisher's exact test. 
Results: A total of 106 were analyzed. Mean age was 4.84 years, and 68 (64.2\%) were boys. SG were 62 (58.5\%), and RG were 44 (41.5\%). 22 (20.8\%) patients had an uncertain conclusion. Among them, SG were 5 (22.7\%), and RG were 17 (77.3\%). (p 0.001). 7 patients underwent additional CT scans with an uncertain conclusion. Only 1(14.3\%) patient in the SG and $6(85.7 \%)$ in the RG. (p 0.001)

Conclusions: Abdominal ultrasound operated after working hours by radiology residents had a more uncertain conclusion and led to more $\mathrm{CT}$ imaging. If the radiology specialist was available at a time other than working hours, unnecessary CT scans might be reduced by about $70 \%$.

\section{3}

\section{Critical / Emergency Care}

Syncope in the Young: Retrospective Review of Emergency Department Presentations

Leona Nertney $^{1,2,4}$, Jessica McGirr ${ }^{2}$, Ciaran Finucane ${ }^{3}$, Roisin McNamara', Patrick Fitzpatrick ${ }^{1}$, Paul McNally ${ }^{2,4}$

${ }^{1}$ Emergency Department, Children's University Hospital, Temple Street, Ireland ${ }^{2}$ Department of Paediatrics, Royal College of Surgeons in Ireland, Ireland ${ }^{3}$ Medical Physics, St James's Hospital, Ireland

${ }^{4}$ NCRC, National Children's Research Centre, Ireland

Background: Syncope is transient loss of consciousness due to global cerebral hypoperfusion, occurring in 1 in 5 children. 1-3\% of visits to Paediatric Emergency services result from syncope or ancillary injury. Clinically challenging, acute unexplained episodes are compounded by lack of collateral history or fear of sinister pathology. Resulting over-investigation may occur, with increased burden on both patient and healthcare system.

Objective: This study sought to determine the current management practices of childhood syncope within a tertiary emergency department.

Methods: Retrospective review of presentations to TSCUH Emergency Department (January-December 2017). Symphony (Patient Information System was interrogated to extract cases with diagnostic codes: "Syncope", "Presyncope", "Vasovagal Episode" or "Faint" in 0-16-year olds. Information on demographics, presentation, event characteristics, clinical management and measures of healthcare utilisation were analysed. All data are reported as proportions, means+/-SD as appropriate and analysed in STATA14.0.

Results: Data from 104 patients diagnosed with syncope were analysed: mean age $11.5+/-3.4 y r s ; 73(70.2 \%)$ female. Family history was documented in $45(43.2 \%$ ) cases. Normal physical examination in 101(97.1\%); abnormalities noted in 3(2.9\%) - cardiac murmur, bradycardia, hypotension. 66(63.5\%) events were witnessed; 47(45.2\%) were recurrent fainters. Event-related injuries were sustained in 26(25\%). Key differentiating symptoms were undocumented in most cases. Events occurred largely in the standing position $57(54.8 \%$ ), followed by situational syncope $14(13.5 \%)$. ECG was the most commonly performed investigation $86(82.7 \%)$, while more patients received urinalysis $29(27.9 \%)$ than lying/standing BP 20(19.2\%). Referrals were initiated by parents in $73(70.2 \%)$ cases, $28(26.9 \%)$ were transported by ambulance. Average attendance duration was 3 hrs 33 mins. Admission occurred in $4(3.9 \%)$ patients with additional $22(21.1 \%)$ referred for outpatient follow-up (highest referral rate to cardiology-50\%).

Conclusion: Syncope is associated with varying levels of case complexity, resulting in high resource use. Adoption and implementation of International Clinical Guidelines which address these complex events should be considered, with proven positive impact on patient management.

\section{7}

\section{Critical / Emergency Care}

Children \& Adolescence Injuries on the "New" Road with Electric Vehicles- COR (Children On Road) Study
Iris Noam Botton ${ }^{1}$, Ayelet Shlez ${ }^{1}$, Dania Takagi ${ }^{1}$, Ehud Rosenbloom ${ }^{1}$ ${ }^{T}$ Pediatric Emergency Department, Meir Medical Center, Israel

Background: Road accidents are a major cause of trauma. In recent years, there has been a significant change in the nature of accidents in children, with the increasing use of electric light vehicles.

Objective: To describe the characteristics of road accidents and injuries among children on "the new road" and to compare between electrical and non-electrical light vehicles accidents.

Method: Retrospective study, analyzing all referrals to pediatric ED for road accidents in children (

Results: 1531 children were included, of these, 205 were pedestrians, 602 were motor vehicle occupants, 504 were light vehicles riders, 203 were light-electric vehicle riders and 17 were motorcycle riders.

Distinct differences between groups were found in the average age, and severity of injuries. While only $2 \%$ of motor vehicle occupants were injured moderate/severe, in the other groups higher rates were found, between $17.3 \%$ (non- electric light vehicle), until 35.3\% (motorcycle).

Light-electric vehicle riders, when compared to non-electric light vehicle riders, were older, more likely to have multiple systemic injuries $(58.6 \%$ Vs. 31.8\%), had higher Injury Severity Scores) 5.8 Vs 4.7), and more head injuries ( $18.7 \%$ vs. $13.1 \%$ ), Chest injuries (3.4\% vs. $1 \%$ ) and external injuries (76.3\% vs. 65.9\%) (P0.05).

Conclusion: All of the unique characteristics of children and teenagers injured by electric bicycles: their age, the mechanism of the accidents, the characteristics and severity of the injuries, indicate that this is a new "player" that may be closer in nature to motorcycles and requires special attention.

There is room for increased awareness and education for safe riding, appropriate legislation and law enforcement. Further research is required in the field.

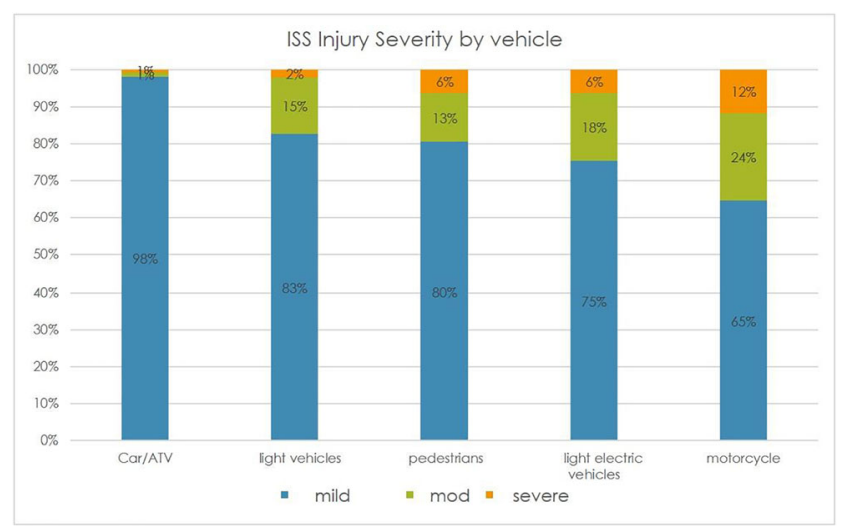

664

Critical / Emergency Care

To Improve the Quality of Paediatric Handovers by Introducing a New Safety Handover Tool and using Modified SBAR Based Handovers

Meenu Pandey $^{1,2}$, Imogen Norton ${ }^{1}$, David Burnside ${ }^{l}$

${ }^{T}$ Paediatrics, Northampton General Hospital, UK

${ }^{2}$ Neonatology, Leicester Royal Infirmary, UK

Background: This Quality Improvement project was conducted with a view to improve the quality of morning handovers in a high turnover paediatric environment. The national recommendations suggest inclusion of a "sick" handover prior to the main handover to allow for a system risk assessment ${ }^{1}$. Our aim was to create a new safety tool, and to introduce a new Modified SBAR (Situation, Background, Assessment, Recommendations) method to increase handover efficiency. 
Objectives: The objectives were to increase the usage of new safety tool and Modified SBAR and in the morning handovers in the paediatric unit. Methods: We conducted a pre-intervention questionnaire to assess the deficiencies in the existing SBAR based handovers. We then devised a new pre-handover safety tool called "SSSS" to discuss the number of Sick patients, Spacing, Staffing levels and Similar sounding names. Also, a new Modified SBAR handover method to include only relevant details of the patients was introduced. The senior trainees were trained in the use of the new handover tools. Two study cycles were conducted which involved modifications to the existing handover sheet. Postintervention staff survey was done to see the results.

Results: The uptake of the SSSS tool was $85 \%$ and that of the Modified SBAR handover was $100 \%$ at the end of the study period. All the parameters to assess handover safety increased. Handover effectiveness increased from $81 \%$ to $95 \%$.

Conclusions: We believe SSSS tool and modified SBAR based handovers are a new way to handover sick paediatric patients. This will also help to minimise errors and improve patient safety. We recommend their use in emergency and acute paediatrics settings for the future.

${ }^{1}$ https://www.rcpch.ac.uk/sites/default/files/2018-03/ facing_the_future_standards_for_acute_general_paediatric_services.pdf

\section{0}

\section{Critical / Emergency Care}

The Prevalence of Bacterial Infection in Recently Vaccinated Febrile Infants

Ali Raba ${ }^{l}$, Ibraheem Krebit ${ }^{1}$

${ }^{T}$ Paediatric Emergency Department, Tallaght University Hospital, Ireland

Background and aims: Evidence is lacking regarding the best approach to evaluating recently vaccinated infants younger than 3 months who present to the emergency department (ED) with fever. There have not been any recent published studies assessing the prevalence of bacterial infections in this population. The aim of the present study is to investigate the prevalence of bacterial infections in young infants presenting with fever within 72 hours after vaccination.

Methods: We reviewed the electronic medical records of infants aged between 6-12 weeks who presented with a fever 38C to paediatric ED, Tallaght University Hospital, Dublin, from January 2018 to December 2018. Febrile infants who recently vaccinated within 72 hours prior to ED presentation were compared with those who did not recently vaccinated. Bacterial infection were diagnosed based on culture results (urine or blood or CSF).

Results: A total of 70 infants (age: $8.8 \pm 1.9$ weeks, male: $40(57.1 \%)$ ) were enrolled in this study. Among 70 infants, $19(27.1 \%)$ had recently vaccinated, of whom $11(57.9 \%)$ presented to ED within 24 hours of vaccination, $7(36.8 \%)$ presented $24-48$ hours after vaccination and $1(5.3 \%)$ presented 48-72 hours after vaccination. The prevalence of bacterial infection in nonrecently vaccinated infants was $17.6 \%$ compared to $10.5 \%$ in recently vaccinated infants. Interestingly, all vaccinated infants who had bacterial infection, were presented to $\mathrm{ED}$ with fever within 24 hours of vaccination and all bacterial infections in this group were urinary tract infection (UTI).

Conclusion: Fever should not be attributed only to the vaccinations in young Infants who present to paediatric ED with fever after recent vaccination. All febrile recently vaccinated infants should be carefully evaluated and at least urine testing should be done regardless of the time of vaccination after the presentation.

585

\section{Critical / Emergency Care}

Post-Enterovirus Polio-like Rhombencephalitis in a 6 Year-old Andreas Trobisch ${ }^{1}$, Raphael Ulreich ${ }^{2}$, Miriam Pocivalnik ${ }^{2}$, Klaus Pfurtscheller ${ }^{2}$, Sebastian Tschauner ${ }^{3}$, Siegfried Rödl ${ }^{2}$
${ }^{1}$ Neonatology, University Clinic for Paediatric- and Adolescent Medicine, Austria

${ }^{2}$ Division of Paediatric Intensive Care, University Clinic for Paediatricand Adolescent Medicine, Austria

${ }^{3}$ Division of Paediatric Radiology, University Clinic for Radiology, Austria

A 6-year old, intubated patient with intermittent CPR-necessity was admitted to the intensive care ward. He had fallen sick one week earlier with flu-like symptoms, which had progressed to head- and neck pain as well as vertigo. Furthermore he developed dyspnoea, hypersalivation, dysphagia and desaturations while failing non-invasive ventilation, leading to his intubation. Guillain-Barré-syndrome was assumed, IVIG given and extended with 3 days Methylprednisolone. Additionally 10 cycles of plasmapheresis were performed leading to a rapid improvement of recurrent reanimation necessary bradycardic-/asystolic events. Cerebral and spinal MRI showed signal alterations/oedema of the cervical anterior horn ( $2^{\text {nd }}$ motor neuron) from $\mathrm{C} 2$ to $\mathrm{C} 5$ as well as signal alterations of tegmentum pontis and medulla oblongata, suspicious for rhombencephalitis and anterior myelitis (Figure 1). At this point, the patient showed (sub)-total paralysis with only movement of a few hand and facial muscles. PCR screening for pathogens in tracheal fluid detected enterovirus and literature associates the MRI lesions with enterovirus D-68 infection. During weaning our patient demonstrated lack of diaphragmatic movement and thus effective breathing, which led to prolonged necessity of nasotracheal intubation and later on tracheostomy with (home)-ventilation. PEG tube was installed since he was unable to swallow effectively without risk of aspiration. Extensive rehabilitative therapies were initiated, leading to significant improvement of movement but remaining home-ventilation dependent. After 3 months on intensive care, he could be transferred to a rehabilitation clinic.

\section{2}

\section{Critical / Emergency Care}

Bulging Fontanelle in Febrile Infants as a Clinical Predictor of Bacterial Meningitis

Amit Ziv ${ }^{1}$, Dania Takagi ${ }^{2}$, Ayelet Shles ${ }^{2}$, Ehud Rosenbloom ${ }^{2}$

${ }^{T}$ Department of Pediatrics, Meir Medical Center, Israel

${ }^{2}$ Pediatric Emergency Unit, Department of Pediatrics, Meir Medical Center, Israel

Background: It is a common practice to perform a lumbar puncture in infants presenting with fever and a bulging fontanelle, in order to rule out bacterial meningitis; however, most of these infants have benign and selflimited diseases.

Methods: We performed a retrospective cohort study of febrile children with a bulging fontanelle who underwent lumbar puncture at the Meir Medical Center between the years 2005-2015. $\chi^{2}$ test and descriptive statistics were used for data analysis.

Results: 764 children aged 2-18 months underwent a lumbar puncture in the Meir Pediatric Department during the study period. A total of 10 infants were diagnosed with bacterial meningitis. Only one infant (10\%) presented with a bulging fontanelle.

304 patients had a bulging fontanelle and fever on their evaluation. Amongst them cerebrospinal fluid pleocytosis was found in 115 cases (37.8\%), including one case of bacterial meningitis $(0.3 \%)$. None of the infants described on admissions as appearing either well or moderate on presentation were found to have bacterial meningitis.

The leading diagnoses for bulging fontanelle in febrile infants in our study were upper respiratory tract infections, aseptic meningitis and acute otitis media.

Conclusions: The finding of a bulging fontanelle, particularly if it's the only relevant sign in the infant's examination, has a very low sensitivity and specificity for bacterial meningitis. Most of the causes for a bulging fontanelle in febrile infants are self-limited diseases. The routine approach of performing a lumbar puncture in febrile infants with a bulging fontanelle should be reconsidered. 
567

\section{Endocrine \& Diabetes}

The Effect of Associated Autoimmune Disease on Metabolic Control in Children and Adolescents with Type 1 Diabetes Mellitus

Simona Cainap ${ }^{l}$, Claudia Bolba ${ }^{l}$, Madalina Ursu ${ }^{l}$, Adriana Bungardi ${ }^{l}$, Georgia Tita', Bogdan Lucian ${ }^{2}$

${ }^{1}$ Second Departament of Pediatrics, University of Medicine and Pharmacy "Iuliu Hatieganu", Romania

${ }^{2}$ Departament of Pediatrics, Emergency County Hospital "Dr Constantin Opris", Romania

Diabetes mellitus type 1(T1DM) is one of the most common chronic diseases in childhood, is caused by insulin deficiency resulting from the destruction of insulin-producing pancreatic beta cells.

Children and adolescents with T1DM are at increased risk for developing other autoimmune diseases (autoimmune thyroid disease, coeliac disease, Addison disease, AIJ, psoriasis, vitiligo, etc.). This could have an effect on the metabolic control of diabetes in the long term. The detection of concurrent autoimmune disease is important to prevent morbidity related to unrecognized disease.

Objective: The aim of this study is to assess the prevalence of different types of autoimmune disorders in a group of children with diabetes mellitus type 1 (T1DM) and to evaluate the effect of concurrent autoimmune condition (AI) on glycaemic control and growth.

Methods: A retrospective cross-sectional study was conducted on 152 patients with T1DM, aged between 1 and 18 years, who were followed up at the Second Pediatric Clinic Cluj-Napoca, from January 2011 to May 2016. The medical records and laboratory findings in the hospital's electronic system of all patients were reviewed.

Results: The prevalence of associated autoimmunity was $26.97 \%$ in our population. Out of the 41 patients, $27(17.6 \%)$ had autoimmune thyroid disease, $9(5,92 \%)$ had coeliac disease, and $3(1,97)$ had juvenile arthritis, $1(0,65 \%)$ had psoriasis and $1(0,65 \%)$ had vitiligo. Addison's disease was not diagnosed in this cohort of patients. There was no significant difference either in growth parameters or HbAlc between children with associated autoimmune disease and T1DM compared with children with T1DM.

Conclusions: The prevalence of concurrent autoimmune disease is greater in children with T1DM than in general population. The effect of having another concomitant autoimmune condition on metabolic control of diabetes and growth is not clear.

\section{7}

\section{Gastroenterology \& Nutrition}

Is Endoscopy Useful in the Investigation of Chronic Abdominal Pain in Children?

David Croaker ${ }^{1}$, Himasha Nanayakkara ${ }^{2}$

${ }^{T}$ Department of Paediatric Surgery, The Canberra Hospital, Australia

${ }^{2}$ Medical School, University of Notre Dame, Sydney, Australia

Background: Investigation for chronic or recurrent abdominal pain is frequently requested in paediatric surgery. The senior author had felt that endoscopy in this setting is rarely useful. Accordingly a retrospective review of our unit's practice was performed to ask the question "how often is endoscopy useful in managing children with chronic abdominal pain?

Objective: To review the usefulness of gastroscopy in the investigation of chronic paediatric abdominal pain.

Methods: Retrospective chart review of all paediatric gastroscopies performed during the last 15 years. This paper focuses on those referred for abdominal pain. Demographic data, reason for referral, and pathological findings were reviewed and compared with children referred for other reasons.
Results: 141 patients referred for investigation of non-specific abdominal pain were reviewed, from a total of 1779 paediatric endoscopies. 75 were female, and 66 male. The average age of those referred for pain was 11.02 years. The average age of 1638 referred for all other reasons was 7.89 years. (p 0.01) $41 \%$ of children had completely negative examinations. $30 \%$ had a gastropathy, and $29 \%$ microscopic findings of oesophagitis, contrasting with $30 \%$ of children gastroscoped for all indications who had a gastropathy. Only those being investigated for coeliac disease were more likely to have reactive gastropathy: $54 \%$.

Specific diagnoses included eosinophilic oesophagitis(EoE): 5; Helicobacter gastritis: 3; other acute gastritis: 5; coeliac disease: 5; gastric or duodenal polyps: 2 .

Conclusion: 141 scopes were performed to make few specific new diagnoses. Low grade chronic changes are commonly reported in both stomach and oesophagus regardless of presentation, and are positively correlated with coeliac disease. The diagnostic yield is not sufficiently high to recommend indiscriminate use of endoscopy in non specific abdominal pain. It is not clear that mucosal changes always explain symptoms of chronic abdominal pain in children.

\section{2}

\section{Gastroenterology \& Nutrition}

The Impact of Oral Nutritional Supplementation on Body Mass Index in Children Treated for Cancer

Ugur Demirsoy ${ }^{1}$, Feza Kirbiyik ${ }^{2}$, Ali Evrim Dogan ${ }^{2}$, Funda Corapcioglu ${ }^{1}$

${ }^{1}$ Pediatric Oncology, Kocaeli University, Faculty of Medicine, Turkey

${ }^{2}$ Advanced Medical Nutrition, Nutricia, Turkey

Objectives and the Study: This was a retrospective single center study where records from malnourished pediatric cancer patients were collected and analyzed from Kocaeli University Hospital, Pediatric Oncology, Turkey. The aim was to assess the impact of oral nutritional supplementation on body mass index (BMI) in malnourished children treated for cancer.

Methods: Inclusion criteria included a diagnosis of cancer and all standard treatments completed in the department according to the standard protocol regimens for the specific indications. Patients were considered malnourished if weight for height was below -2 $\mathrm{SD}$ as determined from gender specific WHO growth charts. The cohorts included patients treated during 2010-2016 time period. Patients who received oral nutritional supplementation (ONS) in addition to their specific anti-cancer regimen were recorded for their nutritional status based on weight, height and BMI during their follow up visits. Records were obtained for a period of 8 months from the start of the treatment.

Results: A total of 60 pediatric cancer patients with malnutrition (\%64,6 male, \%35,4 female) were included in the study. Data were analyzed either for all patients (total) or after clustering in to following tumor groups; CNS tumors $13.3 \%$ (8 patients), lymphoma $18.3 \%$ (11 patients), other tumors $68.3 \%$ (41 patients). Comparison of pre-ONS and post-ONS anthropometric data has shown an increase in bodymass index (BMI) for the total patients group data with an increase in BMI for 36 patients $(60 \%)$ versus decrease in BMI in 24 patients (40\%) (p 0.05) (Figure 1). Statistically significant increase was also observed for other tumors group (24 patients versus 17 patients) (p 0.001) and for lymphoma group (8 patients versus 3 patients $)(\mathrm{p}=0.012)$. Although not statistically significant, there was an increase in BMI for CNS tumors group as well (4 patients versus 4 patients $(\mathrm{p}=0.068)$.

Conclusions: ONS is an important intervention in pediatric cancer patients that increases BMI and improves the nutritional status. The extent of improvement is possibly related to the disease type and location 


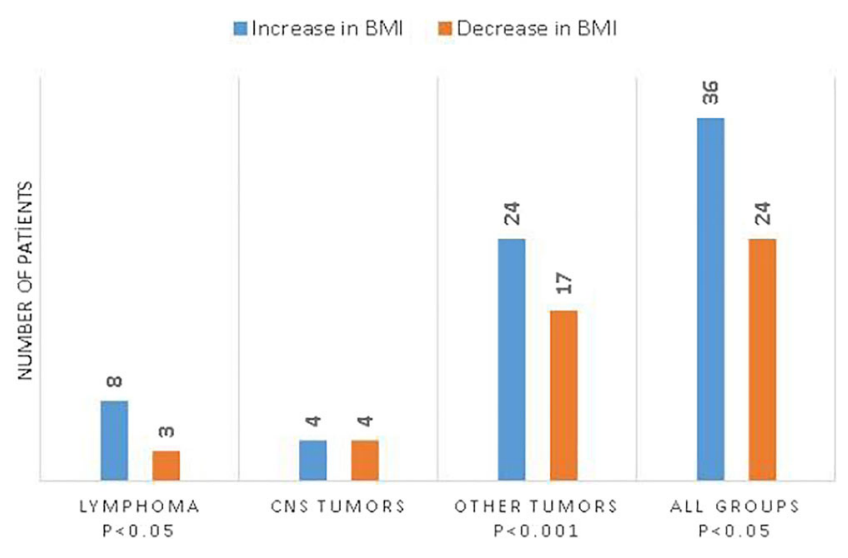

576

Gastroenterology \& Nutrition

Undernutrition in Two Different Communities of School-Children in North of Mozambique

$\underline{\text { Sofia Simões Ferreira }}{ }^{I}$, Joana Caldeira Santos ${ }^{I}$, Andreia Teles ${ }^{1}$, Mulaja Etienne', Carla Rêgo

${ }^{I}$ Department of Pediatrics, Vila Nova de Gaia/Espinho Hospital Center, Portugal

In Africa, the number of stunted children has steadily increased, reaching 58.7 million in 2017, Mozambique showing one of the highest prevalence's. Within the same country there may be nutritional differences depending on the proximity to the cities.

Characterize children from two communities of Mozambique, regarding sociodemographic indicators as well risk factors for malnutrition.

Longitudinal and interventional study in school-children from two communities in northern Mozambique [Ilocone (rural community) and Namicopo (city community)]. Children from 6 months to 12 years were invited to participate during their school-time. Demographic and anthropometric data (weight, brachial perimeter, length) were evaluated. WHO criteria were used to characterize nutritional status. Nutritional education and referral to primary care were provided.

77 children were evaluated: 35 from Namicopo, mean age of 68 months (8-131 months), 65,7\% females; 42 from Ilocone, mean age of 40 months (6-96 months), 59,5\% females. Significant differences (p0.05) were observed between the communities: in Ilocone the children were younger; births occurred more frequently at home; higher consumption of animal protein, beans and fruit; less consumption of grains; father with higher unemployment and age and poorest households. Prevalence of undernutrition was: $74.3 \%$ in Namicopo [with greater prevalence of mild wasting $(54,3 \%)]$ and $52.4 \%$ in Ilocone [mainly stunting (31\%)]. Wasting was significantly (p0,05) associated with less consumption of beans and fruit, prolonged duration of breastfeeding and single-parent families whereas stunting to poorest households, father's unemployment, progenitor's lower level of education and breast milk for shorter periods.

Undernutrition was diagnosed in more than half of this young population, the rural and the urban community showing a higher prevalence of chronic and acute undernutrition respectively. In our sample, poorest households were more significantly associated with stunting and poor feeding with wasting. Implications on present and future health of this population are of concern.

\section{1}

\section{Gastroenterology \& Nutrition}

Maternal Antimicrobial Use at Delivery Affects Gut Microbiota of Infants into the Early Weaning Period $\underline{\text { Naruaki Imoto }}^{1}$, Fumitaka Amanuma $^{2}$, Hidekazu Maruyama ${ }^{3}$, Shin Watanabe $^{I}$, Naoyuki Hashiguchi ${ }^{4}$

${ }^{1}$ Department of Microbiome Research, Juntendo University, Japan

${ }^{2}$ Neonatology, Iwate Prefectural Iwai Hospital, Japan

${ }^{3}$ Pediatrics, Iwate Prefectural Iwai Hospital, Japan

${ }^{4}$ Department of Emergency and Disaster Medicine, Juntendo University, Japan

Background: Intestinal colonization by bacteria such as Bifidobacterium or Bacteroides in infants and their presence in the gut at specific time points are known to affect the health of the host later in life. There are few published studies on the impact of maternal antimicrobial use at delivery on the gut microbiota in infants during the first year of life. To investigate the effect of antimicrobial administration immediately before delivery as intrapartum antibiotic prophylaxis (IAP) on the enteric environment in infancy around the weaning period and one year old.

Methods: A cross sectional study was conducted on healthy Japanese infants aged 6 months $(n=23)$ and 12 months $(n=26)$ to investigate the effect of antibiotic administration immediately before delivery as intrapartum antibiotic prophylaxis (IAP) on the enteric environment in infancy by using next generation sequencing to analyse the diversity and the composition of gut microbiota.

Results: In total, the fecal samples of 23 6-month-old and 26 12-monthold infants were analysed by next generation sequencing. Two 6-monthold infants and one 12-month- old infant were excluded because they had received antimicrobial drugs between birth and the sampling day. Antimicrobial agents were given systemically to the mothers at delivery for cesarean section, Group B Streptococcus-positive status, and premature rupture of membrane cases. IAP at the time of delivery does not influence the overall microbial diversity in the gut microbiota but decreased the occupancy by Bacteroides significantly $(\mathrm{p}=0.03)$, even in a subgroup analysis in infants group who were born by vaginal delivery at 6 months ( $\mathrm{p}=0.02$ ). In contrast, no influence of IAP was observed on gut microbiota in 12 months-old infants group.

Conclusion: Antimicrobial use immediately before the delivery are associated with decreased occupancy of Bacteroides in infants around weaning period. Although the current study provides a significant understanding of the gut microbiota in infancy, a follow-up study for a longer term is needed to identify the clinical significance of the change in the composition of gut microbiota based on the results.

\section{1}

\section{Gastroenterology \& Nutrition}

A Pilot Study of Randomized Controlled Trial of Bowel Preparation for Pediatric Colonoscopy

Pornthep Tanpowpong ${ }^{I}$, Hansa Sriphongphankul ${ }^{1}$, Chatmanee Lertudomphonwanit ${ }^{1}$, Suporn Treepongkaruna

${ }^{1}$ Pediatrics, Faculty of Medicine Ramathibodi Hospital, Thailand

Background: Adequate bowel preparation is crucial for colonoscopy though no standardized regimen exists for children. Polyethylene glycol with electrolyte solution (PEG-ELS) is a widely used osmotic agent due to its good efficacy and high safety profile. However, some children may not tolerate large volume and poor palatability. Previous meta-analyses in adults comparing between a full single dose $v s$. split doses of PEG-ELS showed that the split dose group had a higher rate of successful bowel preparation. Similar study in children is limited.

Objectives: We compared the efficacy (i.e., successful bowel preparation) of using PEG-ELS as a full single dose $v s$. split doses for bowel preparation before pediatric colonoscopy. The secondary outcomes were tolerability, acceptability, and compliance between two regimens.

Methods: Investigator-blinded randomized controlled trial was conducted to enroll children aged 2-18 years who underwent an elective colonoscopy at a teaching hospital between March 2018 and February 2019. Patients were randomly assigned to receive PEG-ELS as a full single 
dose or two split doses. The Boston Bowel Preparation Scale was used for the efficacy (i.e., successful if score $\geq 6$ ). Tolerability, acceptability, and compliance were evaluated by using a standardized questionnaire.

Results: A total of 43 colonoscopies (21 in the full single dose and 22 in the split dose group) were performed. Median age was 9.1 years old (IQR: 5.213.6). We found a higher rate of successful bowel preparation in the split dose group (95\% vs. $71 \%, P=0.046)$. Significant lower rate of nausea/vomiting ( $36 \%$ vs. $71 \%, P=0.02)$ and higher rate of willingness to repeat the protocol ( $82 \%$ vs. $33 \%, P=0.001$ ) in the split dose group were also noted.

Conclusion: The split dose regimen of PEG-ELS for bowel preparation before pediatric colonoscopy provides a superior efficacy, potential tolerability and acceptability as compared to the traditional full single dose regimen.

\section{7}

\section{Gastroenterology \& Nutrition}

Meta-Analysis of 196 Articles: Plasma Phospholipid Fatty Acid Supply of Expecting Women during Pregnancy and at Delivery Eva Szabo $^{1}$, Tamas Marosvolgyi ${ }^{2}$, Laszlo Poto ${ }^{2}$, Tamas Decsi ${ }^{3}$

${ }^{T}$ Department of Biochemistry and Medical Chemistry, University of Pecs, Hungary

${ }^{2}$ Institute of Bioanalysis, University of Pecs, Hungary

${ }^{3}$ Department of Paediatrics, University of Pecs, Hungary

Background: As long-chain polyunsaturated fatty acids play an important role in the maturation of the developing nervous system, maternal fatty acid supply is essential for the fetus as well as the newborn baby. Objective: Our aim was to systematically review available data on fatty acid composition of plasma and erythrocyte membrane lipids in expecting women during pregnancy and at delivery.

Methods: Electronic literature search was performed in July 2017, on Embase, Cochrane Library, and Ovid Medline with a search strategy including the following search expressions: (pregnant* OR gestation* OR deliver*) AND (arachidonic OR docosahexaenoic) NOT animal. We analysed data from clinical trials investigating fatty acid composition of plasma or erythrocyte membrane lipids in healthy expecting women without any fatty acid supplementation during pregnancy. Statistical analysis of fatty acid data was performed by Comprehensive Meta-Analysis v3.0 Software.

Results: After excluding duplicates there were 3351 potentially relevant articles. We excluded 3155 articles because they didn't meet our inclusion criteria, so 196 relevant articles remained publishing fatty acid data of plasma or erythrocyte membrane lipids, while 39 articles published data of plasma phospholipids (PLs). During the first trimester the studies investigated fatty acid status of a great number of mothers $(n=8337-8555)$, while in the three other timepoints the studies investigated somewhat smaller $(n=802-2757)$ populations. Values of arachidonic acid decreased significantly by the $3^{\text {rd }}$ trimester, while its values became significantly higher at delivery. On the other hand, values of docosahexaenoic acid remained quite stable during pregnancy, but decreased by delivery (Table).

Table: Mean values with $95 \%$ confidence interval ( $95 \% \mathrm{Cl})$ of arachidonic acid (AA) and docosahexaenoic acid (DHA) during preznancy and at delivery

\begin{tabular}{|c|c|c|c|c|}
\hline & \multicolumn{2}{|c|}{ Arachidonic acid (AA) } & \multicolumn{2}{|c|}{ Docosahexaenoic acid (DHA) } \\
\hline & mean $(95 \% \mathrm{Cl})$ & $\begin{array}{c}\text { Nr of studies } \\
\text { (Nr of mothers) }\end{array}$ & mean $(95 \% \mathrm{Cl})$ & $\begin{array}{l}\text { Nr of studies } \\
\text { (Nr of mothers) }\end{array}$ \\
\hline 1st trimester & $9.66(9.44 \cdot 9.87)^{2.0 .6}$ & $20(n=8337)$ & $4.34(4.15-4.53)$ & $21(n=8555)$ \\
\hline 2nd trimester & $8.96(8.67-9.24)^{n .0 .0}$ & $11(n=802)$ & $4.15(3.95-4.35)$ & $15(n=1124)$ \\
\hline $3 \mathrm{rd}$ trimester & $8.69(8.42-8.96)^{0.8 .7}$ & $29(n=2479)$ & $4.34(4,03-4.65)$ & $30(n=2757)$ \\
\hline Delivery & $9.14(8.74-9.55)^{\text {tet }}$ & $18(n=1785)$ & $3.97(3.72-4.22)$ & $19(n=1871)$ \\
\hline
\end{tabular}

Conclusion: 1. Our results indicate that on a population level, docosahexaenoic acid status remains remarkable stable during pregnancy 2. However, in plasma phospholipids values of arachidonic acid decreased significantly in the course of pregnancy.

774

\section{Genetic \& Metabolic}

Comparing Cognitive Functions of Children with PKU and Controls using CANTAB Nonverbal Tests

Dora Becsei ${ }^{1}$, Ildiko Erni ${ }^{1}$,Erika Kiss ${ }^{1}$, Erika Simonova ${ }^{1}$, Attila Szabo ${ }^{1,2}$, Ildiko Szatmari ${ }^{1}$, Petra Zsidegh ${ }^{1}$, Janos Bokay ${ }^{1}$

${ }^{I} 1$ st Department of Paediatrics, Semmelweis University, Hungary

${ }^{2}$ Pediatric and Nephrology Research Group, Hungarian Academy of Sciences, Hungary

Background: Phenylketonuria is an inherited error of metabolism caused by the failure of the phenylalanine hydroxylase enzyme. In poorly-treated cases, the patients have mental retardation, psychiatric problems and skin diseases. Objective: We aimed to examine the cognitive functions of children with PKU comparing them with age-related control patients. We examined the correlation between cognitive functions and serum Phe, Tyrosine, Phe/Tyr ratio.

Methods: The children were examined with 5 nonverbal tests of Cambridge Neuropsychological Test Automated Battery including tests of working memory, learning and executive function; visual, verbal and episodic memory; attention, information processing and reaction time. The data were analyzed using Microsoft Excel and R statistical packages. We used Mann-Whitney tests to compare the two groups. We normalized the results of each task and obtained a $0-10$ score system to characterize the mean cognitive functions. The correlations were analysed with Spearman probes. Results: We collected 54 children with PKU and 99 controls. We got the most explicit difference in SOC subsequent thinking time: the children with PKU were 3.12 times slower (1668 ms vs. 533,4 ms). The results show a correlation between cognitive functions with age and Phe. The mean normalized cognitive function was 5,77 in the group of children with PKU, and 7,52 in the control group.

Conclusion: Some CANTAB tests showed a significant difference between PKU and control patients - the most significant one: SOC subsequent thinking time.

According to our results, the nonverbal computer tests can be used very well to examine the fine cognitive dysfunctions caused by PKU, and it has a correlation with the serum Phe. The results confirm the importance of a strict diet.

\section{3}

\section{Genetic \& Metabolic}

Metabolic Syndrome and its Components among Egyptian Obese Adolescents' Girls: Influence of ApolipoproteinE Polymorphism Nayera Elmorsi Hassan ${ }^{I}$, Abeer Atef El Ashmawi ${ }^{2}$, Sahar Abd-El-Rauf El-Masry', Waheeba Ahmed Zarouk ${ }^{3}$, Marwa Farouk Mira' ${ }^{2}$, Gamila SM El-Saeed ${ }^{4}$, Omar Hosni Dwidar ${ }^{1}$

${ }^{1}$ Biological Anthropology Department, National Research Centre, Egypt ${ }^{2}$ Pediatrics Department, Cairo University, Egypt

${ }^{3}$ Molecular Genetics and Enzymology Department, National Research Centre, Egypt

${ }^{4}$ Medical Biochemistry Department, National Research Centre, Egypt

Metabolic complications with obesity are increasing in childhood and extend to adulthood.

Aim: To assess the prevalence of metabolic syndrome (MS) among a sample of Egyptian adolescent girls and investigate its association with Apolipoprotein E. 
Subjects \& Methods: A cross sectional study included 200 Egyptian adolescent girls aged between 12-18 years. They were subjected to lood pressure measurement, anthropometric measurements (weight, height and waist circumference), Laboratory investigations (fasting glucose and lipid profile) and molecular analysis (Apo E).

Results: Overweight/obese girls were suffering significantly more than normal weight girls from hypertension $(66.7 \%$ vs $40.8 \%)$,diabetic $(46.7 \%$ vs $31.2 \%$ ) and low HDL (64\% vs $59.2 \%$ ). Girls withMS had significant higher values of BMI-Z score, WC, blood pressure, cholesterol and triglycerides, and significant lower HDL. Allele E3 was more frequent among girls with MS (59.1\% vs $55.1 \%$ ), while allele E4 was more frequent among girls without MS (41\% vs 36.4). Genotype E3/E4 was more frequent among girls with MS (45.5\% vs $23.1 \%$ ), while genotype E3/E3 was more frequent among girls without MS (43.6\% vs $36.4 \%$ ). MS was the most prominent among E3/E4 genotype (35.7\%) which had the highest frequency of elevated cholesterol, triglycerides, LDL and blood glucose, while genotype $\mathrm{E} 2 / \mathrm{E} 4$; which was rare among both groups; had the highest frequency of elevated blood pressure $(68.8 \%)$ and low HDL $(71.4 \%)$.

Conclusion: MS was significantly more prominent among overweight/ obese girls compared to the normal weight ones, and among E3/E4 genotype; which had the highest frequency of disturbed lipid profile and blood glucose.

\section{6}

\section{Genetic \& Metabolic}

Biochemical Comparation Between Infants with Deficiency of Vitamin B12 Secondary to Maternal Deficiency and due to Genetic Disorder

Sara Teixeira $^{1}$, Teresa Campos $^{1}$, Esmeralda Rodrigues $^{1}$, Teresa Cardoso $^{2}$, Hugo Rocha ${ }^{3}$, Laura Vilarinho ${ }^{3}$, Elisa Leão-Teles ${ }^{1}$

${ }^{1}$ Centro de Referência de Doenças Hereditárias do Metabolismo, Centro Hospitalar de São João, EPE, Portugal

${ }^{2}$ Serviço de Medicina Interna, Centro Hospitalar de São João, EPE, Portugal

${ }^{3}$ Unidade de Rastreio Neonatal, Metabolismo e Genética, Instituto Nacional de Saúde Doutor Ricardo Jorge, Portugal

Background: Vitamin B12 or Cobalamin (Cbl) plays an important role in many metabolic pathways. Its deficiency can lead to a wide spectrum of hematologic and neurological disorders that can be reversed or minimized by early diagnosis and prompt treatment. Cbl deficiency in newborns/ infants may be secondary to nutritional deficits or due to defects in the absorption, transport or intracellular metabolism of $\mathrm{Cbl}$, and can be identified in Portuguese newborn screening by analysis of acylcarnitine profile. Objective: Compare biochemical parameters of infants with deficiency of $\mathrm{Cbl}$ secondary to maternal deficiency to infants with genetic deficit. Methods: Included infants referred due to suspicion of $\mathrm{Cbl}$ deficit, between 2009 and 2018. Data from neonatal metabolic screening and infants and mothers clinical files.

Results: Were identified 8 breastfed infants, 4 with deficiency secondary to maternal cause and 4 with $\mathrm{CblC}$ disorder (an inborn error of the intracellular metabolism of $\mathrm{Cbl}$, secondary to mutations in MMACHC gene). Seven were referred in the first month of life, due to alterations in neonatal screening, and one was diagnosed at 5 months of age when evaluated by growth and development impairment.

In newborn screening, the mean levels of propyonilcarnitine and propyonilcarnitine/acetylcarnitine ratio was two times higher in infants with genetic disorder. In these patients the serum $\mathrm{Cbl}$ was higher (10 times), as well as, the levels of methylmalonic acid (3.5 times) and homocysteine (4 times). Contrariwise, the mean levels of methionine were 1.6 times higher in nutritional deficit.

Conclusion: The mean values of the acylcarnitines, methylmalonic acid, and homocysteine are higher in the genetic $\mathrm{CblC}$ deficiency, when compared to nutritional deficits. However, the size of this sample does not allow conclusions regarding of cutoffs for each parameter.
476

\section{Infectious Diseases}

Prognostic Factors in Late-Onset Streptococcus agalactiae Sepsis and Bacteremia

Serafín Castellano Dámaso ${ }^{1}$, Raquel Gómez Garciá2, José David $\overline{\text { Andrade Guerrero }}^{1}$, Inés De Augusto Claudino ${ }^{7}$, Virginia Santana Rojo $^{I}$, Mariano Silva Hernández ${ }^{I}$, Marta Dorado Criado ${ }^{I}$, Celia Fabra Garrido ${ }^{1}$, María Fátima Ara Montojo ${ }^{1}$, Rosario López López ${ }^{3}$, María de Ceano-Vivas la Calle ${ }^{3}$, Julia Martín Sánchez, María Pilar Romero Gómez ${ }^{4}$, Iker Falces Romero ${ }^{4}$, Juan José Menéndez Suso ${ }^{5}$, Cristina Verdú Sánchez, ${ }^{5}$, Miguel Sáenz de Pipaón Marcos ${ }^{6}$, Laura Sánchez. Garcia $^{6}$, Luis Escosa Garcia ${ }^{1}$, Cristina Ots Ruiz ${ }^{1}$, Fernando Baquero Artigao $^{l}$, Ana Méndez Echevarría ${ }^{1}$, Teresa del Rosal Rabes ${ }^{1}$, Luis Alfonso Alonso Garcia ${ }^{1}$, Francisco Javier Aracil Santos ${ }^{l}$

${ }^{1}$ General Paediatrics and Infectious and Tropical Diseases Department, Hospital Universitario La Paz, Spain

${ }^{2}$ Centro de Salud Alpedrete, Atención Primaria Madrid, Spain

${ }^{3}$ Emergency Department, Hospital Universitario La Paz, Spain

${ }^{4}$ Microbiology Department, Hospital Universitario La Paz, Spain

${ }^{5}$ Pediatric Intensive Care Unit, Hospital Universitario La Paz, Spain

${ }^{6}$ Neonatalogy Department, Hospital Universitario La Paz, Spain ${ }^{7}$ Pediatrics Department, Centro Hospitalar Oeste, Portugal

Background: Streptococcus agalactiae or Group B Streptococcus (GBS) is one of the main causes of sepsis in newborns and young infants. GBS is also a major cause of meningitis in newborns, and it might lead to neurological impairment in those children affected. Disease manifestations are often non-specific, and symptoms are of short duration before diagnosis is made.

Objective: To determine possible prognostic factors of late-onset Group B streptococcal sepsis, diagnosed at a single tertiary care hospital in Madrid, Spain.

Methods: Retrospective review of culture-proven GBS sepsis, after 7th day of life, between 2000 and 2013.

Results: Sixty-eight episodes of late-onset GBS sepsis or bacteremia were diagnosed. Clinical onset started during after-birth hospitalization in $10 \%$ of patients. GBS was identified in blood culture (67 cases) and/or CSF (14 cases). Sixteen (24\%) presented meningitis. Mean age at diagnosis was 34 days (8-125 days). Mother's rectal-vaginal culture was positive for GBS in only $14.5 \%$, negative in $58 \%$ and was not performed in $27.5 \%$. The most common clinical symptoms were: fever (73\%), irritability (58\%) and food rejection (52\%). Three patients died (4.4\%). Seven patients developed severe neurological impairment.

Adverse outcome (death or neurological impairment) in the univariate analysis was related to lower temperature, lower WBC count, lower platelets, lower neutrophils, higher immature/total neutrophil ratio (I/T) and male gender $(\mathrm{p} 0.05)$. With logistic regression analysis, variables related to adverse outcome with $\mathrm{p} 0,1$.

Conclusion: Despite preventive measures, GBS remains a leading cause of sepsis and meningitis in newborns. GBS continues to cause mortality and frequent neurological sequelae. GBS sepsis seems to be more severe in male patients, in those with lower fever, lower WBC, lower neutrophil and lower platelet count and in those with higher immature neutrophil count.

\section{2}

Infectious Diseases

Is Vertical Transmission of Chlamydia trachomatis a Health Problem in Spain?

Marta Dorado Criado ${ }^{I}$, Celia Fabra Garrido ${ }^{1}$, Elena Merino San Martín $^{2}$, Carolina González Arboleya ${ }^{2}$, Patricia González Donapetry, Bartolomé Gómez Arroyo ${ }^{3}$, Fernando Baquero Artigao ${ }^{4}$, María De la Calle Fernández-Miranda ${ }^{2}$, María Inmaculada Quiles Melero ${ }^{3}$, Cristina Calvo Rey ${ }^{4}$ 
${ }^{1}$ Pediatrics, Hospital Universitario La Paz, Spain

${ }^{2}$ Ginecology, Hospital Universitario La Paz, Spain

${ }^{3}$ Microbiology, Hospital Universitario La Paz, Spain

${ }^{4}$ Pediatric Infectious Diseases, Hospital Universitario La Paz, Spain

Background: C. trachomatis infection is the most prevalent sexually transmitted disease (STD) in Europe. Maternal infection increases the risk of premature rupture of membranes and low birthweight and could be transmitted to the newborn through the birth canal, eventually causing nasopharyngitis, conjunctivitis and pneumonia.

Objective: The main objectives were to analyse the prevalence of C.trachomatis infection in young women and the rate of transmission to the newborn. Secondary objective was to evaluate the need for screening programs for C. trachomatis in this population.

Methods: We conducted a prospective and interventional pilot study of 71 pregnant women aged 15-25 years. Polymerase chain reaction (PCR multiplex BDmax) for STD (Chlamydia, Neisseria gonorrhoeae, Trichomonas) was performed in a urine sample during the postpartumperiod.

Newborns from C. trachomatis infected mothers were tested for Chlamydia using PCR in nasopharyngeal aspirate and urine samples. Mothers were interrogated about Chlamydia symptoms in the newborns. Results: Median age was 23 years (IQR 17-23) and $70 \%$ were immigrants, most of them $(60 \%)$ from Central and South America. The prevalence of Chlamydia infection was $19.7 \%$ (14 cases, $70 \%$ from immigrant mothers). We also detected $2 N$. gonorrhoeae and 3 Trichomonas infections. Two infected mothers presented premature rupture of membranes (at 36 weeks gestation) but no newborn had low birthweight. Two children born to infected women (14\%) were positive for Chlamydia. One infant had positive PCR in both urine and nasopharyngeal aspirate, and developed conjunctivitis. The other one had a positive PCR in urine and developed no symptoms. Both mothers and infected infants were treated with azithromycin.

Conclusions: We have found a high prevalence of Chlamydia infection in young pregnant woman, with a $14 \%$ rate of perinatal transmission. Our results support the need for screening programs in this target population.

\section{3}

\section{Infectious Diseases}

The Greatest Impact on Parents Opinion about Children Vaccination is made by Health Care Specialists

Agne Navickaite $^{1}$, Vitalija Mesceriakova ${ }^{1}$, Paulius Kalibatas ${ }^{2}$, Ginreta Valinciute $^{3}$, Stefano del Torso ${ }^{5,9}$, Diego van Esso ${ }^{6}$, Zachi Grossman ${ }^{7}$, Adamos Hadjipanayis ${ }^{8,9}$, Arunas Valiulis ${ }^{2,3,4,9}$, Algirdas Valiulis ${ }^{10}$

${ }^{1}$ Medical Faculty, Vilnius University, Lithuania

${ }^{2}$ Medical Faculty Institute of Clinical Medicine, Vilnius University, Lithuania

${ }^{3}$ Medical Faculty Institute of Health Sciences, Vilnius University, Lithuania

${ }^{4}$ Clinic of Children's Diseases, Vilnius City Clinical Hospital, Lithuania

${ }^{5}$ Pediatra di Famiglia, Pediatra di Famiglia, Italy

${ }^{6}$ Primary Care Service Muntanya, Catalan Institute of Health, Spain

${ }^{7}$ Maccabi Health Services, Maccabi Health Services, Israel

${ }^{8}$ Department of Paediatrics, Larnaca General Hospital, Cyprus

${ }^{9}$ European Academy of Paediatrics, European Academy of Paediatrics, Belgium

${ }^{10}$ Medical Faculty, Institute of Clinical Medicine, Department of Rehabilitation, Physical and Sports Medicine, Vilnius University, Lithuania

Background: Global vaccination is one of the greatest achievements in medicine, which resulted in the decrease of life-threatening illnesses. However, over the past decade, the number of people who doubt the benefits of vaccination has increased.
Objective: To evaluate the opinion about vaccines of parents raising preschool children and how different sources of information shape that opinion. Methods: A questionnaire survey was conducted in Vilnius kindergartens in the period from December 2017 to May 2018. 329 parents of children aged from 1 to 4 participated in the survey. The respondents were compared by gender, education level and primary care provider (paediatrician $v s$ family doctor). The data are processed by SPSS 22.0 program, the difference between the compared groups is considered significant when $\mathrm{p} \leq 0.05$.

Results: Parents who at least once have visited homeopath value vaccines benefit 6.33 out of 10 points. However, parents who did not apply to homeopaths tend to evaluate the benefits of the vaccine more favorably ( 8.25 out of 10 points, $p \leq 0.05$ ).

Majority of respondents have received negative information about vaccination. 229 parents at least once have found negative information on the internet, but tend to rate benefits of vaccines quite well: 8.24 out of 10 points. Furthermore, 17.3 perc. respondents, who at least once received negative information about vaccination from health care specialists, tend to evaluate vaccinations worse: 6.40 out of 10 points. More than 20 perc. of parents are reported at least one episode of negative information about vaccines received during visite of health care specialist.

Conclusions: The greatest impact on parents' vaccines related negativism is done by healthcare specialists, but not internet or other mass media. Future research is needed due to necessity of deeper analysis of primary sources and ways of spreading of vaccines related information in the families of preschool children.

\section{4}

\section{Infectious Diseases}

Prevalence of Hepatitis B Infection in Children of Eastern Nile (Khartoum State)

Amin Alagib Mohamed Musa ${ }^{1}$, Altayeb Abdulmonem ${ }^{2}$, Homeida Mamoun $^{I}$, A.S.Ali Shymaa ${ }^{I}$, A.A.Ibnouf Sondos ${ }^{I}$

${ }^{1}$ Paediatrics, University of Medical Sciences and Technology, Sudan

${ }^{2}$ Department of Surgery, Ibn Sina Hospital Ministry of Health, Khartoum, Sudan

Background: The global prevalence of Hepatitis B is important with2 billion people exposed. The younger the person infected the more serious are the outcomes in term of complications. The World Health Organization estimated that 100 million persons have chronic hepatitis B in Africa.

Screening for Hepatitis B was introduced in Sudan in 2002 as part of the extended program of Immunization. In Sudan, Hepatitis B positivity ranged from $6.8 \%$ to $26.0 \%$.

Methods: A screening for hepatitis B positivity was carried on a sample of 894 school -children (839 from Darfur and 55 from North State). The variables collected were age, area of residence, history of exposure to Hepatitis B, family past and parent history of exposure to risk factors classified as low, average and high. The data collected were summarized in term of frequency tables to estimate prevalence and chi-square tests were used to determine association between variables.

All statistical tests were considered significant when $\mathrm{p} 0.05$.

Results: The overall prevalence was $4.1 \%$ (37/894). Across the age groups the prevalence was higher $(4.7 \%, 30 / 639)$ in those aged 10-14 years with no statistically significant difference between age group $(\mathrm{p}=0.381)$. No positive case was recorded in outside Darfur $(0 / 55)$ whereas in Darfur the positivity was $4.6 \%$ (37/802). No statistically significant association was found between Hepatitis B positivity with past history ( $\mathrm{p}=0.196)$, family $(p=0.117)$ and parent's $(p=0.539)$ history and level of exposure to risk $(\mathrm{p}=0.09)$. 
Conclusions: Our findings revealed a prevalence of $4.1 \%$ which was out of the ranged of the national prevalence of 6.5\%-26.0\%; this difference may be due to the impact of the extended program of immunization.

\section{1}

\section{Infectious Diseases}

Bacteriophage Therapy in Pediatrics: Pros \& Cons

Karaman Pagava ${ }^{I}$

${ }^{T}$ Child \& Adolescent Medicine, Tbilisi State Medical University, Georgia

Introduction: The emergence of antimicrobial resistance and increase in side effects of antibiotic therapy has become a critical problem in modern medicine. As a rule the new antibiotics are more toxic, corresponding damages can have cumulative nature. The above-mentioned points to the necessity of optimization of the stewardship of antibiotic therapy, especially in children. Bacteriophage therapy is considered as an effective tool to improve the treatment of bacterial infections and minimalize its adverse consequences. Pediatricians' knowledge in this area is not sufficient.

The goal of the study was to conduct a survey of the literature regarding bacteriophage therapy (BT), define its Pros and Cons.

Materials: The published literature of last twenty years was analyzed. Our own experience, exceeding more than 30 years was taken into consideration as well. Results: Pros - the bacteriophage therapy does not cause toxicity, allergy, dysbacteriosis, it is safe and can be used even in premature babies and pregnant women, combined with all kind of medicines, it has no contraindications.

It is claimed that bacteriophages can be used effectively instead or with the antibiotics in the treatment of different bacterial infections (sepsis, gastroenteritis, dermatitis, osteomyelitis, pneumonia, urinary tract infections) caused by following agents - Proteus, Klebsiella, Escherichia, Shigella, Pseudomonas, Salmonella, Streptococcus, Staphylococcus. Bacteriophages may have some immunomodulation abilities too.

Cons - The paucity of appropriately conducted placebo-controlled clinical trials,

Regulatory conundrum of BT.

BT (at least when bacteriophages are given PO) can induce production of antibacteriophage antibodies, which might decrease the efficacy of BT; In children, particularty in infants their production is less manifested.

Conclusions: BT is efficient and safe, but further investigations are needed.

\section{3}

\section{Infectious Diseases}

Coronary Arterial Lesions and Intravenous Immunoglobulin Resistance in Kawasaki Disease: A Case-Control Study

Virginia Santana Rojo ${ }^{1}$, Mariano Silva Hernández, ${ }^{1}$ Pablo Fernández. Fraga', Marta Dorado Criado $^{1}$, Celia Fabra Garrido ${ }^{1}$, Serafín Castellano Dámaso ${ }^{1}$, José David Andrade Guerrero ${ }^{I}$, Marta Bautista

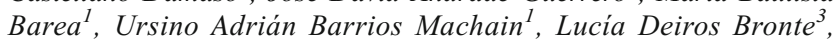
Ángela Uceda Galiano ${ }^{3}$, Antonio J. Cartón Sánchez, ${ }^{3}$, Carlos Labrandero de Lera ${ }^{3}$, Luis García-Guereta Silva ${ }^{3}$, José Antonio Ruiz. Domínguez ${ }^{4}$, Marta Bueno Barriocanal ${ }^{4}$, Miguel Ángel Molina Gutiérrez $^{4}$, Monserrat Bret Zurita ${ }^{5}$, Ana Gómez Zamora ${ }^{6}$, Miguel del Río Garciá, Sara Murias Loza ${ }^{7}$, Luis Alfonso Alonso Garcia ${ }^{6}$, Luis Escosa Garcia ${ }^{2}$, Fernando Baquero Artigao ${ }^{2}$, Talía Sainz Costa ${ }^{2}$, Cristina Ots Ruiz ${ }^{2}$, Cristina Calvo Rey ${ }^{2}$, Ana Méndez Echevarría ${ }^{2}$, Teresa del Rosal Rabes ${ }^{2}$, Francisco Javier Aracil Santos ${ }^{2}$

${ }^{1}$ Department of Paediatrics, Hospital Universitario La Paz, Spain

${ }^{2}$ Department of General Paediatrics and Paediatric Infectious Diseases, Hospital Universitario La Paz, Spain

${ }^{3}$ Department of Paediatric Cardiology, Hospital Universitario La Paz, Spain
${ }^{4}$ Paediatric Emergency Department, Hospital Universitario La Paz, Spain ${ }^{5}$ Department of Paediatric Radiology, Hospital Universitario La Paz, Spain ${ }^{6}$ Paediatric Intensive Care Unit, Hospital Universitario La Paz, Spain

${ }^{7}$ Department of Paediatric Reumatology, Hospital Universitario La Paz, Spain

Background: The most important complication related to Kawasaki Disease (KD) are coronary arterial lesions, mainly aneurisms. Although several drugs have been used for this disease, the only treatment that decreases coronary lesions is intravenous immunoglobulin (IVIG). However, fever persists or reappears in $20 \%$ of the patients with KD treated with IVIG, which is called IGIV resistance. IGIV resistance has been linked to an increased risk of coronary damage.

Objective: The aim of this study was to compare the frequency and the severity of coronary arterial lesions among resistant and responsive KD patients treated with IVIG in a Spanish tertiary hospital.

Methods: We performed a case-control study. It included $38 \mathrm{KD}$ patients who were resistant to IVIG (cases) in a period of 16 years. We chose 2 IVIG responsive KD patients (controls) per each case, the immediately previous and after ones.

Results: The incidence of resistance to IVIG in our KD patients was $18 \%$.

Coronary damage was present in $50 \%$ of our resistant patients, whilst its incidence in responsive patients was $13 \%$. Furthermore, resistant patients suffered severe coronary arterial lesions more frequently compared to responsive patients ( $11 \%$ vs $1 \%)$. Not only severe lesions were more usual, but also the mild ones ( $21 \%$ vs $7 \%$ ).

The extension of injury was higher in resistant patients. $24 \%$ of the unresponsive patients showed 3 or more vessels affected, while none of the responsive patients had such extensive damage.

Moreover, all the coronary arterial lesions were transient in patients with good response, but in $16 \%$ of the unresponsive patients, lesions were permanent.

Conclusion: Resistance to IVIG is quite common in our KD patients. It is related to higher risk, severity, extension and duration of coronary damage. Thus, prediction, early detection and close follow-up are necessary to adequate the treatment and improve the prognosis of these patients.

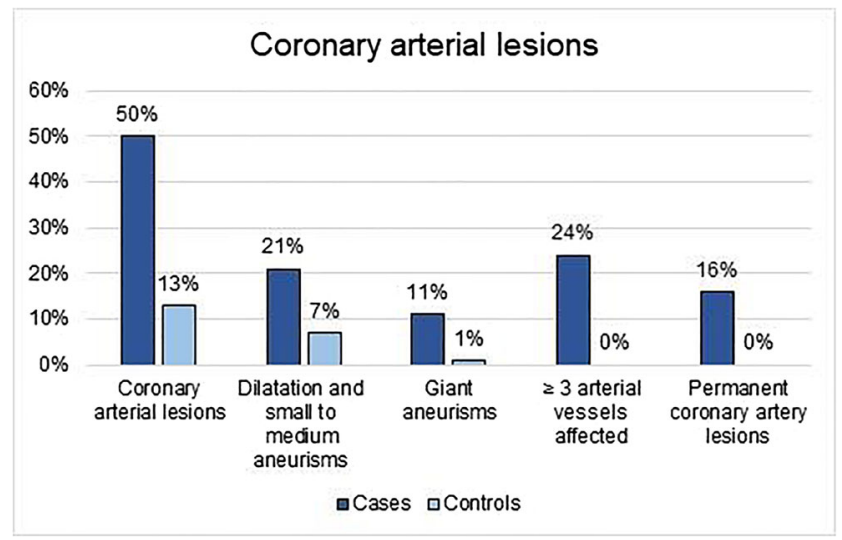

850

Infectious Diseases

EBV or ZVZ... Which one to blame? A case of ADEM

Mariana Simoes $^{l}$, Gonçalo Vale ${ }^{l}$, Maria São Pedro ${ }^{1}$, Rita Matos Parreira', Susana Correial, Elisabete Gonçalves $^{I}$

${ }^{1}$ Pediatrics, Centro Hospitalar Barreiro-Montijo, Portugal

Background: Acute Disseminated Encephalomyielitis (ADEM) is an immune-mediated demyelinating disorder which can occur 
following viral infections or vaccination and spontaneously. Several viruses can be implicated, including Epstein-Barr Virus (EBV) and Varicella Zoster Virus (VZV). Fever, headache, and meningeal irritation signs are the most common initial findings followed by seizures, focal neurological deficits and altered consciousness. Diagnosis is clinical and radiologic, being Magnetic Ressonance Imaging (MRI) the most important imaging exam. With appropriate treatment, prognosis is generally good.

Objective: To alert to less common etiologies and manifestations of ADEM

Methods: Report a clinical case of a child admitted to a suburban hospital in Portugal.

Clinical Report: 4-year old boy, admitted in the Pediatric Emergency Room (ER) with depression of consciousness and conjugate eye deviation to the right. In the previous 10 days, the family reported headache, frequent vomiting and fluctuant prostration, without fever. He also had recent history of chickenpox (20 days before admission). Blood analysis were unrevealing. Examination of cerebrospinal fluid (CSF) revealed pleocitosis $(54$ cells $/ \mathrm{mm} 3)$ with slight predominance of polymorphonuclear cells. He was hospitalized for investigation and started intravenous acyclovir. Cranioencephalic (CE) Computerized Tomography (CT) showed no alterations. Electroencephalogram documented diffuse slow-wave activity and CE-MRI revealed thalamic and subcortical lesions suggestive of ADEM. CSF microbiological culture was negative and multiplex Polimerase Chain Reaction for neurotropic virus was positive for EBV. Acyclovir was suspended at the $8^{\text {th }}$ day of hospitalization. He remained asymptomatic since the $6^{\text {th }}$ day after admission and was discharged after stopping acyclovir. He was posteriorly readmitted for immunoglobulin administration.

Conclusion: ADEM can be difficult to diagnose. Frequently, the etiologic agent remains unknown. In this case, EBV might be considered as an opportunistic agent in the setting of a transient immunosuppression state. We also highlight EBV as an important causative agent of ADEM.

651

\section{Neonatology}

Effect of High-Resolution Computed Tomograhy (HRCT) Results in Prognostic Evaluation of Bronchopulmonary Dysplasia (BPD) Cases Abdullah Baris Akcan ${ }^{1}$, Seyhan Erisir Oygucu ${ }^{2}$, Ahmet Gokhan Arslan ${ }^{3}$, Deniz Ozel ${ }^{4}$, Nihal Oygur 5

${ }^{I}$ Department of Pediatrics, Division of Neonatology, School of Medicine, Aydin Adnan Menderes University, Turkey

${ }^{2}$ Department of Pediatrics, The Kyrenia University, Dr Suat Günsel Hospital, Cyprus

${ }^{3}$ Department of Radiology, Akdeniz University, Turkey

${ }^{4}$ Department of Biostatistics and Medical Informatics, Akdeniz University, Turkey

${ }^{5}$ Department of Pediatrics, Division of Neonatology, School of Medicine, Akdeniz University, Turkey

Objectives: BPD carries a risk of long term pulmonary sequale with changes in pulmonary mechanics and parenchyma. HRCT is a method of detecting such structural changes. Our aim was to characterize these structural abnormalities associated with BPD and to evaluate whether the severity of HRCT changes was associated with clinical findings.

Methods: 28 patients born with a mean gestation age of $30 \pm 2.9$ weeks and diagnosed as BPD in their neonatal period were reevaluated when they were between the post natal ages of 6-12 months. They were divided into three groups as mild (n:8), moderate (n: 10), and severe (n: 10) according to their clinical data. HRCT scans were performed in patients with a history of moderate and severe BPD. One radiologist analysed scans using a structured scoring system.

Results: Their ages were between $9.8 \pm 2.3$ months at time of reevaluation. The average HRCT total score of patients was respectively moderate $7.20 \pm 4.05 ; 7.40 \pm 2.84$ with severe BPD.The differerence between them was not significant $(\mathrm{p}=$ 0.620).Data obtained during the neonatal period, there was no significant correlation between the duration of oxygenation, the duration of mechanical ventilation, birth weights and the total HRCT scores performed at the time of reevaluation, in patients with moderate and severe BPD $(\mathrm{p}=0.408, \mathrm{r}=0.183$ for birth weights).

Conclusions: Those data suggest that the pathology detected in radiological imaging may not always be parallel to clinical findings and that the clinical course of infants with severe radiological data may be better or worse than expected. However, we believe more studies with larger number of patients and with different scoring systems are necessary in order to evaluate the role of HRCT in the clinical evaluation and long term prognosis of BPD.
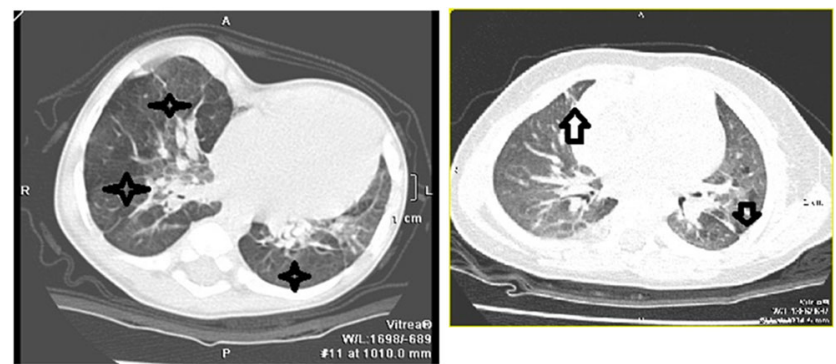

\begin{tabular}{|c|c|c|c|c|}
\hline Category & Variable & 0 & 1 & 2 \\
\hline \multirow{3}{*}{ Hyperexpansion } & Hyperexpansion & Not & Focal & Global \\
\hline & Mosaic pattem & Not & Vaguely & Certain \\
\hline & Intercostal bulge & Not & Vaguely & Certain \\
\hline \multirow{2}{*}{ Emphysema } & Number of Bullae & Not & Single & Plural \\
\hline & Size of Bullae & Not & $\leq 5 \mathrm{~mm}$ & $>5 \mathrm{~mm}$ \\
\hline \multirow{3}{*}{ Fibrosis } & $\begin{array}{c}\text { Triangular subpleural } \\
\text { opacities }\end{array}$ & Not & 1-3lobe & 4-6 lobe \\
\hline & $\begin{array}{l}\text { Deformity and } \\
\text { thickening of the } \\
\text { bronchovascular } \\
\text { structure }\end{array}$ & Mild & Moderate & Heavy \\
\hline & Consolidation & Not & Vaguely & Certain \\
\hline SubjectiveEvaluation & & Mild & Moderate & Heavy \\
\hline
\end{tabular}


Table 2. General characteristics of the cases in the neonatal period

\begin{tabular}{|c|c|}
\hline Features & Cases (n:28) \\
\hline Gender (Male Female) & $13 / 15$ \\
\hline Place of Birth (AUFM Outer Centers) & $15 / 13$ \\
\hline Gestational age ${ }^{*}$ & $30=2.9$ \\
\hline Birth weight** & $1372 \pm 784$ \\
\hline Mechanical ventilation time ${ }^{8}$ & $22.9=16$ \\
\hline CPAP duration" & $15.3=4.2$ \\
\hline Hood oxygen uptake period & $12.0=4.2$ \\
\hline Total oxygen support duration ${ }^{8}$ & $50=16.7$ \\
\hline $\begin{array}{l}\text { Mechanical ventilation duration of moderate and } \\
\text { severe } \mathrm{BPD}^{s}\end{array}$ & $26.6=17.3$ \\
\hline $\begin{array}{l}\text { CPAPduration of cases with moderate and severe } \\
\qquad \text { BPD }^{s}\end{array}$ & $16.7=3.5$ \\
\hline $\begin{array}{l}\text { Hood duration of cases with moderate and severe } \\
\qquad \text { BPD }^{s}\end{array}$ & $11.7 \pm 3.9$ \\
\hline $\begin{array}{l}\text { Total oxygen suppon duration of cases with } \\
\text { moderate and severe BPD }\end{array}$ & $54.9 \pm 17.4$ \\
\hline
\end{tabular}

* Average $\pm \mathrm{SD}$ (week), ${ }^{* *}$ Average $\pm \mathrm{SD}$ (gram), ${ }^{5}$ Day

139

Neonatology

Acute Reduction in Neonatal Brain Injury by Selective Blockage of CB1 and Activation of CB2 Receptors

Daniel Alonso-Alconada ${ }^{1}$, Silvia Carloni ${ }^{2}$, Andrea Duranti ${ }^{2}$, Ainara Pintor-Rial ${ }^{1}$, Antonia Alvarez ${ }^{1}$, Enrique Hilario ${ }^{1}$, Walter Balduini ${ }^{2}$

${ }^{1}$ Department of Cell Biology and Histology, University of the Basque Country, Spain

${ }^{2}$ Department of Biomolecular Sciences, University of Urbino "Carlo Bo", Italy

Background: Cannabinoids ( $\mathrm{CBs}$ ) can modulate a wide range of neurological and physiological processes through the activation of their receptors, $\mathrm{CB} 1$ and $\mathrm{CB} 2$. $\mathrm{CB}$ receptor agonists and antagonists have demonstrated to produce either protective or toxic responses after brain ischemia. URB447, a CB1 antagonist and CB2 agonist, can selectively block $\mathrm{CB} 1$ and activate $\mathrm{CB} 2$ receptor signaling.

Objective: Using an in vivo model of neonatal brain injury, to examine the potential therapeutic effect of URB447, given before or after a hypoxicischemic (HI) event.

Methods: 7-day-old rat pups received a single intraperitoneal injection of URB447, i) 1 hour before starting the ischemic procedure (HI+URB447 1h-PRE), ii) 30 minutes (HI+URB447 30min-POST) or iii) 3 hours (HI+ URB447 3h-POST) after hypoxia. To test if the protective effect of URB447 was mediated by CB1/CB2 modulation, WIN-55212, a CB1/ $\mathrm{CB} 2$ receptor agonist, was injected i.p. $1 \mathrm{~h}$ and 30 minutes before $\mathrm{HI}$ (HI+ WIN-55212) or 30 minutes before URB447 administration (HI+ URB447+WIN-55212). Each HI-injured group (HI) received a corresponding volume of vehicle. $\mathrm{N}=10$ for all groups. The neuroprotective effect of drugs was assessed using histological and molecular techniques in 14 day-old animals.

Results: HI-induced reduction in whole hemisphere $(46.5 \%)$ and cerebral cortex $(65.7 \%)$ areas was avoided after URB447 administration, showing a decrease in brain infarction both before (whole hemisphere: $83.7 \%$; cerebral cortex: $92.7 \%$ ) and $30 \mathrm{~min}$ after (whole hemisphere: $95.7 \%$; cerebral cortex: $95.4 \%$ ) the injury (Fig.1). WIN-55212 completely blocked the neuroprotective effect of URB447 (Fig.2). Of clinical relevance, URB447 injected 3 hours after $\mathrm{HI}$ reduced brain infarction $95.7 \%$ and $88 \%$ in whole hemisphere and cerebral cortex, respectively (Fig.1). Furthermore, delayed cell death, astrogliosis and white matter damage returned to those values observed in sham animals.

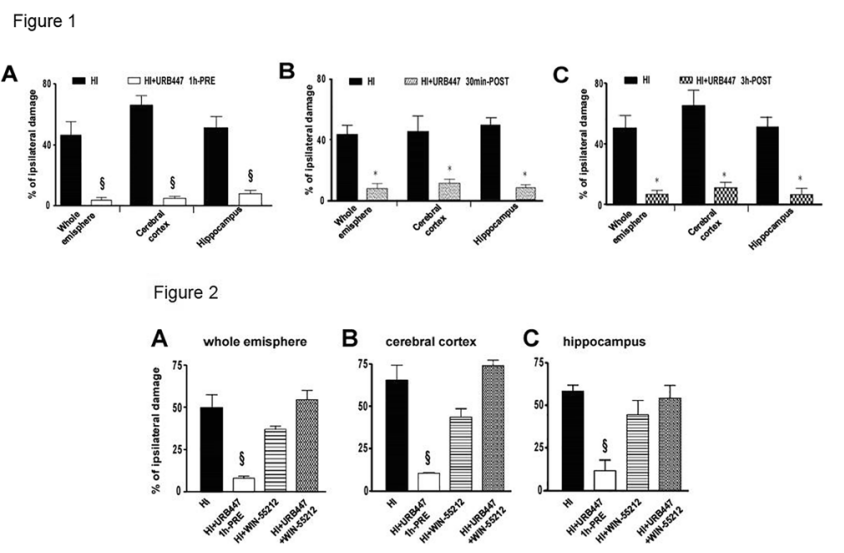

Conclusion: Selective blockage of $\mathrm{CB} 1$ and activation of $\mathrm{CB} 2$ receptors induce an acute reduction in brain injury in the neonatal rat. Grants: UPV/ EHU (GAITUZ2019, GIU17/018).

810

Neonatology

Inhaled Nitric Oxide for Pulmonary Hypertension and Bronchopulmonary Dysplasia in Extreme Prematurity; Benefits with Targeted Use

Deepa Hariharan ${ }^{1}$, Ganesh Veluswami ${ }^{1}$, Velmurugan Kannappan ${ }^{1}$, Pangaluru Sarathkala ${ }^{I}$

${ }^{I}$ Neonatology, Sooriya Hospital, India

Background: Preterm infants are at risk for early pulmonary hypertension $(\mathrm{PH})$ that is associated with high risk of bronchopulmonary dysplasia (BPD) or death. While inhaled nitric oxide (iNO), is an effective treatment for $\mathrm{PH}$ in term infants, there is insufficient evidence for BPD prevention; however, the preterm infants were not screened for early $\mathrm{PH}$ and iNO was not targeted for PH in these studies. We hypothesized that early detection of $\mathrm{PH}$ and targeted iNO therapy will improve respiratory outcomes in extreme prematurity.

Aim/ methods: 164 infants less than 30 weeks gestation who needed ventilation during the 18 month study period were enrolled. On echocardiographic $(\mathrm{ECHO})$ diagnosis by peaditaric cardiologist, 46 infants were detected to have $\mathrm{PH}$ at 72 - 96 hours of age. They were randomized to receive iNO at $5 \mathrm{ppm}$ (study group, $\mathrm{n}=23$ ) or placebo (control group, $\mathrm{n}=23$ ) till 14 days of age or resolution of $\mathrm{PH}$, whichever was earlier. $\mathrm{ECHO}$ was done every 48 hours in both groups. The demographic profile, ventilator and surfactant use, PDA closure, nutrition and inotrope use were similar in the 2 groups. The 2 groups were analyzed for oxygenation index (OI) at 7 days, incidence of BPD and death.

Results: The mean OI on day 7 in the study and control groups were 9.7 and 14.1 respectively (p0.05). Duration of ventilation in the study and control groups were 13.6 days and 19.6 days respectively (p0.05). 4 infants in the study group, and 8 in the control group had BPD (p0.05). There was 1 death in each group.

Conclusion: Early detection of $\mathrm{PH}$ and targeted iNO therapy improves oxygenation and reduces BPD in extreme prematurity. 
455

\section{Neonatology}

Exploring Nurses' Perspective about Helping Babies Survive Programme in Western India - Qualitative Findings

Naveen Thacker ${ }^{1}$, Robert Clark', William Keenan ${ }^{3}$, Deep Thacker ${ }^{1}$, Mayur Shinde ${ }^{4}$, Richard Bell ${ }^{5}$, Kandarp Talati ${ }^{6}$, Somashekhar Nimbalkar ${ }^{4}$

${ }^{1}$ Pediatrics, Deep Children Hospital, India

${ }^{2}$ Pediatrics, Brigham Young University, USA

${ }^{3}$ Pediatrics, Saint Louis University, USA

${ }^{4}$ Pediatrics, Pramukhswami Medical College, India

${ }^{5}$ Pediatrics, NorthBay Medical Center, USA

${ }^{6}$ Department of Interdisciplinary Research, Foundation for Diffusion of Innovations, India

Background: Helping Babies Survive (HBS) program was piloted in five districts of India during 2016-17. Landscape analysis evaluated changes between HBS and non-HBS healthcare facilities to understand how the program had influenced newborn care practitioners and practices.

Objective: To understand nurses' perception towards usefulness of HBS trainings and experiential feedback for further strengthening HBS implementation and scale-up

Methods: Interview guideline was developed and perception explored through in-depth interviews. Total 14 nurses were interviewed (9 HBS trained, 5 untrained). Manual content analysis was done to report the findings under pre-defined thematic domains - Delivery and Newborn Care Practices; and HBS Skill Station/Practice Sessions/Continuous Quality Improvement(CQI).

Results: Nurses perceived that HBS training, CQI initiatives, visits by mentors and program officers(PO) helped in skill improvement. It helped improve birth preparedness, patient communication during labor, delivery per abdomen, skin to skin care(SSC) and early initiation of breastfeeding. HBS training also improved self-confidence to administer initial care, including resuscitation, to babies not crying/unstable who were earlier referred out without any interventions. Nurses learnt that not all babies require bag \& mask or ventilation, and many a times baby responds to rubbing or stimulation.

Nurses were able to practice at designated HBS skill stations within their facilities, however organizing peer practice sessions were challenging. Shortage of staff, shift duty, staff attrition/rotation and workload were cited as primary reasons for inability to regularly practice. Visits by HBS POs were catalytic in facilitating peer practice sessions, discussing real life challenges and having shared insights and hence are of paramount importance to ensure CQI.

Conclusion: Nrses perceived that HBS training and mentoring visits helped deliver essential newborn care even at remote facilities. Further implementation and scale-up of HBS program should emphasize facility visits by HBS POs/mentors on monthly basis and strategies to address known facility-level barriers to CQI.

\section{6}

\section{Neonatology}

Introducing a Lactation Consultant into a NICU Service Improves Early Access to Breast Milk

Margaret Broom ${ }^{1,2}$, Judith Smith ${ }^{1}$, Mary Ellen Youseman ${ }^{1}$, Alison Kent ${ }^{3,4}$ ${ }^{1}$ Dept of Neonatology, Centenary Hospital for Women and Children, Canberra Hospital, Australia

${ }^{2}$ University of Canberra and ACT Health, SYNERGY: Nursing and Midwifery Research Centre, Australia

${ }^{3}$ Division of Neonatology, University of Rochester, Golisano Children's Hospital at URMC, USA

${ }^{4}$ Medical School, Australian National University, Australia
Background: Providing breast milk for extremely premature and sick neonates has documented benefits. Lactation consultants (LC) have become important additions to neonatal intensive care unit teams to improve provision of maternal breast milk, particularly in the early hours after birth.

Aim: To assess the impact of the introduction of a permanent LC on: 1) provision of breast milk for neonates; and 2) education and lactation support for mothers and staff.

Methods: A mixed method study including a retrospective chart audit and a pre and post LC staff survey. Information collected included: first expressed breast milk, episodes of lactation education and support. Survey questions provided staff feedback regarding: education access to support and meeting maternal needs. Data was analysed using descriptive statistics and Chi-square tests.

Results: 161 charts were included ( 82 pre LC and 79 post LC). Post LC service there was a significant increase in maternal access to LC appointments (12/82 vs 63/79; $\mathrm{p} 0.01)$ as well as breast pump education $(53 / 82 \mathrm{vs}$ $64 / 79 ; \mathrm{p} 0.01)$. There was an increase in EBM provision at 12 hours postnatally ( $38 / 82$ vs $48 / 79 ;$ p 0.01 ). Staff surveys ( 36 pre LC and 56 post LC) showed there was a significant increase in staff confidence in providing $\mathrm{BF}$ education to mothers $(25 \%$ vs $46 \%$; $\mathrm{p}=0.013)$ and improved access to LC appointments (5\% vs $89 \%$; p0.01)

Conclusions: LC services can significantly improve the provision of breast milk within the first 12 hours of life for very preterm neonates, and improve maternal access to education and support. Further research is required to assess the effect of LC's on improving long term breast feeding rates.

813

Neonatology

Is Early Oxidative Stress the Common Origin of Both Bronchopulmonary Dysplasia and Impaired Neurodevelopment in Preterm Infants Less Than 29 Weeks of Gestation?

Ibrahim Mohamed, Jean-Claude Lavoie, Francine Lefebvre

${ }^{T}$ Pediatrics/Neonatology and Nutrition, University of Montreal, Canada

Background: Preterm infants are exposed to many oxidants early in life. They have limited antioxidant capacity. Early oxidative stress is involved in bronchopulmonary dysplasia (BPD) development. Studies confirme the association between BPD and impaired neurodevelopmental outcome.

Hypothesis/Objectives: We hypothesis that early oxidative stress has multiorgan negative effects on the lung and the neuro-development. Specific objectives: To examine the effect of early oxidants on the oxidative stress at 36 weeks gestational age (GA) and to test its relationship with both BPD and neurodevelopment. Design and methods: A prospective cohort study included 51 infants 2 $\geq 25 \%$ on day 7 of life and parenteral nutrition (PN) duration 14 days. tstudent test, $\mathrm{Chi}^{2}$ and logistic regression were used as appropriate. Baseline characteristics, BPD and Bayley-III at 18 months corrected age were collected. $\mathrm{P}$

Results: The effect of early oxidants on oxidative stress markers at 36 weeks PMA is shown in Table 1.

Table 1: The effect of maximal $\mathrm{O}_{2}$ on day 7 of life and the duration of PN on GSH, GSSG and the redox potential at 36 weeks PMA.

\begin{tabular}{|c|c|c|c|c|c|c|}
\hline & $\begin{array}{c}\mathrm{FiO}_{2}<25 \% \%^{*} \\
(\mathrm{n}=20)\end{array}$ & $\begin{array}{c}\mathrm{FiO}_{2} \geq 25 \% \\
(\mathrm{n}=19)\end{array}$ & $\mathbf{P}$ & $\begin{array}{c}P N \leq 14 \text { days } \\
(\mathrm{n}=6)\end{array}$ & $\begin{array}{c}\text { PN > 14 days } \\
(\mathrm{n}=33)\end{array}$ & $\mathbf{P}$ \\
\hline GSH $^{\mathbf{a}}$ & $7.6 \pm 0.5$ & $7.4 \pm 0.6$ & 0.79 & $7.5 \pm 1.2$ & $7.5 \pm 0.4$ & 0.99 \\
\hline GSSG $^{\mathbf{a}}$ & $0.18 \pm 0.02$ & $0.29 \pm 0.04$ & 0.03 & $0.13 \pm 0.02$ & $0.26 \pm 0.03$ & 0.04 \\
\hline Redox potential $^{\mathrm{b}}$ & $-198 \pm 2$ & $-191 \pm 2$ & 0.02 & $-203 \pm 5$ & $-193 \pm 2$ & 0.03 \\
\hline
\end{tabular}

There was a significant association between exposure to early oxidants and BPD and impaired neurodevelopment Table 2. 
Table 2: Effects of maximal $\mathrm{O}_{2}$ on day 7 of life and the duration of PN on BPD and neurodevelopmental outcomes.

\begin{tabular}{|l|c|c|c|c|c|c|}
\hline & $\mathbf{F i O}_{2}<25 \%$ & $\mathbf{F i O}_{2} \geq \mathbf{2 5} \%$ & $\mathbf{P}$ & $\mathbf{P N \leq 1 4}$ days & $\mathbf{P N}>14$ days & P \\
\hline BPD (percentage) & $11 / 23$ & $22 / 25$ & 0.006 & $2 / 13$ & $32 / 35$ & .001 \\
& $(52 \%)$ & $(88 \%)$ & & $(15 \%)$ & $(91 \%)$ & \\
\hline Cognitive score & $96 \pm 2$ & $87 \pm 4$ & 0.04 & $96(3)$ & $86(3)$ & 0.15 \\
\hline Language score & $93 \pm 3$ & $81 \pm 3$ & 0.01 & $94(4)$ & $83(3)$ & 0.04 \\
\hline Motor score & $92 \pm 2$ & $81 \pm 3$ & 0.01 & $92(2)$ & $83(2)$ & 0.03 \\
\hline
\end{tabular}

Increased oxidative stress at 36 weeks PMA was associated with both BPD and neurosevelopmental impairment Table 3.

Table 3: The relation between BPD and neurodevelopmental outcome at 18 months and the redox potential at 36 weeks PMA.

\begin{tabular}{|c|c|c|c|}
\hline \multirow{4}{*}{$\begin{array}{c}\text { Redox potential } \\
\text { (in mV) }\end{array}$} & BPD & NO BPD & P \\
\cline { 2 - 4 } & $202.9 \pm 4.4$ & $192.8 \pm 1.7$ & 0.02 \\
\cline { 2 - 4 } & Conitive $\geq \mathbf{8 5}$ & Cognitive $<\mathbf{8 5}$ & \\
\cline { 2 - 4 } & $-197 \pm 2$ & $-193 \pm 5$ & 0.37 \\
\cline { 2 - 4 } & Language $\geq \mathbf{8 5}$ & Language $<\mathbf{8 5}$ & \\
\cline { 2 - 4 } & $-200 \pm 2$ & $-189 \pm 3$ & 0.01 \\
\cline { 2 - 4 } & Motor $\geq \mathbf{8 5}$ & Motor $<\mathbf{8 5}$ & 0.01 \\
\cline { 2 - 4 } & $-199 \pm 2$ & $189 \pm 3$ & \\
\hline
\end{tabular}

* Mean (s.e.m.);

In a logistic regression including GA, illness severity score and maternal education; the redox potential at 36 weeks PMA remained a significant independent factor for motor Bayley score $(\mathrm{P}=0.036)$ and any Bayley score Conclusion: Early exposure to oxidants results in oxidative stress that increases the risk of both BPD and neurodevelopmental impairment. We speculate that strategies to re-establish oxidant -antioxidant balance can improve both short and long-term outcomes in infants less than 29 weeks GA.

767

\section{Neonatology}

Early Postnatal Automated Spectral EEG Analyses of Premature Infants may Help Predict Neurocognitive Outcome at Adolescence Tone Nordvik ${ }^{1,2}$, Eva Margrethe Schumacher ${ }^{2}$, Pål Gunnar Larsson ${ }^{3}$, Are Hugo Pripp $^{4}$, Gro Christine Christensen Løhaugen ${ }^{5}$, Tom Arne Stiris ${ }^{1,2}$

${ }^{1}$ Institute of Clinical Medicine, Faculty of Medicine, University of Oslo, Norway ${ }^{2}$ Department of Neonatal Intensive Care, Ullevål, Oslo University Hospital, Norway

${ }^{3}$ Clinical Neurophysiological Section, Department of Neurosurgery, Oslo University Hospital, Norway

${ }^{4}$ Oslo Centre for Biostatistics and Epidemiology, Oslo University Hospital, Norway

${ }^{5}$ Department of Pediatrics, Sørlandet Hospital HF, Norway

Background: Early postnatal aEEG/EEG of premature infants

Objective: We have previously demonstrated an association between total absolute band power (tABP) during the first three days of life and developmental outcome at 24 months corrected age in premature infants born before 28 weeks gestational age. The aim of this study is to determine whether this association is still present at adolescence.

Methods: Premature infants with a gestational age 28 weeks were continuously EEG-monitored for the first three days of life. The total absolute band powers of the different frequency bands delta, theta, alpha and beta were analyzed. At 10-12 years of age neurocognitive outcome was assessed using the Wechsler Intelligence Scale for Children 4th edition (WISC), Vineland Adaptive Behavior Scales and Behavior Rating Inventory of Executive Function (BRIEF). The mean difference in $\mathrm{tABP}$ was assessed for individuals with normal versus abnormal neurocognitive scores.
Results: 22 infants were included. The infants were assessed at a mean age of 11.5 years of age. tABP in all four frequency bands were significantly lower in infants with pathological results in the main composite score (full intelligence quotient, adaptive behavior composite score and global executive composite score) of all three tests (p0.05).

Conclusion: Early postnatal EEG har the potential to assist in predicting cognitive outcome at 10-12 years of age in extremely premature infants 28 weeks gestation.

542

Neonatology

Excessive Weight Loss in the Perinatal Period: A Case-Control Study Maria Joao Palha ${ }^{1}$, Sara Limão ${ }^{2}$, Mafalda Crisóstomo ${ }^{3}$, David Lito $^{2}$, Mário Paiva ${ }^{2}$

${ }^{1}$ Pediatrics, Hospital de Santa Maria, Centro Hospitalar Universitário de Lisboa Norte, Portugal

${ }^{2}$ Pediatrics, Hospital Vila Franca de Xira, Portugal ${ }^{3}$ Pediatrics, Hospital Dona Estefânia, Centro Hospitalar de Lisboa Central, Portugal

Background: Weight loss is expected in the first days of life. There is lack of population-based data on risk factors for newborn's weight loss. Objective: Determine factors associated with weight loss higher than $10 \%$ at postpartum discharge.

Methods: Prospective case-control study conducted in a second-level hospital during a five-month period, including all the newborns with weight loss $10 \%$ at the third day of life. Gestational age less than 35 weeks, birth weight less than $1900 \mathrm{~g}$ and need for intermediate/intensive care were exclusion criteria. Controls were matched by gestational age, type of delivery and sex. Clinical and demographic variables, and occupancy levels were analyzed. For statistical analysis, we used t-student and qui-square tests ( $p$-value

Results: A total of 59 newborns were included (6.1\% of total deliveries). The majority (96.6\%) was full-term and $44.1 \%$ were delivered via cesarean. Concerning birth weight, no significant difference between groups of cases and controls was found. Mean percentage of weight loss was $11.0 \%$ in the case group. There was a higher percentage of gestational diabetes and hypertension during pregnancy in the study group, but the difference wasn't significant $(10.2$ vs $1.7 \%, p 0.051 ; 8.5$ vs $1.7 \%, p 0.094$, respectively). Parents were older in the case group (median age 33 vs 31 years for mothers and 35 vs 33 years for fathers), without significant difference ( $p 0.294$ and $p$ 0.055). We found a significant higher occupancy rate of the nursery $(66.8 \%$ vs $60.0 \%)$ in the group of cases ( $p 0.012)$. No significant difference was found regarding number of siblings, maternal active smoking, psychiatric conditions or ethnicity.

Conclusion: Occupancy rate of the nursery was the only factor with a strong association with excessive weight loss in our study, demonstrating the importance of nurse counseling of mothers during the first days of the newborn's life.

653

Neonatology

Optimal Time to Apply Combined Pulse Oximetry and Physical Examination Aiming in Detecting Severe and Critical Congenital Heart Diseases in Term Neonates

Andreas Petropoulos ${ }^{1,2}$, Ruslan Huseynov ${ }^{3}$, Nabil Seyidov ${ }^{4}$

${ }^{T}$ Cardiac ICU, Great Ormond Street Hospital, UK

${ }^{2}$ Pediatrics-Cardiology, The "Aziz Aliyev" national postgraduate and CME medical training center, Azerbaijan

${ }^{3}$ NICU, Neonatal ICU of the Ob/G Institute of Ministry of HealthNICU of Azerbaijan, Azerbaijan

${ }^{4}$ Childhood Medical Care Sector, Ministry of Health of the state of Azerbaijan, Azerbaijan 
Background: Up to date, many studies around the world have proven the use of pulse oximetry(pox) as a screening test with high specificity, moderate sensitivity and a low overall false-positive rate, for detecting critical and severe Congenital Heart Diseases (c/s-CHD). Additional to that important neonatal nosology as acute/chronic lung disease, early onset of sepsis or PPHN can be early detected. Few studies have comment on the exact timing and the amount of measurements needed to obtain the optimal results.

Aim: Prospective screening study by pox + physical assessment, aiming to detect the optimal timing amount of measurements for optimal results Population-Method: From June 2016 to August 2018, 2570 term neonates were screened in two major maternity units in Baku-Azerbaijan. Pox levels using a motion-tolerant pulse oximeter where blindly reported on the end of 1st and 3ed day of life by a team of trained nurses, using a simultaneous pre and post ductus arteriosus (pre/post DA) measurement. A second team manded by neonatologists then assessed these neonates on the same dates. Firstly, blinded to existing pox measurements and then combining them together with Physical Assessment(pox+PA). Cut-offs of pathological pox were below $90 \%$ in any measured site or persisting pre/post DA difference $3 \%$, following 2 assessments within 30 minutes apart. At day five from birth, all of them received Echo-2D screening from two examiners both double-blinded to the results of pox, pox + physician assessment and findings of previous Echo-2D.

Findings: From 2570 screened by Echo-2D, 47 CHD's were found. 17/ 47: c-CHD \& 5 s-CHD. 25/47 were simple/ moderate complex noncyanotic CHD.

Pox team; detected 14/22 (63.63\%) c-CHD at $24 \mathrm{~h}$ post-birth. This raised $18 / 22(81.81 \%)$ at $72 \mathrm{~h}$ post-birth. Also detected 65 cases $(2.2 \%)$ of additional neonatal non- cardiac pathology.

Combined team: PA only 12/22(55.5\%) \& 14/ (63.63\%). Pox+PA: $17 /$ $22(77.28 \%) \& 20 / 22(90.91 \%)$ of c/s-CHD at $24 \& 72 \mathrm{~h}$ post-birth respectable. Additional, 75 cases (3\%) neonatal non- cardiac pathology were detected.

Conclusion: Study showed that when the test when applied $72 \mathrm{~h}$ post birth had the highest positive predicted value screening c/s-CHD test. An additional measurement in the end of first $24 \mathrm{~h}$, can determine

559

\section{Neonatology}

Multiple Pregnancy: The Experience of A Level III Neonatology Unit Sara Pinto $^{I}$, Joana Caldeira Santos ${ }^{I}$, Lorena Stella ${ }^{1}$, Ana Marta Pinto ${ }^{2}$, $\overline{\text { Manuela Pinho }}{ }^{2}$, Márcia Gonçalves ${ }^{1}$, Manuela Mateus ${ }^{1}$

${ }^{1}$ Neonatology Unit of the Paediatrics/ Neonatology Service, Centro Hospitalar Vila Nova de Gaia/Espinho, Portugal

${ }^{2}$ Gynecology/Obstetrics Service, Centro Hospitalar Vila Nova de Gaia/Espinho, Portugal

Background: The incidence of multiple gestations (MG) has increased over the last decades, with a consequent increase in the risk of fetal/ neonatal morbidity/mortality.

Objective: Evaluate the approach, clinical evolution, complications and follow-up of newborns from MG.

Methods: Retrospective analysis of newborns admitted to the Perinatology Sector (PS) and Neonatal Unit (NU), resulted from MG, between January 2016 and December 2017.

Results: Ninety-eight gestations were identified, 3 triple, and 95 twin. $70.5 \%$ were bicorionic/biamniotic. $71 \%$ of the pregnancies were older than 31 years and $41 \%$ had medically assisted production techniques (APT). Of the 197 newborns, $52 \%$ were female, $73.5 \%$ premature (2.7\% extreme preterm), and the mean birth weight (BW) was $2062 \mathrm{~g}$. $4.5 \%$ presented extreme low birth weight (ELBW). 58.4\% were admitted directly in NU, $41.6 \%$ in PS. Mean gestational age (GA) of PS group was 36.8 weeks, and BW mean $2569.7 \mathrm{~g} .52 .9 \%$ had hospitalization longer than protocolated.

Median length hospitalization's in NU was 19 days, with $77.2 \%$ requiring intensive care. Mortality rate was $2.5 \%$, with $80 \%$ being extreme premature.
$83.7 \%$ had a follow-up visit, with a poor staturo-ponderal evolution in $2 \%$ and inadequate psychomotor development in $6 \%$.

Extreme prematurity was associated with mortality (p0.001), and intraperiventricular hemorrhage (IPVH) (p0.001). Need for surfactant and mechanical ventilation were correlated with low birth weight $(\mathrm{p} 0.001, \mathrm{p}=0.002)$, very low birth weight (VLW) (p0.001,p=0.027) and ELBW (p0.001,p0.001). Need for intensive care was associated with ELBW $(\mathrm{p}=0.006)$ and VLW (p0.001). Inadequate psychomotor development was associated with VLW ( $\mathrm{p}=0.036$ ), and inadequate staturo-ponderal development with intrauterine growth restriction $(\mathrm{p}=0.001)$.

Conclusion: As described in the literature, maternal age was high and there was a high percentage of APT use.

In addition to complications inherent to prematurity, we found an extension of PS hospitalization time.

BW and GA are important risk factors for neonatal IPVH, respiratory morbidity, inadequate psychomotor development and mortality.

\section{3}

\section{Neonatology}

Maternal Adipokines in Relation to Newborn Weight

Maysa Saleh ${ }^{2}$, Enas Rafaat ${ }^{2}$, Yasmine El Gendy ${ }^{1}$, Lobna Sherif ${ }^{2}$, Hanaa Hamdy $^{3}$

${ }^{1}$ Pediatrics, Ain Shams University, Egypt

${ }^{2}$ Child health, NRC, Egypt

${ }^{3}$ Hormone, NRC, Egypt

Background: Intrauterine exposure and its relation to the offspring metabolic and growth outcome is not clear .

Adipokines; leptin and adiponectin are correlated with adult and childhood obesity, yet early gestational exposure and its relation with offspring growth needs to be determined.

Objectives: To assess the relation between serum maternal and umbilical cord blood level of adipokines with the newborn weight.

Subjects and Methods: 50 pairs of mother and their newborns (N:100) were enrolled in the study. Serum maternal and umbilical cord adipokines ( leptin and adiponectin) level were determined. Maternal and their newborn anthropometric measurement were obtained by trained examiners.

Results: Lower serum maternal and cord blood level of leptin are associated with higher newborn weight compared to appropriate for gestational weight ( $\mathrm{p} 0.05$ ). Moreover increased newborn weight was associated with higher level of cord blood adiponectin ( $\mathrm{p} 0.05)$.

cord blood of leptin was significant increased in newborns whose mother were obese when compared to normal weight mothers ( $\mathrm{p} 0.05$ ).

Conclusions: Maternal adipokines level affects the newborn weight

\section{2}

Neonatology

\section{Effects of Probiotics on Controlling Neonatal Jaundice Devajit Kumar Sarma ${ }^{1}$ \\ ${ }^{T}$ Neonatology, Satribari Christian Hospital, India}

Background: Hyperbilirubinemia remains the commonest cause of hospitalisation and readmission of new-borns. It can also cause considerable morbidity and mortality if not treated timely and properly.

Objectives: The aim of this study is to assess the effect of probiotics on controlling neonatal jaundice.

Methods: 102 consecutive born new-borns, beyond 32 weeks of gestations who met inclusion criteria, were randomly assigned to either control or probiotic group. Those in probiotic group ( group I) were given once daily dose of probiotic with Bacillus clausi, 2 billion unit and exclusive breastfeeding from day 1 until day 7. The control group (group II) were only on breastfeeding. Serum bilirubin was estimated on all babies on day 2, 4 and 7 . 
Results: There were 51 infants in each group. Out of which 24 babies in the probiotics group and 27 babies in the control group were in high-risk categories for neonatal jaundice. On day 2 bilirubin on the group I was 6.5 $\mathrm{mg} / \mathrm{dl}+/-1.7$, and in group, II was $8.6 \mathrm{mg} / \mathrm{dl}+/-2.6(\mathrm{P}=0.0001)$. Similarly on day 4 it was $10.2+/-2.3$ group I and $12.1+/-3$ in group II $(\mathrm{P}=0.0005)$ and $8.6+/-2.6$ and $97+/-3.4$ on day $7(\mathrm{P}=0.983)$

An innovative contribution to research-

Probiotics are effective in controlling neonatal jaundice and subsequent need for hospitalization.

643

\section{Neonatology}

Comparision of Gradual Versus Sudden Weaning of Preterm Neonates from Nasal CPAP

Harshal Wagh ${ }^{l}$

${ }^{T}$ Pediatrics, Bharati Vidyapeeth (Deemed to be) University, Medical College \& Hospital, Sangli, India

Background: Advantages and disadvantages of CPAP are well known but ideal strategy of weaning from CPAP is still unknown. Removing neonate from CPAP may lead to increased $\mathrm{O}_{2}$ requirement, increased work of breathing, need to restart CPAP. Hence safe weaning methods are required to prevent neonatal mortality and morbidity.

Objective: To compare gradual versus sudden weaning of preterm neonates from nasal CPAP.

Methods: Preterm neonates (37weeks, $2.5 \mathrm{~kg}$ ) on CPAP were included; those with major malformations \& air leaks were excluded. Neonates ready to be weaned were randomized. If the neonate was in 'sudden' weaning group, the baby was directly kept off CPAP for next 72 hours. If the neonate was in 'gradual' weaning group, the neonate was kept off CPAP for predetermined hours each day. All babies were closely monitored. If the neonates developed distress while weaning, they were put back on CPAP. Results: Out of 91 neonates, $46(50.5 \%)$ were weaned by sudden weaning, while 45 babies (49.5\%) were weaned gradually. Among the 77 babies $(84.6 \%)$ who succeeded weaning, $44(48.3 \%)$ were from the gradual weaning group as compared to $33(36.2 \%)$ in the sudden weaning group. The chi-square statistic was 11.8476 (p 0.05). Failure rate was more $(14.2 \%)$ in the sudden weaning group $(p 0.05)$. Success rate was more $(58.2 \%)$ in babies with birth weight $1.5-2.5 \mathrm{kgs}$ and gestational age of $32-37$ weeks $(58.2 \%$ ) (p value 0.01 ).

Conclusions: Gradual weaning was found to be better than sudden weaning. Success was more in babies $1.5 \mathrm{~kg} \& 32$ weeks.

\section{9}

\section{Nephrology}

\section{Primary Hyperoxaluria Type 1: A Case Report}

Ines Ganhao ${ }^{1}$, Catarina Borges ${ }^{2}$, Telma Francisco ${ }^{3}$, Marta Amorim ${ }^{4}$, Isabel Gonçalves ${ }^{5}$, Margarida Abranches ${ }^{3}$

${ }^{1}$ Serviço de Pediatria, Centro Hospitalar Barreiro-Montijo, Portugal

${ }^{2}$ Pediatria, Unidade Local de Saúde do Baixo Alentejo, Portugal

${ }^{3}$ Unidade de Nefrologia Pediátrica, Centro Hospitalar Universitário de Lisboa Central, Portugal

${ }^{4}$ Serviço de Genética, Centro Hospitalar Universitário de Lisboa Central, Portugal

${ }^{5}$ Unidade de Hepatologia, Centro Hospitalar e Universitário de Coimbra, Portugal

Background: Primary Hyperoxaluria type 1 (PH1) is a rare autosomal recessive inherited disease, with an estimated incidence of 1:100 000 live births per year in Europe. It is caused by mutations in AGXT gene, leading to liver-specific peroxisomal enzyme alanine-glyoxylate aminotransferase (AGT) dysfunction. Its heterogeneous presentation proves a diagnostic challenge and a high level of suspicion is necessary for an early diagnosis. Over 50\% already present with end stage renal disease (ESRD) at time of diagnosis.

Case report: We report the case of a 14-year-old boy, second child to consanguineous parents, followed in Pediatric Nephrology consultation of a tertiary hospital. No relevant past history, except for enuresis and minor beta-talassemia. He was first referred at the age of 9 for recurrent lithiasis (episodes of stone elimination) and ureteral dilatation. At presentation, estimated glomerular filtration rate (eGFR) was $32.3 \mathrm{~mL} / \mathrm{min} / 1.73 \mathrm{~m}^{2}$ and ultrassonography (US) confirmed bilateral renal lithiasis causing right ureter obstruction and nonspecific parenchymal hyperechogenicity. Stone analysis revealed calcium oxalate monohydrate crystals and 24-h urine had markedly elevated urine oxalate excretion $\left(2.22 \mathrm{mmol} / 1.73 \mathrm{~m}^{2} /\right.$ day $)$. Besides hydration, he was treated with sodium and potassium citrate and pyridoxine, and eGFR transiently improved $\left(93.7 \mathrm{~mL} / \mathrm{min} / 1.73 \mathrm{~m}^{2}\right)$. However, despite increasing doses, urine oxalate excretion remained more than 3-fold higher than reference values. Genetic tests confirmed a mutation in AGXT gene, c.1151TC, in homozygosity. Approximately two years after initial symptoms, nephrocalcinosis was identified in US, consistent with the diagnosis, and eGFR gradually declined while albuminuria and uricemia got elevated. Oxalate deposition in myocardium, retina, bone marrow and thyroid was excluded following a multidisciplinary approach. At 14 years old, eGFR went bellow $40 \mathrm{~mL} / \mathrm{min} / 1.73 \mathrm{~m}^{2}$ and he was then proposed for a sequential liver-kidney transplantation. Liver transplantation of deceased donor was performed with no major complications.

Conclusions: Recurrent urolithiasis and nephrocalcinosis in children along with suggestive family history/consanguinity should raise the suspicion of PH1. Definite diagnosis is based on mutation analysis. Conservative treatment may increase renal survival, therefore it should be started when the disease is first suspected. Sequential or combined liver and kidney transplantation should be planned when eGFR declines below $40 \mathrm{~mL} / \mathrm{min} / 1.73 \mathrm{~m}^{2}$ to avoid systemic complications of systemic oxalosis.

562

Neurology

Pediatric Care of Neurodevelopmental Disorders

Maureen Casper ${ }^{I}$

${ }^{7}$ Speech-Language-Hearing Sciences, City University of New York, USA

Background: Differential diagnosis and monitoring of treatments for children with neurodevelopmental disorders continue to present a challenge in pediatric care. Deficits in the control of coordinated movement of limb, speech and voice production often present in those children diagnosed within the spectrum of neurodevelopmental disorders. The measurement of speech and voice production allows for a sensitive and quantitative description of coordinated movement. Vocal fold vibration and movement of the articulators may be quantitatively assessed with acoustical measures of fundamental frequency (F0) and formant frequencies (F1 and F2) as well as syllable duration. When embedded within a stimuli designed to elicit prosodic contrasts (Casper, et al., 2006) these measures provide an objective index for monitoring speech motor control.

Objective: Normal child development is marked by intelligible speech, gradual increase in length and complexity of utterances and flexible prosody. Both standard assessment and acoustic analyses of speech are reviewed as measures for evaluating neurological status.

Method: Differential diagnosis and treatment protocols directed at improving speech motor control were used to assess and monitor neurological status in pre-school children aged 3-5 years. Case studies presented evaluate verbal apraxia, sensory processing and delayed language development. 
Results and Conclusion: The results of these analyses and the other uploaded clinical data provide objective measures for the rate of progress of a child diagnosed within the spectrum of neurodevelopmental disorder in the areas of intelligibility, length and complexity of utterances, and speech prosody. The data and results can be shared across physicians, clinicians and researchers to improve the efficacy of treatment protocols and identify areas of intervention that need emphasis in developing the child's neurophysiological ability to produce and perceive spoken language.

142

\section{Neurology}

Epidemiology of Autism Spectrum Disorders: A Multifactorial Model for Populations in Transition

Gary Diamond $^{1,2}$, Lutfi Jaber ${ }^{1,2,3}$, Ditza Tsahor ${ }^{4}$

${ }^{T}$ Pediatric Neurology and Child Development, Schneider Children's Medical Center, Israel

${ }^{2}$ Pediatrics, Clalit Health Services, Israel

${ }^{3}$ Pediatrics, Bridge to Peace Community Health Center, Israel

${ }^{4}$ Autism Center, Asaf Harofeh Medical Center, Israel

Background: Autism Spectrum Disorders (ASD) are a group of developmental disabilities characterized by impairments in social communication, restricted areas of interest, and repetitive and stereotyped patterns of behavior. Symptoms typically are apparent by the age of 3 years.

Objective: To determine the prevalence of ASD among children of the largest of 4 health maintenance organizations in Israel.

Methods: Point prevalence and diagnostic trends of ASD over the past 18 years were examined over a wide geographical area of central Israel, encompassing a database of 331,169 children, age 3-18 years. Special importance was attached to statistics from different religious and ethnocultural groups as potentially reflecting discrepancies in diagnosis, reporting, and possibly environmentally-related trigger factors in the causality of an essentially genetically determined syndrome.

Results: Overall prevalence was 0.004 (1/250 live births), well below firgures from other similar studies abroad and in Israel. Prevalence figures for the ultra-orthodox Jewish community were the lowest (0.0022), when compared with the general population and Israeli Arabs (0.0033). Time trends indicated a surge of ASD among Israeli Arabs and Ethiopian Jews between the years 2004 and 2015, in contrast with a general flattening for the ultra-orthodox community ( $\mathrm{p} 0.01$ ).

Conclusions: Results indicated that besides discrepancies in diagnosis and reporting factors, there exists a possible relation between clinical expression of an ASD genetic predisposition and the socioeconomic/ cultural environmental factors at work in impacting on the epigenetics in the causality of autism. Our prevalence rates are currently lower than those for ASD in Europe and the USA. The results should be of special interest to planners of health and educational services for the handicapped in countries with minorities or immigrant groups in social transition.

\section{9}

\section{Neurology}

Nusinersen: a Single-Center Real-Life Experience in Type 1 Spinal Muscular Atrophy

$\underline{\text { Andreia Lomba }}^{1,2}$, Joana A. Ribeiro ${ }^{2}$, Henriqueta Araújo ${ }^{2}$, Vera Ribeiro ${ }^{2}$, Núria Madureira $^{3}$, Filipe Palavra ${ }^{2,4}$, Isabel Fineza ${ }^{2}$

${ }^{1}$ Pediatrics Department, Centro Hospitalar do Baixo Vouga, Portugal

${ }^{2}$ Centre for Child Development - Neuropediatrics Unit, Hospital Pediátrico, Centro Hospitalar e Universitário de Coimbra, Portugal

${ }^{3}$ Pediatrics Department - Pneumology Unit, Hospital Pediátrico, Centro

Hospitalar e Universitário de Coimbra, Portugal

${ }^{4}$ Faculty of Medicine, Coimbra University, Portugal
Background: Nusinersen, an antisense oligonucleotide designed to treat 5q-Spinal Muscular Atrophy (SMA), is currently available in Portugal for SMA patients. In clinical trials, SMA type 1 patients achieved a substantial motor milestone response.

Objective: To report the clinical results of nusinersen in a single tertiary centre, in Portugal.

Methods: We performed a retrospective study of type 1 SMA patients treated with nusinersen. Demographic, clinical (including motor scores CHOP-INTEND), ventilation and feeding parameters were obtained from clinical records.

Results: We are currently treating 5 genetically confirmed SMA type 1 patients - current mean age 16,4 months (range 8-25 months), four with 2 copies of $S M N 2$ and one with 3 copies of SMN2. The mean time from the first symptoms to the diagnosis confirmation was 3,1 months (range 0,3$10,0)$ and from the diagnosis confirmation to the beginning of treatment was 1,1 months (range $0,5-1,7$ ). Only 3 patients had $\geq 6$ months of treatment (5, 6 and 9 administrations of nusinersen respectively), and they all improved at least 4 points in CHOP-INTEND score (mean change 10.0 points). The eldest patient ( 25 months-old) began treatment at 2,3 months and has achieved independent sitting. Prior to treatment, 2 patients required continued non-invasive ventilation (NIV) and one 14 hours per day. After 6 months of treatment they all reduced time of NIV up to 8 hours per day. Gastrostomy was performed in 2 patients during treatment and one required tube feeding. No side effects of treatment were noticed.

Conclusion: We observed an improvement in motor function and ventilatory support in SMA type 1 patients with $\geq 6$ months of treatment with nusinersen. Despite our small sample, our findings are promising and contribute to the increasing evidence that early diagnosis and treatment is paramount for these patients.

401

Neurology

PANS as a Post-Infectious Autoimmune Disease: Benefit of IVIG Isaac Melamed ${ }^{1}$, Andrew Schechterman ${ }^{1}$

${ }^{T}$ IMMUNOe Research Center, IMMUNOe Health Centers, USA

Background: Pediatric acute-onset neuropsychiatric syndrome (PANS) is a clinical diagnosis given to children who have a dramatic - almost overnight - onset of neuropsychiatric symptoms. Recent findings suggest a relationship between a post-infectious response and behavioral changes, which may be a form of post-infectious autoimmunity, through molecular mimicry, resulting in various neurological symptoms. We've coined the term "Alzheimer's of the Immune System," or AIS, to identify this syndrome. As a result of our studies and observations, we've identified a number of common immune defects in patients with this presentation which suggests a new immune deficiency. Given our findings, we hypothesize that AIS is the underlying mechanism that leads to PANS.

Methods: Based on this hypothesis, we proposed a study to explore the use of intravenous immunoglobulin (IVIG)[Octagam 5\%] for the treatment of PANS. The primary objective of the study was to assess the efficacy of IVIG in PANS over a period of 6 months (6 infusions) based on psychological evaluation scores using 6 different assessments (Figure 1). Secondary objectives included evaluation of the immunomodulatory effect of IVIG on key biomarkers associated with PANS.

\section{Figure 1:PANS Study Psychological Assessments}

1. Pediatric Acute Neuropsychiatric Symptom Scale Phone Interview Scores (PANSS-PI)

2. Children's Yale-Brown Obsessive Compulsive Scale (CY-BOCS)

3. Yale Global Tic Severity Scale (YGTSS)

4. Anxiety Disorders Interview Schedule for DSM-IV (ADIS)

5. Clinical Global Impression (CGI)

6. Parent-Rated Symptom Survey (PRSS)

Results: Our results indicate statistically significant reductions in symptoms from baseline to end of study (infusion 6) in all 6 assessments measured. 
Dramatic results can be seen in the PANSS-PI (Figure 2). Statistically significant reductions in symptoms were demonstrated beginning at infusion 3 through infusion 6 compared to baseline, with steady improvement from infusion 1 to infusion 6 . Additional results from CY-BOCS indicated statistically significant reductions in obsessive compulsive symptoms, resulting in $50 \%$ improvement, that were sustained 8 weeks after the final infusion (Figure 3).
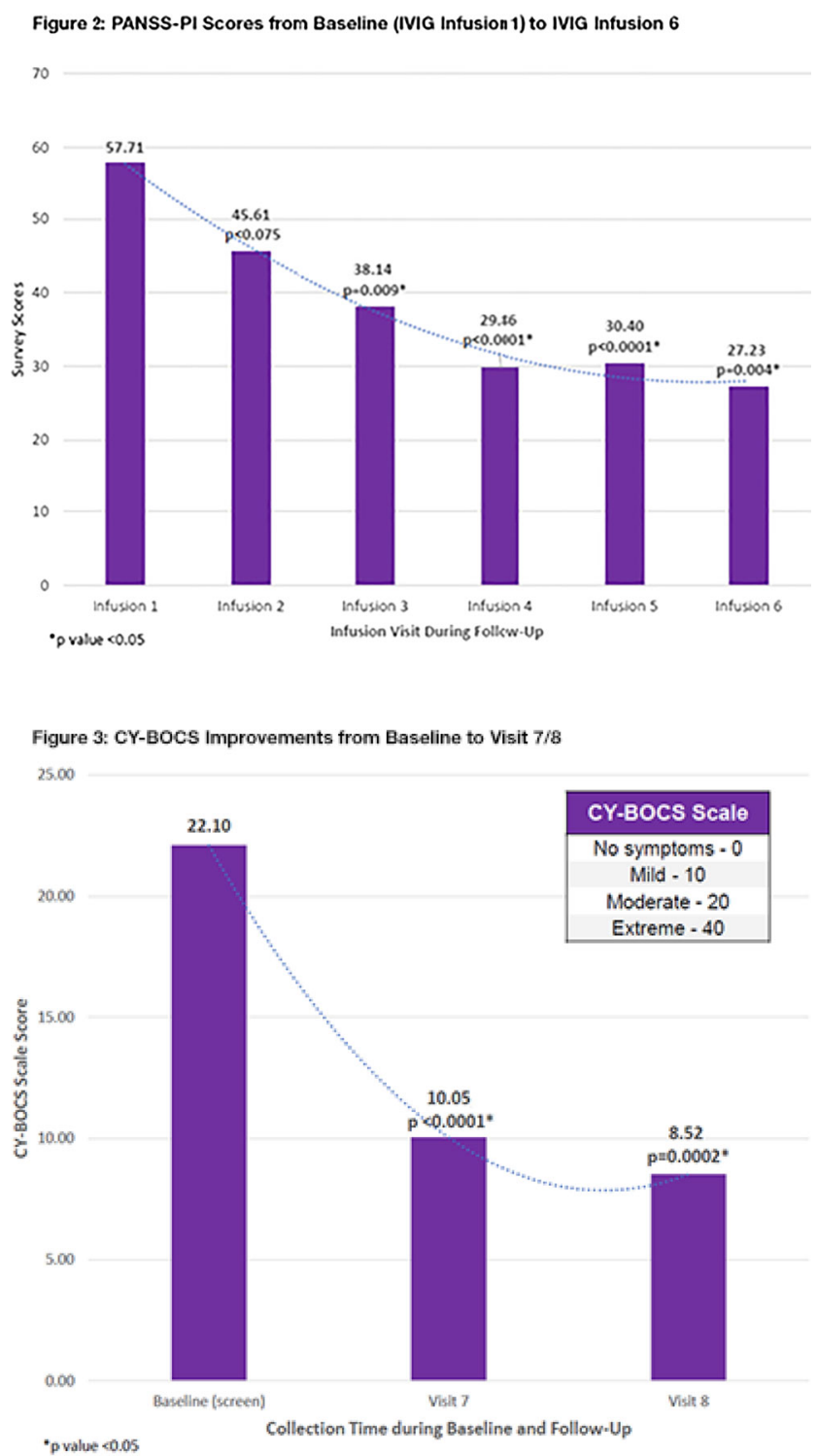

Conclusion: In patients presenting with PANS, which may be associated with an underlying immune deficiency mechanism we term AIS, IVIG [Octagam $5 \%$ ] successfully mediated psychological symptoms and dysfunction.

144

Neurology

Long Term Neurodevelopmental Outcome following Therapeutic Hypothermia for Perinatal Asphyxia

Basavaraj Patil ${ }^{1}$, Pranay Boralkar ${ }^{1}$, Sharanagouda Patil ${ }^{1}$, Roopa Mangshetty ${ }^{I}$, Rohit Bhandar ${ }^{I}$, Apurva Arun ${ }^{I}$, Kudlappa Angadi ${ }^{I}$, Mallikarjun Kobal ${ }^{l}$, Kiran Hosgoudal

${ }^{1}$ Pediatrics, Mahadevappa Rampure Medical College, India
Introduction: Neuroprotective benefit of therapeutic hypothermia in term newborns with hypoxic-ischemic encephalopathy (HIE) was analyzed by survival and neuro-developmental outcome of neonates subjected to this procedure.

Material and Methods: Newborns with gestational age 36 weeks and 6 hours of age with moderate to severe asphyxial encephalopathy underwent cooling protocol at a temperature of $33.5{ }^{\circ} \mathrm{C}$ for 72 hours and rewarming period of 6 hours. Outcome measures assessed were death and neurodevelopmental characteristics, which were compared at the different age using ASQ3.

Results: 42 children were assessed at age 6-8months, 42 children at $12-14$ months. Median gestational age was 38 weeks, birth weight $2.8 \mathrm{~kg}$, Apgar score $2 / 4$ and $\mathrm{pH}$ on admission to the hospital 7.02. Four (8\%) children died. At the first assessment developmental categories of communication were normal in $61.9 \%$, problem solving in $69.1 \%$, personal-social in $61.9 \%$, gross motor in $66.27 \%$, and fine motor in $40.5 \%$ with a high need of retesting in this area. Second assessment was done in 42 patients: developmental categories of communication normal in $78.6 \%$, problem solving in $76.2 \%$, personal-social in $71.4 \%$, gross motor in $81.0 \%$, and fine motor in $50 \%$.

Conclusion: There was no correlation between baseline parameters and outcome. Results of the study are showing that therapeutic hypothermia in term newborns can provide better survival and less neurologic sequels in HIE patients.

Table Baseline data at birth for patients enrolled in study

\begin{tabular}{lllll}
\hline & & & & Range \\
Gestational age-weeks & 38.6 & 1.4 & 40 & $37-42$ \\
Birth weight-grams & 2800 & 450 & 3.25 & $2.5-4$ \\
Head circumference-cm & 35.12 & 1.1 & 35 & $33-37$ \\
Apgar score at 1min & 2.26 & 1.772 & 2 & $0-6$ \\
Apgar score at 5min & 4.37 & 1.363 & 4 & $2-6$ \\
Ph at hospital admission & 6.99 & 0.186 & 7.02 & $6.5-7.3$ \\
Age at starting of cooling-hours & 4.32 & 2.33 & 4 & $2-8$ \\
\hline
\end{tabular}

157

Obesity

Obesity in Relation to Hypertension in a Sample of Egyptian Adolescents with Insulin Resistance

Moushira Zaki ${ }^{1}$, Sanaa Kamal ${ }^{1}$, Karima Bahgat ${ }^{2}$,Walaa Basha

${ }^{1}$ Biological Anthropology, National Research Centre, Egypt

${ }^{2}$ Pediatric, Faculty of Medicine, Al-Azhar University, Egypt

Background: Obesity is a global epidemic disease that goes worse in many countries all over the world and has very serious effects on health status and economy of the community.

Objectives: The aim of this study is to evaluate the association between obesity and high blood pressure levels in a sample of obese Egyptian adolescents with insulin resistance (IR).

Methods: A cross-sectional study was conducted on 200 obese adolescents with (IR), aged between 16 and 17 years (100 males and 100 females) and 200 non-obese healthy controls matched in age and sex. Anthropometric parameters, body composition, fasting insulin and glucose were measured. IR was evaluated by the Homeostasis Model Assessment-Insulin Resistance (HOMA-IR). Spearman correlation and multiple regression analysis were used to assess the relationship. Receiver operating characteristic curve (ROC) analysis was performed to obtain the best cut-off values, sensitivity, and specificity for discrimination of hypertension in obese IR. 
Results and Conclusions: Results show positive correlations between systolic and diastolic blood pressure, HOMA-IR, body mass index (BMI), waist circumference and body fat $\%$. Multiple regression analysis showed that BMI, waist circumference, waist to hip ratio and body fat percentage were independently related to systolic and diastolic blood pressure in IR patients in both genders, suggesting the importance of their use in the evaluation of hypertension among obese adolescents with IR.

328

Obesity

Association between Overweight/Obesity, Serum Gonadotropin and Androgen Levels among Egyptian Pubertal Girls

Sahar El-Masry ${ }^{I}$, Rehab A. Mahmoud ${ }^{3}$, Muhammad Al-Tohamy ${ }^{1}$, Hanaa H. Ahmed ${ }^{2}$, Mahmoud A.S. Afify ${ }^{l}$

${ }^{1}$ Biological Anthropology, National Research Centre, Egypt

${ }^{2}$ Hormones Department, National Research Centre, Egypt

${ }^{3}$ Pediatrics Department, Institute of Postgraduate, Childhood Studies, Ain Shams University, Egypt

Background: Previous studies have suggested an association between adiposity and elevated androgen in late pubertal girls.

Objective: Evaluate the association between overweight /obesity, serum gonadotropin and androgen levels among Egyptian pubertal girls.

Subjects and Methods: It was a cross-sectional one, carried out in the Obesity Clinic of the Diabetes, Endocrine and Metabolism Pediatric Unit (DEMPU), Pediatric Hospital, Cairo University. It included 40 overweight and obese girls and 40 age-matching normal weight (control) girls, aged $12-18$ years. Anthropometric assessment (weight, height, hip and waist circumferences) was recorded, and waist/hip and BMI were calculated. Laboratory investigations: lipid profile. (TG, TC, HDL, LDL), serum gonadotropin (LH, FSH), androgen (free and total testosterone), estradiol, insulin, $\mathrm{HbAlc}$, and fasting blood sugar were measured .Insulin resistance (IR) was calculated.

Results: Hypogonadotropins (FSH and LH) and hyperandrogenaemia (total and free testosterone) were significantly prominent among obese girls. Correlation between gonadotropin (FSH and LH), androgen (Total Testosterone and free Testosterone) and all the studied variables for the 3 groups under study (obese, overweight, control) revealed constant relations. Gonadotropin and androgens had opposing correlations. Gonadotropin had significant negative correlations, and androgens had significant positive correlations with the anthropometric obesity markers (BMI, Waist $\mathrm{C}$, and $\mathrm{W} / \mathrm{H}$ ratio), $\mathrm{HbA} 1 \mathrm{c}$, insulin, insulin resistance and lipid profile (triglycerides, total cholesterol and LDL). In addition, Gonadotropin had significant positive correlations, and androgens had significant negative correlations with estradiol and HDL, and insignificant correlation with age.

Conclusion: Overweight and obesity had no effect on the correlations between gonadotropin and androgen in one side and the anthropometric measurements and laboratory investigations on the other side. The changes in androgen levels were earlier than in gonadotropin among the overweight and obese children.

186

Obesity

Prevalence of Overweight and Obesity in Youth (7-21 Years) with Intellectual Disabilities, without Down Syndrome, in the State of Kansas, USA: 2012-2019

Edwin Loovis ${ }^{1}$, Ken Pitetti ${ }^{2}$, Ruth Ann Miller ${ }^{3}$

${ }^{T}$ Department of Health and Human Performance, Cleveland State University, USA

${ }^{2}$ Department of Physical Therapy, Wichita State University, USA

${ }^{3}$ Department of Electrical Engineering, Wichita State University, USA
Background: For typically developing (TD) youth in the United States (USA), Odgen and colleagues (2014) reported that for combine sexes the prevalence of overweight (OW) was $34.2 \%$ and $34.5 \%$ in ages 6-11 years and 12-19 years, respectively; and prevalence of obesity (OB) (Ogden and colleagues, 2016) of $17.4 \%$ and $20.5 \%$ in ages $6-11$ years and $12-19$ years, respectively. However, there has been no tracking of $\mathrm{OW}$ and $\mathrm{OB}$ rates of youth with intellectual disability (ID) in the USA.

Objective: To provide current estimates of the prevalence of $\mathrm{OW}$ and $\mathrm{OB}$ of youth with ID, without Down syndrome (DS), for ages 7-21 years in a midwestern state (Kansas).

Methods: Two hundred and twenty-two (222) males and 108 females, ages 721 years with moderate to mild ID without physical disabilities were evaluated between 2012 and 2019. Height and weight were measured using standardized protocols and body mass index (BMI: wt $[\mathrm{kg}] \cdot h \mathrm{ht}[\mathrm{m}]^{-2}$ ) was calculated for age groups 7-12 years (children); 13-16 (adolescents); 17-21 (young adults). BMI classifications of $\mathrm{OW}$ and $\mathrm{OB}$ were determined using international standards for age and sex specific cut-off points (Cole et al., 2000).

Results: Results are illustrated in Table 1.

Table 1. Prevalence of OW and OB by Age and Gender

Overweight Obese 7-12 yrs 13-16 yrs 17-21 yrs 7-12 yrs 13-16 yrs 17-21 yrs

\begin{tabular}{lllllll}
\hline $\mathrm{M}$ & $20 \%$ & $18 \%$ & $25 \%$ & $15 \%$ & $30 \%$ & $34 \%$ \\
$\mathrm{~F}$ & $13 \%$ & $31 \%$ & $36 \%$ & $17 \%$ & $21 \%$ & $40 \%$ \\
$\mathrm{M}+\mathrm{F}$ & $17 \%$ & $26 \%$ & $27 \%$ & $15 \%$ & $29 \%$ & $34 \%$ \\
\hline
\end{tabular}

Conclusion: Compared to TD youth in the USA: youth with ID in Kansas reported lower prevalence of $\mathrm{OW}$ and $\mathrm{OB}$ in ages $\leq 12$ years; and a lower prevalence of $\mathrm{OW}$ and a higher prevalence of $\mathrm{OB}$ in adolescents and young adults, respectively. These results challenge previous reports that $\mathrm{OW}$ and $\mathrm{OB}$ are more prevalent in populations with ID, without DS, and suggest that regional differences in obesogenic environments may exist.

\section{0}

\section{Pediatric Cardiology}

Changes in the Cardiovascular System during Therapy with Propranolol of Infantile Hemangiomas

Nataliya Konstantinova ${ }^{1}$, Natalia Kotlukova ${ }^{1,2}$, Ekaterina Karelina ${ }^{2}$, Aleksei Grishkin ${ }^{2}$, Tatiana Lavrova ${ }^{3}$

${ }^{I}$ Tabolin Department of Hospital Pediatrics, Pirogov Russian National Research Medical University, Russia

${ }^{2}$ Department of Pediatric Cardiology, Bashlyaeva Children's City Clinical Hospital, Russia

${ }^{3}$ functional diagnostics department, Bashlyaeva Children's City Clinical Hospital, Russia

Background: Propranololol is undeniably effective as an infantile hemangiomas $(\mathrm{IH})$ treatment, however, the drug acts on the myocardial tissue and heart rate.

Objective: To assess the state of the cardiovascular system in patients before treatment, during therapy with propranolol and after its completion.

Methods: Cardiac examination (ECG, echocardiogram, 24-Hour Holter monitoring), and ultrasound with Doppler of $\mathrm{IH}$ vessels were performed 202 children aged 1 month to 4.5 years with IH different localization. Studies were conducted before the appointment of propranolol therapy, then every 3 months during treatment and after discontinuation of therapy.

Results: According to the ECG, there was not a single case of bradycardia. However, 24-Hour Holter monitoring showed that in all children, 3 months after the start of therapy, the average daily heart rate decreased by $9 \%$ from the norm. Also, according to 24-Hour Holter monitoring, $18 \%$ of children were diagnosed with AV-blockade of grade I-II and $6 \%$ of children were diagnosed the rhythm pauses, significantly exceeding the age norm. Syncopal states 
were observed in 2 children, accompanied by pauses of the heart rhythm with a maximum duration of 18 seconds. In 1 child, according to the echocardiogram, there was a decrease in the contractile function of the myocardium. Doppler ultrasound found that when blood flow in the vessels was

Conclusion: Cardiological examination revealed a small percentage of undesirable effects on the background of propranol. Only a flow-up 24-Hour Holter monitoring allowed timely identification of life-threatening changes in heart rate and timely correction of the dose of the drug. Ultrasound with Doppler allowed us to establish an individual duration of treatment with propranolol and to minimize the percentage of rebound syndrome against the background of discontinuation of therapy with propranolol.

672

\section{Paediatric Cardiology}

Postnatal Echocardiographic Screening of Babies with Positive Family History of Congenital Heart Disease. A Two-Year Retrospective Study

Rachel Hoi Khoon Tan ${ }^{I}$, Hossam Khaliel ${ }^{I}$, Maysara Aziz ${ }^{l}$

${ }^{1}$ Paediatrics Department, St Helens and Knowsley Teaching Hospitals NHS Trust, UK

Background: Congenital heart disease (CHD) is the most common congenital disorder in newborn. The incidence is $1 \%$. There is good evidence that left ventricular outflow tract obstructions have 'high heritability', however there is no consensus guidelines on screening babies with positive family history. Often the family history is vague and unclear.

Aim: To review the family history, and detection rate of CHD, for the babies with family history of CHD.

Method: Retrospective review of two-year data on all babies who were referred to clinic with family history of CHD. The data collected include: gender, pre- and post-ductal saturations, the types of family history, family affected by $\mathrm{CHD}$, investigations performed and the findings on referred babies. Results: Out of 665 of new patients seen in cardiac clinic between January 2017 to December 2018, 75 (11\%) babies were referred for family history of CHD. Only $69 \%$ of these babies have pre- and post-ductal saturations done, and all of them were normal. Heart murmur presented in 20 babies (27\%).

The most common family history is 'hole in the heart', and unspecified $\mathrm{CHD}$. And eight families only declared history of heart murmur. The CHD was most commonly presented in siblings (29\%), followed by mother $(20 \%)$ and father $(20 \%)$.

9 out of $75(12 \%)$ of these babies were found to have abnormal findings on echocardiogram. This is a 10-fold increased in risk if compared to general population. Most common CHD detected is ASD. Out of them, two babies $(22 \%)$ have heart murmur on clinical examination.

Conclusion: Obtaining accurate cardiac family history during newborn check can be challenging and baby who has CHD is often asymptomatic. Screening of babies with family history of CHD is justified, but may need to be more targeted, in light of new studies on inheritability of CHD.

\section{Family Member Affected by CHD}

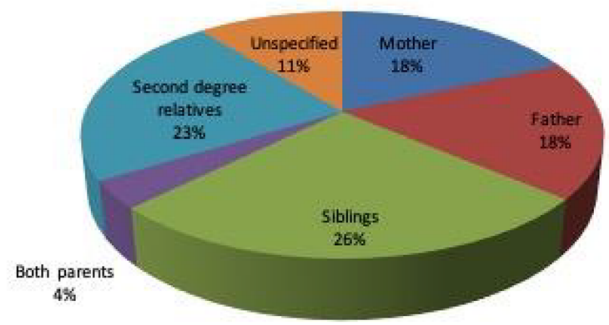

Abnormalities Detected on Screening

\begin{tabular}{|l|c|l|c|c|}
\hline $\begin{array}{c}\text { Abnormality } \\
\text { detected on } \\
\text { screening }\end{array}$ & Number & \multicolumn{1}{|c|}{ Family history } & Murmur & $\begin{array}{c}\text { Pre- Post } \\
\text { ductal sats }\end{array}$ \\
\hline ASD + PDA & 1 & ASD (Mother) & No & Normal \\
\hline ASD & 4 & $\begin{array}{l}\text { Heart murmur (unspecified) } \\
\text { Mitral valve prolapse (mother) } \\
\text { CHD unspecified (Father) } \\
\text { ASD (mother) }\end{array}$ & $\begin{array}{c}\text { Yes In } 1 \text { baby, } \\
\text { No in } 3 \text { babies }\end{array}$ & Normal \\
\hline $\begin{array}{l}\text { Bicuspid Aortic } \\
\text { valve }\end{array}$ & 1 & Heart murmur (Father) & No & Normal \\
\hline $\begin{array}{l}\text { Mild coarctation of } \\
\text { aorta }\end{array}$ & 1 & $\begin{array}{l}\text { Coarctation of aorta (Aunty } \\
\text { and half sister) }\end{array}$ & Yes & Normal \\
\hline Branch PA stenosis & 2 & $\begin{array}{l}\text { Aortic stenosis (Mother) } \\
\text { VSD (Sister) }\end{array}$ & No & Normal \\
\hline
\end{tabular}

Types of Family History

\begin{tabular}{|l|c|}
\hline \multicolumn{1}{|c|}{ Family History } & Number \\
\hline Hole in the heart & 13 \\
\hline CHD required surgery & 9 \\
\hline CHD unspecified & 11 \\
\hline TGA & 3 \\
\hline Heart Murmur & 8 \\
\hline VSD & 8 \\
\hline ASD & 4 \\
\hline ASD+VSD & 6 \\
\hline Coarctation of aorta & 2 \\
\hline Valvular abnormality & 4 \\
\hline Hypoplastic heart & 1 \\
\hline Aortic stenosis & 3 \\
\hline Bicuspid aortic valve & 1 \\
\hline Tetralogy of fallot & 1 \\
\hline Miscellaneous & 7 \\
\hline Cardiomyopathy & 3 \\
\hline
\end{tabular}

*Some families have more than one cardiac abnormality

\section{6}

\section{Paediatric Tropical Medicine}

Rotavirus Vaccine, Knowledge and Practice of Parents and Caregivers in a Teaching Hospital in Southeast Nigeria Chikosolu Okiche $^{I}$, Chineze Okorowo ${ }^{I}$, Lilian Nwobashi ${ }^{l}$ ${ }^{\mathrm{T}}$ Paediatrics, Federal Teaching Hospital Abakaliki, Nigeria

Introduction: Diarrhoeal disease is the second leading cause of death in children under five years old. Rotavirus and Escherichia coli, are the two most common etiological agents of moderate-to-severe diarrhoea in lowincome countries. Therefore, rotavirus vaccination is pivotal in the prevention of severe forms diarrhoea disease. The vaccine is yet to be adopted to be given for free in Nigeria and costs about $\$ 20$ (N7, 000) per dose.

Objectives: This study, which is a pre-intervention study, aimed at understanding the knowledge, perception and practices of parents about rotavirus vaccination and also educate the parents about the vaccine in a tertiary hospital setting in an Eastern State of Nigeria

Methods: In a descriptive cross-sectional study, 186 caregivers of children who came to the children outpatient clinic were enrolled. A researcher administered questionnaire was used to assess the knowledge and practice of parents about rotavirus vaccine. The parents who did not know about the vaccine where also educated on the vaccine and when it's given. Result: The mean age of respondents was 33.4years, $83 \%$ were mothers and the rest fathers. On knowledge, $76.9 \%$ of the respondents didn't know about rotavirus vaccine. Also as regards practice, $91.9 \%$ of the respondents did not vaccinate their children and of them, $21 \%$ did not vaccinate because of the cost of the vaccine. $152(81.7 \%)$ of parents said they will like to vaccinate their children and $64.5 \%$ agreed to do so even if they will have to pay for it with only $36.6 \%$ of the parents saying they will not be willing to pay

Conclusion: Majority of the parents didn't know about the rotavirus vaccine as a means of preventing diarrhoea and were excited to hear about the vaccine, its cost and when it is given. This emphasizes the need for more awareness about the vaccine. 
241

\section{Paediatric Tropical Medicine}

Severe Acute Malnutrition (SAM): A Vicious Socio Economic Problem in South India

Basavaraj Patil ${ }^{1}$, Pranay Boralkar ${ }^{l}$, Sharanagouda Patil ${ }^{1}$, Roopa Mangshetty $^{I}$, Apurva Arun ${ }^{1}$, Rohit Bhandar ${ }^{I}$, Kiran Hosgouda , Mallikarjun Kobal ${ }^{l}$, Kudlappa Angadi ${ }^{l}$

${ }^{I}$ Pediatrics, Mahadevappa Rampure Medical College, India

Introduction: Out of 19 million SAM child in all developing countries, 8 million $(42 \%)$ are in India.

Materials and Methods: Study type: Observational. Study design: Observation model - cohort, Time perspective-prospective. Study centre: Basaveshwar and Sangameshwar teaching hospital. Study duration: august 2017 -september 2018. Informed consent was obtained from the parents of all the children.

Aims and Objectives: To evaluate the clinico-epidemiological profile, complications, and comorbidities of SAM children. To identify the sociodemographic risk factors of SAM and to evaluate the outcome of hospitalized SAM children.

Results: Mean age of admitted children was $17.21 \pm 13.94$ months and mostly belonged to lower socio-economic scale, rural areas and large families. $9.2 \%$ were less than 6 months at time of admission. Male: female ratio was 1:1.24. Most common associated infections were acute respiratory tract infections $(35.27 \%)$, and diarrhoea (31.75\%). oAnaemia was an important co-morbidity (83.52\%). Mean duration of exclusive breastfeeding was 3.1 \pm 1.8 months and mean age of starting semi-solid complementary food was $8.5 \pm 2.5$ months. Most commonly used supplementary food was over diluted cow milk (92\%). Cure rate was $75.24 \%$. Defaulter rate was $13.65 \%$ and death rate was $0.63 \%$.

Conclusion: The problem of SAM is multidimensional and tertiary care centres provide life-saving care for children as demonstrated by the high survival rates.

\section{CHARACTERISTICS OF SAM CHILDREN}

\begin{tabular}{llllll}
\hline 8 & & & & & $48-60 \mathrm{MO}$ \\
& 26 & 31 & 11 & 6 & 3 \\
\hline
\end{tabular}

LITERACY STATUS OF MOTHER

\begin{tabular}{lll}
\hline & & PERCENATGE \\
ILLITERATE & 35 & 41.1 \\
PRIMARY \& MIDDLE SCHOOL & 30 & 35.2 \\
HIGH SCHOOL & 19 & 22.3 \\
GRADUATE \&POST GRADUATE & 1 & 1.1 \\
\hline
\end{tabular}

ECONOMIC STATUS OF SAM CHILDREN

\begin{tabular}{lll}
\hline & & PERCENTAGE \\
UPPER & 0 & 0 \\
UPPER MIDDLE & 2 & 2.35 \\
LOWER MIDDLE & 3 & 3.52 \\
UPPER LOWER & 30 & 35.29 \\
LOWER & 50 & 58.81 \\
\hline AWARENESS OF MALNUTRITION & \\
\hline & & PERCENTAGE \\
YES & 21 & 24.7 \\
NO & 64 & 75.29 \\
\hline
\end{tabular}

IMMUNISATION STATUS

\begin{tabular}{lll} 
& & PERCENTAGE \\
UNIMMUNISED & 10 & 11.75 \\
PARTIALLY IMMUNISED & 70 & 82.35 \\
FULLY IMMUNISED & 5 & 5.84 \\
\hline
\end{tabular}

RESIDENCE(URBAN/RURAL)

\begin{tabular}{lll}
\hline & & \\
URBAN & 21 & 25 \\
RURAL & 64 & 75 \\
\hline
\end{tabular}

378

Primary Care

Parental Beliefs and Attitudes towards Pediatric Vaccination in a Country with High Vaccine Coverage

Luisa Barros $^{I}$, Inês Cabral Fonsecal

${ }^{T}$ Faculdade de Psicologia, Universidade de Lisboa, Portugal

Background: Anti-vaccination movements are rising in different European and Western countries, causing a decline in the rate of pediatric vaccination. Portugal has one of the highest vaccine coverage rates among countries with non-mandatory pediatric vaccination, and overall higher levels of vaccine confidence. However, anti-vaccination ideas are spreading among parents of young children.

Aim: This study aimed to characterize parental beliefs and attitudes towards vaccination in Portugal and to explore its association with vaccine hesitation. Methods: Based on the relevant literature, we developed a questionnaire to assess vaccines`safety and efficacy perception, perception of severity of vaccine-prevented diseases, conspiracy theory ideas, beliefs about immunizations requirements, social norms and relationship with health professionals. This questionnaire was disseminated online using the snowball method and several parenting social media groups. Parents of children $0-12$ years old were invited to participate.

Results: The questionnaire showed good validity and reliability. A sample of 1118 parents aged 18 to 58 years old filled the questionnaire, and only $3.1 \%$ reported vaccine hesitancy regarding an eventual future child. All the evaluated dimensions showed discriminant power. A binary logistic regression was performed to test for discriminative power and predict vaccine hesitation. Perception of vaccine's safety and efficacy, and positive attitudes towards vaccination requirements predicted the intention to adhere to the recommended immunization plan in the event of having another child. Conclusions: The large majority of Portuguese parents of young children hold vaccine-favorable beliefs and attitudes. All dimensions evaluated were significantly different for parents who would not vaccinate a new child compared to those who would. Beliefs regarding vaccine's safety and efficacy and acceptance of school or health services requirements were the most relevant dimensions for this choice.

385

Primary Care

Feasibility Testing of the Core Set of Quality Indicators for Paediatric Primary Care in Europe, COSI-PPC-EU

Dominik A. Ewald ${ }^{1}$, Gottfried Huss ${ }^{2}$, Rike Antje Kraska ${ }^{3}$, Max Geraedts ${ }^{4}$ ${ }^{T}$ Health Services research, German Society of Primary Care Paediatrics, Germany 
${ }^{2}$ Health Services research, European Association of Primary Care Paediatricians, ECPCP, Germany

${ }^{3}$ Institute for Health Systems Research, Faculty of Health, Witten/ Herdecke University, Germany

${ }^{4}$ Institute for Health Services Research and Clinical Epidemiology, Faculty of Medicine, University of Marburg, Germany

There is a need to measure and improve the quality of paediatric primary care in Europe where major differences in the delivery and outcomes of child health care exist. A collaborative panel of paediatric senior experts developed a Core Set of Indicators for Paediatric Primary Care in Europe by compiling 42 quality indicators in a modified consensus process following the RAND/UCLA appropriateness method. The aim of this study was to explore the feasibility of the quality indicator set in European paediatric primary care practices. Seventy-nine practices from eight countries participated in a detailed online interview. The practices rated the applicability, relevance, reliability and acceptance of the 42 quality indicator as well as the availability, technical feasibility and effort to retrieve the needed data from their medical records. Most quality indicators were considered applicable, available, reliable, acceptable and relevant for monitoring quality of care in paediatric primary care. Respondents rated feasibility and effort to retrieve the data lowest because of difficulties collecting the data from the medical records.

Conclusion: European paediatric primary care practices generally agree with the proposed quality indicator set. They document most of the parameters. However, the collection of specific needed values from available routine patient-data is considered technically difficult and time-consuming.

\section{9}

\section{Primary Care}

Early Predictors in Infancy for a Developing Neurobehavioral Disorder - a Pilot Study

Mina Gurevitz ${ }^{I}$

${ }^{T}$ Pediatrics, Maccabi Health Services, Israel

Background: The increasing incidence of Neurobehavioral Disorders (NBDs), such as Autism Spectrum Disorder (ASD), Attention Deficit Hyperactivity Disorder (ADHD) and Developmental Coordination Disorder (DCD), is of major concern in public health. Although early detection of a developing NBD is critical to enable intervention while brain plasticity is prominent, no screening tool for NBD development at infancy is available. Objective: The aim here was to assess whether risk factors that predict forthcoming NBDs can be identified at early infancy.

Methods: The Israeli Health system provides a general developmental screening system from birth through preschool years at special clinics. In a retrospective cohort study the charts of 161 toddlers, who were diagnosed at a later age for NBDs [47 Autism Spectrum Disorder (ASD), 56 Developmental Coordination Disorder (DCD), 58 Attention Deficit Hyperactivity Disorder (ADHD)], were evaluated and compared to the records of 58 children with typical development. Ten covariates including 28 parameters were accumulated, compared and statistically analyzed.

Results: Deviation from trajectories of seven parameters (gestational age, birth weight, head circumference percentile, weight percentile, gross motor development, difficulties in communication and language) seem collectively to predict ASD with $85 \%$ probability. The first five parameters may collectively predict DCD with $72 \%$ probability. Predictors of ADHD with $58 \%$ probability have been described (Gurevitz et al 2014).

Conclusions:This cohort retrospective study illuminates risk factors at infancy that may predict the development of NBDs. Most prominent are deviations from weight followed by deviations in head circumference trajectories, and delay in motor development due to muscle strength and tone irregularities. Identification of risk factors at infancy is crucial for early intervention programs, such as prevention of rapid changes in weight and manipulations to strengthen motor development and the corresponding neural circuits. The sooner risk factors are recognized, more efficient an early tailored bio-psycho-social treatment might be.

166

Primary Care

KKI-NECT: A Neurodevelopmental and Mental Health TeleEducation Network for Paediatric Primary Care Clinicians Mary Leppert ${ }^{1,2}$, Joyce Harrison ${ }^{1,2}$, Nancy Grace ${ }^{1,2}$

${ }^{1}$ Neurodevelopmental Medicine and/or Psychiatry/Behavioral Psychology, Kennedy Krieger Institute, USA

${ }^{2}$ Pediatrics and/or Psychiatry, Johns Hopkins University School of Medicine, USA

Background: There are growing concerns in the U.S. about the increasing prevalence of paediatric neurodevelopmental and mental health disorders, now $15 \%$ and $11-20 \%$ respectively. There is a critical shortage of board certified neurodevelopmental, developmental/behavioral or child psychiatry subspecialists to care for this vulnerable population. Per the American Board of Pediatrics' estimates, there is one board certified subspecialist for every 100,000 U.S. children. Given workforce shortages, paediatric primary care clinicians (PPCCs) are increasingly tasked with identifying and managing neurodevelopmental and mental health disorders. However, surveys of practicing paediatricians indicate gaps in the training, knowledge, and confidence to meet this need.

Objectives: Approaches to preparing the PPCC workforce include high quality continued medical education (CME) and integrated care models. Through guided practice and mentorship, the Kennedy Krieger Institute Network for Early Childhood Tele-Education (KKI-NECT) aims to expand PPCC workforce capacity to identify and manage neurodevelopmental and mental health disorders within the medical home.

Method: KKI-NECT replicates the Project ECHO® hub-and-spoke model, offering CME instruction on neurodevelopmental and mental health disorders. Each virtual clinic combines a case presentation by a spoke member and didactic content aimed at guiding PPCC practice. Didactic presentations are based on a multidisciplinary, multilevel curriculum authored by KKI/Johns Hopkins faculty.

Results: A remarkable number of cases (69\%) are complicated by co-occurring neurodevelopmental and mental health concerns. Further, 59\% of children had at least one known Adverse Childhood Experience (ACE). With KKI-NECT guidance, nearly all cases have been managed within the Medical Home.

Conclusion: Based on KKI-NECT data, PPCCs are increasingly entrusted with caring for children with ACEs, neurodevelopmental and mental health disorders, and very often co-occurring conditions. Project ECHO $\cong$ appears to be an effective strategy for guiding PPCC identification and management of neurodevelopmental and mental health disorders. Thus, KKI-NECT provides an effective solution to the paediatric subspecialty workforce shortage.

\section{Singular and Co-occurring Diagnoses}

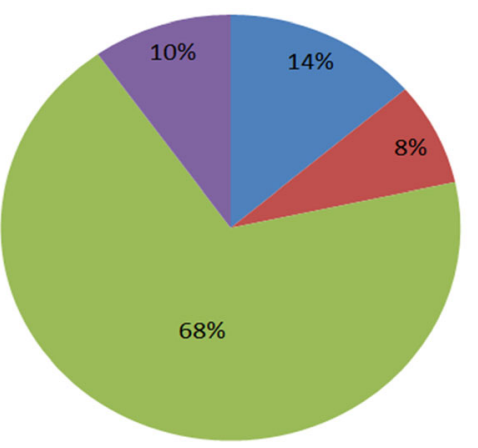


372

\section{Primary Care}

Experiences with Hypnotherapy in Primary Care Pediatric Practice Helena Mole ${ }^{I}$

${ }^{T}$ Primary Care Pediatric Office, Pediatrija Helena Mole, Slovenia

Background: The last 50 years is the era of modern hypnotherapy in children. Thanks to mounting research work hypnotherapy is now succesful evidence-based method of treatment in many pediatric conditions.

Primary care pediatrician interested in the field can achieve proper education on hypnotherapy. In primary care pediatric practice there are many children that can profit if the help is offered on spot and in much less time than referral to the specialist.

Objective: In three years after finishing the national training program on hypnotherapy more than 150 children were offered help with hypnotherapy in my primary care pediatric office.

Methods: The results of the therapy were observed retrospectively. Regarding the cause for using hypnotherapy we observed the results of hypnotherapy in three groups - Nocturnal enuresis, Anxiety and fears and Tics.

Results: Nocturnal enuresis -41 children were treated (age group 4,5 to $11,5$ years, boys in $2 / 3)$, successfully in $28(68 \%)$, on average they needed 3 sessions.

Anxiety and fears - 40 children were treated (age group 4,5 to 18 years, equal by gender), successfully in $35(87 \%)$, on average they needed 2,5 sessions, $12(34 \%)$ of successful cases needed only one visit.

Tics -15 children were treated (age group 5,5 to 13 years, boys in $2 / 3$ ), successfully in $12(80 \%)$, on average they needed 2 sessions.

The children with other diagnoses were also treated with hypnotherapy but the numbers are too small to present the results. Chronic pains, sleep disorders and stuttering are some examples in which hypnotherapy can be very helpful. Conclusion: Hypnotherapy can be a very helpful method in primary care pediatric practice.

\section{4}

\section{Primary Care}

Developing and Implementing Asthma Severity and Control Screening in a Large Urban Health System Marina Reznik ${ }^{1}$, Philip O. Ozuah ${ }^{1}$

${ }^{T}$ Pediatrics, Montefiore Medical Center, USA

Background: Asthma severity or level of control classification is the first step in asthma management. To optimize asthma management, screening must be done at each visit.

Objectives: 1) To develop and implement asthma severity and control screening within electronic health record (EHR) of a large urban health system; 2) To increase asthma screening from $0 \%$ to $80 \%$ by December 2020. Methods: We developed an asthma screening algorithm in EHR based on national guidelines. Screening is conducted by the nursing staff prior to patient being seen by the provider. Based on responses, severity/control classification is calculated and displayed in EHR. Classic Quality Improvement tools such as the Model for Improvement and Plan Do Study Act (PDSA) cycles were used to incorporate changes into daily workflow. Practice Advisory Board meets monthly to discuss barriers to implementation and identify solutions. The Board makes decisions on adopting successful strategies and brainstorm new strategies to address barriers.

Results: This project is ongoing and preliminary results are available from 3 practices: small pediatric, large pediatric and family medicine practices. To date, a total of 4,057 screenings have been completed. Screening rates improved from $0 \%$ to $78.6 \%$ in a small pediatric practice, from $0 \%$ to $66.7 \%$ in a large pediatric practice, and from $0 \%$ to $38.7 \%$ in a family practice (See figures).
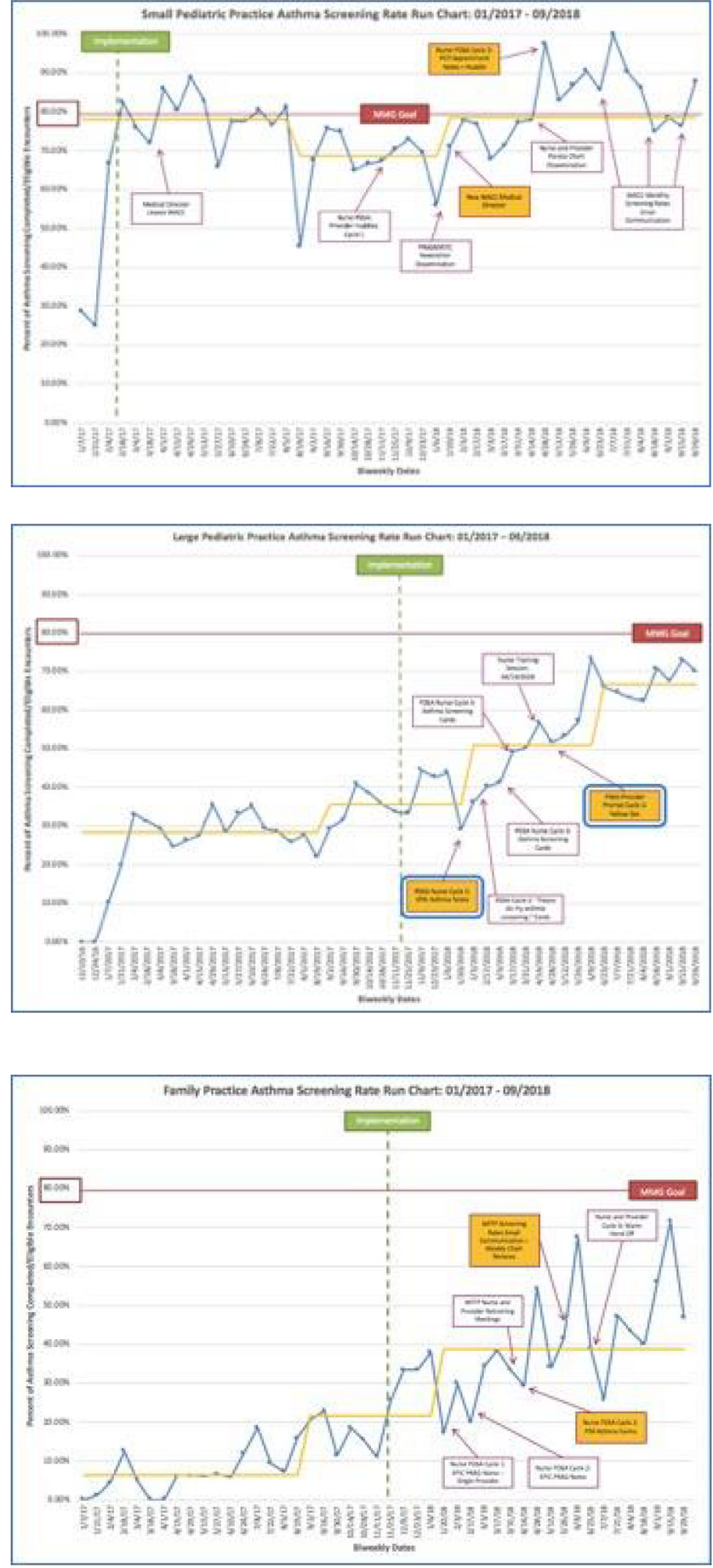

Challenges encountered include: competition with other nursecompleted screenings; screening questions are available in English; nursing staff shortages; nurses forgetting to conduct screening; and caregiver not knowing information about child's asthma.

Conclusion: We developed an asthma screening algorithm within EHR, trained nursing staff and implemented patient screening at the time of clinic visit allowing providers take action on asthma management based on the screening result. Further collaborative efforts are needed to improve and sustain screening rates with the ultimate goal of improving patient clinical outcomes. 
768

\section{Primary Care}

Pocus Aiding in The Diagnosis of Infantile Pompe Disease In Pediatric Practitioner Office: Based Setting

Adib Salim ${ }^{I}$

${ }^{T}$ ATS Bergamo, Italy

Background: Pompe disease is a rare genetic disorder that affects glycogen and lysosome storage secondary to a deficiency in the enzyme acid alfa glucosidase, with a spectrum of severity attributed to the differing amounts of enzyme deficiency.

Objective: Point-of-care ultrasound (POCUS) modality offer a means of access to early diagnosis and appropriate treatment of rare disorders to achieve better quality of life.

Methods: We report the case of incidental bedside echocardiographic finding of mild LV septal wall thickness in 3 months of age infant prompted further investigations led to early diagnosis of Pompe disease. Conclusions: Recognizing earlier this disease by POCUS and initiating enzyme replacement therapy led to improve survival, cardiorespiratory function, growth and development milestones of the patient.

536

\section{Primary Care}

I Have Seen You Before - Frequent Users of the Pediatric Emergency Room

Maria Sao Pedro ${ }^{I}$, Gonçalo Vale ${ }^{l}$, Mariana Simões ${ }^{I}$, Rita Parreira ${ }^{l}$, $\overline{\text { Susana Correia }}^{I}$, Cristina Didelet ${ }^{l}$

${ }^{I}$ Pediatrics, Centro Hospitalar Barreiro-Montijo, Portugal

Background: Patients' overuse of emergency room (ER) services is a serious and growing health care problem in Portugal. Particularly in pediatric age, there is often misuse of resources with overburden of the ER with non-urgent conditions. Recurring visits are also a major concern.

Objective: Characterize the most recurring patients of a pediatric ER service. Methods: Descriptive study, conducted in a suburban hospital in Portugal. Sample consisting of the 20 most frequent users of the pediatric ER per year, in the last 3 years. Data regarding to patient's visits to the ER, medical history and socioeconomic status were collected from their clinical file. Software Microsoft Excel was used for statistical analysis.

Results: 60 patients, 26 female, with median age at the first ER visit of 11 months (IQR 2,04)[4 days-16 years old] and an average of 20 visits each per year (SD 5,97)[15-49]. Almost all continued to be and/or were frequent users of the ER in the previous years, with multiple recurrences during the same acute episode (48 out of 60 ), sometimes more than once per day (31 out of 60 ). A significant number of romanies was registered. Almost half the sample didn't have chronic diseases and among the ones that had, 22 of the 31 had recurrent wheezing/asthma. Although 41 of the 60 attended hospital consultations, 16 of them often missed the appointments. A similar behavior was noted regarding primary care follow-up (15 out of 60$)$. There was a high prevalence (34 out of 60) of patients coming from low income families. Conclusion: Healthcare education programs, especially for at-risk populations, alongside with optimized links between primary and secondary care are needed. Particularly in chronic patients, better management of the disease with empowerment of the patient/caregiver and easier access to ambulatory revaluation should also help reduce ER overflow.

619

\section{Respiratory}

Management of Bronchiolitis in Portugal, 2000-2015: Do Guidelines Have an Impact?

Jose Fontoura-Matias ${ }^{1}$, Diana Moreira-Sousa ${ }^{2}$, Alberto Freitas ${ }^{3,4}$, Inês Azevedo $^{1,2,5}$
${ }^{1}$ Centro Materno-Pediátrico, Pediatrics Department, Centro Hospitalar São João, Portugal

${ }^{2}$ Department of Obstetrics-Gynecology and Pediatrics, Faculty of Medicine, University of Porto, Portugal

${ }^{3}$ Department of Community Medicine, Information and Health Decision Sciences - MEDCIDS, Faculty of Medicine, University of Porto, Portugal ${ }^{4}$ Center for Health Technology and Services Research (CINTESIS), Faculty of Medicine, University of Porto, Portugal

${ }^{5}$ EpiUnit, Institute of Public Health, University of Porto, Portugal

Background: Several studies report an excessive use of diagnostic tests and procedures in bronchiolitis, not supported by guidelines.

Objectives: We aimed to evaluate medical interventions in children hospitalized with bronchiolitis in public Portuguese hospitals, from 2000 to 2015, to evaluate the impact of the national guideline and assess variations between regions.

Methods: Data was collected retrospectively from an administrative database that contains all hospitalizations in mainland public hospitals. Cases were selected using the ICD-9-CM codes 466.11 (bronchiolitis due to respiratory syncytial virus) and 466.19 (bronchiolitis due to other infectious organisms), in children under 2 years of age. For statistical analysis we used Chi-square test and logistic regression.

Results: In the 80,491 hospitalizations due to bronchiolitis, we found a high mean use rate of non-recommended diagnostic and treatmet procedures: chest x-ray $(66.5 \%)$, blood analysis $(56.5 \%)$ and respiratory secretions analysis (12.7\%); nebulized therapy (83.5\%), intravenous corticosteroids (24.2\%), intravenous antibiotics (26.0\%), electrolytes infusion (37.6\%), and chest physiotherapy (20.4\%). Over time, there was a gradual change in attitudes (Ptrend 0.001), with significant variation between regions. Centre region registered the lowest mean rates of routinely non-recommended procedures. Conclusions: In this first national study, rates of the non-recommended diagnostic and treatment attitudes in bronchiolitis were higher than desirable, although there was a modest decrescent trend in their use over time. The observed variations were mainly dependent on the region, with no clear impact of the national guideline in attitude changing, highlighting the need for more active measures.

\section{1}

\section{Rheumatology}

Phenotypic and Genotypic Characteristics and Damage Accrual of Monogenic Autoinflammatory Diseases other than Familial Mediterranean Fever from the Pediatric Rheumatology Arab Group (PRAG)

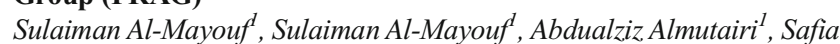
Albrawi $^{2}$, Abdulatif AlEnazi $^{8}, B$ Fatallah $^{4}$, Abdullah Alsonbul ${ }^{1}$, Moahmmed Abu-shukair ${ }^{3}$, Raed Alzyeoud ${ }^{3}$, Adel Alwahadneh ${ }^{3}$, Mubruka Zlenti ${ }^{7}$, Ebtisam Kawaja ${ }^{7}$, Khloud Khawaja ${ }^{5}$, Zakia Almusawi ${ }^{6}$, Wafa Madan ${ }^{6}$, Muna Almuatiri ${ }^{10}$, Nora Almuatiri ${ }^{9}$

${ }^{1}$ Pedaitric Rheumatology, King Faisal Specialist Hospital \& Research Center, Saudi Arabia

${ }^{2}$ Pedaitrics, Royal Hospital, Oman

${ }^{3}$ Pedaitrics, Queen Rania Children Hospital, Jordan

${ }^{4}$ Pedaitrics, Jalilah Children Hospital, United Arab Emirates

${ }^{5}$ Pedaitrics, Mafraq Hospital, United Arab Emirates

${ }^{6}$ Pedaitrics, Salmanyah Hospital, Bahrain

${ }^{7}$ Pedaitrics, Tripoli Children Hospital, Libyan Arab

${ }^{8}$ Pedaitrics, King Fahad Medical City, Saudi Arabia

${ }^{9}$ Pedaitrics, AlSabah Hospital, Kuwait ${ }^{10}$ Pedaitrcs, Adan Hospital, Kuwait

Objective: To report the phenotype, genotype and response to treatment in Arab children with monogenic autoinflammatory diseases (AIDs) other FMF, with focus on accrual damage.

Methods: We retrospectively reviewed patients with clinical and/ or genetically proven monogenic AIDs other than FMF seen between 1990 and 2018 at 10 rheumatology clinics from seven Arab countries. Data 
were collected at the last follow- up visit comprising consanguinity, age at onset and diagnosis, clinical and laboratory findings, treatment, damage accrual and death related to monogenic AIDs.

Results: Seventy (46 female) patients with monogenic AIDs were analyzed. Consanguinity rate was $74.6 \%$ and a family history of AIDs was present in $60 \%$. The mean age at disease onset was $3.2 \pm 2$ years. The initial diagnosis was inaccurate in $47 \%$ and the mean diagnosis delay was $4 \pm 3$ years. The most frequent monogenic AIDs were LACC1 associated monogenic JIA and monogenic Crohn's (23) followed by CAPS (12), TRAPS (12), HIDS (9), Majeed's syndrome (6). Musculoskeletal involvement was the main feature in LACC1 associated monogenic disorders. Genetic testing was performed in 67 patients, $69 \%$ had genetically confirmed disease. Patients with mutation c.T850C p.C284R in exon 4 of LACC1 had severe arthritic changes. Three CAPS patients with NLRP3 mutation had cognitive impairment and one with significant hearing and ocular damage. Two HIDS patients had homozygous p.V3771 mutation and other two patients with p.V3771/compound heterozygous MEFV: p.E148Q/p.P369S/ p.R408G. Three different LPIN2 mutations were recorded for Majeed's syndrome. Overall, growth failure was the most frequent (36\%), followed by cognitive impairment (13\%). There were three deaths due to infection. Conclusion: The number of genetically confirmed patients with monogenic AIDs other than FMF are not uncommon among Arab children probably due to a high consanguinity rate. Diagnostic delay and high damage accrual emphasize the need for more awareness and early referral to specialized centers.

\section{EAP 2019 Congress and Master Course Poster Presentations}

\section{3}

\section{Adolescent Medicine}

Young People in Crisis- Assessing Risk of Mental Health Patients in the Children`s Emergency Eeparment

Adla Alattas $^{1}$, Amit Gite ${ }^{1}$, Kim Rundell ${ }^{1}$, Kim Pierson ${ }^{l}$, Elizabeth Bessell $^{I}$, Ana Merino ${ }^{I}$

Elia $^{l}$

${ }^{1}$ Paediatrics, Lister Hospital, UK

Mental health $(\mathrm{MH})$ disorders in children and young people (CYP) is on the rise with many CYP presenting acutely to the emergency department (CED) in 'crisis'.CYP presenting to CED will be initially triaged and cared for by non-MH professionals while awaiting assessment by a children's crisis team. There are often complex and multiple issues for these CYP in addition to their medical needs. A Mental Health Risk Assessment (MHRA) tool was introduced to help non-psychiatric trained medical staff in their initial and ongoing assessment.

To conduct a demographical analysis of CYP presenting to CED with acute MH issues in 2018 as well as evaluating our MHRA-tool in risk assessing these patients.

Methods: We collected data including age, gender, diagnosis and duration of admission for all CYP presenting to CED with acute $\mathrm{MH}$ problems over a one-year period. Following the introduction of a MHRA-tool we collected feedback from CED nurses and healthcare assistants over a 3-month period. Results: There were $380 \mathrm{MH}$ admissions to CED over a one-year period. Age of presentation was 9-16 years of age. There was a female:male ratio of 2.7:1. 33\% of CYP presented with self-harm. Nearly $40 \%$ of CYP presented with suicidal attempts or ideation. Over $80 \%$ were admitted for 24-hours, with the average wait for crisis team assessment being about 17 hours. Over $60 \%$ of nursing staff found the MHRA-tool a useful means to ask questions relating to potential risk in CYP during admission. Conclusion: CYP with MH disorders who present to CED in 'crisis' often have multiple and complex issues.

Non-MH trained medical staff need a strategy and formal means to make a risk assessment while CYP await formal psychiatric review.

We recommend using a locally approved MHRA-tool to objectively risk stratify and assess such patients whilst waiting for a mental health assessment.
515

\section{Adolescent Medicine}

Pyometra: A Rare Cause of Acute Abdomen in Prepuberal Girls Maria Bauluz-Barcena ${ }^{1}$, Pilar Fernández-Eire ${ }^{2}$, Laura González ${ }^{3}$, Irene Garcia $^{3}$, Vanesa Rodriguez, Emilio Couceiro ${ }^{3}$

${ }^{1}$ Pediatrics, Hospital Alvaro Cunqueiro, Spain

${ }^{2}$ Pediatric Surgery, Hospital Alvaro Cunqueiro, Spain

${ }^{3}$ Gynecology, Hospital Alvaro Cunqueiro, Spain

Background: Pyometra is the accumulation of purulent fluid in uterine cavity. It is a severe, life-threatening condition. This rare entity is mostly described in postmenopausal women with acquired stenoses of external cervical OS. However, some congenital malformations of female genital tract have been reported as an infrequent cause of pyometra.

Objective: To describe a rare entity with a challenging diagnosis.

Method: We report an exceptional case of a 13-year-old patient with generalized peritonitis due to pyometra. She had no previous medical records, neither had she reached menarche. She presented on Pediatric Emergency Department with severe suprapubic abdominal pain and anorexia for the last 4 days. As she would add later, she had been suffering from suprapubic pain, diarrhea and vomiting once in a month, for the last 6 months. Physical exploration: abdominal guarding, intense hypogastrium pain, normal female genitalia, low-grade fever. Analytical: leukocytosis, increased CRP. On suspicion of acute abdomen, an urgent abdominal ultrasound was made, showing fluid collection in uterine cavity that extended to peritoneal cavity through Fallopian tubes. Gynecologists, under general anaesthesia, performed ultrasound-guided introduction of a grooved probe, through a completely stenotic cervical OS into the uterine cavity, in order to drain the uterus (picture 1). Later, a pediatric surgeon performed a laparotomy, profuse lavage and evacuation of purulent fluid from peritoneum (picture 2). Reproductive organs were preserved, and a Foley baloon catheter was left inside the uterus for 2 weeks. No incidents were recorded during postoperative evolution, so she was discharged. One month later, she was asymptomatic and did not reach menarche yet.

Conclusions: Peritonitis is not always appendicular. Although treatment of pyometra in postmenopausal women is well established involving hysterectomy, it might be challenging in pubertal girls, as preservation of reproductive organs is a priority. Long-term effects over fertility in this patient remain still uncertain.

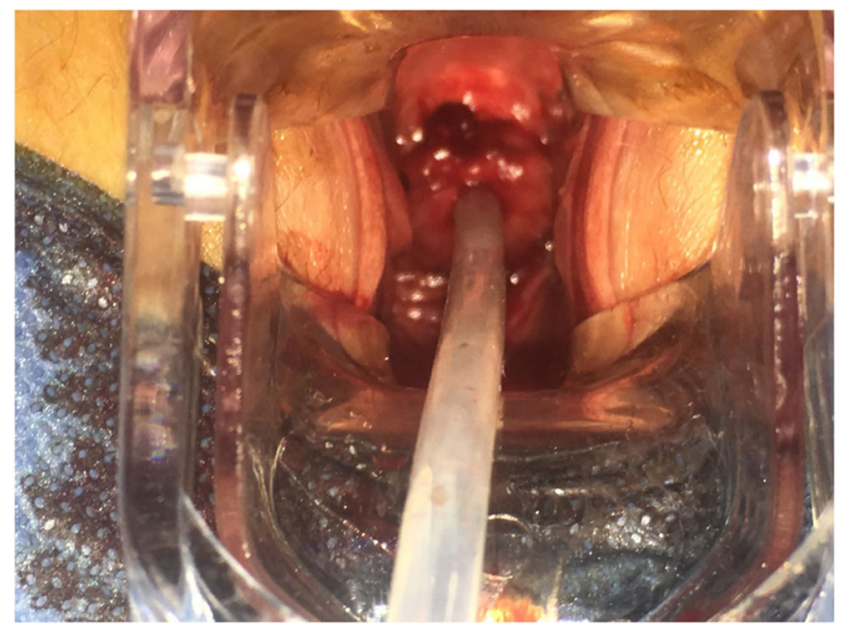

609

Adolescent Medicine

Art Based Support Sessions for Paediatric Inpatients in a District General Hospital

Shavindra Chellen ${ }^{I}$, Yasmin Dosanjh ${ }^{1}$, Su Laurent ${ }^{l}$, Vaseem Hakeem ${ }^{l}$ ${ }^{T}$ Children's Services, Royal Free London NHS Foundation Trust, UK 
Background: In the United Kingdom, there is an increasing emphasis on supporting the additional needs of children and young people in hospitals with the use of schoolteachers and play therapists. Art based support sessions provide a dedicated way for children and young people to express their feelings non-verbally and develop a sense of achievement, positivity and self worth during their inpatient stay.

Objective: Art based support sessions create a sense of achievement and provide a purposeful activity for inpatients, increasing their resilience whilst also encouraging interaction. Personal expression can also help patients process personal issues, which in turn may help with mental health conditions. The positive relationships formed with an art therapist can also enable patients to develop better coping strategies. Art based support sessions induces 'flow' - which is defined as a subjective psychological state of total involvement in an activity. Often cited as bringing about highest sense of wellbeing and overcoming poor concentration.

Methods: Over a one-month period from May 2017 to June 2017, appropriate inpatients were identified by nursing and medical staff and offered the chance to attend an art based support session. Inpatients who volunteered attended one session with a student art therapist. Involvement in these sessions was on a voluntary basis and each session lasted for 1 hour.

Results: 10 patients were involved, ranging from 7 to 16 years old. Written feedback was collected from patients following each session. $70 \%(7 / 10)$ described the session as brilliant. $90 \%(9 / 10)$ wanted another session. 80\% (8/10) would like the session to be longer.

Conclusion: Our feedback shows that children enjoyed these sessions and they distracted them from stressors they faced whilst being hospital inpatients. During these sessions we believe that children experienced a sense of achievement and positivity, developed increased resilience and had an opportunity for personal expression.

303

\section{Adolescent Medicine}

How well are we Involving Children and Young People (adolescents) in Consent to their Surgery? An Audit of Practice Sherine Dewlett ${ }^{l}$, Deborah Davidson ${ }^{l}$

${ }^{T}$ Paediatrics, Royal National Orthopoedic Hospital, UK

Background: It is good practice to involve children and young people (CYP) in consent to treatment. 16-17 year olds are presumed to have capacity. The Royal National Orthopaedic Hospital is a specialist centre performing complex often high risk orthopaedic surgery on CYP, a high proportion of which are adolescents and vulnerable children .

Objectives: We sought to find out how many children are involved in the consent process, if mental capacity was assessed and if parental responsibility was documented with a view to improve the quality of the consent process. Methods: A retrospective audit of electronic notes of children admitted in August 2018.

Results: 108 patients assessed. 22 (20\%) were 16-17 year olds , 78 (72\%) were 10-17 year olds (adolescents) . 72\% of 16-17 year olds countersigned forms but parents gave primary consent. Only 4\% of 16-17 year olds gave primary consent with parents counter signing. Involvement increased with age : $50 \%$ of 10 year olds ; $80 \%$ of 17 year olds countersigning forms. Senior doctors gained informed consent- $70 \%$ being consultant or registrar level. Mental capacity assessment ( MCA) was not documented . Parental responsibility was assessed $94 \%$ of the time. Only 1 child may have lacked capacity; consent was signed by parents and MCA was not documented.

Conclusion: we are involving CYP in consent to surgery but can improve numbers signing consent forms and documentation of MCA. We have tackled this by educational interventions to doctors seeking consent ; a short film with adolescent involvement aimed at CYP and their families ; an opt out policy of consent rather than opt in by using form 1 (primary consent to treatment) in 16-17 year olds rather than form 2 (parental consent ) and added MCA documentation in the admissions booklet and will re-audit.
990

Adolescent Medicine

Shortage of Children and Adolescent Mental Health Service (CAMHs) Beds is the Most Common Reason in Delayed Transfers of Care (DTOCs) in Paediatric Ward

Lucy Walker ${ }_{-}^{\text {, Sanjay Wazir }}{ }_{-}^{1}$, Mojgan Ezzati ${ }^{1}$

${ }^{I}$ Paediatric, Homerton University Hospital NHS Foundation Trust, UK

Background: There is a national shortage in CAMHS beds throughout the UK. Additionally, discharge pathway for adolescent and young children with safeguarding and mental health concerns in paediatric ward encompasses close working relationship between acute, mental health and social care services. Yet, there is increasing pressure on all services in regards to patient flow which compromise this partnership hence patient care.

Objectives: To facilitate discharges of paediatric patients with mental health and safeguarding concerns by counting and collecting data about delayed transfer of care from acute setting to mental health or social care services.

Methods: A retrospective survey was conveyed on all paediatric admissions with mental health and safeguarding concerns over a period of 12 months (March 2018-March 2019) at our paediatric ward at Homerton University Hospital. A total of 25 adolescent patients were admitted in the paediatric ward with mental health and safeguarding concerns during this period. Data were collected from their case notes about the safeguarding, medical and mental history and management. Also, data was collected to compare the dates when they were medically fit for discharge with when they were safe to be discharged including the reasons behind delay for discharge.

Results: There was a total of 57 days over the period of 1 year when patients were medically fit for discharge but not fit for discharge due to shortage of CAMHS beds, awaiting discharge planning meeting, court hearing and housing issues. Of note the most common reason in delay in transfer of care was due to shortage of CAMHS beds followed by housing problem. However, in just less than half admissions, there was no delay in transfer of care.

\section{8}

\section{Adolescent Medicine}

Intentional Drug Intoxication in Adolescents: Who, When, How and Why?

Ana Bernardo Ferreira ${ }^{I}$, Sara Rodrigues ${ }^{I}$, Diana Reis Monteiro ${ }^{I}$, Joana Cardoso', Lúcia Gomes $^{I}$

${ }^{I}$ Pediatric/Neonatology Department, Centro Hospital Entre Douro e Vouga, Portugal

Background: Self-harm behavior in adolescents is a worrying reality. Understanding the patterns and risk factors underlying Intentional Drug Intoxication (IDI) may help prevent it.

Objective: To characterize IDI cases with prescription drugs in adolescents, managed in the emergency department (ED) of a level II hospital, between 2014 and 2018.

Methods: Retrospective study of IDI in adolescents, ages 12 to 17 . Through consultation of medical records, we analyzed patient sociodemographic, time of ingestion, type, number and owner of medication used, management in ED and at discharge, and psychopathology history.

Results: There were 90 episodes of IDI in 85 individuals (71 female). Mean age at first episode was 15 years (similar to median value). Oral intake was the only intoxication route; in 45 cases, only one drug was taken. The most used group of substances were psychiatric drugs $(50,4 \%)$. In $36.6 \%$, the drug belonged to the patient and was a psychotropic medication. Most episodes occurred during the afternoon and at night, and during spring and winter. The main motivation for drugs intake was family conflict $(42,2 \%)$, followed by problems in romantic relationships $(28,9 \%)$. Seventy patients had a psychiatric evaluation before discharge; 4 were admitted to a psychiatric service due to structured suicidal intent. Sixty- 
three percent had a previous history of psychiatric/psychologic follow-up. Psychopathology or history of addiction was present in $35.3 \%$. In $27,1 \%$, parents were divorced. Seven had a history of institutionalization, and other 16 cases were under social services following.

Conclusion: Immaturity, misjudgment, impulsivity and lack of coping strategies are related to self-harm behavior, including IDI, in adolescents. Most times, there is no apparent structured suicidal intent. Emotional conflicts, social problems and a personal and family history of psychopathology are risk factors for this behavior. It is important to implement preventive measures, as follow-up of risk patients in a pediatric/ psychiatric consultation.

\section{8}

\section{Adolescent Medicine}

Internet Addiction, A New Epidemics?

Carmen-Raluca Ghionaru ${ }^{1,2}$, Elena Ruja ${ }^{1,3}$, Vlad-Alexandru Ghionaru,

${ }^{1}$ Family Medicine, Romanian Association for Pediatric Education in Family Medicine AREPMF, Romania

${ }^{2}$ Family Medicine, Family Medicine Practice, Romania

${ }^{3}$ Family Medicine, Family Medicine Practice, Romania

${ }^{4}$ Faculty of Law, University of Bucharest, Romania

${ }^{5}$ Sorbonne Pantheone I, College Juridique franco-roumain d'études européenes, Romania

Background: Being concerned about internet addiction, American researchers have included this condition in compulsive-impulsive disorders. In some countries, officials have set up treatment centers or organized educational programs in schools to stop the spread of the epidemic. In 2016, the World Health Organization included game addiction in the International Diseases Index (ICD11), including in this category also computer game or online addiction. In Romania, we have no real data on the extent of this phenomenon or the need to implement educational programs in schools.

Aims and Objectives: Identify and quantify the forms of internet addiction among secondary school, high school and university students.

Material and Methods: We conducted a qualitative observational survey on a total of 404 subjects, who completed a standardized questionnaire that quantifies internet dependency and classifies it in three mild, moderate, severe forms.

Results and Discussions: $77.6 \%$ of the subjects are internet-dependent to a certain extent $(64.7 \%$ mild dependence, $12.5 \%$ medium and $0.4 \%$ severe). We noticed that the proportion of those who have the medium form of internet addiction is increasing as they get older. (58\% middle school, $67 \%$ secondary school and 74 students), while the rate of no addiction persons decreases with age. There were no forms of severe dependence among high school students.

Conclusions: The study shows that we may be on the verge of an internet abuse epidemic and the necessity to implement educational programs in schools with the goal of acquiring the ability to use the internet for useful purposes, without being at the risk of developing addiction.

488

\section{Adolescent Medicine}

New Trends of Substance Use in Teenagers

Ana Lia Goncalo ${ }^{1}$, Mariana Maia ${ }^{l}$, Ana Luisa Correia ${ }^{l}$, Sara Silva ${ }^{l}$, Ana Aguiar

${ }^{1}$ Pediatrics, Unidade Local de Saúde Matosinhos - Hospital Pedro Hispano, Portugal

Background: Adolescence is a period of important physical, psychological and emotional development. The need to become independent and to experience new sensations is associated with risk behaviors, such as use and abuse of tobacco, alcohol and illicit drugs.

Objective and Methods: To evaluate new trends of substance use in teenagers, by searching medical databases and journals for published articles in the last 5 years, using the keywords "substance", "use", "adolescents"

Results: The most common substances used by adolescents are alcohol and tobacco, as they are more readily available. Although a decline of cigarette smoking in adolescents has been observed in the last decades, ecigarettes are recently gaining popularity and their use has been associated with initiation and then transition to more frequent cigarette smoking. Despite the similar decrease in consumption among teens, alcohol remains the main cause of intoxications admitted to the Pediatric Emergency Department in several European countries (including Portugal) and the differences between genders' consumption habits tend to disappear. Marijuana is increasingly displacing alcohol and cigarettes as the first substance used, and is causally associated with subsequent polysubstance use. Since the mid 2000s New Psychoactive Substances (NPS) have been available on the market, specifically designed to imitate the effects of established illicit drugs, and untruly known as legal. Their use among adolescents is still low, but increasing, as they reported being more likely to try drugs if they are legal.

Conclusion: Adolescent substance use has been declining worldwide. This decreasing trend is probably a result of intensively provided preventive and educational measures, increasing the public awareness of the severity of underage substance use. Of major concern, the more prominent role of marijuana in the early stages of drug use and the rise of NPS, given the considerable potential for harm, including hospitalizations, overdose and fatalities.

\section{1}

\section{Adolescent Medicine}

Post-Traumatic Panhyopituitarism in a Teenager- A Multidisciplinary Challenge

Mihaela Gutu-Robu ${ }^{1}$, Raluca Maria Vlad ${ }^{1,2}$, Irina Dijmarescu ${ }^{1,2}$, Daniela Pacurar ${ }^{\text {I, }}$

${ }^{I}$ Paediatrics, Grigore Alexandrescu Emergency Children's Hospital, Romania

${ }^{2}$ Paediatrics, Carol Davila University of Medicine and Pharmacy, Romania

Background: Post-traumatic panhypopituitarism in children is a rare plurisymptomatic complication of traumatic brain injury, with major impact on the child's development and quality of life. Endocrine screening after trauma is controversial.

Objective: We highlight the steps leading to diagnosis in the case of an adolescent hospitalised for unrelated pathology in order to increase awareness on this rare condition among physicians.

Methods and results: We report the case a 17-year-old boy admitted in March 2019 in „Grigore Alexandrescu” Hospital, Bucharest. The adolescent had a history of severe brain injury (age 13) due to a car accident. He was hospitalized 4 years later in the Orthopaedics Department for surgical correction of right hip chronic epiphysiolysis. The laboratory tests showed liver cytolysis, so he was transferred to the Paediatrics Department. The physical examination revealed coarse features, dry, harsh skin, coarse hair, the absence of face and body pilosity, absent secondary sex characteristics, infantile genitalia, hypopigmented skin lesions, inferior limb inequality with no other pathologic findings. Laboratory investigations showed decreasing transaminases, positive serology for EBV, low values of thyroid hormones, inadequate TSH, hypocortisolaemia, low testosterone and IGF 1. Ophthalmologic evaluation revealed post-traumatic right eye cecity. The endocrinology consult set the diagnosis of panhypopituitarism. Substitution treatment was recommended (Prednisone, L-Thyroxine, Testosterone). The cranio-cerebral MRI showed small pituitary gland, right deviated pituitary stalk, unidentifiable neurohypophysis and right optic nerve, parasphenoidal herniation of the arachnoid. 
Hip surgery was postponed due to potential anesthesia risks. He was referred to the Endocrinology Department for further assessment and treatment.

Conclusion: Post-traumatic rare complications such as panhypopituitarism should be considered even a long time after trauma. Focusing on solving the trauma might lead to a late diagnosis of the condition. A well-established multidisciplinary approach is needed if the child is to safely overcome any acute condition, considering the severe cortisol deficiency.

703

\section{Adolescent Medicine}

Risk Assessment of Liver Pathology in the Treatment of Juvenile Idiopathic Arthritis in Adolescence

Olga Pavloval

${ }^{T}$ Department of Adolescent Medicine, Kharkiv Medical Academy of Postgraduate Education, Ukraine

Background: Juvenile idiopathic arthritis (JA) is the most common type of arthritis in children under the age of 16. Liver disturbance has been attributed not only to the liver disease associated with rheumatoid diseases themselves but also with such factors as fatty infiltration, drug toxicity, thrombotic accidents, or autoimmune liver disease. AST to Platelet Ratio Index (APRI) is relevant for determining the cohort of children that requires further liver examination.

Objective: To study the most vulnerable contingent of patients with liver damage in the treatment of JIA methotrexate using the APRI index

Methods: A total of 73 children aged 10 to 18 years old, 43 girls and 30 boys were observed. The children were divided into five groups by treatment with methotrexate (MTX) (I - did not receive MTX (15 children), II - received up to 1 year (16 children), III - 1-3 years (13 children), IV - 3 -6 years ( 14 children); $\mathrm{V}$ - more than 6 years ( 15 children)). The APRI index was based on the generally accepted formula.

Results: In children with JIA, who did not receive methotrexate APRI index was $0.34 \pm 0.04$. Patients in group II - $0.61 \pm 0.18$ (p

Conclusion: Children receiving methotrexate up to 1 year have highest risk of liver damage. Use of methotrexate more than 1 year reduces risk of liver disease. For a clearer understanding of pathological changes in the liver, further research is needed.

\section{5}

\section{Adolescent Medicine}

Clustering of Health-Related Behaviors and its Relationship with Individual and Context Factors in Portuguese Adolescents: Results from a Cross-Sectional Study

Constanca Santos $^{1,2}$, João Picoito ${ }^{1,3}$, Isabel Loureiro ${ }^{1}$, Carla Nunes ${ }^{1}$ ${ }^{T}$ Escola Nacional de Saúde Pública, Universidade NOVA de Lisboa, Portugal ${ }^{2}$ Department of Pediatrics, Centro Hospitalar Cova da Beira, Portugal ${ }^{3}$ Department of Child and Adolescent Psychiatry, Centro Hospitalar Universitário de Coimbra, Portugal

Background: Health behaviours are shaped early in life, laying the foundations of future health. Unhealthy behaviours tend to occur in complex specific patterns and are influenced by individual and context factors.

Objective: We aimed to identify and characterize patterns of healthrelated behaviours among Portuguese adolescents and correlate them with individual and context factors.

Methods: This study was based in Portuguese 2009/10 survey of Health Beahviour in School Aged Children Study, comprising 4036 adolescents. Individuals were partitioned into groups using two step cluster analysis based on 12 variables regarding diet, physical activity, screen use and substance use. The association between cluster membership and individual, family, school and peer factors was calculated based on crude and adjusted odds ratio using a multinominal regression.
Results: The median age was 13,6 and 54\% were of the female gender. Overweight and obesity were highly prevalent (25\%), as of sedentarism, with only $13 \%$ exercising daily. $78 \%$ lived with both parents and $59 \%$ had medium-low affluent families. We identified four distinct behavioural clusters: "Active screen users" (31,1\%), "Substance users" $(13,3 \%)$, "Healthy" $(26,4 \%)$ and "Sedentary low fruit and vegetable eaters" $(29,1 \%)$. Sociodemographics varied across clusters, showing a positive and significant association between male gender, poor family communication, violent behaviours, poor academic performance and school attachment and the "Substance users" and "Active screen users" clusters and between a low socioeconomic status and the "Sendentary low fruit and vegetable eaters" cluster. We found no association between nutritional status and cluster membership.

Conclusion: Cluster analysis identified several health compromising behaviour patterns. The understanding of these behavioural clusters and its relationship with individual and context factors is of extreme use to Public Health, allowing tailored health-promoting interventions. Further research is needed to understand how cluster membership evolves over time and its influence on nutritional status.

\section{4}

\section{Adolescent Medicine}

Adolescents in the Emergency Room -Three Years in a Pediatric Medical-Surgical Emergency Department

Joana Santos $^{1}$, Silvia Almeida ${ }^{1}$, Inês Sobreira ${ }^{l}$

${ }^{T}$ Pediatric Department, Centro Hospitalar do Baixo Vouga, EPE, Portugal

Background: World Health Organization defines adolescence as the group from 10 to 19 years old. This stage is characterized by fast physical, psychological and social changes. Despite being overall healthy, high prevalence of risk behaviors rises morbidity in this group. It is important to recognize the leading causes of adolescent admission in the emergency services, to invest in prevention, early intervention and promote health.

Objective: Characterize adolescent admissions in emergency department.

Methods: Retrospective analysis of informatic records of adolescent emergency episodes, observed from January, 2014 to December, 2016, at the Pediatric Emergency Department of Centro Hospitalar do Baixo Vouga, EPE. Results: In the analyzed period, there were registered 38363 emergency adolescents episodes. $67 \%$ of the admitions were due to "DISEASE", $24,1 \%$ due to "ACCIDENTS" (road, sports, personal, work, domestic and school accidents), $0,7 \%$ were "INTOXICATIONS", $0,5 \%$ for "ASSAULT" episodes and $0,7 \%$ admissions of "PREGNANT AND PARTURIENT".

One third of the "DISEASE" admissions were due to respiratory disease and one sixth due to digestive disease. Mental health and behavioral issues were responsible for $4 \%$ oh these diagnostics.

$58,3 \%$ of the adolescents was observed only by Pediatrics specialists, and the others needed collaboration from other specialties.

Conclusion: $26 \%$ of the admissions were due to preventable causes, like accidents, intoxications, agressions or pregnancy. It's urgent to act at primary prevention level, but it's also important to take every contact chances to identify those at risk and decrease this group morbidity.

\section{9}

Adolescent Medicine

Health Care Improvement Facing Child Abuse. Mistakes to be Avoided and New Challenges to be Addressed

Eva Maria Navarro Villalba ${ }^{2}$, Mariano Silva Hernandez ${ }^{1}$, Virginia Santana

${ }^{1}$ Pediatría, Hospital Universitario La Paz, Spain

${ }_{2}^{2}$ Pediatría, Centro de Salud Barrio del Pilar, Spain 
Background: Child abuse is an increasing reason of consultation, either in Pediatric Primary Care (PPC) or in Pediatric Emergency Departments (PED). Healthcare professionals typically have not received proper training on the subject at hand. This results in mistakes in patient care that could be avoided.

Clinical case: We herein describe a study case of a 15-year-old male patient who is brought by his mother to our PED, referred from a tertiary-level Hospital after having conducted higher risk intercourse with an adult male. The youngster had arranged a meeting using a mobile phone application. At the first PED he visited, no clinical samples were collected, although medical examination was executed. Instead, the patient was directly referred to the National Police, who took judicial samples with the collaboration of a forensic doctor.At our PED, which he was later referred to, the patient and his mother handed the medical report from the first PED, so no new interrogations or physical examinations were carried out. Following our Hospital's Child Molestation Protocol a blood and urine sample was tested for sexually transmitted diseases and a negative urine toxicity study was carried out. After evaluating the results, the patient began taking the post-exposure prophylaxis and antibiotic prophylaxis medicines. Finally, he was given an appointment with the Pediatric Infectology Consultation so that a proper follow-up could be carried out.

Conclusion: Child abuse is a frequent reason of consultation in PPC services, for which Healthcare professionals (HP) are not completely prepared to assist. Usually, they have not received proper training to do so, and they are not aware of the procedure to be followed in terms of observation, notification and management. Accordingly, a standardized Health Care Protocol should be developed and introduced. HP should receive specific and proper training to successfully address this issue and provide the best patient care possible. The need for the abovementioned is particularly strong, considering the increasing amount of cases taking place,and the new ones arising from the use of the New Technologies.

\section{3}

\section{Allergy \& Immunology}

Improving of the Detection of Primary Immunodeficiency Diseases through Implementation of Educational Programs

Oksana Boyarchuk ${ }^{l}$, Tetyana Hariyan ${ }^{l}$, Maria Kinash ${ }^{l}$, Lyubov Volyanska', Olga Rusanovska ${ }^{2}$, Tetyana Kovalchuk ${ }^{1}$

${ }^{1}$ Department of Children's Diseases and Pediatric Surgery, I.Horbachevsky Ternopil State Medical University, Ukraine

${ }^{2}$ Department of Maternity and Childhood Protection, Uzhhorod National University, Ukraine

Background: Early detection of primary immunodeficiency diseases (PID) is vital for adequate prevention and management of PID infectious complications.

Objective of this study was to improve the primary immunodeficiency diagnostics through implementation of the model combining physician education and public awareness with the infrastructure to diagnose these diseases. Methods: Three strategies combination has been followed from February 2017 in Ternopil region, Ukraine. The first was education of primary-care physicians (pediatricians, GP doctors) and other specialists (rheumatologists, otolaryngologists, pulmonologists, surgeons, etc.) in early PID symptoms and detection using workshops, trainings, and targeted publications. The second was organization of public events, media appearance to raise PID awareness. The third was undertaken immunological testing for patients with suspected PID.

Results: Workshops and trainings for primary care physicians on early PID detection were organized in 2017 in every regional district (15 districts were totally covered). Generally 540 physicians and nurses attended the lectures and workshops, including pediatricians, general practitioners, etc. We also visited the outpatient departments, talking to doctors, patients and their parents about the PID warning signs.
The examination of the children at risk for PID was conducted during the visits. We developed examination cards of children with suspected PID. These children were referred to the regional children's hospital for further examination. We have translated into Ukrainian and published educational materials: warning signs, testing stages. We have also created some educational materials about PID which were disseminated among physicians.

By the beginning of 2017, there were 11 children with PID in our region, at the end of 2018 their number increased to 31 . We also follow 12 patients with suspected PID that need monitoring and examinations to finalize the diagnosis. Conclusion: Implementation of the model combining physician education and public awareness with the infrastructure to diagnose these diseases is effective strategy to increase PID diagnosis.

799

\section{Allergy \& Immunology}

Multimorbidity of Upper Respiratory Tract Pathology in Children with Bronchial Asthma

Tatyana Eliseeva', Svetlana Krasilnikova ${ }^{2}$, Andrei Shakhov ${ }^{2}$, Ivan Balabolkin ${ }^{3}$,Elena Tush ${ }^{1}$, Vilya Bulgakova ${ }^{3}$, Olga Khaletskayal

${ }^{1}$ Department of Pediatrics, Privolzhsky Research Medical University, Russia

${ }^{2}$ Department of Otolaryngology, Privolzhsky Research Medical University, Russia

${ }^{3}$ Institute of Pediatrics, National Medical Research Center of Children's Health, Russia

Background: Bronchial asthma (BA) is a systemic allergic disease and is associated with upper respiratory tract (URT) pathology. In recent years, attention was focused on allergic diseases multimorbidity, while the spectrum of URT pathology in children with BA is not characterized enough. Purpose: To study the structure of URT pathology in children with atopic BA. Method: 358 children with atopic BA were examined, the average age of children was $9,91(9,47 ; 10,35)$ years, of which $67,9 \%$ were boys $(192 /$ 358 ), and 108 children with nasal breathing disorders, comparable in age and sex, but without BA. In addition to the standard all - clinical, allergological, functional examination, all patients underwent video endoscopic examination of the nasal cavity and nasopharynx.

Results: At children with BA were diagnosed with allergic rhinitis (AR) and/ or allergic rhinosinusitis (ARS) with a predominance of persistent forms. The «isolated» course of AR/ARS occurred in 11,7\% (42/358) of patients; other children had nasal symptoms due to a combination of AR/ ARS with other variants of URT pathology. Pharyngeal tonsilhypertrophy occurred in $61,2 \%(219 / 358)$ of patients, hypertrophic rhinitis - in 9,2\% (33/358) of children with BA, nasal architectonics disorders were diagnosed in $50 \%$ (179/358) of patients. The combination of two nosological variants of nose pathology occurred in 47,8\% (117/358) of patients with BA; $40,5 \%$ (145/358) of children with BA had multimorbidity of the nasal pathology - a combination of three or more nosological units.

Conclusion: For children with atopic BA and nasal symptoms often have combined and multimorbid forms of URT pathology. Verification of nasal obstruction causes allows to individualize therapy for patients with BA and minimize negative impact of URT pathology on BA course.

\section{4}

\section{Allergy \& Immunology}

The State of the Autonomic Nervous System in Children with Bronchial Asthma

Elena Tush $^{1}$, Tatyana Eliseeva ${ }^{1}$, Olga Khaletskaya ${ }^{1}$, Vilya Bulgakova ${ }^{2}$, Ivan Balabolkin ${ }^{2}$

${ }^{1}$ Department of Pediatrics, Privolzhsky Research Medical University, Russia ${ }^{2}$ Institute of Pediatrics, National Medical Research Center of Children's Health, Russia 
Background: Violation of vegetative regulation (VR) is a component of the pathogenesis of bronchial asthma (BA).

Purpose: Determine the relationship of VR parameters with the parameters of asthma control level in children using methods available in a wide clinical practice.

Method: 88 patients ( 54 boys and 34 girls) aged 5 to 17 years with atopic bronchial asthma were examined. Quantitative assessment of bronchial asthma control was carried out using questionnaires Asthma Control Questionnaire-5 (ACQ-5), Childhood Asthma control test (ACT-C) in children under 12 years old, and Asthma control test (ACT) in children and adolescents aged 12 years and older. All children underwent a standard examination with determination of blood pressure, pulse, respiratory rate, with the calculation of Kerdo and Hildebrandt indices, characterizing vegetative regulation. Taking into account the age-dependent changes in heart rate, we used for the first time a relative heart rate index equal to the ratio of the patient's heart rate to the median heart rate for this age group. Results: Correlation with ACQ-5 was obtained for the Hildebrandt index $(r=0,45 p=0,0003)$, the respiratory rate $(r=-0,27, p=0,032)$ and the relative pulse index $(r=0.40, p=0,0012)$. The association of Kerdo index with ACT$\mathrm{C}$ test values $(\mathrm{r}=-0,32, \mathrm{p}=0,045)$ was established. In the group of patients with no BA control the Hildebrandt index was statistically significantly higher than in patients with control of the disease. In children with uncontrolled asthma it was 5,23 $\pm 0,25$ units that exceeds normal values and may reflect a mismatch in the work of cardiovascular and respiratory systems. Conclusion: The interrelation of changes of vegetative regulation and the level of control of bronchial asthma in children is established, as well as the mismatch of the functioning of the cardiovascular and respiratory systems in children with uncontrolled asthma.

\section{6}

\section{Allergy \& Immunology}

The Value of Thyroid Status for the Control of Bronchial Asthma in Children

Tatyana Eliseeva ${ }^{l}$, Elena Tush ${ }^{l}$, Elena Bolshoval, Stanislav Ignatov ${ }^{2}$, Vilya Bulgakova ${ }^{3}$, Ivan Balabolkin ${ }^{3}$

${ }^{T}$ Department of Pediatrics, Privolzhsky Research Medical University, Russia

${ }^{2}$ Department of Chemistry, Lobachevsky State University, Russia

${ }^{3}$ Institute of Pediatrics, National Medical Research Center of Children's Health, Russia

Background: Despite numerous studies, there was no consensus about the effect of thyroid hormones on asthma.

Purpose: To assess the relationship between the patient's thyroid status and the level of BA control.

Method: 51 children with BA were examined, of them 15 girls aged $105.1+/-35.3$ and 36 boys aged 114.2+/-14.1 months. All children underwent general clinical examination, spirometry, ACQ5 assessment, determination of thyroid-stimulating hormone (THS) levels, free thyroxine (free T4) test, and levels of antibodies to thyroperoxidase (anti-TPO) by ELISA.

Results: Elevated TSH levels occurred in $12(23 \%)$ patients, while all of them had no clinical symptoms characteristic of hypothyroidism, and the maximum TSH rise was $8.45 \mathrm{mIU} / \mathrm{L}$, which made it possible to treat this condition as subclinical hypothyroidism (cGT). None of the patients had an increase in the level of anti-TPO. Another 15 people had a TSH level in the range of 1.16-1.97 mIU/l, the remaining 24 had 2.01-3.74. 18 - had an excess of T4 free, but it was insignificant - a maximum of $23.1 \mathrm{pmol} / \mathrm{l}$ at the upper limit of the norm of $22 \mathrm{pmol} / \mathrm{l}$, and was not accompanied by symptoms of thyrotoxicosis. Most often (in 33\% of cases) subclinical hypothyroidism occurred in patients with complete control of BA (28, TSH $3.39 \pm 1.96$ ), and was completely absent in patients with uncontrolled disease progression (5, TSH $1,87 \pm 0.73$ ). In patients with partial BA control, TSH levels of 2.0 to $4.1 \mathrm{IU} / \mathrm{L}(18$, TSH of $2.93 \pm 1.5)$ prevailed, which may indirectly indicate iodine deficiency. These differences do not reach the level of statistical significance $(\mathrm{F}=1.74, \mathrm{p}=0.1858)$. Free $\mathrm{T} 4$ levels were comparable in all groups $(16.57 \pm 1.93,16.67 \pm 3.04,15.82 \pm 4.93$, respectively).

Conclusion: Patients with achieved BA control have a rather high incidence of subclinical hypothyroidism (23\%).

\section{8}

\section{Allergy \& Immunology}

Acute Allergic Reactions to Drugs in Pediatric Practice Tatyana Eliseeva ${ }^{l}$,Elena Tush ${ }^{l}$, Polina Trushanina ${ }^{l}$, Vilya Bulgakova ${ }^{2}$, Ivan Balabolkin ${ }^{2}$, Olga Khaletskayal, Stanislav Ignatov ${ }^{3}$, Elena Kareva $^{4,5}$

${ }^{1}$ Department of Pediatrics, Privolzhsky Research Medical University, Russia ${ }^{2}$ Institute of Pediatrics, National Medical Research Center of Children's Health, Russia

${ }^{3}$ Department of Chemistry, Lobachevsky State University, Russia

${ }^{4}$ Department of Pharmacology, Pirogov Russian National Research Medical University, Russia

${ }^{5}$ Department of Pharmacology, I.M. Sechenov First Moscow State Medical University, Russia

Aim: Assessment of the clinical and epidemiological picture of acute allergic reactions to drugs in children.

Methods: A retrospective analysis of the medical documents of 76 patients aged 3-17 years hospitalized in a children`s hospital due to acute hypersensitivity reactions to drugs was carried out.

Results: The average age of patients was 7.6 years [7.0-8.2]: boys - 34, girls - 42. In most children, cutaneous manifestations of hypersensitivity prevailed. Hypersensitive reactions to drugs of the type of acute urticaria are diagnosed in $21 / 76(27.6 \%)$. In $17 / 76(22.4 \%)$ children there was a combined development of urticaria rash and angioedema. All clinical manifestations of acute urticaria occurred in 38/76 (50\%) patients. Various skin rash variants, including spotted-papular and morbiliform exanthema, which were quite rigid to therapy, in some cases prone to pigmentation, occurred in 31/76 (40.8\%) patients, while 19 of them had a combination data of eruptions with symptoms of angioedema. In total, angioedema combined with cutaneous manifestations of hypersensitivity occurred in 36/76 (47.4\%) patients hospitalized for drug hypersensitivity reactions. As the etiological causes of hypersensitive reactions to drugs in the sample of children, antibacterial drugs prevailed - in 45 of 76. In 12 children hypersensitivity reaction developed with the use of cephalosporins, 25 - aminopenicillin or protected aminopenicillin, in the remaining children - after the administration of cotrimoxazole, metronidazole, nitrofurans.

Conclusion: The most common clinical manifestations of side effects on medicines in children are urticaria and morbiiform (coripiform) exanthema. Attention is drawn to the high proportion of Quincke edema in this category of children, which can be very specific for children of childhood.

440

\section{Allergy \& Immunology}

Are Our Children with Asthma and/or Allergic Rhinitis Well Controlled? A Study using CARATKids.

Ana Rita Constante ${ }^{I}$, Zulmira Abdula ${ }^{I}$, Susana Alexandre ${ }^{l}$, Filomena Rebelo $^{T}$

${ }^{1}$ Pediatrics Department, Centro Hospitalar Oeste - Caldas da Rainha, Portugal

Introduction: Around $12.9 \%$ of children and $11.8 \%$ of adolescents suffer from asthma in Portugal, $60-80 \%$ of which have allergic rhinitis concomitantly (ARA). Clinical control evaluation is essential and there is only 
one validated questionnaire for Portuguese children - "Control of Allergic Rhinitis and Asthma Test for Children" (CARATKids). It consists of 8 questions for the child and 5 for their parents, with a maximum score of 13 . The following cut-offs were established: $\leq 3$ : controlled disease; $\geq 6$ : uncontrolled disease.

Objective: Evaluate clinical control of ARA in a group of children attending Paediatric Allergology consults using CARATKids and study the correlation between clinical score and peak expiratory flow (PEF), allergies in skin prick tests (SPT) and medication adherence.

Methods: Observational, descriptive and transversal study, using CARATKids in children diagnosed with asthma and/or allergic rhinitis, data from SPT, PEF and medication adherence. Statistical analysis with SPSS 23.

Results: We studied 31 children, mean age of 9.3 years (min. 6, max. 12). $93 \%$ had a correct inhalator technique and $38.7 \%$ an adequate PEF. $14.8 \%$ did not follow prescribed medication. The most common sensitisations in SPT were to mites. Regarding clinical scores, $38.7 \%$ had controlled disease and $22.6 \%$ uncontrolled disease, with a mean score of 3.97. The parents' score correlated better to the final score than the children's $(p=0,005$ vs $\mathrm{p}=0,024)$. Lower respiratory symptoms were statistically correlated to clinical score $(\mathrm{p}=0,016)$. There was no correlation between PEF or medication adherence and clinical score. Positive SPT to Gramineae and Parietaria were associated with worse clinical scores ( $\mathrm{p}=0,048$ e $\mathrm{p}=0,05$ respectively).

Conclusion: There is a tendency to undermine asthma's and rhinitis' symptoms, which may explain why only one third of the patients have controlled disease. Objective tools for clinical evaluation should be used frequently, such as CARATKids, to improve therapeutic adjustments and increase clinical control.

\section{4}

\section{Allergy \& Immunology}

Child with Insect Sting Induced Anaphylaxis

Marija Dimitrovska-Ivanova ${ }^{1,2}$, Irena Nikolova ${ }^{l}$

${ }^{T}$ Department of Pediatrics, Clinical Hospital Shtip, Macedonia

${ }^{2}$ Department of Pediatrics, Faculty of medical sciences, University "Goce Delcev" Shtip, Macedonia

Background: Anaphylaxis is an acute-onset and potentially lifethreatening allergic reaction that can be caused by numerous allergic triggers. Children with asthma are at higher risk to develop anaphylaxis caused from medicines, foods, insect stings and bites.

Objective: To present a case of insect sting induced anaphylaxis in 6 years old boy with asthma history.

Methods: Six years old boy with asthma history admitted to the hospital due to severe bronchial obstruction. At admission pale, subfebrile $(37,4 \mathrm{C})$, with tachydispnea and weezing, using abdominal muscules, intercostal and jugular retraction. Barrel shaped chest and increased anteroposterior diameter. Auscultation-vesicular breathing with prolonged expirium and weezing. $\mathrm{O} 2 \mathrm{sat}=85 \%$, $\mathrm{WBC}=20 \times 10^{9} / 1, \mathrm{CRP}=20 \mathrm{mg} / \mathrm{ml}$. Child was put on oxygen mask, inhalation with salbutamol, parenteralal corticotherapy and antibiotic. Continued regular inhalation maintenance therapy. On the fifth day, 15 minutes after parenteral application of Ceftriaxone child once felt the stinging pain in his right foot after it occurred aphonia, severe bronchial obstruction, swelling and redness of the face, lips, tongue, eyelids, ears and appearance of hives on the trunk and legs. Blood pressure $=90 / 50 \mathrm{mmHg}$, heart rate $=100 /$ min, respiration rate $=40 / \mathrm{min}$. Treated with oxygen mask, Amp. Adrenalin(1:10000) subcutaneous, anthistamines and corticosteroids intravenous, inhalation therapy with Adrenalin(1:10000), perenteral rehydration.

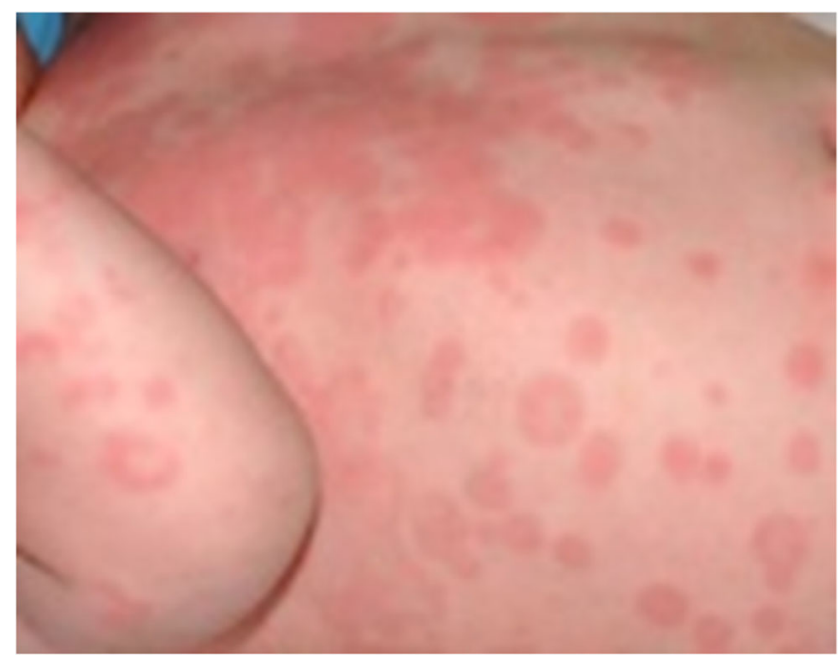

Results: After few hours with significantly reduction of redness and swelling on the face, lips, ears and eyelids, without hives on the trunk and legs and with easy bronchial obstruction treated inhalatory with salbutamol. Near the dorsum of the right foot was noticed insect sting with size of $2 \mathrm{~mm}$. After two days child was discharged in stable condition without respiratory or other allergy symptoms.

Conclusion: Children with allergic background are at higher risk of developing anaphylaxis from various allergens, including insect stings. Those who have experienced anaphylaxis need to be provided and trained how to use auto-injectable epinephrine.

1000

\section{Allergy \& Immunology}

Epidemiological Characteristics of Allergic Rhinitin in Children in Ukraine

Lyudmyla Duda $^{l}$, Olena Okhotnikova ${ }^{l}$, Olena Sharikadze ${ }^{l}$, Olena Usova $^{I}$, Nataliya Yakovleva ${ }^{1}$, Tatyana Tkachova ${ }^{I}$, Olena Ponochevna ${ }^{I}$, Olena Oshlianska ${ }^{1}$

${ }^{1}$ Department of Pediatrics \#1, Shupyk National Medical Academy of Postgraduate Education, Ukraine

Background. The results of recent studies have shown an increase in the prevalence of allergic rhinitis (AR) worldwide. Epidemiological information on AR in childhood proposes new hypotheses for further alethiological research into the genetic, lifestyle, environmental, clinical and medical-care factors for this disease.

Objective. To determine the prevalence of rhinitis in children and examine the age-old features of AR in Ukraine on the example of Kyiv region. Methods. Written questionnaires (methodology by ISAAC) were selfcompleted at school by 13-17-year olds and completed at home by parents of children of younger age groups. 7106 children: group I - 1787 (6 month-5 years), group II - 2080 (6-7 years), group III - 1909 (13-14 years), group IV - 1330 (15-17 years). The gender ratio of the sample is about $1: 1$.

Results. The determination of the rhinitis symptoms prevalence was performed by assessment of the answers to question "symptoms of rhinitis in the past 12 months", selected as the most sensitive criterion. The prevalence of rhinitis symptoms was $24,7 \%$ (759/7106), without significant gender difference $\left(\chi^{2}=0,6509 ; \mathrm{p}=0,419\right)$. In groups: $\mathrm{I}-20,0 \%$ [95\% CI: 18,2-22,0], II - 23,8\% [95\% CI: 21,9-25,6], III - 26,9\% [95\% CI: $24,9-$ 28,9 ], IV $-29,6 \%$ [95\% CI: 27,2-32,2]. The increase of rhinitis prevalence with age is observed $\left(\chi^{2}=44,039 ; \mathrm{p}=0\right)$. The prevalence of symptoms of rhinoconjunctivitis in groups: I - 10,6\% [95\% CI: 9,2-12,2], II 11,3\% [95\% CI: 10,0-12,7], III - 14,8\% [95\% CI: 13,3-16,5], IV - 10,8\% [95\% CI: 9,2-12,6]. AR was diagnosed in 14,2\% [95\% CI: 12,6-15,9] in 
Group I, 19,3\% [95\% CI: 17,7-21,1] in Group II, 20,5\% [95\% CI: 18,7$22,4]$ in Group III , IV - 20,4\% [95\% CI: 18,2-22,6] $\left(\chi^{2}=31,318 ; p=0\right)$. Conclusion. The level of prevalence of AR in children of Ukraine in Kyiv region is quite high, which requires further study.

196

\section{Allergy \& Immunology}

Fatal Streptococcus Pneumoniae Meningoencephalitis in a Fully Vaccinated 4 Year Old Boy

Roberta-Celine Ellul ${ }^{l}$, David Pace ${ }^{I}$, Simon Attard Montalto ${ }^{1}$, Kristian Micallef', Andre Gatt ${ }^{2}$

${ }^{1}$ Department of Paediatrics and Child Health, Mater Dei Hospital, Malta ${ }^{2}$ Department of Radiology, Mater Dei Hospital, Malta

A previously healthy four year old boy, born to non-consanguineous parents, had minor coryzal symptoms, fever and vomiting for 36 hours, treated empirically with oral antibiotics by GP. He was found jerking and unresponsive and rushed to hospital with a temperature of $37.8^{\mathrm{O}} \mathrm{C}$ and status epilepticus refractory to mannitol and repeated doses of midazolam and phenytoin. Shortly after admission, his conscious level deteriorated, requiring intubation and transfer to Intensive Care.

The white cell count was $44 \times 10^{9}$ cells/L, with a neutrophilia of $41.31 \times 10^{9}$ cells $/ \mathrm{L}$. His CRP was $233 \mathrm{mg} / \mathrm{L}$ on admission and $627 \mathrm{mg} / \mathrm{L}$ after 2 days. A CT and MRI brain scans done within hours of admission revealed meningoencephalitis with widespread meningeal pus-infiltrates, cerebral swelling, impaired diffusion, raised intracranial pressure and brainstem herniation ( Refer to Figure 1 and 2). The intracranial bolt pressure was $91 \mathrm{mmHg}$. Serum was PCR positive for Streptococcus pneumoniae, later confirmed as capsulated serotype 19F. He received ceftriaxone, vancomycin, metronidazole, clarithromycin and acyclovir without clinical improvement.Repeated brainstem function was absent after 3 days and life support was discontinued.

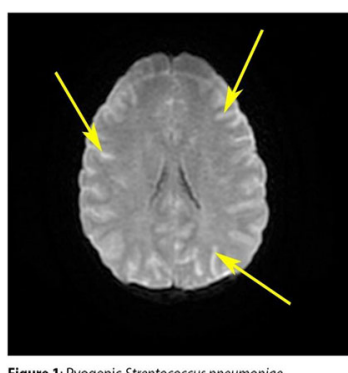

Figure 1: Pyogenic Sterter

meningoenct

secondary to leptomeningeal pus infittentes (yellow

secondary
arrows).

He had received all childhood vaccinations including four doses of a pneumococcal conjugate vaccine. There was no asplenia however immunoglobulins were low: $\operatorname{IgG} 1.2 \mathrm{~g} / \mathrm{L}$ (5.46-17.6), $\operatorname{IgM} 0.13 \mathrm{~g} / \mathrm{L}$ (0.26-1.21), and $\operatorname{IgA}$ $0.23 \mathrm{~g} / \mathrm{L}(0.52-2.84)$. Despite MMR and hepatitis B vaccination, he tested negative for Rubella and Hepatitis B surface IgG antibodies. A diagnosis of agammaglobulinaemia, possibly X-linked (XLA), was considered, particularly since a maternal first cousin had died of severe sepsis in childhood. In fact, subsequent DNA studies showed X-linked agammaglobulinaemia:c.871GC hemizygote in BTK gene. Maternal DNA is still being investigated.

The primary antibody deficiency syndromes, including XLA, usually present with recurrent respiratory tract infections due to an inability to mount an effective immunoglobulin response. Limited awareness of the presenting features commonly gives rise to diagnostic delays or fulminant septicaemia. Therapy consists of Ig replacement therapy and prophylactic antibiotics with resultant increased life expectancy by reducing the frequency and severity of infections. Earlier diagnosis with neonatal screening for those with a family history is the current 'best option', whilst bone marrow transplant and gene therapy offer a potential cure in these patients.

985

Allergy \& Immunology

Pediatric Asthma: Knowledge of a School Community Ana Esteireiro ${ }^{1}$, Maria Gracinda Junqueira ${ }^{2}$

${ }^{T}$ Pediatrics Department, Hospital de Caldas da Rainha, Centro Hospitalar do Oeste, Portugal

${ }^{2}$ Department of Public Health, ACES Pinhal Litoral, Portugal

Background: Asthma is a chronic disease frequent in pediatric patients. The school should have a leading role helping asthmatic students cope with the disease.

Objective: Characterize the knowledge of the school community Gualdim Pais about pediatric asthma.

Methods: Descriptive and transversal study, with application of an anonymous query. The frequency analysis was executed under Microsoft Office Excel囚.

Results: 46 participants were included in this study, most were teachers and assistants $(56.5 \%$ and $30.4 \%$ ), with an average age of 44.3 years. Almost all (93.48\%) recognize that asthma is common in pediatric age, $41.3 \%$ thinks this is an infectious disease and $24.9 \%$ reject that laughing can cause asthma crisis. Most of them (97.8\%) know that asthma causes reversible airway obstruction and recognizes continuous care are required (76.1\%). However, $41.3 \%$ of respondents believe that this disease is curable and $45.7 \%$ report being a rare cause of hospitalization. A minority $(6.5 \%)$ believe that the practice of physical exercise is contraindicated in asthmatics. As for treatment, $87.0 \%$ know that includes medication control and crisis and $33.0 \%$ believes that inhaled therapy is responsible for gaining weight. In the opinion of $80.4 \%$ of individuals preventive measures may be implemented in schools which aim to prevent exacerbations in asthmatics.

Conclusion: Despite the therapeutic evolution, the asthma control is a challenge and this pathology is a frequent cause of hospitalization. The results express many doubts and myths about asthma so the focus on training is essential, taking into account the time spent by the child/adolescent in school. The training allows you to establish preventive measures and act correctly in case of crisis, promoting a better quality of life for asthmatics. Optimizing communication and coordination between health care, family and school promotes the improvement of care and reduction of hospitalizations.

983

Allergy \& Immunology

Physical Development of Children with Nijmegen Syndrome Tetyana Hariyan ${ }^{1}$, Oksana Boyarchuk ${ }^{1}$, Lyubov Dmytrash ${ }^{3}$, Svitlana Nykytyuk ${ }^{2}$

${ }^{1}$ Department of Children's Diseases and Pediatric Surgery, I. Horbachevsky Ternopil National Medical University, Ukraine

${ }^{2}$ Pediatrics Department №2, I. Horbachevsky Ternopil National Medical University, Ukraine Ternopil Regional Children Hospital, Ukraine

Background: Physical development (FD) is one of the indicators of child's health in general and early diagnosis of primary immunodeficiencies.

Nijmegen Syndrome is a combined primary immunodeficiency represented by chromosomal instability and characteristic clinical manifestations: microcephaly, physical development lag, specific dislocations of the facial skeleton, as well as frequent recurrent infections, increased sensitivity to radiation, and predisposition to the implementation of malignant lymphoid processes. Most patients of Slavic origin have a homozygous mutation in the NBS1 gene. About $80-90 \%$ of patients with primary immunodeficiencies remain undiagnosed, including children with Nijmegen Syndrome. 
Objective: It was carried out an assessment of 6 patients with diagnosed Nijmegen syndrome physical development in the Ternopil region ( 5 boys and 1 girl, aged from 1 to 12 ).

Methods: Assessment of physical development was conducted in accordance with:

- norms proposed by WHO (Order No. 149, Ukraine's Ministry of Health, 2008) for children under 5 years old;

- censored schedules of growth and mass (Order No. 254, Ukraine's Ministry of Health of Ukraine, 2006) for children over 5 years old.

Results: All patients had a delay in body weight and growth since birth. There was also a significant difference in head circumference - microcephaly, detected in all patients. It was the main diagnostic criterion for the Nijmegen Syndrome (besides genetic confirmation).

Conclusion: The delay in weight and growth is one of ten alarming primary immunodeficiencies proposed by a number of primary immunodeficiency foundations in the world. This indicator, together with microcephaly, is a clinical criterion for the Nijmegen Syndrome and can help to diagnose the problem in the early period. Child Nijmegen Syndrome is subject to compulsory early diagnosis among the Ukrainian population due to its prevalence in Slovyans. Proper diagnosis of this pathology will allow to start therapy faster, prevent complications and reduce health care costs.

701

\section{Allergy \& Immunology}

Applying LEAP Recommendations In Clinical Practice: Not As Straightforward As It Seems

Gillian Hendriks ${ }^{1}$, Claudine Bryson ${ }^{1}$, David Lacy ${ }^{1}$

${ }^{T}$ Department of Paediatrics, Wirral University Hospital NHS Foundation Trust, UK

Background: Following the revolutionary results from the LEAP (Learning Early about Peanut Allergy) study advising the early introduction of peanuts to children at high risk of this allergy, clinicians and allergists have been trying to implement this safely into clinical practice, with the ultimate goal of reversing the trend of rising peanut allergy within the population. However, despite good intentions and well-thought through methods, this does not always go to plan.

Method: We discuss a 13-month old girl with severe eczema who has a negative skin prick test, $\operatorname{IgE}$ and component blood tests to peanut, and is suitably advised to perform an oral peanut challenge at home. Unfortunately, Mum subsequently reports symptoms of widespread urticaria and watery eyes on ingestion of a small amount of peanut butter. Despite the likelihood that this is an allergic reaction to peanut, because of the discrepancy between her tests and her home challenge, parents request to proceed with an hospital-based oral food challenge.

Conclusion: Applying evidenced-based knowledge within clinical practice may not be as straightforward as clinicians would envisage. In order to work together and made a significant reduction in prevalence of peanut allergy particularly in the UK, clearer national guidelines or recommendations need to be developed to guide clinicians towards the safe introduction of peanuts in children with high risk of developing this allergy.

\section{4}

\section{Allergy \& Immunology}

Kawasaki Disease Shock Syndrome; a Different Subtype of Kawasaki Disease?

Andrea Iglesias Amaya ${ }^{1}$, Giselle Quezada Ortega ${ }^{2}$, Marycarmen Godinez. Victoria $^{2}$, Chiharu Murata ${ }^{1}$, Mónica Adriana Montoya Guzman ${ }^{2}$, María Elisa Drago Serrano ${ }^{3}$, Rafael Campos Rodríguez ${ }^{2}$, Luis Adrián Rosales Hernández, ${ }^{1}$ Marco Antonio Yamazaki Nakashimada ${ }^{1}$

${ }^{1}$ Department of Clinical Immunology, Instituto Nacional de Pediatría, Mexico ${ }^{2}$ Instituto Politécnico Nacional, Mexico

${ }^{3}$ Biological production area, Universidad Autónoma Metropolitana, Mexico
Background. Super antigen (SAg) is suspected to be responsible of Kawasaki Disease Shock Syndrome (KDSS) which is an aggressive complication of Kawasaki Disease (KD). We hypothesize that KDSS is associated with the activation of TCRV $\beta 2$ and TCRV $\beta 8$ lymphocytes through the presence of SAg from bacteria that colonize the gastrointestinal tract.

Objective. To compare the expression of TCRV $\beta 2$ and $\mathrm{V} \beta 8$ receptor variants in lymphocytes of the KDSS and KD groups.

Methods. A case-control study was performed in patients admitted in the National Institute of Pediatrics between January 2018 and July 2019. Case group $(n=8)$, patients with KDSS; control group $(n=56)$, patients with KD without shock. The percentage (\%) of CD4 and CD8 T cells expressing TCRV $\beta 2$ and TCRV $\beta 8$ as well the activated lymphocytes CD25+ were determined in blood samples by flow cytofluorometry. Wilcoxon Rank-Sum Test was used to compare both groups.

Results. The expression of variants CD3+CD4+TCRV $\beta 2+$ and CD3+ $\mathrm{CD} 8+\mathrm{TCRV} \beta 8+\mathrm{CD} 25+$ was significantly higher in the KDSS group, in comparison to non-shock KD group with a median [Q1, Q3]: 27.7 [4.0, 53.2] vs. $2.2[0.3,9.4](\mathrm{P}=0.016)$ and $13.7[3.9,56.3]$ vs. $2.6[0.5,11.2]$ $(\mathrm{P}=0.028)$, respectively.

Conclusion. These preliminary data suggests that a SAg is triggering the excessive response observed in patients with KDSS. Continuing this study with a bigger sample size will be necessary.

620

Allergy \& Immunology

Increased Skin Reactivity to Histamine along with an Increase of Body Mass Index in Korean Children: 2 Years Follow Up Study

Suk Won Chang ${ }^{1}$, Jin-A Park' ${ }^{l}$, Ju Wan Kang ${ }^{1}$, Gil-Chai Lim ${ }^{1}$

${ }^{I}$ Otorhinolaryngology, Jeju National University School of Medicine, South Korea

Background: A skin prick test is the most widely used test to diagnose allergic diseases. However, it is not known much about the effect of the body mass index on skin reactivity. Therefore, we aim to investigate the changes in skin reactivity to histamine and allergens according to the changes in body mass index (BMI) in Korean children.

Methods: The data of 230 children were used in this analysis. And, the data were collected in 2010 and 2012, respectively. Skin prick test was performed for 26 aeroallergens and other variables were obtained, including sex, age, BMI, parental allergy history, and parental smoking. Multivariate analysis was used to confirm the association between the changes in skin reactivity and body mass index.

Results: Mean size of skin wheal induced histamine and BMI were increased for two years. Changes in skin wheal induced by histamine showed significant correlation with changes in BMI (Spearman's Rho=0.209, P-value 0.001). However, changes in the size of the skin response to Dermatophagoides farinae (Df) and Dermatophagoides pteronyssinus (Dp) did not correlate with changes in BMI.

Conclusion: The magnitude of skin response to histamine was correlated with an increase in body mass index. However, the wheal size for Df and Dp was not related to the change in the BMI. The effects of BMI on the interpretation of skin prick test should be considered.

\section{0}

\section{Allergy \& Immunology}

Drug Induced Hypersensitivity Syndrome (DIHS) in a 12 Month Old Child

Ricardo Loureiro $^{2}$, Luisa Prada ${ }^{1}$, Isis Monteiro $^{1}$, Ana Neves ${ }^{1}$

${ }^{T}$ Pediatric Allergology Unit, Santa Maria University Hospital - Lisbon University Hospital Center, Portugal

${ }^{2}$ Pediatric Unit, Federal University of Rio de Janeiro, Brazil 
Introduction: Drug rash with eosinophilia and systemic symptoms syndrome (DRESS)/DIHS is a complex syndrome characterized by a broad spectrum of clinical features, like fever, lymphadenopathy, cutaneous manifestations and visceral involvement, eosinophilia and atypical lymphocytosis.

Beta-lactams, anticonvulsants, allopurinol, sulfonamides are culprit drugs than can trigger this delayed hypersensitivity mechanism. Herpes virusdrug interactions are sometimes observed.

A 12-month-old female baby, with congenital heart disease and chromosomal abnormality (deletion of short arm of chromosome 8) was submitted to undergo cardiac surgery.

On day 6 post-surgery she was prescribed with intravenous vancomycin and gentamycin, for infection.

Due to loss of intravenous line, on day 7 post-surgery, a switch to oral amoxicillin and clavulanic acid was done. On day 8 of antibiotic, she presented an urticarial maculopapular rash with facial edema and fever, hepatomegaly and lymphadenopathy, associated with laboratorial abnormalities $10 \%$ atypical lymphocytes, and elevated AST $429 \mathrm{U} / \mathrm{L}$ and ALT $621 \mathrm{U} / \mathrm{L}$, suggestive of a mononucleosic syndrome. On day 11 of amoxicillin and clavulanic acid, this antibiotic was suspended. Epstein Barr virus, cytomegalovirus and toxoplasmosis were $\operatorname{IgG}$ positive. Parainfluenza virus type 3 was also positive. HHV 6 and 7 serologies and measles virus RNA were negative. On day 18 of admission, the child was seriously ill and vancomycin and ceftriaxone were again started. On day 29 post-surgery, endocarditis was confirmed by cardiac ultrasound, and daptomycin, cefepime and ciprofloxacin was started with clinical improvement. The baby was discharged on day 48 post-surgery, a subtle rash in her legs was still observed.

Four months later, she was inadvertly prescribed amoxicillin and clavulanic acid for an upper respiratory infection. On the $3^{\text {rd }}$ day of antibiotic, she presented again with an urticarial rash that was promptly suspended.

Conclusions: The diagnosis of DRESS/DIHS was proposed, based on RegiSCAR (European registry of severe cutaneous adverse reactions to drugs) criteria.

\section{5}

\section{Allergy \& Immunology}

The Benefit Supplementing with Vitamin D and Omega3 in Atopic Dermatitis in Children

Alina Murgu $^{l}$, Anca Chiriac ${ }^{2}$, Irina Criscov ${ }^{I}$, Paula Popovici ${ }^{1}$, Alice Azoicai ${ }^{I}$, Alice Popescu ${ }^{3}$

${ }^{1}$ Pediatrics, Immuno-Allergology, Children Emergency Hospital "St.Mary", Romania

${ }^{2}$ Dermatology, Recovery and Balneology Hospital, Romania ${ }^{3}$ Pediatric, Privat Pediatric Office, Romania

Atopic dermatitis through clinical expression and evolution is a chronic inflammatory allergic disabling disorder with a major impact on the quality of life of the child and its family. The nutritional assistance of these patients becomes essential to allow for normal growth and development. Supplementation with vitamin D and Omega 3 seems to be beneficial by the complex immunomodulatory and the anti-inflammatory action, by modifying the cytokine production but also by the antiinfectious role Objective: To assess the benefit of dietary supplementation with vitamin $\mathrm{D}$ and Omega 3 on the evolution of atopic dermatitis in the child.

Method: 47 children (0-2 years) diagnosed with atopic dermatitis with varying severity were studied. Two groups were selected: group A -23 cases; Group B - 24 cases. Serum vitamin D levels in both groups were normal. Supplementation with vitamin D3 (1500UI / day) and Omega3 was done only in group A. Group B received the usual dose of Vitamin D3 for the rickets prophylaxis $500 \mathrm{IU} /$ day. The monitoring was performed for 6 months. The treatment for atopic dermatitis during supplementation with the vitamin D and Omega 3 was only with non-cortisone cutaneous topicals and no one calcineurin inhibitors topic.
Results: Initial: group A -SCORAD $\leq 15-8 / 23$; SCORAD: 15-40-12 / 23; SCORAD $\geq 40$-3/23; group B -SCORAD $\leq 15$-9/24; SCORAD: $15-40-13$ / 24; SCORAD $\geq 40-2 / 24$; incidence of skin infections: group A-37\%; group B-35\%; after 6 months: group A-SCORAD $\leq 15-15 / 23$; SCORAD: $15-40-7$ / 23; SCORAD $\geq 40-1 / 23$; group B $-\mathrm{SCORAD} \leq 15$ -10/24; SCORAD: $15-40-12$ / 24; SCORAD $\geq 40-2 / 24$; incidence of cutaneous infections: group A-21\%; group B-36\%;

Conclusions: Supplementing the diet of patients with atopic dermatitis with Vitamin D and Omega 3 improves pruritus, dryness and skin rash, while reducing the rate of cutaneous infections.

\section{6}

\section{Allergy \& Immunology}

ACE Inhibitors: An unusual Cause of Pediatric Angioedema Goncalo Vale $^{I}$, Maria São Pedro ${ }^{I}$, Rita Matos Parreira ${ }^{l}$, Mariana Simões $^{I}$, Patrícia Pais ${ }^{l}$, Joana Extreia ${ }^{1}$

${ }^{1}$ Pediatrics, Centro Hospitalar Barreiro-Montijo, Portugal

Background: Angiotensin converting enzyme inhibitors (ACEI) are widely used to treat hypertension in adults. The use of these drugs in pediatric age is increasing due to the higher prevalence of obesity and its co-morbidities'.

Objective: To alert pediatric community about angioedema as ACEI side effect.

Methods: Report of clinical case from a suburban hospital in Portugal. Clinical Report: Fifteen year-old african descendent female with morbid obesity, hypertension, diabetes mellitus type 2 followed in cardiovascular risk outpatient clinic. Treated with lisinopril since December 2017.

During hospital admission in January 2019, she developed labial and tongue edema followed by asymmetric periorbital involvement, without itching nor urticaria, unresponsive to high dose hydroxyzine. Lisinopril was discontinued and no other therapeutic measures were taken. Clinical manifestations gradually disappeared during the following five days. The patient reported a similar episode five months before that resolved spontaneously, without therapy changes.

Conclusion: In adults, angioedema is an unusual but well-recognized side effect of ACEI therapy, up to five times greater in African descents. However, in the "pediatric world", physicians are less familiar and therefore less alert to possible ACEI side effects. Although benign and selflimited, ACEI induced angioedema can be potentially fatal, therefore physicians should consider this possibility when evaluating patients with acute or recurrent angioedema.

\section{6}

\section{Allergy \& Immunology}

Self and Proxy Reported Quality of Life of Children with Asthma Vaida Taminskiene ${ }^{1}$, Egle Vaitkaitiene ${ }^{2}$, Arunas Valiulis ${ }^{1,3}$

${ }^{I}$ Department of Public Health, Institute of Health Science, Faculty of Medicine, Vilnius University, Lithuania

${ }^{2}$ Medical Academy, Lithuanian University of Health Sciences, Lithuania

${ }^{3}$ Clinic of Children's Diseases, Institute of Clinical Medicine, Faculty of Medicine, Vilnius University, Lithuania

Background: Asthma is a global public health program. It affects patients of different age and is one of the most prevalent chronic diseases among children.

Objective: The aim of our study was to evaluate the quality of life (QoL) of children with asthma by both self- and proxy-reports.

Methods: A total of 527 Lithuanian children with asthma and their parents were enrolled into a cross-sectional study. Participants of the study were asked to fill in anonymous questionnaires during scheduled outpatient visits to pediatric pulmonologist. QoL of children with asthma was assessed using 
PedsQL Asthma Module consisting of 2 scales and 22 questions. Ageappropriate versions of the questionnaire were completed by children and their parents. Scores for each scale and overall score were calculated and ranged from 0 to 100 where higher score referred to better QoL.

Results: The age of children enrolled into this study varied from 2 to 17 years and the mean age was $8.4( \pm 4.3)$ years. A total of $63.2 \%$ of respondents were boys. Children evaluated their asthma related QoL better compared to parents' evaluation (median score $77.3(68.2 ; 86.4)$ and $75.0(63.6 ; 86.4)$ respectively, $\mathrm{p}=0.045)$. The differences between parent and children assessment were the highest at the oldest age group. The most important factors, associated with the QoL of children with asthma by both self- and proxy-reports were asthma severity and control as well as shortness of breath. Parent reported QoL was also related with child's general health and presence of concomitant allergies.

Conclusions: Children with asthma assess their QoL better compared to their parents. The differences in the assessment were the highest at the oldest age group of children and should be taken into consideration during the preparation of individual asthma control plan.

\section{3}

\section{Critical / Emergency Care}

Traumatic Thoracic Aortic Transection After a High-Energy Trauma Mariana Adriao $^{1}$, Elson Salgueiro $^{2}$, Maria José Oliveira $^{3}$, Amélia $^{2}$ Moreira $^{3}$, Augusto Ribeiro ${ }^{3}$

${ }^{1}$ Serviço de Pediatria, Centro Hospitalar Universitário de São João, Portugal

${ }^{2}$ Serviço de Cirurgia Cardiotorácica, Centro Hospitalar Universitário de São João, Portugal ${ }^{3}$ Serviço de Medicina Intensiva Pediátrica, Centro Hospitalar Universitário de São João, Portugal

Background: Deceleration trauma resulting in thoracic aortic contained rupture is uncommon and associated with a high prehospital mortality. Less than $25 \%$ of patients with blunt thoracic aortic injury survive long enough to reach a hospital. Radiological plays an important role in diagnosis and accurate classification of the type of dissection, in order to plan the intervention and optimize the outcome.

Objective: The authors aim to report a rare case of blunt thoracic aortic injury successfully corrected and share the correspondent unique imaging.

Case Report: A 17-year-old high energy accident victim with several traumatic lesions arrived in the emergency room. He was hemodynamically stable and had a Glasgow Coma Scale of 15/15. He presented diminished breath sounds on the left hemithorax and gross hematuria. Chest radiograph revealed left hemidiaphragmatic rupture with stomach herniation and mediastinal enlargement. A thoracic CT scan confirmed visceral herniation through the ruptured diaphragm, fracture of four left ribs and a contained aortic rupture located at the aortic isthmus, associated with perivascular hematoma - type IV. Endovascular treatment was considered unsuitable due to unfavorable anatomical characteristics. Therefore, the patient was submitted to open descending aorta replacement by a vascular prothesis. Distal aortic perfusion was maintained by a Gott shunt. Simultaneously, diaphragm laceration was directly sutured. Post-operative radiological revaluation revealed good resolution of both lesions.

Due to acute renal injury caused by severe rhabdomyolysis, continuous venovenous hemodiafiltration was required for 25 days. Regarding outcome, the adolescent was discharged home 44 days after hospital admission, without any severe sequelae.

Conclusion: This clinical case highlights the importance of considering different types of internal hemorrhage in cases of trauma, particularly life-threatening lesions resulting from blunt trauma. In this particular case, the patient was hemodynamically stable at all times, which was only possible due to the containment of the hemorrhage to the mediastinum.
495

\section{Critical / Emergency Care}

Poisoning and Mucosal Burning by Laundry Detergent Capsules: A Case Report

Jose Andrade Guerrero ${ }^{1}$, Virginia Santana Rojo ${ }^{1}$, Maria Jose Sanchez. Toro ${ }^{\prime}$, Celia Fabra Garrido ${ }^{1}$, Isabel Gonzalez Bertolin ${ }^{1}$, Begoña de Miguel Lavisier ${ }^{1}$, Maria Fernanda Pedrero Escalas ${ }^{2}$, Francisco Javier Aracil Santos ${ }^{3}$

${ }^{1}$ Pediatric Emergency, Hospital Universitario La Paz, Spain

${ }^{2}$ Pediatric Otorhinolaryngology, Hospital Universitario La Paz, Spain

${ }^{3}$ General Pediatrics, Hospital Universitario La Paz, Spain

Background: One of the main sources of poisoning in young children are cleaning products. Among the newest ones, we find laundry detergent capsules (LDC). Because their higher concentration of detergent serious clinical manifestation can be observed.

We present a case of a 12-month-old boy who bit an LDC (Ariel 3in1), and developed metabolic acidosis, digestive and respiratory impairment. Case Presentation: A healthy 12-month-old boy, was brought to Emergency Room vomiting, coughing and dysphonia after biting an LDC. We contacted the National Institute of Toxicology of Spain who told us the main risk of this ingestion is metabolic acidosis, but not chemical mucosal burning. In the physical examination he was irritable with presence of respiratory distress and inspiratory stridor. Laboratory tests on admission revealed: Hemoglobin: 11.4g/dL, WBC 29400/ $\mu \mathrm{L}$ (neutrophils 57\%). The blood gases showed a metabolic acidosis $(\mathrm{pH} 7.29, \mathrm{HCO}$ : $15 \mathrm{mmol} / \mathrm{L}, \mathrm{pCO} 2: 40 \mathrm{mmHg}, \mathrm{BE}:-13 \mathrm{mmol} / \mathrm{L})$. Renal function was normal. A chest X-ray revealed bilateral peribronchial thickening, predominantly at the left base. He was initially treated with dexamethasone, nebulized adrenaline, salbutamol, oxygen and bicarbonate perfusion, which was maintained for 3 days. On the second day, an upper endoscopy showed caustic ulcerations in epiglottis and subglottic regions. Esophageal endoscopy did not show any damage. Hemoglobin gradually dropped to $8 \mathrm{~g} / \mathrm{L}$ at $3^{\text {rd }}$ day. Digestive hemorrhage was suspected and treated with omeprazole.

At discharge respiratory symptoms and metabolic acidosis were solved with no digestive symptoms. Three months after an upper gastrointestinal series showed a membrane in the middle third of the esophagus.

Conclusions: The main component of the Ariel 3 in 1 is Dodecylbenzenesulfonte which may produce gastrointestinal burning. However, combining it with other component, Propylene-gycol, the toxicity may enhance, and appear metabolic acidosis. When bitten by children can burst and spray the content in the respiratory and GI systems, causing mucosal damage leading to several complications.

\section{3}

\section{Critical / Emergency Care}

Pediatric Urgent Care - A Three-year Institutional Experience Magdy Attia ${ }^{I}$

${ }^{T}$ Pediatrics, Division of Urgent Care, Nemours Alfred I duPont Hospital for Children, USA

Background: Pediatric Emergency Departments (PED) evaluate a large number of sub-acute conditions. PED is convenient but expensive resource for sub-acute care to providers and patients alike. Pediatric Urgent Care (PUC) is rapidly reshaping the landscape in delivering subacute care for children. This report is to share our experience.

Methods: Data analyzed included demographics, chief complaints (CCs), throughput, cost, satisfaction and quality. The analysis describes basic operational statistics and compared CCs and volumes during different "seasons". We compared PUC and PED length of visit (LOV) and 
cost. Patient satisfaction was measured by 5-point Likert scale. Data are presented as percentages, means or $p$ values.

Results: We analyzed 15,482 visits. Daily average volume; 18.4 patient (range 7-67). Visits increased 60\% from year 1 to year 2 and a $25 \%$ in year 3. Weekends accounted for 39\% of the visits. Fall/winter season was busier and Sundays were most crowded. The first hour on Sunday mornings had the highest arrival rate. The least arrivals were in the last hour of weeknights. $51 \%$ were males. Age average was 12.9 years $(\mathrm{SD}:+9.9)$. Most common CCs were respiratory infection, fever, extremity injury and sore throat; $14 \%, 13 \%, 12 \%$, and $10 \%$ respectively. There was no significant difference between CCs during fall/winter with more infectious etiologies compared to spring/summer with more injures (P0.5). PUC LOV was 52 minutes and 117 in PED. Percent of 48-hours returns to PUC was $1.1 \%$ (95\% CI: 0.8-1.4) and percent of patients transferred to PED was $1.5 \%$ (95\% CI:-1.1-1.9). Adherence to clinical standard work was $97 \%$. Satisfaction score was $83.5 \%$ using score of 5 . PUC charges for similar visit level was 47-61 \% lower than PED.

Conclusions: PUC are efficient and economical alternative to PED. We described one institution's experience that could prove valuable benchmark to similar practices.

\section{8}

\section{Critical / Emergency Care}

Unintentional Intoxications in Children under 4 Years Old: A Current Reality?

Denise Banganho $^{l}$, Diana Silva ${ }^{l}$, Inês Ferreira ${ }^{l}$, Ana Gomes da Silva ${ }^{l}$, Teresa Gouveia ${ }^{\prime}$

${ }^{1}$ Department of Pediatrics, Hospital de São Bernardo - Centro Hospitalar de Setúbal, Portugal

Background: Young children are particularly susceptible to intoxications due to the natural need of environmental exploration and inability to measure risks. Despite several preventing efforts, intoxications remain a significant cause of admission to the pediatric emergency department (PED).

Objectives: Characterize childhood intoxication cases in the PED and develop prevention strategies.

Methods: Retrospective analysis of clinical cases of children under 4 years old attending to the PED during 10 years.

Results: Between 2008-2017, 383 children with unintentional intoxications attended the PED and accounted for $0,1 \%$ of all emergency visits during the study period. The median age was 2.1 years $( \pm 1.0$ years $)$; $54.3 \% \quad(n=208)$ were female. Ingestion of drugs were involved in $52.5 \% \quad(n=201)$ and antihistamines were the most frequent drug class. Domestic products were the second intoxication agent $(36 \%, \mathrm{n}=138)$ followed by carbon monoxide $(5.7 \%, \mathrm{n}=22)$, poisons $(4.4 \%, \mathrm{n}=17)$ and illicit drugs $(0.8 \%$, $\mathrm{n}=3)$. Forty percent $(40.7 \%, \mathrm{n}=156)$ of the intoxications occurred in the afternoon, 68.1\% ( $\mathrm{n}=261)$ during weekdays. $99.7 \%(\mathrm{n}=382)$ intoxications occurred at home. A total of $50.4 \%(n=193)$ of patients were admitted less than 1 hour after exposure to the toxic agent and only $15.1 \%$ were transported to the hospital through the National Emergency Service. Most children were asymptomatic $(\mathrm{n}=224)$. In symptomatic patients, gastrointestinal manifestations were the most frequent $(58.4 \%, \mathrm{n}=93)$. The National Poisoning and Intoxication Center was contacted in $82.2 \%(n=315)$ of the cases. Gastrointestinal decontamination was performed in $30.3 \%$. Four children underwent hospitalization for more than 36 hours. There were no admissions to the intensive care unit or mortality. Conclusions: The number of children with unintentional intoxication did not change significantly over the last 10 years. Ingestion of drugs that belong to family members accounts for approximately half of the cases. It remains important to ensure prevention strategies in the community.
530

\section{Critical / Emergency Care}

Acute Appendicitis in Children: Postoperative Evolution According to Risk Factors; Single-Centre Retrospective Review

Maria Bauluz-Barcena ${ }^{1}$, Antia Fiel-Ozores ${ }^{1}$, Jessica Lopez-Lopez ${ }^{2}$, Pilar Fernández-Eire

${ }^{I}$ Pediatrics, Hospital Alvaro Cunqueiro, Spain

${ }^{2}$ Pediatric Surgery, Hospital Alvaro Cunqueiro, Spain

Background: Abdominal pain is a frequent complaint in Pediatric Primary Care as well as in the Emergency Department. Acute appendicitis (AA) is the main etiology of surgical abdominal pain in children.

Objectives: Our aim is to describe and analyze the clinical characteristics of our patients who have undergone surgery on suspicion of AA. Secondly, to describe AA evolution according to some prognosis factors. Methods: Retrospective, descriptive and analytical study through review of electronic clinical records. Patients from 0 to 14 years old who have undergone surgery for suspicion of AA, are included. Study period: 8 months. Setting: tertiary hospital.

Results: Sample size $=96$. Median age $=10 ` 8$ years. Male sex: $64.6 \%$. The most frequent symptoms in our study are: pain in low right quadrant $(79.2 \%)$, nausea/vomiting $(72.9 \%)$, fever $(32.3 \%)$ and hyporexia $(22.9 \%)$. Preferred diagnostic method is ultrasound (performed in 91.6\%). Most common intraoperative findings are: phlegmonous AA (37.5\%), gangrenous AA (40.6\%), plastron (10.4\%). 37.5\% appendices are retrocecal. We have analyzed the incidence of complications (days of total admission, admission to pediatric intensive care, need for a central catheter or parenteral nutrition, need of surgical reintervention) in children under 4 years of age, in gangrenous AA, in those with ultrasonography data suggesting complicated AA, and those with increased CRP.

Conclusions: Acute appendicitis is the main surgical diagnosis of abdominal pain in pediatric age. According to literature, some factors are known to be related to worse prognosis, such as: age under 4 years old, gangrenous or perforate AA and CRP increase. However, we have not found a statistically significant association in this respect.

394

Critical / Emergency Care

The Role of Extracorporeal Membrane Oxygenation Support in Malignant Infantile Pertussis

Francisca Calheiros-Trigo $^{1}$, Lurdes Lisboa ${ }^{2}$, Marta João Silva ${ }^{2}$, Roberto Roncon-Albuquerque $\mathrm{Jr}^{3}$, Augusto Ribeiro ${ }^{2}$

${ }^{1}$ Department of Pediatrics, Hospital de Braga, Portugal

${ }^{2}$ Pediatric Intensive Care Unit, Centro Hospitalar Universitário de S. João, Portugal

${ }^{3}$ Department of Intensive Care Medicine, Centro Hospitalar Universitário de S. João, Portugal

Background: Pertussis, or whooping cough, is an acute respiratory infection caused by Bordetella pertussis and Bordetella parapertussis. Malignant pertussis (acute respiratory distress syndrome (ARDS), hyperleukocytosis and pulmonary hypertension) is a severe form of pertussis, more common in young, non-vaccinated children, and almost always a fatal disease. Extracorporeal membrane oxygenation (ECMO) is used as an emergency treatment for children with life-threatening pertussis. However, the success rate is low, with only $30 \%$ survival rate.

Method: We report a clinical case of a newborn admitted at pediatric intensive care unit (PICU) due to malignant pertussis.

Results: A female newborn (28-days of age) with pertussis as a result of B. pertussis infection was admitted in PICU due to hyperleukocytosis $(92030 / \mathrm{uL})$ and worsening respiratory distress. Exchange transfusion was performed with mild improvement. Cardiac ultrasound revealed pulmonary hypertension. By day-2 there was clinical worsening with severe ARDS. After failure of conventional ventilation and high-frequency 
oscillatory ventilation, cannulation of internal jugular vein and common carotid artery was performed and venoarterial ECMO support was instituted. Due to anasarca, dialytic therapy was maintained between days 9 and 32. Serial transfontanelar ultrasounds were performed to access for brain complications. By day-20 ultrasound revealed brain hemorrhage and epileptic activity was noted, with abnormal eletroencephalogram. Antiepileptic therapy was started, with good response. After 41 days of ECMO support the patient was weaned off ECMO, and extubated to noninvasive ventilation 14 days later. She was discharged from PICU at day63 in spontaneous ventilation. She was discharged home at day- 105.

Conclusion: In this patient with malignant pertussis, ECMO was performed for cardiopulmonary support and rescued the infant. During ECMO support, mechanical ventilation with protective settings (high positive end-expiratory pressure and low tidal volumes) provided the opportunity for lung recovery. Despite complications (minor brain hemorrhage) the patient recovery with minor sequelae poses a good prognosis.

\section{0}

\section{Critical / Emergency Care}

Pediatric Hand Injuries Presenting to the Emergency Department: A 6-year Cross-sectional Study in Korea

Joong Wan Park ${ }^{1}$, Do Kyun Kim ${ }^{I}$, Ik Chang Choi ${ }^{1}$, Jae Yun Jung ${ }^{l}$, Se Uk Lee $^{2}$, Soyun Hwang ${ }^{l}$

${ }^{1}$ Emergency Medicine, Seoul National University Hospital, South Korea

${ }^{2}$ Emergency Medicine, Seoul Metropolitan Government Seoul National University Boramae Medical Center, South Korea

Objective: The objective of this study was to evaluate the clinical characteristics of pediatric hand injuries presenting to the emergency department (ED) in Korea and to assess the risk factors associated with severe hand injuries requiring hospitalization or surgery.

Methods: A multicentre cross-sectional study was conducted using the emergency department (ED)-based Injury In-depth Surveillance (EDIIS) registry in Korea between 2011 and 2016. A multivariable logistic regression model was used to obtain the adjusted odds ratios (aORs) for the factors associated with severe pediatric hand injuries.

Results: Among 1,537,617 injured patients, 40,672 (26.5 per 1,000 injured patients) presented to the ED for hand injuries. The prevalence of pediatric hand injuries was highest in teenager (9.9\%) and increased with age. Overall and in each age group, boys were in the majority. In infant, toddler, and pre-schooler, crushing is the most common injury mechanism, while penetrating injury were most common in schooler and teenager. Door related injuries were the most common cause in infant, toddler, and preschooler. Knife related injuries were the most common cause in schooler and teenager. A total of 2,344 (5.8\%) were severe injuries requiring hospitalization or surgery.

Aging (aOR: 1.06, 95\% CI: 1.06-1.07), male (aOR: 1.33, 95\% CI: 1.22 -1.46 ), finger injury (aOR: $1.26,95 \%$ CI: $1.14-1.39$ ), crushing injury (aOR: $1.35,95 \%$ CI: $1.15-1.57$ ), and injury by machine (aOR: $3.91,95 \%$ CI: $2.66-5.75$ ) were associated with severe hand injury.

Conclusion: This study provides a comprehensive overview of the epidemiology of hand injuries in the pediatric population. Preventive strategies are needed to prevent severe hand injuries in children.

\section{8}

\section{Critical / Emergency Care}

Clinical Validation of Affordable, Reusable Novel EKG Monitor for Children in Low-Resource Settings

Judy Chen ${ }^{1}$, Andre Muelenaer ${ }^{2,3}$, John Bird ${ }^{4}$

${ }^{T}$ Virginia Tech Carilion School of Medicine, USA

${ }^{2}$ Department of Pediatrics, Carilion Clinic, USA

${ }^{3}$ Department of Biomedical Engineering and Mechanics, Virginia Polytechnic Institute, USA

${ }^{4}$ Cardinal Mechtronics, USA
Background: Low-income countries severely lack adequate access to devices that accurately measure vital signs which may affect mortality outcome for pediatric patients. Developing an efficient and affordable devices that can accurately determine vital signs can contribute greatly to diagnosing critical pediatric conditions, leading to faster time to appropriate treatment. A team of engineers, industrial designers, and medical professionals developed an affordable, reusable EKG monitor prototype using carbon electrodes.

Objective: The objective of study is to validate the prototype's accuracy compared to a standard EKG monitor.

Methods: 21 healthy subjects, ages newborn to 60 months, were recruited from Carilion Children's General Pediatrics Clinic. Prototype device and standard EKG device were simultaneously attached to subject for 1-2 minutes. Bland-Altman method was used to evaluate if agreement existed between both devices with heartbeats counted obtained per 10-second interval (hpi) as $\mathrm{N}=1$. Results: Prototype was able to detect subject's heartbeat and display distinct EKG waveforms. Loss of EKG detection from prototype was observed during loss of skin contact with carbon electrodes from movement.

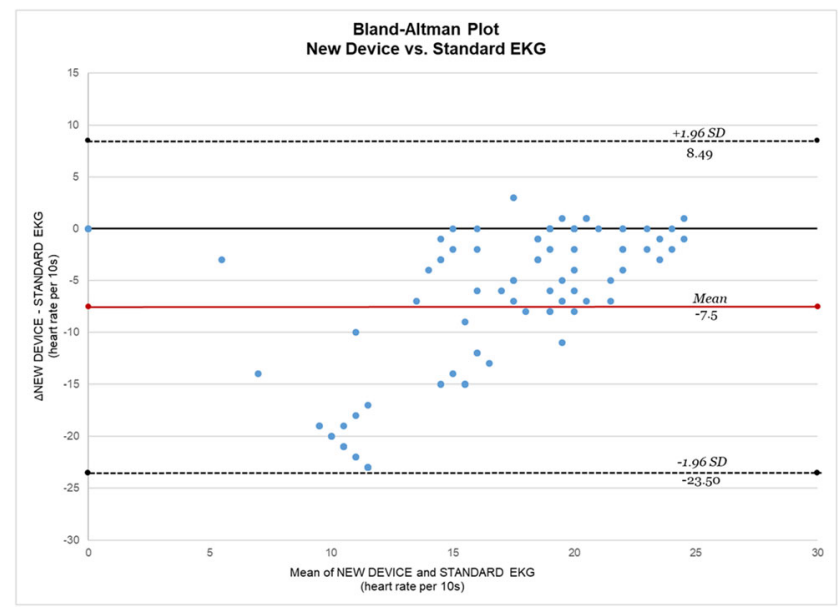

Mean of differences between the two methods clinically translates to signficant difference of 45 heartbeats per minute. Range between limits of agreement demonstrates clinically significant discrepancy in prototype's ability to consistently detect heart rate, presumably from loss of skin contact. However, $35 \%$ of data show difference between -2.2 to 0.4 hpi between the two methods, indicating the prototype can detect heart rate similarly to the standard EKG in presumed instances of constant skin contact.

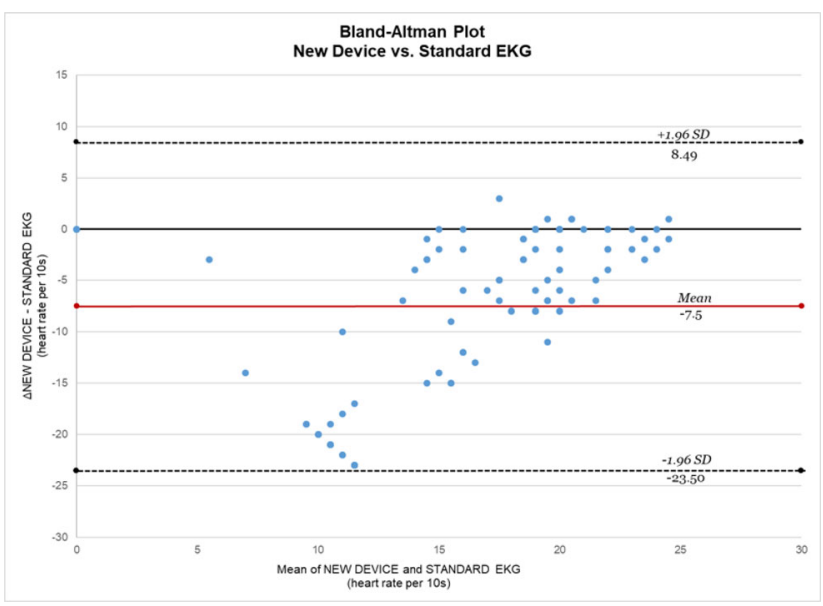

Conclusion: For our prototype, we were able to develop a low-cost, reusable device that can detect heart rate in children and reproduce similar heart rates as the standard EKG monitor, but inconsistently, presumably secondary to loss of skin contact with the carbon electrodes. 
Improvements to optimize carbon electrode contact with skin will be considered for future design.

\section{2}

\section{Critical / Emergency Care}

Audit of Routine Blood Tests on Children's Intensive Care Unit, Leicester Royal Infirmary

Daniel Crankshaw ${ }^{I}$, Jennirfer Mann ${ }^{1}$, Simon Robinson ${ }^{1}$

${ }^{T}$ Children's Intensive Care Unit, Leicester Royal Infirmary, UK

Aim: Over an 8 week period, an audit was performed to analyse the blood tests performed overnight on paediatric inpatients on our unit with the aim of identifying whether more samples are taken than required and the financial impact of this.

Methods: This audit assessed essential (those blood tests which the consultant of the week recommended be taken overnight) versus those actually requested by the paediatric registrar working the night shift. The consultant of the day was asked to complete a short proforma, recording which essential bloods were needed overnight. This consultant recommendation was blinded from the registrar. We then retrospectively looked through iLab to record which bloods had actually been sent overnight. Findings: Data was collected for 62 patients over 16 weekdays on CICU. In total, 165 tests were performed, with 90 of these classed as essential and $75(45 \%)$ as non - essential tests. This resulted in avoidable expenditure of $£ 123.74$ (45\%). Coagulation, urea and electrolytes and liver function tests were the areas of most non-essential expenditure. This is similar to results found when this audit was performed on the Paediatric Intensive Care Unit at Glenfield Hospital, where 115/1059 (48\%) were found to be non-essential, resulting in avoidable expenditure of $£ 647.64$ (44\%.)

Conclusions: This audit has now been performed at both CICU's within our trust and it demonstrates a lack of correlation between which tests are thought to be essential by the paediatric intensive care consultants and which tests are actually being performed by the paediatric registrars covering the units overnight. Therefore, children are likely to be subjected to additional blood investigations which are non-essential to their care and also has a financial impact upon the department. Further consideration of an appropriate quality improvement initiative is needed to address these discrepancies.

\section{2}

\section{Critical / Emergency Care}

Validity and Reliability of the Brazilian Portuguese Version of the Pediatric Confusion Assessment Method for the Intensive Care Unit (pCAM-ICU)

Roberta Castro ${ }^{1}$, Maria Clara Magalhães-Barbosa ${ }^{3}$, Arnaldo PrataBarbosa $^{3}$, Antonio Cunha ${ }^{2,3}$,Elie Cheniaux ${ }^{1,2}$, Heidi Smith ${ }^{4}$

${ }^{1}$ Pediatrics, Universidade do Estado do Rio de Janeiro (UERJ), Brazil

${ }^{2}$ Pediatrics, Universidade Federal do Rio de Janeiro (UFRJ), Brazil

${ }^{3}$ Pediatrics, Instituto D'Or de Pesquisa e Ensino (IDOR), Brazil

${ }^{4}$ Pediatrics, Vanderbilt University Medical Center, USA

Objectives: To determine the validity and reliability of the Brazilian Portuguese Pediatric Confusion Assessment Method for the Intensive Care Unit (BRpCAM-ICU) for diagnosing delirium in patients with chronological and developmental ages from 5 to 17 years in Brazilian Pediatric Intensive Care Units (PICUs).

Methods: Prospective, cross-sectional study performed in eight PICUs, with 116 patients, 5-17 years of age, without developmental delay, submitted to mechanical ventilation or not. To assess the interobserver reliability, two previously trained researchers concomitantly applied the BRpCAM-ICU and independently rated the same patient. To assess the criterion validity, a pediatric neurologist or psychiatrist, blinded to the BRpCAM-ICU assessments, evaluated the same patient within 30 minutes, using the Diagnostic and Statistical Manual of Mental Disorders Fifth Edition - DSM-5, considered the reference standard.

Results: 149 paired assessments were included (some patients had more than one). Delirium was diagnosed in 11 of 149 (7\%) assessments, or 8 of $116(7 \%)$ patients, using both the BRpCAM-ICU and the DSM-5. There was one false positive and one false negative diagnosis, which resulted in $90.9 \%$ sensitivity $(95 \% \mathrm{CI}, 58.7-99.8 \%)$ and $99.3 \%$ specificity $(95 \% \mathrm{CI}$, 96-100\%) for the BRpCAM-ICU. The interrater reliability was considered almost perfect $(\kappa=1.0)$.

Conclusions: The BRpCAM-ICU is a valid and reliable tool for diagnosing delirium in pediatric patients 5-17 years of age in Brazilian PICUs. The implementation of this tool may be useful to reduce underdiagnosis, ensure monitoring and earlier intervention, provide a better prognosis, and improve research on delirium in this age group in Brazil.

521

Critical / Emergency Care

Chest Pain in Children and Adolescents: Reality of an Emergency Department

Ana Barbara De Matos ${ }^{1}$, Nelia Gaspar ${ }^{1}$, Sara Ferreira ${ }^{l}$, Julieta Morais ${ }^{1}$ ${ }^{T}$ Paediatric Service, Centro Hospitalar Médio Tejo, Portugal

Background: Chest pain is a common complaint in children/adolescents visiting the emergency department (ED). The existence of underlying heart disease is rarely verified. It is mostly idiopathic, and in adolescence the psychogenic etiology assumes greater expressiveness.

Objective and Methods: Retrospective analysis of the clinical processes of patients who visited the ED between December 2017 and May 2018 with chest pain. Cases of traumatic etiology were excluded.

Results: A total of 175 children/adolescents (52\% girls; median age 13 years) attended the ED. Five percent had cardiovascular antecedents, and $7 \%$ psychiatric. Regarding pain characteristics, the majority lasted between 1 hour and 1 day (27\%), with preferential retrosternal location $(18 \%)$ or in the left hemithorax (18\%). Associated symptoms occurred in $69 \%$ of patients, including $5 \%$ of palpitations and $5 \%$ of paresthesias in the left upper limb. Physical examination revealed no abnormalities in $39 \%$ of patients, $25 \%$ reported chest wall tenderness and $5 \%$ had altered cardiac auscultation. Eleven percent reported at least one stressful event associated with the onset of chest pain. Fifty-seven percent had chest Xray $(16 \%$ altered), $23 \%$ electrocardiogram ( $7 \%$ altered), $51 \%$ cardiac enzymes ( $2 \%$ altered) and $32 \%$ did not perform any additional investigation. Regarding the final diagnosis, $38 \%$ was idiopathic, $29 \%$ respiratory, $17 \%$ musculoskeletal, $14 \%$ psychogenic and $2 \%$ cardiac. Concerning psychogenic pain, there was a statistically significant relationship with the adolescence phase, female sex and the presence of previous stressful events ( $\mathrm{p} 0.05$ ). Two percent of the patients were admitted in the pediatric unit and $2 \%$ were transferred to a cardiovascular reference center.

Conclusion: The number of children/adolescents who were admitted due to chest pain, highlights the importance of the characterization of this population. Cases of cardiac etiology are rare and it is therefore essential to give young people and families a sense of security in the absence of serious illness.

\section{2}

\section{Critical / Emergency Care}

Utility of Chest Radiographs (CXR) in Paediatric Critical Care Setting

Ramona Dumitru ${ }^{1}$, Paula Avram ${ }^{1}$, Konstantinos Dimitriades $^{l}$

${ }^{T}$ Paediatric Critical Care Unit, Royal London Hospital, UK

Background and aims: XRays have become frequently used imaging tools in the Critical Care setting. CXRs (Chest Xrays) are used either to support clinical decision making and result in therapeutic changes or are 
used to confirm adequacy of current interventions and remain unchanged. This is a retrospective study aimed at evaluating the number of CXRs our patients receive and how the management of patients is influenced by such CXR.

Methods: All patients admitted during Oct-Nov 2017 on Paediatric Critical Care Unit at Royal London Hospital who have undergone at least one CXR per admission in the PCCU. Patients who have received only other XRays - such as abdominal, neck, pelvis, etc. will not be included in the study.

Clinical Data collection: Age of patient, PIM Score, Primary Diagnosis, Length of stay, Type of ventilation - invasive / non-invasive, Number of CXR received during admission, Indication for request, Routine - (e.g. Line/ET check), Urgent Xrays - (e.g. Clinical suspicion of pneumothorax/effusion), Change in management following CXR

Results: There have been 91 patients admitted between 01.10 -30.11.17. Of these, 74 had at least one CXR done. Total 141 of xrays. Average length of admission - 5.2 days/patient. Average of $2.1 \mathrm{CXR} /$ patient. Average PIM score of patients - 33.5\%. 79 of the xrays have been requested for urgent reasons (56\%) whereas (44\%) for routine actions. 47 of the urgent Xray were followed by a change in management $(33.4 \%$ of total xrays and $55 \%$ of the urgent ones)

Conclusions: All our CXR have been requested based on clinical reasons and more than half of them had urgent indication. More than half of the urgent Xrays were followed by a change in management (1/3 of total). Although our study is not large, we conclude that CXR should be performed based on clinical indication only.

\section{9}

\section{Critical / Emergency Care}

The Effect of Simulation-Based Training Prior to Moving to a New Department on the Quality and Safety of Patient Care Boris Fizdel ${ }^{l}$, Evgeni Yakobuvski ${ }^{l}$, Gideon Paret ${ }^{l}$, Tal Sadeh Vered ${ }^{l}$, Itai Pessach ${ }^{\top}$, Ortal Emergy ${ }^{I}$

${ }^{I}$ Pediatric ICU, Sheba-Academic Medical Center Hospital, Israel

Background: The PICU at Edmond and Lily Safra Hospital moved to its new 22-bed facility in February 2017. The transition included several profound changes in the department's operating philosophy that affect daily work, such as closed patient rooms, the possibility of parents staying next to their child and replacement of all monitoring and warning systems while significantly increasing the area of the department. In the face of these extensive changes and the high risk to hospitalized patients during this transition period, a preparatory training program was prepared prior to the actual transition

Method: Analysis of the challenges involved in the transition raised several key points: the need to test systems and work procedures, assimilate the changes in the new work routine, familiarize with new equipment and obtain the ability to provide adequate emergent critical care in the new work environment. In order to meet these challenges, an In-Situ simulation-based training program was created in the new department.

Results: Prior to the opening of the new department, the entire team underwent simulation-based training, which included 258 hours of training and testing of systems. Each simulation examined a different component of the daily work regime and management of extreme situations. Pitfalls were identified and re-examined in subsequent training.

Throughout the simulation-based training, more than 173 potential pitfalls were identified at different levels of severity, including 38 severe pitfalls that could endanger patient care.

Conclusions: Assessments and early simulation-based training in the new work environment allow early detection and correction of significant pitfalls and risks prior to moving to a new work environment. In addition, simulation reduces the level of anxiety and fear from transition, while increasing the responsiveness and readiness of the staff with a significant impact on the quality and safety of patient care.

\section{6}

\section{Critical / Emergency Care}

Role of Salivary Biomarkers in Pediatric Pain Assessment Kristina Ganzijeva ${ }^{I}$, Lina Jankauskaite ${ }^{1,2}$

${ }^{T}$ Medical Academy, Lithuanian University of Health Sciences, Lithuania ${ }^{2}$ Department of Pediatrics, Hospital of Lithuanian University of Health Sciences Kauno klinikos, Lithuania

Introduction: Pain self-reporting is currently a 'golden-standard' tool for pain assessment. However, it varies from patient to patient and could be inaccurately understood by healthcare professionals. According to several studies, acute as well as chronic pediatric pain remains misunderstood, under-diagnosed, and still under-treated. Moreover, accurate pain diagnostic markers are still missing.

Aims: To investigate salivary cortisol and melatonin concentrations in children with acute pain. To compare it with pain severity and vital signs changes. Methods: We conducted pilot observational study in Kauno Klinikos Pediatric emergency department (PED). Twenty-six patients referred to PED due to acute pain were included into the study. We excluded patients with chronical conditions, under pain-killers or hormonal treatment. We recorded patient's gender, age, vital signs (heart rate (HR), blood pressure (BP), respiratory rate $(\mathrm{RR})$, temperature $\left(\mathrm{t}^{\circ}\right)$ and oxygen saturation $(\mathrm{SaO} 2)$ ), pain characteristics (severity and duration of pain). Saliva samples were collected and stored in $-80^{\circ} \mathrm{C}$ till cortisol and melatonin ELISA analysis.

Results: Sixteen boys and 10 girls were involved in our research. Age median was 10 (4-16) years. Fourteen cases were trauma patients, 12 cases referred due to pain of other origin. we observed increased HR and BP due to pain. Other parameters were within age range. The median of cortisol and melatonin were 287.5(68-1330) and 17,6(8.6-46.8) pg/ml respectively. There was tendency of melatonin reduction with increased intensity of pain, however it was not significant $(\mathrm{p}=0.136)$. The longer the pain lasted, the higher cortisol levels were identified $(\mathrm{p}=0.01)$. No link was found between abnormal vital signs and changes in biomarkers.

Conclusion: Primary results show a cortisol rise with regard to pain duration. A drop in melatonin levels was observed with increasing pain. These results show cortisol and melatonin as potential biomarkers in acute pain diagnostics.

\section{6}

\section{Critical / Emergency Care}

\section{A Silent Fate}

Carolina Goncalves ${ }^{I}$, Carolina Fernandes ${ }^{1}$, Alexandra Andrade ${ }^{I}$,
${ }_{\text {Lucilia Aveiro }}$, Francisco Silva
${ }^{I}$ Pediatria, Hospital Dr. Nélio Mendonça, Portugal

Introduction: Carbon monoxide (CO) is an odorless, colorless, non-irritating, yet significantly toxic gas and is considered the leading cause of death by poisoning in the world. The clinical picture is variable and non-specific, ranging from mild to severe. Pediatric age range is a risk group, due to central nervous system immaturity and accelerated basal metabolism. In children, the clinical progression is more aggressive and difficult to detect than in adults. Clinical Case: The following cases describe two siblings who were observed in a Pediatric Emergency Department.

Case 1:

A 10-year-old female, who after awakening suddenly from bitemporal headache, briefly lost consciousness but recovered within minutes. After recovering, she had an episode of vomiting. The patient's physical examination was normal. ABG: $\mathrm{pH} 7.39$, lact $1.4, \mathrm{COHb} 25.2 \%$.

Case 2:

A 7-year-old male, after waking to use the toilet, began having a headache and feeling dizzy with lipothymia, but recovered in minutes. The patient's physical examination was normal. ABG: $\mathrm{pH} 7.37$, lact 1.4, $\mathrm{COHb} 21.7 \%$. Both parents remained asymptomatic. 
The diagnosis of $\mathrm{CO}$ intoxication was made, and the treatment protocol was fulfilled with oxygen therapy in a hyperbaric chamber. Two treatment sessions were performed, with the first treatment at 2 hours after hospital admission and the second occurring 12 hours later.Both patients were discharged 24 hours after the onset of the disease, with no associated symptomatology. Patients were instructed to follow up with a consultation with Hyperbaric Medicine at the 1st, 3rd and 6th month after the occurrence. Conclusion: These two cases demonstrate the importance of clinical judgment to a rather linear history. Correct and timely diagnosis is essential, to ensure rapid action and minimization of possible associated complications. The coordination of the team and between several services is essential.

189

\section{Critical / Emergency Care}

Outcomes following Presentation with Concussion in the Paediatric Emergency Department: Missed Cases of Post-Concussion Syndrome

Laura Harrison $^{1,2}$, Lorcan Duane $^{1,2}$

${ }^{T}$ Paediatric Emergency Department, Royal Manchester Children's Hospital, UK

${ }^{2}$ School of Medical Sciences, University of Manchester, UK

Background: 1 in 5 children will sustain a traumatic brain injury by the age of 16 years, with $90 \%$ of these being concussion. The majority recover to baseline function within 4 weeks, however $31 \%$ suffer from persisting symptoms. There is a lack of clear, consistent guidelines in the UK about management, discharge advice and follow-up of children with concussion. In our Paediatric Emergency department (PED) there was no follow-up arranged for any patients and no patient information leaflet.

Objectives: The aim was to identify any patients with concussion suffering from ongoing symptoms of post-concussion syndrome (PCS), who would benefit from follow-up. Additionals aims were to create an advice leaflet about concussion to be given to patients and make recommendations regarding systematic follow-up for future patients with concussion. Methods: A search was conducted for patients aged 16 or under, discharged from the PED in the last 15 months, with a diagnosis concussion. Their medical notes were reviewed and a telephone survey of parents was conducted asking about current symptoms of PCS.

Results: The search yielded 121 patients. 68 of 121 parents of patients were able to be contacted. Of these, 33 out of 68 reported symptoms of PCS $(48.53 \%)$ of new onset since their head injury, lasting longer than 4 weeks. School-aged children (5-12 years) were most commonly affected. The most common post-concussive symptoms reported were headaches, and problems with temper and impulsiveness.

Conclusion: Concussion management was found to be an area for improvement in this PED. A letter was written to the 33 children with PCS symptoms to arrange follow-up, and a new concussion leaflet for parents and patients was created to ensure follow-up was sought in the case of ongoing symptoms. Recommendations were made about the importance of starting systematic follow-up of all children with a diagnosis of concussion.

\section{7}

\section{Critical / Emergency Care}

Limp in Childhood: Are we Missing Significant Diagnoses? Laura Harrison ${ }^{1}$, Kate Hooper ${ }^{1}$, Sahana Rao ${ }^{1}$ ${ }^{T}$ Department of Paediatrics, Oxford University Hospitals, UK

Background: Limp is a common presentation and accounts for up to 5\% of emergency department visits in children. It is difficult to clinically differentiate benign, self-limiting conditions like transient synovitis, from more serious conditions such as septic arthritis and osteomyelitis. It is therefore imperative to investigate and manage appropriately. Currently, there are no national guidelines for the investigation and management of limp in children in the UK.

Objective: The aim of this audit was to see how many children presenting with limp to the Children's Clinical Decision Unit were being correctly investigated and managed according to local guidelines. Additional aims were to see if any significant diagnoses were missed and to update the guidelines as appropriate.

Methods: A search was done for patients presenting with limp to our Children's Clinical Decision Unit, within the last 6 months. Their electronic medical records were then reviewed for details of their admission, investigations, diagnosis and any missed diagnoses. These were compared to local guidelines at Oxford University Hospitals Trust.

Results: The search found 106 patients. All patient should have had bloods and an x-ray, according to our guidelines. 33 of the 106 patients had the correct initial investigations. Those with 2 or more risk factors for septic arthritis should have had an ultrasound and this occurred in 10 of 15 patients. The most common diagnosis was transient synovitis (45 out of 106) and there were 2 cases of osteomyelitis and 1 case of septic arthritis. There were 4 missed diagnoses including 1 case of osteomyelitis.

Conclusion: Adherence to limp guidelines was poor and as a result significant pathology was missed. Recommendations included increased education of doctors about the limp management guidelines, creation of limp proforma and reinforcing mandatory telephone reviews after discharge. Further ongoing work includes reviewing and updating the limp guidelines.

\section{0}

\section{Critical / Emergency Care}

Paediatric Abdominal Trauma: Variety of Trauma Mechanisms, Resulting Injuries and Treatment

Melanie Kapapa ${ }^{l}$, Katharina Esswein ${ }^{l}$, Alexandre Serra ${ }^{l}$

${ }^{T}$ Department of Surgery University Hospital Ulm, Division of Pediatric Surgery, Germany

The study concentrated on data related to trauma mechanism in children $(n=134)$ with blunt abdominal trauma like clinical findings, affected organs, results of radiologic imaging and clinical outcome. Points of interest were patient's age and sex, time and season of accident, mechanism of injury, involved objects, severity of injuries, treatment and complications. Accident mechanisms varied depending on age and male patients had higher severe grades of injury pattern $(\mathrm{p}=0.008)$. Boys are more often (in $67,9 \%$ ) involved to suffer an intraabdominal injury (IAI) with severe grades of injury pattern $(\mathrm{p}=0.014)$. Depending on age, proportions of various accident mechanisms occurred. Leading trauma was in $56 \%$ a fall, most common less than 1 meter, followed by sport related trauma $(41,8 \%)$ and road traffic accidents $(21.6 \%)$. In children with abnormal laboratory studies the grade injury pattern was more severe $(\mathrm{p} 0,001)$. Focused Assessment with Sonography for Trauma (FAST) was primary performed in $85,8 \%$ and correlation between free abdominal fluid and injury pattern was significant (p0.001). $41,8 \%$ had an IAI with organ laceration and $46,3 \%$ had additionally injuries like head or thorax trauma. Conservative treatment of IAI was performed in $60,7 \%(\mathrm{n}=34)$ and invasive treatment with abdominal surgery in $39,2 \%(\mathrm{n}=22)$. Complications occurred in $34.4 \%$, significantly after spleen $(p=0.001)$, liver $(p=0,001)$ and GI-tract injuries $(\mathrm{p}=0,001)$. Hospital duration stay increased with severity of injury (p0.001), especially after child abuse. Despite rarity of massive IAI after child abuse, it is vital for the affected children that involved medical staff kept this potential trauma issue in mind. We showed that risks for suffering severe IAI are present even during everyday activities, not just in traffic accidents. Focus should be on prevention on risk children, adapted to their age and danger potential. Parents have to be enlightened about risks directly and should train their children to perceive them. 
816

\section{Critical / Emergency Care}

E-Bike-Related Cranial Injuries in Pediatric Population Yevgeny Karepov $^{1}$, Danil A. Kozyrev ${ }^{I}$, Moni Benifla ${ }^{2}$, Vladimir Shapira ${ }^{2}$, Shlomi Constantini ${ }^{l}$, Jonathan Roth ${ }^{l}$

${ }^{1}$ Department of Pediatric Neurosurgery, Department of Pediatric Neurosurgery, Dana Children's Hospital, Tel-Aviv Medical Center, TelAviv University, Israel

${ }^{2}$ Pediatric Neurosurgery Unit, Pediatric Neurosurgery Unit, Rambam Health Care Campus, Haifa, Israel, Israel

Purpose: E-bikes are being used increasingly by all age groups. Children riding e-bikes often do not use safety equipment such as helmets, and are at increased risk for injuries requiring neurosurgery. The most common type of injury among pediatric e-bikers is head and neck trauma. We describe our experience treating cranial injuries.

Methods: Data regarding children (18 years old) with e-bike-related cranial injuries were collected retrospectively from two tertiary centers.

Results: Twenty patients were included. Seventeen were e-bike users, and three were hit as pedestrians. The average age at admission was $11.3 \pm 4.85$ (range 1.5-17) years old. All 17 e-bike users did not wear a helmet. Seventeen of the $20(85 \%)$ suffered from skull fractures $(70 \%$ involving the frontal bone), nine involving more than one region. Six patients $(30 \%)$ had intracerebral contusions, $3(15 \%)$ an epidural hematoma, and $6(30 \%)$ a subdural hematoma. Three patients $(15 \%)$ underwent surgery, two of them for depressed skull fracture reduction, and one for insertion of intracranial pressure monitor. One patient died (5\%); 1 (5\%) had a Glasgow Outcome Scale (GOS) of 3, 5 (25\%) had a GOS of 2, and $13(65 \%)$ were discharged without any neurological deficit (GOS 1).

Conclusion: E-bikes may inflict various cranial injuries, including fractures and intracranial bleeds, and may lead to significant morbidity and mortality. Education of children to use protective gear, wide exposure of younger adolescents to traffic laws, and limiting the use of e-bikes to older children, are all necessary actions.

\section{1}

\section{Critical / Emergency Care}

Predictors of Caregiver Satisfaction in Pediatric Laceration Repair in Pediatric Emergency Department

Soyun Hwang ${ }^{l}$, Hayoung Kim ${ }^{l}$, Jin Hee Lee ${ }^{2}$, Young Ho Kwak ${ }^{l}$, Do Kyun Kim ${ }^{1}$, Jae Yun Jung ${ }^{I}$, Hyuksool Kwon ${ }^{2}$, Dongbum Suh ${ }^{2}$, Yoo Jin Choi $^{2}$, Joong Wan Park', Se Uk Lee ${ }^{1}$, Ik Chang Choi ${ }^{1}$

${ }^{1}$ Emergency Medicine, Seoul National University Hospital, South Korea ${ }^{2}$ Emergency Medicine, Seoul National University Bundang Hospital, South Korea

Introduction: Laceration repair is one of the most common procedures performed in pediatric emergency department (PED). The objective of this study was to define the factors of care that are important to caregivers during a pediatric laceration repair and overall ED satisfaction.

Methods: This was a cross-sectional observation study performed in an urban, tertiary hospital. A convenience sample of patients younger than 18 years old presenting to PED for laceration repair was recognized and their caregivers were enrolled to complete a survey developed for this study. Demographic data of patient and caregivers were analyzed. Univariate and multivariate logistic regressions were used to determine which of these factors are related to satisfaction in the process of laceration repair and the overall ED process.

Results: Total 55 caregivers were enrolled. 39(70.9\%) of the caregivers were fathers. Majority of children had facial laceration $(n=44,80 \%)$ and median ED length of stay was 181 (IQR, 157-208) minutes. the median age of the children was 41.8 (IQR, 23-91) months and the median age of the caregivers were 37 years old (IQR, 35-41). Most of the laceration was repaired by plastic surgeons $(81.8 \%)$. In the multivariate regression analysis, 'preparing before procedure', 'mid-income family', 'caring attitude of nurse', 'cosmetic outcome', and 'measures to control patient's anxiety' were significantly related to the caregiver's satisfaction about the laceration repair ( $\mathrm{p} 0.05$ ), while 'preparing before procedure' and 'ED environment' were significantly related to the caregiver's satisfaction about the overall ED process (p0.05).

Conclusions: 'Preparing before procedure' was significantly related to the caregiver's satisfaction in both pediatric laceration repair and overall PED experience. The strongest predictors were 'cosmetic outcome' for laceration repair process and 'prepare for the procedure' for overall PED experience. Our findings suggest that improvements in various aspects will result in more satisfied parents.

\section{8}

\section{Critical / Emergency Care}

Apnoeic Event in Wolf-Hirschhorn Syndrome: Could this have been a "Tet" Spell?

Kene Maduemem ${ }^{1}$, Gheetha Arumugam ${ }^{1}$, Amol Chingale ${ }^{l}$

${ }^{T}$ Paediatrics, Lincoln County Hospital, UK

Background: Wolf-Hirschhorn syndrome (WHS) is a rare genetic condition caused by partial deletion of short arm of chromosome 4 . Clinical manifestations comprise but not limited to typical facie, mental retardation, growth delay, epilepsy, and congenital cardiac defects. Congenital heart defects occur in about $25-50 \%$ of cases.

Objective: We describe a child with WHS presenting with a femoral fracture who went apnoeic in the course of management resulting in a cascade of events.

Methods: Clinical presentation, laboratory and radiological findings are discussed.

Results: A 4.5-year-old boy known to have WHS was admitted with left femoral fracture. The syndrome was an antenatal diagnosis. Other associations noted were severe growth delay (weight of $6 \mathrm{~kg}$ ), epilepsy, global developmental delay, visual impairment, and tetralogy of Fallot (TOF). His TOF has been managed uneventfully on propranolol with acceptable oxygen saturation $(\mathrm{Sp} 02)$ in the $70 \mathrm{~s}$. The spontaneous fracture required Gallows traction, which provoked distress. He developed hypoxic crisis requiring oxygen therapy to maintain acceptable SpO2. Subsequently, he went apnoeic and dusky needing bag and mask ventilation. Blood gas revealed severe metabolic acidosis, which improved after fluid bolus. Kneechest position could not be attempted due to the fracture. Although he got treated for suspected sepsis due to the dilemma of events, he did have a hypercyanotic spell in hindsight. He was subsequently discharged with conservative management of the fracture.

Conclusion: To the best of our knowledge, this is the first reported case of hypercyanotic (Tet) spell in WHS. This rather uncommon event resulted in a significant learning point for medical and nursing staff.

\section{9}

\section{Critical / Emergency Care}

"Small Things" make a Huge Difference in the Emergency Department

Kene Maduemem ${ }^{1}$, Muhammad Adil Mughal ${ }^{1}$

${ }^{T}$ Paediatrics, Lincoln County Hospital, UK

Background: The assessment of children in a busy mixed adult and paediatric emergency department (ED) can be challenging. Good support from the ED staff not only improves patient safety but also, renders care delivery less daunting. 
Objective: To survey the clinical experiences of junior doctors in the resuscitation room and establish probable factors affecting patient safety before and after implementation of key recommendations.

Methods: An initial survey on the experiences of junior doctors during assessment of children in resus bays was conducted in October 2018. Some recommendations deduced were implemented; provision of sepsis trolley and grab box. A repeat survey in January 2019 was done to evaluate impact of implemented changes.

Results: Twenty doctors participated in the initial survey while 19 in the later survey. First survey was designated T1 and the repeat survey T2. All respondents reported attending at least 2 resuscitations in both surveys. Ten percent in $\mathrm{T} 1 \mathrm{vs.} 35 \%$ in $\mathrm{T} 2$ responded that a nurse was always present during resuscitation events. Ninety percent had performed or assisted in at least 1 procedure in the resuscitation bay in both surveys. Using a 5-point Likert scale, $40 \%$ of respondents in $\mathrm{T} 1$ vs. $63 \%$ in T2 were satisfied with the level of support of ED staff. Easy access to materials for resuscitation was rated good by $10 \%$ in T1 vs. $81 \%$ in T2. Over $70 \%$ in either survey requested resuscitation drugs; promptness of drug retrieval and administration was rated good by $13 \%$ (T1) vs. $45 \%$ (T2). The grab box was used by $65 \%$ in $\mathrm{T} 2$ with excellent feedbacks on its fall-back benefit.

Conclusion: Significant improvement was noted in care delivery following the implementation of the new sepsis trolley and grab box. There still exist areas for continued development in enhancing safe care delivery in this mixed adult and children ED.

\section{2}

\section{Critical / Emergency Care}

Post-extubation Acute Pulmonary Oedema in an Adolescent Catarina Amaro ${ }^{1}$, Joana Mendes $^{1}$, Pedro Protázio ${ }^{2}$, Carlos Rodrigues ${ }^{1}$, Sofia Ferreira ${ }^{1}$

${ }^{1}$ Paediatrics Department, Centro Hospitalar Universitário Cova da Beira, Portugal

${ }^{2}$ Anaesthesiology Department, Centro Hospitalar Universitário Cova da Beira, Portugal

Background: Post-extubation Acute Pulmonary Oedema (PEAPO) occurs in $0.1 \%$ of patients undergoing general anaesthesia, mostly in healthy children and young individuals, usually immediately after tracheal extubation. In paediatric age it has been rarely described, but the actual incidence is thought to be underestimated as many cases of post-operative respiratory distress may be misdiagnosed.

Case report: A former healthy 15 -year-old male was admitted to the emergency department with symptoms and physical examination compatible with acute appendicitis. A laparoscopic appendectomy was performed under general anaesthesia. Immediately after extubation, during which neither aspiration nor vomit were observed, he developed hypoxaemia $\left(\mathrm{SpO}_{2} 82 \%\right)$, intense coughing and polypnea, requiring additional $\mathrm{O}_{2}\left(\mathrm{FiO}_{2} 40 \%\right.$ using Venturi mask). He was hemodynamically stable, with normal cardiac auscultation and without oedema. His thorax expansion was symmetrical, and pulmonary auscultation presented symmetrical decreased breath sounds with dispersed fine crackles. Arterial gasometry showed $\mathrm{pH} 7.36, \mathrm{pCO}_{2} 45 \mathrm{mmHg}, \mathrm{pO}_{2} 48 \mathrm{mmHg}, \mathrm{HCO}_{3}{ }^{-}$ $26.8 \mathrm{mmol} / \mathrm{L}, \mathrm{BE}-0.5 \mathrm{mmol} / \mathrm{L}$, and the chest radiograph revealed bilateral diffuse opacities with ill-defined edges, suggesting acute pulmonary oedema. Intravenous furosemide was administered, and a quick recovery was noticed. The drug was maintained during 3 days, as well as supplementary oxygen, with progressive decreasing. With favourable evolution, the patient was discharged 4 days after surgery, asymptomatic, with normal cardiac evaluation and chest radiograph.

Conclusion: Although rare, PEAPO is a potentially fatal complication, which may worsen the prognosis of low-risk surgical procedures. It is important to recall this diagnosis in children and adolescents who present signs of respiratory distress and hypoxia after general anaesthesia, as prompt intervention is essential for a favourable outcome.
798

\section{Critical / Emergency Care}

The Introduction of Paediatric Cases to Pre-Hospital Grand Rounds Ronan Callanan ${ }^{1,2}$, Kasia Domanska ${ }^{1}$, Anne-Marie Murphy ${ }^{2}$, Alan Watts $^{1}$

${ }^{1}$ Department of Emergency Medicine, University Hospital Limerick, Ireland

${ }^{2}$ Department of Paediatrics, University Hospital Limerick, Ireland

Background: Paediatric emergencies can be challenging and intimidating for providers in the pre-hospital setting. Pre-hospital practitioners require a skill set very different to interventions performed in the adult population. These include challenging intravenous access, medication calculation, potential external stressors and a variety of clinical conditions seen less frequently.

Methodology: Pre-hospital Grand Rounds (PHGR) is a relatively new concept. It was introduced in St Vincent's University Hospital Dublin with the programme in University Hospital Limerick (UHL) commencing in October 2018. Following discussion with pre-hospital colleagues in the midwest region the coordinators of PHGR UHL acknowledged the importance and potential benefit of inclusion of paediatric cases at each session. A survey was conducted to gauge pre-hospital provider views on PHGR UHL as a source of paediatric emergency education.

Discussion: At each meeting a case is presented by a paramedic or advanced paramedic. This includes assessments, interventions on scene and subsequent handover in the Emergency Department. An Emergency Department doctor subsequently discusses the management of the child and outcome. A brief teaching session related to the primary diagnosis follows in an open forum with the audience, consisting of pre-hospital providers, nursing and medical staff. The meeting is supervised by a Consultant in Emergency Medicine with input from the Department of Paediatrics and National Ambulance Service training officers.

When surveyed, participants considered PHGR UHL as an educational resource for paediatric emergencies, considered the meeting to add to their practice and found paediatric emergencies posed different challenges to the adult population.

Conclusion: The introduction of paediatric cases at PHGR UHL has been met with enthusiasm and in its infancy is being considered a beneficial resource. It creates a link of communication between pre-hospital clinicians and hospital providers. The meetings do not follow a template but are developed through feedback and communication between all parties.

836

\section{Critical / Emergency Care}

Transient Loss of Consciousness Resulting in Syncope: Review of International Clinical Practice Guidelines

Leona Nertney ${ }^{1,2}$, Kimran Sidhu ${ }^{1}$, Paul McNally ${ }^{1,2}$

${ }^{T}$ Department of Paediatrics, Royal College of Surgeons in Ireland, Ireland

${ }^{2}$ NCRC, National Children's Research Centre, Ireland

Background: Syncope is a symptom characterised by transient loss of consciousness due to global cerebral hypoperfusion. 30-50\% of children and adolescents experience a faint. Syncope results in 1$3 \%$ of all visits to the Paediatric Emergency Department. While benign pathology dominates, recurrent events, ancillary injuries or anxiety around sinister pathology can bias clinical judgement. Resulting heterogeneity in clinical care drives wasteful investigations or referrals to oversubscribed services. Here we review stateof-the-art International Clinical Practice Guidelines (CPG) which may be used to reduce disease burden.

Methods: Structured review of Cochrane, Pubmed and Web of Science databases performed using search terms: ("Clinical Practice Guideline" 
AND "Paediatric" (OR "Pediatric") AND "Syncope"), ("Patient Algorithm" OR "Diagnostic Algorithm" AND "Paediatric" AND "Syncope").

Results: Our search generated 126 articles, with further review yielding five CPG's: European Society of Cardiology ${ }^{2018}$, American Heart Association $^{2017}$, Canadian Cardiovascular Society/Pediatric Cardiology Association $^{2017}, \mathrm{NICE}^{2011}$, Chinese Pediatric Cardiology Society ${ }^{2018}$. Guidelines used "Class I Evidence" or "Strong Recommendation" to support the role of the following: History (peri-event features), Physical Examination plus Family History (Epilepsy, premature cardiac death in $1^{\text {st }} / 2^{\text {nd }}$ degree relative $\left.40 \mathrm{yrs}\right)$. All but one endorsed ECG as a necessary diagnostic test. In the absence of positive findings, no further investigation is required. While presence of symptoms related to exertion should receive diagnostic work-up with referral to specialist services e.g. syncope unit, cardiology. Reassurance, education/ lifestyle measures remain the cornerstone of clinical management. Guidelines expanded on this to include medication use for refractory cases, maintaining psychological wellbeing and autonomic function training.

Discussion: Adherence to evidence-based guidelines ensures clinically appropriate management of syncope, whilst reducing wasteful investigations or referrals. Although all guidelines reviewed agree on history and examination, specific differences around ECG use, or nonpharmacological treatment exist.

\section{3}

\section{Critical / Emergency Care}

Procalcitonin Useful Diagnostic Marker for Treatment the Asphyxed Preterm Newborns with Sepsis

Danilo Nonkulovski ${ }^{1}$, Aspazija Sofijanova ${ }^{l}$, Silvana Naunova Timovska ${ }^{l}$, Mica Kimovska ${ }^{I}$, Tamara Voinovska ${ }^{l}$, Hristina Mangzukovska ${ }^{l}$, Spasija Neskova $^{1}$, Olivera Jordanova ${ }^{2}$, Aleksandra Janchevska ${ }^{3}$, Vankuver Mancev $^{4}$

${ }^{1}$ Neonatal intensive care unit, University Children Hospital, Macedonia

${ }^{2}$ Clinical laboratory, University Children Hospital, Macedonia

${ }^{3}$ Endocrinology, University Children Hospital, Macedonia

${ }^{4}$ Pediatric, Hospital, Macedonia

Background: Birth Asphyxia can lead to severe hypoxic ischaemic organ damage in newborns followed by neurodegenerative diseases, mental retardation and epilepsies, leading to significant mortality and morbidity in the Intensive Care Unit at the University Children Hospital-Skopje.

Objective: The aim of this study was to determining the corelation between the levels of PCT levels in pretern newborns with asphyxia and term newborns with asphyxia, with sepsis and proven blood culture

Methods: In this study we include the 31 (M:F=14:17) sepsis newborns with asphyxia and proven blood culture. They have been divided into two groups I group included 11 septic preterm newborns with asphyxia and II group - 20 septic term newborns.. Results of WBC, C-reactive protein (CRP) and PCT, were recorded. Procalcitonin levels was assessed before the beginning of the antibiotic treatment and second measurement after 3 days, by using a immunoassay system Vidas based on the Enzyme Linked Fluorescent Assay (ELFA) principles.

Results: We confirmed Staphylococcus, methicillin-resistance gene in all preterm newborns. 14 term newborns were with confirmed Staphylococcus, methicillin-resistance gene and in other 6 term newborns were confirmed Staphylococcus and Streptococcus pneumonia. The values of procalcitonin (PCT) were considerably increased in septic preterm newborns with asphyxia, relative to term newborns. This difference was statistically significant. The values of $\mathrm{C}$-reactive protein gradually increase after 24-36 hours in both groups. The second measurement of PCT after 3 days is a parameter, who show us whether an appropriate antibiotic for the treatment is used and may prevent severe sepsis and septic shock in newborns.
Conclusion: Increased PCT level was significantly associated with preterm newborns with confirmed Staphylococcus, methicillin-resistance gene. The value of PCT is a reliable parameter whether an appropriate antibiotic treatment is used in newborns with asphyxia, and and gram positive blood culture

\section{8}

\section{Critical / Emergency Care}

Factors Associated with Increased Risk for Pediatric Orbital Cellulitis - Who should we scan?

Lea Ohana Sarna Cahan ${ }^{1}$, Noa Hurvitz ${ }^{2}$, Itai Gross ${ }^{3}$, Adi Cohen ${ }^{2}$, Saar Hashavya ${ }^{3}$

${ }^{1}$ Department of Pediatrics, Hadassah-Hebrew University Medical Center, Jerusalem

${ }^{2}$ Faculty of Medicine, Hadassah-Hebrew University Medical Center ${ }^{3}$ Department of Pediatric Emergency Medicine, Hadassah-Hebrew University Medical Center, Jerusalem

Background: Periorbital cellulitis (POC) is a common infection in the pediatric population with minor complications. Orbital cellulitis (OC) carries Potential devastating complications of intracranial infection and vision impairment. Precise evaluation of a child with $\mathrm{POC} \backslash \mathrm{OC}$ is complicated due difficulties in physical examination and risks of imaging by CT. Method: A retrospective review of children aged 0-16 years admitted to the ED for POC or OC from 2009 to 2019.

Results: Over the ten years study period, 247 children 16 years were admitted to the ED with the diagnosis of OC\POC. Mean age $5.2 \pm 4.5$ years of age.

OC documented in 50 patients. The mean age was $7.8 \pm 4.3$ years. Fever (79.6\%), URTI $(42.9 \%)$, swelling of both eyelid (98\%), proptosis (30.6\%), and tenderness on percussion $(24.5 \%)$ were all more common in comparison to POC with $\mathrm{P}$ values of $0.0001,0.03,0.0001$, $0.0001,0.0001$ respectively. All children with diagnosis of OC underwent CT scan. Abscess was documented in $63.3 \%$ of the OC patients.

POC accounted for 247 patients. Mean age was $4.6 \pm 4.3$ years. In $19.8 \%$ of the cases local trauma or bite mark in the infected eye were recorded. Mean leukocyte count $\left(10^{9} \backslash \mathrm{L}\right)$ in OC group 15.2 vs $13.4(\mathrm{P}=0.05)$, ANC was significantly higher in the $\mathrm{OC} 11.3$ vs $7.2(\mathrm{P}=0.0001)$ whereas the lymphocyte count was higher in the POC 4.5 vs $2.4(\mathrm{P}=0.0001)$. Laboratory inflammation signs in OC had mean CRP levels (MG\%) of 11.7 vs $4.9(\mathrm{P}=0.0001)$ and $\mathrm{ESR}$ of 53.6 vs $36.4(\mathrm{P}=0.02)$.

Conclusion: Differentiation between OC an POC is cardinal. while previously findings on clinical examination such as ophthalmoplegia and proptosis were found indicative of OC rather than POC, our study highlights the importance of inflammatory markers including ANC, CRP and ESR in the assessment of infections of the eye.

959

\section{Critical / Emergency Care}

Metamizol Sodic - Milliliters or Drops ?

Carmen Olaru ${ }^{1}$, Valentin Olaru ${ }^{2}$, Tamara Solange Roșu ${ }^{1}$, Ileana ${ }_{\text {Katerina Ioniuc }}^{3}$, Smaranda Diaconescu ${ }^{3}$

${ }^{1}$ Emergency Room, Emergency Children Hospital ,,Sf. Maria", Romania

${ }^{2}$ Intensive Care, Emergency Children Hospital ,Sf. Maria", Romania

${ }^{3}$ Pediatrics, Emergency Children Hospital ,Sf. Maria", Romania

Background: Accidental poisoning with metamizol sodic, by confounding the number of drops with the number of milliliters are going to become an important problem of admission in our emergency department. Objectives: The aim of this study was to describe signs and symptoms of the intoxication, the evolution, the treatment and to established some measures to avoid these situations. 
Material and Methods: A retrospective study was performed. The study group includes 12 children admitted in the emergency room due to January 2017 until December 2018

Results and Discussions: In the study group, age between 7 months and 8 years, the children received a number of milliliters of metamizol sodic equal with the number of drops necessary. Time between ingestion and the admission in the emergency room varied between a few minutes (when the parents took note the incident), until 72 hours. The maximum dose was 15 grams. The symptomatology was severe (seizures, sleepiness) in 3 cases, moderate (vomiting, nausea, abdominal pain, purpura) in 6 cases, 3 cases were asymptomatics. Laboratory investigations revealed leucopenia in 5 cases, trombocitopenia-1, hepatocytolisis - 3, modifications of renal function tests- 3 , and in 3 were cases normal. The treatment consisted in gastric lavage- 4 cases, forced diuresis, hepatoprotectives, corticosteroid therapy - 3 cases. The outcome was favorable in all the cases, 3 children developed cutaneous rash and 2 bronchospasm after few days.

Conclusion: Be careful with metamizol sodic dosage!

\section{1}

\section{Critical / Emergency Care}

Button Battery Ingestion-Case Series from Lakonia, Greece Aikaterini Pana ${ }^{l}$, Olympia-Panagiota Rozakea ${ }^{l}$, Maria-Maroudia Berikopoulou', Panagiotis Panas ${ }^{2}$, Sofia Vasilakou ${ }^{1}$, Afroditi Barmpakou ${ }^{l}$, Ioannis Panagiotou ${ }^{I}$

${ }^{1}$ Pediatrics, General Hospital of Lakonia- Nursing Unit of Sparta, Greece

${ }^{2}$ Obstetrics-Gynaecology, University Hospital Of Patras "Panagia $i$ Voitheia", Greece

Objectives and Study: Button battery ingestions epitomize the challenge among pediatric foreign body ingestions as the outcome can vary from harmless to lethal. The serious outcomes refer to children younger than 5 years old and when ingested batteries are larger than $20 \mathrm{~mm}$ diameter(higher-risk patients).

Esophagus is the target tissue of caustic injury and acute endoscopic treatment is needed. In cases, where battery lies beyond the esophagus (stomach, bowel etc) and the patient is asymptomatic, abdominal radiographs and observation are advocated. Endoscopic removal is suggested only if the battery remains inside the stomach 4 days after ingestion.

Methods: We report two cases of toddlers, presented in our pediatric department, that ingested button batteries with their diagnostic X-rays.

The first was a 3-year-old girl that ingested a battery smaller than $12 \mathrm{~mm}$ diameter $(10 \mathrm{~mm}) 2^{1 / 2}$ hours prior to her admission. Her vital signs were stable (HR:108-114/min) and was asymptomatic. An X-ray was obtained that revealed a radiopaque object(battery) in the bowel. From the laboratory exams, only increased amylase levels were found(221U/1, with normal values:30-118U/1).

The second was a 2.5-year-old girl that ingested a battery larger than $12 \mathrm{~mm}$-diameter $(20 \mathrm{~mm}) 1$ hour prior to her admission. Her vital signs where stable (HR:100/min) and also remained asymptomatic. The X-ray that was performed revealed a battery in the stomach. No laboratory exams were obtained.

Results: Both children received intravenous(iv) fluids and ranitidine as prophylaxis and were immediately transferred to a pediatric hospital with endoscopic unit. They remained asymptomatic throughout their stay and endoscopic intervention was not needed. The battery was dismissed through the stool in both cases, during the second $\left(2^{\text {nd }}\right)$ and third $\left(3^{\text {rd }}\right)$ day after ingestion, respectively.

Conclusion: Button battery ingestions, where battery lies beyond the esophagus and the patient remains asymptomatic, should be managed with caution, as esophageal damage cannot be excluded. Endoscopic intervention should be within immediate reach whether or not it is actually performed in the end.
109

\section{Critical / Emergency Care}

Prevalence and Outcomes of CPR in Pediatric In-Hospital Cardiac Arrest: A 7-year Experience at a Single Center in Thailand

Uthen Pandee $^{I}$, Thita Pacharapakornpong ${ }^{I}$, Jarin Vaewpanich ${ }^{l}$, Nattachai Anantasit ${ }^{I}$

${ }^{I}$ Department of Pediatrics, Faculty of Medicine Ramathibodi Hospital, Mahidol University, Thailand

Background: Children with in-hospital cardiac arrest (IHCA) has variety outcome in difference centers and country.

Objective: The objective in this study was to report the prevalence, characteristic and outcomes of IHCA and also evaluate associated factors in survival.

Methods: A retrospective study including children aged 1 month to 18 years were diagnosed with cardiac arrest and received cardiopulmonary resuscitation between January 2010 and December 2016. Descriptive and logistic regressions analysis was used to analyze factors that related to the return of spontaneous circulation (ROSC) and survival.

Results: Total of admission was 32,122 . Of 88 patients $(0.27 \%)$ were enrolled. Median age of patients was 1.21. The first documented rhythm was pulseless electrical activity ( $43 \%$ ). The overall sustained ROSC and survival rate were $62.5 \%$ and $15.9 \%$, respectively. Of $78 \%$ of those who survived had favorable neurological outcome. The most common cause of IHCA was septic shock. Ischemic cause, development of subsequent asystole and receiving CPR more than 20 minutes had significant higher sustained ROSC rate (p0.05). Multivariate analysis to adjust for the clinical variables significantly associated with failure ROSC confirmed that patients with prolonged duration of cardiopulmonary resuscitation (CPR) and subsequent asystole had higher failure ROSC ( $\mathrm{p} 0.05)$. However, patients who received vasoactive medications less than two drugs had higher hospital survival rate $(\mathrm{p}=0.024)$.

Conclusion: Duration of CPR was the most important factor associated with achieved ROSC, whereas number of vasoactive medications was the factor associated with hospital survival rate.

738

\section{Critical / Emergency Care}

Implementation of the Paediatric Early Warning Score in the Observation Room of the Emergency Department of a Second Level Hospital

Miguel Paiva Pereira ${ }^{I}$, Hugo de Castro Faria ${ }^{I}$, Paula Poção ${ }^{I}$, Ana Serrão Neto

${ }^{I}$ Centro da Criança e do Adolescente, Hospital CUF Descobertas, Portugal

The Paediatric Early Warning Score (PEWS) was initially developed to pre-emptively identify hospitalized children who were at risk of clinical deterioration $^{1,2}$. A study in a paediatric Emergency Department (ED) found that an elevated score was associated with need for Intensive Care Unit (ICU) admission ${ }^{3}$. A lot of PEWS systems have been developed but a recent systematic review noticed lack of consensus on which PEWS is most effective, although improved clinical outcomes were reported $^{4}$. In July 2017 we implemented the Irish PEWS 5 in our paediatric ED aiming to objectify and improve clinical monitoring in our unit.

The purpose of this study is to review our data by verifying if a higher PEWS score correlated to clinical deterioration and if it is applicable to our clinical practice.

To do so we evaluated medical records of patients who were applied the PEWS between July 2017 and March 2019 and registered the maximum score (MS). We defined clinical deterioration as the need for ICU transfer, higher respiratory support or the presence of sepsis criteria.

We evaluated 840 children admitted to the ED observation room, whose age and MS distributions are represented, respectively by the graphics 1 and 2 . 
The MS was $\geq 4$ in 181 (22\%). For a MS $\geq 4$, the sensibility and specificity for clinical deterioration was respectively $96.5 \%$ and $84.0 \%$. Respiratory diseases were the most common diagnosis (227 patients), with 135 (59\%) MS $\geq 4$, whose sensibility and specificity for clinical deterioration were, respectively, $100 \%$ and $48.9 \%$. We also evaluated this score for MS of 5 and 6.

In addition of being a system that allows a standardized surveillance, the use of this score seems to improve patient safety and medical decision, particularly by excluding clinical deterioration, as showed by the high sensitivity values reached using the MS of 4 as a cut-off.

405

\section{Critical / Emergency Care}

Sedation and Analgesia in a Pediatric Intensive Care Unit (PICU): Point of Care Study

Andrzej Piotrowski $^{1,2,3}$, Olga Stańkowska ${ }^{1,2,3}$, Adam Grabowski, ${ }^{1,2,3}$, $\overline{\text { Andrei Trebukhousk }}^{1,2,3}$, Jolanta Gardocka ${ }^{1,2,3}$

${ }^{1}$ Anaesthesia and Intensive Care, Children's Memorial Health Institute, Poland

${ }^{2}$ Anaesthesia and Intensive Care, Children's Memorial Health Institute, Poland

${ }^{3}$ Anaesthesia and Intensive Care, Children's Memorial Health Institute, Poland

Background: Variety of sedation methods are applied for children treated in the PICU without clear evidence about their usefulness. Opioids and benzodiazepines have been a standard analgesic and sedative combination. Currently newer agents, like a2-adrenergic agonists have been proposed.

Objective: To assess the principal sedative and analgesic drug choices for patients treated in a pediatric medical/surgical ICU.

Methods: The study was conducted in a 24 bed complex of two intensive care units, surgical and medical, at one institution. A one day analysis was chosen. The medications, their methods of administration, and doses were analyzed.

Results: There were 24 patients treated in both wards on the day of study, 20 of them required mechanical respiratory support. The majority of patients (13) were up to 3 days after surgery. The most common analgesic drug was morphine used in 16 children (in 9 as bolus, in 5 as infusion) administered for a median of 2 days (range 1-7), followed by non-opioid drugs (paracetamol and metamizole) in 6 cases, and sufentanil by infusion in 2 . The major medication for sedation was midazolam (14 patients, in 5 as an infusion) administered for a median of 3 days (range 1-22), followed by dexmedetomidine infusion in 9 patients (median duration 4 days, range 1-22 days, mean 8 days). The doses of morphine were in the lower therapeutic range (mostly $0,1 \mathrm{mg} / \mathrm{kg}$ bolus), doses of dexmedetomidine were from 0,6 to 1,5 micrograms $/ \mathrm{kg} / \mathrm{hr}$. Oral methadone was administered in 2 children, in one in combination with clonidine. Ten patients were treated concomitantly with 2 analgesic/sedative drugs, five 3 medications, four were receiving 4 drugs, and five one.

Conclusion: In our study morphine, midazolam, dexmedetomidine were main analgesic and sedative drugs used in the PICU. Dexmedetomidine was used for longer periods of time than suggested by the manufacturer and literature.

\section{9}

\section{Critical / Emergency Care}

\section{Familial Hypokalemic Periodic Paralysis} Irina Rosa ${ }^{I}$, Bernardo Camacho ${ }^{I}$, Paulo Sousa ${ }^{2}$, Francisco Silva $^{l}$ ${ }^{T}$ Pediatria, Hospital Dr Nélio Mendonça, Portugal

${ }^{2}$ Neuropediatria, Hospital Dr Nélio Mendonça, Portugal

Introduction: Periodic Paralysis (PP) is a rare neuromuscular disease related to a defect in muscle ion channels, characterized by sporadic episodes of painless muscle weakness that can be precipitated by vigorous exercise, prolonged fasting, or carbohydrate-rich meals. PP is classified as hypokalemic when episodes occur associated with low levels of potassium in the blood. The crises start in late childhood or early adolescence and vary in frequency and duration. Treatment during the acute phase is administration of potassium chloride.

Case Report: A 12-year-old female adolescent appealed to the emergency department due to sudden onset of tetraparesis. She had no difficulty breathing nor signs of respiratory distress. Cardiac, pulmonary and abdomen examination was normal. Conscious and oriented in time and space. Neurological examination showed grade II muscle strength in the lower limbs and grade III in the upper limbs. Osteotendinous reflexes were absent. Facial mimic was present. Upon this presentation the mother promptly revealed a familiar history of periodic hypokalemic paralysis affecting several members.

Blood analysis showed an isolated severe hypokalemia of 1,7-2,0 $\mathrm{mmol} / \mathrm{L}$. ECG showed prolonged PR interval, mild ST depression, prominent $\mathrm{U}$ waves.

Slow correction of potassium was promptly started with gradual recovery of muscle strength and ECG stabilization. She was discharged 20 hours after therapy onset and medicated at home with daily acetazolamide and potassium chloride if onset of symptoms. Genetic study was started for índex familial mutations.

Conclusion: Periodic familial paralyzes are rare conditions, the most frequent being the hypokalemic form. The diagnosis of PP can be confirmed by genetic tests, which are recommended as first line when there is an intermediate to high degree of clinical suspicion. This case report is intended to emphasize the importance of the clinical history and a complete physical examination on diagnosis. Treatment should not be delayed. Genetic family counseling should be offered.

\section{1}

\section{Critical / Emergency Care}

\section{Alkaline Lemonade Poisoning: A Case Report}

Virginia Santana Rojo ${ }^{1}$, Marta Bautista Bareal, Mariano Silva Hernández, José Manuel Caballero Caballero ${ }^{I}$, Isabel González. Bertolin ${ }^{2}$, Paula García Sánchez ${ }^{2}$, Rosario López López ${ }^{2}$, Francisco Javier Aracil Santos ${ }^{1}$

${ }^{1}$ Department of General Paediatrics and Paediatric Infectious Diseases, Hospital Universitario La Paz, Spain

${ }^{2}$ Paediatric Emergency Department, Hospital Universitario La Paz, Spain

Background: First-line treatment for acute gastroenteritis (AGE) are oral rehydration solutions. These prepared solutions are recommended by WHO and ESPGHAN since they assure a right replacement of hydroelectrolitic disbalance, advising against other alternatives.

'Alkaline lemonade' is a home-made alternative to prepared solutions, made with water, sugar, salt, lemon and baking soda. It has been widely recommended and extensively used in Spain due to its availability and effectiveness. We report a case of metabolic alkalosis and electrolytic disturbances in a patient with AGE, who was treated with 'alkaline lemonade'. Case Presentation: A previously healthy 11-month-old boy was brought to the Emergency Department with an AGE caused by Norovirus. On his arrival he was afebrile and hemodynamically stable, with signs of moderate dehydration. Admission blood tests revealed metabolic alkalosis $(\mathrm{pH} 7.54, \mathrm{pCO} 2$ $45.7 \mathrm{mmHg}$ and bicarbonate $38.4 \mathrm{mmol} / \mathrm{L})$, hypokalemia $(2.3 \mathrm{mmol} / \mathrm{L})$ and hypernatremia $(145 \mathrm{mmol} / \mathrm{L})$, with normochloremia, were observed in first blood tests. The electrocardiogram showed flat T-waves in all derivations, without any other changes. He was admitted to the Paediatric Intensive Care Unit, where a fast correction of hypokalemia was performed, with disappearance of electrocardiogram disturbances. He received intravenous fluid therapy until complete recovery of detected alterations.

Due to the importance of disturbances, tubulopathies were ruled out. When the parents were asked about their son's home management, they reported having administered home-made 'alkaline lemonade', without being able to specify the amount of baking soda, but bigger than the 
recommended 'point of knife' quantity. Therefore, the 'alkaline lemonade' poisoning along with the dehydration caused by AGE, explain the magnitude of ionic and acid-base disorders.

Conclusions: 'Alkaline lemonade' recipe is not very accurate, which can lead to elaboration mistakes, as our patient's case. In conclusion, we should recommend oral rehydration solutions for AGE management and inform appropriately about their alternatives.

\section{6}

\section{Critical / Emergency Care}

Falls Under 6 Months of Age: 7-year Period at a Portuguese Hospital $\underline{\text { Ana Sofia Vilardouro }}^{1,3}$, Joana de Brito Chagas ${ }^{2,3}$, Tânia Carvalho ${ }^{3}$, David Marques Lito

${ }^{1}$ Service of Paediatrics, Department of Paediatrics, Santa Maria Hospital - North Lisbon Hospital and Universitary Centre, EPE, Portugal

${ }^{2}$ Department of Paediatrics, Paediatric Hospital, Coimbra Hospital and Universitary Centre, EPE, Portugal

${ }^{3}$ Service of Paediatrics, Vila Franca de Xira Hospital, Portugal

Background: Falls are the third leading cause of death by unintentional injury in European Union children and represent an important cause of incapacity. There is lack of scientific research on the less-than-6-monthold group.

Objective: To describe epidemiologic characteristics, outcomes and factors related to a worse outcome of falls in a specific age group children. Methods: Retrospective descriptive study of all infants younger than 6 months admitted to our Emergency Room (ER) because of unintentional fall. The study was conducted at a second level Portuguese hospital over a 7-year period (2012 through 2018).

Results: There were 112 ER admissions related with falls in this group $(0,045 \%$ of all admissions).

Forty-eight percent had fallen from baby equipment. The majority $(82,4 \%)$ occurred from a $50-100 \mathrm{~cm}$ high and head trauma was present in $94,8 \%$. Symptoms occurred in $23,2 \%$ of infants and head injury was the most common finding on medical examination $(47,3 \%)$. Serious injuries were seen in $16,1 \%$. Imaging abnormalities were identified in $7,1 \%$ (all with head fracture; one third with brain injury) and 6,3\% required hospital admission or transference to another hospital.

Serious injury was more frequent in infants younger than 3 months $(66,7 \%$ vs $33,3 \%, p=0,009)$, in those who had fallen from more than $76 \mathrm{~cm}$ high $(\mathrm{p}=0,013)$ and when medical examination revealed alterations $(72,2 \%$ vs $27,8 \%, p=0,032)$. Radiography with suspected skull fracture was significantly associated with fracture on computed tomography $(p=0,033)$. No deaths or permanent sequelae were found.

Conclusion: In our study the most serious injuries occurred in infants younger than 3 months and from relatively small heights. Although present in almost $10 \%$, fractures and brain injury didn't have long term sequelae. It is important to reinforce the importance of preventive and supervision measures.

\section{0}

\section{Critical / Emergency Care}

Knowledge Translation in Western Australia Tertiary Paediatric Emergency Department: An Audit Cycle of Effectiveness of Standard Education on Updated Bronchiolitis Guideline Yee Lymn Yeo ${ }^{l}$, Meredith Borland ${ }^{l}$, Sharon O'Brien $^{1}$, Natasha Bear ${ }^{2}$

${ }^{T}$ Emergency Department, Perth Children's Hospital (formerly Princess Margaret Hospital), Australia

${ }^{2}$ Telethon Kids Institute, Perth Children's Hospital, Australia

Background: Bronchiolitis is the most common cause of presentation and hospitalization for infants in Australia and New
Zealand. Multiple clinical practice guidelines have been published to minimize unnecessary interventions in infants with bronchiolitis; however, the challenge is translating the knowledge and evidence into clinical practice.

Objective: The objective of this audit cycle was to determine if standard education process of the updated bronchiolitis guideline in a tertiary paediatric emergency department (ED) will reduce unnecessary interventions in infants with bronchiolitis.

Methods: A retrospective chart review was conducted in a tertiary paediatric ED of bronchiolitis presentations in 2015 (pre-education cohort of 465 presentations) and in 2017 (post education cohort of 343 presentations) after implementation of the updated guideline prior to the 2017 bronchiolitis season. The primary outcome was to evaluate the rate of interventions shown to be ineffectual in the updated guideline with secondary outcomes evaluating the rate of hospital, ICU admissions and the rate of multiple interventions versus single intervention.

Results: There was no difference found between 2015 and 2017 for chest $\mathrm{x}$-ray, salbutamol, adrenaline, antibioitics and nasopharyngeal aspirates. There was increased use of glucocorticoids with increased odds of $60 \%$ $(p=0.002)$ which was statistically significant. There was no difference with the number of interventions between 2015 and 2017. Hospital admissions were reduced from 2015 to 2017 (65.2\% versus 56.0\% respectively), with reduced odds of admission of $18 \%(\mathrm{p}=0.008)$ that was statistically significant. There was no difference in ICU admissions.

Conclusion: The updated guideline through standard education process in ED did not show any statistically significant reduction of unnecessary interventions. Community initiated glucocorticoid administration, however, was increased. A larger multi-centered study will be beneficial to determine if the awareness of the guideline to the broader community would reduce unnecessary interventions as well as the challenge of translating current evidence into clinical practice.

\section{3}

\section{Endocrine \& Diabetes}

Management of Diabetic Ketoacidosis in Pediatric Intensive Care Unit

Habib Besbes ${ }^{1}$, Imen Rhouma ${ }^{1}$, Radhia Hadj Salem ${ }^{1}$, Chebil Ben $\overline{\text { Meriem }}^{I}$, Leila Ghedira ${ }^{I}$, Chokri Chouchane ${ }^{I}$, Slaheddine Chouchane ${ }^{I}$ ${ }^{I}$ Pediatric Department, LR12SP17, Fattouma Bourguiba Hospital Monastir University, Tunisia

Background: In recent years, the incidence of type 1 diabetes has been increasing in children. The prognosis is related to acute metabolic complications, mainly ketoacidosis.

Methods: A retrospective descriptive study of patients admitted with inaugural ketoacidosis of type 1 diabetes to ICU of Pediatric Department in Fattouma Bourguiba Hospital of Monastir over 10-year period from 2008 to 2017. Results: A hospital incidence of the inaugural ketoacidosis was 2 per 1,000 . The rate of the inaugural ketoacidosis among new diabetes cases was $22.52 \%$. Fifty patients were collected: 23 girls and 27 boys. The mean age at admission was $6.6 \pm 4.1$ years. The mean time between the onset of symptoms and hospitalization was $15.51 \pm 9.27$ days. Polyuro-polydipsic syndrome and massive ketonuriawas found respectively in $98 \%$ and $82 \%$ of cases. Mean blood glucose at the time of diagnosis was $4.92+/-0.14 \mathrm{~g} / \mathrm{L}$. 16 children were treated according to modified Lestradet protocol and 34 childrenwere treated according to modified ISPAD protocol. Correction of blood glucose, ketonuria, $\mathrm{pH}$ and bicarbonates up to $\mathrm{H} 8$ were without significant difference between the two protocols (p0.05). The most common complication in the acute phase was hypokalemia ( 8 cases). No cases of cerebral edema were observed.

Conclusion: The inaugural ketoacidosis in children remains common in our region. Early diagnosis of type 1 diabetes is important to prevent complications. 
632

\section{Endocrine \& Diabetes}

A Rare Cause of Familial Pubertal Delay

Filipa Briosa $^{1,2}$, Rita Valsassina ${ }^{1,2}$, Joana Soares ${ }^{2,3}$, Marta Amorim $^{4}$, Catarina Limbert ${ }^{2}$

${ }^{1}$ Department of Paediatrics, Hospital Beatriz Ângelo, Portugal

${ }^{2}$ Department of Paediatric Endocrinology, Hospital Dona Estefânia, Portugal

${ }^{3}$ Department of Paediatrics, Hospital de Santo André, Centro Hospitalar de Leiria, Portugal

${ }^{4}$ Department of Genetics, Hospital Dona Estefânia, Portugal

Background: Delayed puberty (DP) is described as central or peripheral whether the problem lies in the hypothalamo-pituitary axis or in the gonads respectively. Idiopathic hypogonadotropic hypogonadism (IHH) is a central cause of DP due to a failure of gonadotrofin releasing hormone $(\mathrm{GnRH})$ secretion impairing the physiological initiation of puberty.

Objective and Methods: We present three intra-familial cases of normosmic IHH (nIHH) related to a new association of two heterozygotic TARC3 mutations.

Case Report: The index case reports to a 16 years-old male with no pubertal development at this age. Later, two of his three sisters also presented complete hypogonadism. All cases had a normal sense of smell. Investigation showed low sex steroid and gonadotropin levels and no evidence of a mass lesion in cerebral MRI. The diagnosis of nIHH was supported by the identification of two heterozygous mutations on TACR3 gene in all subjects.

Conclusion: Congenital IHH is a very rare genetic disorder that if undiagnosed or untreated may lead to infertility associated to complete or partial absence of GnRH.TACR3 mutations have a critical importance on sexual maturation and are an important genetic cause of nIHH. The treatment of nIHH is based on sex steroids replacement, therefore promoting the pubertal development.

273

\section{Endocrine \& Diabetes}

Exercise in Pediatric Type 1 Diabetes Mellitus: Which, Why and How.

Sofia Carneiro ${ }^{1}$, Elisa Galo $^{2}$

${ }^{T}$ Area da Mulher, Criança e Adolescente, Centro Hospitalar Universitário de Lisboa Central - Hospital Dona Estefânia, Portugal

${ }^{2}$ Serviço de Pediatria, Centro Hospitalar do Oeste - Torres Vedras, Portugal

Background: Regular exercise has important health and social benefits for children and adolescents with type 1 diabetes mellitus (T1DM). Different exercise types, intensity and duration have significantly different effects on blood glucose (BG) control, and on the endocrine response to exercise. There have been significant recent Improvements in the knowledge of what underlies these changes and in the appropriate management to support the maintenance of euglycemia. This effects frequently presents additional barriers to exercise - fear of hypoglycemia and loss of glycemic control - increasing sedentary lifestyle in this population. Thus, exercise presents several important challenges to diabetes management, and requires special management by patients and clinicians, based on the knowledge of the impact of exercise on BG homeostasis.

Objective: To systematize the principles of exercise prescription in pediatric population with DMT1, including practical advices in the management of the metabolic control.

Methods: Systematic literature searching in PubMed database. Two types of literature were reviewed: guidance and published studies.

Conclusion: The most recent studies show the positive effects of exercise on cardiovascular risk management, helping to achieve target lipid profile, body composition and fitness and glycaemic goals. This population should be encouraged to practice a wide variety of exercise, with a careful management to ensure a good metabolic control. In general, aerobic exercise tends to lower BG concentrations, while anaerobic exercise tends to increase them. Both exercise types can be performed by children and adolescent with T1DM, and exercise prescription must be individualized, based on a sound understanding of the underlying physiology and adapted to the patient's characteristics. The collaboration between the child/adolescent, healthcare provider, parents, school and the physical education instructor is crucial to a good adherence, promoting an optimal glycemic control during and after exercise.

\section{0}

Endocrine \& Diabetes

Variations in How Youth with Type 1 Diabetes Transition to Adult Care

Roger Chafe ${ }^{l}$

${ }^{T}$ Pediatrics, Memorial University of Newfoundland and Labrador, Canada

Background: Young adults with type 1 diabetes are at increased risk for acute complications during their transition from pediatric into adult care. While there is likely considerable variation in how transition care is structured, there is little evidence about the impact that this variation may have on patient outcomes in early adulthood.

Objectives: To determine how young adults with type 1 diabetes transition into adult care in two Canadian provinces: Newfoundland and Labrador (NL) and Ontario $(\mathrm{ON})$; to examine the impact of different models of transition care on outcomes for patients with type 1 diabetes; and to explore ways to better support patients with type 1 diabetes as they transition into adult care.

Design: Mixed methods: Administrative data and qualitative research.

Results: There are significant variations in how the transition to adult care was structured both within and between provinces. The rate of diabetic ketoacidosis (DKA) in ON youth $(n=2,525)$ was higher in those aged 19-21 years compared to $15-17$ years $(12.0$ vs. $9.3 / 100$ person-years, p0.0018). There was no difference in the rate across transition-age in youth from NL $(n=93)$. In $N L$, there was no dedicated transition program for much of the province. We helped develop and evaluate a single session transfer clinic. We found that such a clinic is a promising step towards improving patient and parent satisfaction without requiring new staff or significant outlays of new resources. It is unclear whether such a clinic will ultimately impact rates of hospitalizations or DKA.

Conclusion: In two Canadian provinces with different transition models and levels of resources, we found consistency in poor outcomes. Adverse events for this vulnerable population are high in both provinces and efforts to optimize system-level transition care are needed.

\section{0}

Endocrine \& Diabetes

Precocious Puberty Caused by Ovarian Dysgerminoma with Gonadoblastoma in a 46,XX Girl: A Case Report Iveta Dzivite $^{1,2}$, Margi Patel ${ }^{1,2}$, Lasma Lidaka ${ }^{2}$

${ }^{T}$ Endocrinology, Obstetrics and Gynaecology, Riga Stradiņš University, Latvia

${ }^{2}$ Endocrinology, Obstetrics and Gynaecology, Children's Clinical University Hospital, Latvia 


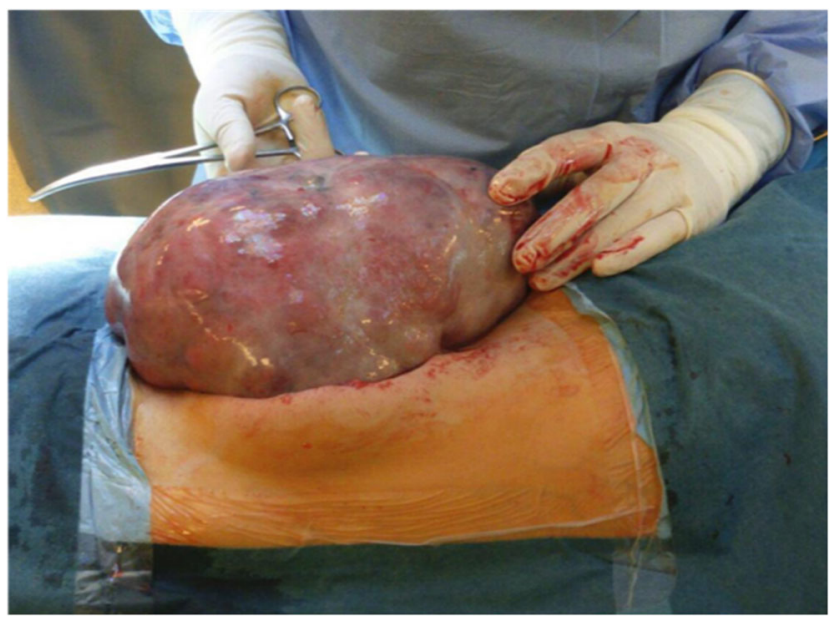

Introduction: Gonadoblastoma is a rare tumour consisting of germ cells resembling granulosa and Sertoli cells. Sometimes these tumours contain stromal elements indistinguishable from lutein and Leydig cells. Almost all patients have 46,XY karyotype or various forms of mosaicism. Dysgerminomas are malignant, undifferentiated germ cell tumours that constitute $1 \%$ of primary ovarian neoplasms. Approximately 5\% of dysgerminomas arise in abnormal gonads(from a gonadoblastoma).

Methods: The clinical case was analysed using patient history and clinical examination, laboratory, genetic, radiological and histological investigations, treatment methods and follow-up procedures.

Results: 6 years 4 months old female presented with early pubertal development, tall stature and a large abdominal mass. Height $135 \mathrm{~cm}(+3.2$ SDS) weight $30.45 \mathrm{~kg}(+2.0 \mathrm{SDS})$. Tanner puberty stage III: Px4, Ax1, Ma3, Me1. Signs of virilization were present.

Laboratory data: significantly elevated estradiol, androstenedione, testosterone, alfa-fetoprotein, beta-hCG and AMH. LH and FSH - prepubertal level.

Bone age(RUS score-598 points) corresponded to an age of 12.2. MRI showed large heterogeneous mass $(\mathrm{CC} 25.1 \mathrm{~cm}, \mathrm{LL} 18.9 \mathrm{~cm}$, AP $10.3 \mathrm{~cm})$ on left ovary. Left periaortic lymph nodes(LN) were increased.

An operation was done after ovarian tumour was confirmed by MRI. Tumour and two additional LN were completely resected, biopsies were taken. Histological report: left ovarian dysgerminoma with metastasis in periovarian LN. However, the tumour was hormonally active, therefore, Sertoli cell component could not be excluded. Consultation from Finnish pathologists suggested dysgerminoma in combination with gonadoblastoma. Karyotype analysis of blood was 46,XX. Skin biopsy excluded mosaicism.

Four courses of chemotherapy were done. Further follow-up was provided by a multidisciplinary team. Blood analysis was taken to monitor hormone levels in each hospital visit. Skeletal scintigraphy, chest CT, abdomen MRI was done after chemotherapy - no metastasis was found. Conclusions: A delayed precocious puberty diagnosis has caused this child significant health issues: hormonal disbalance, prognostic small adult height, therapeutical side effects from chemotherapy, increased risk for metastasis and secondary tumour development. For an early diagnosis of possible malignity, every child needs to be evaluated at least once a year (development, secondary sex traits by Tanner) and parents need to be educated. Successful diagnostic, therapeutic result and follow-up monitoring can only be achieved by a multidisciplinary team.
551

Endocrine \& Diabetes

An Unusual Case of Delayed Puberty

Ana Esteireiro $^{I}$, Ana Ventura ${ }^{I}$, Raquel Machado ${ }^{I}$, Juliette Dupont ${ }^{2}$, $\overline{\text { Brígida Robalo }}^{I}$, Carla Pereira ${ }^{l}$, Maria de Lurdes Sampaio ${ }^{I}$

${ }^{1}$ Unidade de Endocrinologia Pediátrica, Departamento de Pediatria, Hospital de Santa Maria, Centro Hospitalar Universitário Lisboa Norte, Portugal

${ }^{2}$ Serviço de Genética, Departamento de Pediatria, Hospital de Santa Maria, Centro Hospitalar Universitário Lisboa Norte, Portugal

Background: Kallmann syndrome (KS) is a rare congenital form of hypogonadotropic hypogonadism with anosmia/hyposmia.

Description: Female, 14 years old, without relevant personal or family history, referred because of pubertal delay (pubarche at age 13, without thelarche). She denied symptoms of neurological or other organs impairment, practice of strenuous physical exercise, restrictive eating behavior, illicit drug use and history of traumatic brain injury. The investigation performed before showed low estradiol levels and ultrasonography with small uterus, atrophic ovaries and horseshoe kidneys. She had good general condition, no dysmorphism, normal external genitalia and Tanner pubertal stage I (P3, B1, A3). Her growth velocity suffered progressive and slight slowdown after 10 years, but her height was still on the $50^{\text {th }}$ percentile. Complementary study showed low values of gonadotropins, 46XX karyotype and delayed bone age. LH-RH test revealed low values of FSH, LH and estradiol. KS was hypothesized and after a detailed inquiry anosmia/hyposmia was confirmed. Brain MRI showed bulbs and olfactory tracts atrophy and a small pituitary gland. Molecular analysis of the GnRHR and KAL1 gene by cyclic sequencing was normal, but the study proceeded to a Next Generation Sequencing (NGS) multigene panel approach that identified a heterozygous variant of uncertain significance in the KS gene FGF8 gene (c.283CG, p.Gln95Glu). Pubertal induction was performed with transdermal estrogens, and after menarche combined hormonal therapy was started.

Conclusions: In pubertal delay with hypogonadotropic hypogonadism and despite its rarity KS should be considered and anosmia/hyposmia should be searched for. Brain MRI shows typical anomalies in most cases. The genetic cause is recognized in only $30 \%$ of cases. Early diagnosis and hormone replacement prevent the physical and psychological consequences of pubertal delay. NGS approaches will contribute to reveal the molecular basis of a larger number of cases and may bring better understanding and classification of these disorders.

\section{9}

\section{Endocrine \& Diabetes}

A Twenty Month-Old Boy With Russell-Silver Syndrome (RS Sy) Aleksandra Janchevska ${ }^{1}$, Velibor Tasic ${ }^{2}$, Zoran Gucev ${ }^{I}$, Olivera Jordanova $^{3}$, Thomas Eggermann ${ }^{4}$

${ }^{I}$ Endocrinology, University Children's hospital, Macedonia

${ }^{2}$ Nephrology, University Children's hospital, Macedonia

${ }^{3}$ Biochemical laboratory, University Children's hospital, Macedonia

${ }^{4}$ Institute für Humangenetik, Uniklinik RWTH, Germany

Background: The alterations in a telomeric imprinting region 1 (ICR 1) in $11 \mathrm{p} 15$ chromosome and a maternal uniparental disomy of chromosome 7 (upd(7)mat) may not be detected by molecular analyses in approximately $50 \%$ patients with characteristic features of Russell-Silver syndrome. Patient and Methods: A twenty month-old boy with triangular shaped face, midface hypoplasia, prominent forehead, wide, but thin mouth, small chin, low positioned ears, occipitofacial circumference (OFC) (2.3 SDS) had low weight (-3.75 SDS), short stature (-4.6 SDS) and body 
asymmetry with bigger left side and longer left limbs for $1 \mathrm{~cm}$. The boy was born with intrauterine growth retardation (IUGR), birth weight (-4.8 SDS) and birth length (-1.7 SDS), in 38th gestational week (GW). The diagnostic assessment was achieved by clinical features, ultrasound survey, hormonal and molecular analyses.

Results: The IGF1, IGF BP3 and thyroid hormones were within reference range. The tests for pituitary reserve revealed a peak of $8.64 \mathrm{ng} / \mathrm{ml}$. The ultrasound survey revealed hypoplastic right kidney and mild hypertrophy of the myocard. Karyotype was 46, XY. Multiplex ligation probe amplification (MLPA) and a methylation analysis (MS-MLPA) did not detect hypomethilation of the ICR1 or a upd(7)mat. The growth hormone treatment is planned to be commenced.

Conclusions: Herein we present a twenty month-old IUGR born boy with typical clinical features of Russell-Silver syndrome. The molecular analyses unfortunately did not confirm hypometilation in the critical region of chromosome $11 \mathrm{p} 15$ or maternal uniparental disomy of chromosome 7 .

\section{5}

\section{Endocrine \& Diabetes}

Use of FGMS (Freestyle Libre) in Children and Young People with Type 1 Diabetes Mellitus-a UK DGH Experience Surendran Chandrasekaran ${ }^{1}$, Sanjana Kattera ${ }^{1}$, Nosheen Aman ${ }^{1}$ ${ }^{I}$ Paediatrics, East Cheshire NHS Trust, UK

Data was retrospectively collected from Electronic Patient Records for a 12-month period between June 2017 and June 2018. There were 100 CYP's with T1DM, of which 50 were commenced on FGMS. Funding sought appropriately before being commenced on sensor. CYP's and families were also made aware of the fact that funding can be withdrawn after a 6-month trial period if outcomes were unmet. $\mathrm{HbAlc}$ at the start of the trial was compared with $\mathrm{HbAlc}$ at 3, 6 and 9 months.

Results: The $\mathrm{HbAlc}$ at the start of the trial was $65.1 \mathrm{mmol} / \mathrm{mol}$; which showed an $8.1 \%$ improvement at 3 months, $12.3 \%$ at 6 months and $12.8 \%$ at 9 months. $67 \%$ discontinued the use of the FGMS, as qualitative evidence reported it to be unreliable. 2 of these children, recommenced the FGMS.

\begin{tabular}{llll}
\hline & & & Total $(\mathbf{n}=\mathbf{5 0})$ \\
Average age at start of trial & 13.6 & 11.3 & 12.8 \\
HbA1C pre-sensor (mmol/mol) & 75.1 & 64.2 & 65.1 \\
HbA1C at 3 months (mmol/mol) & 88.5 & 62.4 & 59.8 \\
HbA1C at 6 months (mmol/mol) & 58.3 & 58.3 & 57.1 \\
HbA1C at 9 months (mmol/mol) & 52.2 & 57.7 & 56.8 \\
June 2018-19, 6 new patients & & & \\
$\quad$ were commenced $\mathbf{F G M S}$ & & & \\
HbA1C pre-sensor (mmol/mol) & 84.5 & 65 & 71.5 \\
HbA1C at 3 months (mmol/mol) & 40 & 54.3 & 49.5 \\
HbA1C at 6 months (mmol/mol) & 46 & 52.3 & 50.5 \\
\hline
\end{tabular}

Conclusion: Our data shows a reduction in $\mathrm{HbA} 1 \mathrm{C}$ after 3 months of continuous sensor use which is maintained at 6 and 9 months. The improvement in $\mathrm{HbA} 1 \mathrm{c}$ is marked if the sensor was started within 6 months of diagnosis compared to those who have had T1DM for longer. Education is crucial before the start of sensor so that families and CYP's are well equipped to get the best out of it. The use of outcomesbased criteria as set by the CCG's acts to incentivise families, CYP's and HCP's to improve control.

\section{8}

Endocrine \& Diabetes

Precocious Puberty: When the Cause is not Idiopathic Ana Luísa Leite ${ }^{I}$,Elisa Galo ${ }^{3}$, Ana Antunes ${ }^{6}$, Brígida Robalo $^{4}$, Filipa Espada, Sofia Castro $^{2}$, Catarina Limbert ${ }^{5}$

${ }^{1}$ Unidade de Endocrinologia e Diabetologia Pediátrica, Centro Hospitalar de Vila Nova de Gaia / Espinho, Portugal

${ }^{2}$ Serviço de Pediatria, Centro Hospitalar Barreiro Montijo, Portugal

${ }^{3}$ Serviço de Pediatria, Hospital da Luz, Portugal

${ }^{4}$ Unidade de Endocrinologia Pediátrica, Centro Hospitalar Universitário Lisboa Norte, Portugal

${ }^{5}$ Unidade de Endocrinologia Pediátrica, Centro Hospitalar Universitário de Lisboa Central, Portugal

${ }^{6}$ Unidade de Endocrinologia Pediátrica, Hospital de Braga, Portugal

${ }^{7}$ Unidade de Endocrinologia Pediátrica, Hospital Pedro Hispano, Portugal

Precocious Puberty is defined by the onset of pubertal development at an age 2-2,5 standard deviations earlier than the normal population, which means before nine years old in boys and eight years in girls. Central Precocious Puberty (CPP) is diagnosed when the hypothalamicpituitary axis is activated. Although the majority of CPP causes are idiopathic, secondary causes should be discharged because of pathologic conditions with significant risk of morbidity and even death.

Aims: To evaluate the prevalence of secondary CPP in the Portuguese population in the last five years, to characterize anthropometric and imagological features and identify its clinical predictors.

Methods: Cross-sectional study from a National Digital Database, enrolled from eleven Public and Private Pediatric Endocrine Departments. Cases reported from January 2013 to December 2017. Statistical analysis was performed using SPSS ${ }^{\mathrm{TM}} 23.0$ version.

Results: A total of 210 cases presented CPP during the last five years. $187(89 \%)$ were female. The median age of diagnosis was 6,5 years old in idiopathic CPP and 4,8 years old in secondary CPP $(\mathrm{p}=0,002)$. Only 146 $(69.5 \%)$ enrolled hypophyses/cranial image and secondary cases of CPP were identified in 38 patients (26\%). Tumors were diagnosed in ten cases (26,3\% of the secondary CPP) and the most frequent was hamartoma in $60 \%$. From the secondary cases of CPP only 17 were male $(12 \%)$ and there were no significant differences among gender when considering idiopathic/secondary cases. There was a negative correlation between the age of clinical manifestations (p0.001) and secondary CPP.

Conclusions: CPP is more frequent in girls, either in idiopathic or secondary cases. The yearly age of clinical manifestations is a better predictor than gender in the diagnosis of secondary CPP.

\section{4}

\section{Endocrine \& Diabetes}

Thyroid Status and Serotonin Production in Children with Impaired Growth and Neuropsychological Development

Hanna Shlieienkova ${ }^{1}$, Marina Mamenko ${ }^{2}$

${ }^{I}$ Paediatrics № 2, V. N. Karazin Kharkiv National University, Ukraine

${ }^{2}$ Paediatrics № 2, Shupyk National Medical Academy of Postgraduate Education, Ukraine

Thyroid hormones $(\mathrm{TH})$ are essential for the development and maturation of the central nervous system. Serotonin, 5-hydroxytryptamine (5-HT), is an important chemical and neurotransmitter in the human body. It appears to play a key role in neural activities and social behaviors. The effects of $\mathrm{TH}$ and serotonin during development of CNS appear similar. Transient hypothyroidism is common in children with impaired CNS maturation. The levels of serotonin production in these children remain unclear. Objective: To evaluate serotonin levels and thyroid status in children with impaired growth and neuropsychological development. 
Materials and Methods: Target group: 118 children 0-3 years old from orphanage.

Methods: physical, psychomotor development assessment; thyroid status by TSH, TT4, TT3 serum-based measuring (IMA); peripheral blood serotonin measurement («IBL Hamburg», Germany).

Results: All children were accepted in to an orphanage at the age 2.5-3.5 months. They had impaired physical development (44.9\%), delayed psychomotor development $(95.7 \%)$, decreased coefficient of neuropsychological development (82 points). TSH level median was $3.21 \mathrm{mU} / 1$ [QR: $3.1 ; 3.8]$. Negative linear correlation by PPMCC between TSH levels and coefficients of neuropsychological development was detected $(\rho=0.436, p=0.001)$. In children with minimal thyroid dysfunction median serotonin level was 204.1nmol/ml [QR: 202.1; 206.1], vs in children with optimal thyroid function $185.1 \mathrm{nmol} / \mathrm{ml}$ [QR: $177.7 ; 189.7]$, $\mathrm{p}$

Conclusions: In young children, disorders of physical and neuropsychological development are associated with transient hypothyroxinemia and hyperthyrotropinemia. Unlike adults, hyperthyrotropinemia is associated with increased serotonin production. Perhaps such increase in serotonin production is an additional mechanism aimed at completing the maturation of the nervous system.

\section{9}

\section{Endocrine \& Diabetes}

\section{Blurry Vision in Type 1 Diabetes}

Maria Ines Marques ${ }^{1}$, Antonio Guerra ${ }^{1}$, Bruno Pina ${ }^{2}$, Sara Carrasquinho $^{2}$, Isabel Fernandes ${ }^{1}$

${ }^{1}$ Pediatrics, Hospital Santo Espirito Santo, Portugal

${ }^{2}$ Ophthalmology, Hospital Santo Espirito Santo, Portugal

The development of cataracts at the onset of type-1 diabetes mellitus (T1DM) is an uncommon complication in the pediatric population (prevalence of around 1\%). It most often occurs as one of the first manifestations or within 6 months of T1DM diagnosis. Our aim is to describe two cases of adolescents with a recent diagnosis of type-1 diabetes mellitus that were diagnosed with a complication of posterior subcapsular cataracts.

Case 1

A case of an 18-year-old girl, with complaints of reduced visual acuity in both eyes, about one year after T1DM diagnosis, under intensive insulin therapy and excellent glycaemic control $\left(\mathrm{HbA}_{1 \mathrm{C}} 6.1 \%\right)$. After ophthalmological examination, she was found to have bilateral posterior subcapsular cataracts (PSC), that were not present at the time of a routine diabetic eye screening at diagnosis.

The patient underwent cataract surgery and intraocular lens implantation with improved visual acuity.

Case 2

A case of a 17-year-old girl who complained of blurry vision six months after T1DM diagnosis, during an ophthalmological screening. Good metabolic control of diabetes was observed during this time $\left(\mathrm{HbA}_{1 \mathrm{C}} 6.7 \%\right)$.

The patient had a unilateral PSC of the left eye. About one year after the first diagnosis she developed PSC of the right eye. She underwent cataract surgery and intraocular lens implantation with improved visual acuity.

Conclusion: Type- 1 diabetic cataracts have been found to have a female preponderance, while the pathogenesis remains unclear, it seems to be related to poor metabolic control or long history of symptoms prior to diagnosis. In these cases, good metabolic control did not appear to prevent cataract formation.

Visual impairment occurring early in T1DM in pediatric age should raise a suspicion of cataracts and ophthalmologic evaluation should be prompt.

Close monitoring of the fundi for retinopathy following cataract surgery is essential, as proliferative retinopathy may develop rapidly after the surgery.
537

Endocrine \& Diabetes

Recurrent Vomiting in Young Infant: A Challenge Tania Mendo ${ }^{I}$, Catarina Borges ${ }^{I}$, Gabriela Reis ${ }^{I}$, Maria Carlos ${ }^{1}$, Fatima Furtado $^{I}$, Sónia Fernandes ${ }^{I}$, Graça Seves ${ }^{l}$, Aniceta Cavaco ${ }^{I}$

${ }^{1}$ Pediatria Medica, Hospital José Joaquim Fernandes, Portugal

Background: Recurrent vomiting in infants may have several etiologies. Among the most common medical causes are gastroenteritis, urinary tract infections or CNS infections. Besides that, surgical causes such as hypertrophic pyloric stenosis or intestinal obstruction should also be considered. Therefore, diagnosis is not always easy and may require extensive research.

Case Report: Two-months-old male, with previous history of reccurent vomiting, observed in the emergency department with vomiting, poor weight gain, and asymptomatic hyponatremia. Family history of hypothyroidism and rheumatoid arthritis. At physical examination, he was hydrated and hemodynamically stable.

Blood tests showed $\mathrm{Hb} 11.7 \mathrm{~g} / \mathrm{dL}, \mathrm{Ht} 34.6 \%$, WBC $20510 \mathrm{U} / \mathrm{L}, \mathrm{N} 28.6 \%$, $\mathrm{L} 59.1 \%, \mathrm{Na}+120 \mathrm{mmol} / \mathrm{L}, \mathrm{K}+6.94 \mathrm{mmol} / \mathrm{L}$ and the abdominal ultrasound was normal.

He was admitted for IV hydration and correction of hyponatremia.

From the additional investigation performed, the most common causes of vomiting were excluded, as well as tubulopathies, SIADH and hypothyroidism. The diagnosis of congenital hyperplasia of the adrenal gland was considered the most likely, and lately confirmed.

He started therapy with hydrocortisone, and then transferred to a tertiary hospital (Endocrinology Unit) for further care.

Conclusion: With this case the authors aim to draw the attention to a less common cause of recurrent vomiting in young infants. Congenital adrenal hyperplasia is an inherited autosomal recessive disease, caused by the deficiency of enzymes involved in cortisol synthesis. About $95 \%$ of cases are caused by $21-\mathrm{OH}$ deficit. In the neonatal period, the diagnosis can be suspected in the presence of sexual ambiguity or acute salt-losing crisis. Treatment is essential to ensure appropriate growth, puberty and absence of long-term complications.

\section{3}

\section{Endocrine \& Diabetes}

Neurological Symptoms at Presentation: What it could be? Sofia Brandão de Miranda ${ }^{1}$, Joana Vilaça ${ }^{1}$, Sofia Martins ${ }^{1}$, Marina Pinheiro $^{I}$, Vera Baptista ${ }^{I}$, Maria João Magalhães ${ }^{I}, \hat{A}$ ngela Oliveira ${ }^{I}$ ${ }^{1}$ Pediatria, Hospital de Braga, Portugal

Background: Neurological symptoms can have multiple causes, such as structural changes of the central or peripheral nervous system as well as metabolic, hydroelectrolytic or even psychiatric disorders.

Objectives / Methods / Results: A 12-years-old adolescent presented at emergency service (ES) with acute onset of blurred vision, drowsiness, headache and speech difficulties. He was tachycardic but normotensive, emaciated, with slurred speech and dry mouth and objective weight loss of $22 \%$ in a week.

Blood test revealed hyperglycemia (glucose $1763 \mathrm{mg} / \mathrm{dL}$ ), moderate metabolic acidosis ( $\mathrm{pH} 7.169, \mathrm{HCO} 3-12.1 \mathrm{mmol} / \mathrm{L})$, high blood ketone levels (ketone bodies $7 \mathrm{mmol} / \mathrm{L}$ ), acute renal failure (creatinine $2.3 \mathrm{mg}$ / $\mathrm{dL}$, urea $108 \mathrm{mg} / \mathrm{dL}$ ) and sodium level $135 \mathrm{mmol} / \mathrm{L}$ (corrected sodium 161). Head computed tomography without cerebral edema or structural changes.

Based on results it was made the diagnosis of Type 1 Diabetes mellitus (T1DM). Retrospectively, patient's mother reported polyuria, polydipsia 
and polyphagia with several weeks of evolution, and recent pollakiuria that motivated a previous visit to the ES. Fluid therapy and intravenous perfusion of insulin was started. Careful and frequent adjustments were needed, nevertheless he developed important hypernatremia (corrected sodium $175 \mathrm{mmol} / \mathrm{L}$ ). Progressively, he presented improvement in glycemia, ketoacidosis, renal function and dehydration, with disappearance of neurological symptoms.

Subsequent study with decreased C-peptide and insulin, increased GAD65 and anti-insulin antibody, and normal levels of anti-zinc transporter protein 8 autoantibodies, thyroid function and lipid profile.

At discharge he had no analytic changes. Weight gain of $21 \%$, with no other changes at physical examination.

Conclusion: The elevated glycemia and the consequent hydroelectrolytic disorders following the treatment constituted a challenge. It was important to control the rapid rise in hypernatremia to avoid serious consequences as brain shrinkage, intracerebral hemorrhage and demyelination. This case also alert to the importance to a earlier diagnosis of T1DM and to some less frequent and even confounder symptoms in an inaugural episode.

\section{4}

\section{Endocrine \& Diabetes}

Evaluation of Nipple Discharge in Adolescence - A Case Report

Rita Gomes ${ }^{1}$, Alicia Rebelo ${ }^{2}$, Ivete Afonso ${ }^{3}$, Patricia Santos ${ }^{3}$, Marcelo Fonseca $^{3}$, Filipa Espada ${ }^{3}$, Ana Moreira

${ }^{1}$ Pediatrics, Centro Materno Infantil do Norte, Centro Hospitalar Universitário do Porto, Unidade de Saúde Local Matosinhos, Portugal

${ }^{2}$ Pediatrics, Hospital da Senhora da Oliveira Guimarães, Unidade de Saúde Local Matosinhos, Portugal

${ }^{3}$ Pediatrics, Unidade de Saúde Local Matosinhos, Portugal

Background: Nipple discharge is uncommon in children and adolescents. Milky discharge is characteristic of galactorrhea which is typically bilateral and may be secondary to pregnancy, medications, endocrine tumors (pituitary adenoma), endocrine abnormalities (hypothyroidism, hypogonadism), mechanical stimulation or psychological factors.

Case Report: A 17-year-old girl was referred to a Pediatric Endocrinology appointment at ULSM for nipple discharge in the last six months. She had recent complaints of emotional lability and anxiety and a family history of depression. No menstrual irregularities, hot flashes, vaginal dryness, headaches, visual disturbances or other symptoms were mentioned. Pregnancy and medication-related causes were excluded. On physical examination, a bilateral milky nipple discharge was elicited by manipulation. No skin changes, asymmetries or masses were evident on breast examination. No areas of tenderness or pain nor axillary or supraclavicular lymph nodes were palpable. Laboratory tests showed mild elevation of serum prolactin levels, normal LH, FSH, T4 and TSH. Magnetic resonance imaging (MRI) showed a $5 \mathrm{~mm}$ diameter microadenoma of the anterior pituitary and an asymmetry of the sellar diaphragm with a left shift of the pituitary stalk. After discussion with neurosurgery specialists, she was started on cabergoline $0,25 \mathrm{mg}$ twice a week. After 3 months, the galactorrhea was eliminated, there was normalization of the prolactin concentration and there was no significant change in size of the sellar mass.

Conclusions: It has been reported in the literature that depressive symptoms may be associated with mild elevations of serum prolactin levels. Notwithstanding, an MRI of the pituitary should be performed in symptomatic patients with any degree of hyperprolactinemia. Prolactin concentrations $200 \mathrm{ng} / \mathrm{mL}$ generally identify a lactotroph adenoma; values that are between 20 and $200 \mathrm{ng} / \mathrm{mL}$ could also be due to any other sellar mass. Children and adolescents with spontaneous nipple discharge without an obvious cause should be referred to an appropriate specialist.
1003

Endocrine \& Diabetes

Addison's Disease in a Girl with Family History Mafalda Moreira ${ }^{I}$, Diana Alba ${ }^{I}$, Ana Garrido ${ }^{1}$, Mariana Martinho ${ }^{2}$, Ana $\overline{\text { Catarina Chaves }}^{2}$, Carla Brandão ${ }^{I}$

${ }^{1}$ Pediatric Department, Centro Hospitalar Tâmega e Sousa, Portugal

${ }^{2}$ Endocrinology Department, Centro Hospitalar Tâmega e Sousa, Portugal

Background: Primary adrenal insufficiency is a rare condition especially in pediatrics' population and can be fatal if not identified and treated on time. The usual presentation includes constitutional and gastrointestinal symptoms, cutaneous hyperpigmentation and hyponatremia. The treatment consists in hormonal replacement throughout life.

Clinical case: We present the case of a 16 years old female, admitted at the emergency department with asthenia, weight loss and daily vomiting with a month evolution. In admission, she presented cutaneous hyperpigmentation, without dehydration signs. Laboratory results showed hyponatremia with hyperkalemia and hypochloremia, decreased cortisol levels with increased levels of corticotropin and increased levels of renin with decreased aldosterone levels. She started treatment with hydrocortisone and fludrocortisone with favorable response. Additional investigation evidenced a positive family history for autoimmune primary adrenal insufficiency in a sibling.

Discussion: This case is illustrative of an uncommon disease, whose diagnosis requires a high degree of suspicion. The presence of cutaneous hyperpigmentation in a patient with constitutional symptoms and vomiting was the key to the diagnosis, as well as the presence of hyponatremia with hyperkalemia. The family history, although uncommon in this disease, was also a clue to the diagnosis.

\section{3}

Endocrine \& Diabetes

Re-interpreting the Appropriate Growth Hormone Response to Hypoglycaemia

Uzair Athar Khan ${ }^{1}$, Ying Wong ${ }^{1}$, Shahir Ul Azam ${ }^{1}$, Ophelia Blake ${ }^{2}$, Anne Marie Murphy $^{1,2}$, Orla Neylon ${ }^{1,3}$, Clodagh S O'Gorman ${ }^{1,3}$ ${ }^{T}$ Paediatric department, University Hospital Limerick, Ireland

${ }^{2}$ Department of biochemistry, University Hospital Limerick, Ireland ${ }^{3}$ Paediatric department, Graduate Entry Medical School University of Limerick, Ireland

Background: The "Newcastle work-up" is laboratory investigation to elucidate metabolic or endocrine causes of hypoglycaemia. One of the tests it measures is growth hormone GH,which should increase during hypoglycaemia. In our recent clinical experience, GH levels have been sub-optimal during hypoglycaemia. The cut off value for growth hormone levels is $7 \mathrm{mcg} / \mathrm{L}$,reading below is deemed suboptimal and warrant clinician to investigate further.

Aims/Objectives: To identify if the pre-set cut-off value for GH during hypoglycaemia during Newcastle work-up is appropriate and likely or not to identify GH deficiency. The study population was all paediatric patients attending the Paediatrics Department UHL with GH levels over 12 months from January to December 2018, inclusive.

Methods: Retrospective review from a prospectively collected database of glucose and GH,including growth velocity, centiles, mid-parental height ranges and IGF-1 during hypoglycaemia in the Newcastle work-up. GH levels were subdivided into five subgroups: $1 ; 1-2.9 ; 3-4.9 ; 5-6.9 ; 7 \mathrm{mcg} / \mathrm{L}$. Results: Forty Newcastle work-ups were performed on $\mathrm{N}=40$ patients during the study period;GH results according to 5 subgroups are shown in Figure 1. $\mathrm{N}=3 / 39(8 \%) \mathrm{GH}$ levels were $7 \mathrm{mcg} / \mathrm{L}$, which is the pre-defined normal range result. The mean GH was 3.83 and median GH $3.19 \mathrm{mcg} /$ 
L.IGF-1 level was available on 12/40; 11/40 were normal. N=1/40 IGF-1 was low and this patient had decelerating height centile, GHST was subsequently normal.N=19/40 patients had normal growth velocity and growth centiles,Data on remaining patients are incomplete or pending.GHST was performed on 3/40 children.All had normal GHST results.
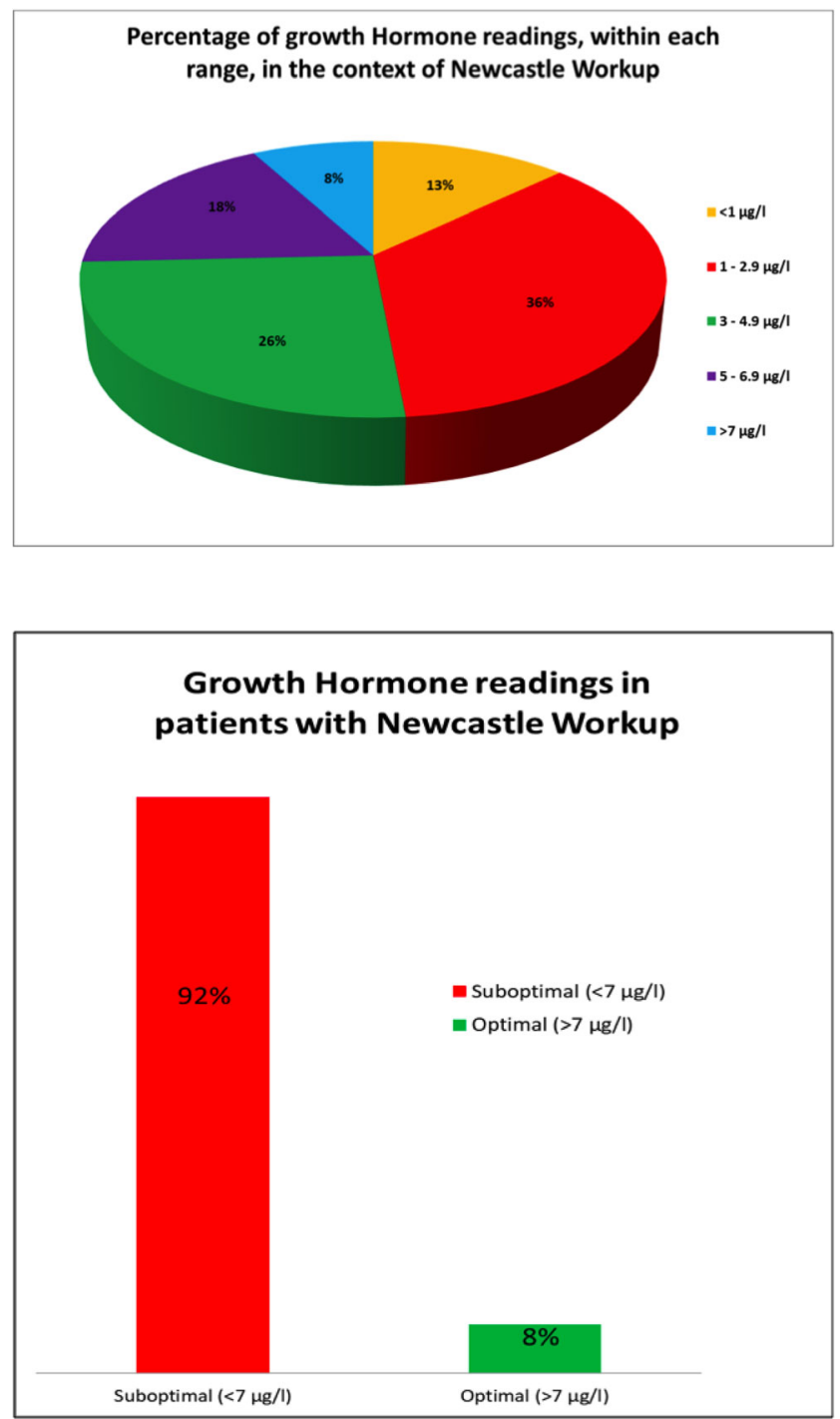

Conclusion: These data are limited and some results are pending. But no patient has documented GH deficiency or loss of height centiles, despite only $\mathrm{N}=3 / 40$ having adequate $\mathrm{GH}$ during hypoglycaemia during Newcastle testing.

Recommendations: These data suggest that the optimal level for GH during testing for causes of hypoglycaemia should be re-evaluated in further and larger prospective studies

\section{4}

\section{Endocrine \& Diabetes}

Hirsutism and Oligomenorrhea, Not Always Polycistic Ovary Syndrome Silvia Gallego Gutierrez' ${ }^{1}$, Eva Pedrero Segura' ${ }^{1}$, Ana Isabel Valdivielso Martinez

${ }^{1}$ Malaga-GuadalHorce District, Primary Care Attention, Spain
Background: Hirsutism is excess terminal hair that commonly appears in a male pattern in women. The most common cause of hirsutism is polycystic ovary syndrome. Other causes are idiopathic hyperandrogenemia, idiopathic hirsutism, adrenal hyperplasia, iatrogenic hirsutism, thyroid dysfunction, Cushing syndrome and androgen-secreting tumors. Congenital adrenal hyperplasia is a group of autosomal recessive disorders encompassing enzyme deficiencies in the adrenalsteroidogenesis pathway that lead to impaired cortisol biosynthesis.

Objective: We present a case of non-classic congenital adrenal hyperplasia $(\mathrm{NCAH})$ in a 13 year old female who presented to us with hirsutism and oligomenorrhea.

Methods: Case reports.

Results: A 13-years-old girl was referred for evaluation for hirsutism. Height $168 \mathrm{cms}$, weight $54 \mathrm{kgs}$ and BMI of 19.13. Distribution and extent of hirsuitism assessed by modified Ferriman-Gallway score was 11 (moderate hirsuitism). She has not rapid onset of symptoms, signs of virilization, and she had not palpable abdominal or pelvic mass. The patient had already consulted on other occasions with the diagnosis of idiopathic hirsutisms. Ultrasonography showed uterus and bilateral ovaries normal in size shape and ecopattern. There was no detectable ovarian or adrenal mass. Androstenedione, dehydroepiandrosterone sulfate and 17-hydroxyprogesteronewere elevated $(8.82 \mathrm{ng} / \mathrm{ml}, 3421 \mathrm{ng} / \mathrm{ml}$ and $38.1 \mathrm{ng} / \mathrm{ml}$ respctively). Estradiol $(54 \mathrm{pg} / \mathrm{ml})$, FSH (10.61 $\mathrm{mIU} / \mathrm{ml})$, T4 $(16.56 \mathrm{pmol} / \mathrm{L})$ and TSH $(4.49 \mathrm{mUI} / \mathrm{ml})$ were normal. A genetic study was carried out, finding a mutation inthe 21hydroxylase (CYP21A2).

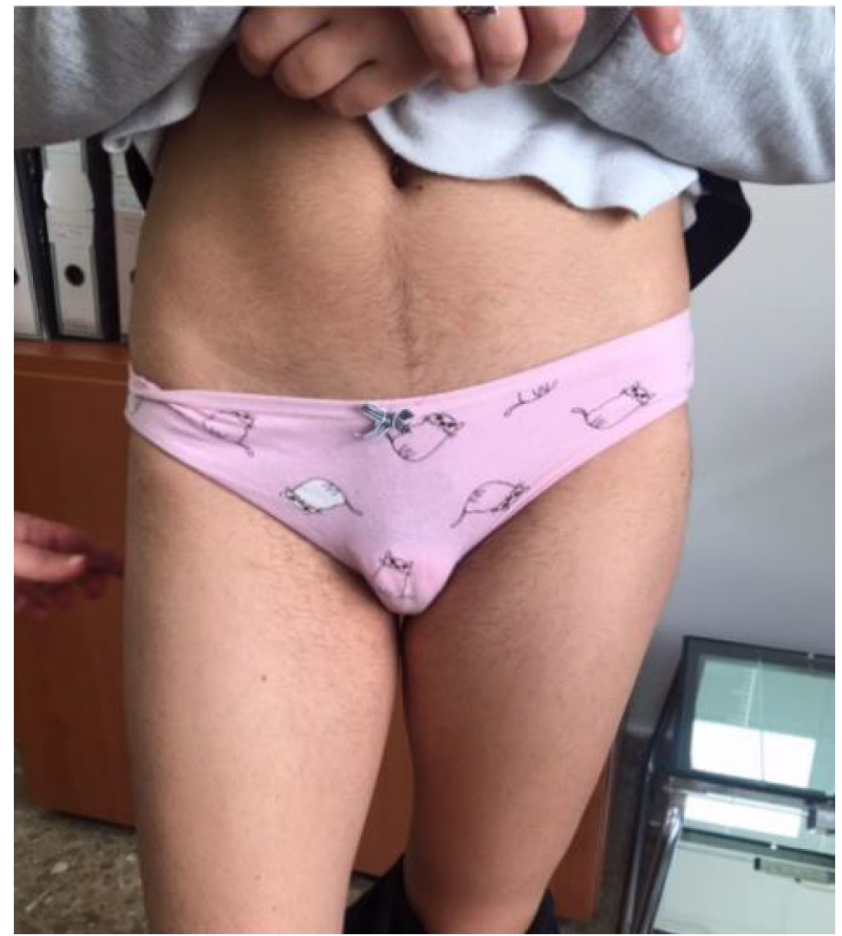

Conclusion: Women presenting with signs and symptoms of androgen excess should be investigated about $\mathrm{NCAH}$. The majority of NCAH can be associated with three distinct enzyme defects: defects of 21-hydroxylase, $11 \beta$-hydroxylase and $3 \beta$-hydroxysteroid dehydrogenase. The first one is the most common. This case emphasizes the importance of making the differential diagnosis of hirsutism with interdisciplinary follow-up in patients with hormonal diseases. 
789

\section{Endocrine \& Diabetes}

\section{A Case Report of a Congenital Adrenal Hyperplasia in a Seven-day- old Boy \\ Miguel Paiva Pereira $^{l}$, Filipa Marujo $^{l}$, Cláudia Fernandes $^{l}$, Ana Cristina Monteiro', Cristina Matos ${ }^{1}$ \\ ${ }^{1}$ Centro da Criança e do Adolescente, Hospital CUF Descobertas, Portugal}

Congenital Adrenal Hyperplasia (CAH) is an inherited autosomal recessive disorder characterized by a deficiency of enzymes involved in the synthesis of cortisol, mineralocorticoids or androgens. Its incidence is $1: 18000$ and the most common type is 21hydroxylase deficiency. Classic $\mathrm{CAH}$ can result in a salt-losing form or a simple virilizing form.

A 7-day-old newborn was brought to the emergency department for prostration and decreased intake. Forty-six-year-old mother, with 2 previous miscarriages. Pregnancy without intercurrences. Elective caesarean section at 38 weeks, Apgar score 6/9/10 and a low birth weight $(2335 \mathrm{~g})$. He was lethargic and pale, with a $9 \%$ weight loss. Urinalysis with 25 leukocytes, hyponatremia $(120 \mathrm{mmol} / \mathrm{L})$, hyperkalaemia $(9.4 \mathrm{mmol} / \mathrm{L})$ with electrocardiographic changes and hypoglycaemia $(25 \mathrm{mg} / \mathrm{dL})$. He was admitted to Neonatal Intensive Care Unit level II, initiating intravenous antibiotics (ampicillin, gentamicin and cefotaxime), bicarbonate, hydrocortisone and ionexchange resins. Uroculture was positive for Escherichia coli. Investigation revealed high levels of adrenocorticotropic hormone $(566 \mathrm{pg} / \mathrm{mL})$, cortisol $(28.8 \mathrm{mcg} / \mathrm{dL})$ and $17-\mathrm{OHP}(241.9 \mathrm{ng} / \mathrm{mL})$, confirming the diagnosis of CAH. Fludrocortisone $(0.3 \mathrm{mg} /$ day $)$ was started. Progressive weaning of intravenous corticosteroid and passage to the oral route according to guidelines, with successive relapses and ionic imbalances. By the $34^{\text {th }}$ day, clinical and analytical stabilization was achieved under hydrocortisone $61,4 \mathrm{mg} / \mathrm{m} 2, \mathrm{NaCl} 6,2 \mathrm{meq} / \mathrm{kg} / \mathrm{h}$ and fludrocortisone, and was discharged home. At present, he remains stable, without adrenal crisis and reducing hydrocortisone $(22,7 \mathrm{mg} / \mathrm{m} 2)$. The genetic study is underway.

$\mathrm{CAH}$ is a serious condition at risk of impending decompensation. Clinical severity and stabilization are related to the initial enzymatic deficit, so corticosteroid doses and the weaning rhythm should be individualized and the patient monitored closely. CAH has non-specific clinical manifestations in newborns, so a high suspicion index is needed facing a presentation before the third week of life with no virilization signs, as in this case, whose severity and precocity were triggered by an infectious event.

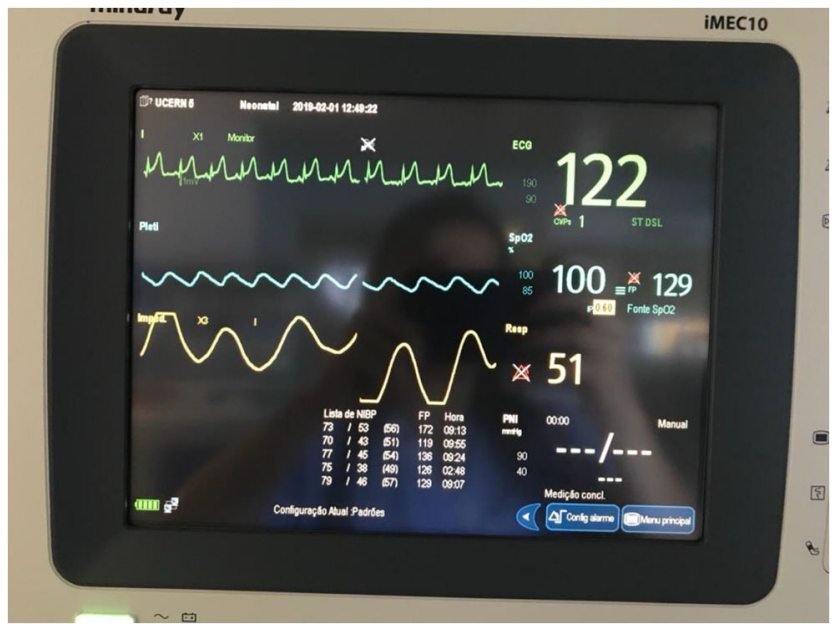

610

Endocrine \& Diabetes

Newborn Pseudohypoparathyroidism: A Case Report Vicente Rey y Formoso ${ }^{I}$, Sofia Fernandes ${ }^{I}$, Rita Santos Silva ${ }^{2,3}$, Carla Costa $^{2,5}$, Miguel Leão ${ }^{4}$, Cintia Castro Correia ${ }^{2,3}$, Manuel Fontoura ${ }^{2,3}$

${ }^{1}$ Pediatrics Department, Maternal and Pediatrics Center - São João University and Hospital Center, Portugal

${ }^{2}$ Pediatric Endocrinology Unit - Pediatrics Department, Maternal and Pediatrics Center - São João University and Hospital Center, Portugal ${ }^{3}$ Pediatrics Department, Porto Medical School, Portugal

${ }^{4}$ Genetics Department, São João University and Hospital Center, Portugal

Background: Pseudohypoparathyroidism(PHP) represents a group of disorders whose main feature consists in a resistance to Parathormone(PTH) action. The most common subtype of PHP is Ia, caused by a mutation in the GNAS1 gene. It is inherited as an autosomal dominant trait but, because of paternal gene imprinting, different phenotypes occur depending on the origin of the mutated gene. When paternally inherited, subjects only manifest the physical syndrome - Albright's Hereditary Osteodystrophy (obesity, rounded face, brachydactyly, subcutaneous ossifications...). When maternally inherited, in addition to those physical characteristics, multiple hormone resistance (PTH, Thyroidstimulating Hormone(TSH), gonadotropin...) occurs - PHP-Ia.

Objectives: Case report of a newborn with PHP-1a presenting with hypothyroidism - the importance of family history, a high suspicion level and thorough laboratory workups.

Methods: Reviews of the subject's and his family's clinical records.

Results: A 27-year-old female gravida (37 weeks gestation), with genetically proven AHO (paternally inherited GNAS1 mutation), gave birth to a $2360 \mathrm{~g}$ male that presented with early signs of respiratory distress, for which he was admitted in the NICU. Respiratory symptoms resolved gradually. Because of the family history, at day 6 , thyroid hormone testing was requested, showing a free-T4 level of $0.67 \mathrm{mg} / \mathrm{dL}$ and a TSH concentration of $13.02 \mathrm{IU} / \mathrm{mL}$. Hypothyroidism/TSH insensitivity was diagnosed - levothyroxine therapy was initiated, and thyroid function levels normalized in the following days - discharge on the 18th day of life. Genetic testing revealed the newborn carried the same mutation as the mother, confirming the diagnosis of PHP-1A. An ultra-sound showed a normal but relatively small thyroid gland. Phosphocalcium metabolism and thyroid function (under levothyroxine) remain normal at 3-month follow-up.

Conclusion: While PTH resistance is the main feature of PHP-Ia, TSH resistance may become apparent before hypocalcemia develops, and may be indistinguishable from primary hypothyroidism. Early treatment and continuous monitoring of these patients' hypothyroidism is paramount in preventing future complications.

383

\section{Endocrine \& Diabetes}

Impact of Growth Hormone Supplementation on Adult Height in Turner Syndrome

$\underline{\text { Liliana Sá }}^{1}$, Sofia Simões Ferreira ${ }^{2}$, Maria Adriana Rangel ${ }^{2}$, Ana Luísa Leite $^{2}$, Rosa Arménia Campos ${ }^{2}$

${ }^{1}$ Pediatrics and Neonatology, Centro Hospitalar de Entre Douro e Vouga, Portugal

${ }^{2}$ Pediatrics and Neonatology, Centro Hospitalar Vila Nova Gaia / Espinho, Portugal

Background: Short stature is the most common phenotypic feature of Turner Syndrome (TS). Treatment with Growth Hormone (GH) is recommended to increase growth velocity (GV) and final adult height. 
Objective: To describe patients with TS who underwent treatment with $\mathrm{GH}$ in a level II hospital in the north region of Portugal.

Methods: Retrospective observational study.

Results: Eight patients with TS underwent treatment with GH with a mean age at diagnosis of $4.5 \pm 3.9$ years. Chromosome analysis demonstrated 3 cases of X-monosomy and 5 cases of mosaicism. None had cardiac pathology and 4 had hypothyroidism. Baseline mean height was $106.1 \pm 16.5 \mathrm{~cm}$ (SDS $-2.0 \pm 0.69$ ), with annualized GV of $4.7 \pm 1.7 \mathrm{~cm} /$ year, and adjusted mid-parental height (MPH) of $158.7 \pm 4,7 \mathrm{~cm}$ (SDS $-0.56 \pm 0.78$ ). Treatment with GH was started on average at $6.7 \pm 3.3$ years of age (subcutaneous daily injection, mean dose of $48 \mathrm{mg} / \mathrm{Kg} /$ day). Currently, the eight patients, with a mean age of $15.0 \pm 4.3$ years, present a mean height of 145.5 $\pm 10.7 \mathrm{~cm}$ (SDS $-1.85 \pm 0.65$ ), with a gain of about $49 \pm 10.7 \mathrm{~cm}$. Throughout the treatment the difference between the SDS of the MPH and the SDS of the stature decreased (1.52 \pm 0.70 to 1.29 \pm 1.22 ), and five patients reached the expected MPH-SDS. The growth was about $5.7 \pm 1.3 \mathrm{~cm}$ per year of treatment, with the greatest increase during the first year (mean of $9.0 \pm 1.8 \mathrm{~cm}$ ). Five patients had already completed treatment at a mean age of $14.6 \pm 0.9$ years. After an average of 9.4 years of treatment they reached a final height of $149.0 \pm 3.9 \mathrm{~cm}$ (SDS $-1.85 \pm 0.66$ ).

Conclusion: Treatment with GH should be instituted as soon as possible in order to optimize adult height.

\section{5}

\section{Gastroenterology \& Nutrition}

Maternal Orthorexia Nervosa Causing Severe Ketoacidosis and Methylmalonic Acidura in Her Offspring

Said Abozaid ${ }^{1}$, Mary Abdo $^{1}$, Galit Tal ${ }^{2}$, Mariam Daher ${ }^{1}$, Noa MandelShorer ${ }^{3}$, Elena Dumin', Wael Nasser ${ }^{2}$, Hanna Mandel ${ }^{1,4}$

${ }^{I}$ Department of Pediatrics, Baruch Padeh Medical Center affiliated to the Faculty of medicine in the Galilee Bar-Ilan University, Israel

${ }^{2}$ Metabolic Unit, Ruth Rappaport children's hospital, Rambam Health Care Center, Israel

${ }^{3}$ Hematology-oncology division, Ruth Rappaport children's hospital, Rambam Health Care Center, Israel

${ }^{4}$ Department of Genetics, Western Galilee Medical Center, Israel

Introduction: Orthorexia Nervosa $(\mathrm{ON})$ is an eating disorder describing patients with obsession for healthy food, in contrast to patients with anorexia and bulimia who worry about its quantity. ON may lead to a state of starvation due to strict diets and shortage of essential nutrients leading to various medical complications as seen in anorexia. To-date, only anecdotal cases on ON in adults were reported. We report for the first time a baby with episodes of apathy and severe ketoacidosis. History of the baby's and his mother's nutrition led us to suspect $\mathrm{ON}$ in the mother causing his critical condition.

Case report: A 17-months-old baby presented with few days history of lethargy and refusal to eat after an attempt of weaning breastfeeding. He had been mainly breastfed and rarely consumed solid food. A detailed dietary history revealed that the mother ate only vegan diet during nursing and provided her baby a strict "vegan, healthy uncooked diet omitting animal products and fortified babys' formulas". The baby was lethargic, pale, hyperpneic, had hypotoia with hyperrexflexia. His growth centiles were below the $3^{\text {rd }}$ percentile. He could not role over nor sit unassisted. Laboratory studies revealed pancytopenia and severe ketoacidosis. Clinical and biochemical features suggested an inborn error of metabolism (IEM). Urinary organic acids revealed severe ketoacidosis and methylmalonic aciduria. Slow refeeding program including multiple vitamins and vitamin-B12 resulted in resolution of all abnormalities.

Conclusion: We describe for the first time $\mathrm{ON}$ in a mother causing life-threatening disorder in her child. We designate this condition as "Maternal ON" similar to the notion of "Maternal phenylketonuria" (PKU) wherein high phenylalanine levels in a PKU mother who does not keep restricted diet, harms her offspring. Pediatricians and dieticians should be aware to Maternal ON mimicking IEM presenting as growth and development delay and/or severe metabolic abnormalities.

\section{2}

\section{Gastroenterology \& Nutrition}

Acute Upper Gastrointestinal Bleeding Associated with Epstein-Barr Virus Infection

Carolina Amaro Goncalves ${ }^{1}$, Ana Lança ${ }^{2}$, Madalena Sasseti ${ }^{3}$, Marta Almeida $^{3}$, Filipa Nunes ${ }^{3}$, Paulo Calhau ${ }^{3}$

${ }^{1}$ Pediatric, Pediatric Department, Hospital de Santa Maria - Centro Hospitalar Universitário de Lisboa Norte, EPE, Lisbon, Portugal, Portugal

${ }^{2}$ Pediatric, Pediatric Department, Hospital de São Francisco Xavier Centro Hospitalar de Lisboa Ocidental, EPE, Lisbon, Portugal, Portugal ${ }^{3}$ Pediatric, Pediatric Department, Hospital Garcia de Orta EPE,Almada, Portugal, Portugal

Epstein-Barr virus (EBV) is a herpes virus responsible for a lifelong latent infection in almost every adult. Primary infection generally occurs subclinically in childhood, but if infection is delayed it frequently causes a self-limited clinical syndrome of infectious mononucleosis.Classic symptoms include pharyngitis, fever and lymphadenopathy. However, EBV infection may exhibit numerous rare, atypical and threatening manifestations involving different organ systems.Predominant gastrointestinal involvement is rarely documented.We present an unusual case of acute EBV infectionassociated hemorrahagic gastropathy.

Case report: A previously healthy 12 -year-old male child presented to the emergency department with a 4-day history of acute onset of malaise, weakness, anorexia and 2 episodes of lypotimia followed by 3 melena stools. There was no fever, weight loss, abdominal pain, vomiting or sore throat.He reported no NSAID, drugs or alcohol history. Physical examination revealed a pale face, afebrile with tachypnea and tachycardia.There was no skin rash, jaundice,lymphadenopathy and the abdomen was soft with no rebound tenderness or hepatosplenomegaly. A complete blood count showed a hemoglobin of $6.8 \mathrm{~g} / \mathrm{dL}$, a normal platelet count, and white blood cell count of $4200 / \mu \mathrm{L}$ with $27 \%$ lymphocytes. The international normalized ratio, partial thromboplastin time and liver function test results were normal. He received transfusion of red blood cells and after being excluded Meckel's diverticulum, an upper GI endoscopy revealed an increased friability of the mucosa and hemorrhagic gastropathy.Treatment with proton pump inhibitors were initiated and was maintained for 8 days with favorable results.An upper endoscopy before the discharge reveled a completely normal gastric mucosa. Gastric biopsy showed lymphoid aggregates without Helicobacter pylori infection. Serologic testing demonstrated a positive $\mathrm{IgG}$ and $\operatorname{IgM}$ antibodies to viral capsid antigen and IgG-antibodies to EBV nuclear antigen 1.Serology for cytomegalovirus, herpes simplex and parvovirus B19 were negative. The present case illustrates the broad spectrum of EBV disease and that it should be regarded as a cause of gastrointestinal lesions. 

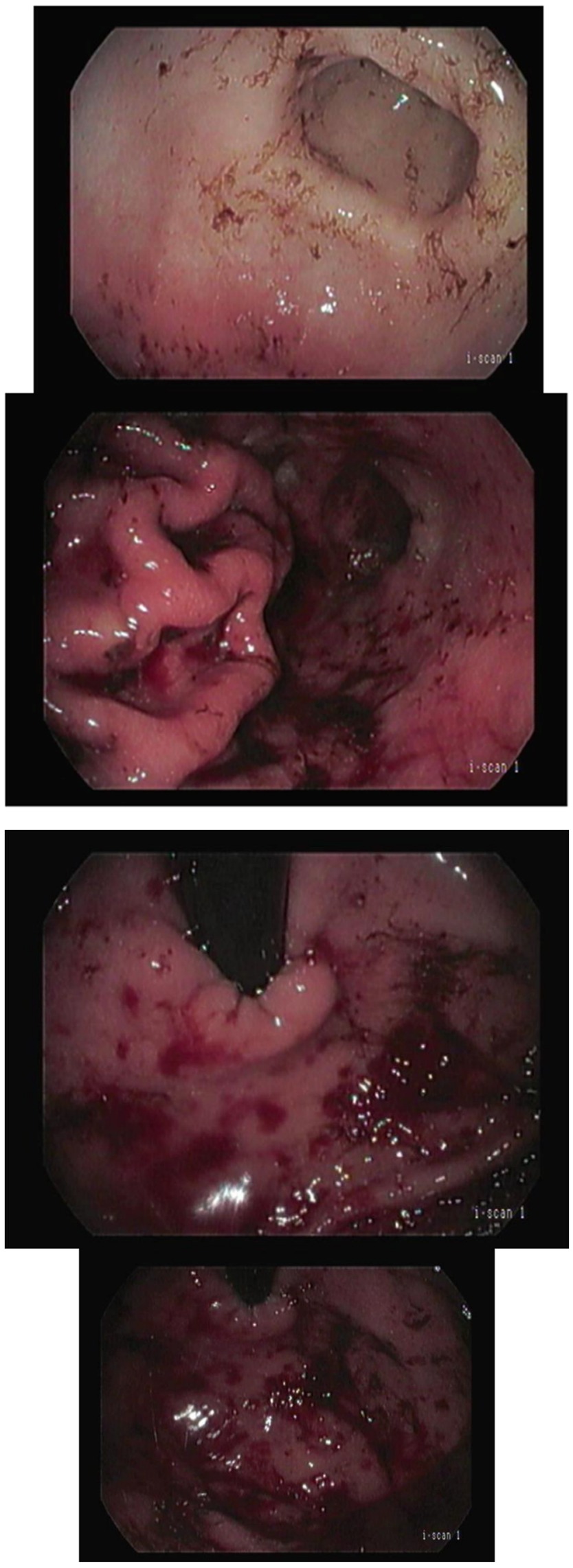

457

\section{Gastroenterology \& Nutrition}

Satisfactory Switch to Infliximab after Adalimumab Loss of Response in a Teenager with Severe Crohn's Disease Andreia Florina Nita ${ }^{1,2}$, Gabriel Becheanu ${ }^{2,3}$, Daniela Pacurar ${ }^{1,2}$ ${ }^{T}$ Paediatrics, Grigore Alexandrescu Emergency Hospital for Children, Romania

${ }^{2}$ Paediatrics, Carol Davila University of Medicine and Pharmacy, Romania

${ }^{3}$ Histopathology, Victor Babes Clinical Hospital for Infectious and Tropical Diseases, Romania

Introduction: Paediatric inflammatory bowel disease is often more aggressive compared to adults and the current tendency is early use of monoclonal antibodies in severe cases, loss of response being the therapeutic challenge.

We present the case of a teenager with Crohn's Disease, naive to antiTNF therapy who developed antibodies to Adalimumab and was switched to Infliximab.

Case Presentation: A 13 years old teenager was admitted for alopecia areata, nummular eczema, abdominal pain and weight loss $(17 \mathrm{~kg})$ lasting for three months. His mother reported repeated episodes of diarrhea but he denied.

He was diagnosed with Crohn's Disease and Pediatric Crohn`s Disease Activity Index score was 60, reflecting a severe disease activity. He was started on exclusive enteral nutrition as induction therapy with a good response. We decided to introduce azathioprine not steroids(CS) based on his reluctance to receive CS due to adverse effects. After six months he presented bloody stools and after nine months he developed anal abscess and perianal fistulae which required surgical intervention. Anti-TNF agent Adalimumab was introduced in the treatment plan. After the first 3 months, the seric level of Adalimumab was low and he developed antibodies to Adalimumab accompanying symptoms. We stopped Adalimumab and initiated treatment with Infliximab which he has been receiving for 20 months now with two relapses addressed by dose increase and rhythm adjustment.

Discussions and Conclusion: In naïve patients loss of response to Adalimumab is expected to be rarer. A loss of response to Infliximab may affect the response to subsequent Adalimumab treatment based on cross-reactivity of antibodies even though the two drugs are different idiothypes. However, there is a general lack of experience on the use of Infliximab after Adalimumab failure.

In Crohn`s Disease treatment strategy, Infliximab might be a solution after Adalimumab loss of response due to Adalimumab antibodies development.

\section{5}

\section{Gastroenterology \& Nutrition}

What about a Negative Serology in an Adolescent with Celiac Disease Case Report

Joanna Ashworth ${ }^{1}$, Carolina Fleming ${ }^{2}$, José Ramon Vizcaino ${ }^{2}$, Gisela $\overline{S i l v a}^{3}$, Helena Silva ${ }^{3}$, Esmeralda Cleto $^{4}$, Marta Tavares ${ }^{3}$, Rosa Lima ${ }^{3}$

${ }^{1}$ Pediatric Department, Centro Materno Infantil do Norte, CHUP, Portugal

${ }^{2}$ Anatomic Pathology Service, Pathology Department, CHUP, Portugal

${ }^{3}$ Pediatric Gastroenterology Department, Centro Materno Infantil do Norte, CHUP, Portugal

${ }^{4}$ Pediatric Hematology Department, Centro Materno Infantil do Norte, CHUP, Portugal

Background: Celiac disease (CD) is an immune-mediated systemic disorder elicited by gluten. Classic diagnosis is based on positive tissue transglutaminase type 2 (TTG2) antibodies and 
histopathological features as villi atrophy. Seronegative celiac patients are very rare in Pediatrics. We describe a unique case of celiac disease in a seronegative adolescent.

Case Report: A 15-year-old girl diagnosed with Blackfan-Diamond anemia at birth was referred to a Pediatric Gastroenterologist due to abdominal pain that started two months prior. She was on immunosuppressive therapy with systemic corticosteroids due to her hematological disease up to three months prior. The pain was more intense in the right upper quadrant and the diagnosis of primary biliary dyskinesia was proposed. Celiac screening workup (TTG2, Gliadin and endomisium antibodies) was negative. During an intense pain episode she was admitted to hospital and underwent an upper endoscopy which showed no relevant features. Gastric and duodenal samples were taken and microscopical examination showed moderate villi atrophy with intraepithelial lymphocytosis corresponding to a grade $3 \mathrm{~b}$ in the modified Marsh classification (Marsh-Oberhuber). Clinical remission was achieved after a gluten-free diet (GFD). One year after diagnosis duodenal/bulb biopsy was repeated showing marked improvement without villi atrophy or crypt hyperplasia but with some intraepithelial lymphocytes (less than 30 per 100 enterocytes) which corresponds to a grade $0 / 1$ in the modified Marsh classification (Marsh-Oberhuber). She remains clinically well on a GFD.

Conclusions: $\mathrm{CD}$ antibodies are not detectable in the blood of all patients with DC, especially in those under systemic immunosuppression. Strong clinical suspicion should always lead to further investigation with histolopathological analysis even in cases with negative serology.

\section{3}

\section{Gastroenterology \& Nutrition}

\section{No Longer an Adult Disease \\ Ruxandra Simona Bacanu ${ }^{l}$ \\ ${ }^{T}$ Pediatrics, General Hospital Tomelloso, Spain}

Background: When caring for children, we are all prepared for evaluating abdominal pain as it seems like every condition leads can cause belly pain.

Aim of Paper: To demonstrate that we have to remain vigilant for those subtle presentations of abdominal pain of more omnious conditions.

Material and Method: We present the case of a 8 years girl we arrived to Emergency Room with a 36 hours history of intensive abdominal pain and vomiting. En ER was realized urgent blood tests which revealed hypertransaminasemia and elevated total bilirubin on account of direct bilirubin. The girl had no history of any moment of posible contagion, signs of infection or other sympotms which could explain a frequent etiology of cytolysis in children.

The physical examination revealed an overweight child with an abundante abdominal pannus, with difuse abdominal pain, predominantly in the epigastrum.

We proceed to an abdominal ultrasonography which revealed a gallbladder with multiple stones inside and a discreet dilatation of coledocian.

Due to these findings, we iniciated medical treatment with antibiotics, with improvement of clinical and analitical tests in 24 hours.

Discussions: Cholelithiasis is being recognized in the children with increasing frequency. In children, there may be any number of possible etiologies for the development of gallstones. In the majority, specific etiologic factor is never established. In our case, we suppose the overweight is directly implicated in the produce of gallstones. Differentiating gallbladder disease in children from other causes of abdominal pain can be very difficult, but probably the single most important factor aiding in the diagnosis is simply main.- taining high index of suspicion that it can and does occur in children.

Conclusions: Not all kid belly pain is benign. Think of cholecystitis, even in kids.
154

\section{Gastroenterology \& Nutrition}

Helicobacter Pylori Infection and Recurrent Abdominal Pain in Children

Ruxandra Simona Bacanu ${ }^{1}$, Mauricio Moreno López ${ }^{1}$

${ }^{T}$ Pediatrics, General Hospital Tomelloso, Spain

Background: Helicobacter pylori (HP) is a gram-negative bacillus responsible for one of the most common infections found in humans worldwide. The incidence is $3-10 \%$ of the population each year in developing countries compared with $0.5 \%$ in developed countries. Most children infected with $\mathrm{H}$ pylori are asymptomatic. The relationship between $\mathrm{H}$ pylori infection and recurrent abdominal pain (RAP) is controversial.

Objective: To determine the incidence of HP infection in children with RAP.

Material and Method: The study is a retrospective one. We have taken in the study all the children with recurrent abdominal pain caused by HP infection that came as "new outpatient" in Pediatrics, between January 2017 - December 2018 and we analized the management they received. Results: From a total of 1004 "new outpatients" with RAP, 37 were confirmed with HP infection (3.68\%), a higher rate of infecion than we found out in different studies. In all the pacients, the first tratment was represented by OAC asociation, with a $67.56 \%$ rate of cure ( 25 pacients). From the other 12 cases, 9 pacients cured after the second tratment (OMA asociation). Two more patients remained asintomatics after the second tratment. We confirmed antibiotic resistence in only 1 case (both metronidazole and claritromicin) and the pacient became asintomatic and cured with OAR asociation.With the two "clasical" tratments, for 14 days, we obtained the cure in $91.9 \%$ patients.

Conclusion: The incidence in our area is not concordante with other studies; that could be explained by the mixture of population here. Despite of this, we obtained very good results with the clasical treatment.

\section{6}

Hepatic Glycogenosis: About a Series of 15 Cases Naima Baddouh ${ }^{1}$, S. Mrhar ${ }^{1}$, N. Hazzab ${ }^{1}$, A. Daoudi ${ }^{1}$, H. Nassih ${ }^{1}$, A. Bourrahouat $^{\prime}$, I. Ait Sab ${ }^{l}$

${ }^{1}$ Pediatrics B Department, Mother and Child Hospital, Mohammed VI University Hospital Faculty of Medicine, Cadi Ayyad University, Morocco

Hepatic glycogenosis are a group of inherited disorders characterized by enzyme defects that affect the glycogen synthesis and degradation cycle. Objective: To describe the characteristics of patients with hepatic glycogenosis, the presentation types and to highlight the diagnostic and therapeutic difficulties in our population.

Methods: The study group consisted of 15 patients admitted in hospital between January 2012 and December 2018.

Results: 11 boys and 4 girls. The sex ratio was 2.75 . Ten patients had type I glycogenosis, four patients had type III and one had type XI. The consanguinity of the parents was found in 11 patients. The referral reason was growth failure in 11 patients and hypoglycemia in 7 cases. Hepatomegaly was present in 13 cases. 5 patients had a facial dysmorphism. Biologically, hepatic cytolysis was present in 10 patients and hypertriglyceridemia in 7 . Liver function was normal in all patients. Hepatic biopsy was performed in all patients and showed histological signs of glycogenosis. Therapeutic management was based on dietary measures. All patients are alive. 3 cases have progressed to cirrhosis.

Conclusion: Hepatic glycogenosis have heterogeneous clinical manifestation. It has to be kept in mind whenever important hepatomegaly and/or hypoglycemia are present. 


\section{7}

Fanconi-Bickel Syndrome: Report of a Case

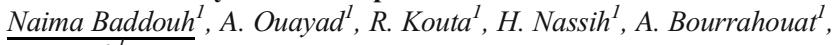
I. Ait $S a b^{I}$

${ }^{I}$ Pediatrics B Department, Mother and Child Hospital, Mohammed VI

University Hospital Faculty of Medicine, Morocco

Fanconi-Bickel syndrome (SFB) is a rare glycogenosis characterized by glycogen accumulation in the liver and kidneys, severe proximal renal tubulopathy, and abnormal glucose and galactose metabolism.

Case presentation: We report the case of a 5-year-old girl from a nonconsanguineous marriage, who presented for growth failure and abdominal distention that progressed gradually from birth. The clinical examination showed a "doll-like" face, a size at -4DS, signs of rickets and hepatomegaly. Biological assessment showed hypoglycemia, hypophosphoremia, normal calcium rate, elevated PAL rate, normal transaminases and TP, hypercholesterolemia and hypertriglyceridemia, metabolic acidosis. In urine analysis we found glycosuria, ketonuria, amino aciduria and phosphaturia. X-rays showed diffuse osteopenia with a typical appearance of rickets. Liver biopsy showed glycogen accumulation in the hepatocytes. The treatment consisted of phosphorus and vitamin D supplementation with low galactose diet and split meals.

Conclusion: This report describes the clinical history of an affected child and highlights the importance of continued follow-up in order to extend our understanding of the history of this rare metabolic disorder.

\section{5}

P1: Gastroenterology \& Nutrition 1 (Friday, September 20, 2019 18:15) Gastroenterology \& Nutrition

Food Parenting Practices and Obstacles: What are Parents of Preschool Children doing to promote their Child's Healthy Eating? Luisa Barros $^{I}$, Cátia Alves ${ }^{1}$, Patricia Nogueira ${ }^{I}$, Eugénia Oliveira ${ }^{I}$, Ana Isabel Pereira ${ }^{l}$

${ }^{I}$ Faculdade de Psicologia, Universidade de Lisboa, Portugal

Background: Eating habits develop early in childhood, and tend to persist into adulthood. Parents play a key role in the development of children`s eating habits. Although most parents are motivated to help their young children eat a healthy diet, their parenting food practices and the perception of barriers may undermine the reaching of these goals. As such, it is critical to develop comprehensive and culturally valid measures of these critical dimensions of food parenting.

Objective: A sequence of two studies aimed to identify food parenting practices and obstacles most reported by parents of Portuguese preschool children, and to develop two validated and culturally adequate questionnaires to be used in future intervention studies.

Methods: Firstly, a semi-structured interview was conducted with 12 mothers of preschool children. Interviews were recorded and transcribed and its contents analyzed according to Thematic Analysis. Secondly, two questionnaires were developed, based on the interview results, relevant literature and previous food parenting instruments; 200 parents of 2 to 6 years old children filled the questionnaire online.

Results: Parenting practices included items related to restriction, pressure to eat, food as reward, permissiveness, restriction of junk food, encouraging fruits and vegetables intake, modeling, negotiation, and selfregulation strategies. Barriers were related to the child's characteristics, parent's characteristics and the context. Two instruments were developed and tested, showing good psychometric properties. Parents of preschool children reported using simultaneously food effective and ineffective parenting practices. Parents reported using more often modeling and selfregulation promotion but also restriction. All barriers were considered moderately important with parent-related barriers considered more important.
Conclusion: Results point to the relevance of advancing our understanding of parenting food practices and difficulties to better tailor preventive interventions to promote healthy eating habits during early childhood.

190

Gastroenterology \& Nutrition

The Impact of a Short-Term Intervention on Adolescent Eating Habits and Food Knowledge

Tal David Berger $^{I}$, Anna Gorodnichenkol, Akiva Fradkin ${ }^{1,2}$, Batia Weiss $^{1,2}$

${ }^{1}$ Division of Pediatric Gastroenterology and Nutrition, Edmond and Lily Safra Children's Hospital, Tel- Hashomer, Israel

${ }^{2}$ Sackler Faculty of Medicine, Tel-Aviv University, Israel

Objective: To evaluate the effect of school-based intervention on the nutritional knowledge, eating habits and physical activity of high school students.

Methods: A prospective questionnaire-based study. Students of the 8$11^{\text {th }}$ grade from one high school in Israel prospectively fulfilled anonymous, previously validated, questionnaires at the beginning of the academic year (September 2014). During the following year, vending machines containing milk products were installed within the school facility, and the students were given 2 informative nutrition lectures regarding proper nutrition for age, calcium requirement and importance, and physical activity. In addition, one active sport-day was performed. At the beginning of the following academic year (September 2015), the students fulfilled the same questionnaires again. We analyzed the results for the whole study group and according to students`age: $13-15$ years (Group I) and age $16-18$ years (Group II).

Results: 330 teenagers, age $15.1 \pm 1.39$ years, $56 \%$ males, were included. Response rate was $83.6 \% \pm 0.4 \%$ to multiple-choice questions, $60.7 \% \pm 0.5 \%$ to multiple section tables, and $80.3 \% \pm 0.9 \%$ to open questions. Post-intervention there was an increase in the habit of eating breakfast $(57.0 \%$ vs. $47.5 \%, p=0.02)$ and a decrease in purchasing food at school $(61.6 \%$ vs $54.3 \%, \mathrm{p}=0.003)$. No changes were observed in consumption of milk products, knowledge regarding calcium and vegetable consumption, or sport activities.

Conclusion: A combination of active and passive nutrition-oriented intervention at high school resulted in a significant change in part of the eating habits, but nutritional knowledge and physical activity remained unchanged.

\section{1}

Gastroenterology \& Nutrition

Malnutrition in Children of Northern Mozambique: Only a Small Picture of a Global Reality!

$\underline{\text { Joana Caldeira Santos }}{ }^{I}$, Sofia Simões Ferreira ${ }^{I}$, Andreia Teles ${ }^{1}$, Mulaja Etienne $^{2}$, Carla Rêgo ${ }^{3}$

${ }^{I}$ Department of Pediatrics, Vila Nova de Gaia/Espinho Hospital Center, Portugal

${ }^{2}$ Department of Pediatrics, Central Hospital of Nampula, Mozambique ${ }^{3}$ Department of Pediatrics, CUF Hospital. CINTESIS - Faculty of Medicin of Porto University. Catholic University of Portugal. Health4MOZ ONGD, Portugal

Background: Malnutrition remains a major cause of morbimortality in Mozambique, being related to $45 \%$ of deaths among children under five years.

Objective: Characterize nutritional status, comorbidities, dietary pattern and clinical evolution of children hospitalized for undernutrition.

Methods: Prospective study conducted in a Central Hospital in northern Mozambique (Nampula) in August and September 2017. WHO criteria were used to characterize nutritional status and a questionnaire was applied. 
Results: 61 children were hospitalized in malnutrition sector, $16.4 \%$ with wasting and $83.6 \%$ with both stunting and wasting, which was severe in $78.7 \%$, predominantly marasmus $(55.7 \%)$. The median age was 17 (IQR 11-25) months, $50.8 \%$ were male. The majority of mothers breastfed their children $(94.7 \%)$ for a mean of $12 \pm 7$ months. Half of parents only spoke Emakhuwa (local language), the majority (53.1\%) were Muslim, unemployment in both parents was present in $45.9 \%$ and $34.4 \%$ reported past use of Mozambican witchdoctors. The majority had neither piped water supply $(80.3 \%)$ nor electricity $(72.1 \%)$. They reported a median of 3 meals each day, based predominantly on roots. The most frequent associated conditions were anemia $(96.4 \%)$, AIDS $(31.1 \%)$ and malaria $(26.2 \%)$. There was an association between AIDS and stunting $(p=0.023)$. Severe malnutrition was associated with younger age $(p=0.008)$ and less consumption of fruit and vegetables with marasmus $(\mathrm{p}=0.004$ and $\mathrm{p}=0.032$, respectively). Hospitalization had a median of 15 days, but $13.1 \%$ of children left the hospital with their parents without medical discharge and other $13.1 \%$ died, mostly as consequence of dehydration/hypovolemic shock.

Conclusion: We emphasize the young age, the severe presentation and the high morbimortality associated with undernutrition, as well as the great prevalence of HIV/AIDS and anemia. Lack of clean water and sanitation in addition to dietary deficiencies are main problems. The high rate of hospital abandonment and witchdoctors attendance reflects the need for health education in Mozambique.

\section{5}

\section{Hemato - Oncology}

Accidental PEComa: Case Report of a Rare Entity Maria Filomena Cardosa', Sofia Lima ${ }^{2}$, Maria Knoblich ${ }^{2}$, Isabel Afonso $^{3}$, Cristina Moreira ${ }^{4}$

${ }^{1}$ Serviço de Pediatria, Hospital Garcia de Orta, Portugal

${ }^{2}$ Serviço de Cirurgia Pediátrica, Hospital Dona Estefânia - Centro Hospitalar Universitário de Lisboa Central, Portugal

${ }^{3}$ Unidade de Gastroenterologia e Hepatologia Infantil, Hospital Dona Estefânia-Centro Hospitalar Universitário de Lisboa Central, Portugal ${ }^{4}$ Serviço de Oncologia Pediátrica, Instituto Português de Oncologia, Portugal

A previously healthy girl was referred to the paediatric gastroenterology consultation at the age of four due to recurrent umbilical pain. During workup, an abdominal ultrasound (US) revealed two gallstones with $4 \mathrm{~mm}$ of diameter without dilation of the biliary tract. She remained in follow-up, asymptomatic, no physical findings and stable US. At the age of ten, a supra-umbilical mass was detected on US, and MRI revealed an umbilical intra-peritoneal tumor $(45 \times 40 \times 45 \mathrm{~mm})$ with well-defined limits, heterogeneous, predominantly solid, highly vascularized, and compressing inferior vena cava without local invasion. Surgical mass resection was performed, and histology revealed morphological and immunohistochemical aspects of a PEComa of the abdominal wall. Given the size of the lesion $(5 \mathrm{~cm}$ ) and admitting uncertain malignant potential, she was referred to the pediatric oncology center and submitted to margin enlargement. Three years later, she remains asymptomatic without clinical or imagiological evidence of relapse.

Perivascular epithelioid cell tumor (PEComa) is a rare mesenchymal tumor with myomelanocytic differentiation. It mainly occurs in middle aged females and can be found at any location, being kidney the most common. The differential diagnosis of PEComas is fairly broad but the immunohistochemical coexpression of melanocytic (HMB45) and smooth muscle (actin and desmin) biomarkers make the diagnosis. They may behave indolently but entail variable malignant potential. When there isn't evidence of metastasis, surgical resection is the recommended approach. The benefits of chemotherapy or radiation haven't been established thus far but some clinical reports show good results with mTOR inhibitors.

With this case we intend to recall the importance of considering this rare etiology in the evaluation of a mesenchymal tumor and enhance the importance of immunohistochemical biomarkers for the definitive diagnosis. The correct histological classification is essential to assess the potential of malignancy and thus assure the most appropriate therapeutic approach.

\section{5}

\section{Gastroenterology \& Nutrition}

Administration of Probiotics Enriched Gut Bacteria Diversity and Glycan Degrading Ability in Small Bowel Transplantees Pi-Feng Chang ${ }^{1}$, Yu-Cheng Lin' ${ }^{1}$, Kevin Liu ${ }^{1}$, Yun Chen ${ }^{1,2}$

${ }^{1}$ Pediatrics and Electronic Engineering, Far Eastern Memorial Hospital and Oriental Institute of Technology, Taiwan

${ }^{2}$ Pediatric Surgery, Far Eastern Memorial Hospital, Taiwan

Background: The role of probiotics among small bowel transplantation (SBT) patients is intriguing because bacteria reside in the transplanted organ per se and hosts' immune functions are inevitably compromised. Glycans are the primary nutritional source for microbes in the human gut. Analysis of glycan degradation by the gut microbiota is an important tool for advancing our understanding of bacterial metabolism in the gut.

Objectives: The aim of this study was to quantify glycan processing capabilities and investigate the relationship between the changes of gut microbiota and glycan metabolism before and after probiotics treatment. Methods: The SBT patients without obvious sepsis, weaning from parenteral nutrition (PN) and normal oral intake were enrolled in this study. During the study period, they were receiving standard doses of Tacrolimus and steroids. These SBT patients received oral probiotics (CBM588: Clostridium butyricum MIYAIRI 588, $1.5 \times 10^{9} \mathrm{CFU} /$ day) for 1 month. Fecal samples were collected at before, 1 week and 1 month after oral probiotics therapy. Next-generation sequencing targeting $16 \mathrm{~S}$ ribosomal sequences from fecal materials was used to evaluate gut microbiota and glycan degradation potency, respectively. We compare the changes of microbiota and glycan degrading capabilities before and after probiotics treatment.

Results: Significant shifts of bacteria compositions were found after one month of CBM588 ingestions. Specifically at the family level, Bacteroidaceae increased with a substantial boost from $4 \%$ to $21 \%$ but Enterobacteriaceae decreased by a large margin from $41 \%$ to $34 \%$. Functional inference with 16S-based microbiome extrapolations suggested characteristic enhancement of glycan degrading capabilities with CBM588 ingestions. Metagenomes from shotgun sequencing confirmed the conjecture, showing both diversified capabilities and increased quantities of carbohydrate (glycan)-active genes. The improvements spread beyond the enzyme categories directly offered by the CBM588 genome. Conclusions: In sum, CBM588-enhanced glycan-processing capabilities well exemplified a metabolic driver potential of probiotics upon the bacteria ecosystem in human bowels.

965

\section{Gastroenterology \& Nutrition}

Meckel's diverticulum: a rare case of rectal bleeding in a teenager Ines Coelho $^{I}$, Sofia Baptista ${ }^{I}$, Teresa Monteiro ${ }^{I}$, Rita Justo Pereira ${ }^{l}$, $\overline{\text { Daniel Tiago }}^{1}$, Stefanie Pereira ${ }^{2}$

${ }^{1}$ Pediatrics, Centro Hospitalar Universitário do Algarve, Unidade de Faro, Portugal ${ }^{2}$ Pediatric surgery, Centro Hospitalar Universitário do Algarve, Unidade de Faro, Portugal

Introduction: Rectal bleeding is a common problem in pediatrics and in the majority of cases the causes are transient, benign and simplytreated. The age of onset relates to certain diagnoses: anal fissures and intussusception in infant, and lymphoid hyperplasia and inflammatory bowel disease in the older child. In children younger than 2 years of age we also have to think in Meckel's diverticulum (MD). In these cases the majority 
of patients don't have symptoms, but the ones who are symptomatic, often present rectal bleeding, which is usually painless.

Case Report: A 12 year-old-boy was admitted to the paediatric emergency room with a 3-day history of abdominal pain with rectal bleeding. No relevant personal or family history. On examination, he presented a BMI $95^{\text {th }}$ percentile, he was pale, but was hemodynamically stable, with no pain on abdominal palpation. The hemogram showed an hemoglobin of $7 \mathrm{~g} / \mathrm{dL}$, so he received a blood transfusion and was admitted to the ward. Throughout his stay he did an upper and lower endoscopy and an MR enterography, which didn't reveal any alteration. We were suspicious of an MD and after having done a technetium- $99 \mathrm{~m}$ pertechnetate scan, the diagnosis was confirmed. He underwent surgical resection of the MD (segmental intestinal enterectomy), which ran without intercurrences. The postoperative period was uneventful and he was discharged on day 6 , clinically well.

Conclusion: Meckel`s diverticulum is a common congenital gastrointestinal malformation in children but is rare in teenagers. It has various clinical presentations and it is necessary to maintain a high suspicion in the paediatric age group. Serious complications rarely happen but when they do, they can cause consequences, such as mechanical obstruction, intestinal perforation and shock. Thus, prompt diagnosis and proper treatment are essential in warranting a better outcome.

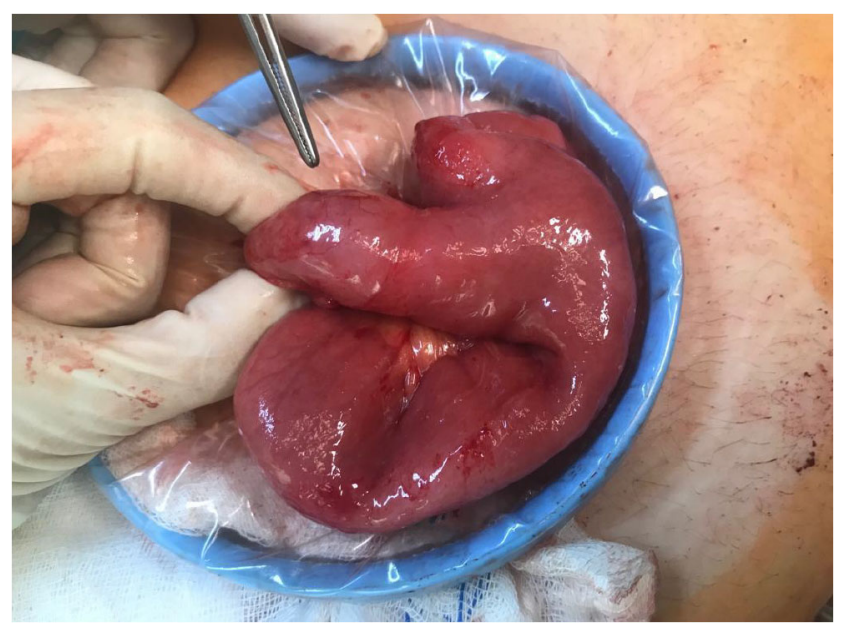

402

\section{Gastroenterology \& Nutrition}

Usefulness of Oesophageal Length Measurement in Paediatric Gastroscopy

David Croaker ${ }^{1}$, Himasha Nanayakkara ${ }^{2}$

${ }^{T}$ Department of Paediatric Surgery, The Canberra Hospital, Australia

${ }^{2}$ Medical School, University of Notre Dame, Sydney, Australia

Background: The senior author has had a routine of including an oesophageal length measurement routinely at paediatric endoscopy. The authors hypothesise that a short oesophagus predicts reflux changes at pathology.

Aim: This audit seeks to assess the utility of oesophageal length measurement.

Methods: The study is a retrospective chart review. At gastroscopy the distance from the teeth to the squamo-columnar junction is noted. Demographics, oesophageal length, clinical background and pathology data were collected and analysed in an Excel spreadsheet.

Results: 1143 endoscopy reports were reviewed. 823 oesophageal length measurements were available. Mean oesophageal length at 12 months is $21.5 \mathrm{~cm}$, and at 10 years is $33.2 \mathrm{~cm}$. The line of best fit has oesophageal growth $\sim 0.1 \mathrm{~cm}$ per month from 0 to 16 years. Some degree of inflammation was noted in 313 of 877 pathology reports. The longest and shortest oesophageal length quartiles normalised for height had similar rates of reflux changes on pathology. As expected oesophageal, length in TOF patients is short, and oesophageal growth slower in TOF than in others. A modest tendency for eosinophilic oesophagitis to correlate with short oesophagus was noted (length difference $\sim 0.7 \mathrm{~cm} ; \mathrm{p}=0.015$ ).

Discussion: Oesophageal length measurements normalised for both height and age failed to correlate with reflux changes which were nonetheless widespread in TOF patients. There was otherwise no strong relationship between oesophageal length and reflux changes in the general population. Conclusions: While our measurements seem valid and accurate, their usefulness in predicting reflux pathology in the general population is limited.

690

\section{Gastroenterology \& Nutrition}

\author{
Is Bile in the Stomach Related to Paediatric Chronic Abdominal \\ Pain? \\ David Croaker ${ }^{I}$, Himasha Nannayakkara ${ }^{2}$ \\ ${ }^{T}$ Department of Paediatrics and Child Health, The Canberra Hospital, \\ Australia \\ ${ }^{2}$ Medical School, Notre Dame University, Australia
}

Background/Aim: While bilious vomiting is regarded as a worrying symptom and sign in acute paediatric surgery, chronic abdominal pain is a common non-acute reason for referral for endoscopy. Chronic nonspecific gastritis is often reported on biopsy in our patients. The authors asked how often bile was seen in the stomach on gastroscopy, and asked whether this correlated with gastric pathology to support the notion of bile gastritis as a cause of chronic abdominal pain in children.

Methods: Retrospective chart review of a paediatric gastroscopy practice. Routine biopsies are usually taken from gastric antrum. Gastric contents were noted. Only patients with recorded gastric contents were included. The pathology report was reviewed. Data were stored in Excel. Presence of gastric bile was compared against histology and presentation. Proportions were compared by Fisher exact test.

Results: 769 patients with available data on the gastric contents were reviewed, 743 of whom had histopathology reports. Results are summarised in the table. These proportions were not significantly different by Fischer Exact test. Patients with bile stained gastric contents however were nearly twice as likely to have been referred for investigation of abdominal pain as those with no bile staining. ( $16 \%$ vs $9 \% \mathrm{p}=0.007$ by Fisher exact test.) No relationship to distal oesophageal reflux changes was found to explain the pain correlation.

Conclusions: While bilious vomiting remains a red flag of the greatest importance, the presence of bile in the stomach without vomiting has no clear relation to gastric histopathology, but may be associated with abdominal pain.

\section{8}

\section{Gastroenterology \& Nutrition}

\section{Alagille Syndrome - The Difficulty of Initial Diagnosis}

Ruxandra Darie $^{1}$, Raluca Maria Vlad ${ }^{1,2}$, Irina Dijmarescu ${ }^{1,2}$, Daniela Pacurar $^{1,2}$

${ }^{1}$ Paediatrics Department, "Grigore Alexandrescu” Emergency Children's Hospital, Bucharest, Romania

${ }^{2}$ Paediatrics Department, "Carol Davila" University of Medicine and Pharmacy, Bucharest, Romania

Background: Alagille syndrome is a rare autosomal dominant disorder with systemic manifestations: paucity of the bile ducts, abnormalities of the heart, eye, skeleton and kidneys. It is diagnosed in children presenting with jaundice in the first 6 months of life (70\%) or cardiac murmur $(17 \%)$. 
Objective: This case presentation emphasises the challenges faced in terms of diagnosis and therapy for one of these patients.

Method and Results: We report the case of a 3 days old boy admitted in the Paediatrics Department of "Grigore Alexandrescu" Children's Hospital, Bucharest for bilious vomiting and intense jaundice. The newborn hadn't pass the meconium. Clinical examination showed abdominal distension and cardiac murmur. Laboratory tests revealed high levels of conjugated bilirubin, normal transaminases, serum proteins and blood coagulation. Viral serologies were all negative. Thoraco-lumbar X-ray of the spine didn't reveal any anomalies. Echocardiography described large pulmonary stenosis. Barium enema failed to pass meconium, exploratory laparotomy was performed for deobstruction. Cystic fibrosis was excluded. Alagille syndrome was the main diagnostic considered, but liver biopsy failed to reveal paucity of the bile ducts. Over the next two years the pacient underwent extensive investigations. A posterior embryotoxon was described. Abdominal CT could not visualise the bile duct. Abdominal MRI + cholangio-MRI showed thin intra/extrahepatic biliary ducts, cirrhotic nodules, the gallblader was not visible. Genetic testing was not available. The pacient associated failure to thrive. Signs of chronic disease began to surface in time: severe jaundice, hepatosplenomegaly, abdominal collateral circulation, persistent hepatic cytolysis and cholestasis, dyslipidemia. Due to progressive chronic hepato-biliary disease and advancing cirrhosis in a 2 years old with failure to thrive, liver transplant is now considered.

Conclusion: Although Alagille should be a straight forward diagnosis, in certain instances it takes time and sustained efforts to reach it and set the course for an optimal therapeutic approach.

\section{2}

\section{Gastroenterology \& Nutrition}

Autoimmune Overlap Syndrome in A 4 Year Old Girl - A Case Report

Irina Dijmarescu $^{1,2}$, Gabriela Mitea ${ }^{l}$, Alexandra Coroleuca ${ }^{l}$, Daniela Pacurar $^{\text {I, }}$

${ }^{I}$ Pediatrics, "Grigore Alexandrescu" Emergency Hospital for Children, Romania

${ }^{2}$ Pediatrics, "Carol Davila" University of Medicine and Pharmacy, Romania

Background: The overlap syndrome is characterized by the simultaneous occurrence of more than one autoimmune disease.

Objective: We aimed to present the difficulties encountered in diagnosing and treating a young child with an overlap syndrome. Case report: We present the case of a 4 year old female patient who was diagnosed with autoimmune hepatitis, primary sclerotic cholangitis and ulcerative colitis. The patient was being investigated for loss of appetite, weight loss, hepatosplenomegaly and bloody stools. The onset was 5 months prior to the diagnosis with bloody stools. Infectious causes were considered and the patient was treated accordingly. She had recurrent fever and hepatosplenomegaly. Laboratory tests showed severe anemia, high transaminases, cholestasis, high inflammatory markers, hypergammaglobulinemia $(50,8 \mathrm{~g} / \mathrm{dl})$ and high fecal calprotectine $(1370$ $\mathrm{mcg} / \mathrm{g}$ ). Viral causes of liver cytolysis were excluded. Due to the persistence of liver cytolysis further investigations were performed and LKM1 antibodies were positive. At the time of the diagnosis the child was completely nonimmunized. Subsequently she received first doses of all mandatory vaccines but no following doses. The colonoscopy showed rectal mucosa with hyperemic patches, histopathological examination being consistent with ulcerative colitis. The liver biopsy displayed advanced hepatic fibrosis and cholangitis. Thus, correlating all data, the diagnostic of an overlap syndrome (autoimmune hepatitis and primary sclerotic cholangitis) associated with ulcerative colitis was established. She received systemic corticotherapy, tapered afterwards (complete exclusion was not possible due to recurrence of bloody stools and liver cytolysis) and azathioprine since the time of diagnosis.
Conclusions: Overlap syndrome is a possibility that should be considered in patients with autoimmune diseases. Further investigations should be conducted in order to establish a complete diagnosis of these patients in order to treat them accordingly.

762

\section{Gastroenterology \& Nutrition}

Efficiency and Safety of GABA Derivatives for Treatment of Functional Dyspepsia in Children

Anna Drokh ${ }^{1}$, Marina Mamenko ${ }^{2}$, Ivanna Romankevych ${ }^{3}$, Katerina Boyarska $^{4}$, Hanna Kozhyna ${ }^{5}$, Raisa Moiseienko ${ }^{6}$

${ }^{1}$ Shupyk National Medical Academy of Postgraduate Education, PhD student, MD, Ukraine

${ }^{2}$ Shupyk National Medical Academy of Postgraduate Education, Professor, Dean of Pediatric Faculty, MD, PhD, DMs, Ukraine

${ }^{3}$ Shupyk National Medical Academy of Postgraduate Education, Assistant Professor of CEMSIT, PhD, MD, Ukraine

${ }^{4}$ Kyiv City Children's Clinical Hospital, Head of the Department of General Pediatrics, MD, Ukraine

${ }^{5}$ Kyiv City Children's Clinical Hospital, MD, Ukraine

${ }^{6}$ Shupyk National Medical Academy of Postgraduate Education, Professor, Vice-Rector for Clinical Work, MD, PhD, DMs, Ukraine

Background and Aims: The quality of life (QL) in children with functional dyspepsia (FD) is often impaired due to the psychological disorders and cognitive dysfunction seen in these patients. The aim of this study was to evaluate the efficacy and safety of phenibut in children with FD.

Methods: 40 children with FD were randomized to 1 month of symptomatic treatment+phenibut $(\mathrm{n}=20,400 \mathrm{mg} / \mathrm{d} 4$ times per day according to the instructions) or exclusively symptomatic treatment ( $\mathrm{n}=20$, Rome IV). QL was determined by a questionnaire "PedsQL". The memory was evaluated by method of "Memory for Numbers" and "RAM". The attention was determined by the method of "Proofreading" and "Technique of Münstenberg".

Results: At 1 month, the active group demonstrated: the improvement of physical functioning $(\mathrm{p}=0.007)$ and vs control group $(\mathrm{p}=0.043)$ : increasing of the emotional functioning $(\mathrm{p}=0.043)$ and $\mathrm{vs}$ the control $(\mathrm{p}=0.033)$; getting better in the functioning at the school $(\mathrm{p}=0.016)$ and $\mathrm{vs}$ the control $(\mathrm{p}=0.045)$. The phenibut group reported the increasing of the level of selective attention $(\mathrm{p}=0.023)$ and vs control group $(\mathrm{p}=0.041)$. The productivity and consistency of attention in the phenibut group was higher after treatment $(\mathrm{p}=0.001)$ and vs control $(\mathrm{p}=0.009)$. The level of short-term visual memory in the active group improved significantly after treatment $(\mathrm{p}=0.047)$ and vs control $(\mathrm{p}=0.038)$. RAM in the phenibut group was higher after treatment $(\mathrm{p}=0.007)$ and vs control group $(\mathrm{p}=0.013)$.

Conclusions: Phenibut was associated with appropriate therapeutic response in cognitive function and QL in children with FD. It was safe and well tolerated in used dosage.

\section{4}

\section{Gastroenterology \& Nutrition}

Tandem Breastfeeding and Human Milk Macronutrients Content

Gilad Rosenberg ${ }^{1}$, Dror Mandel ${ }^{2,3}$, Ronella Marom ${ }^{2,3}$, Laurence Mangel $^{2}$, Ronit Lubetzky ${ }^{1,3}$

${ }^{I}$ Pediatrics, Dana Dwek Children's Hospital, Tel Aviv Medical Center, Israel

${ }^{2}$ Neonatology, Dana Dwek Children's Hospital, Tel Aviv Medical Center, Israel

${ }^{3}$ Sackler Faculty of Medicine, Tel Aviv University, Israel 
Introduction: Little is known about the effect of tandem breastfeeding (the practice of breastfeeding two different age siblings) on human milk (HM) macronutrients content. We aimed to study macronutrients and energy content in HM collected during tandem breastfeeding.

Methods: Prospective-observational study. Mothers who breastfed their infants during pregnancy and intended to continue breastfeeding after delivery were recruited (tandem-group) and compared to a group of mothers who did-not breastfed during pregnancy, gave birth and breastfed one infant (control-group). Milk was collected during the last 4-weeks of pregnancy (tandem-group only), at 72-hours (colostrum, both groups) and at 30-days after delivery (mature-HM, both groups). Analysis was by infrared-transmission-spectroscopy.

Results: Fifty healthy-mothers were enrolled, out of which eighteen continued breastfeeding during term-pregnancy and after labor. They were compared to data from 118 mothers who did-not breastfeed during pregnancy and breastfed only one term-infant. HM during pregnancy had significantly lower fat content compared to $\mathrm{HM}$ in colostrum and mature-milk from the same mother $(2.5 \pm 1.4$ vs. $2.9 \pm 0.9, \mathrm{p}=0.003 ; 2.5$ \pm 1.4 vs. $4.2 \pm 1.2, \mathrm{p}=0.009$, respectively). Colostrum and mature-milk Fat did-not differ between tandem and controls. Protein during pregnancy was significantly higher compared to colostrum and mature-HM (3.5 \pm 1.4 vs. $2.0 \pm 0.8, \mathrm{p}=0.001 ; 3.5 \pm 1.4$ vs. $1.1 \pm 0.3$, p0.0001, respectively). Colostrum and mature-HM protein did-not differ between the 2 groups. HM-lactose during pregnancy was significantly lower compared to mature-HM (6.4 \pm 1.4 vs. $8.1 \pm 0.2$, p0.0001). Colostrum and mature-HM Lactose were significantly higher in tandem-group compared to controls. Energy content during pregnancy was significantly lower compared to mature-HM (63.4 \pm 11.4 vs. $71.9 \pm 10.0, \mathrm{p}=0.047)$. Colostrum Energy was significantly higher in tandem-group compared to controls.

Conclusion: HM expressed during pregnancy has different macronutrients content compared to HM after regular-full-term pregnancy. After delivery, during tandem-breastfeeding, there are minor differences in lactose and energy only in colostrum. Thus, mothers who tandem-breastfeed can be reassured about the macronutrients content of their milk.

\begin{tabular}{|c|c|c|c|}
\hline & $\begin{array}{c}\text { Tandem breastfeeding } \\
(n=18)\end{array}$ & $\begin{array}{c}\text { Non Tandem } \\
\text { breastfeeding ( } n=118)\end{array}$ & $P$ \\
\hline $\begin{array}{l}\text { Energy colostrum } \\
\text { (kCal/100cc) }\end{array}$ & $\begin{array}{l}63.1 \pm 6.8 \\
(53.79)\end{array}$ & $\begin{array}{l}55.0 \pm 17.5 \\
(17-105)\end{array}$ & 0.027 \\
\hline $\begin{array}{l}\text { Energy mature milk } \\
\text { (KCal/100cc) }\end{array}$ & $\begin{array}{c}71.9 \pm 10.0 \\
(53-87)\end{array}$ & $\begin{array}{l}71.4 \pm 15.2 \\
(12-118)\end{array}$ & 0.44 \\
\hline $\begin{array}{l}\text { Energy during pregnancy milk } \\
\text { (kCal/100cc) }\end{array}$ & $\begin{array}{l}63.4 \pm 11.4 \\
(47-86)\end{array}$ & & \\
\hline $\begin{array}{l}\text { Fat colostrum } \\
(\mathrm{g} / 100 \mathrm{cc})\end{array}$ & $\begin{array}{c}2.9 \pm 0.9 \\
(1.3-4.9)\end{array}$ & $\begin{array}{l}2.5 \pm 1.5 \\
(0.1-8.6)\end{array}$ & 0.15 \\
\hline $\begin{array}{l}\text { Fat mature milk } \\
(\mathrm{g} / 100 \mathrm{cc})\end{array}$ & $\begin{array}{l}4.2 \pm 1.2 \\
(2-6.1)\end{array}$ & $\begin{array}{l}4.5 \pm 1.6 \\
(0.7 \cdot 10.6)\end{array}$ & 0.21 \\
\hline $\begin{array}{l}\text { Fat during pregnancy milk } \\
(\mathrm{g} / 100 \mathrm{cc})\end{array}$ & $\begin{array}{r}2.5 \pm 1.4 \\
(1.1-5.5)\end{array}$ & & \\
\hline $\begin{array}{l}\text { Protein colostrum } \\
(\mathrm{g} / 100 \mathrm{cc})\end{array}$ & $\begin{array}{l}2.0 \pm 0.8 \\
(1-4)\end{array}$ & $\begin{array}{l}2.7 \pm 1.9 \\
(0-8.8)\end{array}$ & 0.09 \\
\hline $\begin{array}{l}\text { Protein mature milk } \\
(\mathrm{g} / 100 \mathrm{cc})\end{array}$ & $\begin{array}{r}1.1+0.3 \\
(0.8-1.8)\end{array}$ & $\begin{array}{l}1.2 \pm 0.5 \\
(0.2 \cdot 3.5)\end{array}$ & 0.29 \\
\hline $\begin{array}{l}\text { Protein during pregnancy milk } \\
(\mathrm{g} / 100 \mathrm{cc})\end{array}$ & $\begin{array}{l}3.5 \pm 1.4 \\
(1.1 \cdot 5.8)\end{array}$ & & \\
\hline $\begin{array}{l}\text { Lactose colostrum } \\
(\mathrm{g} / 100 \mathrm{cc})\end{array}$ & $\begin{array}{l}7.1 \pm 1.0 \\
(3.9-7.9)\end{array}$ & $\begin{array}{c}5.0 \pm 1.5 \\
(0.8-7.7)\end{array}$ & $<0.01$ \\
\hline $\begin{array}{l}\text { Lactose } m \text { ature milk } \\
(\mathrm{g} / 100 \mathrm{cc})\end{array}$ & $\begin{array}{c}8.1 \pm 0.2 \\
(7.7-8.4)\end{array}$ & $\begin{array}{l}6.1 \pm 1.3 \\
(2.5-8.8)\end{array}$ & $<0.01$ \\
\hline $\begin{array}{l}\text { Lactose during pregnancy milk } \\
(\mathrm{g} / 100 \mathrm{cc})\end{array}$ & $\begin{array}{l}6.4 \pm 1.4 \\
(2.5-8.3)\end{array}$ & & \\
\hline
\end{tabular}

116

\section{Gastroenterology \& Nutrition}

Audit of Checklist in Recurrent Abdominal Pain Frances Enright ${ }^{1,2}$, Hana Bashir ${ }^{2}$, Dace Balode Novikoda ${ }^{2}$ ${ }^{T}$ Paediatric Outpatients, Mercy University Hosptal, Ireland

${ }^{2}$ Outpatients, Mercy University Hospital, Ireland
Background: Recurrent abdominal pain (RAP) is common at $30 \%$ in childhood especially females 4-6 years of age and 9-11 years. RAP is more commonly non-organic i.e. functional abdominal pain FAP. A symptom-based approach is usually used by clinicians to identify the red flag symptoms of organic disease. In a previous retrospective study in 2015, co-author Dace studied 41 patients with RAP. $63 \%$ had investigation but had no red flag symptoms.

A checklist with sub-typing (attached) was developed to be used by the same doctor at every consult for FAP.

Objective: Reduce unnecessary investigations, keep approach consistent and subtype the FAP.

Methods: A retrospective cross-sectional design was used by Hana in this audit. If FAP is diagnosed the subtype is filled out. The treatment plan signed by parents is filed.

Results: Hana found a total of $23(n=23)$ new patients with recurrent abdominal pain who had the checklist filled. One case (4.3\%) had investigations in the absence of red flag symptoms. Six cases $(26 \%)$ reported no red flag symptoms and did not undergo any investigation. Sixteen of the twenty-three cases $(70 \%)$ reported at least one red flag symptom and received agreed investigations.

3 of the 23 children had organic causes (Gastritis, reflux and Crohns). The subtypes of FAP we identified were $5 \%$ (1) functional dyspepsia (upper abdomen discomfort), 50\% (10) irritable bowel (FAP with alteration in stool consistency), 5\% (1) abdominal migraine (history of maternal migraine and child has paroxysmal episodes of FAP with associated anorexia, pallor, nausea) and 20\% (4) Functional abdominal pain syndrome (by exclusion).

Conclusion: This audit illustrates a significant reduction (63\% to $4.3 \%)$ in the frequency of unnecessary investigations performed in the absence of red flag symptoms following the introduction of the checklist. Irritable bowel functional abdominal pain is commonest subtype. We recommend this method.

\section{9}

\section{Gastroenterology \& Nutrition}

Hypertrophyc Pyloric Stenosis: A Radiographic Finding

Celia Fabra Garrido ${ }^{I}$, Marta Dorado Criado ${ }^{I}$, Ursino Adrián Barrios Machain', Paloma López Ortego ${ }^{2}$

${ }^{1}$ Pediatrics, Hospital Universitario La Paz, Spain

${ }^{2}$ Neonatology, Hospital Universitario La Paz, Spain

Introduction: Hypertrophic pyloric stenosis (HPS) is one of the most frequent causes of non-bilious vomiting in infants. The diagnose is usually carried out by ultrasonography and only a few cases diagnosed by simple radiography are reported in the medical literature. We present a case that was diagnosed incidentally by the "caterpillar sign", a characteristic radiographic sign of HPS.

Clinical Case: A 2-month-old male patient was operated on for a cervical teratoma. Subsequently, he presented weakness of the oropharyngeal musculature and difficulties in swallowing as well as severe gastroesophageal reflux. He was under omeprazole, domperidone and continuous debit nutrition through a transpyloric tube. Despite the treatment, the patient was presenting frequent regurgitation and vomiting and a chest-abdomen $\mathrm{x}$ ray was perfomed to check the correct position of the probe. The radiography showed a distended stomach with increased peristaltic activity and he was diagnosed of HPS (Figure 1). The diagnosis was confirmed by abdominal ultrasound (figure 2) and a submucosal pyloromyotomy was perfomed with successful evolution. 


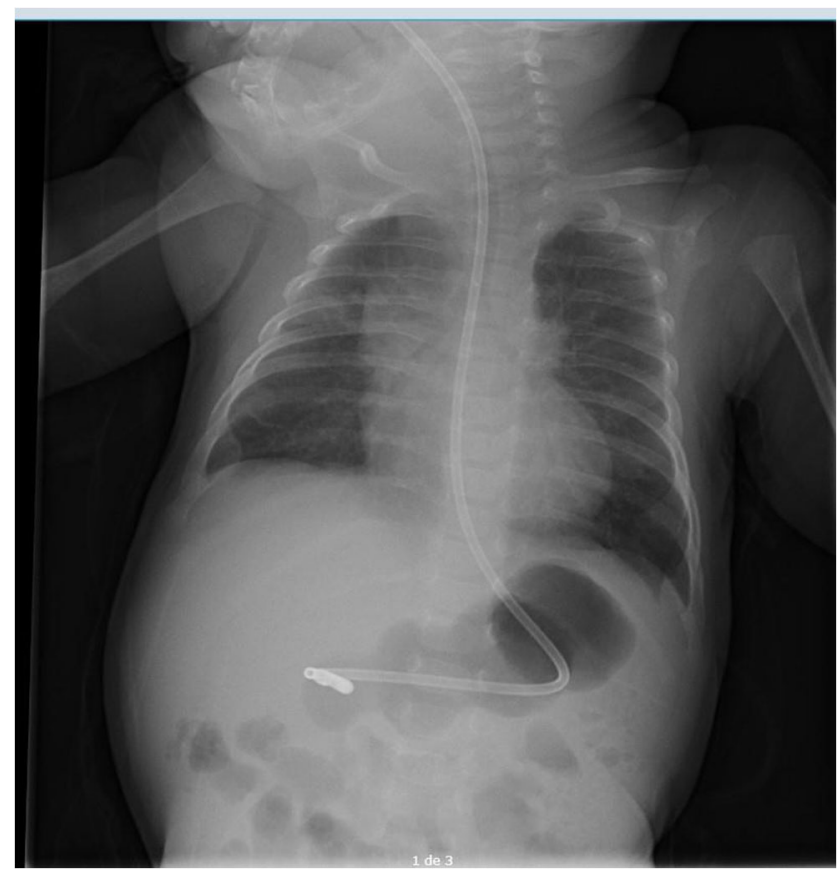

Figure 1. Chest-abdomen x-ray with notable peristaltic waves in the stomach due to HPS.

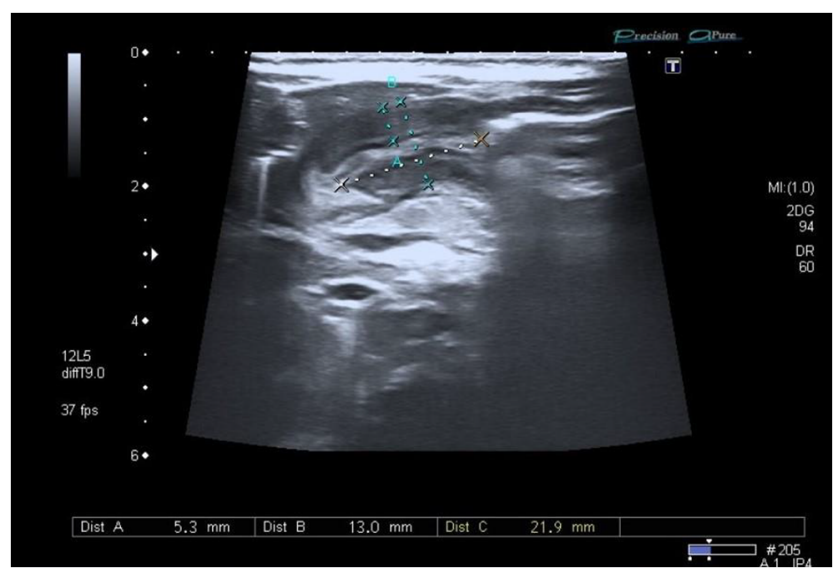

Figure 2. Abdominal ultrasound: Pyloric channel with diagnostic criteria for HPS.

Discussion: The gold standard imaging test for the diagnosis of HPS is abdominal ultrasound. If it does not provide a conclusive result, barium studies or upper gastrointestinal endoscopy are other diagnostic options. Our patient was diagnosed incidentally by simple radiography of the abdomen by the "caterpillar sign". This sign is the result of vigorous peristaltic waves against an abrupt stop at the hypertrophic pylorus.

Conclusion: This clinical case reminds us the importance of the systematic analysis in medical imaging tests, particularly in simple radiography, as despite it being a basic diagnostic tool, it is also a great diagnostic source of information.

\section{6}

\section{Gastroenterology \& Nutrition}

Celiac Disease - A 13-Year Casuistic in a Tertiary Hospital Carolina Freitas Fernandes ${ }^{l}$, Bernardo Camacho ${ }^{I}$, Francisco Silva ${ }^{l}$, Rute Gonçalves

${ }^{1}$ Paediatrics, Hospital Central do Funchal, Portugal
Background: Celiac disease (CD), also known as gluten-sensitive enteropathy is an immune-mediated inflammatory disease of the small intestine caused by sensitivity to dietary gluten and related proteins in genetically predisposed individuals. The disorder occurs in 0.5 to 1 percent of the general population in most countries.

Objective: Evaluating the incidence of celiac disease in the pediatric population in a tertiary hospital.

Methods: A retrospective study was carried out through review of clinical process of the pediatric patients with confirmed diagnosis of celiac disease in a tertiary hospital since 2006 to 2018.

Results: 65 cases of celiac disease were diagnosed, with a mean age of onset at 22 months and mean age of diagnosis between $4-5$ years old, with no gender predominance. In the clinical presentation, 61 patients had gastrointestinal symptoms (GI), with a deficient weight progression (N =45); abdominal distension $(\mathrm{N}=40)$ and diarrhea $(\mathrm{N}=39) .33$ patients also presented non-GI symptoms, where growth retardation $(\mathrm{N}=25)$ and anemia $(\mathrm{N}=18)$ were the most prevalent.

Analytically all patients were positive for $\mathrm{IgA} / \operatorname{IgG}$ anti-transglutaminase antibodies and/or IgA/IgG anti-gliadin antibodies. All patients, except 3, underwent upper digestive endoscopy with biopsies, whose histopathological analysis was suggestive for celiac disease in 58 patients, being classified according to Marsh criteria. $77 \%$ of patients have a positive genetic test for HLA-DQ2.

Conclusion: The diagnosis of $\mathrm{CD}$ is complex, especially with atypical manifestations. The diagnosis may be established after clinical correlation between symptoms, positive serology, intestinal biopsy and response to gluten-free diet. In recent years, the prevalence of $\mathrm{CD}$ has increased because of the greater degree of suspicion and better accuracy of the serological tests.

\section{6}

\section{Gastroenterology \& Nutrition}

Nutrition Support in Pediatric Patients with Malnutrition Aysegul Alpcan ${ }^{1}$, Serkan Tursun ${ }^{1}$, Hacer Fulya Gulerman ${ }^{2}$

${ }^{1}$ Department of Pediatrics, Kirlkkale University, Faculty of Medicine, Turkey ${ }^{2}$ Pediatric Gastroenterology Hepatology and Nutrition, Kırlkkale University Faculty of Medicine Pediatrics, Turkey

Introduction: Malnutrition is an inadequate or irregular intake of energy, protein and other nutrients that can cause disruption of body structure and function If gastrointestinal function is normal, but oral intake is not sufficient, the food nutritives should be supplemented via oral or enteral route with feeding tube.

Methods:Patients who were admitted to Kurikkale University Faculty of Medicine, Department of Pediatric Gastroenterology between January, 2014 and January, 2019 were included in the study. Patient information was obtained from the records retrospectively using malnutrition and enteral nutrition diagnostic codes.

RESULTS: The study included 111patients. Of the patients, 56(50.5\%) were female. Different enteral feeding products were given to the patients according to their clinical status, diagnoses, and recent weight changes. In addition to the patients diagnosed with malnutrition, 23(20.7\%) had GI system disease, $43(38.7 \%)$ had neurometabolic disease, $12(10.8 \%)$ had endocrinological disease, and $2(1.8 \%)$ had hepatobiliary disease. Nine of the patients $(8.1 \%)$ were fed with percutaneous endoscopic gastrostomy. Of the 111 patients examined, $28(25,2 \%)$ were received standard products, $39(35,1 \%)$ were high fiber, $7(6,3 \%)$ were high energy, and $26(23,4 \%)$ were high energy and high-fiber, $5(4.5 \%)$ hydrolyzed nutrition product, others with special diseases received products suitable for diseases they have. 74 of the patients $(66.7 \%)$ were followed up and 37 of them $(33.3 \%)$ were not participate in follow up with their own requests. Patients with neurometabolic disease used the enteral nutrition product continuously. In other patients, enteral nutrition support was continued or discontinued according to nutrition status and weight gain. 
Results: Enteral nutritional support is a major contributor to the prevention of malnutrition, which is an important problem in the chronic disease process, and for better outcome of the actual treatment and follow-up of the disease. It makes it easier for the physician and caregiver / family who follows the patient to cope with the disease.

\section{1}

\section{Gastroenterology \& Nutrition}

An Adolescent Girl with an Extraordinary Crohn Disease Fulya Gulerman $^{I}$, Burcu Guven ${ }^{I}$, Cansu Celik ${ }^{1}$, Zafer Onaran ${ }^{2}$, Banu Acar Celikel $^{3}$

${ }^{1}$ Pediatric Gastroenterology, Hepatology and Nutrition, Kirikkale University, Turkey

${ }^{2}$ Ophthalmology, Kirikkale University, Turkey

${ }^{3}$ Pediatric Nephrology and Rheumatology, Diskapi Yildirim Beyazit Research Hospital, Turkey

Inflammatory bowel disease (IBD) is a comprehensive term used to describe diseases of the gastrointestinal tract resulting from chronic inflammatory processes. Patients with Crohn's disease, a type of IBD, also may have extraintestinal findings. Joint symptoms and mucocutaneous involvement are the most common manifestations. Therefore, the presence of symptoms such as swollen, painful joints, aphthous stomatitis, erythema nodosum, uveitis, and pericarditis should not be avoided.

A 15-year-old girl was admitted to our outpatient clinic with complaints of severe abdominal pain, nausea, vomiting and bleeding in one eye. The patient was diagnosed with acute pancreatitis and uveitis at the first evaluation, followed by bloody diarrhea and arthritis. In endoscopy, antrum, bulbus and second segment of duodenum were seen as hyperemic, nodular and edematous. In the colonoscopy, mucosa of the terminal ileum was granular and edematous. The cecum, the ascending colon, hepatic flexure, descending colon, sigmoid colon, and rectum mucosa were hyperemic, granular in appearance (cobblestone view) and lymphoid hyperplasic in appearance. The patient was diagnosed as multisystemic Crohn's disease. The patient was followed up with prednisolone and mesalazine treatment. At the end of one-year follow-up, the symptoms of pancreatitis improved totally, but there were no response to the treatment for uveitis and arthritis, and patient developed pericarditis and bloody diarrhea continued occasionally. Infliximab, a biological agent (tumour necrotising factor alpha, TNFa blocker), was the second choice of treatment. The expected result could not be obtained and after 6 cure treatments medication was switched to adalimumab. After 6 cycles of treatment, the patient's symptoms were completely regressed.

In conclusion, it should be kept in mind that extraintestinal manifestations of IBD can occur in pediatric patients before the original signs of the disease occur. The management of these findings with new treatment modalities may result in more positive outcomes in the future.

\section{0}

\section{Gastroenterology \& Nutrition}

All Bunged Up - A Service Evaluation of a Nurse-Led Constipation Clinic

Laura Harrison $^{l}$, Ranya Derrick ${ }^{l}$, Furhana Hussein $^{l}$, Brittany Reid ${ }^{l}$, Kim Gordon', Sue Stevens ${ }^{I}$, Peter Sullivan ${ }^{I}$

${ }^{1}$ Paediatric Gastroenterology Department, Oxford University Hospitals, UK
Background: Constipation is extremely common in children. Up to $30 \%$ of children suffer with constipation, lasting longer than 6 months in 5\%. This constipation clinic was set up to provide specialist constipation management with the aim of treating and discharging all children within 2 years. However a significant number of patients are difficult to manage and remain in the clinic beyond 2 years.

Objective: The aim of this service evaluation was to analyse the characteristics of these children for common features that would allow early identification of potentially difficult cases. Additionally, their management within the clinic was evaluated to see if any improvements would aid more rapid treatment and earlier discharge.

Methods: A search was conducted for patients who had been in the paediatric outpatient constipation clinic for greater than 2 years. Their medical notes were reviewed to identify any features of this population and their management in the clinic that could lead to their ongoing difficulties with constipation.

Results: 91 patients were included in the evaluation, ranging in age from 4 to 16 . Particularly noticeable common features of these patients included soiling associated with constipation in $91.2 \%$, withholding/avoidance of using the toilet in $46.1 \%$ and issues with compliance in $34 \%$. Furthermore they had a significant number of changes to their management (many had over 15 medication changes) in clinic, making compliance more difficult.

Conclusion: Recommendations were made for changes to management including giving the patients constipation management plans to aid patient compliance with medication and SOS appointments for those stable on therapy who may relapse. It is clear that this particular subset of paediatric patients with constipation have ongoing difficulties despite intensive outpatient management and, as they would benefit from more individualised therapy, our ongoing work involves developing a risk assessment tool to identify them early.

\section{5}

\section{Gastroenterology \& Nutrition}

Parents and Physicians Perception of Children's Eating Behavior and Actual Body Mass index in Pediatric Ambulatory Setting -Qatar Manasik Hassan $^{1,2}$, Mohamed Sobhy ${ }^{2}$, Hatim Abdulerhman ${ }^{2}$, Ahmed Alhammadi

${ }^{1}$ Peditaric, Hamad Medcial Corporation, Qatar

${ }^{2}$ Peditaric, SIDRA Medicine, Qatar

Background and Aims: Eating behavior in children's considered part of their growth and development; one those behaviors is picky eater. Our aim is to explore and compare parents and physcinics perception of child picky eater and to determine the correlations between their attitude and actual child BMI

Methods: A cross sectional retrospective study done at Hamad Medical Corporation in general pediatrics clinics. Survey include details of demographic, questions to explore perception of picky eater among parents and physicians, compare relation between truly parents concern and the physicians evaluation for the kids regarding feeding habits and how it is affecting the children growth

Results: A total of (136) questionnaire analyzed; mean age of children's 5 years old. (75\%) of mothers were responsible for feeding and scheduling meals. (58\%) of Parents considering their children as picky eater and have concerns on their growth, poor appetite and not getting optimal vitamins from meals compere to physician`s concern (43\%) p value (0.005). family concern matched low BMI among children's (BMI 
Conclusion: Our study showed that parents are more likely to identify their children as being Picky eater than physicians, there was a good correlation between parent's perception and their child's actual BMI.Both group shared same concerns regarding picky eater behaviors. Understanding family dynamics and mealtime interactions will help in improving parenting feeding styles

\section{7}

\section{Gastroenterology \& Nutrition}

\section{A Case Report of Acute Appendicitis caused by Enterobius} Vermicularis

Georgi Ivanov $^{1,2}$, Nikola Kolev ${ }^{I}$, Anton Tonev ${ }^{1}$, Dilyan Petrov ${ }^{I}$, Georgi Valentinov $^{I}, A$ Aleksandar Zlatarov ${ }^{1}$, Polina Ivanova ${ }^{3}$, Valentin Ignatov ${ }^{1}$, Krasimir Ivanov ${ }^{I}$

${ }^{1}$ Department of General and Operative Surgery, University Hospital Saint Marina, Bulgaria ${ }^{2}$ Department of Pediatric Surgery, University Hospital Saint Marina, Bulgaria ${ }^{3}$ Medical University of Varna, Bulgaria

Background: Acute appendicitis is an acute inflammation of the vermiform appendix. It is most likely due to obstruction of the lumen of the appendix and rarely can be caused by parasites. For a long time, the association between parasitic infection of the appendix and appendectomy has been widely investigated.

Objective: To describe a rare case of Enterobious Vermicularis parasitic infection presenting as a standard appendicitis without any clinical indications of parasites. This study aims to highlight the importance of parasitic infections as a deferential diagnosis of a very common condition.

Methods: We present a case report of a 11-years old girl with acute appendicitis caused by parasites. There were no signs of parasitic infection: her eosinophils were at normal range; the CRP protein levels were normal. There were no clinical symptoms as well indicating that it was a parasitic infection of the appendinx. During the laparoscopy, in the appendix were found alive helminths from the species Enterobius Vermicularis which were supposed to be the origin of the inflammation. Results: The results of this study indicate that Enterobious Vermicularis is a rare etiological cause of acute appendicitis. The presence of this parasite can lead to serious changes in the appendix ranging to life-threatening complications.

Conclusion: Parasites are still present inffections and may cause serious inflamation even without any clinical indications. Due to that parasitic infection should be considered in any differential diagnosis of a common appendicitis.

\section{2}

\section{Gastroenterology \& Nutrition}

Bone Health in Adolescents with Anorexia Nervosa: Comparison of $\mathrm{Z}$ score Bone Densitometry with Antropometry and Serum Leves of Osteocalcin and P1NP

Pavel Kabicek $^{I}$, Pavel Kabicek ${ }^{I}$, Jiri Behounek ${ }^{I}$, Klara Borecka $^{l}$, Katarina Jassova', Milan Bayer ${ }^{1}$

${ }^{1}$ Thomayer Hospital Prague - Krč, Pavel Kabicek, Czech Republic

We describe a group of 16 adolescents with anorexia nervosa (AN). We performed bone densitometry and examined serum levels of osteocalcin and $\mathrm{N}$-terminal procollagen 1 (P1NP) peptide. Anthropometric data and a history of the disease were evaluated. The disease lasted for an average of 7.5 months, the age of adolescents was 14-16 years. Amenorea lasted 512 months, twice primary amenorrhea was present. The weight loss was 5-36 kg. Examination of sex hormones has produced findings that are consistent with central hypogonadism. Estradiol was 6 times below 18.4 pmol / L, LH was 5 times below $0.1 \mathrm{U} / 1$. Densitometric examination yielded below average $Z$ scores, in 4 girls the values were below 2 sigma $\mathrm{Z}$ score. The most pronounced changes were in the $\mathrm{Z}$ score of the spine (mean -0,879), the mean Z-score of the femoral neck was -0,518, and the TOTAL Z score was - 0,66 . Both osteocalcin and P1NP values were below average, with two girls achieving osteocalcin less than 2.5 percentile for a given age, and P1NP reached less than 2.5 percentile for a given age in four girls. We will monitor the densitometry for 1 and 2 years after the first examination and compare it with serum osteocalcin and P1NP values and we will try to suggest a treatment scheme for secondary osteoporosis in adolescents with AN. The work is supported by a Thomayer hospital grant: MZd 2018 č.2 RVO-TN/2018: DZ 1816

\section{8}

\section{Gastroenterology \& Nutrition}

Developmental and Behavioral Follow-up at Age 2 Years of Preterm Children Supplemented with Docosahexaenoic and Arachidonic Acid at Age 1 Year: The Omega Tots Trial

Sarah Keim $^{1,2,3}$, Kelly Boone ${ }^{4}$, Krista Pattison ${ }^{5}$, Taniqua Ingol ${ }^{2}$, Grace $\overline{\text { Pelak }^{2}, \text { Kelly Sheppard }}{ }^{2}$, Mary Ann Nelin ${ }^{6}$, Mark Klebanoff ${ }^{1,3,6,7}$, Joseph Rausch $^{1,2}$, Keith Yeates ${ }^{8,9}$, Abigail Norris Turner ${ }^{3,10}$, Lynette Rogers ${ }^{1,6}$

${ }^{1}$ Pediatrics, The Ohio State University College of Medicine, USA

${ }^{2}$ Center for Biobehavioral Health, Nationwide Children's Hospital, USA

${ }^{3}$ Epidemiology, The Ohio State University College of Public Health, USA

${ }^{4}$ Crane Center for Early Childhood Research and Policy, The Ohio State University, USA

${ }^{5}$ Pediatrics, Pennsylvania State University College of Medicine, USA

${ }^{6}$ Center for Perinatal Research, Nationwide Children's Hospital, USA

${ }^{7}$ Obstetrics and Gynecology, The Ohio State University College of Medicine, USA

${ }^{8}$ Alberta Children's Research Institute, University of Calgary, Canada

${ }^{9}$ Hotchkiss Brain Institute, University of Calgary, Canada

${ }^{10}$ Internal Medicine, The Ohio State University College of Medicine, USA

Background: Toddlers consume low amounts of docosahexaenoic acid (DHA) post-weaning. The Omega Tots randomized, blind, placebocontrolled trial of children born at

Objective: To determine whether daily DHA and arachidonic acid (AA) supplementation at age 1 year improves development and behavior at age 2 years among children born preterm.

Methods: Omega Tots participants were followed up at 26-35 months' chronological age to assess longer-term outcomes. Parents remained blind to treatment assignment and completed the Developmental Profile-3 (global cognitive); MacArthur-Bates Communicative Development Inventory (expressive language); Behavior Rating Inventory of Executive Function (BRIEF-P); Child Behavior Checklist 1.5-5 ADHD, PDD, attention; and reported diagnoses. Analyses were intent-to-treat. Mixed models compared treatment groups, controlling for clustering and baseline scores whenever possible. Sub-group effects for language by birthweight and for executive function by income were explored because adverse effects were observed in the original trial.

Results: Parents of 261/328 (80\%) eligible children participated. No benefits of supplementation were observed at age 2 (see Table). The $\mathrm{DHA}+\mathrm{AA}$ group had lower standard cognitive scores than the placebo group, but this was not statistically significant (median=107 vs 114 , $P=0.28$ ). The DHA+AA group was twice as likely to have a current developmental/behavioral diagnosis $(\mathrm{OR}=2.02,95 \% \mathrm{CI}: 1.01,4.05)$. In exploratory sub-group analyses, children with birthweight $\mathrm{P}=0.03$, effect size $=-0.44)$. Effects observed in the original trial on executive function by income were no longer present. 
Conclusion: Long-term, robust assessment is needed to clarify the longterm effects of DHA+AA supplementation in toddlerhood for children born preterm.

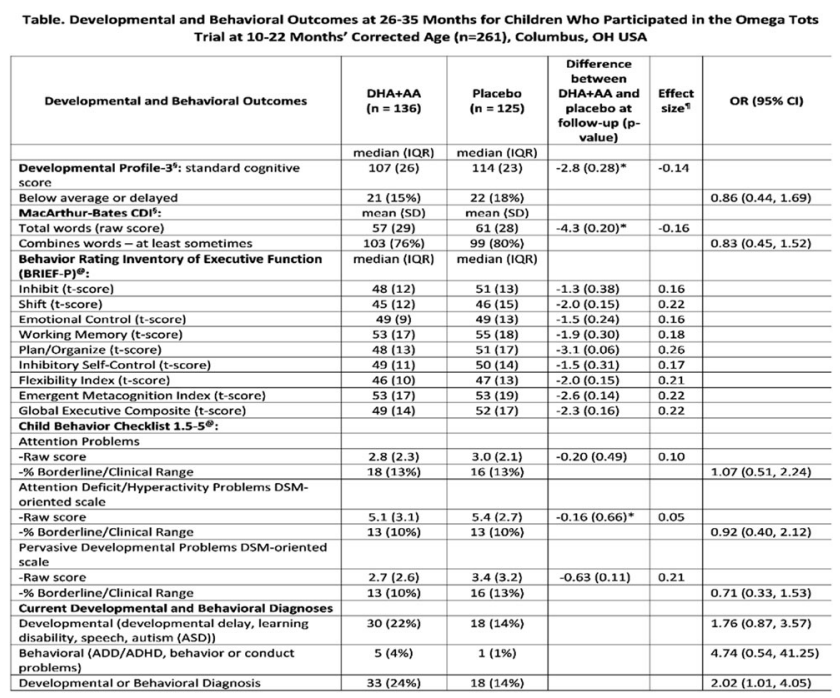

shigher scores indicatemore arvect

Thigher scores indicate greater deficits or behavior problems

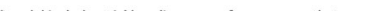

979

Gastroenterology \& Nutrition

A Case of CLD Diagnosed In a Newborn After Surgical Treatment of Ileus Jihye Kim ${ }^{1}$, PilSang Jang ${ }^{1}$

${ }^{T}$ Department of Pediatrics, College of Medicine, The Catholic University of Korea, South Korea

Background: Congenital chloride diarrhea (CLD) is a rare autosomal recessive genetic disorder. The main symptom is osmotic diarrhea with high chloride content due to the mutations in solute carrier family 26 member 3 (SLC26A3) gene encoding the intestinal $\mathrm{Cl}^{-} / \mathrm{HCO}_{3}{ }^{-}$exchanger. In Korea and other East Asian countries, only a couple of cases have been reported. Case report: The patient was born at 34 weeks and 5 days of gestation and had a birthweight of $3.13 \mathrm{~kg}$. He had a history of polyhydramnios and a noticeable dilated fetal bowel loops before birth. On day 1 of birth, the patient exhibited severe abdominal distension, vomiting, and biliary reflux. Because of suspected ileus, surgery was performed, and the surgical findings were ileus caused by Meckel's diverticulum and consequent band formation. Wedge resection (diverticulum resection) of the ileum was performed, and the patient was discharged from the NICU with reduced symptoms. After discharge, however, he was hospitalized several times with intermittent postoperative abdominal distension and metabolite imbalances. The patient, a 5-month-old male infant, was admitted with repeated vomiting and abdominal distension, accompanied with watery stool confused with urine. We suspected CLD and measured chloride level in stool. Watery stool was collected using a rectal tube, and the chloride level was $137 \mathrm{mEq} / \mathrm{L}$, confirming excessive chloride in watery stool. On genetic testing, we confirmed SLC26A3 gene mutation, and CLD was diagnosed. Discussion: This case report describes an infant who initially underwent surgical treatment for ileus induced by Meckel's diverticulum and congenital band. Even after surgery, the patient continued to exhibit abdominal distension. The initial suspicion should be made on prenatal ultrasound, and patients with severe abdominal distension and chronic diarrhea should be tested for the possibility of CLD. Stool examination confirmed high-chloride content diarrhea and genetic testing led to a final diagnosis of CLD.
136

Gastroenterology \& Nutrition

An Audit of Maintenance Intravenous Fluid Therapy in the Paediatric General Wards at Mater Dei Hospital, Malta

Thomas Callejal, Jamie Grech ${ }^{1}$, Iryna Kononenko ${ }^{1}$, Choon Yee Wang ${ }^{1}$, Nikita Taliana ${ }^{l}$, Raymond Parascandalo ${ }^{I}$

${ }^{I}$ Paediatrics and Child Health, Mater Dei Hospital, Malta

Intravenous fluids are one of the most frequently prescribed drugs in hospital, and yet the practice continues to fall short of National Institute for Health and Care Excellence (NICE) guidelines. Paediatric patients are particularly vulnerable to complications of intravenous fluid therapy. We compare current practice in intravenous fluid prescription in the paediatric general wards at Mater Dei Hospital, Malta, to the standard as stipulated by the NICE guidelines. We show how standards of intravenous fluid prescription and subsequent monitoring by staff falls shorts of NICE guidelines, and make recommendations for improvement. Interventions were made to make staff aware of ongoing issues. A second audit cycle is ongoing.

\section{7}

\section{Gastroenterology \& Nutrition}

Complaints, Endoscopic and Histopathological Findings in Patients with Helicobacter Pylori Infection and their Correlation with Each Other

Gunsel Kutluk ${ }^{1}$, Esra Polat ${ }^{1}$,Nermin Gunduz ${ }^{2}$

${ }^{T}$ Pediatric Gastroenterology, University of Health Sciences, Istanbul Kanuni Sultan Suleyman Education and Research Hospital, Turkey

${ }^{2}$ Pathology, University of Health Sciences, Istanbul Kanuni Sultan Suleyman Education and Research Hospital, Turkey

Objectives and study:There is no significant clinical state indicating H.pylori infection in pediatric population.In this study, most common complaints,endoscopic and histopathological findings in children with H.pylori infection were evaluated and their correlation with each other was explicated.

Methods:Patients between 3-18 years,applied to the hospital with dyspepsia,epigastric pain,reflux symptoms, anemia and suspected gastrointestinal(GI)bleeding and underwent upper GI endoscopy were enrolled in this study.Gastric antrum and corpus biopsies were performed in all patients during endoscopy for rapid urease test and histopathological examination.Sydney classification was used to evaluate H.pylori intensity,activity of gastritis,chronic inflammation,atrophy and intestinal metaplasia.Demographical properties, complaints,endoscopic findings and Sydney scores of the patients were recorded.

Results: A total of 339 patients(183 female)were enrolled in the study.The most common complaints were dyspepsia and epigastric pain. $90 \%$ of the patients had antral pathology, $78 \%$ of which was nodularity.Relation between dyspepsia, epigastric pain and antral nodularity is found to be statistically significant.In histopathological examination, intensity score of H.pylori is found to be increasing with the age.Rapid urease test was positive in $90.2 \%$ patients.Relation between rapid urease test and the intensity of H.pylori was statically significant.Relations between detected morphological changes in the antrum and the intensity of H.pylori,activity and chronic inflammation scores were statistically significant.Intestinal metaplasia(IM)and gastric atrophy(GA)was present in $7.1 \%$ and $6.2 \%$ of the patients respectively.No relation was found between IM or GA with the intensity of H.pylori, endoscopic findings and complaints.

Conclusion: The most common complaints of children with H.pylori infection were epigastric pain and dyspepsia.There were significant relations with this complaints,antral morphological changes and with the intensity of H.pylori, and the intensity of H.pylori, increases with age.Since these complaints were related with antral morphological changes and these changes 
were related with activity of gastritis,chronic inflammation and increased intensity of H.pylori, early eradication can be recommended in these children in order to prevent from long term complications.

\section{0}

\section{Gastroenterology \& Nutrition}

Functional Features of the Duodenum in Children with the Syndrome of Bacterial Overgrowth in the Small Intestine

V.P. Novikova ${ }^{I}$, M.M. Guroval, A.P. Listopadova ${ }^{l}$, O.M. Tsekh ${ }^{l}$, E.Yu. Kalinina $^{2}$, E.A. Orishak ${ }^{3}$, A.N. Petrovsky ${ }^{2}$

${ }^{1}$ Laboratory of Medical and Social Problems in Pediatrics SIC, St. Petersburg State Pediatric Medical University, Russia

${ }^{2}$ Department of Pathological Anatomy, North-Western State Medical University named after I.I. Mechnikov, Russia

${ }^{3}$ Department of Medical Microbiology, North-Western State Medical University named after I.I. Mechnikov, Russia

Objective: To study the relationship between the functional state of the duodenum and its microbiocenosis in patients with duodenitis associated with Helicobacter pylori (HP) infection.

Materials and Methods: In our study, we included 60 adolescents aged 14 to 18 years with morphologically proven duodenitis. We detected HP by PCR in feces. Bacteriological examination of the duodenal contents extracted during endoscopy via the endoscope biopsy channel was performed. The activity of enzymes in duodenal biopsy specimens was determined according to the method of A.M. Ugolev and N.M. Timofeev. Results: It was revealed that helicobacteriosis without syndrome of intestinal bacterial overgrowth (SIBO) did not affect the activity of the brush border enzymes. In case of combination of helicobacteriosis and SIBO we found it negative correlation with the activity of lactase $(r=-0.489, p 0.05)$ and aminopeptidase $\mathrm{M}(\mathrm{r}=-0.487, \mathrm{p} 0.05)$. Combination of helicobacteriosis and giardiasis had a negative correlation with the activity of lactase $(r=-0.33, \mathrm{p} 0.05)$ and glycyl-L-leucine dippeptidase $(r=-0.324$, $\mathrm{p}$ 0.05), whereas the combination of helicobacteriosis with SIBO and giardiasis reduced the activity of all three enzymes: lactase $(r=-0.432, \mathrm{p} 0.05)$, aminopeptidase $\mathrm{M}(\mathrm{r}=-0.461, \mathrm{p} 0.05)$ and glycyl-L-leucine dippeptidase $(\mathrm{r}$ $=-0.374, p 0.05$ ). We have identified microorganisms that have a negative effect on the lactase activity: Actinomyces $(r=-0.318, \mathrm{p} 0.05)$, Staphylococcus species $(\mathrm{r}=-0.416, \mathrm{p} 0.05)$, Pseudomonas aeriginosa $(\mathrm{r}$ $=-0.435, \mathrm{p} 0.05)$ Peptococcus species $(\mathrm{r}=-0.455, \mathrm{p} 0.05)$, Candida albicans $(\mathrm{r}=-0.346, \mathrm{p} 0.05)$, Enterococcus faecalis $(\mathrm{r}=-0.426, \mathrm{p} 0.05)$. We found a positive correlation between the presence of lactobacilli in the small intestine and the activity of aminopeptidase $\mathrm{M}(\mathrm{r}=0.341, \mathrm{p} 0.05)$.

Conclusions: The microbiocenosis of the duodenum in adolescents with HP-associated duodenitis affects the activity of the brush border enzymes.

\section{5}

\section{Gastroenterology \& Nutrition}

Battery Ingestion in Children: A Pediatric Tertiary Care Centre Experience

Cristina Lorenzo $^{I}$, Sara Azevedo ${ }^{I}$, Ana Paula Mourato ${ }^{l}$, Helena Loreto ${ }^{l}$, Miroslava Gonçalves ${ }^{2}$, Ana Isabel Lopes ${ }^{l}$

${ }^{I}$ Pediatric Gastroenterology Unit, Department of Pediatrics, Hospital de Santa Maria-Centro Hospitalar Universitário de Lisboa Norte, EPE, Portugal

${ }^{2}$ Pediatric Surgery Unit, Department of Pediatrics, Hospital de Santa Maria-Centro Hospitalar Universitário de Lisboa Norte, EPE, Portugal

Background: Battery ingestion is a frequent problem in pediatric population and can lead to potentially serious complications, especially when located in the esophagus.

Objective: Describe battery ingestion cases requiring endoscopic removal and their complications.
Methods: Retrospective descriptive study of admissions for battery ingestion requiring endoscopic removal in a pediatric emergency department of a tertiary hospital (January 2009/December 2018).

Results: 29 cases, with an increasing number in the last four years (2018: 9 cases). Median age 26 months ( 8 months- 9 years), 52\% male. Witnessed ingestion in $24(83 \%)$ patients; $16(41 \%)$ patients were symptomatic, vomiting $(21 \%)$ was the most frequent symptom. On the X-ray, $8 / 29$ patients $(28 \%)$ had an esophageal battery (EB) and 21/29 (72\%) had a gastric battery (GB). The upper endoscopy revealed the battery (100\% button lithium batteries) located in the esophagus (8), stomach (15), duodenum (1) or was not detected (5); median time until removal of EB was 21 hours (6h-21 days). All patients with EB presented with lesions of different severity: erosion (8), ulceration (6), necrosis (5) and perforation (2); 3 patients presented more than one lesion. Of all the cases of GB only a minor erosion was described. There were no cases of mortality reported.

Patients with EB had a more complicated clinical evolution, with prolonged hospital stay than GB [median 26 days (6days-80days) vs 14 hours (8hours-6days)] and prolonged follow-up [median 3 months (1month-8year)] vs 1.25 month (1month-1.5month)]; $2 / 8$ (25\%) had severe stricture after ingestion and are currently in endoscopic dilation program; one of them is being fed by gastrostomy.

Discussion: There has been an increasing number of battery ingestion in recent years. Esophageal batteries remain responsible for persistent severe lesions, mostly in patients with late removal. This study emphasizes the importance of primary prevention.

538

\section{Gastroenterology \& Nutrition}

Meta-Analysis of 196 Articles: Fatty Acid Supply of Expecting Women during Pregnancy and at Delivery in Erythrocyte Membrane Phospholipids

Tamas Marosvolgyi ${ }^{1}$, Aniko Konig-Peter ${ }^{1}$, Tamas Decsi ${ }^{2}$, Eva Szabo $^{3}$

${ }^{T}$ Institute of Bioanalysis, University of Pecs, Hungary

${ }^{2}$ Department of Paediatrics, University of Pecs, Hungary

${ }^{3}$ Department of Biochemistry and Medical Chemistry, University of Pecs, Hungary

Background: Long-chain polyunsaturated fatty acids are important for the maturation of the developing nervous system. As fetal fatty acids originate from maternal stores, the fetal as well as the neonatal supply are determined by the maternal fatty acids.

Objective: To systematically review available data on fatty acid composition of plasma and erythrocyte membrane lipids in expecting women during pregnancy and at delivery.

Methods: We performed electronic literature search in July 2017 on Cochrane Library, Embase, and Ovid Medline with a search strategy including the following search expressions: (pregnant* OR gestation* OR deliver*) AND (arachidonic OR docosahexaenoic) NOT animal. We analysed data from clinical trials investigating fatty acid composition of plasma or erythrocyte membrane lipids in healthy expecting women without any fatty acid supplementation during pregnancy. Statistical analysis of fatty acid data was performed by Comprehensive Meta-Analysis v3.0 Software.

Results: After excluding duplicates there were 3351 potentially relevant articles. We excluded 3155 articles because they didn't meet our inclusion criteria. 196 relevant articles remained that published fatty acid data of plasma or erythrocyte membrane lipids, from them 19 articles published data of erythrocyte membrane phospholipids. Only 7-9 studies investigated the fatty acid composition of erythrocyte membrane phospholipids during pregnancy, while at delivery there were 13 articles publishing fatty acid data. Values of arachidonic acid remained quite stable in 
the course of pregnancy as well as at delivery. In contrast, values of docosahexaenoic acid were significantly lower during the $1^{\text {st }}$ trimester than in later timepoints and then it remained quite stable (Table)

Table: Mean values with $95 \%$ confidence interval $(95 \% \mathrm{Cl})$ of arachidonic acid $(\mathrm{AA})$ and docosahexaenoic acid (DHA) during pregnancy and at delivery

\begin{tabular}{|l|c|c|c|c|}
\hline \multirow{2}{*}{} & \multicolumn{2}{|c|}{ Arachidonic acid (AA) } & \multicolumn{2}{c|}{ Docosahexaenoic acid (DHA) } \\
\cline { 2 - 5 } & mean $(95 \% \mathrm{Cl})$ & $\begin{array}{c}\text { Nr of studies } \\
(\text { Nr of mothers })\end{array}$ & mean $(95 \% \mathrm{Cl})$ & $\begin{array}{c}\text { Nr of studies } \\
(\text { Nr of mothers) }\end{array}$ \\
\hline 1st trimester & $11.58(10.25-12.92)$ & $7(n=168)$ & $4.06(3.38-4.73)^{\mathrm{a}, 0, c}$ & $7(n=168)$ \\
\hline 2nd trimester & $12.41(11.18-13.64)$ & $8(n=576)$ & $5.05(4.47-5.63)^{\circ}$ & $9(n=725)$ \\
\hline 3rd trimester & $13.67(12.43-14.97)$ & $8(n=339)$ & $5.18(4.56-5.80)^{\circ}$ & $8(n=339)$ \\
\hline Delivery & $12.77(11.81-13.73)$ & $13(n=667)$ & $5.20(4.72-5.68)^{6}$ & $13(n=667)$ \\
\hline
\end{tabular}

Superscript letters denote significant differences between two timepoints: $a, b, c: p<0.05$

Conclusions: 1 . Our results indicate that on a population level both docosahexaenoic acid and arachidonic acid status remain quite stable during pregnancy. 2. Our present data can serve as reference values for future fatty acid supplementation studies in expecting women.

\section{3}

\section{Gastroenterology \& Nutrition}

Food Protein-Induced Enterocolitis Syndrome in a Breastfed Infant Miguel Martins $^{l}$, Isabel Azevedo ${ }^{I}$, Nuno Lourenço ${ }^{l}$, Sofia Ferreira ${ }^{l}$, $\overline{\text { Susana Almeida }}^{2}$, Ricardo Ferreira ${ }^{2}$, Carlos Rodrigues ${ }^{1}$

${ }^{1}$ Pediatrics Department, Centro Hospitalar Universitário Cova da Beira, Portugal

${ }^{2}$ Pediatric Gastroenterology Unit, Hospital Pediátrico de Coimbra, Centro Hospitalar Universitário de Coimbra, Portugal

Background: Food protein-induced enterocolitis syndrome (FPIES) is a non-IgE-mediated food allergy that occurs following the ingestion of a triggering food. Symptoms include incoercible vomiting and diarrhea 1-4 hours post exposure, and in severe cases dehydration and shock. This rare condition usually presents in infants younger than 9 months, with cow's milk protein (CMP) as the main trigger. Breastfed infants seem to be protected against CMP-FPIES, although a few cases have been reported with exclusive breastfeeding. Diagnosis is clinical relying on a high suspicion.

Clinical Case: A 2-month-old girl exclusively breastfed (except for single bottle of regular formula at day-1) was admitted with intermittent bloody and mucous diarrhea, significant weight loss and dehydration. Laboratory findings showed hypoalbuminemia, thrombocytosis, mild hyponatremia and metabolic acidosis. Breastmilk was replaced by a semi-elemental formula (SEF), with clinical improvement. Once breastmilk was reintroduced the symptoms recurred. The infant was kept on a CMP free diet with regular growth and no gastrointestinal symptoms. A first oral food challenge (OFC) to CMP was performed around 9 months and was positive. Last $\mathrm{OFC}$ at 36 months remained positive. Diagnosis of FPIES caused by CMP passed through breastmilk was established. Currently, at age 4 , she continues to thrive well under CMP restriction.

Discussion: Assessment of FPIES is difficult since it's an exclusion diagnosis. Our case enlightens the importance of considering this condition whenever typical symptoms ensue, even in exclusively breastfed infants. Misdiagnosis or delayed diagnosis are common and may lead to a critical life-threatening situation.
212

\section{Gastroenterology \& Nutrition}

BL Whaaat? Baby Led Weaning: The Literature and How Families do it

Rita Matos Parreira $^{l}$, Gonçalo Vale ${ }^{1}$, Mariana Simões $^{I}$, Maria São $\overline{\text { Pedro }}^{\text {, Isabel Bretes }}{ }^{1}$, Cristina Didelet ${ }^{I}$

${ }^{1}$ Pediatrics Department, Centro Hospitalar Barreiro-Montijo, Portugal

Background: For the last 10 years Baby Led Weaning (BLW) has gained popularity as an approach to complementary feeding. It's a hot topic on social media, blogs, parenthood books and forums. Parents keep asking about it in routine appointments.

BLW is a feeding approach that advocates bypassing conventional weaning practices, based in spoon-feeding of puréed food, to presenting whole foods to infants over 6 months.

Objective: There are few studies or long-term outcomes, but the number is increasing corroborating the method's popularity and value.

The main purpose of this presentation is to clarify the method, either in its strict form or mixed with the conventional approach, showing its pros and cons, with the ultimate goal of making it easier for the pediatricians to answer parents' questions.

Methods: Summary of BLW's theoretical basis, literature review and examples of its applicability in Portuguese families, based on clinical practice.

Conclusion: The authors pretend to present a plain and practical perspective, based on what is published in literature and share the heterogeneous experience within Portuguese families.

\section{0}

\section{Gastroenterology \& Nutrition}

A Rare Case of Abdominal Distension

Joana Monteiro $^{I}$, Joana Monteiro ${ }^{I}$, Tânia Mendo ${ }^{1}$, Gabriela Reis ${ }^{1}$, $\overline{M a r i a}$ Carlos $^{1}$, Sónia Fernandes ${ }^{1}$, Isabel Lança ${ }^{1}$, Filipa Santos ${ }^{2}$, Aniceta Cavaco ${ }^{I}$

${ }^{1}$ Pediatric, Hospital José Joaquim Fernandes, Portugal ${ }^{2}$ Pediatric Gastroenterology, Dona Estefânia Hospital, Portugal

Background: There are several causes of abdominal distension, elongation and dilatation of the colon (dolichomegacolon) is a rare diagnosis in pediatric age. It may be congenital or acquired; chronic or acute and may lead to intestinal oclusion and perforation of the intestinal wall, resulting in sepsis and risk of death.

Case Report: A 7 month old male child was referred to the paediatric department for abdominal distension, poor weight progression and abundant stools. First child of young non-consanguineous parents (father with esofagogastric reflux) with no hereditary diseases. His birth and psicomotor development were normal. Under exclusive breast milk up to 4.5 months and because of poor weight (15th centil up to 4 months and 3-15th centil since then) starts infant formula. Introduction of gluten at 6 months. Hospitalization for acute gastroenteritis at 3 months. On examination he had a distended abdomen, soft on palpation with tympanism. Blood investigations showed anemia $(\mathrm{Hb} 10,8 \mathrm{~g} / \mathrm{dL})$, ferritin $27 \mathrm{ng} / \mathrm{mL}$ and PCR $0,2 \mathrm{mg} / \mathrm{dL}$. Hepatic and renal function, total protein and ionogram had normal values, as well as his immunoglobulin concentrations. Celiac disease, cow's-milk protein allergy, cystic fibrosis and giardisis were excluded. Upper endoscopy with biopsy (mild degree of esophagitis and gastritis), high resolution manometry, rectoanal inhibitory reflex and sensitivity to rectal distention were normal. Lower gastrointestinal series showed a dolichomegacolon. He was treated with protonpump inhibitor, laxatives, probiotics, high-fiber, lactose-free diet.

Conclusion: The dolichomegacolon justifies multiple recurrencies to the emergency room presented with constipation or intestinal suboclusion and has a difficult treatment. 
325

\section{Gastroenterology \& Nutrition}

Coeliac Disease (CD) and Gastropathy in Paediatric Practice David Croaker ${ }^{1}$, Himasha Nanayakkara ${ }^{2}$

${ }^{1}$ Department of Paediatric Surgery, The Canberra Hospital, Australia

${ }^{2}$ Medical School, University of Notre Dame, Sydney, Australia

Background: Reactive gastropathy is a defensive change in the stomach. In adults, it is caused inter alia by NSAID use and bile reflux. Histological features of reactive gastropathy include foveolar hyperplasia, mucosal congestion and hyperplastic smooth muscle . Reactive gastropathy is associated with various inflammatory conditions.

Objective: The authors sought to characterise the association between reactive gastropathy and coeliac disease in children.

Methods: A retrospective chart review on paediatric gastroscopies conducted between 2003-2018 was used. The data was stored in Excel. All patients with coeliac disease or any gastropathy were identified by histopathology. The presence of gastropathy was noted in those with proven coeliac disease. The presence of bile in gastric contents was also recorded as this could act as a potential confounder.

Results:1779 gastroscopes were reviewed. 1279 results were available for the histopathological diagnosis of the stomach. 437 had reactive gastropathy, a prevalence of $24 \%$. Of those with reactive gastropathy, 143 had confirmed $C D .842$ did not have gastropathy. Of this group, 149 had confirmed coeliac disease. Fisher's exact test showed a significant difference between the results $(\mathrm{p} 0.05)$.

Of the 143 confirmed $\mathrm{CD}$ diagnoses within the gastropathy group, there were 81 cases of gastric contents recorded. In 231 cases recorded overall, there was no association between the presence of bile in the stomach and gastropathy. (NS using Fisher's exact test.) Overall 195 of 294 with CD recorded an abnormal gastric biopsy, 3 of them lymphocytic gastritis. 12 of 294 (4\%) had eosinophilic oesophagitis (EoE).

Discussion: Gastropathy of various types is common at endoscopic pathology. Reactive gastropathy, distinct from lymphocytic gastritis, is common in $\mathrm{CD}$ in paediatrics, and EoE may also be more common than observed background rates. Bile stained gastric contents did not explain the gastropathy, and reactive gastropathy is not confined to $\mathrm{CD}$ patients.

997

\section{Gastroenterology \& Nutrition}

Is This Caroli Disease?

Irena Nikolova $^{1,2}$, Marija Dimitrovska-Ivanova ${ }^{1,2}$, Luiza Doneva ${ }^{1}$, Marija Karagozoval

${ }^{1}$ Department of Pediatrics, Clinical hospital Shtip, Macedonia

${ }^{2}$ Department of Pediatrics, University Goce Delcev Shtip, Macedonia

Introduction: Caroli disease is rare congenital disorder of the intrahepatic bile ducts. It is characterized by dilatation of intrahepatic biliary tree and with renal cysts. Caroli disease is inherited autosomal dominant.

Aim: To present a case of seven months old infant with icterus of the skin and sclera.

Material and methods: Seven month old infant with gray-yellow skin color and fatigue for a month. Positive family history of policystic renal disease and carcionama of the liver. At admittion afebrile, pale, yellow sclera. Abdomen above torax. On palpation with hepatosplenomegaly. From examinations: WBC $-17,07 \mathrm{CRP}=12.9 \mathrm{mg} / 1$. Tot.Bill $=$ 141,5umol/1, Dir. Bill= 93.3umol/1, AST=142U/L, ALT=143U/L, $\mathrm{ALKP}=790 \mathrm{U} / \mathrm{L}, \mathrm{LDH}=415 \mathrm{U} / \mathrm{L}, \mathrm{CK}=330 \mathrm{U} / \mathrm{L}, \mathrm{CA}-19-9=399,7 \mathrm{U} / \mathrm{ml}$.
Abdominal ultrasound showed more cystic formations in the liver that are connected in parts and a larger cyst in the hepatoiduodenal ligament and the liver hilus. The finding goes in addition to Caroli disease. CT scan of abdomen and MRI cholangiopancreatography showed hepatomegaly, cystic and sacular dilated intra and extrahepatic bile pathways. Both kidneys are without signs of obstruction, but with cortical cysts, right in the upper parts and left in the interpolar region with diameter $15 \mathrm{~mm}$. Mild intestinal distension.

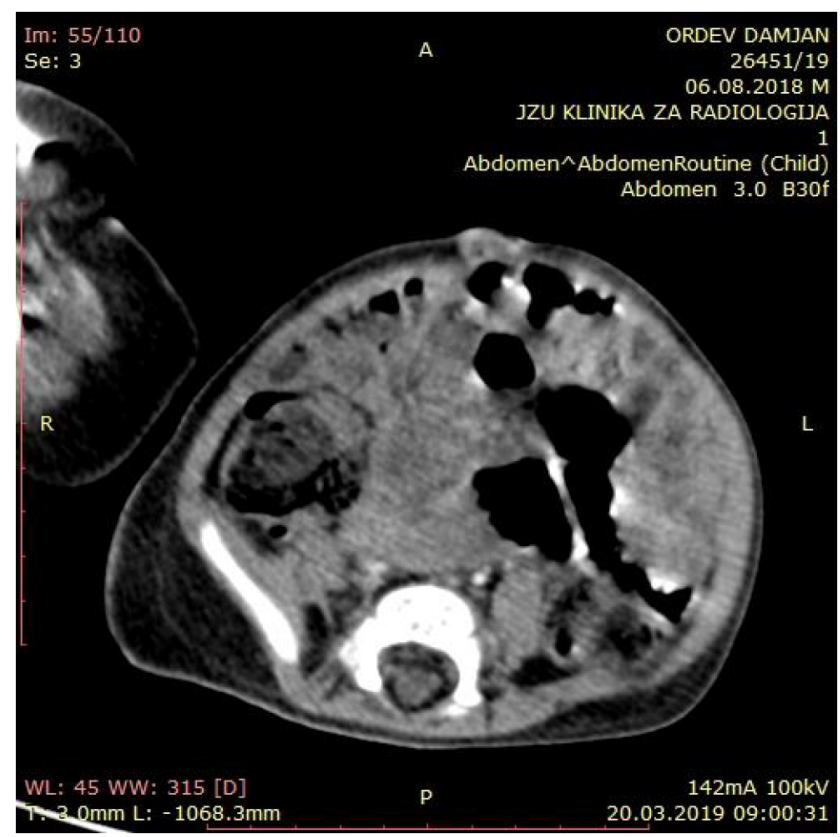

CT scan of abdomen

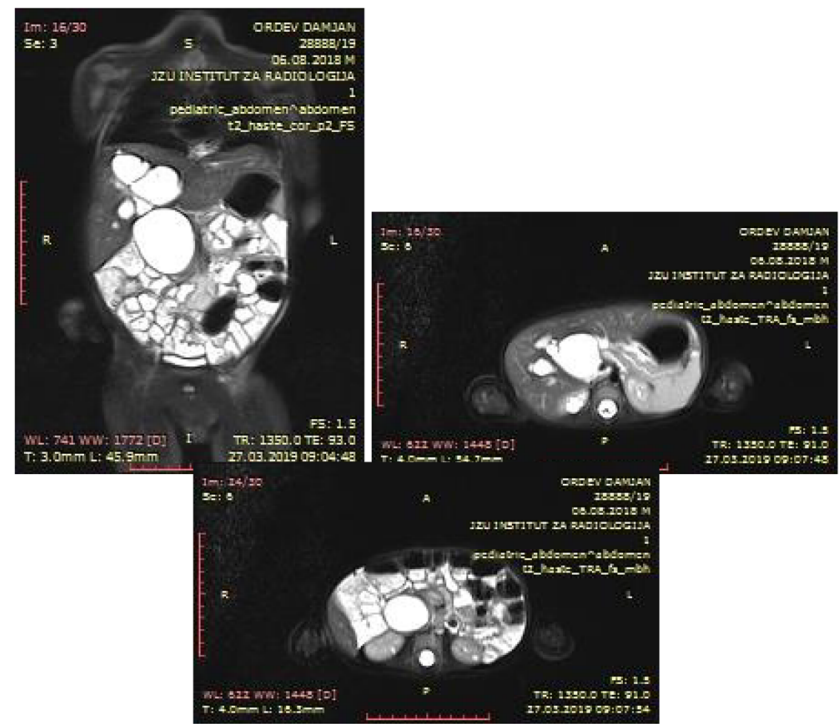

MRI cholangiopancreatography

Results: Child is outpatient followed by a Pediatric gastroenterohepatologyst and Pediatric surgeon. Child recives Ursodeoxycholic acid and the last results with withdrawl of liver enzymes and bilirubin. HLA typisation is planed for liver transplatation in the future. Conclusion: Although this disease is rare, hyperbilirubinaemia of direct type and a positive familial history of polycystic kidney should lead us to think of Caroli disease. 
294

\section{Gastroenterology \& Nutrition}

Late Diagnosis of Right Congenital Diaphragmatic Hernia: Why Didn`t I Think About It Sooner?

Andreea Stefania Nedelcu ${ }^{1}$, Raluca Maria Vlad ${ }^{1,2}$, Daniela Pacurar ${ }^{1,2}$

${ }^{I}$ Pediatrics, "Grigore Alexandrescu”" Emergency Hospital for Children,

Bucharest, Romania, Romania

${ }^{2}$ Pediatrics, "Carol Davila" University of Medicine and Pharmacy, Romania

Background: Diaphragmatic hernia is not a common disease. The severity of symptoms may vary depending on the size of defect and the organs involved. Objectives: The authors aim to raise the awareness regarding this congenital condition as it may not be clinically obvious for a long time.

Methods: We present two cases from the Pediatrics Department of "Grigore Alexandrescu" Hospital, patients with different types of presentation incidentally diagnosed with congenital diaphragmatic hernia after the age of three.

Results: Case 1: 8 year old boy presented with jaundice. The blood tests revealed elevated levels of total bilirubin, normal liver enzymes. The abdominal ultrasound described a liver mass, possibly a vascular malformation. The patient underwent MRI that excluded this supposition. A thoracic $\mathrm{x}$-ray revealed a right, paracardiac, dome-shaped opacity. The diagnosis was right diaphragmatic hernia with liver thoracic dislocation. Case 2: 3 years 6 months old girl is admitted for persistent cough. An antenatal diagnosis of agenesis of the aortic arch had been set. She was born premature, required intensive care during the neonatal period, one diagnosis being right pulmonary hemorrhage. Postnatal ultrasound examination diagnosed venous duct anomaly (hepatic veins draining in the right atrium). She had a good clinical course with normal growth, cardiac and liver functions. The clinical examination was unremarkable. A thoracic $\mathrm{x}$-ray described homogeneous, well-defined opacity in the in the right lower pulmonary lobe. She underwent antibioticotherapy for pneumonia (7 days) with no change of the thoracic image. Abdominal ultrasound confirmed complex malformation of the venous duct (azygos continuation of the inferior vena cava). Thoracic computed tomography demonstrated Morgagni's hernia with liver thoracic dislocation.

Conclusion: Diaphragmatic hernia is a challenging diagnosis to be made. Although a congenital condition, it may be asymptomatic for a long time and have many different clinical "faces" at presentation.

\section{4}

\section{Gastroenterology \& Nutrition}

Preventive Treatment of Various Complications in the Management of Short Bowel Syndrome and Gastrointestinal Neuromuscular Diseases

Dionisiy Petrov ${ }^{2}$, Iuliia Aver'ianova ${ }^{1}$, Sergey Makarov ${ }^{1}$, Elena Zhelezoglo, Anastasia Ermolaeva

${ }^{1}$ Department of Pediatric Surgery, Russian Children's Clinical Hospital of Ministry of Health of Russia, Russia

${ }^{2}$ Department of Pediatric Surgery, Pirogov Russian National Research Medical University, Russia

Aim: To demonstrate the approach for prediction and management of different complications of chronic intestinal failure (IF) caused by short bowel syndrome (SBS) and gastrointestinal neuromuscular diseases (GINMD).

Methods: 58 patients with SBS and 44 with GINMDs aged 1 month to 17 years have been treated in the Department of Pediatric Surgery at Russian Children's Clinical Hospital. We provide a full range of non-transplant options for IF management: home parenteral nutrition (HPN) program, autologous intestinal reconstructions (AIR), absorption-enhancing drug therapy etc. The most common complications included: catheter-associated bloodstream infections (CABSI) $(33,3 \%)$, invasive Candidemia $(20,4 \%)$, Intestinal Failure Associated Liver Disease (IFALD) (4,9\%), metabolic bone disease $(24,5 \%)$, small intestinal bacterial overgrowth (SIBO) and translocation $(11,8 \%)$, recurrent D-lactic acidosis $(5,9 \%)$.

Multiple measures are being made to predict and prevent these complications. Thorough training of parents on central line care and PN administration, routine use of antibacterial locks have helped to minimize CABSI. The use of new-generation lipid emulsions and choleretic agents allowed to reduce the number of IFALD. For metabolic bone disease the total serum $25(\mathrm{OH}) \mathrm{D}$ level and the level of trace elements are being monitored; parenteral Vitamin D and its active metabolites, biphosphonates and calcium/ phosphorus oral supplements are prescribed in accordance with clinical presentation. A combination of pre- and probiotics with rifaximin is used to manage SIBO and recurrent D-lactic acidosis.

Results: SBS patients who underwent AIR revealed a statistically significant dynamics of a decrease in the number of catheter-associated complications, IFALD, invasive Candidemia. In patients with severe GINMDs with recurrent intestinal obstructions we put G-tube and create a T-shaped enteroenteroanastomosis which allows to improve gas evacuation and reduce SIBO.

Conclusions: patients with SBS and GINMDs require a multidisciplinary approach. Prediction and prevention of complications improve the quality of life and shows a great economic value.

\section{0}

\section{Gastroenterology \& Nutrition}

Knowledge, Attitude, and Intention of Breastfeeding among Male and Female University Students

Hadia Radwan, Mona Hashim, Sara Mustafa, Sarah Idris, Layan Al Shalabi, Noor Madan Madan

${ }^{I}$ Clinical Nutrition and Dietetics, University of Sharjah, United Arab Emirates

Background: Breastfeeding is an important public health strategy for improving short-term and long-term health consequences of the mothers and their infants. Paternal breastfeeding knowledge and intention may influence the maternal choice of breastfeeding.

Objective: The purpose of this study was to examine university undergraduate females' and males' knowledge, attitudes and intention toward breastfeeding.

Methods: A cross-sectional study was conducted among 492 university students included 300 female and 192 male. A questionnaire with sociodemographic information and validated scales to explore: Exposure to breastfeeding, knowledge about Breastfeeding, Iowa Infant Feeding Attitude Scale, and Infant Feeding Intentions was administered online to the university students.

Results: The participants' exposure to breastfeeding was considerably high $(89.8 \%)$. There was a highly significant positive correlation between sex and the knowledge and attitude scores $(\mathrm{r}=0.22, \mathrm{r}=0.26, P=0.001)$ where females had more knowledge and positive attitude towards breastfeeding compared to males. Whereas males scored higher on intention scale (P0.001). Furthermore, there was a highly significant positive correlation between knowledge and attitude among the participants ( $\mathrm{r}=0.46, \mathrm{P} 0.001$ ). More than half $(57.5 \%$ ) of the students agreed that breast milk is the ideal food for babies and that breastfeeding is healthier than formula feeding and would increase mother-infant bonding. However, the majority of the participants reported low intention to breastfeed and that they will only formula feed their babies $(75.8 \%)$. There was a highly significant inverse association between knowledge scores and intention $(\mathrm{r}=-0.26, P 0.001)$.

Conclusions: Overall, the findings from this study revealed that although both males and female university students had good knowledge and attitude about breastfeeding, their intention to breastfeed was low. This is alarming and necessitates that educational programs should promote breastfeeding in schools and universities in order to increase awareness and knowledge about the short term and long term benefits of breastfeeding 
586

\section{Gastroenterology \& Nutrition}

A Girl with Jaundice in The Emergency Department Gabriela Reis ${ }^{I}$, Tânia Mendo ${ }^{I}$, Joana Monteiro ${ }^{1}$, Maria Carlos ${ }^{1}$, Sónia Fernandes $^{I}$, Ana Fernandes ${ }^{2}$, Ana Isabel Lopes ${ }^{2}$

${ }^{1}$ Serviço de Pediatria, Unidade Local de Saúde do Baixo Alentejo, Portugal ${ }^{2}$ Unidade de Gastrenterologia Pediátrica, Departamento de Pediatria, Hospital de Santa Maria - Centro Hospitalar e Universitário de Lisboa Norte, EPE, Portugal

Background: Autoimmune hepatitis (AIH) is an inflammatory liver disease, characterized by circulating autoantibodies, increased IgG levels and liver biopsy with parenchymal inflammation. In the absence of early treatment, it may progress to cirrhosis and liver failure. The acute clinical presentation with asthenia, abdominal pain, anorexia, vomiting, jaundice and headache, occurs in $50 \%$ of patients.

Case Report: Previously healthy 11 -year-old girl, observed in the emergency department for fever (temperature $38.6^{\circ} \mathrm{C}$, every 6 hours), cough, rhinorrhea, frontal headache, weariness and anorexia for 8 days. No relevant personal, family or medical history. No history of recent infections. She had subicteric sclerotics, with no other relevant findings. Blood tests showed mild elevation of inflamatory parameters (WBC'S $13.160 \mathrm{U} / \mathrm{L} ; \mathrm{N}$ 75.3\%; CRP $1.1 \mathrm{mg} / \mathrm{dL}$ ), cytolysis (AST 433 UI, ALT 708 UI), without cholestasis (FA 264 UI, GGT 82 U/L, Total Brb 1.97mg/dL, Direct brb $0.95 \mathrm{mg} / \mathrm{dL}$ ), LDH $288 \mathrm{U} / \mathrm{L}$ and normal tests for clottig time. Chest x-ray was normal. Abdominal ultrasound showed hepatosplenomegaly. The diagnosis of viral hepatitis was presumed. Due to the absence of severity criteria, she was discharged, and reassessed 24 hours later. From the additional investigation performed, the virulogical study was negative, and Wilson's disease, alpha-1-antitrypsin deficit and hemochromatoses were excluded. Autoimmunity studies showed positive ANA, doubious anti-Dsdna antibodies and positive anti-LKM1 (LC1) antibodies. The patient was transferred to a tertiary Hospital, where underwent liver biopsy, whose result was compatible with autoimmune hepatitis. She started therapy with prednisolone and hyposaline diet, followed by azathioprine, with clinical and analytic improvement.

Conclusion: Although a large group of patients present with thypical symptoms, sometimes the ones that bring them to the ER are not the most common. Therefore, the authors intend to demonstrate the importance of a thorough physical examination and high degree of clinical suspicion, for a good diagnostic orientation.

\section{6}

\section{Gastroenterology \& Nutrition}

Carotenoids Intake and Skin Carotenoids Level in Asthmatic Thai Children

Lakkana Rerksuppaphol ${ }^{1}$

${ }^{7}$ Department of Preventive Medicine, Srinakharinwirot University, Thailand

Background: The prevalence of asthma is a phenomenon dramatically increasing worldwide. Several antioxidant nutrients including carotenoids have been described to inversely correlate with asthma. The majority of studies quantify carotenoids in relation to the amount of food intake rather than direct measurement of tissue carotenoids.

Objective: The study aims to assess carotenoids intake and skin carotenoids levels in asthmatic children compared to control group.

Methods: A cross-sectional school-based study was conducted in 423 schoolchildren, aged 3.5-17.8 years, in one school at Ongkaluck district, a rural region in central Thailand. Asthmatic children were diagnosed accordingly to Health Interview for Asthma Control questionnaire. Skin carotenoids level was measured with Raman spectroscopy. Demographic data were obtained by directly interviewing children and their parents, whereas anthropometric parameters were measured by trained staff. The intakes of carotenoids were evaluated by a food frequency questionnaire. Pearson chi-squared test and Student's t-test were used to compare categorical and continuous variables between asthmatic and non-asthmatic children, respectively. Correlations between skin carotenoids level and carotenoids intake were measured by Pearson's correlation test.

Results: Seventy three children (17.3\%) had diagnosed asthma. There were no significant differences in term of anthropometric parameters, dietary intakes of $\alpha$-carotene ( 904 vs $1,159 \mathrm{mcg} /$ week; $\mathrm{P}=0.65$ ), $\beta$ carotene $(5,232$ vs $5,189 \mathrm{mcg} /$ week; $\mathrm{P}=0.60), \beta$-cryptoxanthin $(1,028$ vs $1,045 \mathrm{mcg} /$ week; $\mathrm{P}=0.39)$, lycopene $(4,417$ vs $4,951 \mathrm{mcg} /$ week; $\mathrm{P}=0.54)$, lutein and zeaxanthin $(6,077$ vs $6,098 \mathrm{mcg} / \mathrm{week} ; \mathrm{P}=0.54)$ between asthmatic and non-asthmatic children. The mean skin carotenoids level was 12,295.6 RU in asthmatic children and 12,529.6 RU in control group $(\mathrm{P}=0.95)$. Skin carotenoids level significantly positive correlated with all carotenoids intake in a mild to moderate degrees $(\mathrm{r}=0.20-0.42$, P0.05).

Conclusions: Carotenoids intakes and skin carotenoids levels were not associated with of the risk of asthma in Thai children. Skin carotenoids correlated with all carotenoids intake in a mild to moderate degrees. Raman spectroscopy was a useful tool to determine skin carotenoids level.

\section{4}

\section{Gastroenterology \& Nutrition}

\section{Zinc Supplementation in Thai Children with Dengue Fever} Sanguansak Rerksuppaphol ${ }^{l}$

${ }^{T}$ Department of Pediatrics, Srinakharinwirot University, Thailand

Background: Zinc deficiency is common in developing countries and increases the risk for several infectious diseases. Low serum zinc levels have been reported in children with dengue virus infection (DVI).

Objective: To assess the effects of zinc supplementation on DVI outcomes.

Methods: A double blinded, randomized trial was conducted in children with dengue fever (DF)/ dengue haemorrhagic fever (DHF). Bisglycinate zinc or placebo was orally administered 3 times a day for 5 days or until defervescence. Primary outcome was to evaluate the DVI defervescence phase; secondary outcome was to assess hospitalization length, presences of severe DVI and zinc deficiency.

Results: Fifteen children with DHF and 35 with DF were randomized 1:1. Overall prevalence of zinc deficiency was $46 \%$. Serum zinc levels increased from baseline to the end of study: the mean gain was $26.4 \mathrm{mg} / \mathrm{dL}$ (95\% CI: 13.6 to $39.1 \mathrm{mg} / \mathrm{dL}$ ) in the supplementation group and $14.4 \mathrm{mg} / \mathrm{dL}$ (95\% CI: 7.4 to $21.3 \mathrm{mg} / \mathrm{dL}$ ) in placebo group. Mean time of defervescence was not significant different between groups $(29.2 \pm 24.0$ and $38.1 \pm 31.5$ hours; $P$-value $=0.270$ ). Hospital staying was shorter for children with zinc supplementation than for those with placebo (mean difference 22.2 hours, 95\%CI: 5.5 to 38.5 hours; $P$-value $=0.010$ ). No signs of severe DVI were observed in both groups. Zinc supplementation was well tolerated. Conclusion: Overcoming zinc deficiency among Thai children may reduce DF duration and limit the hospitalization, in addition to other advantages that normal serum zinc levels have on overall children health.

514

\section{Gastroenterology \& Nutrition}

Dietary Habits and Body Esteem in Adolescents Survey Ivanna Romankevych ${ }^{1}$, Marina Mamenko ${ }^{1}$, Olexiy Rykov ${ }^{2}$, Stanislava Gaponova $^{I}$, Yuliia Bondarets ${ }^{l}$, Hanna Drokh ${ }^{l}$

${ }^{1}$ Shupyk National Medical Academy of Postgraduate Education, Ukraine $^{2}$ Dobrobut, Ukraine 
Background: There are a lot of studies dedicated to anorexia nervosa in teens, but its general frequency isn't so high. Still, adolescents are risk group for development of imbalanced diet as well as body dysmorphic disorders and low body-esteem. However, nutritional pattern and bodyesteem in adolescents aren't so well established.

The Aim: to evaluate dietary habits and body self-esteem in adolescents. Material and Methods: 36 own design questionnaires, filled in by adolescents by their own. The questioner includes 3 blocks of questions: own evaluation of body composition and weight, dietary habits and adherence to a specific diet.

Results: After analyzing of data we received following results from $53 \%$ (19) boys and 47\%(17) girls with mean age $14.75 \pm 0.46$ years. There were $10.8 \%$ (3) adolescents with underweight, $7.2 \%(2)$ with overweight and $82 \%(31)$ with normal weight. However, all children with underweight considered their weight as normal, 25\%(9) teens with normal weight count their weight as an overweight, but not as obesity. The $83 \%$ (30) of children evaluated body weight of parents as normal. Parents prepared food to all respondents. The 58\%(21) teens consumed food 3-4 times, 25\%(9) 2 times and 8\%(3) 1 time daily. Consuming food without hunger was noted by $58 \%(21)$ of adolescents and eating in front of a TV was found in $75 \%(27)$ cases. There were $66.7 \%(24)$ ordered fast food at least 1 time weekly, 92\%(33) drank soda or sweet beverages daily. However, 92\%(33) and 83\%(30) of children ate fruits and vegetables respectively every day. There were $18(50 \%)$ teens, which follow hypocaloric diet on purpose of weight reduction. We did not have respondents practiced vegetarian or vegan, or other specific diet.

Conclusions: survey showed features of misinterpretation of own weight and body composure, simultaneously with suboptimal dietary habits and adherence to hypocaloric diet in adolescents.

\section{1}

\section{Gastroenterology \& Nutrition}

Utility of Probiotics in Gastroenteritis Treatment in the Paediatric Population

Abidur Rahman ${ }^{1}$, Darolyn Tan ${ }^{1}$, Nnedimma Ozoani ${ }^{1}$, Sarah Koscic ${ }^{1}$, Jia Jun Foo ${ }^{l}$, Craig Duffy ${ }^{I}$, Laken Boochoon ${ }^{l}$, Allison Bell ${ }^{1}$, Eleanor Molloy ${ }^{1}$

${ }^{1}$ Paediatrics, Trinity College Dublin, Ireland

Background: Acute gastroenteritis is the sudden onset of diarrhoea with passage of 3 or more loose stools in a 24 hour period, or the passage of 1 or more bloody stools in the presence or absence of abdominal pain, fever, nausea and vomiting. Acute gastroenteritis is self-limiting lasting 2 weeks or less, however, it is a major cause of morbidity and mortality worldwide. Current primary treatment is restoration of the acid-base balance, correction of electrolyte disturbances, and oral rehydration. The treatment helps to decrease morbidity and mortality, however, it seldom impacts the duration and symptoms of the infection. Probiotics may help reduce both the duration and severity of symptoms of gastroenteritis, however strain specific efficacy and inter-strain comparison has yet to be established.

Objective: This systematic review examines original research and the different strains of probiotics used to determine the efficacy of probiotics in treating acute gastroenteritis in the paediatric population.

Methods: An Embase search was carried out and the articles were screend by two reviewers. All reviewer conflicts were resolved by a third reviewer. Data was extracted from the included articles to determine correlation and effects of probiotics.

Results: 581 results obtained from the search. 11 studies were included for data extraction after applying the inclusion and exclusion criteria. Majority of the studies showed probiotics reduced duration of diarrhoea ( 8 of 11 studies), and duration of hospitalisation ( 6 of 11 studies). 2 papers reported adverse effects, such as fungaemia, in immunocompromised and
ICU patients. The different strains of probiotics that were examined in the selected papers include Saccharomyces boulardii, Lactobacillus casei, and Lactobacillus acidophilus.

Conclusion; Probiotics reduced the duration of diarrhoea symptoms and hospitalization. Usage of probiotics was however accompanied by minimal side effects but not indicated in immunocompromised patients with gastroenteritis. Further research needs to be conducted to determine the strain specific efficacy and dosage requirements for treatment of gastroenteritis using large scale double blinded RCTs.

\section{8}

\section{Gastroenterology \& Nutrition}

Paediatric Inflammatory Bowel Disease from 2008 to 2018: An Expending Problem in our Tertiary Unit Cristina Becheanul, Gabriela Leşanu ${ }^{I}$, Daniela Păcurar ${ }^{I}$, Roxana Smădeanu ${ }^{l}$, Evelina Cerbureanu ${ }^{l}$, Iulia Țincu ${ }^{1}$

'Gastroenterology Department, "Grigore Alexandrescu" Children Emergency Hospital, Romania

Background: The last two decades rapidly risen in the incidence and prevalence of childhood-onset inflammatory bowel disease (IBD) has demanded many research debates. Both developed and developing countries are facing the burden of early onset IBD, although previously it was considered to be rare in children.

Objectives: The aim of our study was to evaluate the characteristics of paediatric IBD patients recorded over a 10 year period, 2008-2018.

Methods: We identified children diagnosed with IBD using the database of „Grigore Alexandrescu” Clinical Emergency Hospital, a tertiary unit, from Bucharest, the capital of Romania.

Results: The total number of IBD paediatric cases investigated in our Department over a 10 years period is 74 . In this interval, 62 patients $(20$ males $/ 42$ females $=0.477$ ) were diagnosed with $U C$ and 12 subjects had $\mathrm{CD}$ ( 7 males $/ 5$ females $=1.4)$. The sex difference was not significant for neither CD nor UC (p0.05). The median age in years was $11.4 \pm 4.83$ for IBD, $11.32 \pm 4.86$ for UC, $11.81 \pm 4.87$ for $\mathrm{CD}$. The median age at diagnosis did not differ between $\mathrm{CD}$ and $\mathrm{UC}(\mathrm{p}<0.005)$, and evaluation regarding sex showed no significant differences between the age at onset in boys and girls $(\mathrm{p}<0.005)$. The mean duration of time between age at first symptoms and diagnosis was $5.02 \pm 7.96,4.96 \pm 8.33$ and $5.33 \pm 6.00$ months for IBD, UC and CD respectively. We report $17.6 \%$ patients with very early onset IBD, while the previous data from 2004 to 2015 showed $33 \%$ being diagnosed before the age of 6 . In the last 3 years we identified 40 newly diagnosed IBD cases with mean age at diagnosis of $13.61 \pm 3.07$ years.

Conclusion: Future studies should investigate the epigenetic factors, such as antibiotics use in the first years of life, feeding patterns and infections, among our paediatric IBD cohort, considering its increasing incidence and changing patterns over time.

\section{3}

\section{Gastroenterology \& Nutrition}

Areas of Action against Later Childhood Obesity: Result of a 5 Years Prospective Study

Cristina Becheanu ${ }^{1}$, Virginia Antoci ${ }^{I}$, Andreea Gârbea ${ }^{l}$, Iulia Tincu ${ }^{l}$

${ }^{I}$ Gastroenterology Department, "Grigore Alexandrescu" Children Emergency Hospital, Romania

Background: Obesity is one of the greatest public health challenges in the world with childhood prevalence rates between 20-26\% and numerous associated health risks. 
Objective: The aim of this study was to analyze a spectrum of factors contributing early in life to overweight and obesity in a Romanian paediatric population, followed from 1 to 6 years.

Methods: Data on breastfeeding, early feeding patterns, individual anthropometric measurements and social characteristics were collected in a population-based prospective cohort study among 382 children. Of the original sample, only $113(29.5 \%)$ subjects participated in the 5-year follow-up.

Results: The distribution according to BMI in terms of underweight, normal weight, overweight/obese children were $11.5 \%$ (13), $74.3 \%$ (84) and $14.2 \%$ (16) respectively, at 12 months old, while the proportions were $3.5 \%$ (4), $73.5 \%$ (83), and $23 \%$ (26) respectively, at the age of 6 . The BMI evolution for 1 year underweight children was to normal weight in $76.92 \%$ cases and to overweight/obesity in $15.38 \%$ subjects, while 1 individual remained underweight. Sixty two per cent $(62.5 \%)$ of the 1 year obese infants were still obese after 5 years. Univariate predictors of BMI in subjects aged 6 were identified as: 12 months BMI ( $\mathrm{r}=0.36$, $\mathrm{p}=000)$, duration of breastfeeding $(\mathrm{r}=-0.45, \mathrm{p}=0.000)$, timing of complementary feeding $(\mathrm{r}=-0.54, \mathrm{p}=0.000)$, amount of meat during weaning $(\mathrm{r}=0.63, \mathrm{p}=0.000)$, while living area, family income, maternal age or education had no impact.

Conclusion: Timing of introduction of solid foods and breastfeeding duration were associated with child obesity at 6 years in our sample. Since reducing obesity is a difficult task once it appeared, preventing interventions should be implemented in the first years of life.

\section{5}

\section{Gastroenterology \& Nutrition}

Recurrent Intestinal Invagination : Atypical Manifestation of Coeliac Disease

Ana Isabel Valdivielso Martnez ${ }^{I}$, Silvia Gallego ${ }^{1}$, Eva Pedrero Segura ${ }^{1}$ ${ }^{T}$ Pediatrics, Primary care Attention, Spain

Background : Intestinal invagination usually presents accutely in infants under 2 years after gastro-intestinal tract infection, when it occurs in older children or with chronic symptomatology, secondary causes must be ruled out such as polips, tumours or rarely, coeliac disease.

Objective: We present here an atypical case of recurrent invagination in a 9 years old girl wih malnutrition.

Methods: Clinical report of a case, clinical and laboratory data have been collected.

Results: 9 years old girl who lived in Morocco with her mother with low socioeconomic status. She used to have pica, failure to thrive and episodes of acute abdominal pain wih distension and vomits. She had no access to healthcare. She was transfered to emergency department in Spain hospital because of abdominal pain, vomiting and bloody diarrhea with ultrasound diagnosis of invagination. During surgery enlarged intestinal lymph nodes were seen and removed for biopsy and the invagination was reducted. Several malnutrition was noticed, with body mass index , Waterlow and Shukla index very low for his age; she presented with no eyelashes and eyebrows thinning (Picture1). In blood test, iron deficiency, low leves of vitamine A, E, B12, zinc and folic acid were measured, and very high levels of anti-transglutaminase antiboides $\operatorname{IgA}(128 \mathrm{U} / \mathrm{ml})$. Duodenal biopsy was carried out and resulted in marked atrophy of villi and increased criptes (Marsh IIIb). After gluten-free diet the patient did not have invagination any more and gained weight.

Conclusion: Although coeliac disease is well-known and easily diagnosed in developed-countries, atypical and severe manifestations can occur when there is no access to medical services in non-developed countries.

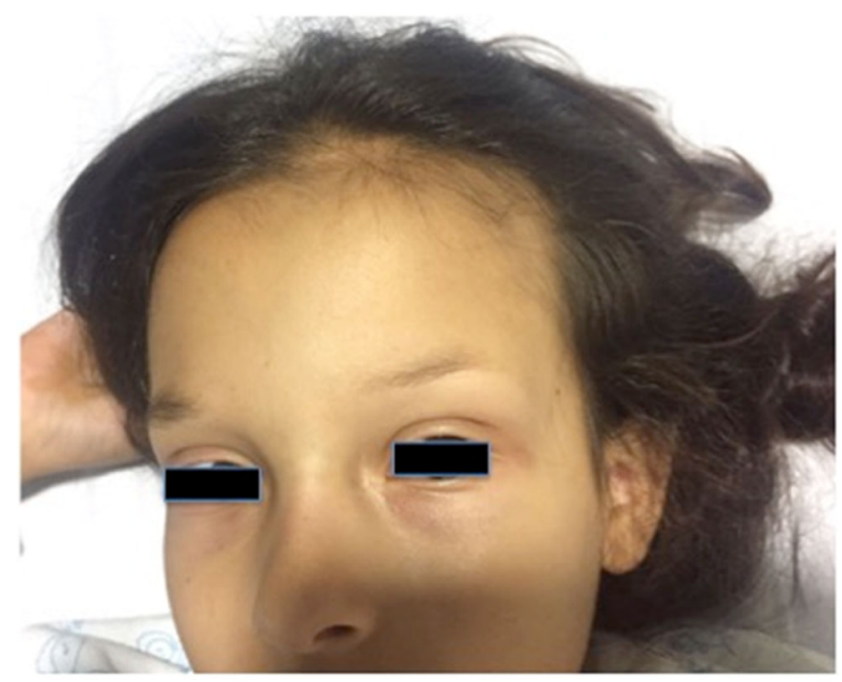

\section{Picture 1}

1005

Gastroenterology \& Nutrition

Severe Iron Deficiency Anemia - Consequences of Excessive Intake of Dairy Products in Children

Goncalo Vale $^{I}$, Rita Matos Parreira ${ }^{1}$, Mariana Simões ${ }^{I}$, Maria São ${ }_{\text {Pedro }}$, Joana Extreia ${ }^{I}$, Sofia Castro ${ }^{I}$

${ }^{1}$ Pediatrics, Centro Hospitalar Barreiro Montijo, Portugal

Background: Iron deficiency can have several clinical consequences such as anemia or short and long-term developmental delay in children. Iron deficiency anemia is multifactorial. It can occur due to problems of intestinal absorption, including excessive intake of dairy products, which interfere with the absorption of iron from other food.

Objective: Remind severe possible drawbacks of excess dairy food in pediatric age.

Methods: Report of a clinical case from a suburban hospital in Portugal.

Clinical Report: Male, two years and eight months old, ex premature with delayed psychomotor development. Gypsy ethnicity, consanguineous parents, family history of Favism. Artificial milk-based feeding since birth due to poor adaptation to breastfeeding and food diversification since eight months of age. Sent to an appointment due to failure to thrive, with all parameters inferior to percentile three according to WHO curves. Prostration and extremely pale mucosa were observed, being decided hospital admission for investigation. Analytical evaluation: Hemoglobin $4.8 \mathrm{~g} / \mathrm{dl}$ (normal 11,5-13,5g/dl), Hematocrit 20.3\% (normal 34-40\%), Iron 19ug/dl (normal 60-170 $\mu \mathrm{g} / \mathrm{dl}$ ), Ferritin1ng/mL (normal 15-300ng/ml); transferrin saturation $4 \%$ (normal 20-50\%); Total capacity fixing iron $503 \mathrm{ug} / \mathrm{dl}$ (normal 250-400 $\mu \mathrm{g} / \mathrm{dl}$ ). Peripheral blood with marked microcytosis and hypochromia, elliptocytes and dacryocytes. Abdominal ultrasound was unremarkable. Blood transfusion was performed and iron therapy was initiated with an improvement of all analytical parameters. During hospitalization, health professionals verified continuous intake of milk, instigated by parents, with detriment and refusal of other food by the child. Parents later admitted that infant feeding is milkbased at all meals.

Conclusion: Excessive milk ingestion is a common cause of iron deficiency in preschool age children. After six months of age, it is necessary to supply iron with complementary food and limit milk intake. Reinforcing this information and stimulating its practice to parents is 
extremely important for the prevention of anemia and its consequences in childhood.

\section{4}

\section{Genetic \& Metabolic}

Contributing Factors to the Profound Incidence of Down Syndrome in the United Arab Emirates and Health Outcomes for Children with Down Syndrome in the UAE

Alya Ahmad ${ }^{l}$, Alya Ahmad

${ }^{T}$ Pediatric, University of California San Francisco at Fresno, USA

Purpose Down Syndrome rates are twice as high in the UAE. It is important to obtain the array of demographic, co-morbidities, and health outcomes of children with Down Syndrome (DS) in the UAE, Down Syndrome Gulf Registry

Methods: After IRB approval, a 3-year retrospective chart review of health data for 178 patients presenting to the DS Clinic were collected and analyzed using SPSS software. Systems-based medical comorbidities associated with Down syndrome and 20 demographic, socio-economic, educational, and maternal age indicators were documented.

A Standardized template of their care plan was incorporated into an EHR system using 2011 AAP DS Health supervision guidelines in a UAE Down Syndrome Clinic. The children with DS were enrolled in the DS registry preventative health and complex care coordination was provided. Results: DS in the UAE is starkly 2-2.5 times greater as global incidence of DS.

Conclusions: Children with Down syndrome living in the UAE share similar characteristics with other populations. However, there are some interesting differences in both demographics and co-morbidity patterns. High rates of parental consanguinity, no prenatal screening, and increasing maternal age maybe a factor in increased incidence. Genitourinary anomalies occur frequently, as do the other commonly described medical co-morbidities. Similar cardiac malformations occur, but AVC is not the most common. Higher co-morbidity rates and surgical are noted in the DS clinics of a tertiary hospitals. Select cases with severe compromise highlight the need to follow medical guidelines to optimize outcomes. Further development of the registry to improve outcomes in sleep apnea monitoring, obesity, and other long-term progressive conditions needs to occur

\section{5}

\section{Poster presentation}

Effect of Pamidronate Therapy in Children with Osteogenesis Imperfecta

Naima Baddouh ${ }^{1}$, L. Bourkhisi ${ }^{l}$, S. Mghar ${ }^{1}$, M. Lagrine ${ }^{1}$, A. Ouayad ${ }^{l}, H$. Nassih $^{I}$, A. Bourrahouat ${ }^{I}$, I. Ait Sab ${ }^{I}$

${ }^{1}$ Pediatrics B Department, Mother and Child Hospital, Mohammed VI University Hospital Faculty of Medicine, Cadi Ayyad University, Morocco

Osteogenesis imperfecta (OI), an inherited connective tissue disorder of remarkable clinical variability, is caused by a quantitative or qualitative defect in collagen synthesis and is characterised by bone fragility. A multidisciplinary team approach is essential for diagnosis, and to tailor treatment needs to the severity of the disease and the age of the patient. Objective: To analyze the clinical and radiological aspects of this condition and to evaluate the response to pamidronate therapy in children with moderate to severe osteogenesis imperfecta.

Methods: This work is a retrospective study with a prospective follow-up of 18 patients, collected at the Pediatrics B department, Mother and Child Hospital, Mohammed VI university hospital, Marrakech, Morocco, during a period spanning $4 \frac{1}{2}$ years, from January 2013 to June 2017 .
Results: clinical presentation was characterized by multiple fractures and deformities in $89 \%$ with $11 \%$ of cases of antenatal diagnosis. Dentinogenesis imperfecta was present in $56 \%$ and blue or gray sclera was found in $94.5 \%$.

All our patients were treated with pamidronate at age-appropriate doses. During the follow-up we found clinical and radiological improvement with a decrease in the fracture rate in all of our patients. Genetic counseling for parents was done.

Conclusion: This study suggests that treatment with pamidronate improves mobility in children with moderate to severe osteogenesis imperfecta and decreases the frequency of fractures.

\section{2}

\section{Genetic \& Metabolic}

How do we Train Clinicians to Integrate Genomics into Paediatrics? Bryony Coupe $^{1,2}$, Maurizio Brotto ${ }^{1,2}$, Rhiannon Clegg ${ }^{1,3}$, Helen Daniels ${ }^{I}$, Jason Griffiths $^{1,4}$, Torsten Hildebrandt ${ }^{1,5}$, Rachel Thomas ${ }^{1,6}$, Ruth Young $^{1,7}$, Claire Morgan ${ }^{1}$

${ }^{1}$ Institute of Life Science, Swansea University Medical School, UK

${ }^{2}$ Singleton Hospital, Abertawe Bro Morgannwg University Health Board, $U K$

${ }^{3}$ Royal Gwent Hospital, Aneurin Bevan Local Health Board, UK

${ }^{4}$ Hywel Dda University Health Board, Withybush General Hospital, UK

${ }^{5}$ Princess of Wales Hospital, Abertawe Bro Morgannwg University Health Board, UK

${ }^{6}$ Wrexham Maelor Hospital, Betsi Cadwaladr University Health Board, $U K$

${ }^{7}$ University Hospital of Wales, Cardiff and Vale University Health Board, UK

Background: Approximately 1 in 17 people is affected by a rare disease in the UK, many of which are genetic in origin. Using DNA sequencing, these and many other conditions will soon be diagnosed antenatally or early in life, having major implications on paediatrics in terms of diagnosis, treatment, and prognosis. Many European governments are investing in Genomic Medicine and paediatricians are well placed to recognise these new opportunities and integrate them into their practice.

Objective: In 2017 the Welsh Government introduced the Genomics for Precision Medicine Strategy, aimed at developing genetics and genomics to improve health and healthcare provision in Wales. To help fulfil this strategy, Swansea University launched an MSc in Genomic Medicine in May 2018, the first University in Wales to provide this opportunity to multi-disciplinary health care professionals, imparting the skills and knowledge necessary to interpret genomic data and understand its impact on patient care.

Methods: Based on the indicative curriculum of Health Education England and Genomics England, this 2 year part-time Masters course integrates lectures, workshops, tutorials, interactive group learning and guest lectures, while encouraging and facilitating workplace learning. Modules include bioinformatics, genomics of common and rare inherited diseases, ethics, techniques and optional modules such as pharmacogenomics. Module assessments are summative and students must complete a dissertation describing an applied genomics project, research, or literature review.

Results: Eight students completed their first year of study in January 2019. A variety of healthcare professionals enrolled (e.g. laboratorybased microbiologists, geneticists, pathologists, oncologists and paediatricians) which fostered collaborative work and learning. The formal and informal feedback to all modules has been excellent, with students ranking all aspects as "highly satisfactory".

Conclusion: The MSc in Genomic Medicine plays a vital role in educating healthcare professionals seeking knowledge and skills in this emerging discipline. 
338

\section{Genetic \& Metabolic}

49XXXXY Syndrome: A Case Report of a Rare Genetic Disorder Sara El Badri ${ }^{1}$, Nuha Elmamoun ${ }^{1}$, Babu Paturi ${ }^{1}$

${ }^{T}$ Paediatrics, Our Lady of Lourdes Hospital, Drogheda, Ireland

Introduction: Boys with 49XXXXY have an additional three X chromosomes, giving them a total of 49 chromosomes. The most common features of 49XXXXY syndrome include: learning difficulties, speech difficulties, hypotonia, underdeveloped sex organs and infertility. The diagnosis can be made by karyotyping in cell samples taken from placental tissue by chorionic villus sampling, or from amniocentesis. CGH array is another method for diagnosis of 49XXXXY.

Case Summary: T.O. is an 11 months old male infant born at $36+1$ weeks' gestation by elective $\mathrm{C} / \mathrm{S}$ for severe IUGR, prematurity and breech. His mother is a 26 years old Irish lady, and she had no antenatal issues. His birth weight was $2.28 \mathrm{~kg}$. He was admitted to NICU after delivery for mild respiratory distress. On examination he was hypotonic and had micropenis. On day of life (DOL) 2 his sodium level was found to be 147 , and continued to be raised until DOL 3 with high serum osmolality. He had a cranial USS on DOL 3 which showed several subependymal cysts in the frontal lobe suspicious for a congenital cyst formation. He also had a renal USS which was normal.

At 4 weeks of age he had an MRI Brain which showed connatal cysts adjacent to the frontal horns of both lateral ventricles.

He was discharged on DOL 16 from the NICU. He had 4 monthly pediatric outpatients follow up as well as physiotherapy follow up.

T.O. had recurrent chest infections requiring admission. On his admission at 8 months of age he had genetic and metabolic work up, and a diagnosis of 49XXXXY was reached. An early intervention referral was made for his persistent central/peripheral hypotonia and his developmental delay. Conclusion: Rare diagnosis like 49XXXXY can often be missed or diagnosed late due to lack of specific features. Features such as underdeveloped genitalia and hypotonia should always raise concerns of underlying cause.

\section{6}

\section{Genetic \& Metabolic}

Hypobetalipoproteinemia: Two Clinical Cases

Ana Esteireiro $^{1,2}$, Blanca Sánchez ${ }^{2}$, Miriam Ortega ${ }^{2}$, Fabiola Guerrero ${ }^{2}$, José Riveró, María Ruiz ${ }^{2}$

${ }^{I}$ Pediatrics Department, Caldas da Rainha Unit, Centro Hospitalar do Oeste, Portugal ${ }^{2}$ Adolescence Unit, Hospital Universitario La Paz, Spain

Background: The familial hypobetalipoproteinemia (FHBL) belongs to a heterogeneous group of monogenic diseases characterized by plasma levels of LDL cholesterol and apolipoprotein B (apoB)

Clinical Cases: Female of 12 years (Patient 1) and male of 20 years (P2) treated in the unit of Adolescent Medicine. In control analytics:

P1: Total cholesterol $85 \mathrm{mg} / \mathrm{dL}$, LDL Cholesterol $37 \mathrm{mg} / \mathrm{dL}$, apoB $26 \mathrm{mg}$ / dL. GOT: 30UI/L, GPT 48 UI/L, GGT 17UI/L.

P2: Total cholesterol 66mg/dL, LDL 13mg/dL, apoB 26mg/dL, GOT: 25 IU/L, GPT 50UI/L, GGT 38UI/L. Ultrasound: diffuse increase in echogenicity compatible with steatosis.

Pending results of parental lipid screening and genetic study in both patients.

In both cases, secondary causes of hypolipemic were excluded.

Conclusion: LDL low cholesterol forces you to rule out secondary causes of hypolipemia. FHBL is due to mutations in the gene apoB or with less frequency of PCSK9 although in $50 \%$ of the cases does not become affiliated with any mutation. Diagnosis is important to prevent the emergence of homozygous FHBL in future generations, which is associated with degenerative neuromuscular diseases and retinal problems.
555

Genetic \& Metabolic

Noonan Syndrome: A Syndrome with Wide Clinical Spectrum Sofia Simões Ferreira ${ }^{1}$, Liliana Sá ${ }^{2}$, Joana Grenha ${ }^{1}$, Isabel Pinto Pais ${ }^{1}$, $\overline{\text { Andreia Teles }{ }^{I}, \text { Helena Santos }}{ }^{I}$, Fátima Santos ${ }^{I}$, Ana Luisa Leite ${ }^{I}$ ${ }^{1}$ Department of Pediatrics, Vila Nova de Gaia/Espinho Hospital Center, Portugal ${ }^{2}$ Department of Pediatrics, Entre Douro e Vouga Hospital Center, Portugal

Noonan syndrome (NS) is a genetic disorder with an estimated incidence of 1/1000-2500 live births. Owing to multiple organ involvement, a wide spectrum of symptoms and signs may occur in this syndrome as well as a high variable expressivity.

We present two cases of NS with different presentations.

4-year-old boy, with prenatal diagnosis of cystic hygroma, pleural effusion and bilateral hydronephrosis. Amniocentesis was performed at 21 weeks for extended genetic and infectious study including Noonan Syndrome and Noonan spectrum mutation panel, which was negative. Admitted at birth due to prematurity of 32 weeks. During hospitalization, craniofacial dysmorphisms (CD) suggestive of NS were observed as well as multiple organ involvement hence he performed genetic study detecting a pathogenic heterozygosity variant c.1391GT (p.G464V) exon 11 in the BRAF gene. He is currently medicated with propanolol for hypertrophic cardiomyopathy. Maintains delayed psychomotor development and feeding problems, exclusively fed through gastrostomy and growing below -2SDS (NS Growth Charts).

16-year-old girl, referred to hospital consultation at 3 years of age due to CD. Performed a metabolic study, karyotype, muscle biopsy and mitochondrial DNA study that were normal. Subsequently she abandoned follow-up and was referral again at age 13 due to pubertal delay and short stature. She presented $\mathrm{CD}$, unilateral mammary bud, very scarce pubic hair, pulmonary stenosis, syndactyly, interventricular comunication, pielic ectasia and intellectual disability. NS was suspected and confirmed by the finding of a heterozygosity variant c.4AG(pSer2Gly) in the SHOC2 gene.

The severity of NS varies widely, from presentations that are lethal prenatally to mildly affected individuals. Because presentation can be mild and the typical facial features recede with age, the diagnosis might be overlooked, while in severe cases the diagnosis is made sooner and multidisciplinary approach is often needed to address the medical and developmental complications.

\section{9}

\section{Genetic \& Metabolic}

Stickler Syndrome, a Genetic Inheritance: Case Report Joana Filipe Ribeiro $^{l}$, Íris Santos Silva ${ }^{I}$, João Virtuoso ${ }^{I}$, Leonor Salício ${ }^{I}$, António Mendes ${ }^{I}$

${ }^{I}$ Department of Pediatrics, Hospital de Sousa Martins - ULS Guarda, Portugal

Background: Stickler syndrome (SS) is a connective tissue disorder affecting the normal collagen production. Majority of cases present an autosomal dominant inheritance pattern. Clinical presentation is characterized by ocular (myopia, vitreoretinal degeneration and retinal detachment), skeletal (premature osteoarthritis, mild spondyloepiphyseal dysplasia and joint hypermobility) and orofacial findings (midfacial underdevelopment, cleft palate and micro/retrognathia), along with neurosensorial hearing loss.

Case Report: A 5-years-old female child was born from a supervised gestation in which a mandibular hypoplasia was suspected in the echography screening of the $2^{\text {nd }}$ trimester (16 weeks). For this reason, she underwent prenatal testing which showed a normal karyotype (46XX) with no further abnormalities. Her father had a previous diagnosis of SS (molecularly confirmed) displaying ocular and osteoarticular manifestations. More recently a patient's brother was also diagnosed with SS in a prenatal 
genetic screening. Our patient was born from a full-term pregnancy with an Apgar index of 8/9/10. On examination, she presented a Pierre Robin sequence and conventional mechanical ventilation was needed during the first 72 hours due to airway obstruction. The patient was hospitalized during 24 days for feeding support. At 12 months of age she was submitted to a surgical correction of cleft palate with a favourable clinical outcome. Subsequent ophthalmological examination depicted a high-grade myopia. Taking into consideration the presence of a Pierre Robin sequence, highgrade myopia, joint hypermobility (Beighton score of 8/9) and positive first-degree SS family history, the diagnosis of SS was established and posteriorly confirmed with a genetic study. The patient is currently under a multidisciplinary follow-up including speech therapy and psychology. Conclusion: SS diagnosis is clinically based, encompassing variable phenotypic expressions depending on the inheritance pattern. In the presence of a Pierre Robin sequence a high index of suspicious is needed for the diagnosis of SS, which requires a multidisciplinary management and follow-up.

\section{3}

\section{Genetic \& Metabolic}

Cri du Chat Syndrome: Case Report Catarina Freitas ${ }^{1}$, Paula Rendeiro ${ }^{2}$, Maria José Costa ${ }^{3}$

${ }^{T}$ Serviço de Pediatria, Hospital Pedro Hispano, Portugal ${ }^{2}$ Centro de Genética Clínica, Portugal Serviço de Neonatologia, Hospital Pedro Hispano, Portugal

Background: Cri du Chat or cat cry syndrome is a rare disorder resulting from a deletion, of variable size, on the short arm of chromosome $5(5 \mathrm{p}-)$. The incidence is estimated between 1:15.000 and 1:50.000 live-born infants. Literature data report $10 \%$ of mortality, $75 \%$ of which occurs within the first year. The most important clinical features are the high-pitched cat-like cry early on life (apparently related to vocal cord abnormalities) as well as low birth weight, microcephaly, round face, broad nasal bridge, epicanthal folds, micrognathia and psychomotor delay and intellectual disability. Moreover, other malformations, although not very frequent, may be present: cardiac, neurological and renal abnormalities. With advancing age, the clinical manifestations could become less striking, making diagnosis more difficult.

Clinical Case: A female infant, of 8 months, institutionalized, with unknown parents, present from the birth high-pitched cat-like cry, broad nasal bridge, and microcephaly, being followed in consults of neonatology. According to clinical records, it was a single term pregnancy, with normal evolution and eutotic delivery with Apgar Index 9/10. Given the suspicion of Cri du chat syndrome, a genetic study (FISH) was done that was negative for $5 p$. Then, the chromosomal array study $(\mathrm{aCGH})$ was performed, which revealed deletion in the terminal region $5 p$, confirming the possibility of the etiology of the patient's phenotype.

Conclusion: Although there is no specific therapy for this syndrome, this case highlights the importance of timely diagnosis for effective educational and rehabilitation interventions, encouraging the performance of the genetic study, namely the chromosomal array study. Moreover demonstrates that make a diagnosis for a genetic or rare disease can often be challenging and thus the valuation of the clinical findings is very important.

220

\section{Genetic \& Metabolic}

Newborn Screening Pilot Study Showed a High Incidence of Cystic Fibrosis in Republic of North Macedonia

Stojka Fustik ${ }^{1}$, Violeta Anastasovska ${ }^{2}$, Dijana Plaseska Karanfilska ${ }^{3}$, Lidija Spirevska $^{1}$, Ana Stamatova ${ }^{1}$

${ }^{I}$ Department for Cystic Fibrosis, University Children's Clinic, Macedonia ${ }^{2}$ Laboratory for Newborn Screening, University Children's Clinic, Macedonia ${ }^{3}$ Research Center for Genetic Engineering and Biotechnology "Georgi D. Efremov", MANU, Macedonia
Background: Cystic fibrosis $(\mathrm{CF})$ is the most frequent, life limiting, genetic disease in Caucasians, caused by mutations in the CFTR gene. The incidence varies in different geographical areas and ethnic groups. The last two decades newborn screening (NBS) for CF has been implemented in the majority of countries where CF is common as a wellestablished public health strategy.

Objective: In May 2018, NBS for CF was introduced in R.N.Macedonia as a pilot study (in 13out of 30 maternities), included in National program for mothers and children's care of the Ministry of Health. Methods: Two steps IRT-IRT algorithm were performed, and then a sweat test for confirmation/exclusion of the CF diagnosis when IRT values were both over the cut off. In cases of positive or borderline sweat tests, mutation analysis of CFTR gene were performed (snapshot reaction for 11 most common regional CFTR mutations or extended gene analysis).

Results: During the period of 6 months 9332 newborns were screened for CF. For IRT1 the cut-off level of $70 \mathrm{ng} / \mathrm{ml}$ was established (99.5th percentile) and for IRT2 a fixed cut off was used at $40 \mathrm{ng} / \mathrm{ml}$. Out of 11 screening positive cases, diagnosis of CF was confirmed in 5 (sweat test results: 90. 102..101..106..102 mmol/L). All diagnosed cases at the end of the first month of life, already had symptoms consistent with the diagnosis of CF. The patient's genotypes were: F508del/ F508del (3), F508del/G1349D and F508del/G542X. In the same period, two symptomatic cases were diagnosed (mean age of diagnosis 4.5 months), born in maternities where the pilot study was not conducted (genotypes: F508del/R1158X and F508del/ CFTRdel2,3).

Conclusion: The pilot study showed a high incidence of $\mathrm{CF}$ in our region of 1:1860 live births. From 2019, NBS for CF will be implemented on the whole newborn population in the country, since early diagnosis of $\mathrm{CF}$ through NBS and the appropriate preventive and curative care management of affected children has a proven benefits with respect to clinical outcomes and health economics.

\section{6}

\section{Genetic \& Metabolic}

Early Maternal Deprivation and Epigenetic Modifications of Later Childhood Emotional Status.

Ketevan Gogberashvili ${ }^{1}$

${ }^{T}$ Pediatrics, Tbilibi State Medical University, Georgia

Background.There is now well documented that environmental agents can modify health by disruption of the homeostatic regulatory mechanisms between the nervous, endocrine and immune systems. During early postnatal life the brain exhibits high plasticity which allows environmental signals to alter the trajectories of rapidly developing circuits. Adversity in early life is able to shape the experience-dependent maturation of stress-regulating pathways such as the hypothalamo-pituitary-adrenal (HPA) system.

Objective. Present study was performed to make clear the influence of early maternal deprivation on formation the emotional status in later childhood; To understand the effect of early deprivation on the regulatory mechanisms of HPA axis.

Methods. The blood neurotransmitters (Norepinephrine NE, Dopamine DA, Serotonin SE) levels were investigated in healthy institutionalized children at age 6 to 36 months from Tbilisi Infant's Orphanage. Emotional status was assessed in institutionalized children at age 3 to 6 years by Leusher's Color Test. Control group formed the same age healthy children from Tbilisi mother-child shelters.

Results. Elevation in blood NE and low levels of DA and SE levels were established in the institutionalized infants to compare with control group. The higher was the percentage of children with anxiety and behavioral 
disorders in the group of orphan children, than in children with family care.

Conclusion. In this study early life stress has been shown to be a strong predictor of impaired inhibitory feedback regulation of the HPA axis. Thus we have proposed that conditions of early life environment can evoke changes in DNA methylation facilitating epigenetic programming of critical genes involved in regulating stress responsivity that may in turn manifest with neuroendocrine and behavioral symptoms in later childhood periods. So, it is recommended to support alternative ways of abandoned children's care against children`s institutions, such as biological mothers' financial support or foster care.

797

\section{Genetic \& Metabolic}

Diabetes Mellitus and Optic Atrophy: Not Just a Coincidence Ana Gomes ${ }^{l}$, Filipa Pinto ${ }^{1}$, Joana Figueirinha ${ }^{l}$, Sérgio Silva ${ }^{2}$, Cláudia Melo $^{3}$, Jorge Pinto Basto ${ }^{4}$, Soraia Tomél, Paula Fonseca ${ }^{1}$

${ }^{I}$ Department of Pediatrics, Centro Hospitalar do Médio Ave, Portugal ${ }^{2}$ Department of Ophthalmology, Centro Hospitalar de São João, Portugal

${ }^{3}$ Department of Pediatrics, Centro Hospitalar de São João, Portugal

${ }^{4}$ Laboratory of Molecular Diagnostics and Clinical Genomics, Centro de Genética Clínica e Patologia (CGC Genetics), Portugal

Wolfram Syndrome (WS1) is a rare autosomal recessive genetic disorder characterized by diabetes mellitus, diabetes insipidus, optic nerve atrophy, hearing loss and neurodegeneration. WS1 may be caused by variants in the WFS1 gene that encodes a protein called wolframin. A 10-year-old boy presented to the emergency department for dysuria, polyuria and polydipsia with four days duration. Recent changes in appetite or weight were denied. He had a history of syncope episodes and progressive vision loss since the age of 7 and was recently diagnosed with bilateral optic nerve atrophy. His physical exam was normal and he had a Tanner stage I. Capillary blood glucose measurement was $388 \mathrm{mg} / \mathrm{dL}$ and urine test strip presented glycosuria without ketonuria. His blood $\mathrm{pH}$ was 7.38 , potassium level was $3.7 \mathrm{mEq} / \mathrm{L}$ and bicarbonate level was $27.2 \mathrm{mmol} / \mathrm{L}$. He had a normal lipid profile and thyroid function, negative antithyroid antibodies, negative screening for celiac disease and negative anti-islet cell and anti-glutamic acid decarboxylase antibodies. Considering the combination of optic nerve atrophy and diabetes mellitus, a genetic study for Wolfram Syndrome was performed and revealed 3 variants: 2 of uncertain clinical significance and one probably pathogenic variant. He had a normal tympanogram and audiogram and abdominal ultrasonography did not reveal any abnormalities. A multiple dose insulin injection therapy was started and he was referred to diabetes and genetics consultation. WS1 has poor prognosis and currently there is no effective therapy for this syndrome. Regular audiologic evaluation will be essential to early identify hearing loss. Therefore, a timely recognition and diagnosis is essential in order to minimize the complications and reduce morbidity and mortality.

795

\section{Genetic \& Metabolic}

Rhabdomyolysis' Etiology: A Challenging Diagnosis For A General Pediatrician

Cátia Leitão ${ }^{l}$, Isabel Ayres Pereira ${ }^{l}$, Joana Tenente ${ }^{l}$, Margarida $\overline{\text { Peixoto }}^{I}$, Marta Vila Real ${ }^{l}$, Helena Santos ${ }^{l}$

${ }^{1}$ Pediatric Department, Centro Hospitalar de Vila Nova de Gaia/Espinho, Portugal
Background: Rhabdomyolysis is a muscular necrosis with release of its constituents into the circulation. It can be a manifestation of multiple disorders of different origins. A thorough clinical history and laboratorial investigation, with a high index of suspicion are of paramount importance to an accurate diagnosis.

Case: A 6 year old female was admitted to the emergency department (ED) with fever and odynophagia. She had a previous history of intermittent toe-walking and creatine kinase (CK) elevation. A blood test in the ED revealed mild anemia, elevated $\mathrm{CK}$, and serology compatible with Epstein-Barr Virus (EBV) infection. During follow-up, a past history of myalgia and exercise intolerance was found. The patient also presented gastrocnemius hypertrophy, negative Gowers sign and an otherwise normal neurological examination. Continued elevation of CK was detected, so she did baseline metabolic studies and Pompe disease screening with no alterations. A muscle biopsy revealed glycogen deposits and an absence of myofosforilasis, suggestive of McArdle disease, confirmed by the presence of c.148CT (p.Arg50*) and c.40delA (p.Ile14Serfs*12) variants in PYGM gene. Patient was started on a carbohydrate enriched diet and light to moderate aerobic exercise regimen, and information on rhabdomyolysis was given. A progressive normalization of CK levels and physical activity were observed.

Conclusion: McArdle disease, or type V glycogen storage disease is a rare, recessive autossomic pathology caused by a mutation on the PYGM gene, resulting in a myofosforilasis deficiency. Only $4 \%$ of the cases are diagnosed before the age of 10 . Its clinical presentation and severity is highly variable, ranging from severe hypotony, gait abnormalities, exercise intolerance, myalgia and muscle fatigue. Patients usually present with elevated CK levels and episodic rhabdomyolysis, myoglobinuria and acute renal failure. This case highlights the importance of investigating rhabdomyolysis' possible etiologies, in order to prevent or allow an early detection of acute decompensations.

\section{5}

\section{Genetic \& Metabolic}

Down Syndrome - A Neurodevelopmental Perspective

Joana Lorenzo $^{1,2}$, Ariana Teles $^{2}$, Diana Gonzaga ${ }^{2}$, Inês Vaz Matos ${ }^{2}$, Ana Catarina Prior $^{2}$

${ }^{1}$ Neurodevelopmental unit, Joana Lorenzo, Portugal

${ }^{2}$ Neurodevelopmental Unit, CMIN-CHP, Portugal

Background: Down syndrome(DS) is the genetic leading cause of intellectual disability. Its worldwide incidence is $1: 700-800$ pregnancies, with 10000-12000 estimated cases in Portugal. The main characteristics include physical features, specific medical conditions and neurodevelopmental disorders.

Objective and Methods: Retrospective and descriptive study, with the aim of analyzing patients with DS in a Neurodevelopment Unit of a Level III Hospital in 31/01/2019.

Results: 29 patients with DS were included, 3(10,3\%) with prenatal diagnosis; median maternal age of 29 years; $18(62,1 \%)$ male gender, between 1-18 years-old (mean and median 7 years). The first neurodevelopment consultation occurred between 1 month-14 years old. $21(72,4 \%)$ patients had cardiopathy, $17(58,6 \%)$ endocrine, $16(55,2 \%)$ respiratory, $7(24,1 \%)$ gastrointestinal, $2(6,9 \%)$ neurologic and $2(6,9 \%)$ haematological disorders.

Regarding neurodevelopmental assessment, all patients had communication disorders, 5(17,2\%) presenting Attention Deficit Hyperactivity Disorder(ADHD) under methylphenidate and 1(3,4\%) Autism Spectrum Disorder(ASD). Concerning interventions applied, 28(96,6\%) attended speech therapy, 25(86,9\%) occupational therapy and $9(31,0 \%)$ physiotherapy. The adopted strategies were based on the learn to read to 
learn to talk program, phonomimic method (Teles P, Portugal) and Dolman Method in 2 patients each and Makaton method in 1 patient. In the remaining the intervention method was not specified. 18 patients $(62,1 \%)$ were schoolers (median 8,5 years), benefitting from inclusive education.Conclusion: This review presents a holistic understanding of DS. In our sample the referral to Neurodevelopmental consultation was late, when it should occur precociously with the remaining specialties. Diagnosing neurodevelopmental disorders in DS patients is challenging, since their learning disabilities, maturity delay and the syndrome itself. Therefore, an ASD diagnosis is often late, which worsens the outcome. Besides, most demonstrate attention deficit, restlessness and impulsivity, with ADHD in only $17,2 \%$ of our sample. The prognosis is influenced by the onset, type and frequency of the intervention. The ideal method should be multi-modal, flexible and focused on the subject, family and community.

\section{8}

\section{Genetic \& Metabolic}

Burnside-Butler Syndrome: A Diagnosis in Neurodevelopmental Delay

Pedro Marinho $^{I}$, Ana de Carvalho Vaz ${ }^{1}$, Mariana Bastos Gomes ${ }^{1}$, Virgínia Sampaio $^{1}$, Mariana Costa ${ }^{1}$

${ }^{1}$ Pediatrics Department, Unidade Local de Saúde do Alto Minho, Portugal

The authors report a 15 -year-old Caucasian male, with a history of learning/intellectual disability (very low verbal and full-scale IQ $(<70)$ ), combined type attention-deficit/hyperactivity disorder (ADHD), an unusual happy appearance, overweight, mild tricuspid valve regurgitation and facial pilosity asymmetry. Karyotype XY, 46 and PCR revealed a FMR1 allele of normal size. Through microarray analysis, he was identified with a copy number variation - a $537 \mathrm{~Kb}$ deletion between BP1 and BP2 on 15q11.2, involving various genes, including CYFIP1, TUBGCP5, NIPA1 and NIPA2.

Patients with 15q11.2 BP1-BP2 microdeletion (termed Burnside-Butler) syndrome, according to recent literature, can present with developmental delay (speech delay, writing and reading difficulties, verbal IQ $\leq 75$ ) and neurobehavioral abnormalities including ADHD and unusual happy expression; less commonly, patients present mild dysmorphic features and congenital heart defects. These features are present in our patient. However, overweight or obesity have not been directly associated. Specific involvement of facial pilosity or tricuspid valve defects have not been previously described in this emerging cytogenetic syndrome, whose phenotypic features are still in expansion.

The authors aim to further the awareness of this etiologic entity, believing that achieving a diagnosis offers a basis on which to delineate the approach both to the patient and to their families, who in this case were oriented to Medical Genetics evaluation.

\section{7}

\section{Genetic \& Metabolic}

Partnering with Patients in the Creation of Online Resources for Interprofessional Education

Leona Nertney ${ }^{3}$, Daire O'Leary $^{3}$, John Hayden ${ }^{1}$, Helen French ${ }^{2}$, Frances Horgan $^{2}$, Judith Strawbridge

${ }^{1}$ School of Pharmacy, Royal College of Surgeons in Ireland, Ireland

${ }^{2}$ School of Physiotherapy, Royal College of Surgeons in Ireland, Ireland

${ }^{3}$ School of Medicine, Royal College of Surgeons in Ireland, Ireland
Rationale: Collaborative practice happens when healthcare professionals work together with patients and families to deliver the highest quality of care. This is the optimal model for paediatric care, with children and families included as partners within the healthcare team. Interprofessional education (IPE) is seen as a necessary step towards collaborative practice. We sought to explore how best to prepare our students for paediatric collaborative practice, harnessing the unique interprofessional learning opportunities in paediatric care. This also required navigating logistical IPE challenges such as asynchronous curricula, unequal class sizes and delivery across international campuses.

Methods: We collaborated with a parent in creating case-based online IPE. Videos of the parent/child in consultations with a paediatrician, pharmacist, physiotherapist and speech and language therapist were recorded and embedded into an interactive Articulate Storyline ${ }^{\circledR}$ package. Pre-post MCQs and key teaching points covered a range of issues including developmental stages, medication administration and complex communication.

Findings: Medical students are currently engaging with the online IPE on a rotational basis and student reaction to the learning is ongoing. Delivery in the pharmacy and physiotherapy curriculum is planned. Currently the learning outcomes for interprofessional collaborative practice are, therefore, obtained unprofessionally.

\section{Discussion / Conclusion}

The patient-centred online interprofessional activity has provided an opportunity to begin to prepare our students for paediatric collaborative practice. We intend to explore further progression towards learning with others and evaluate the outcomes at a higher level.

\section{7}

\section{Genetic \& Metabolic}

Patient with De Novo Mutation of Waardenburg Syndrome Eva Pedrero Segura ${ }^{1}$, Silvia Gallego Gutiérrez ${ }^{I}$, Anabel Valdivielso Martinez ${ }^{\prime}$

${ }^{I}$ Pediatrics, Primary Care Attention Málaga-Guadalhorce District, Spain

Background: Waardenburg syndrome (WS) is an auditory-pigmentary syndrome caused by absence of melanocytes. Melanin contributes to skin, hair, and eye color and it plays an essential role in the normal function of the inner ear. Based on the phenotypic or genotypic characteristics, WS is clasificated in four types. The prevalence of WS is estimated to be $1 / 42,000$ and it is responsible for $1 \%$ to $3 \%$ of cases of profound congenital deafness.

Objective: Describing a clinical case of WS type I with de novo mutation of PAX3 gene that associates other malformations that are not included in this syndrome.

Method: A 2 years old boy with WS type I who associates small ventricular septal defect, epispadias and congenital scoliosis with two hemivertebra. These two last defects are not described in WS clinical features. He has three mayor WS criteria (dystopia cantorum, congenital sensorineural hearing loss, white forelock) and two minor criteria (synofrys and broad and high nasal root with narrow nose), also bilateral blue iris. $\mathrm{He}$ has a profound bilateral hearing loss so he wears headphones with bilateral ventilation tubes pending of cochlear implant. Nowadays he is also pending of correction surgery of epispadias. Our patient has a healthy 5 years old brother and parents without WS features. The genetic study of parents was normal so his mutation has arisen de novo in heterozygosis c.667CT (p.Arg222Ter) in PAX3 gene. 


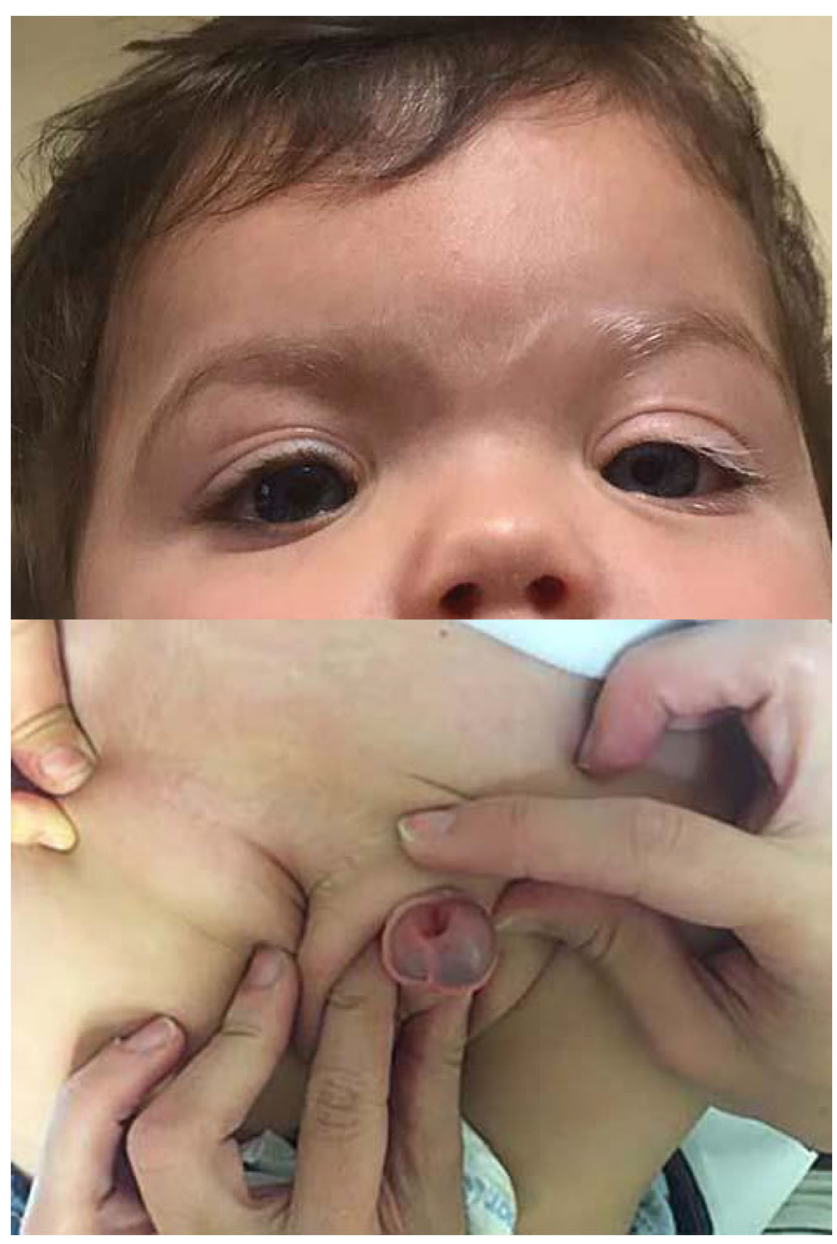

Conclusions: All patients with white forelock, hyperthelorism, iris heterocromia or distopia cantorum should be investigated for hearing loss. Patients with any malformation should be widely studied to rule out other associated malformations. The remarkable finding is that this patient associates abnormalities that we have not found associated with WS in the current literature.

\section{2}

\section{Genetic \& Metabolic}

A Novel Pathogenic Mutation of Vitamin-D-dependent Rickets Jacopo Norberto Pin ${ }^{1}$, Micol Cossettini ${ }^{2}$, Francesco Fabris ${ }^{2}$, Stefano Martelossi $i^{2}$

${ }^{I}$ Dipartimento di Salute della Donna e del Bambino, Università degli Studi di Padova, Italy ${ }^{2}$ UOC di Pediatria, Ospedale Ca' Foncello, Italy

Backgound: Vitamin-D-dependent rickets 1A (VDDR-1A) is an extremely rare, autosomic recessive genetic form of rickets caused by a defect in vitamin $\mathrm{D} 1 \alpha$-hydroxylase enzyme which leads to low levels of 1,25-(OH) vitamin D. Herein, we report two Moroccan sisters R.E. and N.E. (respectively 3 and 15 months old), born from consanguineous parents, who presented with psychomotor retardation and failure to thrive.

Methods: Biochemical analyses in serum and urine were conducted using the automatic analyzer Cobas immediately after collection. Serum intact $\mathrm{PTH}$ was measured by chemiluminescence assay. $25-\mathrm{OH}$ vitamin $\mathrm{D}$ and $1,25-(\mathrm{OH})$ vitamin $\mathrm{D}$ levels were measured by enzyme immunoassays.

The entire coding region of CYP27B1, CYP2R1 and VDR genes were sequenced through NGS technology. Relevant genetic variations were resequenced through Sanger technology. The in silico prediction models used were Polyphen2, SIFT, MutationTaster, PHRED, MutationAssssor.

Results: Both sisters showed typical laboratory findings of VDDR-1A including hypocalcemia, hypophosphatemia, elevated ALP and PTH. Further assesments demonstrated normalelevated levels of $25-\mathrm{OH}$ vitamin D with low $1,25-(\mathrm{OH}) 2$ vitamin D.

Radiological workup revealed osteopenia, widened and irregular epiphyseal plates and in the older sister rachitic rosary.

We therefore sequenced the entire coding region of genes that has been associated to this clinical condition. Both sisters showed the homozigotic mutation p.Leu169Pro (c.596TC) of CYP27B1 gene that has never been described before. All prediction models consider this mutation harmful.

Both sisters immediately started 1,25-(OH) Vitamin D supplementation (N.E. took a galenic formulation of Calcium too).

They both showed an adequate but incomplete treatment response with psychomotor retardation improvement, weight gain, normalization of serum $\mathrm{Ca}$ and $\mathrm{P}$ levels and a significant decrease of PTH and ALP levels.

Conclusion: We report a novel pathogenic mutation of CYP27B1 that leads to VDDR-1A. An early diagnosis is necessary to prevent serious complications of rickets that are only partially reversible.

\section{1}

Genetic \& Metabolic

\section{Piebaldism: A Cutaneous Pigmentation Defect} Sara Pinto $^{I}$, Lúcia Rodrigues ${ }^{I}$, Rui Dourado ${ }^{1}$, Márcia Gonçalves ${ }^{l}$ ${ }^{T}$ Pediatria, Centro Hospitalar Vila Nova de Gaia/Espinho, Portugal

Introduction: Piebaldism is a rare inherited autosomal dominant disease caused by a mutation in the C-KIT gene located on chromosome 4q1213. In the presence of this mutation, a defect occurs in the migration and subsequent proliferation of melanoblasts in some body areas during the embryonic development, causing acromy due to the absence of melanin production.

The most suggestive clinical finding is a triangular acromic area in frontal region associated with hair depigmentation (poliosis), present in $80-90 \%$ of the cases. Other areas such as limbs, chest and abdominal region may also be affected. Skin changes are present from birth and remain stable over time.

The differential diagnosis is made with other cutaneous pigmentation disorders, such as vitiligo, total albinism, Tietz Syndrome and Waardenburg Syndrome.

Case Presentation: A female newborn presented at birth with an acromic macula at the frontal region with associated local hair depigmentation and macular hypochromic lesions in previvial regions, forearms, and abdomen. Physical examination was otherwise unremarkable. She was born of an uneventful first gestation of 39 weeks, had good adaptation to extrauterine life and appropriate to gestational age somatometry at birth.

Familial history was positive for piebaldism (mother and maternal aunt). Ophthalmological and auditory evaluation were normal.

Currently she is 5 months-old and has adequate psychomotor and statoponderal development. 
In the case described, the presence of circumscribed poliosis associated with areas of depigmentation in the limbs and abdomen since birth, the stable course and positive family history, helped in the diagnosis and differentiation from vitiligo. The remaining entities are excluded, taking into account the objective examination and auditory and ophthalmologic evaluations performed.

Conclusion: The conjugation of the clinical history, genealogical tree and objective examination is crucial to the diagnosis of this pathology, obviating the need for complementary tests.

\section{5}

\section{Genetic \& Metabolic}

\section{Gangliosidosis: Diagnosis and Management Challenges} Andreea Madalina Popescu ${ }^{l}$, Alina Burcutal, Bianca Gabriela Olaru ${ }^{l}$, Alexandra Faleschini ${ }^{2}$, Laura Carasava ${ }^{1,3}$, Carmen Magdalena Burloiu ${ }^{2}$ ${ }^{I}$ Pediatric, Grigore Alexandrescu Children's Emergency Hospital, Bucharest, Romania, Romania

${ }^{2}$ Pediatric, Alexandru Obregia Clinical Hospital of Neurology and Psychiatry, Pediatric Department, Bucharest, Romania, Romania

${ }^{3}$ Pediatric, Carol Davila University of Medicine and Pharmacy, Bucharest, Romania, Romania

Introduction: Gangliosidosis is an autosomal recessive lysosomal storage disease, expressed by excessive accumulation of gangliosides in central nervous system and reticulo-histiocytar tissue, caused by lysosomal hydrolysis deficiency.

Objectives: Gangliosidosis is a pathology that affects both neurologic and physical development with progressing regression, involving severe complication and unfavorable prognosis, requiring multidisciplinary collaboration for management and therapeutic attitude.

Case report: We present the case of a male patient aged 18 months, admitted to the Pediatric Neurology Clinic "Alexandru Obregia "for global developmental delay including cognitive and motor regression. He had multiple intercurrent respiratory infections during the first months of life, and at the age of 5 months was admitted in "Grigore Alexandrescu Children's Emergency Hospital", and at abdominal examination hepatomegaly was reported. After excluding other causes, giving the evidence of the medical history, the lysosomal enzyme activity was measured, highlighting the low value of betagalactosidase. After discharge of the hospital, the child had multiple admissions for respiratory intercurrences, presenting also cognitive and motor regression. Upon admission to the neurology clinic, the child was athrepsic, with dysmorphic features, generalized telengiectasis, proeminent abdomen, hepatomegaly, severe developmental disorder, generalized hypotonia, development level below 3 months of age. The ophthalmologic examination revealed the appearance of cherry red-spot. Cardiac ultrasound has not shown any changes at that time. Repeating enzyme dosing revealed low activity of betagalactosidase, which associated with clinical and paraclinic signs confirmed suspected gangliosidosis - most likely type I. In the following month the child presented cardiomyopathy requiring admission in teritorrial unit.

Conclusion: Gangliosidosis is one of the rare causes of global development disorder with regression in the first two years of life. Diagnosis is a challenge, requiring multidisciplinary collaboration. Prognosis is unfavorable because of the associated complications, with no curative treatment so far, life expectancy usually is only two years in type I gangliosidosis.
313

\section{Genetic \& Metabolic}

\section{Bloch Sulzberger Syndrome Case}

Vira Semianchuk ${ }^{l}$, Irina Parandii ${ }^{2}$, Yaroslav Semkovych ${ }^{l}$

${ }^{T}$ Department of Children Diseases of Postgraduate Medical Education Faculty, Ivano-Frankivsk National Medical University, Ukraine

${ }^{2}$ Allergology department, Ivano-Frankivsk Regional Children Hospital, Ukraine

Background: Bloch Sulzberger Syndrome (incontinentia pigmenti) is hereditary X-linked disease with skin lesion and generalized ectodermal and mesodermal dysplasia.

The disease affects 1:91,000 children. It is caused by NEMO/IKK-gamma gene mutation, which is located on chromosome Xq28, however, the implication of mutation in Xp11 and Xq21 segments is not excluded in case with other phenotypes of that syndrome.

In the clinical course of the disease, 4 stages can be singled out: bullous or vesicular, hypertrophic, pigmental and atrophic.

Extracutaneous changes are found in 50\% of patients. The most common are: dental anomaly (dentin deficiency or absence of a teeth row); visual organs disorders (cataract, vascular anomalies and profound changes of retina epithelium); CNS injury (spastic paralysis, convulsive disorder, mental retardation). Congenital heart disease, anomalies of skull, anomalies of kidneys, nail dystrophy, etc. occur less frequently.

Case Report: Eight-month-old girl was admitted in allergology department with numerous erythematic-pigmental and papularbullous linear rash forming along Blaschko lines. Some bullous elements were covered with hard crust (photo 1). Clinical blood and urine analysis, biochemical blood test and immunogram showed no abnormality. Neurosonography, ultrasonic diagnosis and echocardiography did not find any pathology. The patient was initially diagnosed with multiforme bullous erythema. Differential diagnosis was made with herpes zoster, bullous impetigo, bullous mastocytosis. According to anamnesis, the first symptoms occurred in the maternity department, where the child was diagnosed and treated for herpes. Genetic confirmation is not available in Ukraine. However, classic clinical criteria confirm the diagnosis.

Today, the girl is 3 years old. CNS injury and visual organs disorders were not discovered. Bullous rash occasionally recur associated with respiratory condition. Pigmentation areas are present on skin along Blaschko lines (photo 2,3), as well as atrophic skin changes. There are areas of cicatrix alopecia on the skin of the head.

Conclusion: Although Bloch Sulzberger Syndrome is a rare disease, classic symptomatology is indicative of the right diagnosis. These children require case follow-up for early detection of optic nerve disorder and CNS injury.

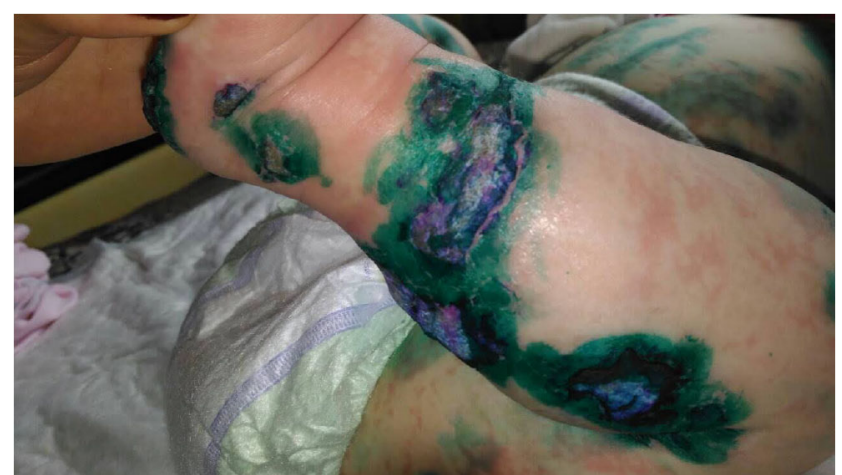




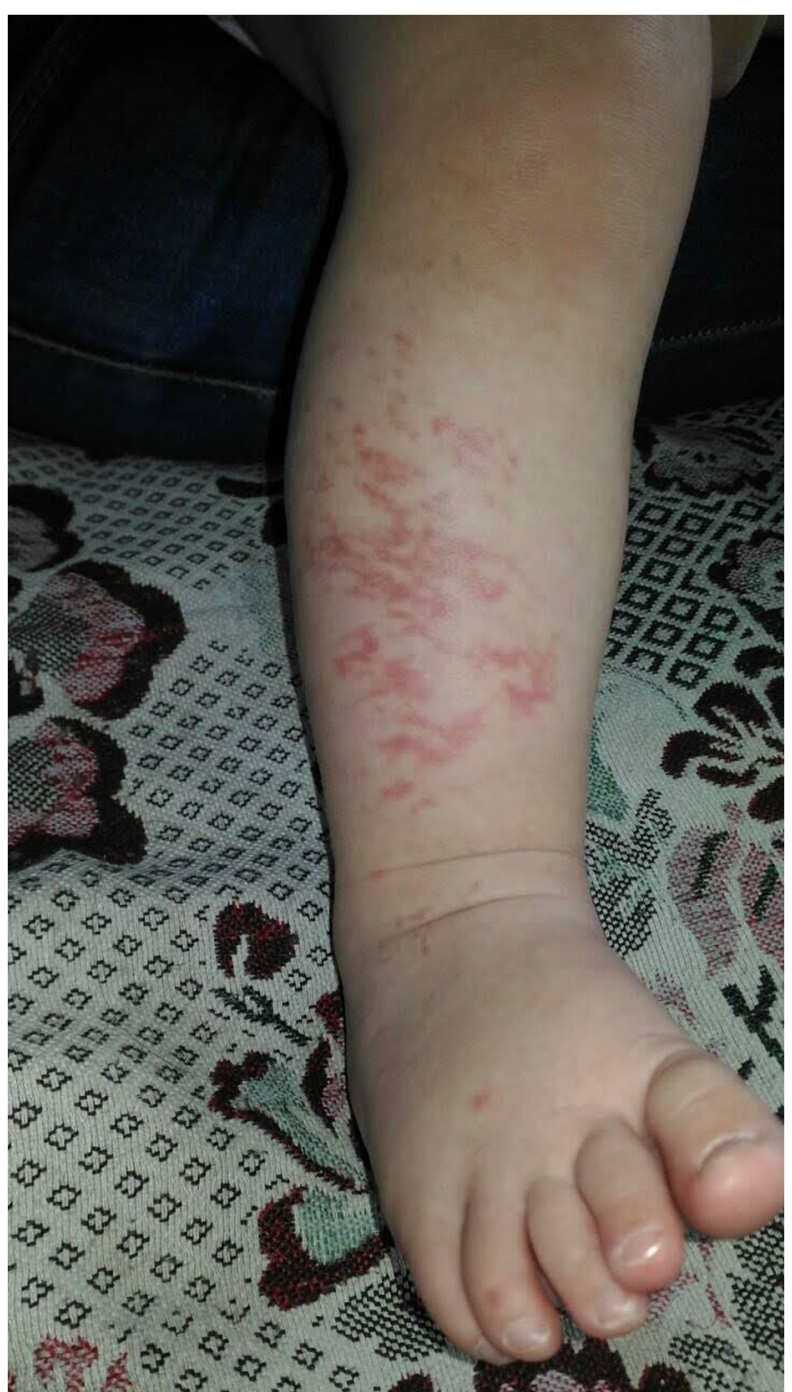

706

\section{Genetic \& Metabolic}

Anhidrotic Ectodermal Dysplasia: Different Fenotype in the Same Family

Ana Isabel Valdivielso Martnez ${ }^{I}$, Eva Pedrero Segura ${ }^{I}$, Silvia Gallego Gutierrez ${ }^{I}$

${ }^{1}$ Pediatrics, Primary care Attention, Spain

Background: Anhidrotic ectodermal dysplasia (AED) is a rare hereditary disorder with a triad os sparse hair, dental hypoplasia and anhidrosis. Most cases of AED are the X-linked form, while others are dominant $\mathrm{o}$ autosomal recessive form.

Objective: To report 4 cases of mild-moderate AED in four sisters, whose father also suffers from AED with the complete phenotype.

Methods: Clinical data were collected from a family with this disease.

Results: We report 4 cases of AED in four sisters. The genetical test revealed X-linked mutation of AED gen in all of them.

Case 1: 1 year old girl, she has had 2 bacterial pneumonias, recurrent wheezing and recurrent episodes of high grade fever. She has sparse hair, hypotricosis and small and pointed teeth. She is able to sweat.

Case 2: 2 years old girl, she has had 4 bacterial pneumonias, recurrent wheezing and recurrent episodes os high-grade fever. She has sparse hair, hypotricosis (image 1)and small and pointed teeth (image 2 ). She has very dry skin and she is not able to sweat, so it is very difficult to treat fever. Case $3: 3$ years old girl, asimptomatyc.

Case $4: 4$ years old gir, she never had pneumonias but she has pointed teeth (picture 3) and sparse hair (picture 4)

Conclusion: the same genetic mutation presents with different grades of phenotypic alterations in the same family. It is important to suspect AED in children with rare teeth and sparse hair and freqent fever episodes.
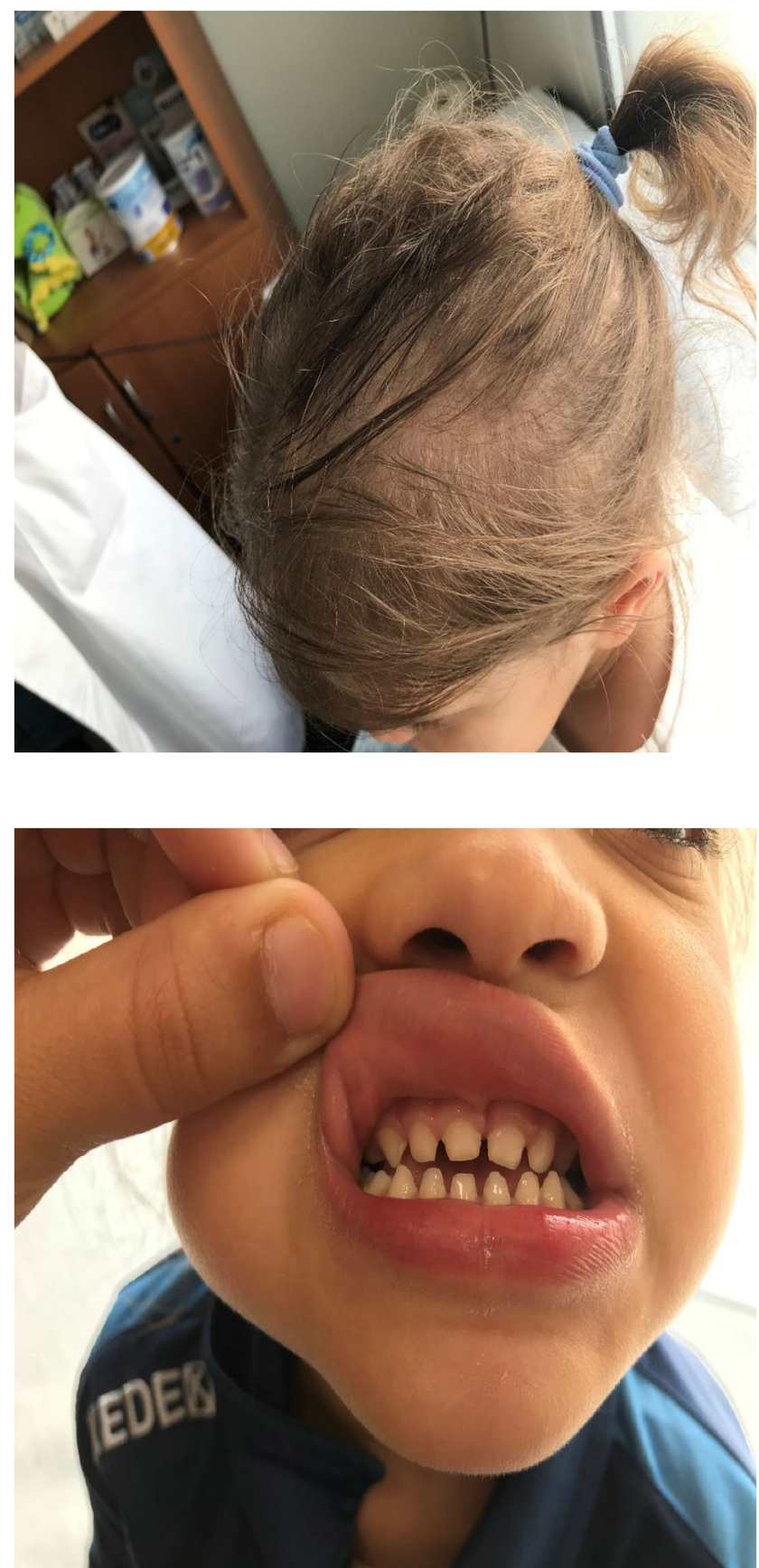

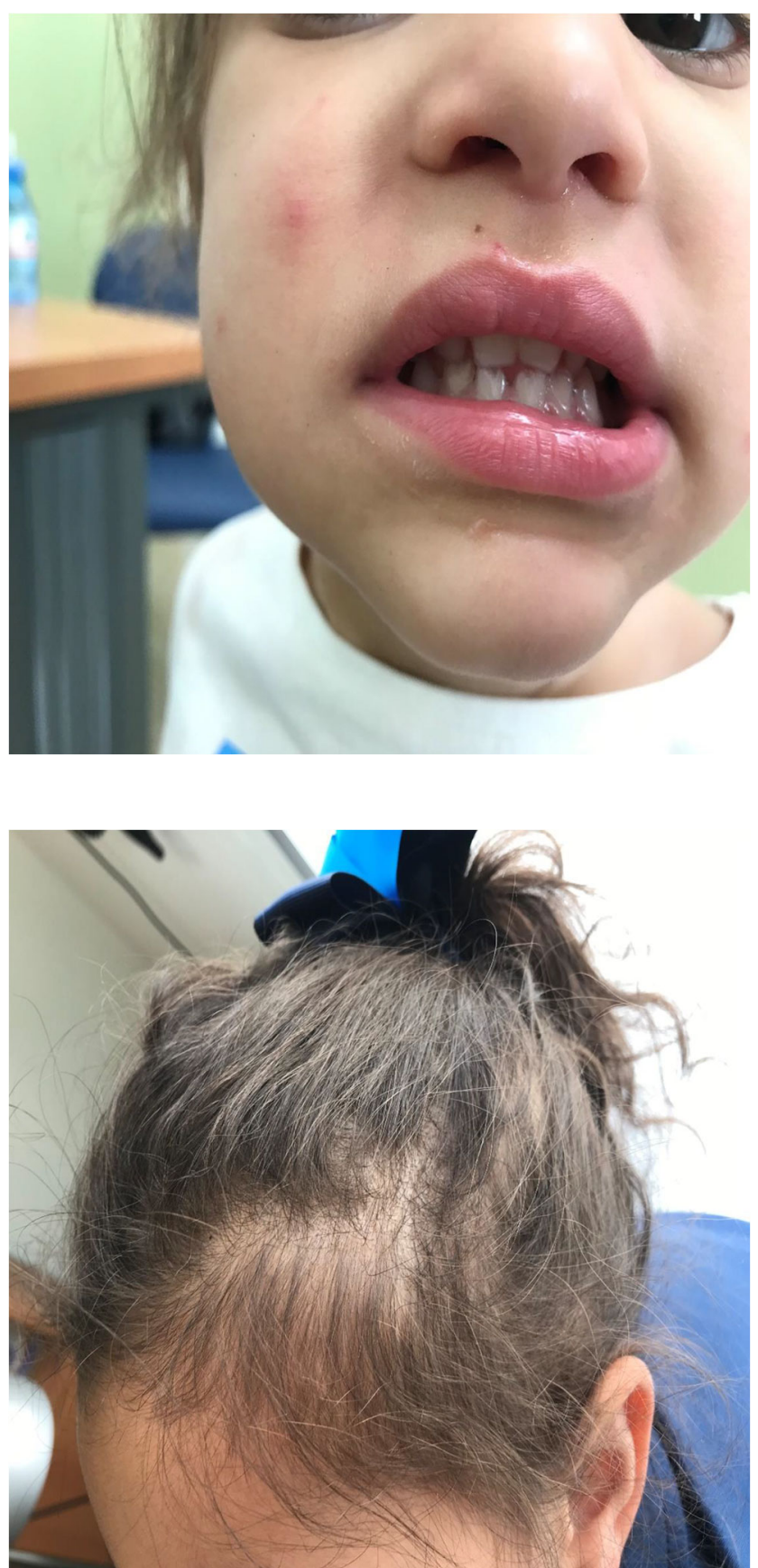

1039

Genetic \& Metabolic

The Language Difficulties Leading to Mucopolysaccharidoses Joana Vilaçal ${ }^{1}$, Sofia Miranda ${ }^{1}$, Anabela Bandeira ${ }^{2}$, Clara Alves Pereira $^{\prime}$, Manuela Costa Alves ${ }^{I}$

${ }^{1}$ Paedriatic Service, Hospital de Braga, Portugal

${ }^{2}$ Paedriatic in the Metabolic Unit of the Reference Center for Inborn Errors of Metabolism, Centro Hospitalar do Porto, Portugal

The mucopolysaccharidoses (MPS) is a lysosomal storage disorder caused by the deficiency of enzymes required for the stepwise breakdown of glycosaminoglycans (GAGs). MPS is a rare condition, with an estimated total incidence of approximately 1 in 20000 live births. MPS type
II or Hunter syndrome is a hereditary genetic disease linked to the $\mathrm{X}$ chromosome and is caused by the enzymatic deficiency of iduronate-2sulfatase.

A 28-month-old boy, was admitted in paediatric with language difficulties. He has no relevant obstetric and family history. Normal growth with cephalic perimeter always in P95. Bilateral inguinal hernia surgery at 15 months. Chronical nasal obstruction with adenoid hypertrophy. Normal motor development with small delay in expressive language.

We observed macrocephaly, rough facial features (including a broad nose, thick lips and enlarged mouth and tongue), excessive body hair growth and hepatomegaly. The abdominal echography revealed hepatomegaly without splenomegaly or other alterations. Electrical and echocardiogram were normal. Without hearing loss or cornea opacity. The analytical study showed normal hemogram, lipid panel, hepatic and thyroid function. From the remaining investigation is important to emphasize the increased levels of GAG's in urine excretion. He was sent to Metabolic Unit, where was confirmed and diagnosed the enzymatic deficiency of iduronate-2sulfatase, MPS type II. He began enzyme replacement therapy at 32 months with regression of hepatomegaly and improved facial features. The MPS is a lysosomal disease with large spectrum of presentation with severe types manifested in first year or attenuated forms with diagnostic in adult. There for it is necessary a high suspicion to do the diagnosis. In the present case, the early diagnosis was possible due to a detailed clinical history and a meticulous objective examination. The early enzyme replacement therapy leads to a better quality of life whereas after the establishment of the sequels these are not reversible.

\section{7}

\section{Hemato - Oncology}

Development and Validation of a Predictor Score of Persistent Thrombocytopenia in Primary Immune Thrombocytopenia in Children.

$\underline{\text { Habib Besbes }}^{I}$, Chokri Chouchane $^{I}$, Radhia Hadj Salem ${ }^{I}$, Saber Hammami $^{1}$, Islem Khefecha ${ }^{3}$, Bahri Mahjoub ${ }^{2}$, Slaheddine Chouchane ${ }^{I}$ ${ }^{1}$ Pediatric Department, LR12SP17, Fattouma Bourguiba Hospital Monastir University, Tunisia

${ }^{2}$ Pediatric Department, Taher Sfar Hospital, Monastir University

${ }^{3}$ Research Laboratory, Statistics, Economics and FinanceFSEG Mahdia, Monastir University

Aim: To develop a prognostic score predictive of persistent thrombocytopenia in acute primary immune thrombocytopenic (PIT) in children.

Methods: Two retrospective cohorts of PIT collected from two pediatric departments over a similar period of 25-year. One cohort served for the derivation of the score and the second served for the external validation. Inclusion criteria were: PIT age between 2 months and 14 years and persistent thrombocytopenia less than $100,000 / \mathrm{mm} 3$ after 6 months of follow-up. The data was analyzed using statistical software SPAD version 5.5. The predictive index was done through a discriminant analysis.

Results: 219 cases were collected in the two studies, respectively: 149 cases (mean age $=5.7$ years, sex ratio $\mathrm{M} / \mathrm{F}=1.48$ ) from the derivation population and 70 cases from the validation population (mean age $=5.4$ years, sex ratio $\mathrm{M} / \mathrm{F}=1.5$ ). The developed score had 6 parameters: beginning onset, failure of the first line treatment, the presence of a triggering factor, age, platelet rate at 1 month and platelet rate at 3 months. The sum of the different coefficients for each patient provides a result convertible to probability of persistence of thrombocytopenia after 6 months. The ROC curve (Receiver operating characteristic) showed a good specificity $(93 \%)$ and a good sensitivity of $(90 \%)$ for a threshold of 35 . The external validation study showed a good specificity e (100\%) and good sensitivity $(83.3 \%)$.

Conclusion: This prognostic clinical score validated by an external study requires further prospective studies to improve validation level and to explore its practical utility. 
509

\section{Hemato - Oncology}

To "B" or not to "B": Cervical Lymphadenopathy in a Previously Healthy 16-year-old Boy

Mafalda Castelão ${ }^{1,2}$, Ana Raquel Claro ${ }^{1}$, Pedro Silva ${ }^{3}$, Ana Barbosa Rodrigues $^{1}$, Ana Catarina Amorim ${ }^{4}$, Ana Isabel Fernandes ${ }^{2,5,6}$, Daniela Macedo ${ }^{5}$, Isabel Esteves ${ }^{1,2}$, Ana Mouzinho ${ }^{1,2}$, José Gonçalo Marques $^{1,2}$

${ }^{1}$ Department of Paediatrics, Hospital de Santa Maria, Centro Hospitalar Universitário de Lisboa Norte, Portugal

${ }^{2}$ Faculty of Medicine, University of Lisbon, Portugal

${ }^{3}$ Department of Paediatrics, Hospital de Santo André, Centro Hospitalar de Leiria, Portugal

${ }^{4}$ Department of Paediatrics, Hospital Fernando da Fonseca, Portugal

${ }^{5}$ Oncoloy Service, Hospital de Santa Maria, Centro Hospitalar Universitário de Lisboa, Portugal

${ }^{6} I M M$, Instituto de Medicina Molecular João Lobo Antunes, Portugal

Background: Lymphadenopathy in children is common and generally benign and self-limited. Specific accompanying systemic symptoms can call for attention for a manifestation of serious underlying disease.

Case description: A 16-year-old male presented to the Emergency Department with a two-month history of a rapidly progressing nontender unilateral cervical mass, with no fever or other complaints. On thorough history, there was an objective weight loss of 8 percent of body weight and increased night sweats over the last few weeks. There were no complaints of pruritus and no past medical history of previous infections, exposures to toxins or evidence of underlying immune deficiencies or familial cancer. On physical examination a localized bulky, firm, rubbery, nontender lymphadenopathy in the lower cervical and supraclavicular region was found. There was no facial edema, dysphagia or dyspnea. He had a clear oropharynx and no evidence of hepatosplenomegaly or skin lesions. His blood tests revealed leukocytosis $(22600 / \mathrm{uL})$ with elevated C-reative protein $(9,45 \mathrm{mg} / \mathrm{dL})$ and sedimentation rate $(42 \mathrm{~mm} / \mathrm{h})$. A CT scan revealed cervical lymphadenopathy in relation with a large mediastinal mass surrounding the aorta, causing complete obstruction of the superior vena cava (SVC) and significant compression of the trachea (axis $5 \mathrm{~mm}$ ). He underwent an excisional biopsy of the lymph node, diagnosing classic Hodgkin Lymphoma (HL). He was referred to Hematology to initiate treatment.

Conclusion: HL is the most common childhood cancer in the 15- to 19year-old age group. Most patients present with only painless lymphadenopathy, usually cervical or supraclavicular. Seventy-five percent have a mediastinal mass at the time of presentation with risk of SVC syndrome. Nonspecific systemic symptoms including night sweats and weight loss, classified as "B" symptoms, are important indicators of malignant disease and warrant a detailed history and complete physical examination for early detection.

617

\section{Hemato - Oncology}

Human Papilloma Virus E7-loaded Fetal Macrophages Could Trigger E7-specific Th1 Immunity against TC-1 Tumorigenesis Jeng-Chang Chen ${ }^{1}$, Liang-Shiou Ou ${ }^{I}$

${ }^{T}$ Department of Surgery, Chang Gung Children's Hospital, College of Medicine, Chang Gung University, Taiwan

Background: In our previous study, fetal exposure to ovalbumin caused Th2-skewed immunity. It was attributed to fetal macrophages that sequestered endocytosed ovalbumin, differentiated toward dendritic cells and later instruct T-cells as they fully developed to more efficiently deal with antigens.

Objective: This study was aimed to examine whether fetal macrophages could elicit Th1 immunity against tumor cells.
Methods: Fetal phagocytes from gestational day 14 C57BL/6 fetal liver and peritoneal cavity were in vitro pulsed with human papillomavirus (HPV) E7 peptides overnight.Then, they were transferred intraperitoneally into 4-6 week-old C57BL/6 mice. One month later, recipient mice were assessed for their T-cell activation by IL-2 release and T-cell polarization by IL-4, IL-5 and IFN $\gamma$ secretion in the culture system under HPV E7 stimulation using ELISA. Lymphocyte proliferation was also measured by the incorporation of tritiated thymidine. Recipients were further evaluated for their resistance to TC-1 tumor cell challenge by monitoring tumor growth and recipient survival following subcutaneous inoculation of $10^{5} \mathrm{TC}-1$ cells.

Results: After adoptive transfer of HPV E7-loaded fetal macrophages,recipient lymphocytes exhibited the activity of lymphocyte activation and proliferation specifically in response to HPV E7 stimulation as evidenced by significant IL2 secretion and tritium incorporation. Recipient lymphocytes displayed a Th1-skewed phenotype as evidenced by significant secretion of IFN $\gamma$ rather than IL-4 and IL-5 under HPV E7 stimulation. Adoptive transfer of HPV E7-loaded fetal macrophages sufficed to protect the recipients from TC-1 tumorigenesis with tumor-free survivals of $70 \%$ at 2-month follow-up, but failed to completely eliminate pre-existing TC-1 cells despite perceptible attenuation of local TC-1 tumor growth.

Conclusion: Following endocytosis of tumor antigens, fetal macrophages could trigger tumor antigen-specific T-cell immunity to preclude tumorigenesis. It shed light on the crucial role of fetal macrophages in tumor immune surveillance, thereby eliminating transformed cells egressing during embryogenesis.

\section{8}

\section{Hemato - Oncology}

Glucose-6-Phosphate Dehydrogenase Deficiency: A Case Series $\underline{\text { Sofia Cochito Sousa }}^{1,2}$, Nélia Costa ${ }^{2}$, Cristina Trindade ${ }^{2}$, Teresa Ferreira', Alexandra Dias ${ }^{2}$

${ }^{1}$ Departamento de Pediatria, Hospital de Santa Maria - Centro Hospitalar Universitário Lisboa Norte, EPE, Portugal

${ }^{2}$ Departamento da Criança e Jovem, Hospital Prof. Doutor Fernando Fonseca, EPE, Portugal

Background: Glucose-6-phosphate dehydrogenase (G6PD) deficiency is the most common human enzyme defect and has a wide variety of clinical manifestations. The highest frequencies are seen in Africa, Asia and Mediterranean region, but with world globalization it's becoming prevalent worldwide.

Objective: Characterization of a pediatric G6PDH deficient cohort followed in a suburban area of an European capital.

Methods: Retrospective study of children with G6PD deficiency in a Pediatric Hematology consultation (2013 to 2018). Record data: demographic, clinical and laboratory information.

Results: 34 children were included, most of them male (82\%) and with African ancestry (74\%). The median age at diagnosis was 1.1 years (IQR 28days-7.8years). Diagnosis was made after: acute hemolytic anemia episode $(n=13 ; 38 \%)$, neonatal hyperbilirubinemia $(n=10 ; 29 \%)$, family screening $(n=6 ; 18 \%)$, neonatal anemia $(n=3 ; 9 \%)$ or investigation of anemia $(\mathrm{n}=2 ; 6 \%)$. Median enzymatic activity was $1.3 \mathrm{U} / \mathrm{gHb}$ (range $0.3-$ $4.8 \mathrm{U} / \mathrm{gHb}$; reference $5 \mathrm{U} / \mathrm{gHb}$ ) and there was no difference between gender. Concomitantly, 3 patients had alpha-thalassemia, 2 sickle-cell disease and 2 hemoglobin Hope.

$76 \%$ had neonatal hyperbilirubinemia, $75 \%$ of these with phototherapy criterion and 1 needed exchange-transfusion; $35 \%$ had neonatal anemia and $41 \%$ had at least one episode of acute hemolytic anemia, $50 \%$ trigged by ingestion of fava beans and $43 \%$ by infection. At the acute hemolytic episode, mean minimum hemoglobin was $6.4 \pm 2.7 \mathrm{~g} / \mathrm{dL}$ and $50 \%$ needed blood transfusion. 
All children were followed in hematology pediatric consultation: one has chronic hemolytic anemia and only one (with sickle-cell disease) had another episode of acute hemolysis, trigged by infection and immune process Mutation test was performed in a minority $(\mathrm{n}=6 ; 18 \%)$.

Conclusion: In this study, neonatal period and infection were important triggers of hemolysis and high prevalence of females was found. We should keep in mind the possibility of concomitance of different causes of hemolysis and that early diagnosis is crucial to prevent future episodes of acute hemolysis and consequent morbidity.

\section{5}

\section{Hemato - Oncology}

\section{Sacro-Coccygeal Teratoma (SCT) in a Pacific Island Nation}

Douglas Greer ${ }^{1,2}$, Benjamin Wagstaff ${ }^{1,4}$, Rajay Rampersad ${ }^{1}$, Celine Hamid $^{1}$, Rooney Jagilley ${ }^{3}$, David Croaker ${ }^{1,2}$

${ }^{1}$ Department of Paediatrics and Child Health, The Canberra Hospital, Australia

${ }^{2}$ Department of Paediatric Surgery, Sydney Children's Hospital, Australia

${ }^{3}$ Department of Surgery, National Referral Hospital, Solomon Islands

${ }^{4}$ Department of Surgery, Coff's Harbour Hospital, Australia

Background: SCT is a rare tumour most common in children with quoted textbook incidences ranging from 1 in 27,000 to 1 in 40,000 live births. Several recent series suggest a higher incidence close to $1 / 10,000$. Since 2015 our unit has been providing paediatric surgical support services to a developing tropical nation, and we have also noted an incidence of approximately $1 / 10,000$.

Objective: To review the incidence and details of our series of Pacific Island SCT.

Methods: Retrospective review of SCT cases 2015- April 2019 inclusive was performed, including patient demographics, diagnosis, operation performed, and histopathology (where available). Incidence was calculated using World Bank population data. Statistical analyses were performed using Stata $\odot$.

Results: In total 8 patients presented with SCT in the study period. Mean age was 1 year (range $0-4)$. Five $(62.5 \%)$ were female. Four (50\%) were type 1 SCT, with two Type 2, and two Type 3. All eight were surgically typical of SCT, and were treated with primary excision. One is lost to follow-up. Of the eight operated on, three have been brought to Australia, and five had their operations at home. Available pathology showed benign mature SCT in all. One report has been lost, and one is awaited. Given a local birth rate of 28.7 per 1000/year, the approximate incidence of SCT regionally was 1 per 9840 births.

Conclusion: SCT remains a rare tumour, but local real incidence may be higher. Given that our observed incidence fits with the higher recently published incidences, we ask whether there has been a real worldwide increase in incidence of SCT over recent decades, or whether there is significant regional variability in incidence. We incidentally note the benefits of links between rich and poor world health services.

492

\section{Hemato - Oncology}

Parotid Swelling: A Rare Presentation of Leukemia

Nelia Gaspar ${ }^{1}$, Ana Barbara de Matos ${ }^{1}$, Marlene Salvador ${ }^{1}$, Filipa Dias Costa $^{I}$, Julieta Morais ${ }^{1}$, Ana Teixeira ${ }^{2}$

${ }^{1}$ pediatric service, Centro Hospitalar Médio Tejo E.P.E, Portugal

${ }_{2}^{2}$ pediatric service, Instituto Português de Oncologia Francisco Gentil

E.P.E, Portugal
Background: Acute leukemia represents the main cause of neoplastic disease in pediatric age. The most frequent clinical presentation of acute lymphoblastic leukemia (ALL) are hepatomegaly and/or splenomegaly, lymphadenopathy, fever, anorexia, weight loss, asthenia and sweating. Although other forms of extramedullary involvement may exist, initial parotid infiltration is very rare.

Clinical Case: A previously healthy nine-year-old boy was admitted to the emergency department due to progressive bilateral preauricular swelling over the last 10 days, with no other associated systemic symptomatology. Bilateral exuberant and painless parotid swelling was observed, with hard consistency and adhesion to the deep planes, as well as submandibular adenopathy, the larger with $3 \mathrm{~cm}$, hard and painless, without other changes on clinical examination. Blood tests showed 27810/uL leukocytes [normal (N) 5000-15000/uL], 18080/uL lymphocytes (N 1500-7000/uL), hemoglobin $9,9 \mathrm{~g} / \mathrm{dL}$ (N 11,5-15,5g/dL), mean globular volume $80,2 \mathrm{fL}$ (N 72-84fL), mean corpuscular hemoglobin 26,9pg (N 25-33pg), platelets $111000 / \mathrm{uL}$ (N 150000-500000/uL), peripheral blood smear with atypical lymphocytes, erythrocyte sedimentation rate $9,2 \mathrm{~mm} / \mathrm{h}(\mathrm{N} 0-10 \mathrm{~mm} / \mathrm{h})$, LDH $567 \mathrm{IU} / \mathrm{L}(\mathrm{N}$

Conclusion: Bilateral parotid swelling constitutes a rare initial presentation form of acute leukemia. The present case underlines the importance of clinical suspicion of neoplastic disease in the presence of parotid swelling with atypical evolution and in the absence of associated inflammatory signs.

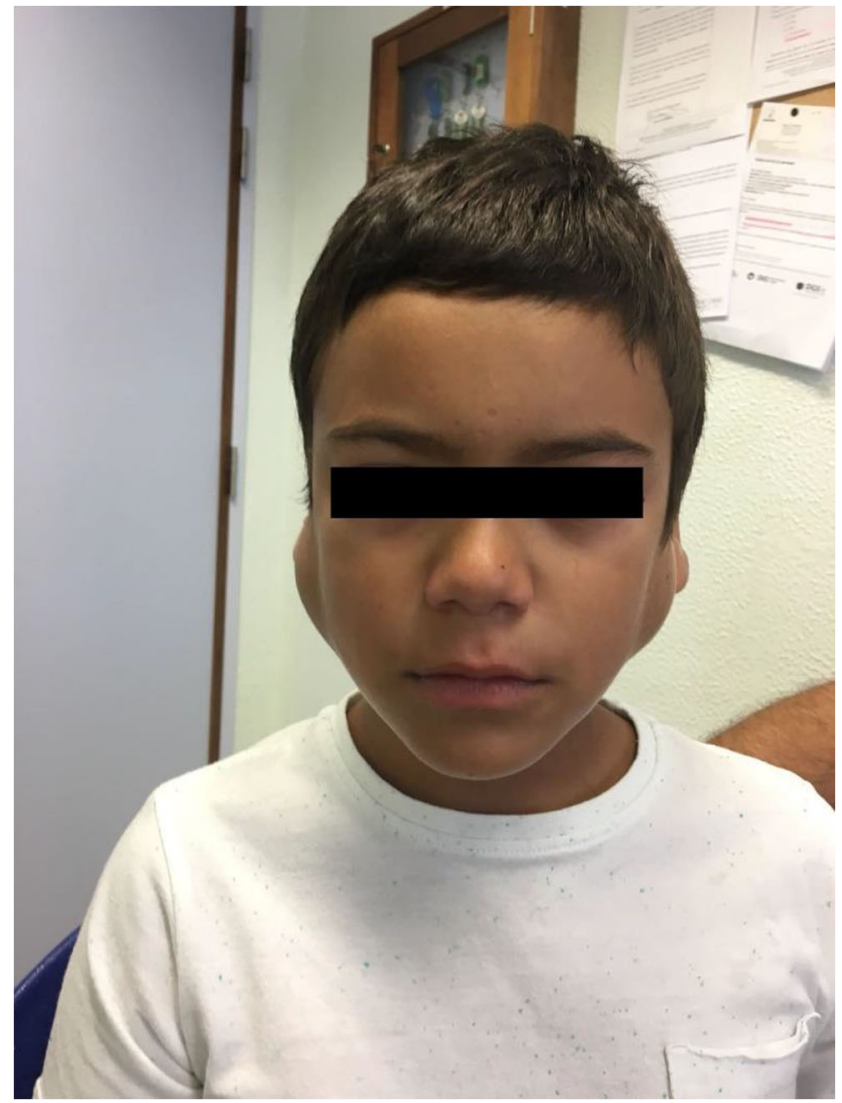


561

\section{Hemato - Oncology}

Classical Hodgkin Lymphoma: A Case Study of a Female Adolescent Presenting with Systemic Symptoms and Microcytic Anaemia Catarina Granjo Morais ${ }^{1}$, Ana Rita Curval ${ }^{l}$, Joana Pereira-Nunes ${ }^{l}$, Catarina Sousa', João Luis Barreira ${ }^{1}$, Artur Bonito Vitor ${ }^{1}$, Teresa Nunes ${ }^{1}$

${ }^{1}$ Pediatrics Department, Centro Materno Pediátrico, Centro Hospitalar e Universitário São João, Portugal

${ }^{2}$ Pediatric Oncology Department, Instituto Português de Oncologia do Porto, Portugal

Hodgkin lymphoma (HL) accounts for approximately seven percent of childhood malignancy. It should be suspected in a child with lymphadenopathy, systemic complaints and mediastinal mass. Differential diagnosis includes other malignant, infectious and inflammatory diseases.

A 12-year-old female, with a six-month background of 15 percent weight loss, night sweats and fatigue and a recent diagnosis of microcytic hypochromic anaemia (Hgb $8.2 \mathrm{~g} / \mathrm{dL}$ ), presented at our emergency department due to aggravated anaemia (Hgb $7.9 \mathrm{~g} / \mathrm{dL}$ ) after a 30-day trial of iron therapy. Respiratory, gastrointestinal and bleeding symptoms were denied. Dietary restrictions, sick contacts and family history of autoimmune diseases were excluded. On examination, she presented fever, pallor and no palpable peripheral lymphadenopathy or organomegaly. Initial laboratory results confirmed microcytic hypochromic anaemia refractory to iron therapy. Blast cells were not seen on blood film, nor other morphologic abnormalities except microcytosis and atypical lymphocytes. It was also apparent an increase of acute-phase reactants (ferritin 515.3ng/mL, ESR $118 \mathrm{~mm} /$ hour), without elevated faecal calprotectin. Abdominal ultrasound was normal, however chest radiography revealed a subtle mediastinal widening. CT scan displayed an anterior mediastinal conglomerate of lymph nodes $(8.7 \times 6.5 \times 9 \mathrm{~cm})$ and mild splenomegaly $(12.6 \mathrm{~cm})$. She was transferred to an oncology center and excisional biopsy revealed a classical HL. Bone marrow involvement was excluded on aspiration and biopsy and FDG-PET scan confirmed supradiaphragmatic nodal involvement.

About three-quarters of paediatric HL cases display a mediastinal mass on chest radiograph at the time of presentation. Mediastinal widening should never be overlooked particularly in the setting of classic B symptoms (fever, night sweats and $\geq 10$ percent loss within six months before diagnosis). It should also be noted that malignant diseases as HL can produce microcytic anaemia, which may be misinterpreted as iron deficiency. Prompt referral to an oncology center is mandatory for diagnosis, staging and management.

723

\section{Hemato - Oncology}

A Case of Severe Autoimmune Hemolytic Anemia

Maria Gutu ${ }^{I}$, Ruxandra Vidlescu ${ }^{1}$, Cristian Scurtu ${ }^{l}$, Mihaela $\overline{\text { Smarandoiu }}^{1}$, Diana Derewicz ${ }^{1,2}$, Andreea Giurgiuveanu ${ }^{1}$, Doinita Sfrijan $^{1,2}$

${ }^{I}$ Department of Pediatric Oncology, "M.S. Curie" Emergency Children's Hospital, Romania

${ }^{2}$ Department of Pediatrics, "Carol Davila" University of Medicine and Pharmacy, Romania

Introduction: We present the case of a 5 months old baby with severe hemolytic anemia.

Case presentation: The infant was admitted through transfer from another hospital with the diagnosis of severe anemia (Hemoglobin $(\mathrm{Hb})=$
$3.6 \mathrm{~g} / \mathrm{dl})$ - apparently with sudden onset, no infections or treatments administered within the last 7 days.

The clinical examination revealed a baby in poor clinical condition with intense pale skin, scleral icterus, tachycardia (150 -170 $\mathrm{b} / \mathrm{min}$ ), systolic murmur of $2 / 6$ in the V-th parasternal left space, hepatosplenomegaly.

The blood analysis indicate severe autoimmune hemolytic anemia $(\mathrm{Hb}=$ $3.6 \mathrm{~g} / \mathrm{dl}$, with high reticulocyte count, hyperbilirubinemia, hypersideremia, positive Coombs test).

The blood transfusion was postponed (due to the presence of several classes of autoantibodies - IgG 3+; IgM 2+; C3c+/-; C3d $3+$ ) and treatment with corticoids was started (first intravenously with Methylprednisolone $30 \mathrm{mg} /$ day, slowly tapered and then orally with Prednisone)

Under the treatment, the evolution was favourable - from both clinic (good general condition, normal skin colour, no jaundice or hepatosplenomegaly) and biologic point of view (with progressive increase of the hemoglobin until the normal value for the age, negative direct Coombs test and normal values of sideremia and bilirubinemia).

Conclusion: It is essential that patients with severe anemia be admitted in a department were they can be completely investigated in order to get the appropriate treatment.

\section{9}

Hemato - Oncology

Another Acute Media Otitis, Now What?

Miguel Labrusco $^{l}$, Joana dias, Joana Dias ${ }^{1}$, Sara Almeida ${ }^{1}$, Luis Rodrigues $^{2}$, Lígia Paulos ${ }^{I}$, Paulo Oom ${ }^{1}$

${ }^{1}$ Department of Pediatrics, Beatriz Ângelo Hospital, Portugal

${ }^{2}$ Department of Otorhinolaryngology, Beatriz, Angelo Hospital, Portugal

Background: Rhabdomyosarcoma is the most common soft tissue sarcoma in children, however its location on the ear or temporal bone is quite rare. We present a case report of an uncommon clinical presentation of this disease.

Clinical Case: Male child, 6 years old, healthy, presenting right ear otalgy, homolateral face oedema and lip commissure asymmetry. A suggestive examination prompted an initial diagnose of an Acute Media Otitis complicated with facial palsy.

Oral treatment was started, initially with amoxicillin $90 \mathrm{mg} / \mathrm{kg} / \mathrm{day}$ and prednisolone $2 \mathrm{mg} / \mathrm{kg} / \mathrm{day}$, after which, due to lack of improvement, with cefuroxime $40 \mathrm{mg} / \mathrm{kg} / \mathrm{day}$, and a new course of prednisolone.

Because of progressive worsening symptoms, a hospital admission was proposed for intravenous antibiotics (Ceftriaxone $100 \mathrm{mg} / \mathrm{kg} /$ day + Clindamycin $20 \mathrm{mg} / \mathrm{kg} /$ day) and corticotherapy (prednisolone $2 \mathrm{mg} / \mathrm{kg} /$ day) and further investigation.

Urgent Computerized Tomography of the ear showed a right erosive media and external otitis, with total opacification of the ear, and mastoid osteitis on various locations, and Magnetic Resonance revealed a bulky tumefactive lesion, involving the right temporal bone.

An exploratory tympanomastoidectomy was proposed, revealing a bulky obliterative polypoid lesion, that was partially removed, with pericortical, cortical, tegmen tympani and mastoid invasion from a polypoid-like neoformation submitted to biopsy. Anatomopathological examination, revealed a Rhabdomyosarcoma of the ear. Chemotherapy was started, with clinical improvement.

Conclusion: Rhabdomyosarcoma presentation symptoms can often mimic Chronic Suppurative Acute Media Otitis, making it a challenging, but important differential, as an early diagnosis can dramatically influence the overall survival rates. 


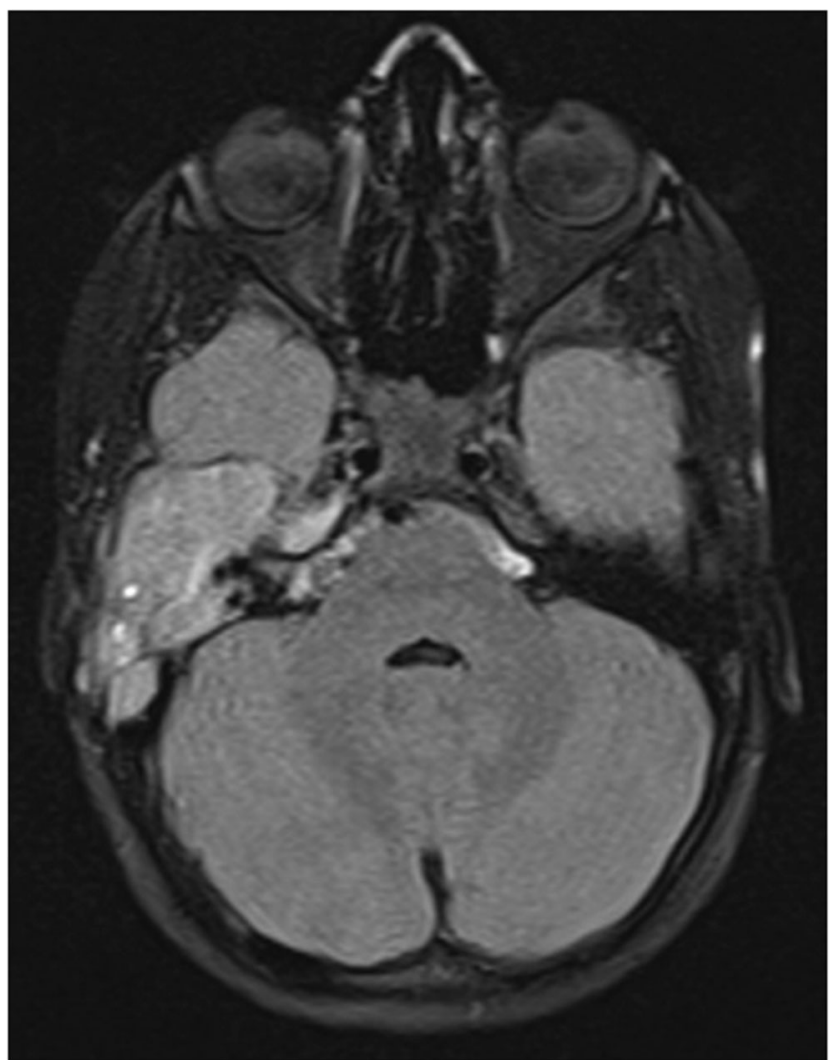

486

Hemato - Oncology

Neurotoxicity Associated with Methotrexate: An Alarming Benign Entity

Cristiana Martins $^{1}{ }^{\text {, Bruno Loureiro }}{ }^{1}$, Catarina Magalhães ${ }^{l}$, Isabel ${ }_{\text {Rodrigues }}{ }^{1}$, Mónica Costeira ${ }^{1}$, Ana Maia ${ }^{1}$, Cátia Sousa ${ }^{I}$, Tereza Oliva ${ }^{l}$

${ }^{I}$ Department of Pediatrics, Portuguese Oncology Institute Porto, Portugal

Introduction: Methotrexate is an essential drug in the treatment of children with acute lymphoblastic leukemia diseases. In this population, neurotoxicity has a prevalence range between $2.3-15 \%$ and can be characterized by a broad range of signs and symptoms (from headache to strokelike symptoms).

Case Report: We present a young girl (11-year-old) with lymphoblastic lymphoma (in phase IIA) who as a history of leukoencephalopathy, which mimicked stroke (a transient episode of mild right central facial paresis, right hemiparesis with flexion of the finger and ataxia) one week after treatment with high doses intravenous methotrexate. Brain magnetic resonance imaging revealed "probable hyperacute ischemic lesion in the territory of perforating arteries of the left middle cerebral artery"; cervical eco-doppler and Bubber test were normal; thrombophilia study at the beginning of treatment of oncologic was normal. The patient's symptoms completely improve within two days without sequels and was medicated with antiplatelet agent. Ten days after a new administration of methotrexate, she presented with right-side hemiparesis and dysarthria. Again with full resolution. The patient was discharged home without neurological symptoms and continued to the maintenance phase under prophylaxis with destometrophan ( $20 \mathrm{mg}, 3$ times daily) without intercurrences.

Conclusions: Neurotoxicity associated with methotrexate is transient and most patients continue to receive this therapy without intercurrences. Symptoms can be treated previously by administration of destometrophan and it is important that healthcare professionals recognize this entity.

547

\section{Hemato - Oncology}

ALPS: The Rare Diamond among Lymphadenopathies Inês Rosinhal ${ }^{l}$, Sónia Lemos ${ }^{2}$, Lea Santos ${ }^{1}$

${ }^{T}$ Pediatrics, Centro Hospitalar do Baixo Vouga, Portugal

${ }^{2}$ Pediatrics, Hospital Pediátrico de Coimbra; Centro Hospitalar e Universitário de Coimbra, Portugal

Introduction: Autoimmune Lymphoproliferative Syndrome (ALPS) is a rare primary immunodeficiency belonging to the subgroup of immune dysregulation diseases. It's a disorder of lymphocytic apoptosis' mediators, with subsequent generalized chronic non-malignant and noninfectious lymphoproliferation, and expansion of an unusual population of T lymphocytes (double-negative T cells). Chronic lymphadenomegaly and/or splenomegaly and autoimmune cytopenias are common characteristics.

Clinical Case: Four-year-old male, without personal relevant background, observed in paediatric consultation, due to cervical lymphadenopathy detected by two years of age, exacerbated in infectious contexts and in the last six months. Splenomegaly noticed during an acute febrile episode. Without constitutional symptoms. Maternal family history: autoimmune haemolytic anaemia. At physical examination, he had elastic and mobile cervical bilateral adenopathy conglomerates and splenomegaly. Complementary diagnostic tests (in fasting and without disease): Cervical echography: multiple ganglionic formations probably of reactive nature. Abdominal ultrasonography: globous liver and splenomegaly, with multiple ganglionic formations adjacent to splenic thread and pancreas. Blood test: Haemoglobin $12,3 \mathrm{~g} / \mathrm{dL}$, Total leucocytes $6,4 \times 10^{9} / \mathrm{L}$, Platelets $144 \times 10^{9} / \mathrm{L}$ (150-450), Peripheral blood smear "reactive 
lymphocytes and slight anisocytosis", C-reactive protein $0,46 \mathrm{mg} / \mathrm{dL}$, Cholesterol HDL $18 \mathrm{mg} / \mathrm{dL}$ (45), Triglycerides $207 \mathrm{mg} / \mathrm{dL}(\leq 90)$, IgA $197 \mathrm{mg} / \mathrm{dL}, \mathrm{IgG} 2150 \mathrm{mg} / \mathrm{dL}$ (350-1620), IgM $20 \mathrm{mg} / \mathrm{dL}(30-$ 265).Considering the diagnosis of ALPS, he was sent to a Tertiary Hospital's primary immunodeficiency consultation. The subsequent study reveals: B12 8124 pg/mL (295-1769); Immunophenotyping 10\% lymphocytes $\alpha / \beta C D 3+C D 4-C D 8-;$ Genetic study: Fas deficit heterozygosity for mutation c.817CA (p.Q273K).

He completed two prednisolone cycles. Currently (7 years old), under sirolimus, with frank improvement in lymphadenopathy dimensions and hepatosplenomegaly.

Conclusion: This clinical case illustrates the importance of the high level of clinical suspicion regarding generalized chronic lymphadenopathies and/or splenomegaly. Despite ALPS's non-malignant nature, the diagnosis may be quite challenging and requires long-term clinical surveillance, particularly because of the increased risk of malignancy.

\section{0}

\section{Hemato - Oncology}

Osler-Weber-Rendu, an Early Presentation

Cristina Rodrigues ${ }^{l}$, Joana Vilaça ${ }^{I}$, Ana Ribeiro ${ }^{l}$, Joana Macedo ${ }^{l}$, Helena Silva', Almerinda Pereira ${ }^{l}$

${ }^{I}$ Department of Pediatrics, Hospital de Braga, Portugal

Background: Osler-Weber-Rendu syndrome is a rare autosomal dominant disease characterized by telangiectasias and arteriovenous malformations, which may affect multiple organs. Symptoms develop over time and are more likely to appear in adulthood.

Case Presentation: Ten-year-old girl with a medical history of atopic dermatitis, Henoch-Schonlein purpura at 3 years of age and positive Phadiatop test for inhalant allergens. Her mother, uncle and maternal grandmother had Osler-Weber-Rendu syndrome. At the age of 8 presented frequent spontaneous epistaxis, several times a week, even while sleeping, without seasonal predominance. There were no visible telangiectasias and no anemia was detected. Right anterior rhinoscopy showed vascular ectasia. It was decided in Genetics appointment to perform molecular study, which confirmed the presence of the family mutation of the gene ACVRL1 C. $1232 \mathrm{G}$ A p.arg411Gln, at 9 years old. The results of abdominal and vesical ultrasound, electrocardiogram and echocardiography were normal. She had menarche at the age of 10 years. At 10 years old she was evaluated because of easy fatigability, mainly at afternoon. Bilateral vascular ectasias throughout septal mucosa were detected at anterior rhinoscopy. Blood tests indicated an iron deficiency anemia $(\mathrm{Hb} 10.1 \mathrm{~g} / \mathrm{dl}$, with presence of target cells in peripheral blood smear and ferritin $3 \mathrm{ng} / \mathrm{ml}$ ). She started iron oral supplementation $6 \mathrm{mg} / \mathrm{Kg} /$ day. One month later control hemogram indicated an hemoglobin rise to $13.3 \mathrm{~g} / \mathrm{dl}$ and ferritin $16 \mathrm{ng} / \mathrm{mL}$. Actually she is waiting for a contrast echocardiogram, brain angio-magnetic resonance imaging and new abdominal ultrasonography for arteriovenous malformations screening.

Discussion: Epistaxis is the most common symptom of the disease. If it appears in pediatric age, it is typically after 10 years old, and rarely requires invasive interventions to control bleeding. This child presents an exuberant phenotype, and therapeutic experience is scarce in the approach of the pathology at such an early age.

1046

Hemato - Oncology

A Rare Case of Neuroblastoma Presenting with Skin Findings Sirin Sonmez ${ }^{l}$, Uzuner Selcuk ${ }^{l}$, Anil Gulsel BahaliI ${ }^{2}$, Fatma Betul Cakir ${ }^{3}$, Tahaoglu Irmak

${ }^{1}$ Department of Pediatrics, Bezmialem Vakif University, School of Medicine, Turkey
${ }^{2}$ Department of Dermatology, Benzmialem Vakif University School of Medicine, Turkey

${ }^{3}$ Department of Pediatrics, Division of Pediatric Hematology and Oncology Disease, Benzmialem Vakif University School of Medicine, Turkey

Neuroblastoma is the most common extra-cranial solid tumor in infants and children, Neuroblastoma tends to behave quite differently in infants as compared to children over 12-18 months.

We present a rare case of neuroblastoma presenting with skin rash as a preliminary finding.

Case:

A 39-day-old male patient with no medical history was admitted to our hospital with yellow bullous rash that started on the face and spread throughout the body in 15 days. The patient was born at term by caesarean section. His neurologic development is appropriate for his age. There is no family history and his parents are not related. On physical examination, he had yellow bullous rash all around his body, inspiratory stridor was auscultated and respiratory distress was seen.

The patient was consulted to dermatology clinic for his bullous rash and skin biopsy was performed. Bullous pemfigoid was detected pathologically, which is rare condition in infancy. Because the patient had inspiratory stridor and respiratory distress chest X-Ray was performed and reported normal. For further evaluation the patient was consulted to pediatric pulmonary specialist and with their suggestion thorax CT was performed. A solid mass that was suspected to be a neuroblastoma was found. So we performed abdominal MRI, and taken tru cut biopsy and determined neuroblastoma. İmmunoglobulin levels and lymphocyte subgroups were analyzed and found normal due to suspicion of immune deficiency.

Conclusion: Rashes are extremely common in infancy. Most of them are innocent and transient. However, serious infectious, congenital skin diseases and sometimes malignant tumors should be kept in mind when dealing with a rash in infancy.

We recommend that every infant with a skin rash and respiratory distress should be investigated for a infancy neuroblastoma.

The clinician should keep in mind that skin rash may be the first clinical finding of neuroblastoma.

288

Hemato - Oncology

Dental Morbidity in Children with Iron Deficiency Anemia Svetlana Tuleutayeva ${ }^{1}$, Bibigul Abeova ${ }^{2}$, Zhadyra Ashirbekova ${ }^{3}$, $\overline{\text { Kulzhakhan Shauyenova }}^{4}$, Aigul Kurdabayeva ${ }^{5}$

${ }^{I}$ Dentistry, Medical university of Karaganda, Kazakhstan

${ }^{2}$ Children Diseases, Medical university of Karaganda, Kazakhstan

${ }^{3}$ Dentistry, Medical university of Karaganda, Kazakhstan

${ }^{4}$ Dentistry, Medical university of Karaganda, Kazakhstan

${ }^{5}$ Dentistry, Medical university of Karaganda, Kazakhstan

Study of the dental disease prevalence in children with iron deficiency anemia (IDA).

Objective: to study the prevalence of caries in children with type iron deficiency anemia

Materials and Methods: A study of 1,500 people of children and adolescents in rural areas of Karaganda region aged from 3 to 17 years with IDA and dental diseases was conducted. $765(51 \%)$ of them are boys and $735(49 \%)$ are girls. Total number of children with IDA was $812(54 \%$ of the total number surveyed). Mild IDA was in 288 (35.5\%) children, moderate - in $181(22.3 \%)$, severe - in $51(6.4 \%)$.

Results: The prevalence of caries was $69.4 \%$ in patients with anemia, the prevalence of periodontal disease was $39 \%$, dental-anomalies occurred most of all in children with anemia - 96,1\%. The highest prevalence of caries in children with anemia was observed in preschool (73.9\%) and 
younger school age (77.2\%). In IDA, a mildly chronice form of caries was found in $54.3 \%$ of cases, the subacute form of caries was $9.51 \%$, and the acute form - in $0.84 \%$. With moderate IDA $39.1 \%, 14.8 \%$ and $12.2 \%$ respectively. When severe IDA $-32.4 \%, 32.4 \%$ and $19.1 \%$, respectively. With an increase in the severity of IDA, a dynamic increase in caries was observed, indicating a direct correlation.

Conclusions: The prevalence of caries in 3-6 year old children on the background of IDA is $75.6 \%$ and without IDA - 71.3\% (p 0.001), in 7-11 year old children $79.2 \%$ and $74.9 \%$ ( 0.001 ), in $12-17$ year old children $52.6 \%$ and $52.6 \%$ (p 0.001 ), respectively.

\section{5}

\section{Infectious Diseases}

Predicting Factors for Hospitalization due to Odontogenic Infections in the Pediatric Population

Mary Abdo $^{1}$, Michael Joachim ${ }^{2}$, Said Abozaid ${ }^{1}$, Yasmine Ghantous ${ }^{2}$, $\overline{\text { Wael Nasser }}^{1}$, Imad Abu El Na'aj ${ }^{2}$

${ }^{1}$ Department of Pediatrics, Baruch Padeh Medical center, Poriya affiliated to the Faculty of medicine in the Galilee Bar-Ilan University, Israel ${ }^{2}$ Oral and Maxillofacial Surgery Department, Baruch Padeh Poriya Medical Center, Israel

Background: Orofacial infections of odontogenic origin are a common and important health concern in the pediatric population. Incorrect or late treatment may lead to serious and possibly life threatening complications. Objective: The aim of this study is to analyze the data on pediatric hospitalizations due to odontogenic infections and to isolate parameters predicting prolonged length of stay (LOS).

Methods: Data was collected from the records of patients (age 15 years) who were hospitalized due to odontogenic infections in Pediatric/ Oral and maxillofacial surgery department in Baruch Padeh Poriya Medical Center- Israel between 1/2010-12/2015. Facial infections of other origin was excluded.

Results: A total of 411 patients were included. $58.7 \%$ of them were males, $66.9 \%$ of non-Jewish ethnicity. Roughly half were discharged after one day of hospitalization. $42.8 \%$ of patients were not previously treated with antibiotics. $79.8 \%$ of patients did not present $38^{\circ}$ body temperature at admission and $98.8 \%$ of them were treated by drainage with or without extraction of the involved tooth. $91 \%$ of patients received antibiotics after discharge and $4.6 \%$ were admitted for rehospitalization. The calculated OR for odontogenic infections in patients from Arab background was 2.5 significantly higher ( $\mathrm{p} 0.0001)$ than their Jewish counterparts. The mean age in the study was $6.53 \pm 2.59$ years. There was significantly higher (P0.05) values of White Blood Cells (WBC) and platelets in patients with 3 days LOS, patients with 3 days LOS were significantly older (8.36 vs 6.28) and OR for a prolonged LOS in Arab patients was 2.55 comparing to Jewish patients $(\mathrm{p}=0.01)$.

Conclusion: Risk factors for prolonged LOS were: older age, Arab ethnicity and higher WBC count and platelets values at admission.

\section{8}

\section{Infectious Diseases}

Viral Pathogen Association with Community Acquired Pneumonia in Hospitalized Children- Report from the State of Qatar Manasik Hassan ${ }^{1,2}$, Amal Alnaimi ${ }^{2}$, Aji Varughese ${ }^{1}$, Magda Youssef ${ }^{2}$, Ahmed Alhammadi ${ }^{2}$

${ }^{T}$ Peditaric, Hamad Medcial Corporation, Qatar

${ }^{2}$ Peditaric, SIDRA Medicine, Qatar
Background: Community acquired pneumonia is the leading cause of childhood morbidity and mortality globally, although the etiology of CAP varies by age, viruses remain the most etiologic agent and may lead to sever disease especially in children with co-morbidities. Data on community acquired pneumonia in children were found to be limited in Qatar.

Objectives: This study aimed to determine common virus associated with CAP in hospitalized children and to identify risk factor for pediatric intensive care unit (PICU) admission.

Methods: A cross-sectional single institutional retrospective descriptive study was performed at Hamad Medical Corporation, the only tertiary and academic medical center in the State of Qatar. The study included children admitted with communityacquired pneumonia from Dec 2017-June 2018, details of demographic and respiratory viral as detected by molecular assays were analyzed

Results: Total of 59 hospitalized children with diagnose of CAP between 3 months-14 years were included,mean age at diagnosis 3 years. 43 patients $(73 \%)$ had positive PCR nasal swab,single virus isolated in 31 case,multiple viruses in 12 cases. The most isolated virus was Rhinovirus(21\%),Human Meta-pneumovirus(17\%),Boca virus(15\%),H1N1 influenza virus(8\%),Respiratory Syncytial Virus and corona viruses were the least common with(7\%) each. Nearly $20 /$ $59(33 \%)$ of children admitted to PICU,15(25\%) had positive viruses in which the most frequent were H1N1influnza and Rhinovirus with longer PICU stay (median 10 days). Common risk factor associated with PICU hospitalization;prematurity and bronchial asthma.

Conclusions: Our study revealed that Rhinovirus and HMP Virus were the most common causative agents in hospitalized children with CAP. This finding in contrasts with a previous report from WHO in which RSV was the most frequently isolated virus. H1N1influanza virus play a major role in PICU admission, we need to increase public and healthcare providers knowledge to annual vaccine.

213

\section{Infectious Diseases}

The Journey to the Pediatric Intensive Care Unit Following Vaccine Refusal

Nukhet Aladag Ciftdemir ${ }^{1}$, Ridvan Duran ${ }^{1}$, Tuba Eren ${ }^{1}$, Mustafa Torehan Aslan'

${ }^{I}$ Department Pediatrics, Trakya University Faculty of Medicine, Turkey

Background: Vaccines are the most effective public health interventions against many vaccine-preventable diseases. In recent years, the number of parents refusing recommended childhood vaccination increased significantly. The concept of increasing vaccine refusal has emerged in the world in the 1990s and in Turkey in the beginning of 2010. Here, we report a clinical case of an infant with severe pertussis with vaccinerefusing parents.

Case Presentation: A 13 months-old girl was admitted to emergency department with a 10-day history of cough, which was gradually progressive. She was diagnosed with viral upper respiratory tract infection in another hospital. On examination, body temperature was $38.2^{\mathrm{c}}$, oropharynx was hyperemic and bilateral crackles were detected by lung auscultation. The remaining findings on her physical examinations were normal. Laboratory investigations showed the following results: WBC: $66.900 / \mathrm{mm} 3$ (80\% lymphocyte), CRP negative, ESR $19 \mathrm{~mm} / \mathrm{h}$, blood biochemistry, liver and kidney function tests were normal. Bilateral infiltrations were seen on chest radiography. Patient was diagnosed with pneumonia, and 
treated with intravenous ceftriaxone. Pertussis was suspected because of the infant's persistent cyanotic cough and apnea, intravenous clarithromycin was added to antibiotherapy. Two days later, her WBC count had increased to $79.800 / \mathrm{mm} 3$ (85\% lymphocyte). No tumor cells was detected in peripheral blood smear. She was transferred to the pediatric intensive care unit with, generalized tonic seizure after coughing. Brain computed tomography examinations and EEG were normal. The culture of nasopharyngeal secretions and polymerase chain reaction (PCR) were both positive for B. Pertussis. During his follow-ups, on 14th day of hospitalization, her WBC count gradually dropped, physical examination was normal and she was discharged from the hospital. The patient had been given all the missing vaccinations in the follow-up after discharge of the baby as a convinced family vaccination after discussing her illness with her parents and explaining all the facts of the event.

Conclusion: Children of parents who refuse immunizations are at increased risk of in for vaccine-preventable diseases. Vaccine refusal also increases the risk for the whole community. All parents should be informed about the benefits of vaccination and risk of severe infection deseases.

\section{6}

\section{Infectious Diseases}

\section{Intermittent Irritability in a Toddler}

Susana Alexandre ${ }^{1}$, Pedro Fernandes ${ }^{3}$, Ana Mouzinho ${ }^{2}$, Mafalda Castelão $^{2}$, Catarina Gomes $^{1}$, Sara Santos

${ }^{1}$ Paediatrics, Centro Hospitalar do Oeste - Caldas da Rainha, Portugal ${ }^{2}$ Paediatrics - Infecciology Unit, Centro Hospitalar Lisboa Norte Hospital Santa Maria - Centro académico de Lisboa, Portugal

${ }^{3}$ Orthopedic Unit, Centro Hospitalar Lisboa Norte - Hospital Santa Maria - Centro académico de Lisboa, Portugal

Background/Objective: Spondylodiscitis is a rare entity in pediatrics. The low specificity of symptoms and laboratory data, the scarcity of physical examination findings and the late radiographic changes justify the usually late diagnosis.

Methods: 15-month-old boy with irrelevant personal and family history. Presented to our emergency department with notion of pain in the previous 15 days, characterized by periods of polypnea and irritability. Progressive worsening in the previous week, with nocturnal awakening, improvement with ibuprofen, and antalgic position in dorsal decubitus or lumbar hyperextension, with gait refusal in the last days. History of diarrhea 2 weeks before admission. He looked prostrate, complaining while sitting but had no pain in passive mobilization of the lower limbs or in palpation/percussion of the spine. He started fever at the hospital that persisted for 2 days. The blood panel showed ESR 55mm and ALP $1076 \mathrm{U} / \mathrm{L}$ and no increase in CRP or leukocytosis. CT showed vertebral irregularity of D9-D10 with possible relation to bone destruction secondary to spondylodiscitis.

He was transferred to a tertiary hospital, where the MRI confirmed the diagnosis.

Serologies for Brucella, Bartonella and Coxiella, blood culture, IGRA and PCR for bacterial DNA were negative. After 8 days of intravenous ceftriaxone IV he was asymptomatic and was discharged with oral amoxicillin and clavulanic acid for 5 weeks.

Conclusion: Spondylodiscitis usually has an insidious onset of symptoms. Although children may report low back and walking pain, infants may initially only show nonspecific signs such as irritability. Therefore, it is important to remember this illness, especially as it may have serious complications. MRI is the key standard for an early diagnosis and followup of disease progression.

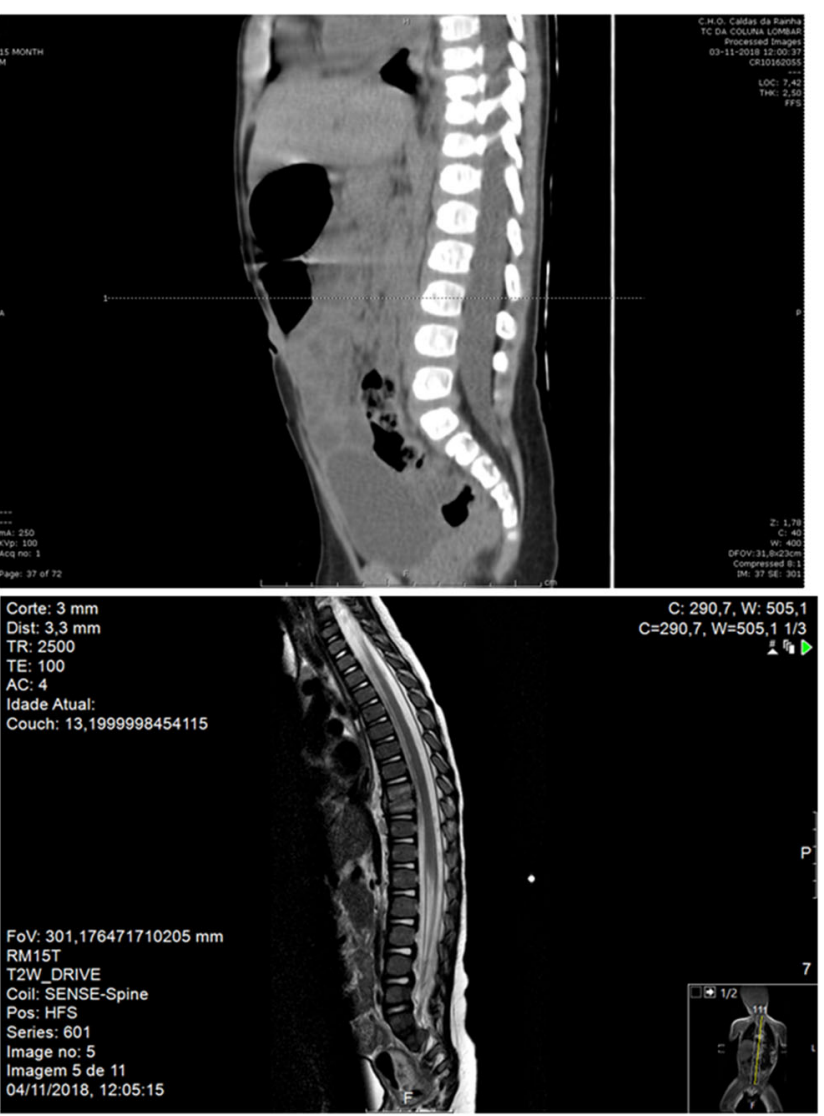

805

Infectious Diseases

\section{Review of CNS Abscess in Children}

Jose Andrade Guerrero ${ }^{1}$, Serafin Castellano Dámaso ${ }^{1}$, Marta Bautista Barea $^{\prime}$, Ines de Augusto Claudino ${ }^{2}$, Mariano Silva Hernández ${ }^{1}$, Virginia Santana Rojo ${ }^{1}$, Marta Dorado Criado ${ }^{1}$, Celia Fabra Garrido ${ }^{1}$, Fernando Carceller Benito ${ }^{3}$, Javier Saceda Gutiérrez ${ }^{3}$, María Laplaza González, Jesus Domínguez Riscart ${ }^{1}$, Carmen Román Hernández ${ }^{4}$, Miguel del Rio Garcia $^{4}$, Marta Garcia-Fernández de Villalta ${ }^{1}$, Francisco Climent Alcalál$^{l}$, Ana Méndez Echevarríal, Luis Alonso Garcial ${ }^{l}$, Cristina Ots Ruiz $^{1}$, Fernando Baquero-Artigao ${ }^{1}$, María Fátima Ara Montojo ${ }^{1}$, Luis Escosa Garcia ${ }^{I}$, Francisco Javier Aracil Santos ${ }^{I}$

${ }^{1}$ General Pediatrics and Infectious Diseases, Hospital Universitario La Paz, Spain

${ }^{2}$ General Pediatrics and Infectious Diseases, Centro Hospitalar Oeste, Portugal

${ }^{3}$ Pediatric Neurosurgery, Hospital Universitario La Paz, Spain

${ }^{4}$ Pediatric Intensive Care Unit, Hospital Universitario La Paz, Spain

Background: CNS abscesses are rare and severe conditions. These infections are categorized based on their location: epidural abscess, subdural empyema and brain abscess. Treatment requires a combination approach with antibiotic therapy and frequently surgical drainage.

Objective: To describe the clinical manifestations, predisposing factors, diagnosis, treatment and outcome of pediatric patients with CNS abscesses

Methods: Retrospective single-center study of pediatric patients with CNS abscesses in the 2000-2018 period. Neonatology patients were excluded. 
Results: A total of 32 patients were included in the study (brain abscess $31 \%$, subdural empyema $31 \%$, brain + subdural $6 \%$, epidural abscess $25 \%$ ). Age range was 1,5 months to 16 years (median 6 years). Male/female ratio was 2:1. Predisposing factors were identified in all patients. Most important predisposing factors were mastoiditis and sinusitis $(56 \%)$, complex congenital cardiopathy $(16 \%)$ and bacterial meningitis (13\%). Etiologic agents were identified in 18 patients (Staphylococcus aureus $28 \%$; Streptococcus pneumoniae 28\%; Streptococcus intermedius $22 \%$; gram-negative bacilli $17 \%$ ). Fever, headache, earache, vomits and seizures $(41 \%)$ were the signs and symptoms most often described. Half of patients underwent surgery of the CNS abscess (brain abscess $75 \%$, epidural abscess 25\%). Two main initial antibiotic schemes were used, cefotaxima-vancomycinmetronidazole (42\%) and meropenem-vancomycin (48\%). More frequently used oral antibiotics were quinolones, metronidazole and linezolid. Median of duration of antibiotic treatment (intravenous + oral) was 69 days (range 40-120). One patient died and seven had permanent sequels.

Conclusions: Congenital heart diseases and otorhinolaryngological infections were the main underlying disorders. Antibiotic therapy was enough to resolve small epidural abscesses and small subdural empyema. But most patients with brain abscesses and big subdural empyema also required surgical treatment.

\section{5}

\section{Infectious Diseases}

Drug Related Adverse Events During Treatment of CNS Abscess in Children

Marta Bautista Barea ${ }^{1}$, Serafín Castellano Dámaso ${ }^{1}$, Jose Andrade Guerrero $^{I}$, Ines de Augusto Claudino ${ }^{2}$, Mariano Silva Hernández, Virginia Santana Rojo ${ }^{I}$, Marta Dorado Criado ${ }^{I}$, Celia Fabra Garrido ${ }^{I}$, Fernando Carceller Benito ${ }^{3}$, Javier Saceda Gutiérrez, María Laplaza González ${ }^{4}$, Jesus Domínguez Riscart ${ }^{1}$, Miguel del Rio Garcia ${ }^{4}$, Carmen Román Hernández ${ }^{4}$, Marta Garcia-Fernández de Villalta ${ }^{l}$, Francisco Climent Alcalàl, Ana Méndez Echevarríal, Luis Alonso García ${ }^{l}$, Cristina Ots Ruiz ${ }^{l}$, Fernando Baquero-Artigao ${ }^{1}$, Luis Escosa Garcial, María Fátima Ara Montojo ${ }^{l}$, Francisco Javier Aracil Santos ${ }^{1}$

${ }^{1}$ General Pediatrics and Infectious Diseases, Hospital Universitario La Paz, Spain

${ }^{2}$ General Pediatrics and Infectious Diseases, Centro Hospitalar Oeste, Portugal

${ }^{3}$ Pediatric Neurosurgery, Hospital Universitario La Paz, Spain

${ }^{4}$ Pediatric Intensive Care Unit, Hospital Universitario La Paz, Spain

Background: Treatment of brain abscesses and other intracranial infections requires long lasting antibiotics, glucocorticoids and antiepileptic therapy. Antibiotics with good diffusion to CNS and inside abscesses are the preferred ones. Medicaments related adverse events are frequent in these patients. Generally, they force to change the planned treatment.

Objective: To describe drugs related adverse events during treatment of pediatric patients with CNS abscesses.

Methods: Retrospective single-center study of pediatric patients treated for CNS abscesses.

Results: A total of 32 patients were included in the study (20002018). Presented with brain abscess $31 \%$, subdural empyema $31 \%$, brain abscess + subdural empyema $6 \%$ and epidural abscess $25 \%$. Age range was 1,5 months to 16 years (median 6 years). Male/female ratio was $2: 1$.
Usually, these patients received sequential intravenous and oral treatment. Median of duration of antibiotic treatment (intravenous + oral) was 69 days (range 40-120). Intravenous treatment median was 28 days (range 7-90 days). Received glucocorticoids $47 \%$ and antiepileptic drugs $62 \%$.

Three patients developed neutropenia (related to linezolid and cotrimoxazole). Four patients developed exanthems. Three patients developed DRESS syndrome (one due to phenytoin and two related to antibiotics). Two patients had knee arthritis related to fluoroquinolones. One patient developed linezolid related peripheric neuropathy. Cushing syndrome was related to prolonged glucocorticoid therapy.

Conclusions: Patients treated for CNS pyogenic infections frequently develop drug related adverse events. Careful monitoring of blood counts, liver and renal function and skin disorders is mandatory.

435

Infectious Diseases

Intelligent IT System Developed to Reduce the Risk of TORCH Congenital Infections

Andreia Florina Nita $^{1,2,3}$, Loredana Sabina Cornelia Manolescu ${ }^{1,2}$, Gabriela Loredana Popa ${ }^{1,2,4}$, Mircea Ioan Popa ${ }^{1,2}$

${ }^{I}$ Microbiology, Carol Davila University of medicine and Pharmacy, Romania

${ }^{2}$ Microbiology, Cantacuzino National Medico-Military Institute for Research and Development, Romania

${ }^{3}$ Paediatrics, Grigore Alexandrescu Emergency Hospital for Children, Romania

${ }^{4}$ Parasitology, Colentina Clinical Hospital, Romania

Introduction: TORCH congenital infections have a substantial burden of disease, leading to non-genetic, preventable long-term sequelae. Effective interventions for prevention of mother-to-child transmission of TORCH infections are not implemented in Romania. Furthermore, there is a lack of national protocols and guidelines regarding screening and diagnosis of TORCH congenital infections.

Methods: The current project was carried out as a collaboration between medical experts from Carol Davila University of Medicine and Pharmacy and an information technology team from Stefan S Nicolau National Institute of Virology. The project was supported from UEFISCDI Bridge Grant: PN-III-P2.1-BG-2016-0384 and performed during 20162018.

Results: We have developed an intelligent system for pregnant women and medical staff, gathering information about the infections tests in TORCH panel and enclosing algorithms for correct screening and diagnosis of TORCH infections in order to reduce the risk of congenital infections. Using practical and non-invasive electronic resources widely available such as smartphones, the authors have created a website portal (https://www.torch.ro/) and an application for mobile phones (MAMI) which allows women to have quick access to correct information about TORCH infections and tests and more important, to obtain an individualized answer regarding TORCH screening and diagnosis which can contribute to further planning and case management carried out by the physician.

Conclusion: TORCH-IT portal and MAMI app for smartphone, developed to reduce the risk of congenital infections while helping to obtain a correct diagnosis and management, are expected to become valuable resources for both the patient and the physician, as they are unique in Romania. 


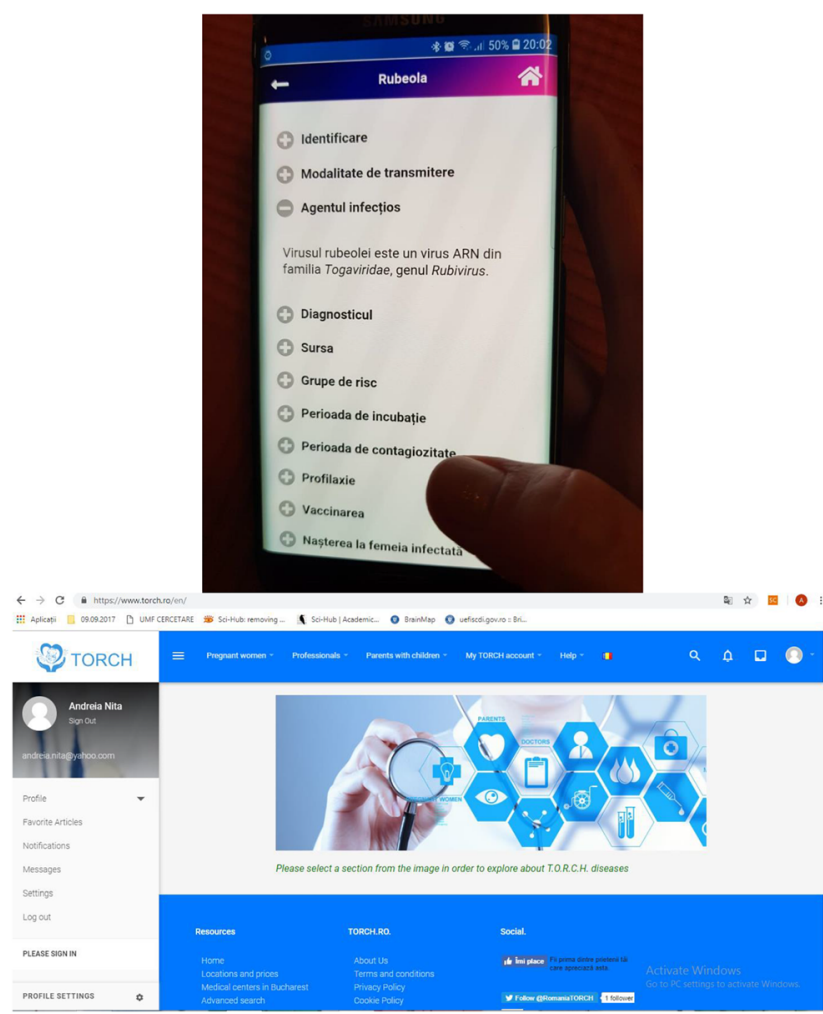

161

\section{Infectious Diseases}

\section{Scalp Cellulitis of Unexpected Cause}

Ruxandra Simona Bacanu ${ }^{1}$, Vianney Marienela Cuba Mamani ${ }^{2}$

${ }^{T}$ Pediatrics, General Hospital Tomelloso, Spain

${ }^{2}$ Emergency Department, General Hospital Tomelloso, Spain

Background: Cellulitis is usually caused by a bacterial infection of an area of skin that is no longer intact. Other causes may include human or animal bites, or injuries that occur in water.

Aim of Paper: To demonstrate that there are weird causes that lead to a cellulitis and the importance of a exhaustive differential diagnosis.

Matherial and Method: We present the case of a 5 years old girl who arrived to Emergency Room with scalp cellulitis. Six days before, the parents noticed the presence of a wart in the girl's scalp that during later days associated pain and redness in the area, accompanied by fever. The girl was attended in Primary Care and was in treatment with Azitromicin. The girl did not had history of trauma or other event. The physical exam revealed a healthy child with a painfull, red scalp area about $5 \mathrm{~cm}$, covered by a crust.

We asked for urgent blood tests which revealed elevated acute phase reactants. It has been iniciated intravenous treatment with Augmentine. 2 days later, after scratching, expels a tick.

We asked for complete serology tests for Rickettsia and Borrelia, that luckily were negative. The microbiology test informed us about the infection of the bite area with Pantoea agglomerans.

The patient had a favorable evolution. After 2 months the ulcer has healed completely but with the persistence of a central scarring alopecia.

Disscusion: As we previous comment, there were no history of trauma or other event. The presence of a tick was a surprise for everybody (the tick was previously interpreted as a wart). The luck was that by scratching it fell into bed, and also that all the serology tests were negative.

Conclusion: We must always think of all the possible etiologies, no matter how weird it would seem to us.

615

Infectious Diseases

A Peculiar Scalp Lesion

Gabriela Botelho $^{I}$, Susana Gomes ${ }^{1}$

${ }^{T}$ Pediatria, Hospital do Espirito Santo de Évora, Portugal

Background: Tinea is a fungal infection and it is an increasing problem worldwide. Kerion is a severe manifestation of tinea capitis developing from an intense immune response to the infection, which is less common than the non-inflammatory variants. It can often mimic other conditions, leading to inaccurate diagnosis and delay in treatment with scarring alopecia.

Objective: Our goal is to report a case of atypical presentation of tinea capitis and highlight the importance of a careful clinical history and examination and timely diagnosis.

Methods: We analyzed the atypical presentation of a tinea capitis scalp infection and reviewed the literature.

Results: A three-year-old girl presented with a painful, soft plaque in the scalp, $3 \mathrm{~cm}$ of larger diameter, with thick crusting, pustules, dark spots, broken hair strands and some drainage areas, evolving for two weeks. She had no other skin lesions nor fever. There had been no trauma, contact with animals nor contacts with similar lesions. After inspection of the lesion, our hypothesis was a kerion. Empirical treatment with oral fluconazole and topic cetoconazol was begun. There were not any lasting lesions nor scarring, after four weeks of treatment.

Conclusion: Kerion is often misdiagnosed. Our choice of antibiotic was fluconazole and cetoconazol, as griseofulvin is not available in our country and terbinafine is not available as oral solution. Timely diagnosis and treatment led to recovery without scarring or residual alopecia.

\section{1}

\section{Infectious Diseases}

Evaluation of Antiviral Therapy for Influenza and Influenza-Like Diseases in Risk Groups

Vilya Bulgakova ${ }^{1}$, Natalya Pshenichnaya ${ }^{2}$, Artem Poromov $^{3}$, Antonina Grekova $^{4}$, Elena Kareva ${ }^{5,6}$

${ }^{1}$ Institute of Pediatrics, National Medical Research Center of Children's Health, Russia

${ }^{2}$ Scientific Department, Central Research Institute of Epidemiology, Russia

${ }^{3}$ Scientific Department, Lomonosov Moscow State University, Russia

${ }^{4}$ Department of Infectious diseases, Smolensk State Medical University, Russia

${ }^{5}$ Department of Pharmacology, Pirogov Russian National Research Medical University, Russia

${ }^{6}$ Department of Pharmacology, I.M. Sechenov First Moscow State Medical University, Russia

Background: Bacterial complication and prolonged course of influenza and influenza-like illnesses (ILI) are often observed among patients with risk factors for severe course of disease. The aim of study was to evaluate the efficacy of antiviral therapy with arbidol (umifenovir) in hospitalized patients with influenza and ILI who had risk factors for severe course of disease. 
Methods: 3285 medical charts of patients with influenza and ILI have been retrospectively reviewed. Laboratory confirmation of disease was available in $31.0 \%$ patients; of these, influenza viruses were found in $60.7 \%$ samples (influenza A/H1N1 - 48.8\%, A/H3N2 - 16.3\%, influenza A subtype $-10.6 \%$, influenza B - in $24.3 \%$, other respiratory viruses $39.7 \%$ ). Patients were divided into 2 groups depending on therapy. Antivirals were not administered to patients in the 1th group. 2th group of patients was treated with umifenovir from the first 24-48 hours after symptom onset. Patients in subgroups $1 b(n=155)$ and $2 b(n=628)$ had risk factors (children younger than 2 years old and adults over 65 , pregnant women, people with chronic somatic diseases and obesity).

Results: Duration of fever and frequency of complications in patients without risk factors were significantly lower in 2a group versus 1a group: $2.86 \pm 1.57$ days and $3.76 \pm 2.83$ days, 13.1 $\pm 1.3 \%$ and $20.0 \pm 3.2 \%$, accordingly. Duration of fever and frequency of complications in patients with risk factors were significantly lower in $2 \mathrm{~b}$ group versus $1 \mathrm{~b}$ group: $3.0 \pm 1.48$ days and $4.1 \pm 2.2$ days, $9.9 \pm 1.2 \%$ and $42.6 \pm 3.6 \%$, accordingly.

Conclusion: The efficacy of antiviral therapy was higher in patients with risk factors than in patients who are not at risk. In patients treated with umifenovir the duration of fever and frequency of complications were lower than in patients who did not receive antiviral therapy.

\section{6}

\section{Infectious Diseases}

Characteristics of Influenza and Influenza-Like Diseases in Risk Groups

Vilya Bulgakova ${ }^{1}$, Natalya Pshenichnaya ${ }^{2}$, Artem Poromov ${ }^{3}$, Antonina Grekova $^{4}$, Elena Kareva ${ }^{5,6}$, Elena Obraztsova ${ }^{7}$

${ }^{I}$ Institute of Pediatrics, National Medical Research Center of Children's Health, Russia

${ }^{2}$ Scientific Department, Central Research Institute of Epidemiology, Russia ${ }^{3}$ Scientific Department, Lomonosov Moscow State University, Russia

${ }^{4}$ Department of Pediatrics, Smolensk State Medical University, Russia

${ }^{5}$ Department of Pharmacology, Pirogov Russian National Research Medical University, Russia

${ }^{6}$ Department of Pharmacology, I.M. Sechenov First Moscow State Medical University, Russia

${ }^{7}$ Department of Pediatrics, Research Institute of Influenza, Russia

Aim: To identify risk factors (RFs) for the development of bacterial complications and the prolonged course of influenza and other acute respiratory viral infections (ARVIs) among inpatients treated in Russian healthcare facilities in the post-pandemic period; to determine the clinical presentation of the disease (flu-like syndrome) in risk-group people and to evaluate the efficacy of antiviral therapy with arbidol (umifenovir).

Methods: The investigators retrospectively analyzed randomly selected medical records of inpatients with influenza and other ARVI in 88 hospitals from 50 regions of the Russian Federation: those of 5287 patients, respectively, by applying parametric and nonparametric statistical methods.

Results: Single-factor analysis of variance revealed factors influencing the course of flu-like syndrome and identified risk groups: children younger than 3 years old and adults over 65, pregnant women, and people with chronic somatic diseases and obesity. The high-risk groups exhibited a more severe course of flu-like syndrome than did the patients outside the risk groups. The incidence of complications was higher, especially in the under 2-year-year-old children and in patients with endocrine, metabolic, or respiratory diseases, with a large proportion of complications being pneumonia. The efficacy of antiviral therapy (umifenovir) was higher in patients with chronic diseases than in patients not at risk. The duration of fever and frequency of complications proved to be lower than those in patients who did not receive antiviral therapy.

Conclusion:The RFs for influenza and ARVI complications are patient's age (children under 3 years of age and adults older than 65 years), the presence of chronic somatic diseases, and pregnancy. Patients with endocrine, eating, metabolic (including obesity), circulatory, and respiratory disorders are at high risk for influenza and ARVI complications. Umifenovir therapy substantially reduces the duration of fever and risk of complications, especially in patients with laboratory-confirmed influenza infection.

\section{9}

\section{Infectious Diseases}

Incomplete Kawasaki Disease on a 4 Month Old Child: When Fever is the only Clinical Sign

Bernardo Camacho ${ }^{1}$, Alexandra Andrade ${ }^{1}$, Carolina Freitas Fernandes $^{\prime}$, Carolina Ferreira Gonçalves ${ }^{1}$, Joana Oliveira ${ }^{1}$, Cristina Freitas $^{I}$, Conceição Freitas ${ }^{1}$

${ }^{1}$ Department of Pediatrics, Hospital Central do Funchal, Portugal

Background: Kawasaki disease (KD) is one of the most common vasculitis of childhood and remains the main cause of acquired heart disease in children in developed countries. It most commonly $(90 \%)$ affects children before 5 years of age and less frequently (10\%) before 6 months of age. The pathophysiology is still unknown, but immunologic factors appear to play an important role.

Objective: We present a case that highlights the importance of clinical suspicion of KD, even in the small infant.

Methods - case report: A four-month-old female infant was admitted for fever and irritability for 5 days. Vaccination with Meningococcal Group B Vaccine had been instituted 2 days before the show of symptoms. On admission, there were no remarkable physical findings. Laboratory evaluation showed elevated white blood cell count (WBC) and C Reactive Protein (CRP), urinalysis was normal. Blood and urine cultures were obtained and intravenous Ceftriaxone was started. On the 4th day of admission the patient was afebrile, but because of maintained irritability and tympanic temperatures constantly above $37.5^{\circ} \mathrm{C}$, laboratory evaluation was repeated. WBC and CRP values remained elevated, now with associated thrombocytosis and elevated liver enzyme values. Cultures showed no bacterial growth. On the $8^{\text {th }}$ day, due to the maintained clinical status, laboratory evaluation was once again repeated, and continued to show a rise of the inflammatory markers. KD was considered and echocardiographic evaluation was performed, showing diffuse ectasia of the right coronary arteries $(3 \mathrm{~mm})$. Treatment with IV immunoglobulin and acetylsalicylic acid was started, with clinical improvement.

Conclusion: KD remains an infrequent illness before 4 months of age and its aetiology a cause of debate. This case report highlights the importance of clinical suspicion of $\mathrm{KD}$, even when fever is the only positive diagnostic criteria.

\section{9}

\section{Infectious Diseases}

Pediatric Haemophilus Infuenza Invasive Disease in a Portuguese Second-Level Hospital: 2010-2018

Mafalda Casinhas Santos ${ }^{1}$, Sara Limão ${ }^{1}$, Florbela Cunha ${ }^{1}$

${ }^{T}$ Pediatric Service, Hospital Vila Franca de Xira, Portugal

Introduction: Haemophilus influenza is a nasopharynx colonizer in children, responsible for upper respiratory tract infections and rarely causes invasive disease, namely meningitis, pneumonia, arthritis or bacteremia. Before routine immunization against Haemophilus influenza type b (Hib) was included in National Vaccination Program (NVP) in 1994, this was the main responsible for invasive disease. Afterwards, non-capsulated (NC) strains have been the most frequently implicated. 
Methods: Retrospective observational study describing all pediatric cases of invasive Haemophilus influenza disease in a second-level hospital between 2010 and 2018 .

Results: Seven cases were identified: three Hib and four NC strains. There were five boys and two girls and a mean age was 18 months ( $3 \mathrm{~m}-11 \mathrm{y})$. Hib vaccination was complete in all children except for one, who wasn't vaccinated. Major risk factors were prematurity (2), asthma (1), psychomotor development retardation (1), recurrent acute otitis (1), recent tonsillectomy (1) and IgA deficiency (2). Two vaccine failures occurred, a girl with a recent tonsillectomy and a boy with IgA deficiency.

Diagnosis was confirmed by isolation of Haemophilus from blood (6), cerebrospinal fluid (3) and pleural fluid (1). Clinical presentations were meningitis (4), three of them with septicemia, pneumonia (2) and occult bacteremia (1). Of the Hib infection cases, two presented with meningitis and one with occult bacteremia. One patient with Hib meningitis developed permanent neurosensorial hear loss and ataxia. Three patients were admitted to Intensive Care Unit for a medium of four days.

Third generation cephalosporins (5) and ampicillin (2) were the chosen antibiotics. Ampicillin resistance mediated by beta-lactamases was identified in one patient.

Discussion: Haemophilus influenza invasive disease represents a potential serious condition. Although NC strains are the most prevalent, Hib is still present and responsible for some cases

National epidemiological surveillance is of crucial importance and may help the development of new preventive strategies.

\section{3}

\section{Infectious Diseases}

Risk Factors Associated with Bacteremia Due to Multi-Drug Resistant Pseudomonas Aeruginosa in a Pediatric Tertiary Referral Hospital in Mexico City

Adrian Ceballos Bocanegra ${ }^{1}$, Gabriela Guzman Gutierrez, Martha Aviles Robles $^{2}$

${ }^{T}$ Pediatrics, Centro Medico Dalinde, Mexico

${ }^{2}$ Infectious Diseases, Hospital Infantil de México Federico Gómez, Mexico

${ }^{3}$ Pediatrics, Hospital Infantil de México Federico Gómez, Mexico

Background: The incidence of multi-drug resistant P. aeruginosa (MDRPA) has increased in recent years. There is an association between antimicrobial resistance and the increased in mortality and prolonged hospitalization. Objective: To identify risk factors associated with the acquisition of bacteremia due to MDR-PA in pediatric patients

Methods: We performed a retrospective case -control study from 2012 to 2018 in Hospital Infantil de México Federico Gómez in Mexico City. Cases: patients with positive cultures for MDR-PA. Controls: patients with cultures positive for $\mathrm{P}$. aeruginosa susceptible to at least 3 groups of antibiotics. To assess for possible risk factors we perform crosstabs and calculation of odds ratio (OR). A multiple logistic regression model was conducted with significant variables.

Results: We analyzed 130 subject (46 cases and 84 controls). Mean age 6.6 years. The Most frequent basal condition was oncologic (42\%) and the most frequent diagnosis septic shock $(53 \%)$. Cephalosporins $(56 \%)$ was the previous antibiotic more frequently used before the event of bacteremia by MDR-PA. Meropenem was the antibiotic most used as empirical treatment (58.4\%).: Previous use of broad spectrum antibiotics (OR 8.2, 95\% CI 1.86 - 36.65, p 0.01) and previous stay in PICU (OR $4.9,95 \%$ CI 1.21- 20.39, p 0.01) were associated to bacteremia due to MDR-PA.. There were more cases of complication in the group of MDR-PA bacteremia: septic shock $(97.8 \%, \mathrm{P}$ 0.01) and admission to UTIP $(63.3 \%$

Conclusion: In our population, risk factors for acquisition of bacteremia due to MDR-PA were previous use of antibiotics and previous stay in PICU. Mortality from bacteremia due to MDR-PA was high (51.8\%).
1085

Infectious Diseases

Indications for and Outcomes of Lumbar Punctures Performed in Infants less than 3 Months Old

E. Clyde ${ }^{l}$, J. Orr ${ }^{l}$, S. Alexander ${ }^{I}$

${ }^{T}$ Royal Hospital for Sick Children, UK

Background: Infants under 3 months often present with nonspecific signs and symptoms making it a challenge to diagnose CNS infection in this population. Yet missing the diagnosis can result in significant mortality and morbidity. A recent large review showed low rates of bacterial meningitis $(\mathrm{BM})$ in the under two month population, contributing to increased interest in whether low risk infants can be identified to reduce the need for invasive investigations.

Objectives: To look at indications for and results of LP in the less than 3 months population presenting to our hospital and determine whether features exist to aid in stratifying infants as low risk of BM.

Methods: Data was retrospectively collected on all infants under 3 months who had LP performed between September 2017 and September 2018. Using the hospital's electronic database we gathered demographic data, initial observations, presenting features and documented indication for LP alongside results from biochemical and bacteriological investigations.

Results: 134 LP were performed, 43 in the less than 1 month group and 91 in the 1-3 months group. There were no positive bacteriology results with $26(19.4 \%)$ confirmed cases of viral meningitis. $3(0.02 \%)$ patients were treated for presumed BM (1 was clinically suspected with no LP performed, 1 had raised WCC with positive CSF microscopy and 1 was found to have meningeal thickening on cranial ultrasound scan). See chart 1 for summary of diagnosis at time of discharge.

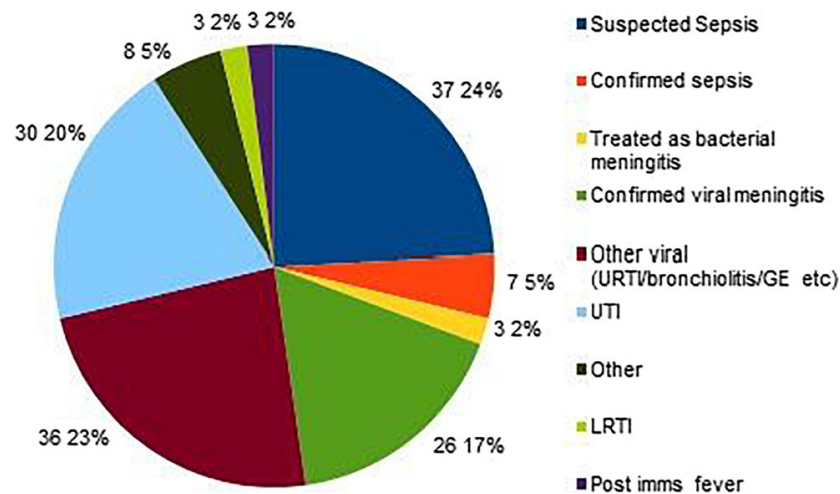

Chart 1: Diagnosis at the time of discharge for all infants under 3 months of age on whom an LP was performed between September 2017 and 2018

Conclusion: This data adds to a growing body of evidence of low rates of positive results from LP in the under 3 month group. Further research is required to better stratify this population and avoid unnecessary invasive investigation without missing the diagnosis in the small proportion of infants who do have BM possibly allowing a more individualised approach to febrile under 3 month old patients.

\section{8}

\section{Infectious Diseases}

Acute Osteomyelitis: A Retrospective Study Sofia Cochito Sousa ${ }^{1}$, Patrícia Lipari Pinto ${ }^{1}$, Paula Correia ${ }^{1}$, Teresa Ferreira ${ }^{I}$, Pedro Beckert ${ }^{2}$, Helena Cristina Loureiro ${ }^{I}$

${ }^{I}$ Departamento da Criança e Jovem, Hospital Prof. Doutor Fernando Fonseca, EPE, Portugal

${ }^{2}$ Serviço de Ortopedia, Hospital Prof. Doutor Fernando Fonseca, EPE, Portugal 
Background: Acute osteomyelitis(AO) is a rare but serious infection in children, as it can cause significant morbidity.

Objective: Characterization of children with $\mathrm{AO}$ in a suburban area outside Lisbon.

Methods: Retrospective study of children (18years) hospitalized for AO between 2012 and 2018. Record data: demographic, clinical, laboratory, imagiologic and therapeutic information. Statistical analysis performed with IBM-SPSS $®$.

Results: 40 children were included, the majority male $(60 \%)$ and of African origin $(63 \%)$ with mean age of $7.9 \pm 5.4$ years.

Lower limb was the most affected (75\%), with predominance of femur $(35 \%)$. Concomitant septic arthritis was present in $28 \%$, being coxofemoral the most affected joint.

Main symptoms at presentation were pain at mobilization $(90 \%)$, spontaneous pain $(85 \%)$, fever $(60 \%)$ and local inflammatory signs $(55 \%)$, with median duration before admission of 2.0 days (IQR 1.0-6.0). 45\% had leukocytosis, 3\% leukopenia, 79\% elevated erythrocyte sedimentation $\operatorname{rate}(20 \mathrm{~mm} / \mathrm{h})$ and $58 \%$ elevated Creactive $\operatorname{protein}(2 \mathrm{mg} / \mathrm{dL})$.

$53 \%$ had risk factors: sickle-cell disease (43\%), acute mastoiditis (5\%), smallpox $(2.5 \%)$ and preceding fracture $(2.5 \%)$.

Agent identified in 18 (45\%): S. aureus 7 (MSSA 5, MRSA 2), Streptococcus pneumoniae 3, Escherichia coli 3, Streptococcus pyogenes 2, Neisseria meningitidis 1, Mycobacterium bovis 1, Candida famata 1. Methods: culture media (89\%) and detection of bacterial DNA by PCR $(11 \%)$.

Magnetic resonance(MRI) was performed in $95 \%$. In all, signs of OA were present.

All were treated with antibiotics with median duration of 6.1weeks (IQR 6.0-8.6). Mycobacterium bovis infection was treated with anti-tuberculosis drugs and fungal infection with antibiotics and antifungal drugs. $35 \%$ needed surgery and $8 \%$ hyperbaric oxygen therapy.

There were 11 complications: subperiosteal abscess $(n=6 ; 15 \%)$, deep soft tissue abscess $(n=1 ; 3 \%)$, venous thrombosis $(n=4 ; 10 \%)$.

Conclusion: We found a high prevalence of african children and sickle-cell disease. In a child with fever and bone pain, especially if elevation of acute phase reactants, we should consider OA and perform a MRI.

\section{8}

\section{Infectious Diseases}

Guillain-Barré Syndrome in a Level II Hospital: Pre and Post Protocol Sara Completo', Marta Veríssimo ${ }^{1}$, Carlos Escobar ${ }^{11}$, Paula Correia, Catarina Luis ${ }^{1}$

${ }^{I}$ Pediatrics Department, Hospital Prof. Doutor Fernando Fonseca, E.P.E., Portugal

Background: Guillain-Barré Syndrome (GBS) is a heterogeneous group of acute immune-mediated polyneuropathies. Incidence is lower in children than in adults and there is usually complete recovery. In 2011 the Portuguese Neuropediatric Society issued a diagnosis and management protocol for GBS that allows a more systematic orientation of patients and earlier treatment (when criteria are met), avoiding possible complications.

Aim: Characterize GBS pediatric patients in a level II hospital; compare the approach before and after the protocol.

Methods: Retrospective analysis of the epidemiological, laboratory, clinical, treatment and follow-up data of the cases admitted to our hospital with GBS between 2011-2018 (second group - postprotocol), and comparison with a previous study (2010) which included patients from 1997 to 2009 (first group - pre-protocol). Data was analyzed with Excel.
Results: The first study group had six patients, medium age 4,5 years, and the second, four patients, medium age 4,3 (total: seven masculine, three feminine). All had lower extremity weakness and areflexia. Other symptoms included: pain, paresthesia, cranial nerve involvement, and fever. Eight progressed to superior extremity weakness, and three had dysautonomic disfunction. One required mechanical respiratory support.

Most (9/10) had previous infection. All patients underwent lumbar puncture (LP), 8/10 had albuminocytologic dissociation. 9/10 had electromyography (EMG) compatible with peripheral polyneuropathy. Pre-protocol, 5/6 were treated with Immunoglobulin (Ig) and 2/6 with corticosteroids; Post-protocol, all patients were treated with Ig; Two repeated Ig, due to clinical worsening. 9/10 had complete recovery and one abandoned the follow-up, with partial areflexia after three months.

Conclusion: According to the protocol, corticosteroids are contraindicated and weren't used on any patient from the second group. With atypical symptoms, LP and EMG can be helpful. Almost all patients had total recovery of the symptoms, which is frequent in children. The protocol permits a more uniformized approach to GBS.

\section{6}

\section{Infectious Diseases}

Cervical Lymphadenopathies Refractory to Antibiotic Treatment Ana Luisa Correia, Ana Lia Gonçalo, Ana Carolina Castro, Rui Almeida, M. Sofia Aroso Pediatrics, Hospital Pedro Hispano, Portugal

Background: Cervical lymphadenopathies are a common occurrence among children. They are often associated with an infectious process and can be caused by multiple pathogens. Identifying an etiology can be challenging, as well as choosing the appropriate treatment.

Case Report 1: 11-month-old girl with a 2 days complaint of limitation of neck movement, without fever or other symptoms. She presented with voluminous and hardened bilateral cervical tumefaction despite having a good general appearance. Blood tests showed leukocytosis and a high C-reactive protein. Cervical ultrasound revealed unspecific features and the neck $\mathrm{CT}$ exhibited numerous reactive adenomegalies, the largest one with necrotic center. She started antibiotic therapy with ceftriaxone, clindamycin and azithromycin. Even so, she developed fever that lasted 10 days.

Case Report 2: 10-month-old girl with a 9 days history of left submandibular growing mass, with no history of fever or any other symptoms. Physical examination identified a palpable indurated left submandibular mass. Cervical ultrasound revealed findings consistent with bilateral adenitis. Blood work revealed leukocytosis and a high C-reactive protein. She started amoxicillin/clavulanic acid and azithromycin. She developed fever from day 1 until day 3 .

Etiological investigation was negative for both patients, including EBV, CMV, Bartonella, Toxoplasmosis and tuberculosis, as well as blood cultures. After more than 15 days without clinical improvement, they underwent surgical drainage of the lymph node, and bacteriologic exam of pus identified a Staphylococcus aureus (sensitive for the antibiotics used empirically).

Conclusion: Staphylococcus aureus is one of the main pathogens implied on lymphadenitis. Generally, there is a sudden onset of inflammatory signs, with appearance of poorly mobile cervical lymphadenopathies. About $30 \%$ of the infected nodes suppurate within two weeks. With these cases we pretend to emphasize that treatment of Staphylococcus aureus lymphadenitis can be difficult, even when appropriate antibiotics are used resulting in a $\mathrm{n}$ eed for surgical drainage. 


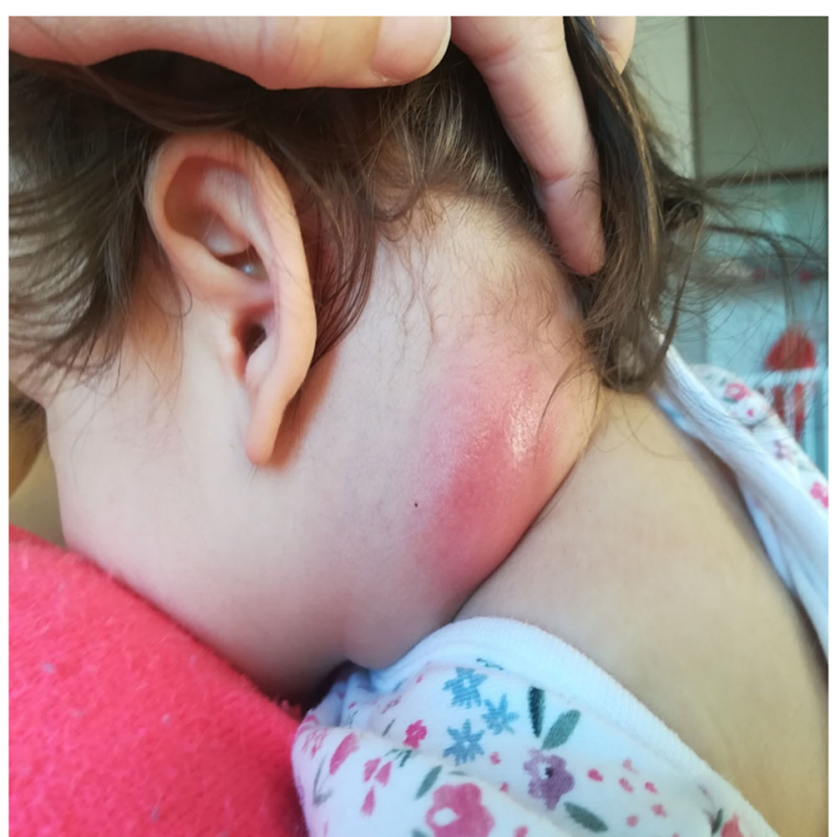

523

\section{Infectious Diseases}

Mastitis in Adolescents: Acknowledging New Risk Factors

Vanessa Costa ${ }^{1}$, Raquel Alves ${ }^{2}$, Filipa V Espada ${ }^{2}$

${ }^{\mathrm{T}}$ Paediatrics, Centro Hospitalar e Universitário do Porto, Portugal

${ }^{2}$ Paediatrics, Unidade Local de Saúde de Matosinhos - Hospital Pedro Hispano, Portugal

Introduction: Mastitis is uncommon in pediatrics and can be associated with breast abscess. In young nulliparous women there is a causal relationship with smoking habits, due to ductal and periductal inflammation. Disease severity and invasion is higher in the presence of risk factors such as cystic breast lesions. Clinical severity and treatment resistance results from poor penetration of antibiotics into breast tissue.

Case report: 17-year-old adolescent, nulliparous, menarche at age 12, with regular menses and not on oral contraceptive medication. Denied tobacco or other consumptions but exposed to second-hand smoke since early childhood. History of breast asymmetry since puberty, with an epidermoid cyst in her right breast diagnosed at 16 years of age. The patient presented acutely to the emergency department in March 2018, with painless heat and swelling of the right breast. Physical examination revealed a firm and well-defined tumefaction of the right breast, painful on palpation. No axillary lymph nodes were palpable. Breast ultrasound was performed due to the adolescent's medical history and breast inflammatory signs, which revealed a $13 \mathrm{~mm}$ epidermoid cyst with adjacent fat densification and fluid collection suggestive of mastitis. Started on oral flucloxacillin, the lesion presented a slow and gradual, however incomplete, reduction in size and inflammatory signs, with resolution only after 6 weeks of antibiotic treatment and surgical drainage.

Discussion: We present this case report as it is uncommon for a nulliparous adolescent to develop mastitis, thus considering the likely impact of second-hand smoke on its pathogenesis, as active smoking is considered a major risk factor for breast inflammatory lesions. In conclusion, we emphasize how tobacco exposure not only has a role on the development of comorbilities such as lung cancer and chronic pulmonary disease, but also other less frequent but equally severe pathological conditions in younger age groups, reinforcing smoke-free environments for children.
772

Infectious Diseases

Roseoloviruses In Patients With Neurological Disorders Tamara Amvrosieva ${ }^{1}$, Katerina Divakova ${ }^{2}$, Elena Kishkurno ${ }^{1,3}$, Alexandra Arinovich ${ }^{1}$, Alexandr Kudin ${ }^{4}$

${ }^{1}$ Laboratory of Natural Reservoir Infections, The Republican Research and Practical Center for Epidemiology and Microbiology, Belarus

${ }^{2}$ Pediatric Infectious Diseases Department, Belarusian State Medical University, Belarus

${ }^{3}$ Infectious Diseases Department, Belarusian Medical Academy of PostGraduate Education, Belarus

${ }^{4}$ Infectious Diseases Unit №5, City Children's Infectious Clinical Hospital, Belarus

Background: Recent studies indicate that HHV-6 and HHV-7 may trigger some neurological disorders.

Methods: We detected HHV-6 and HHV-7 in 121 samples of clinical material (serum, peripheral blood cells, urine, saliva/buccal swabs, and CSF) from 31 patients with the following clinical diagnoses: encephalitis/meningoencephalitis, febrile seizures, epilepsy, children with unspecified febrile fever, psychotic disorders, chronic fatigue syndrome.Qualitative detection of HHV-6 DNA was carried out by real-time PCR with a commercial kit, and HHV-7 primers and probe (Wada et al., 2009) were used for detection of HHV-7 DNA.

Results: HHV-6 DNA was found in $6.45 \%$ patients ( 2 of 31 ): in saliva, cells and urine $(n=1)$, in cells and serum $(n=1)$. HHV-7 DNA we detected in $12.9 \%$ patients ( 4 of 31$)$ : in CSF with blood $(n=1)$, in saliva and urine $(n=1)$, in cells and saliva $(n=1)$, in cells and CSF $(n=1)$. Both HHV- 6 and HHV-7 were found in $25.8 \%$ patients ( 8 of 31 ): 3 of them had both viruses in saliva, 1 - in blood cells; in other 4 children roseoloviruses were detected in variants - HHV-6+ HHV-7 in saliva and HHV-7 in blood cells, HHV-6 in saliva and HHV-7 in cells, HHV-6 in urine and HHV-6+HHV-7 in buccal swabs, HHV-6+HHV-7 in saliva and HHV-7 in CSF and urine.The quantity of HHV-6 in urine was Conclusion: Roseoloviruses are often detected in the clinical material of patients with neurological disorders. This can't indicate its etiopathogenic role, since persistent carriage of latent roseoloviruses occurs in healthy controls. This requires further research.

\section{9}

\section{Infectious Diseases}

\section{Evaluation of Diagnostic Tests in Pediatric Brucellosis}

Marta Dorado Criado ${ }^{1}$, Ursino Barrios Machain ${ }^{1}$, Lucia Vigara Rey ${ }^{4}$, María Penín Antón ${ }^{4}$, Sara Murias Loza ${ }^{3}$, Rosa María Alcobendas Rueda ${ }^{3}$, Agustín Remesal Camba ${ }^{3}$, María Cruz Vecilla Rivelles ${ }^{4}$, Francisco Javier Aracil Santos ${ }^{2}$

${ }^{1}$ Pediatrics, Hospital Universitario La Paz, Spain

${ }^{2}$ Pediatric Infectious Diseases, Hospital Universitario La Paz, Spain

${ }^{3}$ Pediatric Reumathology, Hospital Universitario La Paz, Spain

${ }^{4}$ Pediatrics, Hospital Universitario Príncipe de Asturias, Spain

Introduction and Objectives: Brucellosis is a worldwide zoonosis which is currently in clear regression in Europe. In Spain, the incidence has been decreasing since 1990, although some human cases persist. The historical importance of this disease and the current lack of experience on its management, make us present this retrospective study.

The objective of our study is to assess the clinical validation of diagnostic tests performed in pediatric patients with brucellosis.

Methods: Retrospective study of 13 pediatric patients diagnosed from 1989 to 2018 (30 years), in two hospitals of Madrid province. The cases have been identified from the registries of Microbiology Services, hospital admissions and outpatient clinics.

Results: Cases were diagnosed from 1992 to 2003. All patients except of one, recognized consumption of not pasteurized milk products. Twelve out of 
thirteen patients presented arthralgias and / or arthritis; the most frequent locations were hip and sacroiliac joints. The median duration was 20 days for joint symptoms and 18 days for fever (median maximum temperature $38.7^{\circ} \mathrm{C}$ ). The diagnosis was initially carried out wtih Rose Bengal test in all patients (100\% positive). Table 1 shows results of main diagnostic tests.

\begin{tabular}{l|ccc}
\multicolumn{1}{l}{ Tests } & Criteria & Found & Median \\
\hline Rose Bengal & agglutination & $100 \%$ & + \\
Wright's agglutination & $\geq 1 / 160$ & $100 \%$ & $1 / 640$ \\
Anti-Brucella Coombs test & $\geq 1 / 80$ & $100 \%$ & $1 / 1280$ \\
Ervthrocvte sedimentation rate & $>22 \mathrm{~mm} / 1 \mathrm{hr}$. & $55 \%$ & $32 \mathrm{~mm} / 1 \mathrm{hr}$. \\
Creactive protein & $>20 \mathrm{mg} / \mathrm{L}$ & $20 \%$ & $7.5 \mathrm{mg} / \mathrm{L}$ \\
ALT/AST & $>40 / 45 \mathrm{U} / \mathrm{L}$ & $42 / 50 \%$ & $39 / 42 \mathrm{U} / \mathrm{L}$ \\
Platelets & $<175000 / \mu \mathrm{L}$ & $33 \%$ & $228000 / \mu \mathrm{L}$
\end{tabular}

Table 1|

Blood culture and bone scintigraphy weren't carried out in all patients. Blood culture was positive in $50 \%$ and bone scintigraphy showed pathological findings in $60 \%$ of tested patients. Rifampicin combined with cotrimoxazole or doxycicline were the adopted treatment in most patients. One patient fell on a relapse after the initial treatment.

Conclusions: The number of patients diagnosed from brucellosis was small. We have not diagnosed any case of pediatric brucellosis in the last 15 years. Serological tests were positive in all cases. Erythrocyte sedimentation rate and $\mathrm{C}$ reactive protein were near normal in most patients. Blood cultures and bone scintigraphy were useful diagnostic tools in half of the patients.

\section{4}

\section{Infectious Diseases}

Streptococcus Pyogenes: An Emerging Cause of Severe Pneumonia Celia Fabra Garrido ${ }^{1}$, Marta Dorado Criado ${ }^{1}$, Paloma Troyano Hernáez $^{2}$, Virginia Santana Rojo ${ }^{1}$, Serafin Castellano Dámaso ${ }^{1}$, Jose David Andrade Guerrero ${ }^{1}$, Ursino Barrios Machain ${ }^{1}$, Mariano Silva Hernández ${ }^{1}$, Marta Bautista Barea ${ }^{1}$, Adela García Perea ${ }^{2}$, Iker Falces Romero $^{2}$, Teresa Del Rosal Rabes ${ }^{3}$, Fernando Baquero Artigao ${ }^{3}$, Luis Escosa Garcia ${ }^{3}$, Cristina Ots Ruiz, Talia Sainz Costa ${ }^{3}$, Clara Udaondo Gascón ${ }^{3}$, Luis Alfonso Alonso García ${ }^{3}$, Cristina Calvo Rey ${ }^{3}$, Ana Méndez Echevarría ${ }^{3}$, Ana Pérez ${ }^{4}$, Manuel Parrón Pajares ${ }^{4}$, María LaPlaza González ${ }^{5}$, Belén Calderón Iopis ${ }^{5}$, Jose Antonio Ruiz Dominguez $^{6}$, Begoña De Miguel Lavisier ${ }^{6}$, Francisco Javier Aracil Santos $^{3}$

${ }^{1}$ Pediatrics, Hospital Universitario La Paz, Spain

${ }^{2}$ Microbiology, Hospital Universitario La Paz, Spain

${ }^{3}$ General Pediatrics and Infectious Diseases, Hospital Universitario La Paz, Spain

${ }^{4}$ Radiology, Hospital Universitario La Paz, Spain

${ }^{5}$ Pediatric Intensive Care Unit, Hospital Universitario La Paz, Spain

${ }^{6}$ Pediatric Emergency, Hospital Universitario La Paz, Spain

Background and Aims: Streptococcus pyogenes is an uncommon cause of community-acquired pneumonia(CAP) in children, but it seems to have increased in the last years. It is associated with severe disease, including longer duration of fever and hospital stay, development of complications (nectrotizing pneumonia, bacteremia, toxic shock syndrome) and larger pleural effusions, with higher rates of bacterial isolation in pleural fluid, compared with other bacteria. The aim of this study is to review the clinical aspects of S. pyogenes-CAP diagnosed in in our hospital during the last two decades.

Methods: Retrospective descriptive study of pediatric inpatients with S. pyogenes-CAP in the period 2000-2019.

Results: We included 21 patients (16 boys and 5 girls), median age [interquartile range-IQR] at diagnosis was 25 [20-43] months. Yearly distribution of cases is shown in Figure 1. Median hospital stay [IQR] was 22 [11-26] days. A total of 17 patients $(80 \%)$ were admitted to the Intensive Care Unit (median [IQR] stay 4 [2-8] days). All patients but two developed pleural effusion that required chest tube insertion. Sixteen patients (76\%) required respiratory support. Pleural fluid culture was positive in 17/18 (94\%). Blood cultures were positive in 6/20 (30\%). Twelve patients out of $21(57 \%)$ had positive viral detections in nasopharyngeal aspirates (identified by multiplex PCR), being influenza the most frequent one (5 cases). Regarding complications, 8 patients (38\%) developed necrotizing pneumonia, 9 (43\%) had hemodynamic instability and $5(23 \%)$ toxic shock syndrome. All patients received intravenous antibiotics, followed by oral antibiotics with complete resolution.

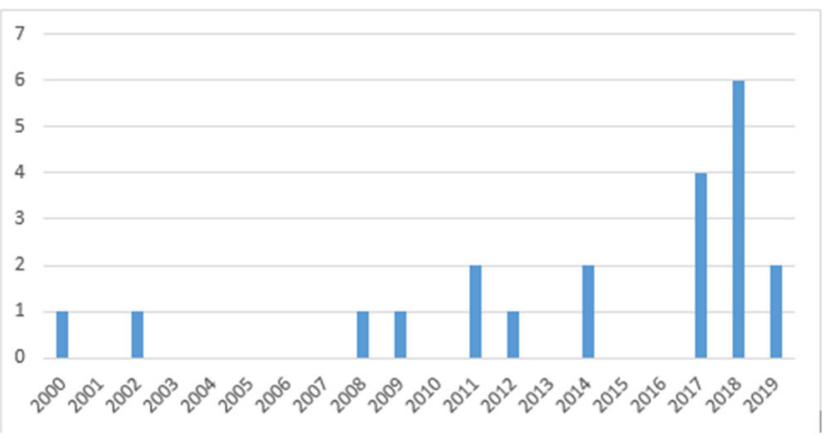

Conclusions: We have observed an increase in the number of cases of S. pyogenes-CAP in the last years. Most patients were preschoolers with severe pneumonia and pleural effusion. These infections require prolonged hospital stay and frequent Intensive Care Unit admission, as complications occur often. However, all patients achieved a full recovery.

\section{9}

\section{Infectious Diseases}

Tuberculosis Meningitis: A Case Report

Carolina Figueiredo $^{I}$, Ana Teresa Raposo ${ }^{1}$, Juan Gonçalves ${ }^{1}$, M. Rita Soares $^{I}$, Fernanda Gomes ${ }^{l}$

${ }^{I}$ Pediatric Service, Hospital Divino Espirito Santo, Portugal

Background: Currently, Portugal presents low risk of Mycobacterium tuberculosis infection. In 2014, the incidence of the disease reached the limit of low incidence $(20 / 100,000$ inhabitants) that remains until the present time. In 2016, BCG vaccination strategy was changed from a universal strategy to selective (risk groups).

Objective: Case report of a 17-year-old adolescent with iron deficiency anaemia, who had been medicated for 3 months with oral iron, that went to urgency due to night-time holocranial headache that interfered with sleep associated with photophobia, phonophobia and night fever. He presented with anorexia, asthenia and weight loss of $8 \mathrm{Kg}$ with 1 month of evolution. At admission, a decrease in the vesicular murmur in the right pulmonary base was identified. He did not present alterations in the neurological examination, namely meningeal signs. The blood count did not reveal changes and the inflammatory parameters were negative. A chest $\mathrm{X}$-ray revealed small pleural effusion on the right. CT of the chest and molecular research of Mycobacterium tuberculosis in respiratory secretions were requested. He was hospitalized for an etiological study. An infectious context with risk contact was identified: the patient's mother had a chronic cough with 1 year of evolution and a maternal uncle had recently been hospitalized for pulmonary tuberculosis. The patient under study would have been called for tuberculosis screening but did not attend. In day 5 of hospitalization, for maintenance of intense headache with little relapse to the therapy of relief, and appearance of stiffness of the neck, a lumbar puncture was performed that allowed identification of Mycobacterium tuberculosis complex by the DNA PCR technique.

Conclusion: Tuberculosis meningitis is a rare disease with a challenging diagnosis due to its insidious evolution. Targeted treatment should be initialized as soon as possible. 
771

\section{Infectious Diseases}

Difficulties in the Diagnosis of Extrapulmonary Tuberculosis on a Refugee Teenager. A Case Report

Marie Bilger ${ }^{l}$, Mathilde Arabska ${ }^{1}$, Laurence Long ${ }^{l}$, Houria Demil ${ }^{l}$, Jean Stefaniuk ${ }^{l}$, Stamatia Gkouliavoudi ${ }^{l}$

${ }^{I}$ Pediatric Departement, Sainte Catherine Hospital of Saverne, France

Background: Over recent years in France, the rate of tuberculosis remains stable with a declining tendency. $12.9 \%$ of tuberculosis cases concern teenagers and young adults from 15 to 24 years-old.

Objective: To reveal the importance of Polymerase chain reaction (PCR) in the diagnosis of bone tuberculosis.

Methods: A case report of a teenager with bone tuberculosis

Results: A 17-year-old Somalian boy without medical history was referred to our pediatric department with a 3-month history of fever, abdominal pain, vomiting, diarrhea, dysphagia to solids and serious anorexia. Refugee, his BCG vaccination status was unknown. He appeared unwell with dyspnea class II NYHA, bilateral inguinal and cervical painless lymphadenopathy. Pulmonary auscultation found decreased breath sounds on right lung. The Creactive protein level was $57.4 \mathrm{mg} / \mathrm{L}$. The thoracic computed tomography (CT) found medium lobar consolidation with right-sided pleural nodules and bilateral inguinal lymphadenopathy. An abdominopelvic CT scan confirmed a 6-centimeter abscess in the spinal canal (T11-T12) and a 2-centimeter cystic lesion in the left iliac crest. QuantiFERON was positive. Neither a tuberculin purified protein derivative test nor a chest XR were performed. Expectorated sputum and gastric catheterization showed no sign of Mycobacterium tuberculosis (MT). First bone biopsy under local anesthesia failed due to pain and lack of patient cooperation. Meanwhile symptoms worsened. Any movement of the left hip became unbearable especially at night and while exercising. Lumbar spine and pelvic MRI revealed spondylitis involving T12, cold epidural and paravertebral abscess with compression sign and a cystic lesion of the right-sided sacrum. As the neurological exam was normal, no surgical decompression was recommended. A second biopsy was scheduled under general anesthesia. PCR confirmed MT, sensitive to rifampin. The patient regained normal function within a month. Conclusion: PCR was very useful for the diagnosis of bone tuberculosis of a spinal lesion.

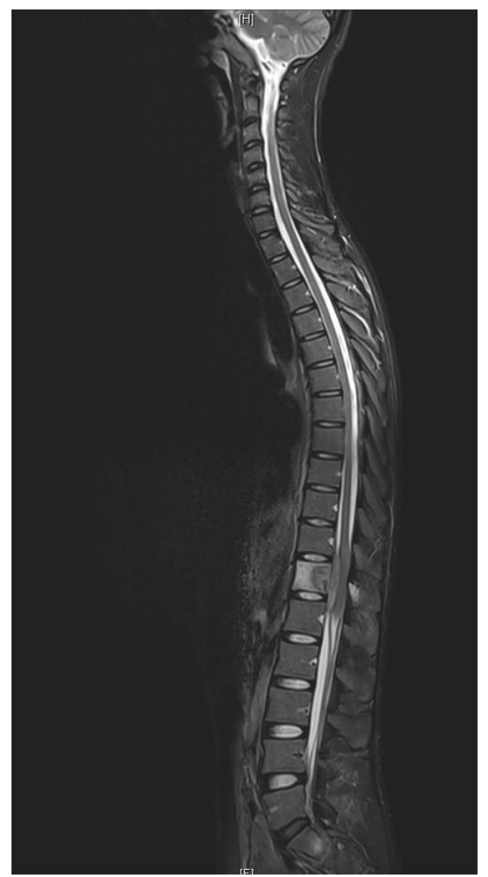

516

Infectious Diseases

Prepatellar Septic Bursitis in an 11 year old Girl Joana Gonçalves ${ }^{1}$, Ana Lançal ${ }^{1}$, Vivian Gonçalves ${ }^{1}$, Paula Nunes ${ }^{I}$ ${ }^{T}$ Department of Pediatrics, Centro Hospitalar Lisboa Ocidental, Portugal

Background: Prepatellar bursitis is an inflammation and fluid collection within the prepatellar bursa, typically due to trauma and less frequently related to infection, leading to septic bursitis. It is an extremely rare condition in the pediatric population.

Case Report: An 11-year-old obese girl presented to the emergency department reporting fever and an erythematous, swollen, and painful right knee following a trauma four days earlier. Two days before, she had been evaluated by an orthopedist who diagnosed a post-traumatic prepatellar bursitis. On admission she had normal vital signs, difficulty in gait and a knee with signs of inflammation and tender to palpation. There were insect bites in the skin overlying the area. Blood tests revealed: white cell count $13,6 \times 10^{9} / \mathrm{L}$, neutrophils $66,3 \%$, sedimentation rate $52 \mathrm{~mm} / \mathrm{h}$ and C-reactive protein $17,2 \mathrm{mg} / \mathrm{dL}$. There were an increased volume of the soft tissue anterior to the patella at the $\mathrm{x}$-ray. She was hospitalized and 24 hours later spontaneously drained purulent and serohematic content through a blister with $7 \mathrm{~cm}$ in diameter and the wound was cleaned. The ultrasonography showed a superficial abscess with a fistula to the exterior, with no intra-articular extension. The culture of bursal fluid revealed a methicillin-susceptible Staphylococcus aureus. She completed 7 days of endovenous flucloxacillin at the hospital and another 7 days orally at home. She had a favorable evolution, with no long-term complications.

Conclusion: If not treated correctly, septic bursitis can result in osteomyelitis with bone destruction. Early diagnosis with Gram staining and imaging is essential both to provide early adequate treatment and to exclude joint or bone involvement.

\section{8}

\section{Infectious Diseases}

Altered Consciousness Attributable to Unexpected Early-Onset Neurological Manifestation in Typhoid Fever: Case Report from Limited Resources Setting in East Nusa Tenggara, Indonesia Angela Grace ${ }^{I}$, Leonardus Wibowo Hidayat ${ }^{l}$

${ }^{T}$ General Medicine, St. Rafael General Hospital, Cancar, Indonesia

Background: Typhoid fever inflicts a significant burden of disease in endemic areas. While generally manifesting at a later course, multiorgan complications may occur prematurely. Typhoid delirium state, also known as typhoid encephalopathy or typhoid toxemia, is the most common and yet, under-recognized neurological complication.

Objective: To underline the challenges of diagnosing a severe typhoid fever with neurological complications in a limited resource setting.

Methods: A case of severe typhoid fever with early-onset neurological manifestations, initially suspected of acute psychosis, in a local low-resource private hospital in East Nusa Tenggara, Indonesia is presented. A 15-year-old undernourished boy presented with incoherent speech, generalized weakness, and disorganized behavior since the preceding 4 days. Previously, he had had headache and fever, followed by a seizure. An unidentified medication was administered a day earlier. Previously, he had always been a normal kid. Upon examination, he appeared delirious, with unremarkable physical examination. Widal test discovered S. typhi $O$ and $H$ titres of 1:320 and 1:160 respectively, with normal results of other feasible blood tests. A diagnosis of severe typhoid fever with neurological manifestation was decided. As his consciousness had not improved by third day of admission despite 
adequate antibiotics, antipsychotic medication was administered. The next day he was completely alert and subsequently discharged. Follow-up at the clinic 2 weeks post-discharge revealed reemerging neurologic symptoms subsequent to discontinuation of antipsychotic medication for 8 days. Repeat Widal test showed S. typhi $O$ titre of 1:320 and negative S.typhi $H$ titre. He was then diagnosed with typhoid carrier with a sequelae of typhoid delirium state. Despite being completely alert, he suffered from mild cognitive impairment.

Conclusion: Careful evaluation is needed in patients with altered mental status to discover underlying causes, including typhoid fever. Furthermore, typhoid fever's neurological complications may exhibit early, hence prompt diagnosis and treatment are paramount.

\section{3}

\section{Infectious Diseases}

Cerebral Vasculitis in Meningeal Tuberculosis: A Case Study Catarina Granjo Morais ${ }^{I}$, Joana Pereira-Nunes ${ }^{I}$, Ana Reis-Melo ${ }^{I}$, Margarida Tavares $^{1,2}$, Carolina Faria ${ }^{1,2}$, Joana Oliveira ${ }^{3}$, Natalina Miguel $^{4}$, Irene Pinto-Carvalho ${ }^{1}$

${ }^{1}$ Pediatrics Department, Centro Materno Pediátrico, Centro Hospitalar e Universitário São João, Portugal

${ }^{2}$ Pediatric Infectious Diseases Department, Centro Materno Pediátrico, Centro Hospitalar e Universitário São João, Portugal

${ }^{3}$ Department of Neurosurgery, Pediatrics - Neurosurgery Unit, Centro Hospitalar e Universitário de São João, Portugal ${ }^{4}$ Pediatrics Department, Centro Hospitalar de Trás-os-Montes e Alto Douro, Portugal

Meningeal tuberculosis (MTB) is particularly frequent in children less than five years old and it is often overlooked due to non-specific symptoms. The Portuguese annual incidence of MTB in children aged under five years was less than 1:10.000.000 inhabitants $(2011-2016)$.

An 18-month-old girl was admitted due to poor feeding and lethargy, with a four-month history of intermittent fever, enlarged cervical lymph nodes and weight loss. Primary care follow-up was maintained by different general practitioners. She had not been immunized with $B C G$ vaccine. Cranial CT scan displayed normal ventricular size and lumbar puncture showed pleocytosis, polymorphonuclear neutrophils predominance and hypoglycorrhachia. Empirical treatment for CNS infection was initiated and she was transferred to our tertiary hospital. Due to clinical suspicion of MTB, tuberculostatic therapy (four-drug regimen) was initiated within 24 hours, as well as adjunctive therapy with glucocorticoids. Over the next 48 hours, she presented tonic-clonic seizures and progressive impaired consciousness. Repeated CT scan revealed hydrocephalus requiring admission in intensive care unit for external ventricular drain (EVD). Subsequent MRI revealed a large vessel vasculitis. Positive results for Mycobacterium tuberculosis were obtained by cerebrospinal fluid (CSF) culture and by PCR in CSF, bronchoalveolar lavage and gastric aspirate specimens. Chest CT scan exhibited a miliary pattern. Prompt screening of household contacts identified the index-case (her father) in collaboration with public health department. Rapid progression of vasculitis lead to refractory intracranial hypertension with need of decompressive hemicraniectomy. Ventriculoperitoneal shunt was necessary due to unsuccessful EVD removal. She maintained severe neurologic deficits on oneyear follow-up.

In the reported case, stage of disease at presentation and complications (especially cerebral vasculitis) were possibly the major determinants of neuropsychomotor sequelae. It is of paramount importance recognizing early MTB stages for prompt management. Screening close contacts is vital, reinforcing the need of optimizing collaboration with public health departments.

\section{3}

\section{Infectious Diseases}

Pediatric Borreliosis is not Just Small Borreliosis: Similarities and Differences in Tick Borne Relapsing Fever in Children and Adults Saar Hashavya ${ }^{1}$

${ }^{T}$ Department of Pediatrics, Hadassah Medical Center, Israel

Tick borne relapsing fever (TBRF) is a spirochete infection and is characterized mainly by recurring episodes of fever. Our objective was to characterize pediatric TBRF in comparison to adult TBRF. Data from the medical records of all patients diagnosed with TBRF in the 3 largest hospitals in Jerusalem, Israel, between the years 2004-2018 were collected and analyzed. During the study period, 92 patients were diagnosed with TBRF of whom 21 (22.8\%) were children. Children had longer periods of fever compared to adults: $15.6( \pm 10.5)$ VS $7.9( \pm 9.9)$ days (p0.01) and had $2.2( \pm 1.3)$ VS $0.6( \pm 0.8)$ relapses of fever at presentation. Children had a lower neutrophil count $\left(4.8 \pm 2.7 * 10^{9} /\right.$ liter VS $7 \pm 2.9 * 10^{9}$ / liter; P0.01) and lower C- reactive protein levels $(8.1 \pm 4.3 \mathrm{mg} / \mathrm{dl}$ VS 15.6 $\pm 10.1 \mathrm{mg} / \mathrm{dl}$; P0.01). In conclusion, children exhibited a distinctly different course of TBRF, characterized by more episodes of fever and lower levels of inflammatory markers.

\section{1}

\section{Infectious Diseases}

Evaluation of Diagnostic Panel for Rapid Blood Culture Identification of Pathogenes in Bloodstream Infection at The University Children Hospital- Skopje

Olivera Jordanova $^{1}$, Liljana Gurzanova Durnev ${ }^{1}$, Aspazija Sofijanova ${ }^{2}$, Silvana Naunova Timovska ${ }^{2}$, Danilo Nonkulovski ${ }^{2}$, Aleksandra Janchevska ${ }^{3}$, Rodna Jordanova ${ }^{4}$

${ }^{1}$ Clinical laboratory, University Children Hospital, Macedonia

${ }^{2}$ Neonatal intensive care unit, University Children Hospital, Macedonia

${ }^{3}$ Endocrinology, University Children Hospital, Macedonia

${ }^{4}$ General medicine, Medical center, Macedonia

Background: Bloodstream infection is a leading cause of morbidity and mortality in critically ill children in the University Children HospitalSkopje.

Objective: The aim of study is an early diagnosis and rapid therapeutic intervention of sepsis during the critical first hour of develop the clinical signs in paediatric patients, is crucial to stop progression into severe sepsis and septic shock.

Methods: We investigated 350 patiens with clinical signs suspected for sepsis in the Univetsity Children Hospital-Skopje. The new multiplex polymerase chain reaction-based rapid diagnostic test (BioFire FilmAray Blood Culture Identification (BCID) was used for early recognition of 27 targets of sepsis, 24 pathogenes and 3 antibiotic resistance genes (mecA, blaKPC, and Van A/VanB), associated with bloodstream infection sample 200ul from positive blood culture detected on BACT/ALERT 3D 60. The result report indicating the detected microorganism is obtained after 60 minutes.

Results: We detected 51 positive blood culture, 30 were single blood culture isolate. Twenty eight of the isolates were Gram positive bacteria Staphylococcus aureus, which twenty five were methicillin resistant, two were Streptococcus pneumonia. None of the Gram positive isolates were vancomycin resistant. Twelve of the isolates were Gram negative bacteria, seven were Seratia marcescens, five were Enterobacteriae. Nine had two or three bacteria at the same time. Rapid diagnosis of the causing microorganism and relevant resistance determinants is important for early 
administration and modification of appropriate antimicrobial therapy, as well as reduced antimicrobial resistance selection.

Conclusion: FilmAray Blood Cell Identification quickly identifies the pathogen in the blood culture of pediatric patients with suspected sepsis, a short time to the results can be of added value to optimize the clinical management of patients, improves the outcome of the potential complications for severe sepsis and septic shock and reduces hospital treatment costs.

\section{8}

\section{Infectious Diseases}

Fever of Unknown Origin in Young Children: Think Kawasaki Ana Lança ${ }^{1}$, Carolina Amaro Gonçalves ${ }^{2}$, Madalena Sasseti ${ }^{3}$, Marta Almeida $^{3}$, Filipa Nunes ${ }^{3}$, Paulo Calhau ${ }^{3}$

${ }^{1}$ Pediatric Department, Hospital de São Francisco Xavier, Centro Hospitalar de Lisboa Ocidental, EPE, Portugal

${ }^{2}$ Pediatric Department, Hospital de Santa Maria, Centro Hospitalar de Lisboa Norte, EPE, Portugal

${ }^{3}$ Pediatric Department, Hospital Garcia de Orta, EPE, Portugal

Background: Kawasaki disease (KD), a vasculitis of unknown etiology, is an important cause of prolonged fever in childhood. Fever lasting more than five days and at least four signs of mucocutaneous inflammation characterize it. It's main complication are coronary artery aneurysms being the primary cause of acquired heart disease in children in developed countries.

Case-report: A 22-month-old girl is admitted to our hospital with a persistent fever for eight days accompanied by a generalized maculopapular exanthema that affected palms, to which bilateral cervical adenopathies are associated at the sixth day of disease. The serial investigational tests performed revealed leukocytosis with neutrophilia and a reactive-C protein maximum $26,5 \mathrm{mg} / \mathrm{dL}$ and she was medicated with amoxicillin for the suspicion of an occult bacteremia, without improvement. At admission she fulfilled three clinical criteria for $\mathrm{KD}$ and the remainder laboratory results supported the diagnosis: a normocytic normochromic anemia (minimum of 7,4 $\mathrm{g} / \mathrm{dL}$ ) and hypoalbuminemia (minimum of 2,8 g/dL). The urinary exam, chest radiography and blood cultures were negative. An echocardiogram was performed revealing a dilation of the anterior descendent coronary artery (Z-score $+2,35)$. She was administered intravenous immunoglobulin (IVIG) in a single dose of $2 \mathrm{~g} / \mathrm{kg}$ and initiated aspirin in an anti-inflammatory dosage. After the initial clinical improvement the fever reappeared in two days. She repeated the administration of IVIG without improvement. At the tenth day of hospitalization she initiated methylprednisolone pulses for three days, with resolution of the fever and progressive clinical improvement. She was discharged with an overlapping echocardiographic assessment and antiplatelet dosage aspirin.

Conclusion: The diagnosis of incomplete KD may be a clinical challenge especially in the first 24 months of life. With this report we aim to alert to the differential diagnosis of a child with fever of unknown origin and the importance of the echocardiographic assessment in the diagnostic evaluation.

\section{0}

\section{Infectious Diseases}

Prevalence of Community Acquired Pneumonia C And D among Patients Aged 6 Months to 5 Years who had Previously Received Hib Vaccinations: A 1 Year Retrospective Study Ma. Monica Mampusti ${ }^{1}$, Jennie Wong ${ }^{1}$

${ }^{7}$ Pediatrics, Ospital ng Maynila Medical Center, Philippines
Background: Among children under five years old, pneumonia is still one of the leading cause of mortality globally and Haemophilus influenza type $\mathrm{b}$ (Hib) is one of the most common causative agent for bacterial pneumonia. Locally, the Expanded Programme for Immunization included Hib vaccination given for free in health centers. Data on clinical outcome of pneumonia after widespread use of Hibvaccination is important to document its protective effect against pneumonia and in improving its management.

Objective: To describe the prevalence of PCAP C and D among patients aged 6 months to 5 years admitted from June 2016 to June 2017, who had received Hib vaccinations.

Materials and Methods: Retrospective, descriptive study done in a tertiary government hospital.

Results: Among the children admitted for PCAP C and D from June 2016 to June 2017, 22\% received at least one dose of Hib vaccination with majority $(56.3 \%)$ aged 6 months -12 months, mostly male. As to the Hib immunization status, $10.4 \%, 19.4 \%$ and $70.1 \%$ received 1,2 , and 3 doses of Hib vaccination respectively and none received a booster dose.The mean length of hospital stay for the patients vary.Complications were notably decreased in those who received Hib vaccine.

Conclusion: The prevalence of PCAP C and D among patients was comparable to other studies, with Hib vaccine providing a protective effect for PCAP C and D as the number of given dose increases. The mortality and complications in these group of populations were observed to be low.

939

Infectious Diseases

"Who forgot to brush their teeth?" - A Retrospective Study of Dental Abscesses in a Suburban Hospital in Portugal

Rita Matos Parreira ${ }^{l}$, Patrícia Pais ${ }^{l}$, Catarina Espirito Santo ${ }^{l}$, Joana Extreial, Susana Correia $^{l}$

${ }^{1}$ Pediatria, Centro Hospitalar Barreiro-Montijo, Portugal

Background: Odontogenic infection is a polymicrobial infection with different degrees of clinical severity. It is the most common type of oral infection and its treatment involves up to $10 \%$ of all antibiotic prescriptions leading to important costs in public health, parents and school absenteeism, loss of quality of life and miss of dental pieces. The costs of this evitable health problem are high.

Objective: The purposes of this study were to retrospectively assess characteristics of pediatric patients with odontogenic-based facial cellulitis that needed in-hospital treatment and describe management in a suburban Hospital in Portugal.

Methods: Data from clinical records of 34 patients who were admitted for in-hospital treatment of orofacial infection at the pediatric department from January 2015 to May 2019. Medical records were reviewed for all patients with a discharge diagnosis of facial cellulitis and tooth decay or buccal cellulitis.

Results: Thirty-four patient records were reviewed, 50\% girls with a mean age of 11 years (4-17 years). The mean length of stay was six days. Thirteen of the patients had, at least, one parent with economic insufficiency.

In $62 \%$ antibiotic treatment was started prior to admission.

Treatment with intravenous antibiotics was needed in $94 \%$ of them, twothirds using two or more different antibiotics. Cooperation with other specialities was frequent, mainly Stomatology (22 out of 34).

Conclusion: Tooth decay is a major public health problem and it's a target of health programs. Nevertheless, many children and adolescents do not use these programs maintaining high incidence of dental abscesses that motivate hospital admission. There is a need for more motivation and information for parents and children regarding this important public health issue. 
450

\section{Infectious Diseases}

\section{Painful Finger}

Jacinta Mendes ${ }^{I}$, Nuno Vilas Boas ${ }^{I}$, Cristina Novais ${ }^{l}$, Anabela Bicho ${ }^{l}$, Sara Santos

${ }^{1}$ Paediatrics, Centro Hospitalar do Oeste - Caldas da Rainha, Portugal

Background: Herpetic whitlow is a rare cause of finger infection most commonly affecting children. It is a type 1 or 2 Herpes simplex virus infection characterized by significant pain and erythema with overlying non-purulent vesicles. Herpetic whitlow should be suspected based on clinical signs. It is caused by inoculation of the virus through a break in the skin barrier. Eczema and other skin lesions can increase the risk of its appearance.

Case Presentation: We report a case of a previously healthy 16-year-old male adolescent admitted to the hospital with erythema and swelling of the fingers of his both hands. The right fifth finger was very painful and showed, in terminal phalanx, grouped vesicular lesions with clear fluid and surrounding erythema; along multiple other fingers of both hands he showed dyshidrotic eczema lesions. No treatment was applied in the right fifth finger as the diagnosis of herpetic whitlow was assumed. Dyshidrotic eczema finger lesions were treated with topical corticosteroids. At ten-day follow up all skin lesions resolved completely. There were no previously history of herpetic infection and there were no recurrences in the next ten months.

Conclusion: We draw attention to the typical clinical signs and correct management of herpetic whitlow as it may go underdiagnosed and its appearance may mimic a bacterial infection. Treatment is usually symptomatic as lesions are self-limited and unnecessary surgical interventions should be avoided since they can exacerbate symptoms or lead to complications.

\section{1}

\section{Infectious Diseases}

Acute Hepatitis in a 6 Year Old Boy: Who`s to Blame? ${ }_{\text {Joana Mendes }}{ }^{2}$, Adriana Formiga ${ }^{l}$, Sandra Mesquita ${ }^{l}$, Sofia Ferreira ${ }^{1}$, Nuno Lourenço

${ }^{1}$ Pediatrics Department, Centro Hospitalar Universitário Cova da Beira, Portugal

Background: Cat scratch disease (CSD) is a common infectious disease, caused by Bartonella Henselae, typically characterized by self-limited regional lymphadenopathy. CSD rarely causes disseminated illness, like hepatosplenic disease.

Hereditary spherocytosis' (HS) presentation can vary from mild to severe depending on the degree of haemolysis and complications. HS can remain undetected until challenge by an infectious illness.

Case Report: A previously healthy 6-year-old boy, with family history of spherocytosis and exposure to cats, presented to our hospital with a 3-day history of fever, right cervical lymphadenomegaly, nonspecific rash, jaundice and choluria. He was previously medicated with amoxicillin/ clavulanate for adenitis and suspected streptococcal tonsillitis. Laboratory test revealed: haemoglobin $11.1 \mathrm{~g} / \mathrm{dL}$; mean corpuscular volume 88 fl; mean corpuscular haemoglobin concentration (MCHC) 37.2 $\mathrm{g} / \mathrm{dL}$; reticulocytosis $0.21 \times 10^{\wedge} 6 / \mathrm{uL}$; peripheral blood smear (PBS) with anisocytosis, toxic granulation and vacuolated neutrophils; C-reactive protein $1.17 \mathrm{mg} / \mathrm{dL}$; hyperbilirubinemia (direct $3.21 \mathrm{mg} / \mathrm{dL}$; indirect $5.28 \mathrm{mg} / \mathrm{dL}$ ); aspartate aminotransferase $117 \mathrm{U} / \mathrm{L}$; alanine aminotransferase $227 \mathrm{U} / \mathrm{L}$; lactate dehydrogenase $578 \mathrm{U} / \mathrm{L}$. Ultrasonography revealed mild splenomegaly and no parenchymal liver changes or structural cause of obstructive cholestasis. During hospital stay, there was an improvement in symptoms with supportive treatment followed by decreasing levels of aminotransferases and bilirubin. Serologic testing for putative infectious agents were negative. He was discharged four days later, asymptomatic. Post-discharge results were positive for Bartonella henselae IgM titers. No specific treatment was prescribed since the patient was asymptomatic by the time of results. Further studies on suspected spherocytosis were performed at a tertiary hospital. Discussion: We considered a diagnosis of acute hepatitis due to infection of Bartonella Henselae, according to laboratory results, lymphadenomegaly and exposure to cats. Although there were no red blood cell abnormalities in PBS, HS is suspected because of a positive family history, increased MCHC and hemolysis.

773

\section{Infectious Diseases}

Paediatric Tuberculosis: The Demographic Characteristics and Spectrum of Disease in Children in University Malaya Medical Centre $\frac{\text { Basri Mohd Azahari }}{\text { Muhammad Nor Asiah }}{ }^{3}$, Mia Tuang Koh ${ }^{2}$, Anuar Zaini Azriyanti ${ }^{2}$,

${ }^{I}$ Department of Paediatrics, Faculty of Medicine, Universiti Teknologi MARA (UiTM), Malaysia

${ }^{2}$ Department of Paediatrics, Faculty of Medicine, Universiti Malaya (UM), Malaysia

${ }^{3}$ Center for Communicable Disease Research, Institute of Public Health, National Institute of Health, Malaysia

Background: Childhood tuberculosis remains a common and often fatal vaccine preventable disease worldwide. At this point of time, there is limited data available on tuberculosis among Malaysian paediatrics population. The present study is designed to analyse the spectrum of the disease and demographic patterns of affected children. This is a retrospective study is conducted among paediatric population seen in University Malaya Medical Centre from 2008 - 2017.

Objectives: To describe the spectrum of paediatric tuberculous (TB) disease in UMMC. To describe the demographic characteristics of children diagnosed with tuberculosis.

Methods: This is a retrospective, cross - sectional descriptive study on all paediatrics patients who were diagnosed with TB in UMMC over period of ten years from January 2008 until December 2017. All patients were identified through records obtained from attendance to DOTS clinic, Paediatric Infectious Disease clinic, and Ministry of Health CDCIS eNotifikasi website. Results: There were a total of $156(75.4 \%)$ eligible candidates recruited into the study; $102(65.4 \%)$ and $54(34.6 \%)$ patients in the confirmed TB and clinical compatible TB groups respectively. The majority of affected patients predominantly were female $(\mathrm{n}=91 ; 58.3 \%)$. Paediatric population aged between 12 to 18 years of age contributed the higher number of patients $(n=99)$. In terms of ethnic groups Malays $(n=92)$ were predominantly affected followed by Indians $(n=27)$ and Chinese $(n=25)$. The pulmonary TB is the commonest form of the TB spectrum followed by extrapulmonary, and miliary / disseminated TB.

Conclusion: Children and adolescents who were exposed to TB patients should be screened so that early detection and intervention could be carried out. Tuberculosis in paediatric population has specific characteristics that are important to understand for health personnel working in clinical medicine. Pulmonary TB is still the commonest form tuberculosis in children.

\section{1}

\section{Infectious Diseases}

Streptococcos Pyogenes Meningitis in an Imunnocompetent Child Teresa Monteiro ${ }^{I}$, Inês Coelho ${ }^{I}$, Sofia Baptista ${ }^{l}$, Rita Pereira ${ }^{l}$, Daniel Tiago $^{I}$, Luísa Gaspar ${ }^{1}$, Maria João Virtuoso ${ }^{l}$

${ }^{1}$ Pediatrics, Centro Hospitalar e Universitário do Algarve, Portugal

Background: Group A streptococci (GAS) infections of the central nervous system are extremely rare in the antibiotic era, accounting for less 
than $1 \%$ of all childhood meningitis. However, in the last decades, recent reports suggest an increase in invasive SGA infections.

Case Presentation Summary: The authors present a case of a 3-year-old boy admitted to the emergency room, with a history of high fever, cough, vomiting and lethargy. He had been diagnosed with varicella 5 days before and medicated with acyclovir.

On examination, he presented paleness, positive Kernig's and Brudzinski's sign, and nuchal rigidity. He also had several crusted lesions spread across the whole body and some petechiae. Vital signs were normal.

Laboratory tests results showed a white blood cell count of $17.1 \times 10^{\wedge} 9 / \mathrm{L}(82 \%$ neutrophils, $11.4 \%$ lymphocytes and $6 \%$ monocytes) and a C-reactive protein of $212 \mathrm{mg} / \mathrm{l}$. Lumbar puncture revealed a nucleated cell count of $27 \mathrm{cel} / \mathrm{mm}^{3}$, a protein of $36.9 \mathrm{mg} / \mathrm{dL}$ and a glucose of $80 \mathrm{mg} / \mathrm{dL}$.

Therapy with intravenous (IV) ceftriaxone and acyclovir was started. Twenty-four hours later, GAS was isolated in the cerebrospinal fluid (CSF). Antibiotics were switched to IV penicillin G and clindamycin.

The patient was discharged after 16 days. He was asymptomatic, with no sequelae.

Learning Points/Discussion: In paediatric patients, varicella is a welldocumented risk factor for invasive GAS, associated with approximately $15 \%$ to $30 \%$ of such infections.

Post-varicella GAS meningitis has rarely been reported and it seems to be related to skin lesions. However the pathogenesis of GAS meningitis has not been entirely explained.

\section{2}

\section{Infectious Diseases}

Carotid Space: A Rare Location of Cervical Abscesses

Teresa Monteiro ${ }^{1}$, Sara Monteiro ${ }^{1}$, Luisa Gaspar ${ }^{1}$, Guida Gama ${ }^{1}$, César Anjo $^{2}$, Ilídio Gonçalves ${ }^{2}$

${ }^{1}$ Serviço de Pediatria, Centro Hospitalar e Universitário do Algarve, Portugal

${ }^{2}$ Serviço de Otorrinolaringologia, Centro Hospitalar e Universitário do Algarve, Portugal

Introduction: Deep cervical spaces infections are rare entities in pediatric age and may be associated with significant percentages of morbidity and mortality.

Case Presentation Summary: We present the case of a four-yearold girl, previously healthy, admitted to our emergency room with a 3 days history of neck pain and fever. She had odynophagia and fever, two weeks before. Physical examination showed tenderness of the cervical region, without trismus, and bilateral cervical infracentimetric adenopathies. Otoscopy showed signs of acute otitis media on the right and serous otitis on the left, hyperemia and tonsillar hypertrophy with tonsillar exudate. On blood analysis presented leukocytosis, neutrophilia and Creactive protein $79 \mathrm{mg} / \mathrm{L}$. She started intravenous (IV) antibiotics with amoxicillin and clavulanic acid. During the hospitalization a cervical ultrasound revealed an increase in the echogenicity of the adipose planes of the right carotid space (RCS), a finding that led to a cervical CT scan, which showed a liquid collection at the level of the upper third of the RCS, with $25 \times 15 \mathrm{~mm}$ compatible with abscess, with significant reduction of the right carotid artery diameter. Significant clinical improvement was observed after she started IV antibiotis, but due to the location of the abscess and the absence of improvement in the imaging tests, methylprednisolone IV was started and the patient was transferred to a Central Hospital. During hospitalization clindamycin was added to the therapeutic scheme, with progressive improvement and without the need for surgical drainage.
Discussion: Carotid space infections, due to their location and structures, are associated with potentially fatal complications. Early diagnosis is essential. However, as it is a rare pathology, with varied clinical features and nonspecific findings on physical examination, it becomes a diagnostic challenge that requires a high degree of clinical suspicion.

\section{8}

\section{Infectious Diseases}

Oral Ulcerations after Respiratory Symptoms

Sara Mosca $^{1}$, Mafalda Moreira ${ }^{2}$, Margarida S. Fonseca ${ }^{2}$, Susana Lira ${ }^{2}$, Leonilde Machado ${ }^{2}$, Gisela Silva ${ }^{1}$

${ }^{1}$ Pediatric Department, Centro Materno-Infantil do Norte, Centro Hospitalar Universitário do Porto, Portugal ${ }^{2}$ Pediatric Department, Centro Hospitalar Tâmega e Sousa, Penafiel, Portugal, Portugal

Background/Objective: Mycoplasma pneumoniae is frequently associated with respiratory infections in pediatric patients, but can also cause mucosal and cutaneous lesions. It has a wide spectrum of manifestations including Kawasaki disease, erythema multiforme, StevenJohnson Syndrome (SJS), toxic epidermal necrolysis and Mycoplasma pneumoniae induced rash and mucositis (MIRM). The authors' objective was to illustrate two possible presentations of MIRM.

\section{Case description:}

Case 1: Female, 15-years-old, was admitted to the emergency room with diffuse ulcerations of the oral mucosa (lips, palate and gums) partially covered by hemorrhagic crusts, as well as conjunctiva's erythema. During the previous month, she presented nasal obstruction, rhinorrhea and cough. No fever and no cutaneous involvement. Complementary exams included a chest X-ray consistent with an atypical pneumonia, negative DNA protein chain reaction but the serum enzyme immunoassay revealed a positive $\mathrm{M}$. pneumoniae IgM.

Case 2: Male 17-years-old, presented to the emergency room with a 7-days history of fever, cough and rhinorrhea. One day before to the admission, he developed edema and painful erosions of the oral mucosa, purulent bilateral conjunctivitis and minor urethral meatus erythema. Absence of cutaneous involvement. Chest Xray was consistent with an atypical pneumonia and the DNA protein chain reaction was positive for $\mathrm{M}$. pneumoniae; the enzyme immunoassay is in progress.

Both were treated with a 3-days azithromycin and supportive care and pain management. Following presented a noticeable clinical improvement and favorable prognosis.

Discussion/Conclusion: The MIRM was described as a new entity with mucosal findings consistent with SJS, but without or sparse cutaneous involvement, preceded by a recognized $\mathrm{M}$. pneumoniae infection. These cases evidence clinical and diagnostic features previously described in MIRM which include a predominance of two or more mucosal sites, relatively sparse cutaneous involvement and evidence of an atypical infection. An accurate diagnosis is of utmost importance for the correct management and prognosis.

\section{8}

Infectious Diseases

\section{EBV Infection and Leptospiroses in Children}

Wael Nasser $^{1}$, Heba Abozayed ${ }^{2}$, Sami Shehade ${ }^{2}$, Boshra Nasser ${ }^{1}$, Haia Naser', Said Abozd ${ }^{2}$, Susan Nasser ${ }^{1}$

${ }^{1}$ Pediatric Nephrology, Faculty of Medicine in the Galilee - Bar Ilan University, Israel

${ }^{2}$ Pediatric, Faculty of Medicine in the Galilee, Baruch Padeh Medical Center, Bar Ilan University., Israel 
Introduction: Leptospirosis is a zoonotic disease caused by leptospira, and is transmitted to humans by direct or indirect exposure to urine or other body fluids of infected animals. The clinical presentation in humans ranges from a mild flu-like illness to a severe illness with jaundice, meningitis, renal failure, pulmonary hemorrhage. Epstein-Barr virus can induce complex humoral and cellmediated immune responses in humans, which impair liver function, we describe and adult girl with leptospirosis associated with active EBV infection.

Case Report: A 14-year-old girl was admitted because of a 1-week history of general malaise. Lymphadenophaty no history of travel and had not taken any medicine in the previous weeks. The patient's body temperature was $39^{\circ} \mathrm{C}$, blood pressure was 110 / $70 \mathrm{mmHg}$ normal HR and normal RR. his skin and sclera were icteric, cervical lymphadenopathy, splenomegaly. Laboratory leukocytosis with high atypical lymphocytes, no anemia, mild thrombocytopenia, liver enzyme abnormality and cholestatic jaundice, normal renal function. Abdominal sonography showed mild splenomegaly without other specific findings. The patient was treated with parenteral ceftriaxone and doxycycline. Serum antibodies against hepatitis $\mathrm{A}$ and serum surface antigen of hepatitis $B$ were negative. EBV serum immunoglobin $\operatorname{IgG}$ and $\operatorname{IgM}$ were positive. PCR in urine for leptospira was positive.

Cystitis Cystica in Children In general, the diagnosis is initially based on clinical suspicion, and is confirmed later by laboratory results, usually by the detection of PCR IN URINE. The coinfection with EBV attributable to immunosuppression caused by active EBV infection, and increased the chance of hepatitis and another symptoms of liptospera.

\section{0}

\section{Infectious Diseases}

Helicobacter Pylori Infection Associated with Henoch-Schonlein Purpura

Susan Nasser ${ }^{1}$, Sami Shehade ${ }^{1}$, Boshra Nasser ${ }^{1}$, Ehsan $\mathrm{Na}^{1}$, Said

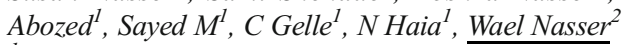

${ }^{1}$ Pediatric, Faculty of Medicine in the Galilee, Baruch Padeh Medical Center, Bar Ilan University., Israel

${ }^{2}$ Pediatric Nephrology, Faculty of Medicine in the Galilee, Barcuh Padeh Medical Center, Bar Ilan University., Israel

Introduction: Henoch-Schonlein-purpura is a systemic vasculitis, of small vessels, resulting in skin, joint, gastrointestinal and renal involvement. The pathogenesis of HSP $t$ is postulated that an unknown chronic antigenic stimulus, In children the prognosis is good, as HSP typically resolves rapidly and without complication. Helicobacter pylori is of the most common bacterial infections may cause some extra intestinal manifestations some of which are dermatological conditions, including Henoch-Schönlein purpura.

Case Report: A previously healthy 10year-child was admitted to our department, because of-week history of abdominal pain, and purpura on his lower extensor extremities. Physical examination revealed purpuric papules, on the legs, thighs, buttocks,. On admission, his temperature was $37.5^{\circ} \mathrm{C}$, , and blood pressure $110 / 60$ $\mathrm{mmHg}$. showed a white blood cell count of $18200 / \mathrm{mm}^{3}$. The hemoglobin concentration was $11.8 \mathrm{~g} / \mathrm{dL}$, the platelet count was 400000 , while normal results for serum creatinine level Urinalysis revealed microscopic hematuria and proteinuria 200 $\mathrm{mg} / 24 \mathrm{hrs}$ ). Occult blood was found in the feces., breath test for H. pylori showed a positive result. the patient received a two-week treatment course consisting of omepradex+, amoxicillin, and clarithromycin without steroid treatment. After the treatment, the abdominal manifestations dramatically subsided within three days and the purpuric skin lesions resolve.

Conclusion: H.pylori infection has been associated with certain extradigestive dermatological conditions, including chronic urticaria, systemic sclerosis, and atopic dermatitis The symptoms of HSP disappeared after H. Pylori eradication therapy, In conclusion, H. pylori may be an etiological factor in pathogenesis of HSP and breath test fo H. pylori examination should be considered.

\section{4}

\section{Infectious Diseases}

Lyme Diseases of Children in Ternopil Region (Ukraine) Svitlana Nykytyuk ${ }^{l}$, S.S Podobivsky ${ }^{l}$, S.I Klymnyuk ${ }^{l}$, L.Ya Fedoniuk ${ }^{l}$ ${ }^{T}$ I. Horbachevsky Ternopil National Medical University, Ukraine

Summary: The results of the monitoring the child with contamination of tick bite during the spring-autumn of 2018 in Ternopil and Ternopil region were summarized, according to which the peak of such lesions occurred in May-June and AugustSeptember. It was found that the percentage of ticks infected with pathogens of infectious diseases, namely Borrelia burgdorferi s.l., Borrelia miyamotoi and Anaplasma phagocytophilum, was about $37 \%$. There were cases where the ticks were infected with multiple pathogens (B. burgdorferi s.l. with A. Phagocytophilum, B. miyamotoi and A. rhagocytophilum) simultaneously. Regarding the importance of different stages of the tick Ixodes ricinus in the transmission of infectious agents, it has been shown that a greater number of vaccinated individuals were among females and nymphs. Although the role of males and larvae in this process can not be ruled out either. There were 47 females, 76 nymphs, 1 male and 4 larvae among 128 infectes ticks.

Clinical trials of children affected by ticks have shown that the most common initial manifestation of Lime borreliosis is a typical skin disorders, known such as erythema migrans. The necessity of appointing preventive treatment of Lyme borreliosis in children who have been exposed to ticks has been proved.

\section{0}

\section{Infectious Diseases}

The Global Point Prevalence Survey of Antimicrobial Consumption and Resistance (GLOBAL-PPS): Results of Antimicrobial Prescribing in Paediatric Units in Georgia

Karaman Pagava ${ }^{1}$, Irma Korinteli ${ }^{1}$, Ann Versporten ${ }^{3}$, Herman Goossens ${ }^{3}$, Helen Phagava ${ }^{2}$

${ }^{1}$ Department of Child and Adolescent Medicine, Tbilisi State Medical University, Georgia

${ }^{2}$ Department of Epidemiology and Biostatistics, Tbilisi State Medical University, Georgia

${ }^{3}$ Laboratory of Medical Microbiology, Vaccine and Infectious Diseases Institute, University of Antwerp, Belgium

Background: We used a uniform and standardized method for surveillance of antimicrobial use to assess overall quantity and quality of antibiotic prescribing in Georgia (Eastern Europe). bioMérieux provided unrestricted funding support for the survey.

Methods: The Global-PPS was conducted in four, nine and six different Georgian hospitals in 2015, 2017 and 2018 respectively. 
The survey included all paediatric inpatients receiving an antimicrobial on the day of PPS. Data included details on antimicrobial agents, reasons and indications for treatment and a set of quality indicators. A web-based application was used for data-entry, validation and reporting as designed by the University of Antwerp, Belgium (www.global-pps.com). Because of the different hospitals included, merged results are presented.

Results: In total 20 paediatric wards with 250 inpatients were surveyed of which $61.4 \%$ admitted to hospitals in Tbilisi. Overall antimicrobial prevalence was $84.6 \%$ with highest rates found in paediatric medical wards $(63.1 \%)$ and neonatal intensive care units $(20.0 \%)$. The most frequent indications for antimicrobial use were pneumonia $(44.8 \%, 101$ patients). In total 223 antibacterials for systemic use (ATC J01) were prescribed of which ceftriaxone accounted for $35.5 \%$, followed by ampicillin/sulbactam (20.4\%) and azithromycin (16.0\%). Out of all patients treated for pneumonia, $14.8 \%$ got meropenem. Out of all patients on surgical prophylaxis for the gastrointestinal tract, $76.0 \%$ received ceftriaxone, $84 \%$ used more than 3 day. With respect to antimicrobial quality indicators, the reason in notes was documented in $81.7 \%$; a stop review date in $18.2 \%$. Guidelines were missing in $9.7 \%$ and overall compliance to antibiotic guidelines was $90.2 \%$. Empiric therapy was most common $(92.8 \%)$. In $88.4 \%$ of patients CRP was used for treatment decision making.

Conclusions: The high prevalence of overall antibiotic use, particularly ceftriaxone and meropenem, the irrational antimicrobial prophylaxis in surgery and the high rate of empirical therapy are worrisome findings. Application of CRP for decision making indicates an attempt of rational antibiotic use. It is reasonable to set up feasible antimicrobial stewardship interventions to enhance appropriate antibiotic prescribing.

\section{2}

\section{Infectious Diseases}

\author{
About Leukemoid Reactions in Pediatrics \\ Maria João Palha ${ }^{1,4}$, Joana Chagas ${ }^{2,4}$, Luísa Queiró ${ }^{3,4}$, Florbela \\ Cunha \\ ${ }^{1}$ Pediatrics, Hospital de Santa Maria - Centro Hospitalar Universitário \\ de Lisboa Norte, EPE, Portugal \\ ${ }^{2}$ Pediatrics, Hospital Pediátrico - Centro Hospitalar Universitário de \\ Coimbra, EPE, Portugal \\ ${ }^{3}$ Pediatrics, Hospital de São Francisco Xavier - Centro Hospitalar de \\ Lisboa Ocidental, EPE, Portugal \\ ${ }^{4}$ Pediatrics, Hospital de Vila Franca de Xira, Portugal
}

Background: Leukemoid reaction (white blood cells [WBC] of $30000 / \mathrm{mL}$ or more) is rare in children and adolescents. It is commonly associated with infections and may indicate serious conditions. There is a lack of data regarding the underlying causes and the necessary work-up.

Objective: Investigate the clinical and laboratory characteristics of children and adolescents with leukemoid reaction.

Methods: Retrospective study consulting the clinical files of children and adolescents (1 month to 17 years old) presenting with WBC of $30000 / \mathrm{mL}$ or more, in a second level hospital, in 2017. Demographic, clinical and laboratory parameters were analyzed.

Results: A total of 29 patients were included, aged between 3 months and 9.7 years (median 1.6 years), with equal gender distribution. The majority $(65.5 \%)$ were previously healthy. Fever was present in $79.3 \%$ (31\% on the first day; $17.2 \%$ on the second; $13.8 \%$ on the third) and vomiting in $41.1 \%$. The WBC count ranged from 30000 to $56100 / \mathrm{mL}$ (mean $34628 / \mathrm{mL}$; neutrophilia was predominant in $93.1 \%$, lymphocytosis in $6.9 \%$ ) and reactive-C protein ranged from 0.5 to $38.8 \mathrm{mg} / \mathrm{dL}$ (mean $10.3 \mathrm{mg} / \mathrm{dL}$ ). The most common diagnosis were: pneumonia $(17.2 \%)$, vomiting $(17.2 \%)$, skin and soft tissue infection $(13.8 \%)$, urinary tract infection $(10.3 \%)$ and others (febrile convulsion, acute otitis media, adenoiditis, tonsillitis, mononucleosis-like illness, appendicitis). In seven cases, viral etiology was likely. Sixty-two percent were hospitalized, with a median hospitalization length of three days. Antibiotic therapy was performed in $65.5 \%$. Four of the urine cultures were positive $(4 / 15$, mostly Escherichia coli). All the blood $(n=18)$ and cerebrospinal fluid $(n=2)$ cultures were sterile. There were no cases of hemato-oncological disease, glucocorticoid administrations or polyethylene glycol intoxications.

Conclusion: Fever and/or vomiting were present in a significant number of leukemoid reactions. Almost two-thirds were hospitalized and treated with antibiotic. Leukemoid reactions in healthy children can be related to benign and common diseases as we find in our study.

403

\section{Infectious Diseases}

Clinical Predictors to Estimate the Probability of Urinary Tract Infection in Pediatric Febrile Patients Younger than 2 Years Old: Retrospective Analysis of Fever Registry

Jin Hee Lee ${ }^{l}$, Yun Seong Park ${ }^{l}$, Hyuksool Kwon ${ }^{l}$, Dongbum Suh ${ }^{l}$, Yoo Jun Choi ${ }^{l}$, Youngho Kwak

${ }^{1}$ Emergeny Medicine, Seoul National University Bundang Hospital, South Korea

${ }^{2}$ Emergency Medicine, Seoul National University Hospital, South Korea

Background: Urinary tract infection (UTI) is a significant issue in febrile young patients because it may cause harm irreversible complications in kidneys. Early detection of urinary tract infection is crucial. Urinary culture is a gold standard to diagnose UTI, but it takes time. Clinical predictors are needed to predict UTI.

Objective: To find clinical predictors that can predict UTI.

Methods: We analyzed the registry of pediatric febrile patients less than 24 months of age collected in two pediatric emergency departments from Aug. 2016 to Feb. 2018. UTI was defined as 5 X $10^{4}$ in urine culture with bacteriuria or pyuria according to AAP criteria. Viral infection (VI) is defined as the case in which the bacterial infection is clearly not detected through the medical records and laboratory tests among the patients who were discharged with the diagnosis of viral infection. We analyzed the difference in demographic and clinical findings and performed a logistic regression analysis.

Results: There were $1003(74.2 \%)$ patients in VI group and 348 (25.8\%) patients in UTI group. UTI group had more males, younger, had a history of renal diseases, and had fewer rashes. There was no significant difference on body temperature, but fever duration of UTI group was shorter than that of VI group (31.7 \pm 34.1 hours vs. $37.8 \pm 47.2$ hours, $\mathrm{p}=0.03$ ). The logistic regression revealed that the likelihood of UTI was greater in men, when less than 12 months of age, when the amount of food is reduced by $50 \%$ or more, and if a rash is present, it is unlikely to be a urinary tract infection.

Conclusion: The likelihood of urinary tract infection increases with men, when less than 12 months, when the amount of food is reduced by $50 \%$ or more, and decreases when there is a rash 


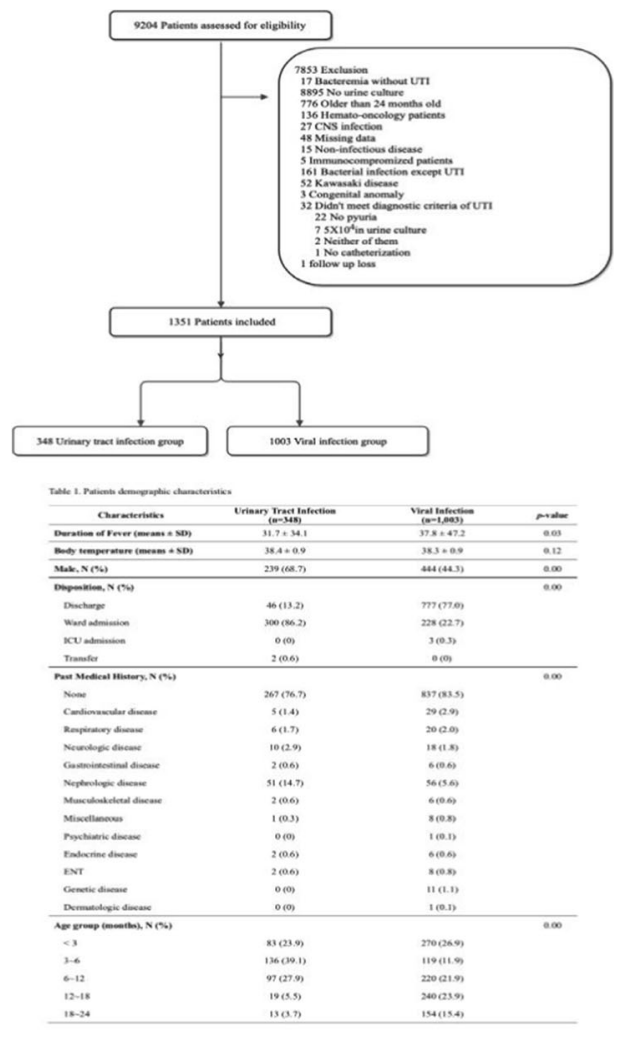

721

\section{Infectious Diseases}

Salmonella Enterica Serovar Paratyphi A Carrying the CTX-m Gene Sepsis

Surendran Chandrasekaran $^{1}$, Jamie Peoples ${ }^{1}$, Lukas Huhn ${ }^{1}$, Rajesh Rajendran ${ }^{I}$, Ignatius Losa'

${ }^{I}$ Paediatrics, East Cheshire NHS Trust, UK

We report a case of a 9-year old girl who presented with fever, recurrent gastrointestinal disturbances, and consequent dehydration, after a visit to Pakistan 3 months previously.

From her blood culture, Salmonella Enterica serovar paratyphi A was isolated, carrying the CTX-m gene and demonstrating resistance to amoxicillin, co-amoxiclav, pipericillin+tazobactam, ceftazidime, cefepime, cefotaxime, tobramycin, ciprofloxacin and tigecyclin.

This is the first case of its kind in the UK, underlining a worrying trend both toward higher proportions of enteric fever caused by S. paratyphi (an organism for which no vaccine is available), and toward increasing loss of antimicrobial effectiveness against the organism in South Asia and elsewhere. This is the first organism of its kind in the UK to be found to be resistant to all cephalosporins and narrower-spectrum beta lactamases such as amoxicillin. Further typing revealed that production of cephalosporinases by this organism contributed to this.

As geographical context typically provides the predicted bacterial sensitivities, which in turn permit our empiric antibiotic treatment (while we await lab-confirmed cultures), our empiric antibiotic selection in these cases must appropriately reflect these resistance patterns. Moreover, they starkly illustrate our vital need for appropriate antibiotic stewardship now and in the future, to preserve our ability to treat the potentially-fatal condition.

As an additional point of interest, we have identified in this case the coexistence of entamoeba histolytica species with this S. paratyphi, which we believe may be the first such finding documented in the literature. Further study is required to elucidate their pathophysiological relationship and its effects on the clinical picture.

911

Infectious Diseases

The Use of Bacterial DNA From Saliva for The Detection of GAS Pharyngitis

Naama Pines ${ }^{2}$, Saar Hashavyal, Ayelet Michael-Gayego ${ }^{3}$, Abraham Schechter $^{4}$, Itai Gross ${ }^{1}$, Allon Moses ${ }^{3}$

${ }^{1}$ Department of Pediatric Emergency Medicine, Hadassah Medical Center, Israel

${ }^{2}$ Department of Pediatrics, Hadassah Medical Center, Israel

${ }^{3}$ Department of Microbiology and Infectious Diseases, Hadassah Medical Center, Israel

${ }^{4}$ Pediatrics, Meuhedet Health Services, Israel

Background: Acute tonsillitis is one of the most common medical conditions for which children and young adults seek medical advice. Despite different methods of detection, all tests are based on GAS sampling using a throat swab. However, obtaining the swab can elicit vomiting and is often accompanied by fear and apprehension in children. The aim of this study was to find a non-invasive method for the detection of GAS pharyngitis.

Methods: A classic throat swab was obtained for culture, and a saliva sample was taken by voluntary spitting from 100 subjects recruited from Hadassah Medical Center and a Meuhedet Health Care Organization clinic (HMO). Real time PCR (RT-PCR) was performed to detect GAS dnaseB specific gene in the saliva samples. Throat swabs were sent for bacterial culture.

Results: $56 \%$ of the throat cultures were positive for GAS and $48 \%$ of the PCR samples were positive. The overall sensitivity and specificity of the 
saliva PCR method was $79 \%$ and $91 \%$ respectively; NPV and PPV were $77 \%$ and $92 \%$ respectively. When excluding patients who presented on the first day of fever, sensitivity and specificity increased to $90 \%$ and $100 \%$ respectively. No other anamnestic or clinical findings increased the yield of the test.

Conclusion: Saliva- based PCR amplification of GAS DNA method is effective in detection of GAS pharyngitis. Further studies are needed to achieve detection rates to replace the traditional throat swab- based approach.

529

\section{Infectious Diseases}

Abdominal Pain: An Unlikely Diagnosis

${ }_{\text {Diana Pinto }}{ }^{1}$, Teresa Pinheiro ${ }^{I}$, Alexandra Martins ${ }^{l}$, Joana Cardoso ${ }^{l}$, $\overline{\text { Teresa Oliveiral }}^{l}$, Susana Tavares ${ }^{I}$

${ }^{I}$ Serviço de Pediatria e Neonatologia, Centro Hospitalar Entre Douro e Vouga, Portugal

Pyomyositis is a rare infection of the striated muscle. Generally results from hematogenous spread of septic emboli. It is caused mainly by Staphylococcus aureus. The clinical presentation is unspecific and the diagnosis is often difficult and delayed.

We present the case of a previously healthy 14 years old boy, who was admitted at Emergency Department for localized pain in the left inguinal region for 2 days. No fever, urinary complaints and no history of trauma. He presented with left lower limb flexed and pain on the left iliac fossa and anterosuperior iliac spine. X-ray and ultrasound of the pelvis did not revealed any abnormal find. Analytically he had leukocytosis, with predominance of neutrophils, C-reactive protein $79.6 \mathrm{mg} / \mathrm{L}$ and sedimentation velocity of $81 \mathrm{~mm}$ in the first hour. He performed an abomino-pelvic computed tomography scan that was normal, however, he was admitted in our hospital for surveillance. In day 1 he developed fever and inflammatory signs in the left inguinal region, therefore empirical antibiotic therapy was started with intravenous Flucloxacillin and Clindamycin. Subsequently, he performed an magnetic resonance imaging that identified multiple abscesses adjacent to the left anterior-superior iliac spine and left oblique and iliac muscles. Drainage of the abscesses was performed but no etiological agent was identified. This patient completed 14 days of intravenous antibiotic therapy and was than discharged with indication to complete five weeks of antibiotic therapy. Of the cultural exams requested the patient presented three negative blood cultures and immunological study revealed to be normal.

Early diagnosis of pyomyositis is crucial and requires a high level of suspicion. In this patient it was not possible to isolate the etiologic agent probably because the abscess drainage was already performed under antibiotic therapy. Its evolution was favorable, with no associated complications and no functional limitations at the time of discharge.

587

\section{Infectious Diseases}

\section{Bacterial Acute Gastroenteritis: A 6 Year Study in a Tertiary Hospital \\ $\underline{\text { Mariana Portela }}^{1}$, Ana Catarina Oliveira ${ }^{3}$, Ana Sara Silva ${ }^{4}$, Ana Sofia $\overline{C o s t a}^{5}$, José António Moreira ${ }^{4}$, Paulo Capelo ${ }^{5}$, Bruna Malheiro ${ }^{2}$, Marina Majar ${ }^{2}$, Filipa Neiva ${ }^{1}$, Manuela Costa Alves ${ }^{1}$ \\ ${ }^{1}$ Pediatrics, Hospital de Braga, Portugal \\ ${ }^{2}$ Clinical Pathology, Hospital de Braga, Portugal \\ ${ }^{3}$ Family Medicine, ACES Cávado I - USF do Minho, Portugal \\ ${ }^{4}$ Family Medicine, ACES Cávado I - USF M. Rocha Peixoto, Portugal \\ ${ }^{5}$ Family Medicine, ACES Cávado I - USF Gualtar, Portugal}

Background: Acute gastroenteritis (AGE) is an important cause of morbidity in developed countries. Indications for coprocultures are restricted and symptomatic therapy is indicated in most cases. However, the knowledge of epidemiology is fundamental in guiding therapeutic decisions. The objective of this study is to know the evolution of the local epidemiology of acute intestinal bacterial infections of children observed in a level III hospital in the last 5 years.

Objective and Methods: A total of 247 positive stool samples were obtained, between 2012 to 2017 . The peak of positive tests occurred during Summer, most of them in preschool age. Campylobacter spp. was the most prevalent agent (55.1\%), followed by Salmonella spp. $(34.8 \%)$, Yersinia (4\%) and Shigella sonnei (3.6\%). Faeces with blood have been detected frequently in Campylobacter spp. and the duration of diarrhea were prolonged in Salmonella spp cases. Fever were present in $69.1 \%$. Vomits were frequent $(41.1 \%)$. Bacterial co-infections were in $2 \%$ of the cases and viral ones in $12.6 \%$, mostly due to rotavirus. References of a familiar context of AGE were present in 51\%. Dehydration was the main cause of complication. Hospital admissions (39\%) were superior compared with other studies, mainly due to Salmonella spp infections. Antibiotics were given in 32 cases, with resistance profile pattern similar to other national studies.

Conclusion: The aetiology of AGE has been changing, depending on the country. There is a lack of studies giving us a local pattern of bacterial AGE, allowing the possibility to initiate targeted preventive measures. It is also a concern to know the principal clinical features of bacterial AGE, in order to minimize unnecessary tests or treatments, once antibiotics are still used in a high percentage of cases.

\section{0}

P3: Infectious Diseases 3 (Sunday, September 22, 2019 10:30)

\section{Infectious Diseases}

Pertussis Infection; Recent Concerns and New Recommendations Ali Raba ${ }^{1}$, Ibraheem Krebit ${ }^{1}$

${ }^{T}$ Paediatric Emergency Department, Tallaght University Hospital, Ireland

Pertussis is a highly contagious bacterial disease which can affect people at any age, and which is significantly underdiagnosed. Its epidemiology has recently changed with a shift in distribution from infants and young children towards older age groups. Immunity from vaccination and from illness wanes over time, leaving adolescents and adults susceptible. These are the main source of infection in infants, in whom significant complications are most common. In order to improve individual and herd immunity and thus reduce transmission of infection to young infants, an adolescent pertussis booster vaccine is recommended. This review discusses recent findings in B. pertussis epidemiology and recent advances in the diagnosis, treatment and prevention, and vaccination recommendations for the ultimate elimination of pertussis disease.

389

\section{Infectious Diseases}

Not Just a Pneumonia...

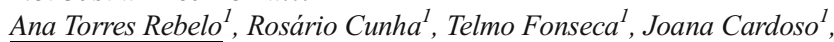
Joana Monteiro $^{I}$, Mariana Pinto ${ }^{l}$

${ }^{1}$ Centro Hospitalar entre o Douro e Vouga, Pediatrics/ Neonatology, Portugal

Introduction: A lung abscess is an uncommon pediatric problem, characterized by a thick-walled cavity (larger than 2 centimeters) in the pulmonary parenchyma that contains purulent material resulting from a pulmonary infection. The pathogenesis of lung abscess involves an area of initial pneumonitis that leads to necrosis, cavitation and abscess formation. It is classified as primary, or secondary, based upon the existence of pre-existing conditions. The diagnosis is usually made on chest 
radiography, however, the use of computed tomography scan may facilitate the distinction between a lung abscess and other conditions such as necrotizing pneumonia.

Clinical Case: A 2-year-old boy, previously healthy, was admitted to the pediatric department following 10 days of fever and cough despite 7 days of treatment with oral amoxicillin/clavulanic acid. Examination revealed tympanic temperature $37,4{ }^{\circ} \mathrm{C}$, oxygen saturation $96 \%$ in ambient air, auscultation with soft crackles over the right chest. Examination of other systems was normal. The peripheral blood count showed a hemoglobin of $10,1 \mathrm{~g} / \mathrm{dL}$, leukocytosis of $16,000 / \mathrm{uL}$ with a differential neutrophil count of $73,8 \%$ and a C-reactive protein of $247 \mathrm{mg} / \mathrm{L}$. Blood cultures were negative. The chest radiograph revealed a rounded cavity involving the left hemithorax and the possibility of lung abscess was considered. The computed tomography scan described a parenchymal consolidation of the left lower lobe with necrosis and parenchyma cavity with hydro aerial level. The clinical signs resolved within 24 hours of initiation of therapy with intravenous ampicillin 400 $\mathrm{mg} / \mathrm{kg} /$ day and clindamycin $30 \mathrm{mg} / \mathrm{kg} / \mathrm{day}$. After 2 weeks of treatment, the chest radiograph showed a marked reduction of the abscess. This treatment was continued for 3 weeks, when it was changed to oral clindamycin for an additional week.

Conclusion: In conclusion, lung abscess is infrequent in infants and children.

The initial choice of treatment is conservative management and the prognosis is usually very good.

\section{6}

\section{Infectious Diseases}

\section{A Differential Diagnosis of Painful Hip}

${ }_{\text {Ana Torres Rebelo }}^{I}$, Diana Pinto ${ }^{I}$, Diana Reis Monteiro ${ }^{I}$, Sara Freitas Oliveira $^{I}$

${ }^{1}$ Centro Hospitalar entre o Douro e Vouga, Pediatrics/Neonatology, Portugal

Introduction: Obturator internus muscle abscess is an uncommon clinical entity and should be considered as a differential diagnosis in the child who presents with a painful hip. The clinical presentation can be similar to bacterial infection of the hip joint and it generally presents as fever, hip pain with decreased range of motion, and limp.

Clinical Case: We report the case of a 10-year-old boy, previously healthy, that resorted to the emergency department because of right hip pain, limp and a three-day fever. In the physical examination, there was pain over the right thigh area which aggravated with hip flexion and rotation. He played football, but there was no history of recent trauma. In the laboratorial tests performed, there was an increase of the erythrocyte sedimentation rate and C-reactive protein (ESR $23 \mathrm{~mm} / \mathrm{h}$, CRP 99,5 mg/L). Blood cultures were negative. Ultrasound of the soft tissues revealed hip joint effusion. On suspicion of septic arthritis, surgical drainage of the joint was performed, and this hypothesis was not confirmed. A magnetic resonance imaging (MRI) of the pelvis revealed an abscess of the right internal obturator muscle and acetabular osteitis.

The child completed 6 weeks of cefuroxime antibiotic therapy with favorable clinical and analytical evolution. Six months after discharge, the patient is asymptomatic and without changes to the objective examination.

Conclusion: The diagnosis of obturator internus muscle abscess is frequently late because of its rarity and is often mistaken for septic arthritis of the hip. Small collections may not be detected on ultrasound, especially when they affect the obturator internus muscle, because of its deep location. MRI is a very sensitive examination for the diagnosis. Appropriate antibiotic therapy initiated early is critical in the resolution of this pathology.
987

\section{Infectious Diseases}

Ramsay Hunt Syndrome: a Pediatric Case Ana Isabel Ribeiro ${ }^{1}$, Cristina Rodrigues ${ }^{1}$, Isabel Costa ${ }^{2}$, Ana Antunes ${ }^{1}$, $\overline{S o f i a}_{\text {Martins }}{ }^{\prime}$, Susana Carvalho ${ }^{1}$, Teresa Pontes ${ }^{1}$

${ }^{1}$ Paediatric Department, Hospital Braga, Portugal

${ }^{2}$ Otorhinolaryngology Department, Hospital de Braga, Portugal

Background: Ramsay Hunt Syndrome (RHS) is characterized by peripheral facial palsy, periauricular pain, vesicular eruptions of the external canal and pinna and vestibulocochlear dysfunction. RHS is rare in children but it is the second most common cause of nontraumatic facial palsy in childhood. The diagnosis is primarily clinical and treatment is a combination of high-dose corticosteroids and acyclovir.

Case Report: A 13-year-old boy was admitted in the emergency room due to aggravation of right earache in the last five days, without fever. Seven days before he started treatment with amoxicillin and clavulanate for right acute media otitis. He was diagnosed with type 1 diabetes mellitus at 10 years of age and had varicella infection at 8 years old. The physical examination revealed: right auricular edema, pain at mastoid palpation and redness of ipsilateral eardrum at otoscopy. Few hours later, he developed a vesiculobullous rash on the right ear canal and on the right half of the palate in addition to a right peripheral facial palsy (House-Brackmann scale grade II) and vestibulocochlear symptoms (hearing loss and vertigo).

The complete blood count, the inflammatory markers and the computed tomography scan were normal. Pure tone audiogram confirmed a mild sensorineural hearing loss on the right side. The patient was hospitalized and started intravenous acyclovir $(30 \mathrm{mg} / \mathrm{Kg} /$ day), ceftriaxone $(50 \mathrm{mg} / \mathrm{Kg}$ / day) and prednisolone (60 $\mathrm{mg} /$ day) for seven days. The prednisolone dose was tapered during the following two weeks. Facial physiotherapy and eye care were also prescribed. On discharge day, the audiogram was normal. The adolescent showed a complete recovery of facial nerve function within the 20 days after discharge.

Discussion: The authors want to emphasize the importance of careful examination and early initiation of therapy in suspected cases of RHS, which improves the recovery rate of the facial nerve palsy and have a positive effect on disease prognosis.

842

\section{Infectious Diseases}

Acute Pediatric Mastoiditis: A Single-Center Experience. Pre and Post Pneumococcal Vaccination and "Watchful-Waiting" Era Goran Ristic $^{I}$, Andrea Otero ${ }^{1}$, Josep Maria Gairi ${ }^{1}$, Matilde Vinas ${ }^{1}$, Bergona De Frias Berzosa $^{2}$, Ivan Domenech ${ }^{2}$, Victoria Montoro ${ }^{2}$ ${ }^{1}$ Pediatrics, University Hospital Quiron Dexeus, Spain

${ }^{2}$ Otorhinolaryngology, University Hospital Quiron Dexeus, Spain

Background:A suppurative infection of the mastoid air cells, complication of acute otitis media, is defined as acute mastoiditis.With the introduction of pneumoccocal vaccination, the incidence of otitis media in pediatric population has dropped and new treatment guidelines have been introduced, but it remains unclear whether this decrease has been also seen in acute mastoiditis.

Objective:To compare and evaluate the demographics and clinical characteristics ofchildrenwith acute mastoiditisin pre and post pneumoccocal vaccination and "watchful-waiting" era.

Methods: We reviewed retrospectively medical charts of childrenaged 1 month to 16 years admitted at the University Hospital Quirón Dexeus with acute mastoiditis from January 2014 to January 2019 and compared it with a group collected and published in a period from January 1998 to January 2002. Results:A total of twenty-two cases were collected, 14 (68\%) males and 8 $(32 \%)$ females, with the mean age of 5.4 years and $6(27.3 \%)$ patients younger than 2 years. We found a statistically significant decrease in the 
incidence, use computerized tomography as a mean on diagnosis, less use of third generation cephalosporins and faster diagnosis compared with the previous group.

Conclusions:After the introduction of pneumococcal vaccination, the incidence of acute mastoiditis in our population has been reduced, but this remains to be proven in larger studies. The more prudent use of antibiotics and the vaccination will prove to be rightful pathway to further reduce this potentially harmful complication.

\section{3}

\section{Infectious Diseases}

\section{Acute Miliary Tuberculosis}

Ana Barbosa Rodrigues ${ }^{1}$, Mafalda Castelão ${ }^{1}$, Carina Cardoso ${ }^{2}$, Catarina Lacerda ${ }^{3}$, Filipa Prata ${ }^{1,4}$, Ana Mouzinho ${ }^{1,4}$, Francisco Abecasis ${ }^{1,5}$, José Gonçalo Marques ${ }^{1,4}$

${ }^{1}$ Department of Paediatrics, Hospital de Santa Maria - Centro Hospitalar Universitário Lisboa Norte, Portugal

${ }^{2}$ Department of Paediatrics, Hospital de Cascais, Portugal

${ }^{3}$ Department of Paediatrics, Hospital do Barreiro, Portugal

${ }^{4}$ Pediatric Infecciology Unit, Hospital de Santa Maria - Centro Hospitalar Universitário Lisboa Norte, Portugal

${ }^{5}$ Pediatric Intensive Care Unit, Hospital de Santa Maria - Centro Hospitalar Universitário Lisboa Norte, Portugal

Introduction: Miliary tuberculosis is a clinical disease resulting from hematogenous dissemination of Mycobacterium tuberculosis. Clinical manifestations are most likely subacute or chronic and are nonspecific. Risk factors may be absent. Diagnosis is confirmed through bacteriologic identification. Case Description: A 16-year-old healthy female was admitted at our hospital with a 10-day history of daily fever, cough, night sweats and $20 \%$ estimated weight loss. Her symptoms failed to improve with previous azithromycin and amoxicillin-clavulanate treatment. Originating from Angola, she had a household positive history for bacilliferous pulmonary tuberculosis three months before symptoms onset. On physical exam she presented tachypnea, hypoxemia and crepitations throughout both lung fields. Chest radiograph showed a bilateral reticulonodular opacification pattern; laboratory tests revealed C-reactive protein of $7.92 \mathrm{mg} / \mathrm{dL}$ and erythrocyte sedimentation rate of $35 \mathrm{~mm} / \mathrm{sec}$, with normal blood cell count. HIV serology was negative. Smear and culture of sputum were negative for acid-fast bacilli. Initial treatment included supportive measures, however within 72 hours she gradually developed acute respiratory distress syndrome (ARDS), requiring management at the pediatric intensive care unit through ventilatory support and higher oxygen supply. Treatment was iniciated with piperacilin-tazobactam, isoniazid, rifampicin, pyrazinamide, ethambutol and prednisolone. A bronchoalveolar lavage was performed and Mycobacterium tuberculosis was identified. Piperacilin-tazobactam was stopped with a favorable evolution. Fever and hypoxemia relapsed after prednisolone withdrawal and a more prolonged course with slow tapering was needed. Mycobacterial blood cultures were negative. No extrapulmonary disease was identified. Her overall outcome was good.

Conclusion: We present an immunocompetent patient with miliary tuberculosis and a severe acute presentation. Tuberculosis must be considered in any patient with epidemiologic risk. A negative sputum smear may be expected in miliary tuberculosis. Since this is a potentially lifethreatening condition, prompt diagnosis and early intervention are keys to survival. Though controversial, steroid therapy may play an important role in patients with ARDS.

\section{2}

Infectious Diseases

A Fatal Case of Haemophilus Influenzae Type B Epiglottitis in Vaccinated Child
Joana Rosa ${ }^{1}$, Raquel Amaral ${ }^{1}$, António Paiva ${ }^{2}$, Anuschka Langner ${ }^{3}$, Marta Oliveira ${ }^{4}$, Vera Brites ${ }^{4}$, João Estrada ${ }^{4}$

${ }^{1}$ Pediatric Department, Divino Espirito Santo Hospital, Portugal

${ }^{2}$ Anesthesiology Department, Divino Espirito Santo Hospital, Portugal

${ }^{3}$ Intensive Care Unit, Divino Espirito Santo Hospital, Portugal

${ }^{4}$ Pediatric Intensive Care Unit, Hospital Dona Estefânia, Portugal

Background: Haemophilus influenzae type b (Hib) can be responsible for severe and life-threatening infections, such as epiglottitis. Since the introduction of Hib conjugate vaccine, it was observed a considerable reduction in Hib invasive disease and a decrease in community nasopharyngeal carriage.

Case report: A healthy and fully immunized 5-year-old girl was admitted to the pediatric emergency department with sore throat, dyspnea, and stridor. Physical examination revealed respiratory distress and diminished vesicular sounds. The general condition worsened after nebulized Lepinephrine and dexamethasone, culminating in an ill-looking appearance. Epiglottitis was suspected and intubation material was being prepared when she went into cardiopulmonary arrest. Advanced pediatric life support maneuvers were carried out for 15 minutes, which included difficult intubation and neuroprotective measures. She was transferred to the intensive care unit and connected to mechanical ventilation. Laboratory analysis revealed leukocytosis $31.46 \times 10^{9} / \mathrm{L}$, neutrophilia $29 \times 10^{9} / \mathrm{L}$, C-reactive protein $7.88 \mathrm{mg} / \mathrm{dL}$ and procalcitonin $46.7 \mathrm{ng} / \mathrm{mL}$. She was medicated with cefotaxime, clindamycin, and metronidazole. Multiplex PCR detected Haemophilus influenza and microbiologic analysis confirmed Hib.

She also had myoclonic movements, after cardiopulmonary arrest, and cranioencephalic CT scan together with Transcranial Doppler ultrasound (TCD) showed cerebral edema. The electroencephalogram presented status epilepticus and as so phenobarbital, propofol, levetiracetam, and 3\% sodium chloride were initiated.

The patient was referred to a pediatric intensive care unit in a tertiary hospital. She was evaluated by pediatric neurology and MRI exam revealed severe hypoxic-ischemic brain injury. The neurologic exam showed a progressive deterioration, reporting an irreversible loss of brain function, including the brain stem. Electroencephalogram showed isoelectric trace and TCD demonstrated cerebral circulatory arrest, having been decided to withdraw life support.

Conclusion: Despite the high efficacy of Hib vaccine, invasive Hib disease can occur and be fatal in an apparently immunocompetent and immunized child. Epiglottitis should continue to be considered in patients with dyspnea and stridor.

\section{6}

Infectious Diseases

Epidemiology of Hospitalizations Related to Coding (ICD-9-CM) of the Influenza Diagnosis in Infants up to 6 Months of Age in Spain (2009 - 2015)

Jesus San Roman $^{1}$, Ruth Gil Prieto ${ }^{1}$, Angel Gil de Miguel ${ }^{1}$

${ }^{T}$ Departamento de Medicina y Cirugía, Psicología, Medicina Preventiva y Salud Pública e Inmunología y Microbiología Médica y Enfermería y Estomatología, Universidad Rey Juan Carlos, Spain

Background: Influenza is a vaccine-preventable respiratory infection that results in substantial morbidity and mortality each year. Although infants below 6 months of age have the highest hospitalization rates for their increased risk of developing severe influenza, the existing influenza vaccines are not licensed for this group of age.

Objectives: To analyze hospitalization episodes with an ICD-9 diagnosis code of influenza (codes 487 and 488) in any diagnostic position in infants up to 6 months of age from 2009 to 2015 in the Spanish hospital surveillance system.

Methods: Retrospective survey by reviewing data of the National Surveillance System for Hospital Data including more than $98 \%$ of 
Spanish hospitals and $99.5 \%$ of the population in Spain. It contains data about admission and discharge date, age, sex, diagnosis and discharge status for all hospitalizations in our country. All hospitalizations related to coding (ICD-9-CM; 487, 488) of the influenza diagnosis in infants up to 6 months of age were analyzed.

Results: A total of 2,739 hospital admissions were obtained and analyzed. Of those, 2,676 admissions corresponded to diagnoses ICD-9 code $487(97.7 \%)$ and $63(2.3 \%)$ corresponded to ICD-9 code 488 . Males were $59.6 \%$ and females were $40.4 \%$. The global median age was 1.7 months and the average inpatient length of stay was 6.8 days (95\% CI, $6.5-7.1)$. A total of ten deaths were recorded among the inpatients throughout the entire period, corresponding to an inpatient hospital fatality rate of $0.4 \%$. Conclusions: In our study, ICD-9-CM codes 487 and 488, related to influenza diagnosis, are present in a high number of hospitalizations in infant below 6 months of age. Improving preventive strategies such as increasing vaccination coverages in elderly population, cocoon immunization and vaccination strategy of the pregnant woman may reduce these hospitalizations.

\section{6}

\section{Infectious Diseases}

Multiple Brain Abscesses Due to Actinomyces Odontolyticus: A Rare İnfection In a Previously Healthy Child

Betul Saritas $^{1}$, Burca Bursal Duramaz ${ }^{2}$, Osman Yesilbas $^{3}$, Oznur Gokce Nizam $^{1}$, Mustafa Namik Oztanir ${ }^{4}$, Abdurrahim Tekin ${ }^{4}$, Ozden Turel ${ }^{2}$, Safa Baris $^{5}$

${ }^{1}$ Department of Pediatrics, Bezmialem Vakif University School of Medicine, Turkey

${ }^{2}$ Department of Pediatrics, Division of Pediatric Infectious Disease, Bezmialem Vakif University School of Medicine, Turkey

${ }^{3}$ Department of Pediatrics, Division of Pediatric Intensive Care, Bezmialem Vakif University School of Medicine, Turkey

${ }^{4}$ Department of Neurosurgery, Bezmialem Vakif University School of Medicine, Turkey

${ }^{5}$ Department of Pediatrics, Division of Pediatric Allergy and Immunology, Marmara University, Turkey

Actinomyces spp. are commensal gram-positive, non-sporulating, anaerobic bacteria which may cause infections in both immunocompetent patients and in patients with impaired host defenses. Actinomycosis of the central nervous system (CNS) is a rare manifestation affecting between $1-$ $15 \%$ of cases with actinomycotic infection. We present a child with multiple brain abscess due to $A$. odontolyticus.

Case: A $2^{6 / 12}$ years-old male patient with neither previous history of hospitalization or trauma nor family history of early death or serious illness (except prematurity-32 gestation week and twin) was admitted (five and one day ago before admission to our pediatric intensive care unit (PICU)) with complaints of fatigue, vomiting and nasal discharge (no fever). At secondary admission, brain CT and MRI scan were performed because of weakness of the extremities on the left side revealing three brain abscesses in the right hemisphere and a shift to the left hemisphere. He was transferred to our PICU for further treatment. In addition to neurosurgical drainage of the abscess, vancomycin plus ceftriaxone and metronidazole intravenously were initiated. Also decompressive craniectomy was performed. Actinomyces odontolyticus was isolated from the pus obtained during surgery. Since he had lymphopenia $\left(1300 / \mathrm{mm}^{3}\right)$ primary and secondary immunodeficiencies were searched. HIV serology was negative. Serum immunoglobulins, lymphocyte subset analysis, and dihydrorhodamine (DHR) test results were normal. The blood specimen for $\mathrm{CH} 50$ was sent, all exons analyzes were performed in terms of genetic defects.

Conclusion: Antimicrobial treatment following neurosurgical evaluation of the CNS abscess resulted in complete neurofunctional recovery. The clinicians should keep in mind that $A$. odontolyticus may be a causal agent in brain abscesses even in healthy children.
829

\section{Infectious Diseases}

Severe Malaria in a Spanish Tertiary Hospital: A Descriptive Study Mariano Silva Hernandez ${ }^{5}$, Virginia Santana Rojo ${ }^{5}$, Inés De Augusto Claudino $^{I}$, José David Andrade Guerrero ${ }^{5}$, Celia Fabra Garrido ${ }^{5}$, Marta Bautista Barea ${ }^{5}$, Patricia Bote Gascón ${ }^{2}$, Serafín Castellano Dámaso ${ }^{5}$, Marta Dorado Criado ${ }^{5}$, Jesús Domínguez Riscart ${ }^{5}$, Luis Escosa Garcia ${ }^{5}$, Cristina Ots Ruiz, Milagros García Hortelano, Fernando Baquero Artigao, Teresa Del Rosal Rabes ${ }^{5}$, José Antonio Ruiz Dominguez, Silvia García Bujalance ${ }^{3}$, Cristina Verdú Sánchez ${ }^{4}$, María De Ceano-Vivas La Calle ${ }^{2}$, Juan José Menéndez Suso ${ }^{4}$, Francisco Javier Aracil Santos 5

${ }^{1}$ Paediatrics, Centro Hospitalar Oeste, Portugal

${ }^{2}$ Paediatric Emergency, Hospital Universitario La Paz, Spain

${ }^{3}$ Microbiology, Hospital Universitario La Paz, Spain

${ }^{4}$ Paediatric Critical Care, Hospital Universitaria La Paz, Spain

${ }^{5}$ Hospitalary Pediatrics infectious and tropical diseases, Hospital

Universitario La Paz, Spain

Background: In recent years there has been an increasing in number of tropical stays in children. They are related to tourism, migration and international adoption. Malaria in one of the main diseases that should be ruled out in this kind of patients. Children under 5 years old have an increased risk of severe illness. In fact, malaria is the fourth cause of children mortality worldwide. Therefore, we should study malaria and its treatment, specially its features in children and its severe form.

Objective: The aim of the present study was to describe epidemiological, clinical, analytical, microbiological, radiological and treatment characteristics of patients with severe malaria in a tertiary Spanish hospital.

Methods: We performed a case series study. It included 12 patients who suffered from severe malaria and were admitted from 2004 to 2018.

Results: The average age of patients was 4 years old. Eight patients were female. Four patients were transferred from other hospitals and two by the airport sanitary services.

All severe cases were caused by Plasmodium falciparum. All patients contracted the disease in west Africa. Seven of the them lived there and travelled to Spain. The other five patients travelled from Spain to visit relatives. Eight patients (75\%) got malaria in Equatorial Guinea. Just one received prophylaxis with mefloquine.

Fever and digestive symptoms were the most frequent. One patient, treated with intravenous quinine, suffered black water fever. Table shows main features. Five patients showed a parasitaemia equal or higher than 5\%. The highest parasitaemia detected was $12 \%$. Intravenous quinine plus clindamycin and intravenous artesunate were the most frequently used initial treatments. None of the patients presented sequelae related to malaria or died. Conclusion: Malaria should be suspected in every patient with fever traveling from endemic countries. Most children with severe malaria had parasitaemia under $10 \%$. These patients present severe complications and must be initially treated in critical care settings.

\section{2}

\section{Infectious Diseases}

Significance of the Pneumococcal Vaccine to Children's Health: Research Conducted in Drachevo and its Environment (2015-2018) Svetlana Smugreska ${ }^{1}$, Mitka Trencheva ${ }^{3}$, Velibor Tasic ${ }^{2}$

${ }^{T}$ Paediatrics, PZU D-r Smugreska, Macedonia

${ }^{2}$ Nephrology, Children's Clinic, Macedonia

${ }^{3}$ Paediatrics, PZU D-r Trencheva, Macedonia

Introduction: Streptococcus pneumoniae is an invasive bacillus and a trigger of upper and lower respiratory infections. The invasive strain and the child's immune response are the focal factors to whether it will spread to a disease or will remain within the frame of a carriage status. 
Aim: With the introduction of a vaccine that is not on a regular vaccine calendar, the number of children infected with rhinosinusitis, otitis and pneumonia, has dropped significantly.

Methods and Investigations: A study of 325 children has been conducted in the period of 2015-2018, out of which, 191 males and 134 females, carriers of pneumococci in nasopharynx. 242 children attend kindergarten and 83 are taken care of at home. Depending on the clinical presentation, a symptomatic and antibiotic treatment has been provided. After receiving 1 to 2 doses of the vaccine, the number of paediatrician visits decreased dramatically. Also, outstanding effect has been detected to the sample who received 2 doses.

Conclusion: Although the pneumococcal vaccine is not mandatory in the vaccine calendar, it delivers great benefits including reduced usage of antibiotics. It is my personal regret that it's not available yet in my country and the children remain unprotected, therefore, I appeal for its procurement since it's vital for the health of children between the age of 9 months and 3 years.

\section{9}

\section{Infectious Diseases}

\section{Cystitis Cystica in Children}

Magdalena Szymanek-Szwed $^{l}$, Katarzyna Zateska-Oracka ${ }^{l}$, Joanna Samotyjek', Beata Jurkiewicz'

${ }^{I}$ Pediatric Surgery and Pediatric Urology Clinic, Centre of Postgraduate Medical Education, Poland

Infections of the urinary tract are the second frequent among all bacterial infections in the pediatric population.

Recurrent infections lead to chronic inflammatory changes. If the symptoms of cystitis last for more than 2-3 weeks or recurrences appear after a short period of remission, chronic cystitis may be assumed. The most common form of chronic inflammation in children is cystitis cystica, diagnosed in approximately $10 \%$ of patients with urinary tract infections, with a definite female predominance $(80 \%)$.

Macroscopically, in the endoscopic examination small cysts of pearl, pink, brown or yellow color are found. The lesions are usually located in the area of the bladder triangle, neck and around the ureteral mouth, but they can occupy the entire surface of the bladder, and are rarely described in the ureter and pelvis. The bladder wall trabeculation, swelling and congestion of the mucous membrane, incorrect location and widening of the the ureters orifices are often found.

The pathogenesis of the disease is unclear.

The most frequently isolated inflammatory agent is E.coli, and other Gram-negative bacteria: Pseudomonas, Klebsiella, Enterobacter and others.

According to some authors, cystitis cystica is considered a precancerous condition.

Clinically, the inflammation may be asymptomatic or proceed in the form of active infection. Chronic inflammation can lead to urinary incontinence, urgency, pollakiuria, dysuria and even urinary retention.

In urodynamic studies, a decrease in the volume of urine given per portion in relation to the expected values, irregular curves of tubular flows, their flatness, residual urine after micturition, are observed, which is the cause of subsequent infections.

Treatment of cystitis cystitis is difficult and long-lasting. A multi-month alternation treatment with furazidine and trimethoprim with sulphamethoxazole administered orally and intravesical instillations of antibiotics (gentamicin) and corticosteroids (prednisone) are applied. The results of treatment depend on the severity of changes in the bladder, and full recovery is obtained only in the case of lesions of low and medium intensity.

The authors present their own experience regarding the diagnosis and treatment of patients with cystitis cystica.
398

Infectious Diseases

Resurging Measles Attendances from the Perspective of a Paediatric Emergency Department

Sung Shin Teng

${ }^{T}$ Children's Emergency, KK Women's and Children's Hospital, Singapore

Background: Viral exanthemas in the paediatric age group are common. The worldwide resurgence of measles infection in recent years and its impact on the paediatric population of a small cosmopolitan city-state, with high volume of migrational traffic like Singapore is unknown. Our study aims to describe epidemiological trends of proven measles infection cases that presented to our emergency department and identify risk factors that can influence our emergency department isolation policies.

Methods: This study is a retrospective observational cohort study, looking at laboratory-confirmed measles infection in patients. Patients admitted through our paediatric emergency department from January 2010 to December 2016 were included. Data was collected on demographics, clinical features at presentation, isolation precautions implementation, admission duration, and vaccination records.

Results: A total of 277 patients were hospitalised for measles infection. Of these, $90(32 \%)$ patients were isolated at triage at initial presentation. Eighty-one (29\%) patients attended the emergency department more than once, prior to admission. Patients presented to the emergency department at an average of 4.3 day of illness at presentation. At initial presentation, $75(27 \%)$ patients did not have rash. Out of all the admitted patients with measles, only 1 patient had completed 2-dose measles vaccination.

Conclusions: Our study found that it is difficult to adequately identify and isolate all patients with measles infection, even with strict isolation strategies. As such, there is an urgent need for global healthcare systems to promote vaccination uptake to increase herd immunity and curb the spread of infection, especially to at-risk population groups.

\section{5}

\section{Infectious Diseases}

Effectiveness of Probiotics in the Treatment of Acute Gastroenteritis Caused by Rotavirus in Children Under 5 Mariia Teslenko ${ }^{1}$, Lyudmila Chernyshova ${ }^{2}$, Alexander Kasyan ${ }^{3}$ "Paediatric Department, Medical Center "Dobrobut-Clinic" Llc, Ukraine

${ }^{2}$ National Medical Academy Of Postgraduate Education, Ukraine ${ }^{3}$ Pediatric Hospital \# 1, Ukraine

Introduction: Acute Gastroenteritis(AGE) is a second common cause of death in children under 5.Probiotics are being used in the treatment of AGE, addition to the rehydration. Saccharomyces boulardii( S.bulardii)and (Lactobacillus rhamnosus $G G$ )LGG have a moderate level of evidence and strong recommendation, Lactobacillus reuteri DSM17938(L.reuteri) has a low level of evidence and weak recommendation. Objective: Compare the effectiveness of probiotics with a strong recommendation and moderate level of evidence (L.rhamnosus GG and S.boulardii) and probiotics with a low recommendation and low level of evidence (L reuteriDSM-17938) as adjuvant therapy for AGE caused by rotavirus in children under 5 .

Methods: 122 children under 5 with symptoms of AGE were examined. Determination of the antigen of the rotavirus was performed by ELISA. The children were divided into groups depending on which probiotic were used. The first group received a LGG or S.boulardii. The second group was treated with L. reuteri. The number of episodes of stools/day, the length of stay in a hospital and the total duration of diarrhea syndrome were estimated.

Results: The first group was 59 children, in the second group- 63 children. In the $1^{\text {st }}$ group the number of episodes stool/day(Me[QI 25;75]) was 
$5[4 ; 8]$, the length of stay in a hospital(Me[QI 25;75])-3[2;4] and the total duration of diarrhea syndrome(Me[QI 25;75])-7[6;8].

In the $2^{\text {st }}$ group the number of episodes stool/day(Me[QI 25;75]) was $8[5 ; 12]$, the length of stay in a hospital(Me[QI $25 ; 75])-4[2 ; 4]$ and the total duration of diarrhea syndrome(Me[QI 25;75])-7[5;10].

We compared two groups of children. A significant difference was in the number of diarrhea stool episodes $(\mathrm{p}=0.02)$. There were no statistically significant difference in the others criteria $(\mathrm{p}=0.43)$ and $(\mathrm{p}=0.78)$ respectively.

\section{Conclusions:}

1. LGG and S.bullardi has better affect the reduction of stool frequency then L.reuteri, and thus ease the symptoms of the disease.

2. LGG,S.bullardi and L.reuteri have the same effect on the duration of the disease and hospitalization.

\section{4}

Influenza Profile on Otherwise Healthy Children Hospitalized in a District Hospital over Winter Season 2018-2019 in Greece

Efitsek Tsekoura ${ }^{1}$

${ }^{T}$ Pediatric Department, Asklepieion General Hospital, Greece

Background: Influenza cases profile is of epidemiologic interest in order to take measures controlling infection spread and reducing severe morbidity and mortality. In Greece epidemiologic influenza surveillance is contacted by the Hellenic Center for Diseases Control and Prevention HCDCP.

Aim: To study influenza like episodes in pediatric patients admitted during winter period 2018-2019 in a District General Hospital, Athens Greece, which serves 1.500.000 inhabitants.

Materials and Methods: Retrospective analysis of influenza like cases admitted in children's ward during winter 2018-2019. Outcome measures included virus type, main symptoms, complications, sex, ethnicity and the presence of other children at home.

Results: During January-March 2018-2019, n=61 children hospitalized with febrile respiratory tract infection, RTI. Influenza virus confirmed in $23 / 61(37.7 \%), 13 / 23$ boys $(56.52 \%)$ and $10 / 23$ girls (43.47\%). Greek nationality had $17 / 23(73.91 \%)$. No siblings at home reported in $13.05 \%$ (3/23), two and three in $43.47 \%(10 / 23)$ and in $30.43 \%$ (7/23) respectively and four in $13.05 \%(3 / 23)$. Type A influenza virus identified in $21 / 23$ (91\%) and type B in $2 / 23(9 \%)$. Fever $39.5^{\circ} \mathrm{C}$ had $17 / 23$ children $(73.91 \%)$ and $39.5^{\circ} 6 / 23(26.08 \%)$. Rhinitis with couphing had $86.95 \%$ (20/23) cases, vomiting $13.04 \%$ (3/23), diarhoea 8.69\% (2/23) and limp walking $4.34 \%(1 / 23)$. Main complications were pneumonia $(4 / 23$, $17.39 \%$ ), the remaining being myositis, dehydration, neutropenia in one case respectively.

Conclusions: One out of three children hospitalized with febrile RTI during influenza period were identified as influenza illness. Main virus type was A. Having none or two many siblings seemed to be a favorable protective factor.

\section{6}

\section{Infectious Diseases}

Evaluation of Effectiveness of Tuberculin Skin Test Screening Programme in Lithuania

Ginreta Valinciute $^{1}$, Izabele Juskiene ${ }^{1}$, Algirdas Valiulis ${ }^{2}$

${ }^{1}$ Vilnius University Medical Faculty, Institute of Clinical Medicine, Clinic of Children's Diseases, Lithuania

${ }^{2}$ Vilnius University Medical Faculty, Institute of Clinical Medicine, Department of Rehabilitation, Physical and Sports Medicine, Lithuania

Background: The incidence of tuberculosis (TB) in Lithuania is one of the highest in the European Union. According national legislation the BCG vaccine is given obligatory during first 3 days after the birth and tuberculin skin test (TST) carried out for all children of 7-years-old and higher TB risk children at different age (not vaccinated children, TB contacts, children from social risk families including migrants, etc.).

Objective: The aim of the research was the assessment of the effectiveness of TST screening program of 7-year-old children and children of higher TB risk groups in Lithuania.

Material and Methods: The retrospective descriptive analysis of the use of TST in Lithuania between 2013 and 2018 was conducted. The data are processed by SPSS 22.0 program, the difference between the compared groups is considered significant when $p \leq 0.05$.

Results: In the period of 2003 to 2017 BCG vaccination coverage was 98.5 perc. and it was the highest rate in the Baltic States. From 2013 to 2018 TST was performed in 123936 (72.4 perc.) of all 171284 7-yearsold Lithuanian children and in TB contact group TST was performed in 14045 of 14414 (97.4 perc.) children. The positive TST was more common in TB contacts than in 7-year-old children $\left(\chi^{2}=6220.5 ; \mathrm{p} 0.001\right)$. The detection of positive TST is more often in contacts than in group of 7year-old children (risk ratio -3.71 ; 95 perc. CI 3.59, 3.83). Positive TST was detected in 9593 (7.7 perc.) of 7-year-old children and 4029 (28.7 perc.) of TB contact children. The same trend was observed when calculating positive TST of children from social risk families. It was found more often positive TST in social risk group children $\left(\chi^{2}=484.2 ; p\right.$ 0.001 ; risk ratio $-1.67 ; 95$ perc. CI $1.60,1.75)$, if compare with the group of 7-year-old children.

Conclusions: The obligatory national TST screening for tuberculosis infection in the group of 7-year-old children is not cost effective and clinically relevant. TST screening programme should focus on risk group children only.

\section{3}

\section{Infectious Diseases}

Pediatric Tuberculosis Under Six Years Old: a 15-Year Experience of a Level II Hospital

Joana Vieira, Andreia Ribeiro, Paula Correia, Rosário Ruivo, Helena Loureiro

${ }^{1}$ Child and Youth Department, Hospital Professor Doutor Fernando Fonseca, Amadora, Portugal, Portugal

Background: Tuberculosis (TB) disease under 6 years old is an indicator of recent transmission. Strategies to control TB are influenced by its incidence. Portugal became a low incidence country in 2014. In 2016, BCG vaccination switched from universal immunization to risk groups recommendation.

Objectives: Characterization of TB cases in children under six years old in a Pediatric Department of a level II hospital in Lisbon metropolitan area, which serves a high percentage of immigrants from Portuguesespeaking African countries.

Methods: Retrospective and descriptive study of TB disease in patients under six years old treated in our Department between January 2004 and December 2018 (15 years). Demographic, clinical, laboratory, imagiologic and therapeutic data were analyzed. Statistical analysis was performed with SPSS Statistics 25.

Results: 19 patients were included, nine (47\%) were diagnosed between 2016 and 2018. Median age of 22 months (min: 0.3, max: 70), 57.9\% female. 12 patients had immigrant families from countries with high TB incidence and another three were born in those countries. Seven out of nine patients not immunized with BCG had recommendation to do so. Although a close contact with TB was known in 12 patients at the time of referral to our center, three of those didn't start on chemoprophylaxis and two were not screened. At diagnosis: four patients had disseminated TB (three miliary TB and one congenital TB), 10 cases had microbiological confirmation by PCR or culture and one case had multirresistant TB. None had HIV infection. Four patients had sequelae (bronchiectasis in one, atelectasis in two, scoliosis in one). 
Conclusion: The increase in TB cases under six years old in the last three years points to some pitfalls in TB surveillance and transmission prevention. Although living in a low incidence country, the migration flux must be taken into consideration regarding the prevention of TB.

\section{2}

Infectious Diseases

One child, Difference Complications of Acute Mastoiditis ${ }_{\text {Joana Vilaca }}{ }^{1}$, Ivo Neves ${ }^{I}$, Margarida Reis Morais ${ }^{I}$, Carla Garcez ${ }^{I}$, $\overline{\text { Daniel Miranda }}{ }^{2}$, Carla Moreira ${ }^{1}$, Helena Silva ${ }^{l}$

${ }^{1}$ Paedriatic Service, Hospital de Braga, Portugal

${ }^{2}$ Otorhinolaryngology Department, Hospital de Braga, Portugal

Acute mastoiditis (AM) is a suppurative infection of the mastoid air cells with symptoms of less than one month's duration.

The clinical spectrum of mastoiditis ranges from a lack of symptoms with spontaneous resolution to progressive disease with life-threatening complications. A 28-month-old girl, with updated immunization schedule and history of recurrent acute otitis media (AOM), was evaluated in day (D1) of fever, rhinorrhea and frontal headache and treated symptomatically for upper airway infection.

In $\mathrm{D} 4$, for persistence of fever, was re-evaluated and diagnosed with bilateral AOM. Started treatment with oral cefuroxime and betamethasone, with apyrexia since D6.

In D7 noticed left retro-auricular inflammatory signs and poor feeding. She was admitted with the diagnosis of left AM and started intravenous ceftriaxone, nasal and topical corticosteroids

In D9 restarted fever and in D11 worsened left retro-auricular inflammatory signs with significant swelling and fluctuation. Neurological examination was normal. Cranial computed tomography with intravenous contrast revealed bilateral mastoiditis, left subperiosteal abscess, left temporal extra-axial empyema and suspicion of ipsilateral partial thrombosis, all findings confirmed by cranial magnetic resonance.

She was submitted to left mastoidectomy and myringotomy, without isolation of agent in the exudate. Ceftriaxone dose was increased to meningeal doses and started on intravenous metronidazole and dexamethasone and subcutaneous enoxaparin.

She had progressive clinical, analytical and imaging improvement. Completed 4 weeks of antibiotic and enoxaparin treatment and 10 days of dexamethasone and was discharged with oral hypocoagulation.

Currently, after four months of multidisciplinary follow-up, she had no complications or sequels and keeps a normal psychomotor development. Complications of mastoiditis, although rare, may be serious with significant morbidity and mortality.

An unfavorable clinical course of AM should raise the suspicion of complications and the need of imaging evaluation.

820

\section{Infectious Diseases}

Successful Eradication of Candida Parapsilosis Fungemia and Hickman Catheter Salvage with Dual Antifungal Lock Therapy Eleni Volakli ${ }^{l}$, Asimina Violaki ${ }^{l}$, Menelaos Svirkos ${ }^{l}$, Elpis Chochliourou ${ }^{l}$, Eleni Karakeke', Anastasia Anastasiou' ${ }^{1}$, Anna Kalaitzi ${ }^{1}$, Parthena Georgiadou ${ }^{l}$, Eirini Kotzapanagiotou ${ }^{l}$, Maria Sdougka ${ }^{l}$

${ }^{I}$ Pediatric Intensive Care Unit, Hippokration General Hospital, Greece

Introduction: Antifungal Lock Therapy (AfLT) in patients with fungemia and valuable long-term CVCs is an acceptable treatment with catheter salvage about $75 \%$. The common approach locks the catheter with an antifungal agent of a proper solution, at least for $12 \mathrm{~h} / 24 \mathrm{~h}$, in conjunction with systemic antifungal therapy. Ethanol $70 \%$ lock is an alternative approach which could augment the success rate. The aim of the current case report is to present the successful eradication of Candida parapsilosis fungemia and Hickman catheter salvage with Dual AfLT in a pediatric PICU patient.

Patient presentation: A 7mo, 8kg infant (F) was admitted in PICU with severe bronchiolitis. Due to prolonged PICU stay and mechanical complications of prior CVCs (DVT on both femoral veins), a two lumen, 7F, Hickman catheter was inserted on $40^{\text {th }}$ day. On the $50^{\text {th }}$ day a Candida parapsilosis fungemia was developed in a screening blood culture $(\mathrm{BC})$ followed by 3 more positive $\mathrm{BC}$ till the $54^{\text {th }}$ day. Peripheral veins were inserted and systemic Liposomal amphotericin B (L-AMB) therapy was started in parallel with AfLT $(2 \mathrm{mg} / \mathrm{ml}, 4 \mathrm{ml} /$ each lumen, changed $/ 12 \mathrm{~h})$. One day later, to enhance results, ethatol $70 \%$ was added $(2 \mathrm{ml} /$ each lumen) interchangeably $(12 \mathrm{~h} \mathrm{~L}$ $\mathrm{AMB} / 12 \mathrm{~h}$ ethanol), plus systemic voriconazole.

Results: $\mathrm{BC}$ became negative on $2^{\text {th }}$ treatment day and therapy was continued for additional 14 days. No serious adverse effect was recorded. The patient was discharged with a tracheostomy, spontaneously breathing on $98^{\text {th }}$ day and remains infection free.

Conclusions: Given the integral role of long-term CVC and the limited venous access in PICU pts, dual AfLT appears to be a promising strategy as an adjunctive therapy for fungemia treatment and catheter salvage.

\section{5}

\section{Infectious Diseases}

\section{A Rare Case of Sweet Syndrome}

Harshal Wagh ${ }^{1}$

${ }^{T}$ Pediatrics, Bharati Vidyapeeth (Deemed to be) University, Medical College \& Hospital, Sangli, India

11 yrs female, $2^{\text {nd }}$ issue of non-consanguineous marriage with H/O Joint Pain \& swelling since 8 months, fever since 4 days was admitted with us.

Our provisional diagnosis was Polyarticular Arthritis to r/o Mixed Connective tissue disorder.

Patient was investigated and treated symptomatically. Peripheral smear showed dense neutrophilia. Serological investigations like ANA, ASO and RA factor were negative. Skin biopsy confirmed the final diagnosis of 'Sweet Syndrome'. Symptoms relieved dramatically after MethylPrednisolone Pulse Therapy. Guttons' papules also resolved on the day of discharge. Patient was discharged on oral glucocorticoids and was advised follow up.

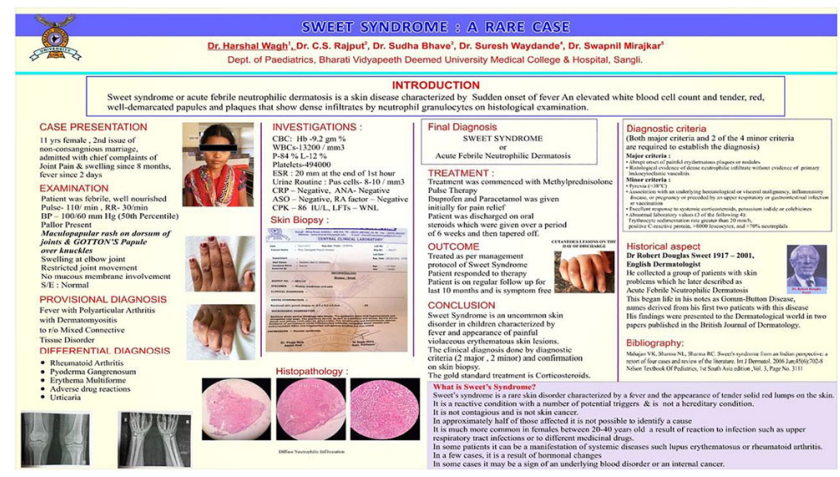

954

Infectious Diseases

Infection Screening in Asylum Seeking Children, Are We Getting It Right?

Jennifer Ward $^{l}$, Varsha Sadavarte ${ }^{I}$

${ }^{T}$ Community Paediatric Department, University Hospital North Midlands, $U K$ 
Background: In 2017 in the UK, there were 2,206 asylum applications from unaccompanied asylum seeking children (UASC). Our local population had the third highest numbers of asylum seekers in the region. They are at an increased risk of health complications including blood borne viruses and tuberculosis.

Objective: The aim of this audit was to compare the current practice of infection screening of UASC in our department with the screening advice set out in the migrant health guide by Public Health England.

Methods: The 'looked after children assessment' reports of all UASC in 2018 were retrospectively analysed. The recommendations regarding screening for blood borne viruses and TB were noted. A search was conducted on the trust's pathology system to confirm what testing the patient had received and what the results were. The patient's country of origin was then referenced with the recommended screening set out by Public Health England.

Results: Out of 23 children, more than half had screening recommendations that were incorrect. This included both under and over-testing. Most frequently, testing was requested when it not necessary. Infrequently it was not requested when it was required. 7 of the 23 patients who were recommended to have screening for blood borne viruses did not have the necessary tests. 16 of the 23 patients did not have TB screening despite it being recommended.

Conclusion: The infection risk for asylum seeking children varies greatly by country and so the screening advice needs to reflect this. Over testing can have negative impacts to a child by subjecting them to unnecessary blood tests, and has cost implications. It is equally important that patients at a higher risk are screened appropriately. Improvement interventions have included follow up letters for missed tests and addressing the referral process for TB screening.

\section{1}

\section{Infectious Diseases}

Assessment of Health Education as a Tool in Improving the Knowledge, Attitude and Practices among Child "Bulilit" Health Workers on Leptospirosis

Ma. Monica Mampusti ${ }^{1}$, Jennie Wong ${ }^{1}$
${ }^{1}$ Pediatrics, Ospital ng Maynila Medical Center, Philippines

Background: In the Philippines, approximately 14 per 100,000 become affected with leptospirosis. A spike of $60 \%$ in leptospirosis case in the National Capital Region during January 2018 to July 2018 alone. Worldwide, it was estimated that 500,000 severe cases of Leptospirosis occur yearly. Health prevention is an inexpensive way to prevent diseases, and in such, the health sectors tapped the community workers in aiding in information dissemination. There have been many health workers and even including the young population worldwide. In the Philippines, the term "Bulilit" means child, hence the name Bulilit health workers . Most studies done on evaluation of health education in prevention of diseases focused on other diseases. Data with the use of KAP tool in Leptospirosis and more so on younger population are scarce, hence this study.

Objective: To assess health education as a tool in improving the knowledge, attitude and practices among bulilit health workers on leptospirosis. Design: A prospective cross-sectional analytical study was used. Settings: The study was done in a public grade school in Manila Participants: Bulilit Health Workers of a public school in Manila were the participants of the study Interventions: A PowerPoint lecture regarding leptospirosis was given.

Main Outcome Measures: Pretest questionnaire was given followed by the lecture, after which, a post-test questionnaire was done. Statistical method used was paired t-test.

Results: Participants were ages 7-11 years, with almost equal distribution between male and female. Majority go to school by riding a vehicle. The television is the most common source of information. There was an increase in the mean scores comparing the baseline to the post test scores $(\mathrm{p}=0.0001)$. The total mean score for knowledge regarding leptospirosis is $86.36 \%$ for the pretest and $95.45 \%$ to post-test; for attitude $71.33 \%$ and $78.67 \%$; and $82.9 \%$ and $88.33 \%$ for practices.

Conclusions: There is a significant improvement in knowledge, attitude and practices for leptospirosis after an informative lecture among the Bulilit Health Workers. Therefore, health education even on younger subjects can improve knowledge, attitude and practices towards Leptospirosis.

329

\section{Infectious Diseases}

Epidemiological and Clinical Characteristics of Bacterial Gastroenteritis in the Newborns

Alexander Yelak ${ }^{1}$, Ronella Marom ${ }^{1,2}$, Dror Mandel ${ }^{1,2}$, Laurence Mangel $^{\prime}$, Galia Grisaru-Soen ${ }^{2,3}$, Amos Adler ${ }^{2,4}$

${ }^{1}$ Department of Neonatology, Lis Maternity Hospital,Tel-Aviv Sourasky Medical Center, Israel

${ }^{2}$ Sackler Faculty of Medicine, Tel-Aviv University, Israel ${ }^{3}$ Pediatric Infectious Disease Unit, Dana Dwek children Hospital, Tel-Aviv Sourasky Medical Center, Israel

${ }^{4}$ Clinical Microbiology Laboratory, Tel-Aviv Sourasky Medical Center, Israel

Aim: Acute bacterial gastroenteritis is a major cause of morbidity and mortality, especially in the developing countries. We examined the incidence, clinical features and outcomes in the first week of life.

Methods: This was a retrospective study of culture-proven bacterial gastroenteritis in newborn infants that were diagnosed between January 2011 and September 2018 in a tertiary centre in Israel.

Results: There were 10 cases of culture-proven bacterial gastroenteritis, detected out of 91 stool cultures. All infants were born vaginally and nine were full-term infants. The annual incidence was 0.096 per 1000 live births. The responsible pathogen was Campylobacter in six patients, Salmonella in two and Shigella sonnei in two. The mean age of disease onset was two days of life. Antibiotics were given to five patients, but were inappropriate in two cases. Only one patient with the Shigella sonnei infection required respiratory support. All patients fully recovered.

Conclusion: One in ten newborn infants with bloody stools had bacterial gastroenteritis, contradicting the low rates found in other studies and indicating the importance of considering this diagnosis. Antimicrobials active against Salmonella or Shigella should be given to newborn infants who have bloody stools and look ill.

\section{4}

\section{Neonatology}

\section{Heritability of Tongue Tie: Role of Family History in Assessment of the Problem \\ Abhay Vats ${ }^{l}$, Laurie Jones ${ }^{I}$, Damara Vasquez ${ }^{I}$, Rajeev Agarwal $^{l}$ \\ ${ }^{I}$ Newborn Medicine, Agave Pediatrics, USA}

Introduction: Tongue-tie (TT), also known as ankyloglossia, is diagnosed when the lingual frenum is short and restricts the mobility of the tongue and can be an isolated finding in upto $5 \%$ of infants. It can have an adverse effect on oromuscular function, breast feeding (BF) and speech later on. While the existence of tongue- tie has never been doubted, there have been sporadic reports of familial TT that are non syndromic.

Methods: We performed a retrospective analyses of infants treated or followed for TT at a large pediatric private practice in Phoenix and its suburbs in AZ to look for patterns of inheritance of this condition.

Results: We followed 6982 patients with TT over a 13 year period from 2006 to 2019. An analyses of the patients attending the clinic over 1 year 
period (2018-19) was done. We identified multiple families with several members affected by TT. The patterns of inheritance included Autosomal Dominant $(n=5)$, Autosomal Recessive $(n=23)$ and $X$ linked $(n=1)$. Both males and females were equally affected. A significant number of patients had symptoms of dysfunctional BF, diastema or speech problems (in older children / adults).

Conclusion: TT can be a very common and often underdiagnosed problem in infants. Untreated TT can cause significant problems in post natal period that can lead to failure of BF. A family history should be taken to assess and identify additional family members with TT

\section{7}

\section{Neonatology}

Development of an Intra-Oral Vacuum Pressure Measuring Device to Assess Breastfeeding

Abhay Vats ${ }^{I}$, Peter Vitruk ${ }^{l}$, Damara Vasques ${ }^{l}$, Rajeev Agarwal ${ }^{1}$

${ }^{I}$ Newborn Medicine, Agave Pediatrics, USA

Background: There are numerous advantages of breastfeeding (BF) for both the mother and the newborn and every effort should be made to prevent its failure. Nipple trauma and latching problems, often seen in infants with tongue tie (TT) can contribute to development of poor intraoral vacuum pressure and lead to BF failure. This dysfunctional BF could be to quantitatively assessed by measuring the inter-oral cavity vacuum pressure.

Methods: We developed a simple and portable oral manometer for measuring intra-oral vacuum pressures using off the shelf components and without the need for a complex setup or operator skills.

Results: The working manometer prototype has 3 main components: 1) pressure transducer, 2) tubing assembly, 3) a laptop computer.Further details are as follows: The pressure transducer: This is the main component and uses an absolute pressure transducer with DAQ system (National Instruments) and is connected to the tubing assembly on one end and a computer on the other. Tubing assembly: It consists of disposable Silicone Rubber Tubing (5F); Blunt Tip Dispensing Needle with Luer Lock and has an in line hydrophobic Filter (PTFE 13mm)that acts as barrier to fluids's entry into the transducer assembly. Computer: A laptop with a dedicated software developed utilizing NI LabVIEW for data acquisition Preliminary testing: It was performed on a full-term healthy infant and a adult volunteer. In infant the sucking process was characterized by a burst-pause sucking pattern where a bursts occurring at a typical frequency of $\sim 1 \mathrm{~Hz}$, separated by pauses of $\sim 2 \mathrm{~s}$. This pattern manifested in three stages: continuous, intermittent and paused.

Conclusion: We report the development of oral manometer for quatitative assessment of BF. It needs futher studies and validation and can be potentially used for assessement of BF in infants with and with out TT and other cranio-facial malformations.

566

\section{Neonatology}

Measuring 4-Limb Blood Pressure is not a Reliable Discriminator in Neonatal Heart Murmur Assessment Leila Ahmed ${ }^{1}$, Mahvash Rastegari ${ }^{1}$, Mojgan Ezzati

${ }^{T}$ Neonatology, University Hospital Lewisham, UK

Background: Heart murmurs are commonly found during routine neonatal examination at postnatal ward, with an incidence of $0.6-1.4 \%$. Measuring 4 limb blood pressure alongside of checking femoral pulses and pre and postductal saturation was part of initial assessment of babies with heart murmur. Objectives: To assess the effectiveness of 4 limb blood pressure as a reliable discriminator between pathological and innocent heart murmurs in Newborn babies with heart murmur.
Methods: We found heart murmur in 52 babies over a period of 12 months (2017-2018) during routine baby check at postnatal ward at University Hospital Lewisham. Following thorough physical examination of all babies with neonatal heart murmur, we checked femoral pulses, pre and post ductal oxygen saturations and 4 limbs blood pressure as part of their assessment for heart murmur. All babies with normal saturation and femoral pulses were assessed again at neonatal follow up clinic after 4 weeks. Babies who had still heart murmur were referred to paediatric cardiology clinic for further assessment including echocardiography.

Results: The incidence of heart murmur in babies was $1.3 \%$. All babies had normal pre and postductal saturation and femoral pulses. Almost in $50 \%$ of babies' upper limb mean blood pressure (MBP) was higher than lower limb MBP. Almost all babies with heart murmur had a patent foramen ovale (PFO). Only 1 patient had aortic regurgitation on echocardiography.

Conclusion: Measuring 4 limb blood pressure in babies with heart murmur who have normal oxygen saturation and normal femoral pulses does not add any additive value in assessment of babies with heart murmur.

517

\section{Neonatology}

\section{Ichthyosis: A Challenging Case Report}

Maria Almendra $^{1}$, Ana Isabel Carvalho ${ }^{1}$, Cláudia Silva ${ }^{1}$, Inês Carvalho ${ }^{2}$, Telma Francisco $^{3}$, Rodrigo Carvalho ${ }^{4}$, Helena Ramos ${ }^{l}$, Teresa Tomé ${ }^{1}$

${ }^{1}$ Women, Children and Adolescents Department, Maternidade Dr. Alfredo da Costa, Centro Hospitalar de Lisboa Central, Lisbon, Portugal, NICU, Portugal

${ }^{2}$ Medical Genetics Department, Hospital Dona Estefânia, Centro Hospitalar de Lisboa Central, Lisbon, Portugal, Portugal

${ }^{3}$ Women, Children and Adolescents Department, Hospital Dona Estefânia, Centro Hospitalar de Lisboa Central, Lisbon, Portugal, Nephrology Unit, Portugal

${ }^{4}$ Department of Dermatology and Venereology, Centro Hospitalar de Lisboa Central, Lisbon, Portugal, Centro Hospitalar de Lisboa Central, Lisbon, Portugal, Portugal

Background: Inherited ichthyoses are a group of genetic disorders characterized by generalized dry skin, scaling and hyperkeratosis, and often associated with erythroderma. These manifestations are due to mutations in genes mostly involved in skin barrier formation, particularly in keratinization. Multiple causes have been associated with ichthyosis that may or may not be related to genetic syndromes.

Case Report: A newborn female of inbreeding Roma parents was admitted to the Neonatal Intensive Care Unit due to prematurity $(33 w+6 d)$ and low birth weight $(895 \mathrm{~g})$. Intrauterine growth restriction was detected at $21^{\text {th }}$ weeks of gestation and an emergent caesarean section was performed at 33 weeks as fetal bradycardia was seen in continuos cardiotocography. At birth, she presented with dysmorphic facial features, general dermatosis and global alopecia with cutaneous fragility and erythema spots. Humeral fracture was diagnosed immediately after delivery. Since the first 24 hours of life she suffered from hydroelectrolyte imbalances with hypernatremic dehydration (maximum $167 \mathrm{mmol} / \mathrm{L}$ ) and hypokalemia (minimum $2.2 \mathrm{mmol} / \mathrm{L}$ ) associated with polyuria and later, hypomagnesemia (minimum $1.06 \mathrm{mmol} / \mathrm{L}$ ), hypocalcemia and hypophosphatemia. Simultaneously there was weight loss $(23.5 \%$ of birth weight). Due to the long-lasting polyuria together with the electrolyte changes she needed high fluid intakes and ion supplemented. She also had recurrent Staphylococcus aureus sepsis with multiple prolonged antibiotic courses. It was requested Dermatology, Primary Immunodeficiency and Genetics collaboration and the genetic tests are still in course. We compared this case to a previous similar case back in 2017 presented with ichthyosis and severe hydroelectrolyte imbalances (including hypomagnesemia) born to consanguineous Roma parents, which is associated to epidermal growth factor receptor (EGFR) homozygous pathogenic mutation.

Conclusion: Face and skin features together with ion imbalance and family history fit in EGFR mutation clinical cases. There is only a very 
few reported cases, all of them with poor prognosis.

\section{0}

\section{Neonatology}

Reducing Term Admission from Respiratory Conditions by Decreasing Separation of Mother and Baby (Re-TARC). A quality improvement initiative at the Neonatal Unit, Univeristy Hospital Wishaw

Augusta Anenih ${ }^{1}$, Lorna Lennox ${ }^{1}$

${ }^{T}$ Neonatal Intensive Care Unit, University Hospital Wishaw, UK

Background: Unexpected term admission, a proxy indicator of harm occurring along the maternity-neonatal pathway form the largest proportion of admissions to neonatal units. On our unit, they account for just under $50 \%$ of admissions, leading indications being respiratory conditions and hypothermia. Skin to skin (STS) immediately after birth is known to enhance the natural bonding process and facilitate smooth postnatal adaptation. We describe our experience of reducing term admissions from respiratory condition (TARC) through implementation of in-theatre skin to skin (ITSTS) and NEWTT escalation pathway bundle.

Methods: Primary target was mothers presenting for elective Caesarean Sections (EL-CS) at term. Change process involved using Improvement methodology including process maps and cause and effect diagrams. Review of background term admission data (Badger-Net database) detailing leading admission indications, CS type, admission duration, type/length of respiratory support and whether ITSTS was offered and received was undertaken. Parental education on benefits of STS, staff training on providing ITSTS and offering ITSTS to mothers presenting for EL-CS. In theatre, monitoring leads were placed on mother's back, blood pressure cuff on non-dominant arm and operating table placed at a slight head-up tilt to increase comfort. Once cord was cut, baby was dried, immediately placed in direct contact with mother's chest and covered with pre-warmed blankets. STS continued for at least an hour. A NEWTT escalation pathway codesigned with staff was introduced to guide management of term babies with respiratory conditions following CS.

Results:

$48 \%$ reduction in TARC following EL-CS

$77 \%$ of babies who would otherwise have been separated remained with their mothers.

$19 \%$ reduction in total term admission/1000 deliveries.

$85 \%$ of EL-CS mums offered ITSTS and $85 \%$ achieved it.

Conclusion: ITSTS and NEWTT escalation pathway has significantly decreased TARC, decreased separation of mothers and babies, increased parent satisfaction and fostered collaborative working. Requires ongoing QI work to sustain and spread.

\section{1}

\section{Neonatology}

Hypoxic Ischaemic Encephalopathy (HIE): An Audit of Assessment, Management and follow up In a Level 2 Neonatal Unit

Charlotte Avann ${ }^{1}$, Jessica Sarah Bowen ${ }^{1}$, Subramanian Mahadevan ${ }^{1}$

${ }^{T}$ Paediatrics and Neonates, Russells hall Hospital, The Dudley Group NHS Foundation Trust, UK

Background: HIE is a significant cause of neonatal morbidity and mortality affecting 1.5 per 1000 live births. For moderate to severe HIE, evidence supports therapeutic hypothermia in improving survival and neurological outcomes.

Objective: Assessment of diagnosis and management of babies with HIE, and their neurodevelopmental outcome at 1 and 2 years.

Methods: Retrospective analysis of infants born at a level 2 unit in 2014 2016 with a diagnosis of HIE on the Badger system. Duplicates and babies
Results: 38 ( $0.3 \%$ of live births) were identified as HIE: grade $1-34 \%$; grade $2-37 \%$; grade $3-29 \% .53 \%$ were cooled. $47 \%$ had seizures. 3 infants with grade 3 died.

For grade 2 babies, $90 \%$ of the 10 cases where babies were cooled had normal MRI's. $50 \%$ of the 4 babies not cooled had MRI changes and developmental delay.

The most common developmental concern in Grade 2 HIE was speech delay $(15 \%)$.

1 patient with grade 2 had poor cord gases, but AGPARS $7^{5,} 9^{10}$ and was initially neurologically intact so was not cooled, but developed seizures at 8 hours. MRI changes and mild developmental delay was observed at 2 years. $26 \%$ of HIE patients followed, including 1 with grade $1 \mathrm{HIE}$, had developmental delay.

$18 \%$ of all grade 3 babies had profound delay, but $36 \%$ had a normal Baileys assessment at 2 years following cooling.

Conclusion: Developmental follow up is very important in HIE, particularly grades 2 and 3 .

Grades 1 and 2 have good outcomes for survival and neurodevelopment. Cooling should be carefully considered in grade 2 babies. Neurology should be observed closely in patients with poor gases, ensuring the window of opportunity is not missed.

Grade 3 babies can do well with cooling.

\begin{tabular}{|c|c|c|c|c|}
\hline & HIE Grade & Gr 1 & Gr 2 & Gr 3 \\
\hline Total Number & & $13(34 \%)$ & $14(37 \%)$ & $11(29 \%)$ \\
\hline Ther. Hypothermia & Cooled & 0 & 10 & $10^{11}$ \\
\hline \multirow[t]{2}{*}{ Apgar at 10mins } & $0-5$ & 2 & 4 & 9 \\
\hline & $6-10$ & 11 & 10 & 2 \\
\hline \multirow[t]{5}{*}{ Max resuscitation } & Inflation breaths & 3 & 3 & 0 \\
\hline & Ventilation breaths & 8 & 3 & 2 \\
\hline & Intubated & 1 & 4 & 4 \\
\hline & Chest compressions & 1 & 4 & 3 \\
\hline & Drugs & 0 & 0 & 2 \\
\hline Resus at $10 \mathrm{mins}(\%)$ & Yes & $3(23 \%)$ & $7(50 \%)$ & $9(82 \%)$ \\
\hline \multirow[t]{3}{*}{ TOBY } & Criteria A & 10 & 13 & 10 \\
\hline & Criteria b & 2 & $11^{5}$ & 11 \\
\hline & $A+B$ & $1^{1}$ & $10^{6}$ & 10 \\
\hline \multirow[t]{3}{*}{ CFAM } & Normal & 5 & 4 & 0 \\
\hline & Abnormal & 0 & 8 & 11 \\
\hline & Not done & 8 & 2 & 0 \\
\hline Seizures (\%) & Yes & $1^{2}(8 \%)$ & $8^{7}(57 \%)$ & $9^{11}(82 \%)$ \\
\hline \multirow[t]{3}{*}{ MRI } & Not done & 10 & 2 & $2^{12}$ \\
\hline & Normal & $1^{5}$ & 9 & 6 \\
\hline & Abnormal & $2^{2,3}$ & $3^{8}$ & 3 \\
\hline Survival & Died & 0 & 0 & $3^{13}$ \\
\hline \multirow[t]{3}{*}{ F/U 1 year } & Normal & 7 & 9 & 2 \\
\hline & Devel. concerns & 0 & 1 & 4 \\
\hline & Not done & 6 & $4^{9}$ & 2 \\
\hline \multirow[t]{3}{*}{$\mathrm{F} / \mathrm{U} 2$ years } & Normal & 2 & 4 & 2 \\
\hline & Devel. concerns & $1^{4}$ & 2 & $4^{14}$ \\
\hline & Not done & 10 & 8 & 2 \\
\hline \multirow{3}{*}{ Baileys } & Normal & 3 & 3 & 4 \\
\hline & Mild delay & $1^{4}$ & $1^{10}$ & 0 \\
\hline & Not done & 9 & 10 & 4 \\
\hline
\end{tabular}

Poor gas, satisfactory APGARS, passively cooled, abnormal neurology quickly resolved, no MRI, Baileys Norma 'Required chest compressions, APGARS, 6 at 10 mins. pH 7.06. Did not meet criteria A, seizures required phenobarbitone. MRI subacute haemorrhage. Not followed up

${ }^{3}$ Watershed ischamia (poor gases \& apgars, rapidly improving neurology, not cooled, no seizures, development normal ?no indication)

Speech only

1 only poor tone documented for criteria B - but poor gases, APGARS ok, seizures on rewarming, MRI ok. No F/U ${ }^{6} 1$ not cooled -poor gases, satisfactory APGARs, neurologically ok, but seizures at 8hours, MRI showed HIE changes, mild speech delay - importance of monitoring poor gases

2 not cooled. -1 did not fulfil criteria A, developed seizures. MRI showed HIE seizures mild language delay. \& as per ${ }^{6}$ HIE cortical and dee p grey matter involvement (as $\left.{ }^{\circ}\right)$,

Occipital ischaemia \& small SAH (poor gases\& APGARs, cooled, seizures D2, no f/u)
Cystic encephalomalacia in it parietal lobe (good gas \& APGAR, neuro ok, not cooled seizures-phenobarb), mild language delay

delay

${ }_{10}^{10}$ mild speech only

${ }^{11}$ Did not meet criteria A, abnormal neurology \& seizures, MRI suggesting HIE, no F/U

${ }^{12}$ Both deceased

Age 1 day, 3 days and 5 days old

${ }^{4} 2$ profound, 2 mild 
955

\section{Neonatology}

Intra- and Inter-Observer Variability in PDA Size Measured on Transthoracic Echocardiography (TTE) in Extremely Preterm Babies.

$\underline{\text { Kunal Babla }}^{l}$, Ramona Dumitru ${ }^{I}$, Donovan Duffy ${ }^{l}$, Justin Richards ${ }^{l}$, Anay Kulkarni ${ }^{l}$

${ }^{I}$ Neonatal Unit, St George's University Hospitals NHS Foundation Trust, $U K$

Background: The internal diameter of patent ductus arteriosus (PDA) measured on transthoracic echocardiography (TTE) is commonly used in determining the need for treatment in clinical practice and enrolment in research trials. PDA diameter measured on TTE has not been well validated.

Objective: To assess the intra- and inter-observer variability in PDA internal diameter measured on TTE on the same examination in babies born at $\leq 30$ weeks' gestation.

Methods: We included 56 babies born $\leq 30$ weeks gestation that had clinically indicated TTEs. TTEs were performed by a neonatologist (AK) certified in echocardiography. PDAs were visualised on high parasternal views, and cineloops were stored without measurement annotations.

PDAs were measured at the narrowest diameter on 2-Dimensional (2D) and Colour Doppler echocardiography. A second certified neonatologist (DD) measured the internal diameter of PDA on the same cineloops. They were blinded to each other's measurements. AK repeated measurements at a later date, blinded to his original measurements.

Results: Table 1 shows results for inter-observer comparisons. Fig 1 and 2 show intra-observer measurement comparisons. Comparison of all measurements showed colour measurements a mean of $0.29 \mathrm{~mm}$ higher than 2D.

Table 1. Bland-Altman Analysis of inter-observer measurement comparisons.

\section{Repeatability Index}

$\begin{array}{lllllllll}2 \mathrm{D} & 0.7 & 2.5 & 0 & -0.04 & 1.67 & -1.75 & 1.75 & 81 \% \\ \text { Colour } & 0.96 & 2.5 & 0 & -0.83 & 0.63 & -2.3 & 1.49 & 62 \%\end{array}$

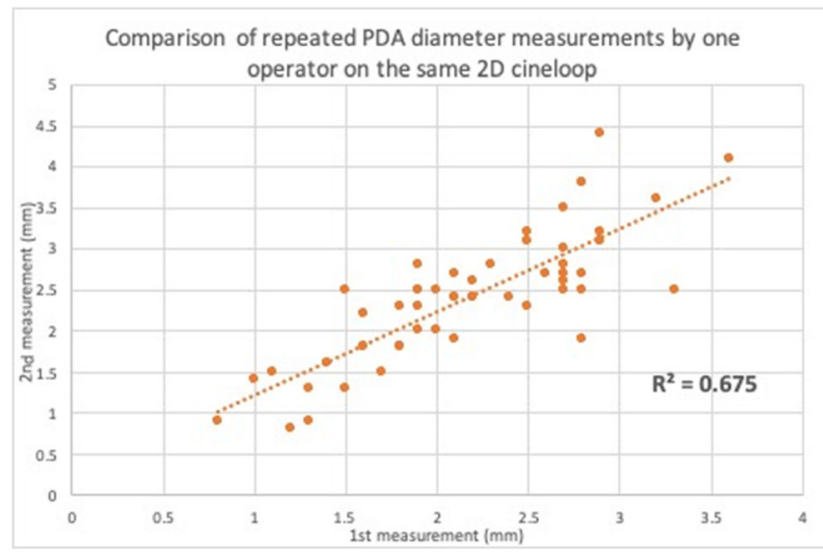

Fig 1. Intra-observer measurement comparisons (2D).

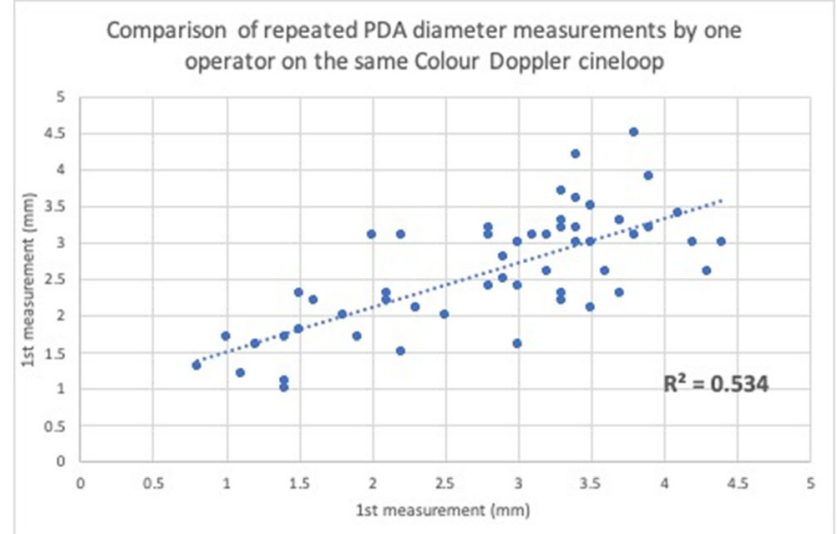

Fig 2. Intra-observer measurement comparisons (colour).

Conclusion: The poor repeatability coefficients and repeatability indices lead us to conclude that there is significant inter- and intra-observer variability in 2D and Colour echocardiography measurements of PDA diameter, even on the same cineloops. Thresholds and timing of treatment for PDA remain controversial. We advise caution when using diameter as a surrogate marker for degree of shunting.

696

\section{Neonatology}

Assessment of Environmental Radiofrequency Electromagnetic Field Levels in a Neonatal Intensive Care Unit

Dimitri Besset $^{l}$, Brahim Selmaoui ${ }^{1,3}$, Pierre Tourneux ${ }^{1,2}$, Stephane Delanaud $^{I}$, René de Seze $e^{1,3}$, André Leke ${ }^{1,2}$, Erwan Stephan-Blanchard ${ }^{1}$

${ }^{1}$ Péritox UMR_I 01, Université de Picardie Jules Verne, France ${ }^{2}$ Neonatal Intensive Care Unit, University Medical Centre, France ${ }^{3}$ TOXI Unit, INERIS, France

Preterm neonates represent a vulnerable population with a high sensitivity to their environment. While hospitalized in Neonatal Intensive Care Units (NICU), they can possibly be exposed to radiofrequency electromagnetic fields (RF-EMF) due to the increased number of devices using wireless communications (mobile phones, DECT, WIFI). Strikingly, data on RFEMF levels in NICU are currently not available in the literature.

The aim of the present study was to quantify the RF-EMF levels in a NICU by performing a mapping of environmental RF-EMF levels and by measuring spot emissions from medical devices.

Measurements of RF-EMF levels were performed in a tertiary NICU including 34 beds using three-axis dosimeters. A first set of $24 \mathrm{~h}$ measurements aimed at evaluating the RF-EMF levels in the general environment and in children's rooms. A second set of measurements, in faradised chamber, assessed RF-EMF emitted by medical devices commonly used in the NICU and identified as potential sources of exposure.

For a total of 102 measurement points were analysed. Mean RFEMF levels were significantly higher in the staff environment than in children's rooms (p0.0011), and were also significantly higher during the day than during the night (p0.001). The highest RF-EMF levels (above the mean value $\pm 2 \mathrm{SD}$ ) represented $3.5 \%$ of the measurement duration. Very high peak values above $6 \mathrm{~V} / \mathrm{m}$ were also detected. The measurements with isolated NICU medical devices showed that they do not emit RF fields.

Globally, mean RF-EMF levels in the NICU were low. The detection of RF-EMF levels was more probably related to the parents and staff activities rather than to medical devices. A chronic exposure combined with high peak values in this specifically vulnerable population should require particular attention. Decreasing the preterm neonates' exposure to RFEMF would include parental and staff intervention to reduce the wireless communication and others devices in the NICU. 


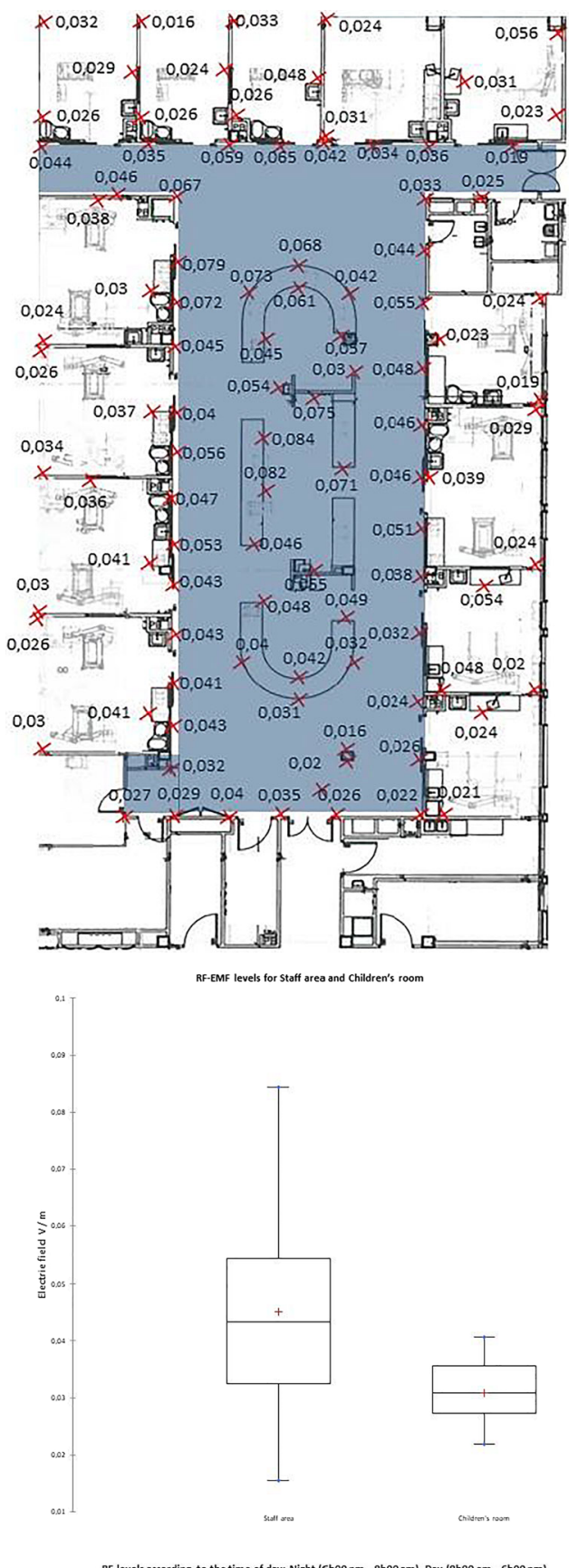

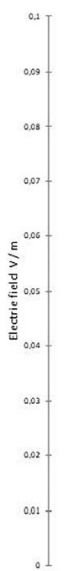
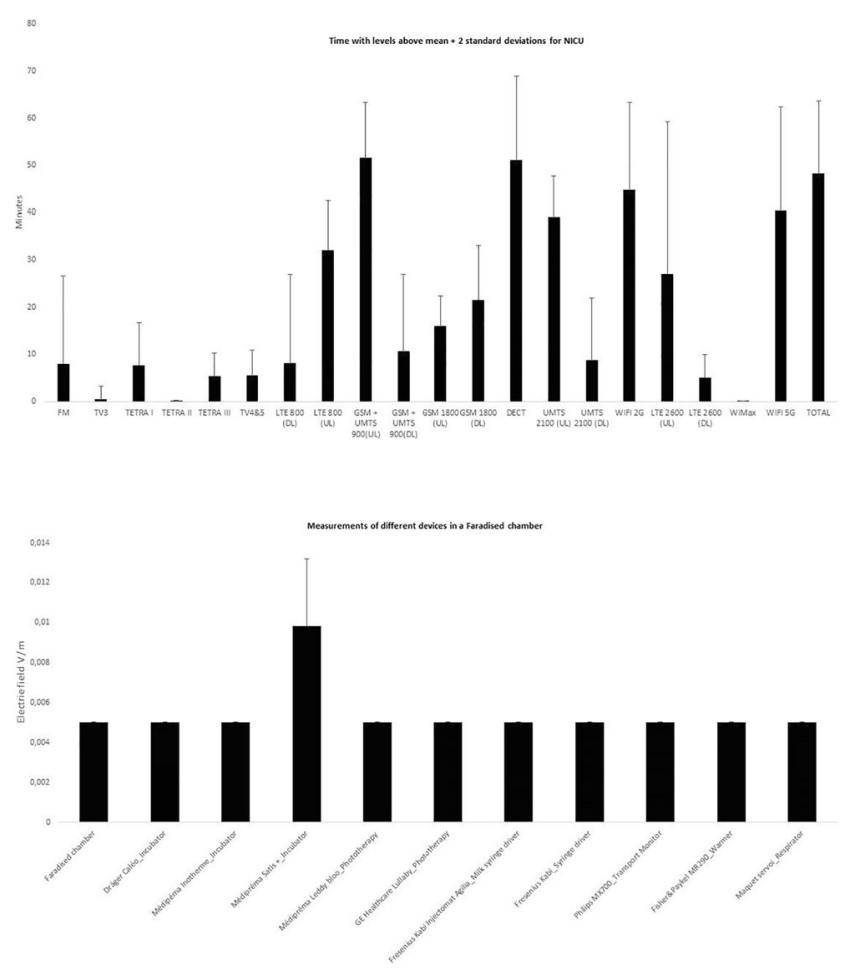

446

Neonatology

Selenium Status in SGA Newborns

Teofana-Otilia Bizerea-Moga ${ }^{1,2}$, Claudiu Angelescu ${ }^{3,4}$, Otilia Marginean ${ }^{1,2}$, Otilia Bizerea ${ }^{2}$, Constantin Ilie ${ }^{3,4}$

${ }^{1}$ Pediatrics Department, "Victor Babes" University of Medicine and Pharmacy, Romania

${ }^{2} 1$ st Pediatric Clinic, "Louis Turcanu" Children's Clinical and Emergency Hospital, Romania

${ }^{3}$ Neonatology Department, "Victor Babes" University of Medicine and Pharmacy, Romania

${ }^{4}$ Clinic of Obstetrics, Gynecology and Neonatology, "Pius Brânzeu" Emergency County Hospital, Romania

${ }^{5}$ Faculty of Chemistry, Biology, Geography, West University, Romania

Introduction: Adaptive physiological changes during pregnancy lead to an increase of pro-oxidant and antioxidant activity. Oxidative stress that occurs when antioxidant mechanisms are overcome and exerts a negative effect on fetal growth and development. Seleno-compounds such as glutathione-peroxidase and thioredoxin-reductase, are endogenous antioxidants that play a key role in defence against oxidative stress.

Aim of the Study: The aim of the study was to identify a correlation between the serum and urinary selenium levels and the neonatal mechanisms of response to intrauterine hypoxia and subsequent oxidative stress. Materials and Methods: A prospective case-control study was conducted over a 3 month period ( $1^{\text {st }}$ of January $-31^{\text {st }}$ of March 2017 ), at the Clinic of Obstetrics, Gynecology and Neonatology of the Emergency County Hospital Timisoara. The study group consisting of 32 SGA neonates was matched 1:1 for gestational age with AGA newborns, representing the control group. After obtaining the written consent from the caregivers, venous blood samples of $2 \mathrm{~mL}$ were collected at birth from the umbilical cord and $5 \mathrm{~mL}$ of urine were collected using sterile urine collecting bags in all neonates included in the study. The selenium concentration was determined using atomic absorption spectrometry.

Results and Discussions: Mean serum selenium concentrations were lower in SGA neonates compared to the control group ( $29.44 \mu \mathrm{g} / \mathrm{L}$ 
versus $43.26 \mu \mathrm{g} / \mathrm{L}$ ). Neonates from the study group also had significantly lower levels of urinary selenium compared to controls $(18.88 \mu \mathrm{g} / \mathrm{L}$ and $29.15 \mu \mathrm{g} / \mathrm{L}$ respectively).

Conclusions: Selenium concentration in both serum and urine is decreased in SGA infants and depends on intrauterine exposure to hypoxia and subsequent oxidative stress.

\section{4}

\section{Neonatology}

RECAN Software Reliability Assessment for Heart Rate Variability (HRV) Analysis in a French Neonatal Intensive Care Unit (NICU) Thibault Blache ${ }^{1}$, Andrei Cividjian ${ }^{5}$, Roxana Diehl ${ }^{2}$, Muriel Rabilloud ${ }^{4}$, Olivier Claris $^{1,2}$, Franck Plaisant ${ }^{1,2}$, Kim-An Nguyen ${ }^{1,2,3}$

${ }^{I}$ Neonatalology, Hospices Civils de Lyon (HCL), Hôpital Femme Mère Enfant (HFME), France

${ }^{2}$ Neonatalology, Hospices Civils de Lyon (HCL), Centre Hospitalier Lyon Sud (CHLS), France

${ }^{3}$ Pharmacotoxicology, Hospices Civils de Lyon (HCL), France

${ }^{4}$ Biostatistic, Hospices Civils de Lyon (HCL), France

${ }^{5}$ Alpha 2 Society, Hospices Civils de Lyon (HCL), France

Background: Heart rate variability (HRV) consists of changes in the time intervals between consecutive heartbeats called interbeat intervals (IBIs). HRV constitutes a relevant noninvasive tool to assess the autonomic nervous system (ANS) function especially in preterm infants nearby their term corrected age. HRV would be a rational way to assess cardiovascular system maturation in this population. A stable and mature cardiovascular condition is essential before discharge of preterm infants. RECAN software were developed to precisely obtain the indices of HRV for routinely use by neonatologist. RECAN is based on an algorithm that reduces noises and artefacts from the 24h cardiac records and providing an automatic calculation for HRV indices. Objective: To assess RECAN reliability in estimation of HRV indices comparing to the $24 \mathrm{~h}$ Holter ECG interpreted by cardiologist.

Methods: We realised a prospective cohort study in a French NICU between 2015 and 2018. For any preterm newborn consecutively included, a holter ECG and RECAN were started at the same time during $24 \mathrm{~h}$. HRV indices were independently analysed by cardiologists and RECAN software. In this pilot study, we chose SDNN for the comparison. SDNN is one of heart rate variability time-domain indices. The standard deviation of the IBI of normal sinus beats (SDNN) is measured in ms. "Normal" means that abnormal beats, like ectopic beats (heartbeats that originate outside the right atrium's sinoatrial node), have been removed. Results: 30 correct $24 \mathrm{~h}$-records in 15 patients were included for analysis. For the primary result, the concordance correlation coefficient (CCC) in SDNN index was 0.94 (0.87- 0.98; CI 95\%), showing a strength of agreement, almost perfect. Conclusion: RECAN is a reliable software for SDNN analysis compared to ECG-24h interpreted by cardiologist. Further research in large scale is necessary to validate this tool and its application in a secure discharge of preterm infants.

247

\section{Neonatology}

Gastroschisis: Epidemiology and Management in a Roumanian Tertiary Centre Ana Maria Bradeanu ${ }^{1}$, Camelia Taran ${ }^{1}$, Laura Balanescu ${ }^{2}$

${ }^{T}$ Neonatal Intensive Care Unit, , Grigore Alexandrescu” Emergency Children's Hospital, Romania

${ }^{2}$ Pediatric Surgery, ,, Grigore Alexandrescu” Emergency Children's Hospital, Romania

Background: Gastroschisis is a ventral body wall defect trough which protrude mainly large and small intestines. The disease's clinical course and prognosis depend on perinatal factors, surgical techniques, accompanying anomalies and complications.

Objective: The aims of this study were to evaluate the anomaly's incidence, management and outcome of patients in our unit.

Methods: We performed a review of patients with gastroschisis admitted over a nineteen-year (2000-2018) period. The analyzed data was collected from from our hospital files and databases.

Results: The overall incidence of gastroschisis was $5.34 \%$ with an average birth gestational age of 35.87 weeks, average birth weight $2192.5 \mathrm{~g}$; only $15.73 \%$ of cases had an antenatal diagnose. $16.08 \%$ of cases presented associated anomalies: 12 cases of atresia /stenosis of intestinal segments, 6 cases with renal anomalies and 5 cases with cardiac defects. Surgical techniques performed were: primary closure 35 cases $(24.47 \%)$, staged closure 12 cases (8.39\%), Gross technique 44 cases $(30.76 \%)$ and manual reduction and closure (Bianchi procedure) 52 cases $(36.36 \%)$. The mean length of stay in NICU was 32.35 days with an average period of digestive pause of 13.9 days and a median duration of parenteral nutrition of 25.2 days. The most frequent complication was late on set sepsis $(37.8 \%)$ but $18.88 \%$ of cases underwent unplanned reoperation and 7 neonates $(5 \%)$ remained with short bowel. The average mortality in our group was $32.8 \%$ and constantly decreased from $84 \%$ in 2000 to $0 \%$ in 2018 . Sepsis, prematurity, low birth weight and associated defects were identified as major risk factors for an unfavorable outcome.

Conclusions: The optimal surgical management of gastroschisis has not been yet well determined and practice patterns are variable. The complex form of gastroschisis (involvement of other intestinal annomalies, ischemia, and perforations) was associated with a longer hospital lenght of stay and septicaemia was the commonest cause of death but the outcome of our patients had an obvious improvement during the studied period.

As the result, the care of these patients must involve specific antenatal ultrasound exams, an efficient multidisciplinary team and suitable surgical and nutritional protocols in order to improve neonatal survival.

\section{4}

Neonatology

Stickler Syndrome: A Possible Presentation of Pierre Robin Sequence

Filipa Briosa $^{l}$, Sara Oliveira ${ }^{l}$, Rodrigo Sousa ${ }^{l}$, Paulo Oom ${ }^{l}$

${ }^{T}$ Department of Paediatrics, Hospital Beatriz Ângelo, Portugal

Background: Stickler syndrome (SS) or hereditary progressive arthroophthalmopathy is a rare connective tissue disorder with a predominantly autosomal-dominant inheritance. It is characterized by orofacial, ocular, skeletal and auditory abnormalities. Affected children can initially present only with a Pierre Robin sequence (PRS) and a high index of suspicion is required. Objective: We report a case of SS in a child with PRS.

Methods: Case-report

Results: A preterm female neonate with a positive combined first trimester screening at 12 weeks of gestation (W) was diagnosed with micrognathia, mandibular hypoplasia and retrognathia on prenatal ultrasonography. The serological screening was negative. Her older sister had retrognathia and cleft of the soft palate. Due to the patient's prenatal history and her older sister's malformations she was submitted to a prenatal genetic screening. Amniocentesis revealed a normal cariotype with a positive array for SS. She was born by eutocic delivery at $32 \mathrm{~W}$ and needed no resuscitation. Upon examination she presented with PRS (figures 1 and 2). She presented respiratory distress within 24 hours of life, needing mechanical ventilation until the $14^{\text {th }}$ day of life. Enteric nutrition was initiated with a nasogastric tube. Red eye reflex and newborn hearing screening were normal. A complete eye examination revealed no alterations. She presented episodes of bradycardia and apnea secondary to glossoptosis, which reverted with manipulation. She was submitted to a successful mandibular distraction at 3 months of age and is currently being followed-up by a multidisciplinary team from plastic surgery, physical rehabilitation, ophthalmology, 
otolaryngology and orthopaedics. The patient's older sister was also subsequently screened and diagnosed with SS.

Conclusion: Although SS can present initially with an isolated PRS, abnormalities in the vitrous gel, joint hypermobility, osteoarthritis, sensorineural deafness and mitral valve prolapse may occur in the future leading to a wider therapeutic approach when compared with isolated PRS.
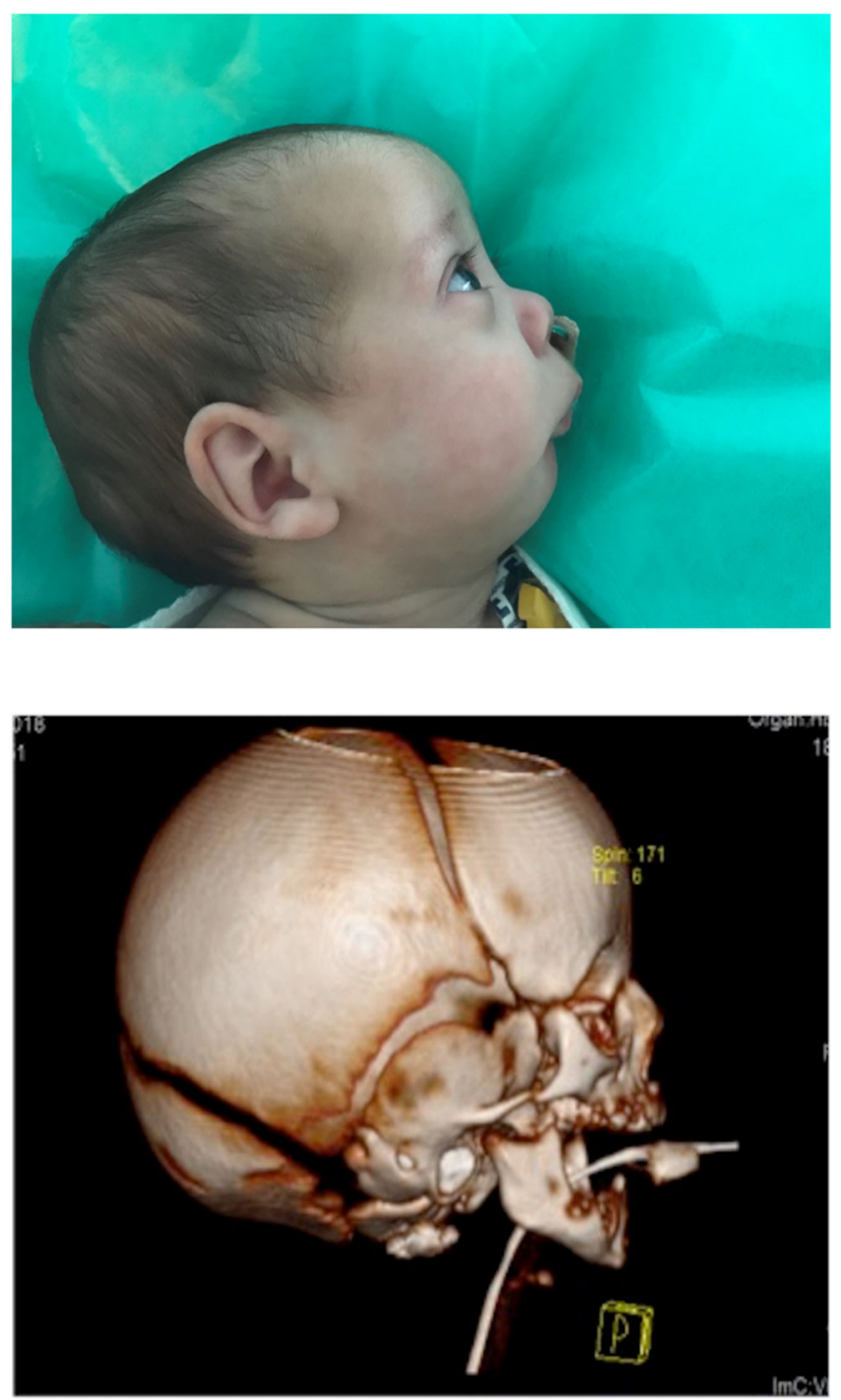

744

\section{Neonatology}

Apgar Score at $\mathbf{1}^{\text {st }}$ Minute: Early Prognostic Value?

Joana de Brito Chagas ${ }^{1,2}$, Mafalda Casinhas Santos ${ }^{1}$, Vanessa Albino ${ }^{l}$, Ana Sofia Vilardouro ${ }^{1,3}$, David Marques Lito ${ }^{1}$, Mário Paiva ${ }^{1}$

${ }^{1}$ Serviço de Pediatria, Hospital Vila Franca de Xira, Portugal

${ }^{2}$ Departamento de Pediatria, Hospital Pediátrico, Centro Hospitalar Universitário de Coimbra, EPE, Portugal

${ }^{3}$ Serviço de Pediatria, Departamento de Pediatria, Hospital de Santa Maria - Centro Hospitalar Universitário de Lisboa Norte, EPE, Portugal

Background: Apgar score (AS) provides a standardized reporting method for newborn status. Low 5-minute-AS correlates with neonatal complications. Although a low 1-minute-AS does not predict any individual infant's long-term outcome, there is lack of scientific data for the implications in the first days.
Objective: To evaluate low 1-minute-AS implications in the newborn first days.

Methods: Retrospective case-control study, matched by gestational age (1:2). All newborns with 1-minute-AS $\leq 5$ from a Portuguese level II hospital, between 2017 and 2018, were included. A 5-minute-AS $\geq 8$ subgroup was considered to exclude bias from known poor prognosis of a low 5-minute-AS. Results: Included 85 cases with no differences in sex, gestational age and birth weight from the controls (170). Deaths occurred only in the sample group ( $\mathrm{n}=3$ ).

Sample group had more alterations in cardiotocography $(41 \%$ vs $4 \%$, p0.001), meconium ( $24 \%$ vs $5 \%$, p0.001), intubation ( $29 \%$ vs $1 \%$, p0.001), intermittent positive pressure ventilation (IPPV) (88\% vs $1 \%$, $\mathrm{p} 0.001)$ and compressions ( $5 \%$ vs $0 \%, \mathrm{p}=0.011)$.

First-minute-AS $\leq 5$ was associated with more respiratory distress syndrome ( $31 \%$ vs $2 \%$, p0.001), Neonatal Intermediate Care Unit (NICU) needed (57\% vs $11 \%, \mathrm{p} 0.001$ ) and longer hospitalization (median 4 vs 3,p0.001). They required more umbilical catheterization ( $20 \%$ vs $4 \%$,p 0.001 ), intravenous fluids ( $54 \%$ vs $11 \%, \mathrm{p} 0.001)$, ventilation support ( $13 \%$ vs $5 \%, \mathrm{p}=0.03)$, antibiotherapy (33\% vs $9 \%$, p0.001), nasogastric tube feeding (16\% vs $7 \%, \mathrm{p}=0.016)$ and phototherapy ( $31 \%$ vs $15 \%, \mathrm{p}=0.003)$. These newborns were less exclusively breastfeeding than controls ( $53 \%$ vs $75 \%$, p0.001). In the 5-minute-AS $\geq 8$ subgroup ( $n=40$ ) differences remained statistically significative $(\mathrm{p} 0,05)$ regarding to cardiotocography, meconium, intubation, IPPV and compressions. This subgroup experienced more respiratory distress syndrome, NICU stay, intravenous fluids, antibiotherapy and longer hospitalization.

Conclusion: First-minute-AS $\leq 5$ (even when 5-minute-AS $\geq 8$ ) has important implications on the first days of life. Newborns needed more medical care which possibly increased costs. Other factors such as long maternal breastfeeding and microbiome may have been compromised.

693

Neonatology

Automated Thermal Management of the Neonatal Incubator during Phototherapy

Fatima Chahin Yassin $^{1,2}$, Stéphane Delanaud ${ }^{1}$, Pierre Tourneux ${ }^{1,2}$, Estelle Durand ${ }^{I}$

${ }^{1}$ PériTox Laboratory UMR-I 01,, UFR de Médecine, University of Picardie Jules Verne, Amiens, France

${ }^{2}$ Pediatric intensive care,, CHU Amiens, Picardie,, France

The incidence of jaundice is about $80 \%$ in preterm newborns, and Phototherapy (PT) is considered the first-line treatment. The efficacy of PT depends not only on the device characteristics but also on the modalities of use such as the distance between the infant and the device, the light source, the body surface area exposed, and the duration of exposure. However, risks such as hyperthermia also exist during PT, and the evaluation of the parameters that influence PT efficiency and the overheating risk is not easy in clinical practice.

The aim of this study is to evaluate the risk of overheating during PT. A thermal mannequin representing a preterm newborn was used to simulate heat exchange and to propose a new optimal air temperature setpoint during PT in various clinical situations.

We evaluated the impact of 3 different incubator models, the distance between the PT device and the preterm newborn(incubator roof or $5 \mathrm{~cm}$ above), and the time required to reach thermal equilibrium during PT. Preliminary data point out that there is a risk of hyperthermia related to decreased heat loss in the premature newborn during PT.

The power of the mannequin decreased significantly during PT (before PT:6.491 \pm 1.444 vs during PT:5.295 $\pm 1.533 \mathrm{~W} ; p 0.0001)$. A significant increase was measured in the incubator's air temperature(before: $31.3 \pm 0.9$ vs during: $\left.31.690 \pm 0.911^{\circ} \mathrm{C} ; p 0.0001\right)$, in the incubator's roof temperature (before:25.883 \pm 7.209 vs during: $30.938 \pm 3.076^{\circ} \mathrm{C} ; p 0.0001$ ) and in the radiation temperature(before: $30.480 \pm 0.685$ vs during: 31.390 
$\pm 0.797^{\circ} \mathrm{C} ; p 0.0001$ ). The simulation results suggest that optimal air $\mathrm{T}^{\circ} \mathrm{C}$ should be decreased between $1.64 \pm 0.20$ and $2.70 \pm 0.07{ }^{\circ} \mathrm{C}$ according to the clinical situation, the model of the incubator, the PT device, the distance between the neonate and the device, and the gestational age.

Results show that the overheating risk associated with PT is correlated with the incubator, the PT device and its use. Therefore, we propose a correction of the incubator's air temperature setting during PT.

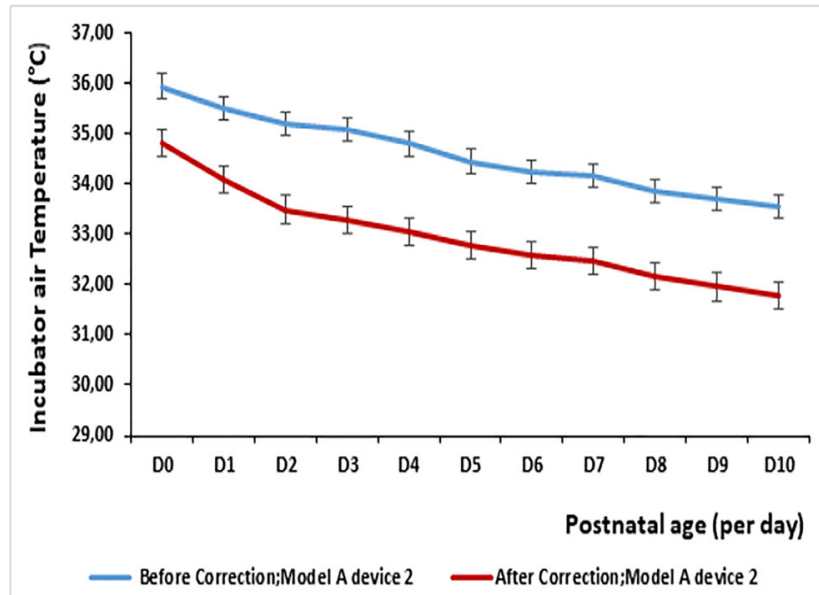

356

Neonatology

The Impact of Early Empiric Antibiotics use on Gut Microbiota in Very Low Birth Weight Preterm Infants

Hung-Yang Chang ${ }^{I}$, Jen-Shiu Chiang Chiau ${ }^{I}$, Hung-Chang Lee ${ }^{I}, Y$ Hsuan $\mathrm{Ho}^{2}, \mathrm{Kun}-\mathrm{Nan} \mathrm{Tsai}^{2}$, Chia- Yen Liu ${ }^{2}$, Jui-Hsing Chang ${ }^{1}$, Chyong-Hsin Hsu ${ }^{1}$, Chia-Ying Lin ${ }^{1}$

${ }^{1}$ Department of Pediatrics, MacKay Children's Hospital, TAIWAN

${ }^{2}$ Delta Research Center, Delta Electronics Inc., TAIWAN

Introduction: Antibiotics are frequently prescribed to preterm infants and are known to disrupt microbial balance. Our objective was to determine the impact of different empiric antibiotics use in the first week of life on microbial colonization and diversity in very low birth weight (VLBW) preterm infants.

Methods: Breast fed VLBW infants were divided into two groups, including those who received $3 \mathrm{~d}$ of combination treatment of ampicillin and gentamicin (AG group), and those received $7 \mathrm{~d}$ of ampicillin and cefotaxime (AC group). Infants with any antibiotics use after age of $7 \mathrm{~d}$ and infants on exclusive formula feeding were excluded. Stool samples were collected at age of $7 \mathrm{~d}, 14 \mathrm{~d}$, and $30 \mathrm{~d}$. The $16 \mathrm{~s}$ ribosomal DNA community profiling was used to compare the microbiota between two groups.

Results: Twenty-four infants were enrolled in our study (AG group $=10$, AC group =14). The gestation age and birth weight were $30.0 \pm 2.5$ weeks and $1286 \pm 190 \mathrm{gm}$ in the AG group versus $28.9 \pm 2.5$ weeks and $1099 \pm$ $280 \mathrm{gm}$ in the AC group, respectively. Infants of AC group had significantly increased abundance of Enterococcus in the 7th day of life compared to those of AG group ( $12.3 \%$ vs $0.6 \%, \mathrm{P}=.032)$. The richness of species in AC group significantly decreased in the $14 \mathrm{~d}(\mathrm{p}=.038)$ and 30 $\mathrm{d}(\mathrm{p}=.030)$ samples as compared to that in the $7 \mathrm{~d}$ sample. The evenness was lowest in the $7 \mathrm{~d}$ sample of AC group comparing to the $14 \mathrm{~d}$ and $30 \mathrm{~d}$ samples $(\mathrm{p}=.041)$. The diversity within group had no significant difference between two groups in each time period samples.

Conclusions: Different antibiotic treatments affect the early development of gut microbiota in VLBW preterm infants. A combination of ampicillin and cefotaxime resulted in an overgrowth of Enterococcus and a decreased in richness and evenness.
207

Neonatology

Two-Step Mask Continuous Positive Airway Pressure Improves Transient Tachypnea of the Newborn

Hiroo Chiba ${ }^{1}$, Hiroshi Watanabe ${ }^{1}$

${ }^{T}$ Department of Pediatrics, National Hospital Organization Sendai Medical Center, Japan

Background: Recent studies indicate mask continuous positive airway pressure (mCPAP) improves the neonatal respiratory stabilization. However, it is not clear whether mCPAP is effective against term infants with transient tachypnea of the newborn (TTN) delivered by elective cesarean section (eCS). In December 2017, we decided to treat central cyanotic and forced breathing term infants delivered by eCS with mCPAP in theater within 15 minutes, instead of administering oxygen. Moreover, we transferred the infants to the treatment room and treated them with mCPAP until 60 minutes after birth, if their central cyanosis and forced breathing either appeared or was prolonged.

Objective: The objective of this study was to evaluate whether two-step mCPAP until 60 minutes after birth is effective for decreasing the number of term infants hospitalized for TTN delivered by eCS.

Methods: Among infants with 36 weeks of gestation and more who were delivered by eCS in our hospital, we retrospectively compared 160 delivered from 1 December 2016 to 30 October 2017 (Oxygen Group) with 161 delivered from 1 December 2017 to 30 October 2018 (MC Group). Results: Baseline characteristics of all infants were comparable, but the Apgar score at 5 minutes was statistically less in the MC Group than in the Oxygen Group (9.0, p0.05). Oxygen administration was statistically less in the MC Group than in the Oxygen Group (12/160 vs 57/161, p0.01) and mCPAP was used statistically more often in the MC Group than in the Oxygen Group (54/160 vs 0/161, p0.01). The number of infant hospitalized for TTN in the MC Group was significantly less than in the Oxygen Group (17/161 vs 5/161, p0.05).

Conclusion: Two-step mCPAP until 60 minutes after birth might decrease the number of term infants hospitalized for TTN delivered by eCS.

\section{Table1. Baseline Characteristics}

$\begin{array}{lrrr} & \text { Oxygen(n=161) } & M C(n=160) & \text { P value } \\ \text { Gestational age(weeks) } & 37.9 \pm 0.5 & 37.9 \pm 0.5 & 0.313 \\ \text { Birth Weight(g) } & 2838 \pm 316 & 2915 \pm 394 & 0.054 \\ \text { Males ( } \mathrm{n} \text { ) } & 75 & 77 & 0.435 \\ \text { Multiple births } & 6 & 2 & 0.144 \\ \text { FGR } & 1 & 4 & 0.186 \\ \text { Apgar scorc at 1 min } & 8.1 \pm 0.7 & 8.1 \pm 0.5 & 0.300 \\ \text { Apgar score at 5 min } & 9.0 \pm 0.3 & 8.9 \pm 0.4 & <0.05 \\ \text { Mask Bagging/Tube Bagging } & 4 & 2 & 0.345 \\ \text { Oxygen administration } & 57 & 12 & <0.01 \\ \text { Mask CPAP } & 0 & 54 & <0.01\end{array}$

\section{0}

Neonatology

Role of Ultrasonography in the Assessment of Correct Endotracheal Tube Placement in Neonates. A Prospective Single Center Study Claire-Sophie Descamps ${ }^{1}$, Anne Beissel ${ }^{1}$, Philippe Vo Van ${ }^{1}$, Nicolas Stacoffe $^{2}$, Sébastien Blanc ${ }^{1}$, Franck Plaisant ${ }^{1}$, Olivier Claris ${ }^{I}$, Kim-An Nguyen $^{1,3}$

${ }^{I}$ Neonatal Intensive Care Unit, Hôpital Femme Mère Enfant, Hospices Civils de Lyon, France

${ }^{2}$ Radiology Department, Hôpital Femme Mère Enfant, Hospices Civils de Lyon, France

${ }^{3}$ Pharmacotoxicology Department/LBBE/EMET/UMR5558, Université Claude Bernard, France 
Background: Endotracheal intubation remains a complex procedure in neonatal care as a malposition may lead to pulmonary complications. To confirm correct endotracheal tube (ETT) position, chest X-Ray (CX) is the reference investigation. However it is often delayed and exposes to ionizing radiations. Bedside ultrasonography (US) is a faster non-invasive technique which might supply CX. Objective: To compare US and CX in order to confirm an endotracheal intubation and evaluate the appropriate ETT position.

Methods: All neonates admitted in our level III neonatal ward and intubated were included. Immediately after intubation, the radiology unit was called for a CX as well as the neonatologist to perform a bedside tracheal US which consisted in two views:

- a coronal view with direct visualization of the trachea to confirm the tracheal intubation

- a parasternal view to determine the distance between the tip of the tube and the aortic arch. A distance between 0.5 and 1 centimeter was considered as optimal.

On X-ray, ETT was in good position if visualized in relation to the body of the first thoracic vertebra.

Number of bradycardia and desaturations were recorded. Sensitivity, specificity, positive and negative predictive value of US to determine the same position of ETT as CX were calculated.

Results: Twenty five infants were included with a mean corrected gestational age of 32.3 weeks $+/-6.2$ and mean birth weight of $1796 \mathrm{~g}+/-676$. Endotracheal intubation was confirmed for all the patients by CX and US. The positive predictive value to confirm the intratracheal position of the tube was $100 \%$. Sensitivity of US to detect the same ETT position as CX was $77 \%$, specificity $29 \%$. No adverse effects were recorded during the US. Conclusion: Tracheal US, performed by neonatologists, is a fast investigation to confirm the tracheal intubation and appears feasible to evaluate ETT position.

\section{0}

\section{Neonatology}

Neonatal Macrosomia: What are the Risk Factors and Implications? Ana Dias Curado ${ }^{l}$, Sara Costa ${ }^{l}$, Cristina Lorenzo ${ }^{I}$, Joana Gil ${ }^{2}$, Sandra Valente $^{2}$, Joana Saldanha ${ }^{2}$

${ }^{1}$ Department of Pediatrics, Santa Maria Hospital - Centro Hospitalar Universitário Lisboa Norte, Portugal

${ }^{2}$ Neonatal Intensive Care Unit, Santa Maria Hospital - Centro Hospitalar Universitário Lisboa Norte, Portugal

Background: A birth weight over $4000 \mathrm{~g}$, regardless of gestational age, is the definition of macrosomia. It affects about $3-15 \%$ of the babies born worldwide and is associated with neonatal and maternal complications.

Objective: To describe the risk factors and complications present in macrosomic children born in a tertiary center.

Methods: Review of clinical records of children born between July and December 2018. Descriptive analysis of demographic data, risk factors for macrosomia and neonatal and maternal complications.

Results: There were a total of 1346 births, 4,9\% ( $n=66)$ of them corresponding to macrosomic newborns, with male predominance $(74 \%)$. Regarding maternal factors: maternal mean age was 30,2 $\pm 6,2$ years; $1,6 \%$ presented pre-eclampsia; $10,9 \%$ had diabetes (1/7 pre-gestational and $6 / 7$ gestational diabetes); 9,4\% were obese; $4,7 \%$ smokers and $31,3 \%$ had a previous macrosomic newborn. In relation to newborn factors: $15,6 \%$ had a prenatal diagnosis of macrosomia; the mean gestational age was $39,9 \pm 1$ weeks; mean birth weight of $4356,7 \pm 304,4$ grams with $41 \%$ large for gestational age (P90). At least one complication was present in $81 \%$ of the newborns (hyperbilirubinemia in $28,1 \%$, neonatal hypoglycemia in $23,4 \%$, shoulder dystocia in $10,9 \%$, neonatal respiratory distress syndrome in $10,9 \%$, brachial plexus palsy in $4,7 \%$, clavicle fracture in $3,1 \%$, weight loss greater than $10 \%$ in 3,1\%, and $6 \%$ needed hospitalization in a neonatal care unit. Maternal postpartum hemorrhage occurred in $1,6 \%$ and $56,3 \%(n=48)$ had perineal laceration.
Conclusion: As according to the literature, our rate of macrosomic newborns was similar to the worldwide data and there was also a male predominance. There was a high neonatal complication rate in our sample. The prevention of modifiable risk factors for macrosomia and its prenatal diagnosis is crucial to reduce associated newborn and maternal morbidity.

\section{2}

Neonatology

Sudden and Unexplained Neonatal Death with Group a Streptococcus Infection

Sara El Badri ${ }^{l}$, Syed Rizvi ${ }^{l}$, Babu Paturi ${ }^{l}$

${ }^{T}$ Paediatrics, Our Lady of Lourdes Hospital, Drogheda, Ireland

Introduction: Group A streptococcus (GAS) is a rare infection in neonates. A 2004 review of GAS invasive infection in the neonates has described 39 patients since 1966 in the world literature. The mode of transmission in the majority of invasive late onset cases is unknown. Vertical transmission or postnatal acquisition of focal GAS infection such as pharyngitis and episiotomy abscess are probable sources of transmission.

Case Report: A 5 days old baby boy, born term to a healthy mother by vaccum delivery, was vigorous at birth and no resuscitation was required. $\mathrm{He}$ was brought to the emergency department with poor feeding, reduced activity and difficulty in breathing. On examination, initially he was pink, afebrile, vitals were normal, he had intermittent grunting and marked hypotonia with poor response to stimulation. Septic work up was done, bloods showed WCC $=1.6$, Neutrophils $=1.02$, Platelets were out of range, Prolonged PT of 22.8, CRP 186. He received IV Cefotaxime, Amoxicillin and Gentamicin. He had a chest xray which showed consolidation in both lung bases. He deteriorated clinically after 1 hour of presentation, he started having apnoeic episodes, capillary refill was 4 secs, mean blood pressure 30 , he had a full anterior fonanelle and weak pulses. He was fully resuscitated with Endotracheal intubation and ventilation. He received a total of 3 IV Normal saline $20 \mathrm{ml} / \mathrm{kg}$ boluses. He also received dopamine, noradrenaline, and mannitol. He was then transferred to a tertiary ICU. His blood culture was positive for Streptococcus pyogenes Group A at 9 hours. His clinical condition continued to deteriorate; ventilatory support was withdrawn after parental consent and was pronounced RIP after 12 hours of presentation.

Conclusion: Even though Group B strep is more common in neonatal sepsis, group A strep also needs to be considered in the differentials as it can be fatal.

\section{2}

\section{Neonatology}

Introduction of Care Bundle for Non-invasive Respiratory Support for Neonates Admitted to a Tertiary Neonatal Unit in the United Kingdom: A Quality Improvement Project

Katie Evans ${ }^{I}$, Anay Kulkani ${ }^{1}$, Tessa Sigola-Rowett ${ }^{1}$, Sandeep Shetty ${ }^{1}$

${ }^{T}$ Department of Neonatology, St George's University Hospitals NHS Foundation Trust, UK

Background: It is widely recognised in neonatal practice that there is variation in the instigation, switching and weaning of infants between various forms of non-invasive ventilation. With continuous positive airway pressure (CPAP) circuits lasting seven days and high flow, high temperature, humidified nasal cannula oxygen (HFNCO2) circuits lasting thirty days there is a clear financial incentive to minimise circuits used.

Objective: Introduction of care bundle to standardise initiation and switching of non-invasive respiratory support in babies needing respiratory support will lead to reduction of respiratory circuits being used for non-invasive support, making its use more efficient.

Methods: Pre-intervention data was analysing using Badger data from all babies born in 2016 looking at the number of CPAP and HFNCO2 circuits utilised. A comprehensive teaching package was introduced to the 
nursing and medical teams around circuit usage and costs (£93 per CPAP circuit and $£ 101$ per HFNCO2 circuit). Specific guidelines were developed to guide initiation, switching and weaning of respiratory support. Post-intervention data was collected from all babies born in 2017 for comparison.

Results: There was decreased use of CPAP and HFNCO2 circuits in babies requiring respiratory support. Regarding CPAP there was a reduction from 1.53 circuits per baby to 1.0 and with $\mathrm{HFNCO} 2$ this was 0.64 circuit per baby to 0.49 . In lower gestational ages this was more significant; under 28 weeks gestation it went from 2.44 to 1.59 circuits per baby with CPAP and 1.02 to 0.58 circuits per baby with HFNCO2. This equates to a gross yearly saving of $£ 22,800$ (assuming a similar patient population with 3000 annual admissions). Conclusion: Introduction of a care bundle involving an education package, clear written guidelines and increased awareness of durations and costs of CPAP and HFNCO2 circuits amongst medical and nursing staff leads to considerable cost savings when incorporated into clinical practice.

\section{1}

\section{Neonatology}

An Integrated Discharge Planning Pathway Between Acute and Social Care Services is Needed to Prevent Delay in Care Transfer for Babies with Safeguarding Concerns

Saara Masood ${ }^{l}$, Rajani Viswanathan ${ }^{l}$, Susan Summers ${ }^{1}$, Mojgan Ezzati ${ }^{1}$

${ }^{1}$ Neonatology, University Hospital Lewisham, UK

Background: Discharge pathway for babies with safeguarding concerns in neonatal unit encompasses close working relationship between acute and social care services. Yet, there is increasing pressure on both services in regards to patient flow which compromise this partnership hence patient care. Objectives: To facilitate neonatal discharges with safeguarding concerns by counting and collecting data about delayed transfer of care from acute setting to social care services.

Methods: A retrospective survey was conveyed on all neonatal admissions with safeguarding concerns over a period of 12 months (2017-2018) at our neonatal unit at University Hospital Lewisham. A total of 21 babies were admitted at neonatal unit during this period. Data were collected from their case notes about the safeguarding and medical history and management. Also, the gap between the dates when they were medically fit for discharge versus when they were safe to be discharged was analysed including the reasons behind delay for discharge.

Results: There was a total of 210 days over the period of 1 year when babies were medically fit for discharge but not fit for discharge due to awaiting discharge planning meeting, court hearing and housing issues. Of note the delay in discharge was longer in babies who were discharged home compared discharged to foster carer. There was only 1 baby with no gap between medically fit and being safe discharge date.

Conclusion: Discharge planning pathway for safeguarding cases should be in parallel with medical management rather than being sequential. There is a need to develop a robust and consistent guideline to integrate discharge pathway from acute care to social care services from the very first day of admission of babies with safeguarding concerns.

713

\section{Neonatology}

Association of Low-Grade Intraventricular Haemorrhage with MRI Findings at Term Equivalent Age and Severe Neurodevelopmental Impairment

Aikaterini Drougia ${ }^{1}$, Dimitrios Rallis ${ }^{l}$, Vasileios Xydis ${ }^{2}$, Maria Fintzou ${ }^{1}$, Anastasia Giantsouli ${ }^{2}$, Meropi Tzoufi ${ }^{3}$, Maria Argyropoulou', Vasileios Giapros $^{I}$

${ }^{1}$ Neonatal Intensive Care Unit, Medical School, University Hospital of Ioannina, Greece
${ }^{2}$ Department of Radiology, Medical School, University Hospital of Ioannina, Greece

${ }^{3}$ Pediatric Neurology, Department of Pediatrics, Medical School, University Hospital of Ioannina, Greece

Background: In premature neonates, the evidence regarding the risk of white matter damage and neurological impairment following an uncomplicated intraventricular haemorrhage (IVH) remains inconclusive.

Objective: To evaluate any association of IVH I-II with the brain MRI findings at term equivalent age (TEA), and the neurodevelopmental outcome. Methods: We retrospectively reviewed the medical records of all neonates $\leq 32$ weeks' gestation admitted to the Neonatal Unit of University Hospital of Ioannina, during 2010-2017. Neonates with IVH I-II were included, and compared with neonates without IVH. All neonates had neurodevelopmental assessment at two years of corrected age. Those with congenital anomalies, IVH III-IV, cystic periventricular leukomalacia (cPVL) or infarct detected in ultrasound were excluded.

Results: Totally 133 neonates were enrolled; 40 neonates of $29 \pm 2$ weeks' gestation with IVH I-II, and 93 controls of $29.5 \pm 1.7$ weeks' gestation $(\mathrm{p}=0.163)$.

Among the perinatal characteristics examined between the two groups, neonates with IVH had haemodynamically significant PDA in higher proportion ( 23 over $8 \%, p=0.004)$ and required ventilation for longer ( 10 over 4 days, $\mathrm{p}=0.032$ ) compared to controls, however, they had similar rates of bronchopulmonary dysplasia and were discharged in similar corrected age. The $113(85 \%)$ neonates had an MRI; 33 with IVH and 80 controls. Abnormal findings detected in $2(6 \%)$ neonates with IVH (1 had diffuse PVL-dPVL and 1 infarct), and in $8(11 \%)$ controls (6 had dPVL and 2 cPVL).

Cerebral palsy was diagnosed in one infant of 30 weeks' gestation with IVH I and normal MRI, and another of 24.3 weeks' gestation with normal ultrasound but cPVL in MRI.

Conclusions: Premature neonates with IVH I-II have similar findings in MRI compared to neonates without IVH. Furthermore, they are in the same low risk for cerebral palsy.

\section{4}

\section{Neonatology}

The Association of Fluid Overload During the First Postnatal Day with Patent Ductus Arteriosus and Bronchopulmonary Dysplasia in Preterm Infants

Dimitrios Rallis ${ }^{1}$, Aikaterini Drougia ${ }^{1}$, Foteini Balomenou ${ }^{1}$, Maria Fintzou $^{I}$, Thomas Benekos ${ }^{2}$, Antonios Vlahos ${ }^{2}$, Vasileios Giapros ${ }^{I}$

${ }^{7}$ Neonatal Intensive Care Unit, Medical School, University Hospital of Ioannina, Greece

${ }^{2}$ Pediatric Cardiology, Department of Pediatrics, Medical School, University Hospital of Ioannina, Greece

Background: Fluid balance in premature infants is critical, however evidence examining the association of over-hydration during early life with haemodynamically significant patent ductus arteriosus (hsPDA) and bronchopulmonary dysplasia (BPD) is limited.

Our aim was to evaluate the association of fluid overload during the first postnatal day with hsPDA and BPD in preterm infants.

Methods: The medical records of neonates $\leq 30$ weeks' gestation and1500 grams' bithweight, admitted to the Neonatal Unit of University Hospital of Ioannina during 2010-2017 were reviewed. Additional fluid intake above the prescribed maintenance goals was calculated [(Total fluid intake-Target fluid intake)/Target fluid intake \%]. Also, fluid overload (5\%) was calculated by (Total fluid intake-Total output)/kg \% . HsPDA was defined according to echocardiographic indicators of ductal diameter and shunt pattern, degree of pulmonary overflow and magnitude of systemic hypoperfusion.

Results: Fifty-two infants identified; 15 (29\%) developed fluid overload, and were of similar gestational age $(27.2 \pm 1.5$ versus $27.5 \pm 1.3$ weeks $)$ 
and birthweight $(981 \pm 209$ versus $1051 \pm 240 \mathrm{~g})$, compared to $37(71 \%)$ infants without. Also, no differences were noted regarding the remaining perinatal characteristics.

The total fluid intake $/ \mathrm{kg}(102 \pm 21$ versus $86 \pm 11 \mathrm{ml} / \mathrm{kg})$, additional fluid intake (23\% versus $12 \%)$, and fluid balance/kg ( $80 \pm$ 40 versus $30 \pm 15 \mathrm{ml} / \mathrm{kg}$ ) were significantly higher in infants with fluid overload; accordingly, the total output $/ \mathrm{kg}$ was decreased (30 \pm 17 versus $60 \pm 17 \mathrm{ml} / \mathrm{kg}$ ).

Infants with fluid overload developed hsPDA in 53\% and BPD in $80 \%$, compared to $22 \%(\mathrm{p}=0.044)$, and $35 \%(\mathrm{p}=0.005)$ of those without, respectively. The length of stay, rates of intraventricular haemorrhage and survival were similar between the two groups.

Conclusions: In preterm infants, fluid overload $5 \%$ is significantly associated with hsPDA and BPD, therefore, fluid management during the first postnatal day should be closely monitored and regulated.

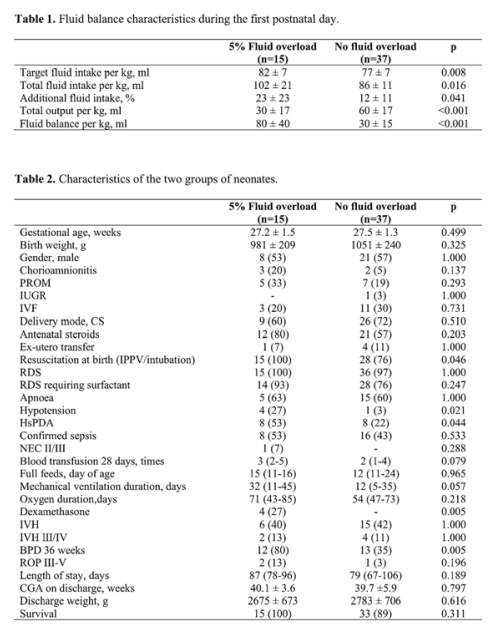

715

Neonatology

The Risk Factors for Late Onset Sepsis on Very Preterm Neonates and the Impact on Neuroimaging At Term Equivalent Age Dimitrios Rallis ${ }^{l}$, Aikaterini Drougia ${ }^{l}$, Theodoros Gouvias ${ }^{1}$, Maria Fintzou $^{l}$, Maria Fintzou ${ }^{l}$, Vasileios Xydis ${ }^{2}$, Anastasia Giantsouli ${ }^{2}$, Maria Argyropoulou ${ }^{2}$, Vasileios Giapros ${ }^{1}$

${ }^{1}$ Neonatal Intensive Care Unit, Medical School, University Hospital of Ioannina, Greece

${ }^{2}$ Department of Radiology, Medical School, University Hospital of Ioannina, Greece

Background: Neonatal sepsis remains significant reason of increased mortality among extremely preterm neonates, and has been associated with white matter injury.

Objective: To examine the risk factors for late-onset sepsis (LOS) and the outcomes in extremely preterm neonates. Also, to evaluate any association between LOS and the brain MRI findings at term equivalent age.

Methods: The medical records of neonates $\leq 28$ weeks' gestation, admitted to the Neonatal Unit of University Hospital of Ioannina during 20062017 were reviewed. Neonates with LOS, confirmed with blood culture, were compared with neonates without sepsis. Neonates with early onset sepsis were excluded.

Results: Totally 155 neonates were enrolled; 55 (35\%) with LOS and 100 $(65 \%)$ controls.

Multiple LOS episodes were recorded in 17 neonates; among 79 sepsis episodes, Gram(+) pathogen detected in $61 \%$, fungi in $22 \%$ and Gram(-) in $11 \%$. Neonates with sepsis were predominantly males (71 versus $46 \%$, $\mathrm{p}=0.002$ ), had a longer duration of mechanical ventilation (37 versus 16 days, $\mathrm{p}=0.001)$, developed bronchopulmonary dysplasia in higher proportion ( $64 \%$ versus $34 \%, \mathrm{p}=0.001$ ), required prolonged parenteral nutrition ( 35 versus 15 days, p0.001), received more blood transfusions ( 8 versus 5 times, p0.001), and had longer length of stay ( 95 versus 82 days, $\mathrm{p}=0.002)$. Survival rates were similar $(71 \%$ versus $73 \%)$. Male gender (2.992, $\mathrm{p}=0.026,95 \% \mathrm{CI} 1.142-7.842)$ and blood transfusion $(0.765$, $\mathrm{p}=0.030,95 \% \mathrm{CI} 0.601-0.974)$ were predominant risk factors for LOS.

The $68 \%$ of neonates with LOS had a normal MRI, versus $90 \%$ controls $(\mathrm{p}=0.030)$. The commonest abnormal finding was diffuse periventricular leukomalacia (dPVL), in $18 \%$ of neonates with LOS versus $4 \%$ of controls $(\mathrm{p}=0.021)$.

In multivariate analysis, sepsis was a strong predictor for developing dPVL (3.958, p=0.048, 95\% CI 1.011-15.491).

Conclusions: In extremely preterm neonates, multiple factors predispose for the development of LOS. Nonetheless, in this cohort, sepsis remains an independent risk factor for the development of dPVL.

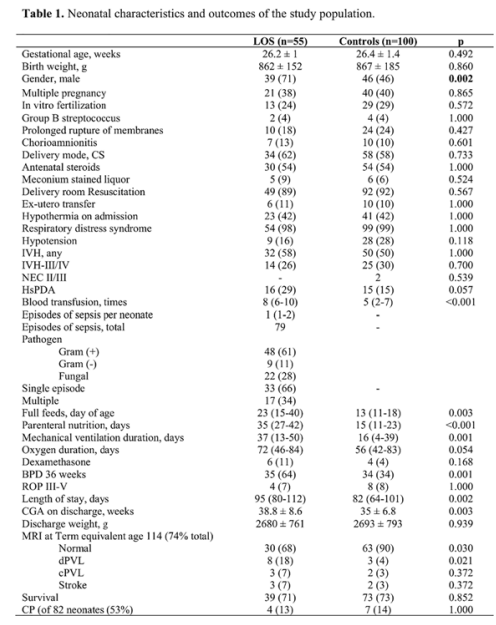

359

Neonatology

Congenital Knee Dislocation: Importance of Early Diagnosis

Catarina Freitas $^{1}$, Joana Morais ${ }^{1}$, Andreia Martins ${ }^{2}$, Ana Cristina Barros $^{2}$, Claudia Ferraz 2

${ }^{1}$ Pediatria, Hospital Pedro Hispano, Portugal

${ }^{2}$ Neonatologia, Hospital Pedro Hispano, Portugal

Background: Congenital knee dislocation (CKD) is a rare anomaly, with an incidence of about $1 \%$, that mostly affects female sex (ratio 10:3). In CKD there is a variable anterior displacement of the tibia under the femur, frequently associated with other musculoskeletal abnormalities (hip developmental dysplasia, equine varus foot) or syndromes (Larsen syndrome). Fibrosis and shortening of the quadriceps muscle with supra-patellar hypoplasia is a pathognomonic characteristic of CKD. Even though treatment is difficult and controversial, most authors advocate conservative treatment.

Clinical Case: Female new-born, first child of healthy and nonconsanguineous young parents. Single term pregnancy, with normal evolution. Maternal serologies were negative and prenatal ultrasound scans did not detect any anomalies. Eutocic delivery, anthropometry was appropriate to gestational age, with Apgar Index 9/10. Physical examination at birth detected an abnormal position of the knees and legs, with no other associated anomalies. Those findings were interpreted as a congenital knee dislocation and the newborn was evaluated by orthopedics, confirming the diagnosis. The treatment of choice was conservative and, therefore, a plaster cast was applied. Control radiography was 
performed and the patient was discharged, with neonatal and orthopedic referral in order to guarantee an adequate follow-up.

Conclusion: The conservative approach, with immediate reduction of the dislocation within the first hours of life, is in agreement with most of the literature. The detection of other associated orthopedic anomalies on physical examination is still of primary importance. This case highlights the role of the pediatrician in the identification, diagnosis and early intervention of these situations.
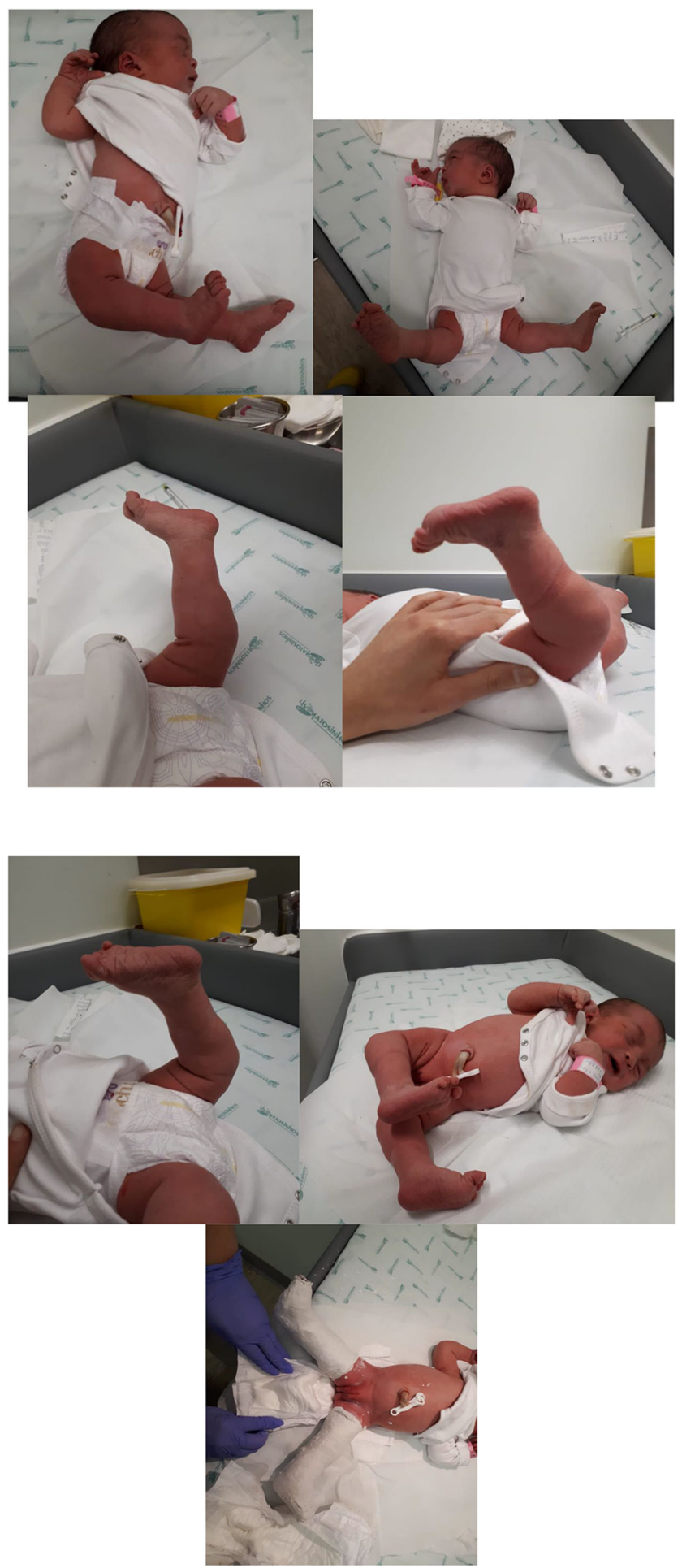

618

\section{Neonatology}

Petechiae in the Newborn: A Sign to Consider Maria Gaia ${ }^{1}$, Joana Brandão Silva ${ }^{1}$, Mariana Capela ${ }^{1}$, Manuela Mateus $^{I}$, Rui Pinto ${ }^{1}$

${ }^{1}$ Serviço de Pediatria Médica / Neonatologia, Centro Hospitalar de Vila Nova de Gaia/Espinho, Portugal

Background: Neonatal alloimmune thrombocytopenia (NAIT) occurs when the mother forms antiplatelet antibodies against fetal platelets. Clinical findings depend on the severity of thrombocytopenia, including petechiae, bruising and bleeding, in mostly otherwise well-appearing newborns. The diagnosis is confirmed by the presence of maternal antihuman platelet antibody.

Case Report: Full-term newborn routinely examined on the first day of life. First pregnancy, without complications. Routine serologic testing and prenatal ultrasounds without alterations. Vacuum-assisted delivery, Apgar score of 9/10/10 and anthropometric measuring at the 10-50 percentile. During observation, the newborn appeared well. Multiple petechiae were detected on the axillary, inguinal and popliteal folds and inferior area of the abdomen, with no other physical examination findings, including signs of bleeding or mucosal affection. A blood test was performed, revealing thrombocytopenia of $28000 /$ ul, with normal remaining blood cell lines and negative $\mathrm{C}$ Reactive Protein. A dose of intravenous immunoglobulin was administered given the hypothesis of NAIT. Control blood test showed a minimum platelet count of 25000/ul 9h after immunoglobulin, increasing to $44000 / \mathrm{ul}$ after $18 \mathrm{~h}$. A second dose was administered after 24 hours. Platelet count then progressively increased, with $176000 /$ ul by the 7 th day. Diagnosis confirmation was possible by the 5 th day. Parents' study revealed antiplatelet anti-HPA-1a alloantibodies in the mother. Parents' platelet compatibility test had a strong positive (4+) result. Platelet HPA-1 genotypes of mother, father and newborn were $1 \mathrm{~b} 1 \mathrm{~b}, 1 \mathrm{a} 1 \mathrm{a}$ and $1 \mathrm{a} 1 \mathrm{~b}$, respectively. Presently, the infant is 2 months old, is clinically well with a platelet count of $415000 / \mathrm{ul}$.

Conclusion: An exhaustive physical examination of the newborn is essential, as some serious conditions may manifest with subtle alterations. In the differential diagnosis of isolated petechiae of the newborn, NAIT must be considered. The serologic and genetic testing are important to confirm diagnosis and to properly provide genetic counselling to the couple.

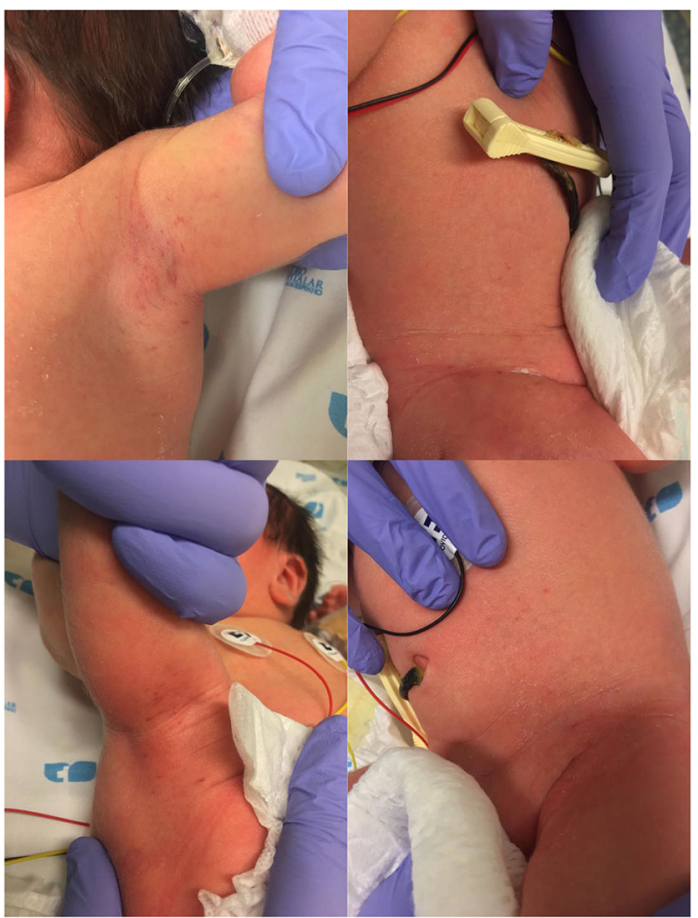


710

\section{Neonatology}

Reducing Investigations in Suspected Early onset Sepsis (RISE)Quality Improvement Project

\section{Anip Garg ${ }^{1}$, Prakash Loganathan ${ }^{2}$}

${ }^{T}$ Department of Paediatrics, University Hospital of North Tees, North Tees and Hartlepool NHS Foundation Trust, UK

${ }^{2}$ Neonatal Unit, The James Cook University Hospital, South Tees Hospitals NHS Foundation Trust, UK

Background: Full blood count (FBC) is used commonly in evaluation of early onset sepsis (EOS). In this project we evaluated validity of FBC as screening tool in EOS.

Methodology: Retrospective review of babies ( $\geq 34$ weeks GA) born at North Tees University hospital and started on antibiotics for suspected EOS in six months duration (January 2018 June 2018). "Presumed sepsis" [True Positives] was defined as clinically ill (Respiratory/Haemodynamic instability requiring support or encephalopathy or any persistent physiological abnormality) on examination or any culture positive.

Results: Total 169 babies were started on antibiotics in the six months duration for suspected EOS. White cell count (WCC) of $20 / \mu \mathrm{l}$, absolute neutrophill count (ANC) of $5 / \mu$ land C-reactive protein (CRP) value of $10 \mathrm{mg} / 1$ showed poor sensitivity $(26.87 \%$, $10.45 \%$ and $29.85 \%$ respectively) and negative predictive value $(56.64 \%, 61.54 \%$ and $63.28 \%$ respectively) for evaluation of sepsis. The area under Reciever Operating Characterstics (ROC) curve for WCC, ANC and CRP were $0.59(\mathrm{p}=0.05), 0.58$ $(\mathrm{p}=0.074)$ and $0.58(\mathrm{p}=0.076)$ respectively and considered as 'poor test'. There was no difference in mean WCC and ANC in babies with 'presumed sepsis' versus babies with 'no sepsis'. Significant difference in CRP levels was found in babies with 'presumed sepsis' versus babies with 'no sepsis' ( $21 \pm 49$ vs. 7.7 $\pm 12, p=0.032$ ). Correlation of duration of antibiotics with CRP levels $\left(\mathrm{r}_{\mathrm{s}}-68 \%, \mathrm{p}=0.00\right)$ was strong, while it was poor with WCC $\left(\mathrm{r}_{\mathrm{s}}-12.8 \%, \mathrm{p}=0.10\right)$ and ANC $\left(\mathrm{r}_{\mathrm{s}}-11 \%, \mathrm{p}=0.16\right)$. Abnormal clinical examination had a poor correlation with antibiotics duration 48hours (kappa 0.20).

Conclusion: In well babies with suspected EOS, FBC is not a useful screening test. The decision to prolong duration of antibiotics was predominantly based on CRP.

Recommendations: Abandoning practice of doing FBC in well babies with suspected EOS and performing FBC only in admitted babies to evaluate severity of sepsis

$10 \mathrm{mg} / \mathrm{l}$ ) in clinical or culture positive sepsis" width="455" height="299" /

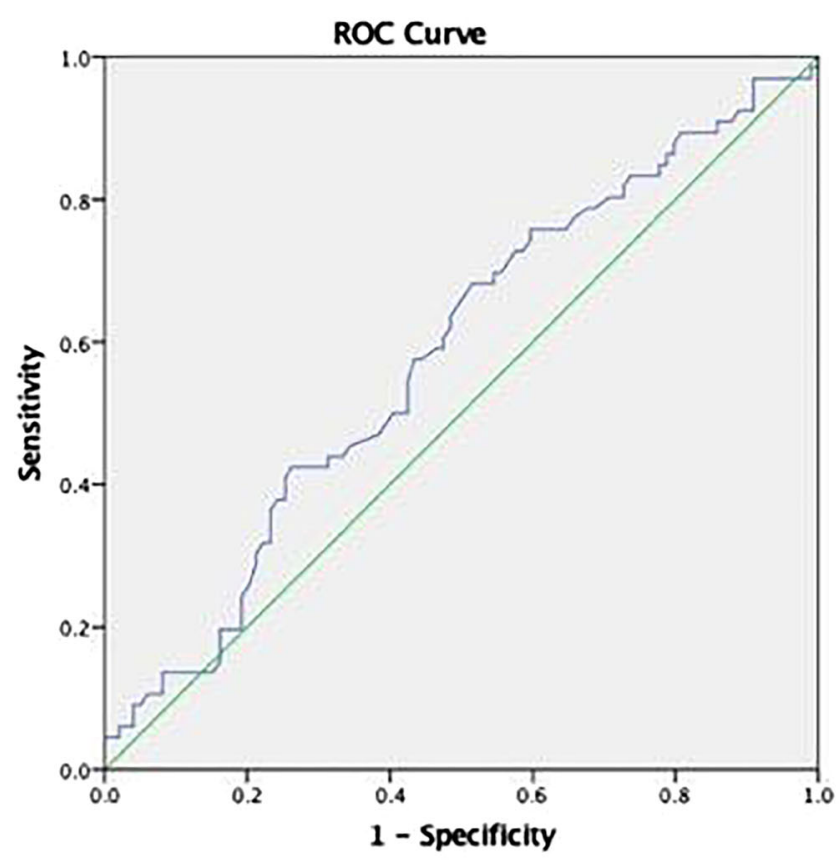

Diagonal segments are produced by ties.

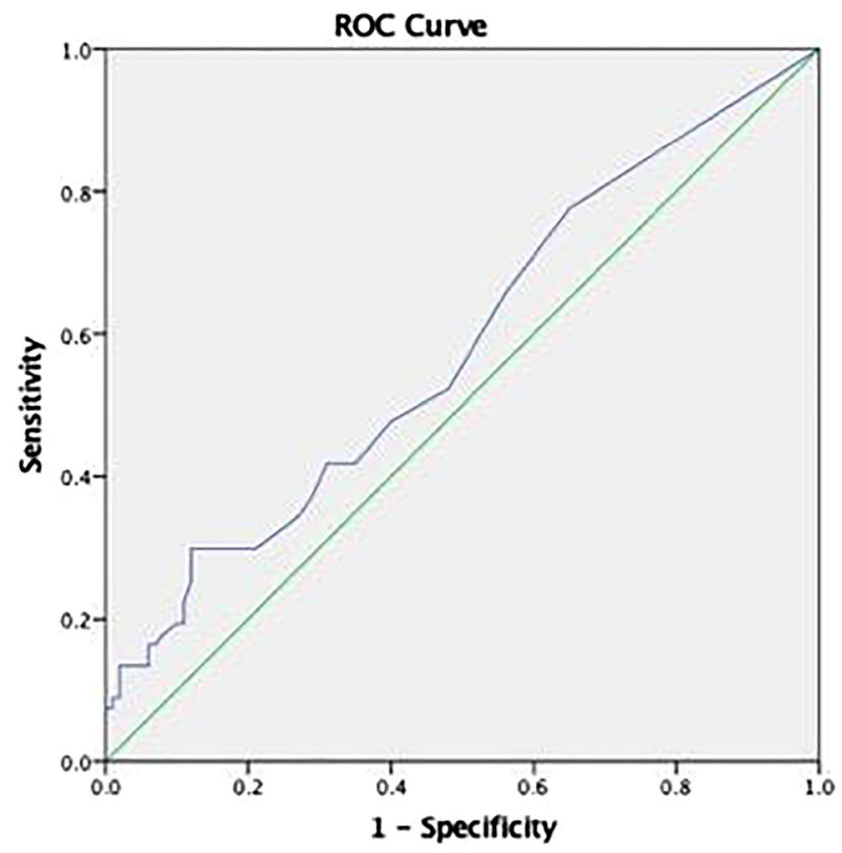

Diagonal segments are produced by ties. 


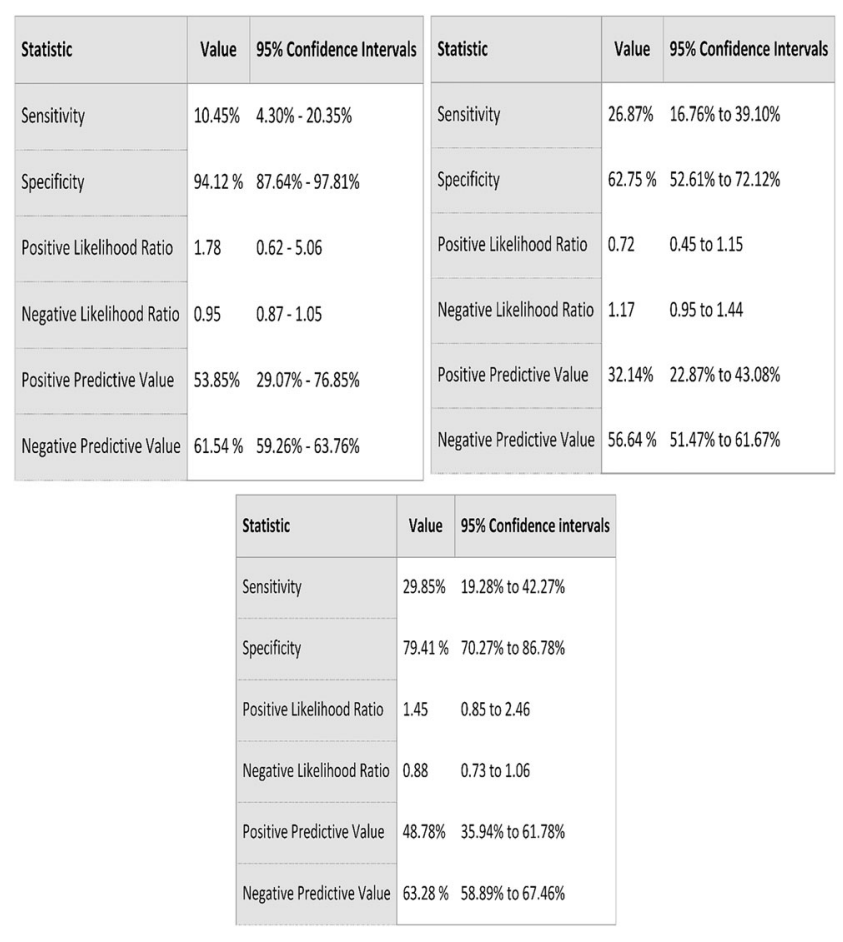

961

\section{Neonatology}

Early Onset Neonatal Infection: Review of Antibiotic Guideline in Practice

Iona Gilmour ${ }^{1}$, Lucy Paterson-Brown ${ }^{1}$, Lubna Siddiqui ${ }^{I}$, Sijo Francis ${ }^{1}$, Anay Kulkarni ${ }^{l}$

${ }^{I}$ Neonatal Unit, St George's University Hospitals NHS Foundation Trust, $U K$

Background: National Institute of Clinical Excellence (NICE) implemented a guideline which is well used in Neonatal departments to prevent infection within 72 hours of life for healthy babies. "It aims to reduce delay in recognising and treating sick babies and prevent unnecessary use of antibiotics."

Objective: To review practice for well, term babies who are started on antibiotics due to risk factors as suggested by NICE.

Methods: Data was collected retrospectively over a six month period from October 2018 to March 2019 at St George's Hospital, a tertiary neonatal service in London, UK. All well asymptomatic babies $\geq 37$ weeks gestation, started on antibiotics due to risk factors were included. Of these we reviewed which:

Met NICE criteria

- Had laboratory evidence of infection, indicated by $\mathrm{C}$ reactive protein (CRP) rise $\geq 10$

- Had clinically significant culture results - blood or CSF

- All babies who were admitted to the neonatal unit were excluded.

Results: A total of 149 babies were included. 104 of these met NICE criteria for screening. Of these, $53(51 \%, 36 \%$ of total) had a CRP rise of $\geq 10$, none had a positive culture. 45 babies did not meet the criteria, of these $24(53 \%, 16 \%$ of total) had a CRP rise and one had a positive culture.

Conclusion: Only half of babies who met the criteria had a CRP rise, none of these babies had a positive culture. Half of the babies who did not meet the criteria, but were still screened had a CRP rise and one baby had a positive culture. This audit recommends a call to review the guideline as despite not meeting criteria babies are still being treated for and are found to have signs of infection. There also remains a large proportion of well babies who receive antibiotics and remain in hospital unnecessarily.

\section{6}

Neonatology

Neonatal Inflammatory Skin and Bowel Disease Type 2: A Case Report

Joana Gonçalves ${ }^{1}$, Mariana Lemos ${ }^{2}$, Cátia Correia ${ }^{1}$, Ana Teresa Maria ${ }^{1}$ ${ }^{T}$ Department of Neonatology, Centro Hospitalar Lisboa Ocidental, Portugal

${ }^{2}$ Department of Pediatric Cardiology, Centro Hospitalar Lisboa Ocidental, Portugal

Background: The homozygous EGFR mutation is associated with neonatal inflammatory skin and bowel disease type 2 . So far only a hand few of cases have been reported.

Case Report: Preterm female infant, born at 28 weeks of gestation via emergency cesarean due to non-reassuring fetal status, weighing 650 grams. Her Apgar score was 4/7/7. She was intubated in the delivery room and transferred to the NICU. On physical examination she had several malformations (sparse scalp hair, craniofacial abnormalities, thin and long limbs, arachnodactyly, absence of subcutaneous fat, arthrogryposis) and a very fragile skin with extensive erythema. While in the NICU she required continuous mechanical ventilation due to bronchopulmonary dysplasia. She presented with recurrent infections, anemia and thrombocytopenia requiring multiple courses of antibiotics and transfusions. Echocardiography revealed a severe congenital heart disease with ventricular disproportion with hypoplastic left ventricle, severe aortic arch hypoplasia, complete atrioventricular septal defect and a patent ductus arteriosus. She developed persistent hypernatremia, hypokalemia, hypophosphatemia and severe hypomagnesemia requiring frequent replacement therapy. Cranial ultrasounds showed tetraventricular hydrocephalus with severe periventricular and subcortical white matter lesions, cerebellum atrophy and a mega cisterna magna. Genetic testing identified the c.1283G-A, p.(Gly428Asp) mutation in homozygosity, in exon 11 of the EGFR gene. At 3 months of post-menstrual age the infant died of progressive cardiac failure, despite optimization of medical therapy.

Conclusion: Homozygous EGFR mutation is associated with neonatal inflammatory skin and bowel disease type 2 which presents with a complex and severe phenotype and overall bad prognosis, with no reports of successful therapy. The inheritance of this disease seems to be autosomal recessive, and thus consanguinity is a high risk factor. With this case we expect to help characterizing this extremely rare syndrome. Neonatologists and pediatricians should consider EGFR mutation in neonates presenting with inflammatory skin disease from birth.

519

Neonatology

Congenital Cataract in the Newborn: Report of an Early Diagnosis Vanessa Gorito $^{l}$, Gorett Silva $^{l}$, Raquel Bragança ${ }^{l}$, Renato Silva ${ }^{2}$, $\overline{\text { Augusto Magalhães }}{ }^{2}$, Jorge Breda ${ }^{2}$

${ }^{1}$ Neonatology and Pediatrics, Hospital São João, Portugal

${ }^{2}$ Pediatric Ophthalmology, Hospital São João, Portugal

Introduction: Congenital cataracts are a malformation of the lens that develop during the embryonic life. Its etiology can be explained by the presence of congenital infections, genetic alterations or other diseases manifested during gestation. The diagnosis should be early, for timely referral to Ophthalmology, being the treatment weighted case by case. Although treatment is decided on a case by case bases, surgical intervention is often required in the first months of life 
Objective: We present a case of congenital cataract with early in utero diagnosis.

Case report: Mother, 30 years old, primiparous, past history of hypothyroidism (positive antibodies), medicated with levothyroxine. Pregnancy was non-complicated. Serological markers were negative. Five fetal ultrasounds were carried out. On the ultrasound performed at $301 / 7$ weeks an opacity of the lens was identified raising the possibility of congenital cataract. This image was not present at last ultrasound (35 4/7 weeks). Delivery occurred at 41 weeks. APGAR 10/9/10. Measures at birth: weight $3680 \mathrm{~g}$ (P85); length $51 \mathrm{~cm}$ (P50-85); head circumference $34 \mathrm{~cm}$ (P50). Physical examination was normal with the exception to the absence of red reflex in the left eye in D1 of life. An Ophthalmological evaluation as carried including echography that revealed left eye microphthalmia $(15.9 \mathrm{~mm})$ and poor and irregular dilatation, thus confirming the pre-natal diagnosis.

At 2 months of age, she maintains follow up visit in Neonatology and Ophthalmology. At the last visit, a total cataract with vascularized membrane was identified. Currently active surveillance is maintained and surgical intervention is delayed.

Discussion: Congenital cataracts, although rare, should be considered. A careful prenatal ultrasound study, complemented by the objective examination, namely with the evaluation of the red reflex, can lead to an early diagnosis. A possible association between maternal hypothyroidism, medicated during pregnancy and the occurrence of congenital cataract should be further clarified.

953

\section{Neonatology}

Folic Acid and Autism: A Systematic Review of the Current State of Knowledge

Bianka Hoxha ${ }^{1}$, Malvina Hoxha ${ }^{1}$, Bruno Zappacosta

${ }^{T}$ Department for Chemical-Toxicological and Pharmacological Evaluation of Drugs, Catholic University Our Lady of Good Counsel, Albania

Background: Autism spectrum disorders (ASDs) prevalence has increased over time. Different studies indicate folic acid (FA) importance in improving childhood behavioral outcomes and underline its role as a modifiable risk factor for ASDs. Modulations dependent reduced folate uptake and auto-antibodies against folate receptor alpha (FRAA) were associated with neurodevelopmental disorders.

Objective: A systematic review was conducted in both animal and human clinical studies to determine the association of FA with ASDs.

Methods: Different keywords in PubMed, Scopus, Medline, Embase databases were used to identify and select the eligible studies without language and date limitation. Two authors independently screened potentially eligible articles and extracted relevant data.

Results: 43 studies met the inclusion criteria, of which 8 were conducted in animals. 14 clinical trials investigated the association between maternal vitamin supplementation and the risk of ASDs in offspring; 10 studies investigated the role of methylenetetrahydrofolate reductase (MTHFR) C677T polymorphism in ASDs risk; 9 studies observed the clinical benefits after folate supplementation in ASDs diagnosed children; 2 studies investigated the frequency of serum FRAA in ASDs children. All of them associated maternal FA use to a significant reduction risk of the ASDs in offspring, particularly when assumed at least from 4 weeks before the start of pregnancy. Serum FRAA were present in $77.5 \%$ (31/40) of children with ASDs compared with 54.8\% (23/42) of neurotypical development children in a 2018 cohort study. MTHFR 677T-allele frequency was found to be significantly higher in ASDs cases compared with nonautistic children. A recent study concluded that maternal FA supplementation $(600 \mu \mathrm{g})$ during first month of pregnancy may reduce ASDs recurrence in families with ASDs children.
Conclusion: Biological, genetic and epidemiological evidence underline the important role of folate supplementation in reducing the ASDs risk and behavioral improvement in offspring.

709

\section{Neonatology}

Neonatal Acute Kidney Injury

Silvana Naunova Timovska ${ }^{1}$, Aspazija Sofijanova ${ }^{1}$, Mica Kimovska ${ }^{l}$, Tamara Voinovskal, Hristina Mangzukovska ${ }^{l}$, Spasija Neskova ${ }^{l}$, Danilo Nonkulovski ${ }^{1}$, Olivera Jordanova ${ }^{2}$, Vladimir Timovski ${ }^{l}$

${ }^{1}$ Neonatal Intensive Care Unit, University Children Hospital, Macedonia

${ }^{2}$ Clinical laboratory, University Children Hospital, Macedonia

Objective: Acute kidney injury is common condition in neonatal intensive care unit and is associated with poor outcome. The incidence of neonatal kidney injury is the highest incidence followed by adults and children, depending of different factors such as gestational age, birth weight, contributing conditions and the facilities of the NICU.

The aim of the study: The aim of the study was to determine the incidence and risk factors of neonatal kidney injury.

Methods: This was a clinical, prospective study that was performed in a referentt NICU at the University Children's Hospital in Skopje. All neonates admitted from the period of 3 years with documented acute kidney injury were included. Medical data records of admitted neonates with AKI were analyzed. The material was statistically processed using methods of descriptive statistics.

Results: During the study period 770 neonates were admitted to NICU and $6.5 \%$ develop acute kidney injury. The male to female ratio was 2.1:1. Most of the neonates involved in the study were born at term (62\%). Oliguric AKI was found in $56 \%$ and nonoliguric in $44 \%$. The prevalence of prerenal, renal and postrenal AKI were $78.5 \%, 19.5 \%$ and $2.0 \%$. Perinatal asphyxia was the most common predisposing factor for AKI and was evaluated in $38 \%$ of cases with predominance of term infants and male. The mortality rate was $32 \%$ and was significantly higher in the group of patients with congenital heard diseases.

Conclusion: Acute kidney injury is a life threatening condition with still high mortality rate. Early recognition of risk factors and rapid effective treatment of contributing conditions will reduce AKI in neonatal period

Key words: acute kidney injury, neonates, risk factors

\section{3}

\section{Neonatology}

Quality of Life of Children with Anorectal and other Associated Malformations

Melanie Kapapa $^{l}$, Nastassja Becker ${ }^{I}$, Doris Henne-Bruns ${ }^{2}$, Alexandre Serra

${ }^{I}$ Department of General Surgery, Division Pediatric Surgery Ulm University, Germany Department of General Surgery, Ulm University, Germany

Background: Incidence of anorectal malformation was 1 of 5000 livebirth new-born, $64 \%$ suffered of associated malformations. Surgical reconstruction was performed in early childhood and complications like incontinence and constipation affected long term outcome. Aim was to evaluate the quality of life and complications after surgical reconstruction. We hypothesized that they had disadvantages in daily life and worthier 
quality of life in spite of adequate pediatric surgical treatment with correct anatomical reconstruction.

Methods: Between 2004 and 2014 forty-four children with anorectal malformation were treated at university hospital. $65,8 \%$ had associated malformations. Used surgical technique was posterior sagittal anorectal plasty. We analyzed pre- and postsurgical development, recorded quality of life and clinical outcome with questionnaires.

Results: Parents specified common quality of life averages with $83,2 \%$, their children with $90,1 \%$ of $100 \%$ via questionnaires. Parents of children with associated cardio vascular disease estimated with $81,7 \%$, with associated urological diseases $82,7 \%$ and with associated genital diseases $83,2 \%$. In case of vertebral or spinal associated malformation parents evaluated an average of quality of life with $84,6 \%$. In children with caudal regression syndrome parents specified $82,8 \%$, the children themselves with 93,1\%. Physical well-being in children with associated malformations was estimated with $83,7 \%$ by parents, in children with isolated anorectal malformation we found $77,7 \%$. Psychological well- being war scored with $90,2 \%$ in children with associated malformations, and with $84,4 \%$ in children with isolated anorectal malformation. The results for self-esteem was higher without associated malformations $(84,7 \%)$, than in children with associated malformations $(74,6 \%)$.

Conclusion: Postsurgical outcome of anorectal malformations was good independently of associated malformations. None of these patients had a significant limitation for quality of life. The sub score of self-esteem was lower in those patients and because of these findings psychological support should be added to pediatric standard examinations. Sensitization of parents and surgeons is necessary.

\section{8}

\section{Neonatology}

Outcome of NEC and FIP: Surgical Treatment or Wait and See? Melanie Kapapa $^{I}$, Janina Hahne ${ }^{I}$, Doris Henne-Bruns ${ }^{2}$, Alexandre Serra

${ }^{1}$ Department of General Surgery, Division Pediatric Surgery Ulm University, Germany

${ }^{2}$ Department of General Surgery, Ulm University, Germany

Background: Necrotizing enterocolitis (NEC) and focal intestinal perforation (FIP) affect almost exclusively premature new-borns. Incidence of NEC is $0.3 \%$ and rises up to $15 \%$ in birth weight

Objective: Aim of this study is to evaluate surgery rate, complications, clinical outcome and hospital stay after NEC and FIP.

Methods: Seventy-six children treated for NEC or FIP during study period (2003-2013) were included in 3 groups: Group 1: NEC without perforation, group 2: FIP, and group 3: NEC and perforation. All data recorded during hospital stay till discharge including surgical procedures were collected.

Results: In group 1, 44\% underwent surgery, in group 2 and 3, 100\% were surgically treated. Complications like hematocrit decrease occur in $36.1 \%$, and in $57.4 \%$ of these children blood transfusions were necessary: group 3 was affected in $62.2 \%$, followed by group 2 and 1 in $50 \%$ each. Postsurgical bleeding or perforation occurred in group 1 and 3. Postsurgical short bowel syndrome $(9,8 \%)$ and malnutrition $(6,6 \%)$ were present in group 1 and 3. Cholestasis was present in $41 \%$ of all children; in $45.9 \%$ of group 3 , in $41.7 \%$ of group 2 and in $25 \%$ of group 1 . Complications like stoma prolapse was present in group $2(58,3 \%)$ and in group $3(33.3 \%)$. The hospital duration stay differs, in group 1 the average stay was 85,4 days, in group $2,111,1$ days and in group 3 , 114,1 days.

Conclusion: NEC with perforation was treated surgical more frequently, experienced significantly higher levels of complications and had extended hospital stay, followed by FIP and at least by
NEC without perforation. Post-operative complications and length of stay correlate with the children's outcome. To optimize clinical outcome recognition of NEK is necessary before occurrence of gut perforation.

471

Neonatology

Intestinal Fatty Acid-Binding Protein (I-FABP) as a Diagnostic Early Marker for Necrotizing Enterocolitis in Prematuraly Born Infants Anastasiia Konoplitska ${ }^{1}$, Olga Yablon ${ }^{1}$, Nataliia Chornopyshchuk ${ }^{1}$, Olena Mazur ${ }^{\prime}$, Yaroslava Kulyk ${ }^{I}$

${ }^{1}$ Paediatric Department \#1, National Pirogov Memorial Medical University, Ukraine

Background: I-FABP is mainly located in the enterocytes of the small intestine and released into the blood after NEC-associated inflammation. Objective: To establish diagnostic level of I-FABP for the study of necrotizing enterocolitis of premature infants.

Methods: The study group - 95 premature infants with necrotizing enterocolitis (NEC) who were treated in the NICU. The first group consisted of 27 preterm infants with NEC I stage, the second group - 35 preterm infants with NEC II stage, the third group - 8 preterm infants with NEC of the 3rd stage. The control group - 25 preterm infants without NEC. IFABP measurements in the blood serum were obtained using the Human I-FABP (Hycult Biotech, Netherlands) ELISA Test System. The statistical processing was carried out using the software package Statistica 6.1.

Results: In the first group, the I-FABP content was 831,0 [582-974] pg/ $\mathrm{ml}$, in second group - 1037,0 [862-1846] pg/ml, in third group - 1856,5 [1627-2756] pg/ml, which significantly exceeded the value of I-FABP in the control group - 269,0 [226-346] pg/ml (p0.01). The serum I-FABP value of $\geq 727.50 \mathrm{pg} / \mathrm{ml}$ allows to identify NEC in premature babies with a sensitivity of $73.6 \%$ and a specificity of $72.2 \%$, obtained by ROC analysis, the area under the ROC curve (AUC) is 0.883 [95\% CI $0.806-$ $0.961]$, indicating a high diagnostic value of the model.

Conclusions: The content of I-FABP in the blood serum of children in groups first, second and third was significantly different from the I-FABP content in the control group at 3, 4 and 7 times ( $p$ 0.01). Thus, the serum IFABP value of $\geq 727.50 \mathrm{pg} / \mathrm{ml}$ allows to identify NEC in premature babies at early stages of the disease, even before the clinical manifestation of NEC.

\section{5}

\section{Neonatology}

Improving Performance: A Multidisciplinary Approach to NICU Breast Milk Utilization Pre and Post Discharge

Ravi Krishnan $^{1}$, Irfan Ahmad ${ }^{2}$, Purificacion Tumbaga ${ }^{1}$, Jessica Nordeck', Rohun Krishnan ${ }^{l}$, Shawna Leeper ${ }^{l}$, Stacy Mott ${ }^{l}$, Rosa Ortega $^{l}$, Mendy Hickey ${ }^{l}$, Wendy Johnson ${ }^{1}$, Lisa Mieir ${ }^{I}$, Nora Straw $^{I}$, Victoria Anene ${ }^{I}$, Dushant Oza $a^{1}$, Christine Bixby ${ }^{2}$

${ }^{1}$ NICU, St Mary Medical Center, St Joseph Health, Providence, USA

${ }^{2}$ NICU, Children's Hospital of Orange County, St Joseph Health, Providence, USA

Setting: At St Mary Medical Center, which is a 14 bed Community level 3 NICU with 300 admissions annually.

Smart Aim: Increase breastmilk utilization for all NICU admissions and promoting post discharge breastmilk utilization from our 2016 baseline of $52.84 \%$ and achieving and maintaining the goal of $62.84 \%$ by December 2018 which would be a $10 \%$ absolute gain.

Drivers of Change: Alignment with NICU nursing leadership, education of RNs on supporting lactation in the NICU, lactation team involvement and physician engagement. 
Methods: Multidisciplinary team was established and processes developed, PDSA cycles initiated and audited. Processes developed included breast pump initiation within 6-12 hours of NICU admission with lactation consult including hand expression education, referral to Mommy and Me Breast Feeding Support Clinic at discharge and prescription for multivitamins at discharge.

Measurements: Lactation nurses completed daily audit for breast pump. Audited mandatory lactation consults, physician prescriptions, referral to clinic and any use of breastmilk at discharge for all infants discharged from the NICU.

Results: Breast pump initiation improved from a baseline of $33 \%$ to $89 \%$ with PDSA cycles. Lactation consult including hand expression reached $100 \%$. Physician prescription for multivitamins went up from $52 \%$ to $100 \%$ Physician referral to Mommy and Me clinic went from $93 \%$ to $100 \%$. Any Breast milk within 24 hours of discharge improved from 52.84 to $62.84 \%$.

Discussion: Improvements were driven through nursing education during the first PDSA cycle and through lactation audits in subsequent PDSA cycles. Lactation support for hand expression, education, and facilitating physician`s referrals to breastfeeding support clinic led to significant improvements in use of breastmilk at discharge. Regular audits, staff education and teamwork were the key drivers to success.

Next Steps: Our future goals include monitoring of colostrum usage, lactation follow-up survey, and implementing donor human breastmilk.

\section{7}

\section{Neonatology}

Effects of Sepsis and Necrotizing Enterocolitis on Neurodevelopment in Preterm Infants

Eun Sun Lee ${ }^{I}$, Ee Kyung Kim ${ }^{l}$, Seung Han Shin ${ }^{1}$, Han-Suk Kim ${ }^{l}$, Yoo Jinie Kim ${ }^{I}$, Jung Ha Yun ${ }^{1}$

${ }^{1}$ Pediatrics, Neonatology, Seoul National University Children's Hospital, South Korea

Background: In premature infants, white matter injury (WMI) of brain is major cause of neurodevelopmental impairment. Several clinical and animal studies have suggested that WMI could be induced by systemic inflammation in this population.

Objective: To investigate whether preterm infants who experienced systemic inflammation such as sepsis or necrotizing enterocolitis (NEC) would have abnormal findings in brain MRI at term equivalent age and impaired neurodevelopment at 18 months of corrected age (CA).

Methods: This was a prospective cohort study including infants born before 30 weeks of gestation or less than $1,250 \mathrm{~g}$ at birth. They were initially assigned as the $\mathrm{N}$ group and further assigned according to the presence of sepsis and NEC; G0 (clinical systemic inflammation without proven sepsis or NEC, $n=32$ ), G1 (culture proven sepsis or NEC, $n=12$ ) and $\mathrm{N}$ (no systemic inflammation, $\mathrm{n}=51$ ). Findings from laboratory results, cerebral oximeter and amplitude-integrated EEG (aEEG) at each event were collected. Brain MRI at term equivalent age (TEA) and aEEG at 35 weeks of postmenstrual age (PMA) were evaluated and the BayleyIII was performed at 18 months of CA.

Results: Infants in the G0 and G1 group had more WMI in the brain MRI at TEA and lower maturation scores in the aEEG at PMA 35 weeks than infants in the $\mathrm{N}$ group. Developmental scores of language, motor and adaptive behavior domains of the Bayley-III were significantly lower in the G0 and G1 groups. Among the laboratory findings at the episode of systemic inflammation, C-reactive protein and proportion of immature cells were negatively correlated with developmental scores in the Bayley-III and hemoglobin level was positively correlated with developmental scores.
Conclusion: Systemic inflammatory conditions were associated with WMI at TEA and adverse neurodevelopmental outcomes at 18 months of CA. Several markers of systemic inflammations at the episode were correlated with developmental scores.

Table 1. Perinatal Characteristics and Neonatal Complications

\begin{tabular}{|c|c|c|c|c|c|}
\hline & $\begin{array}{c}\text { Total } \\
(\mathrm{n}=95)\end{array}$ & $\begin{array}{c}\text { Normal } \\
(\mathbf{n}=51)\end{array}$ & $\underset{(\mathrm{n}=32)}{\mathrm{G} 0}$ & $\underset{(n=12)}{G 1}$ & P-value \\
\hline Gestational age (week) & $27^{+6}\left(23^{+0}, 34^{+2}\right)$ & $29^{+2}\left(24^{+1}, 34^{+2}\right)$ & $27^{+1}\left(24^{+3}, 33^{+1}\right)$ & $25^{+5}\left(23^{+0}, 33^{+0}\right)$ & 0.000 \\
\hline Birth weight (g) & $\begin{array}{c}940(420, \\
1400)\end{array}$ & $\begin{array}{c}1090(540 \\
1340)\end{array}$ & $\begin{array}{c}790(420, \\
1400)\end{array}$ & $\begin{array}{c}685(490 \\
1400)\end{array}$ & 0.000 \\
\hline Gender (male) (\%) & 49.5 & 41.2 & 59.4 & 58.3 & 0.219 \\
\hline Multiple bitth (\%) & 43.2 & 41.2 & 50.0 & 33.3 & 0.774 \\
\hline \multicolumn{6}{|l|}{ Apgar score } \\
\hline At $1 \mathrm{~min}$ & $4(1,8)$ & $5(1,8)$ & $4(1,8)$ & $3(1,8)$ & 0.194 \\
\hline At $5 \mathrm{~min}$ & $7(1,9)$ & $7(1,9)$ & $7(3,9)$ & $6(3,9)$ & 0.065 \\
\hline Cesarean section (\%) & 66.0 & 68.6 & 59.4 & 72.7 & 0.583 \\
\hline PROM (\%) & 17.9 & 21.6 & 9.4 & 25.0 & 0.373 \\
\hline Chorioamnionitis (\%) & 42.1 & 39.2 & 43.8 & 50.0 & 0.862 \\
\hline Preeclampsia (\%) & 5.3 & 3.9 & 9.4 & 0.0 & 0.658 \\
\hline Meconium stain (\%) & 1.1 & 2.0 & 0.0 & 0.0 & 0.862 \\
\hline $\begin{array}{l}\text { Received antenatal steroid } \\
(\%)\end{array}$ & 89.0 & 87.2 & 90.6 & 91.7 & 0.610 \\
\hline SGA (\%) & 31.9 & 30.0 & 34.4 & 33.3 & 0.912 \\
\hline RDS, surfactant (\%) & 66.0 & 28.0 & 75.0 & 83.3 & 0.083 \\
\hline Air leak (\%) & 2.1 & 0.0 & 6.5 & 0.0 & 0.125 \\
\hline $\mathrm{BPD}, \geq$ moderate $(\%)$ & 43.6 & 23.5 & 61.3 & 83.3 & 0.000 \\
\hline PDA, operation (\%) & 16.8 & 5.9 & 21.9 & 50.0 & 0.001 \\
\hline $\mathrm{NEC}, \geq$ stage I (\%) & 5.4 & 0.0 & 9.7 & 18.2 & 0.023 \\
\hline ROP, $\geq$ stage $Ш ~(\%)$ & 18.3 & 4.0 & 29 & 50 & 0.000 \\
\hline PVL (\%) & 12.9 & 0.0 & 12.9 & 0.0 & 0.014 \\
\hline
\end{tabular}

Table 2. Laboratory finding, aEEG and Brain MRI

\begin{tabular}{|c|c|c|c|c|c|}
\hline & $\begin{array}{c}\text { Total } \\
(\mathrm{n}=95)\end{array}$ & $\begin{array}{c}\text { Normal } \\
(\mathrm{n}=\mathbf{5 1})\end{array}$ & $\underset{(\mathrm{n}=32)}{\mathbf{G 0}}$ & $\begin{array}{c}\mathrm{G} 1 \\
(\mathrm{n}=12)\end{array}$ & P-value \\
\hline \multicolumn{6}{|l|}{ CBC } \\
\hline \multicolumn{6}{|l|}{$\mathrm{WBC}\left(\times 10^{3} / \mu \mathrm{R}\right)$} \\
\hline DO & $\begin{array}{c}11.08(1.20, \\
38.90)\end{array}$ & $\begin{array}{c}11.05(3.0, \\
28.0)\end{array}$ & $\begin{array}{c}9.71(1.20 \\
38.90)\end{array}$ & $13.33(4.0,34.0)$ & 0.314 \\
\hline D2 & $\begin{array}{c}9.95(4.70 \\
55.06)\end{array}$ & $\mathrm{NA}$ & $\begin{array}{c}9.46(4.70 \\
24.25)\end{array}$ & $14.78(7.0,55.0)$ & 0.111 \\
\hline \multicolumn{6}{|l|}{ L/T ratio } \\
\hline Do & $0.00(0.00,0.89)$ & $0.00(0,0)$ & $0.0(0.0,0.89)$ & $0.01(0,0.22)$ & 0.094 \\
\hline D2 & $0.20(0.00,1.00)$ & NA & $0.01(0.0,1.0)$ & $0.09(0,0.34)$ & 0.087 \\
\hline \multicolumn{6}{|l|}{ Platelet $\left.\times 10^{3} / \mu \ell\right)$} \\
\hline Do & $190(30,811)$ & $219(53,499)$ & $175(30,811)$ & $77(43,413)$ & 0.011 \\
\hline D2 & $173(11,727)$ & NA & $182(39.727)$ & $89(11,323)$ & 0.547 \\
\hline \multicolumn{6}{|l|}{ Hemoglobin } \\
\hline Do & $11.15(7.2,19.4)$ & $11.7(9,17)$ & $11.1(7.2,19.4)$ & $11.45(7.4,15.5)$ & 0.800 \\
\hline D2 & $11.2(8.1,17.0)$ & NA & $10.4(8.1,17.0)$ & $11.20(9.9,14.5)$ & 0.709 \\
\hline \multicolumn{6}{|l|}{ Hematocrit } \\
\hline Do & $\begin{array}{c}33.25(21.1 \\
56.4)\end{array}$ & $34.6(26,49)$ & $32.5(23.1,56.4)$ & $33.4(21.1,46.9)$ & 0.889 \\
\hline D2 & $32.9(22.3,48.1)$ & NA & $30.9(22.3,48.1)$ & $32.9(27.8,40.5)$ & 0.856 \\
\hline \multicolumn{6}{|l|}{ ANC } \\
\hline Do & $\begin{array}{c}4328(210 \\
30303)\end{array}$ & $\begin{array}{c}2851(880, \\
11819)\end{array}$ & $\begin{array}{c}3889(210 \\
30303)\end{array}$ & $9786(794,29308)$ & 0.070 \\
\hline D2 & $\begin{array}{c}4015(682 \\
30283)\end{array}$ & NA & $3781(682,1311)$ & $\begin{array}{c}7070(1426 \\
30283)\end{array}$ & 0.175 \\
\hline \multicolumn{6}{|l|}{ CRP } \\
\hline Do & $\begin{array}{c}1.11(0.01, \\
18.21)\end{array}$ & $0.05(0,1)$ & $\begin{array}{c}1.36(0.03 \\
18.21)\end{array}$ & $2.72(0.07,9.10)$ & 0.000 \\
\hline D2 & $\begin{array}{c}0.68(0.04 \\
19.16)\end{array}$ & NA & $0.68(0.04,8.17)$ & $0.79(0.06,19.16)$ & 0.368 \\
\hline \multicolumn{6}{|l|}{ aEEG score } \\
\hline Do & $5(1,10)$ & NA & $5.5(1,10)$ & $4(2,8)$ & 0.335 \\
\hline D2 & $5.5(1,11)$ & NA & $5.5(1,10)$ & $4(1,7)$ & 0.192 \\
\hline D6 & $6(2,11)$ & NA & $\sigma(4,11)$ & $5(2,9)$ & 0.201 \\
\hline PMA 35wks & $9(4,13)$ & $9(4,13)$ & $8(4,12)$ & $8(4,11)$ & 0.006 \\
\hline \multicolumn{6}{|l|}{ MRI } \\
\hline Normal :WMI $(\%)$ & $84.2: 15.8$ & $92.2: 7.8$ & $71.9: 28.1$ & $83.3: 16.7$ & 0.048 \\
\hline Normal:focal:diffuse(\%) & $84.2: 8.4: 7.4$ & $92.2: 3.9: 3.9$ & $71.9: 12.5: 15.6$ & $83.3: 16.7: 0$ & 0.076 \\
\hline
\end{tabular}


Table 3. Neurodevelopmental Outcomes

\begin{tabular}{lccccc}
\hline & $\begin{array}{c}\text { Total } \\
(\mathrm{n}=\mathbf{9 5})\end{array}$ & $\begin{array}{c}\text { Normal } \\
(\mathrm{n}=51)\end{array}$ & $\begin{array}{c}\mathbf{G} \text { 0 } \\
(\mathbf{n}=\mathbf{3 2})\end{array}$ & $\begin{array}{c}\text { G1 } \\
(\mathbf{n}=\mathbf{1 2})\end{array}$ & P-value \\
\hline Head circumference & $30.0(24.5,33.0)$ & $30.5(26.5,33.0)$ & $29.5(25.5,32.5)$ & $28.5(24.5,32.5)$ & $\mathbf{0 . 0 0 7}$
\end{tabular}

\begin{tabular}{|c|c|c|c|c|c|}
\hline \multicolumn{6}{|l|}{$\begin{array}{l}\text { Bayley score } \\
\text { (CA 18mo) }\end{array}$} \\
\hline \multicolumn{6}{|l|}{ Cognition } \\
\hline Score & $95(55,125)$ & $95(85,125)$ & $90(55,120)$ & $92.5(60,115)$ & 0.061 \\
\hline $\begin{array}{l}\text { Percent below } \\
\text { score } 85(\%)\end{array}$ & 10.7 & 0.0 & 25.0 & 16.7 & 0.003 \\
\hline \multicolumn{6}{|l|}{ Language } \\
\hline Score & $94(50,121)$ & $97(74,121)$ & $89(56,121)$ & $84.5(50,106)$ & 0.031 \\
\hline $\begin{array}{l}\text { Percent below } \\
\text { score } 85(\%)\end{array}$ & 25.0 & 15.9 & 32.1 & 41.7 & 0.107 \\
\hline \multicolumn{6}{|l|}{ Motor } \\
\hline Score & $94(46,112)$ & $98.5(82,112)$ & $91.0(46,110)$ & $89.5(64,110)$ & 0.009 \\
\hline $\begin{array}{l}\text { Percent below } \\
\text { score } 85(\%)\end{array}$ & 15.5 & 6.8 & 25.0 & 25.0 & 0.071 \\
\hline \multicolumn{6}{|l|}{ Social, emotional } \\
\hline Score & $92.5(55,115)$ & $95(80,110)$ & $90.0(55,115)$ & $90(65,110)$ & 0.174 \\
\hline $\begin{array}{l}\text { Percent below } \\
\text { score } 85(\%)\end{array}$ & 12.2 & 4.7 & 25.9 & 8.3 & 0.027 \\
\hline \multicolumn{6}{|l|}{ Adaptive Behavior } \\
\hline Score & $87(48,112)$ & $90(61,112)$ & $81.0(48,111)$ & $84.5(50,97)$ & 0.012 \\
\hline $\begin{array}{l}\text { Percent below } \\
\text { score } 85(\%)\end{array}$ & 35.4 & 23.3 & 51.9 & 41.7 & 0.046 \\
\hline
\end{tabular}

Table 4. Correlation Between Bayley Score, Infection Marker (D0) and aEEG Score

\begin{tabular}{|c|c|c|c|c|c|c|c|c|c|c|c|}
\hline $\begin{array}{c}\text { Total } \\
(\mathrm{n}=95)\end{array}$ & WBC & $\underset{\text { ratio }}{\mathrm{I} / \mathrm{T}}$ & $\mathrm{Hb}$ & Het & platelet & ANC & CRP & $\begin{array}{l}\text { aEEG } \\
(\mathrm{D} 0)\end{array}$ & $\begin{array}{l}\text { aEEG } \\
\text { (D2) }\end{array}$ & $\begin{array}{l}\text { aEEG } \\
\text { (D6) }\end{array}$ & $\begin{array}{l}\text { aEEG } \\
\text { (PMA } \\
\text { 35wks) }\end{array}$ \\
\hline Cognitive & $\begin{array}{c}0.201 \\
(0.149)\end{array}$ & $\begin{array}{l}-0.296^{*} \\
(0.031)\end{array}$ & $\begin{array}{l}0.276^{\circ} \\
(0.0+5)\end{array}$ & $\begin{array}{c}0.243 \\
(0.079)\end{array}$ & $\begin{array}{c}0.046 \\
(0.743)\end{array}$ & $\begin{array}{c}0.219 \\
(0.115)\end{array}$ & $\begin{array}{l}-0.285^{*} \\
(0.038)\end{array}$ & $\begin{array}{c}-0.193 \\
(0.428)\end{array}$ & $\begin{array}{c}-0.159 \\
(0.517)\end{array}$ & $\begin{array}{c}-0.096 \\
(0.688)\end{array}$ & $\begin{array}{c}0.183 \\
(0.11 S)\end{array}$ \\
\hline Language & $\begin{array}{c}0.157 \\
(0.262)\end{array}$ & $\begin{array}{l}-0.177 \\
(0.204)\end{array}$ & $\begin{array}{l}0.290^{\circ} \\
(0.035)\end{array}$ & $\begin{array}{c}0.251 \\
(0.070)\end{array}$ & $\begin{array}{c}0.104 \\
(0.460)\end{array}$ & $\begin{array}{c}0.129 \\
(0.359)\end{array}$ & $\begin{array}{c}-0.257 \\
(0.063)\end{array}$ & $\begin{array}{l}-0.326 \\
(0.173)\end{array}$ & $\begin{array}{c}-0.039 \\
(0.876)\end{array}$ & $\begin{array}{c}-0.203 \\
(0.391)\end{array}$ & $\begin{array}{c}0.158 \\
(0.179)\end{array}$ \\
\hline Motor & $\begin{array}{c}0.192 \\
(0.168)\end{array}$ & $\begin{array}{l}-0.457^{+} \\
(0.001)\end{array}$ & $\begin{array}{c}0.258 \\
(0.062)\end{array}$ & $\begin{array}{c}0.235 \\
(0.091)\end{array}$ & $\begin{array}{c}0.129 \\
(0.357)\end{array}$ & $\begin{array}{c}0.189 \\
(0.175)\end{array}$ & $\begin{array}{c}-0.392^{*} \\
(0.00+t)\end{array}$ & $\begin{array}{l}-0.051 \\
(0.836)\end{array}$ & $\begin{array}{c}-0.064 \\
(0.796)\end{array}$ & $\begin{array}{l}-0.057 \\
(0.811)\end{array}$ & $\begin{array}{c}0.164 \\
(0.164)\end{array}$ \\
\hline Social & $\begin{array}{c}0.189 \\
(0.181)\end{array}$ & $\begin{array}{l}-0.314^{*} \\
(0.023)\end{array}$ & $\begin{array}{l}0.298^{4} \\
(0.032)\end{array}$ & $\begin{array}{c}0.269 \\
(0.054)\end{array}$ & $\begin{array}{c}-0.012 \\
(0.534)\end{array}$ & $\begin{array}{c}0.176 \\
(0.212)\end{array}$ & $\begin{array}{c}-0.253 \\
(0.069)\end{array}$ & $\begin{array}{l}-0.208 \\
(0.409)\end{array}$ & $\begin{array}{c}-0.142 \\
(0.573)\end{array}$ & $\begin{array}{l}-0.136 \\
(0.579)\end{array}$ & $\begin{array}{c}0.113 \\
(0.343)\end{array}$ \\
\hline Adaptive & $\begin{array}{l}0.277^{\circ} \\
(0.0+7)\end{array}$ & $\begin{array}{c}-0.222 \\
(0.114)\end{array}$ & $\begin{array}{c}0.263 \\
(0.059)\end{array}$ & $\begin{array}{c}0.238 \\
(0.089)\end{array}$ & $\begin{array}{l}0.120 \\
(0.397)\end{array}$ & $\begin{array}{c}0.206 \\
(0.144)\end{array}$ & $\begin{array}{l}-0.397^{\circ} \\
(0.00 t)\end{array}$ & $\begin{array}{l}-0.312 \\
(0.207)\end{array}$ & $\begin{array}{c}-0.052 \\
(0.836)\end{array}$ & $\begin{array}{c}-0.185 \\
(0.448)\end{array}$ & $\begin{array}{l}0.241^{\circ} \\
(0.0+1)\end{array}$ \\
\hline
\end{tabular}
The values are Pearson correlation coefficient with p-value.

\begin{tabular}{|c|c|c|c|c|c|c|c|c|c|c|c|}
\hline$\underset{(\mathrm{n}=61)}{\mathrm{G}}$ & WBC & $\begin{array}{c}\mathrm{L} / \mathrm{T} \\
\text { ratio }\end{array}$ & $\mathrm{Hb}$ & Hct & platelet & ANC & CRP & $\begin{array}{l}\text { aEEG } \\
\text { (D0) }\end{array}$ & $\begin{array}{l}\text { aEEG } \\
\text { (D2) }\end{array}$ & $\begin{array}{l}\text { aEEG } \\
\text { (D6) }\end{array}$ & $\begin{array}{l}\text { aEEG } \\
\text { (PMA } \\
35 \mathrm{wks})\end{array}$ \\
\hline Cognitive & $\begin{array}{c}0.270 \\
(0.096)\end{array}$ & $\begin{array}{l}-0.313 \\
(0.053)\end{array}$ & $\begin{array}{c}0.287 \\
(0.077)\end{array}$ & $\begin{array}{c}0.253 \\
(0.120)\end{array}$ & $\begin{array}{l}0.045 \\
(0.783)\end{array}$ & $\begin{array}{c}0.298 \\
(0.065)\end{array}$ & $\begin{array}{l}-0.278 \\
(0.086)\end{array}$ & $\begin{array}{l}-0.193 \\
(0.428)\end{array}$ & $\begin{array}{l}-0.183 \\
(0.467)\end{array}$ & $\begin{array}{l}-0.096 \\
(0.688)\end{array}$ & $\begin{array}{c}0.157 \\
(0.367)\end{array}$ \\
\hline Language & $\begin{array}{c}0.166 \\
(0.312)\end{array}$ & $\begin{array}{l}-0.167 \\
(0.308)\end{array}$ & $\begin{array}{c}0.305 \\
(0.059)\end{array}$ & $\begin{array}{c}0.278 \\
(0.086)\end{array}$ & $\begin{array}{l}0.105 \\
(0.525)\end{array}$ & $\begin{array}{c}0.168 \\
(0.307)\end{array}$ & $\begin{array}{c}0.238 \\
(0.145)\end{array}$ & $\begin{array}{l}0.326 \\
0.173\end{array}$ & $\begin{array}{l}-0.123 \\
(0.628)\end{array}$ & $\begin{array}{l}-0.203 \\
(0.391)\end{array}$ & $\begin{array}{l}0.127 \\
(0.467)\end{array}$ \\
\hline Motor & $\begin{array}{c}0.217 \\
(0.185)\end{array}$ & $\begin{array}{l}-0.462^{*} \\
(0.003)\end{array}$ & $\begin{array}{l}0.335^{*} \\
(0.037)\end{array}$ & $\begin{array}{c}0.307 \\
(0.058)\end{array}$ & $\begin{array}{c}0.090 \\
(0.588)\end{array}$ & $\begin{array}{c}0.327 \\
(0.146)\end{array}$ & $\begin{array}{l}-0.372^{*} \\
(0.020)\end{array}$ & $\begin{array}{c}-0.051 \\
(0.836)\end{array}$ & $\begin{array}{c}-0.071 \\
(0.781)\end{array}$ & $\begin{array}{l}-0.057 \\
(0.811)\end{array}$ & $\begin{array}{c}0.142 \\
(0.417)\end{array}$ \\
\hline Social & $\begin{array}{c}0.217 \\
(0.191)\end{array}$ & $\begin{array}{l}-0.325^{*} \\
(0.0+6)\end{array}$ & $\begin{array}{c}0.239 \\
(0.149)\end{array}$ & $\begin{array}{c}0.214 \\
(0.197)\end{array}$ & $\begin{array}{l}0.007 \\
(0.968)\end{array}$ & $\begin{array}{c}0.226 \\
(0.173)\end{array}$ & $\begin{array}{l}-0.247 \\
(0.135)\end{array}$ & $\begin{array}{c}-0.208 \\
(0.409)\end{array}$ & $\begin{array}{l}-0.199 \\
(0.443)\end{array}$ & $\begin{array}{l}-0.136 \\
(0.579)\end{array}$ & $\begin{array}{c}0.096 \\
(0.589)\end{array}$ \\
\hline Adaptive & $\begin{array}{l}0.332^{+} \\
(0.0+2)\end{array}$ & $\begin{array}{c}-0.203 \\
(0.222)\end{array}$ & $\begin{array}{c}0.218 \\
(0.189)\end{array}$ & $\begin{array}{c}0.205 \\
(0.217)\end{array}$ & $\begin{array}{c}0.097 \\
(0.563)\end{array}$ & $\begin{array}{c}0.284 \\
(0.084)\end{array}$ & $\begin{array}{l}-0.366^{4} \\
(0.023)\end{array}$ & $\begin{array}{l}-0.312 \\
0.207\end{array}$ & $\begin{array}{l}-0.128 \\
(0.625)\end{array}$ & $\begin{array}{l}-0.185 \\
(0.448)\end{array}$ & $\begin{array}{c}0.327 \\
(0.059)\end{array}$ \\
\hline
\end{tabular}

975

Neonatology

A Case of Saccharomyces cerevisiae Fungemia in a Premature Infant treated with Probiotics

Hye Mi Lee ${ }^{l}$, Pil sang Jang ${ }^{l}$

${ }^{T}$ Department of Pediatrics, The Catholic University of Korea, South Korea

Background: Probiotics are live microorganisms that confer a health benefit on the host when administered in adequate amounts. Probiotics affect the intestinal ecosystem by impacting mucosal immune mechanisms. Enteral probiotics are commonly used in premature neonates with antibiotic associated diarrhea, necrotizing enterocolitis. Saccharomyces boulardii, a subtype of $\mathrm{S}$. cerevisiae, is widely used as a biotherapeutic agent. However, fungemia with Saccharomyces cerevisiae can occur in weak and immunocompromised patients. We present the case of a premature infant in neonatal intensive care unit who was treated with probiotics and developed S. cerevisiae fungemia.

Case presentation: A Korean female infant with a birth weight of $1,560 \mathrm{~g}$ was born at $29+3$ weeks of gestation via cesarean section delivery. The patient had feeding intolerance and abdominal distension, requiring parenteral nutrition by a peripherally inserted central catheter. At the age of 37 days, we started on the infant twice-daily treatment with probiotics (Saccharomyces boulardii). After 14 days of receiving probiotics, the patient developed sepsis, and the Saccharomyces cerevisiae was isolated in peripheral blood cultures. We removed the contaminated central venous catheter and used a Fluconazole $10 \mathrm{mg} / \mathrm{kg} /$ day. After antifungal treatment (fluconazole), the blood cultures were negative and the patient recovered clinically.

Conclusion: Probiotics containing S. boulardii are commonly used to treat many diseases. In neonates, we should be cautious about the indication of their use, preparation, and administration, particularly if a central intravenous catheter has been inserted.

\section{6}

\section{Neonatology}

Cerebral Abscesses and Serratia Marcescens: A Case Report Cátia Leitão $^{I}$, Sofia Ferreira ${ }^{l}$, Sónia Almeida ${ }^{1}$, Andreia Teles ${ }^{I}$, Márcia Gonçalves ${ }^{1}$, Anabela João ${ }^{l}$

${ }^{I}$ Pediatric Department, Neonatal Intensive Care Unit, Centro Hospitalar de Vila Nova de Gaia/Espinho, Portugal

Background: Cerebral abscesses in neonates are a rare clinical condition, with high morbidity and mortality rates, and usually arise in the setting of neonatal sepsis or meningitis.

Case: Preterm male, delivered by caesarian section at 35 weeks of gestation due to intra-uterine growth restriction. He weighed $1515 \mathrm{~g}$, and the Apgar scores were 9 and 10 at 1 and $5 \mathrm{~min}$ of life, respectively. On the $6^{\text {th }}$ day, the clinical status deteriorated and laboratory tests showed thrombocytopenia and leucopenia. The patient started an antibiotic regimen (cefotaxime, amikacin and vancomycin), even though the cerebroespinal fluid (CSF) analysis was normal. A Serratia Marcescens was isolated in hemoculture. In 48 hours the patient developed hypertonia and irritability. The lumbar punction was repeated: CSF analysis confirmed the diagnosis of meningitis but cultures remained negative. Antibiotics were modified accordingly (amikacin and meropenem). A transfontanellar ultrasonography revealed hyperechoic circular lesions in the periventricular regions. A brain magnetic resonance imaging (MRI) showed multiple supratentorial abscesses, evolving to cavitation accompanied by incipient porencephalic dilation of the left ventricle and diffuse cerebral edema (image 1). The neurosurgical council suggested conservative treatment with antibiotics (10 weeks) and follow-up MRI every two weeks. The final MRI showed cavitation areas (previous abscesses) and a Rhatke's pouch cyst. The baby was discharged at the 81 st day of life with a normal neurologic examination. At 12 months, the patient has a mild psychomotor and staturo-ponderal retardation; the brain MRI shows chronic cavitary lesions.

Conclusion: Serratia marcescens is a known cause of sepsis in neonatal intensive care units, frequently involving preterm patients with low birth weight. When there is neurological involvement by the infection, the prognosis is usually poor. The diagnosis and treatment of cerebral abscesses is a challenge for any neonatologist, given the absence of guidelines for the duration of treatment on this subject. 


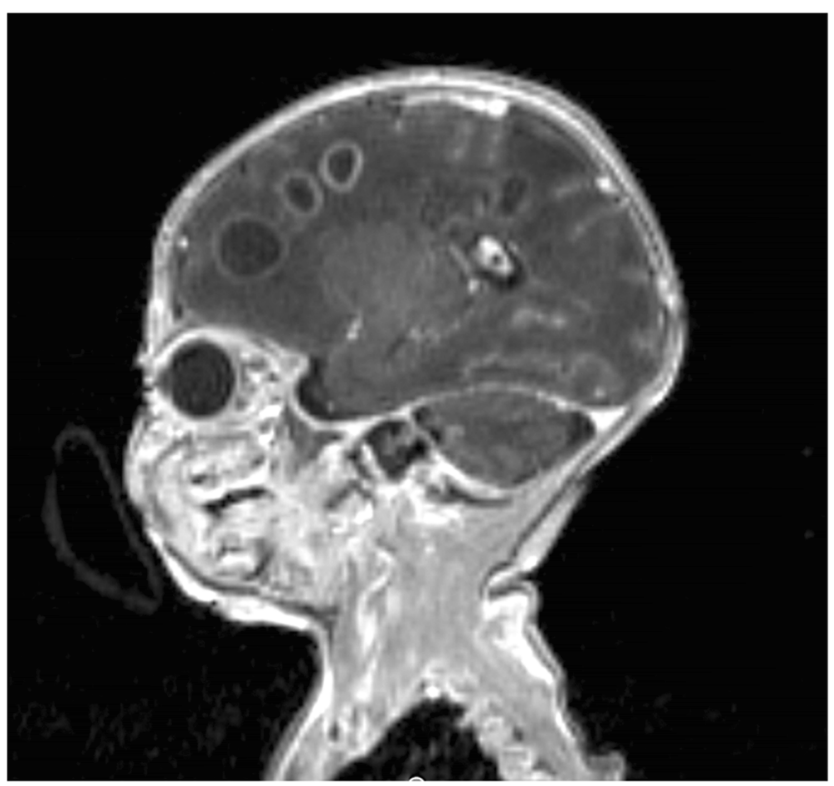

137

\section{Neonatology}

Insertion Depth of Endotracheal Tube in Extremely Low-BirthWeight Infants

Cheung Leung ${ }^{I}$

${ }^{T}$ Division of Neonatology, Far Eastern Memorial Hospital, Taiwan

Objective: To elucidate the relationship between gestational age, body weight, body length, head circumference, chest circumference, and depth of oral ETT in ELBW infants.

Materials and Methods: This retrospective study was conducted at the Far Eastern Memorial Hospital in New Taipei city, Taiwan using data collected from January 2012 to December 2016.We enrolled newborn infants who were intubated in the delivery room or neonatal intensive care unit and who had a body weight of $\leq 1000 \mathrm{~g}$ at birth. The following data were retrieved from medical records: gestational age, birth body weight, body length (crownheel length), head and chest circumferences, and final ETT depth. Data were analyzed using the SPSS version 20.0 software. Linear regression analysis was performed to assess the associations between each variable and the ETT placement depth. Results were considered to be statistically significant if the $\mathrm{P}$ value was 0.05 . Results: A total of 52 candidates were enrolled. The mean (range) gestational age was 25.1 weeks ( 22 to 32 weeks) and mean body weight (range) was $724.5 \mathrm{~g}(400-1000 \mathrm{~g})$. There were $8(15 \%)$, $22(42.5 \%)$, and $22(42.5 \%)$ patients with body weights of $500 \mathrm{~g}$ or less, 501-750 g, and $751 \mathrm{~g}-1000 \mathrm{~g}$, respectively. In linear regression, the relationships of ETT depth to gestational age, body weight, head circumference, chest circumference, and body length were all significant (P 0.001). Regarding the coefficient of determination (r2), body weight ranked first (0.497) and body length second (0.458), and all other parameters fell below 0.4 .

Conclusion: In ELBW infants, linear associations were observed between ETT insertion depth and assessed variables that are typically available at birth, including gestational age, body weight, body length, and head and chest circumferences. Although body weight is the most accurate parameter for predicting ETT insertion depth, body length is an equally useful alternative that is preferable during delivery room resuscitation.
399

Neonatology

Neurodevelopment Outcomes and Cardiac Output Changes following Patent Ductus Arteriosus Ligation in Preterm Infants

Chih Lin ${ }^{1}$, Kai-Hsiang Hsu ${ }^{1}$, Reyin Lien ${ }^{1}$

${ }^{T}$ Division of Neonatology, Department of Pediatrics, Chang Gung Memorial Hospital, Linkou Branch, TAIWAN

Objective: Infants receiving PDA ligations are at risk for subsequent neurodevelopmental impairment. We had conducted a prospective study to monitor cardiac output $(\mathrm{CO})$ changes in preterm infants undergoing surgical ligation for patent ductus arteriosus (PDA) and found a significant decline in $\mathrm{CO}$ following the surgery. Because $\mathrm{CO}$ is positively correlated with cerebral blood flow, we speculated that the degree of $\mathrm{CO}$ changes was associated with neurodevelopmental outcome. In this study, we aimed to correlate post-ligation $\mathrm{CO}$ changes with later neurodevelopment outcome. Methods: Based on previous study, $\mathrm{CO}$ were monitored at pre-surgery, 1minute following ligation, and 1, 6, 12, 18, 24, 48 hours after ligation, and were compared to pre-surgery baseline. We enrolled infants with Bayley III evaluation at 1-year corrected age. Developmental impairment was defined as Bayley III

Results: After excluding 2 infants with intraventricular hemorrhage grade 33, 2 expired before discharge, 1 lost follow-up, 25 infants who had Bayley III score at 1-year corrected age were enrolled and $14(56 \%)$ of them had developmental impairment. Their gestational age, birth weight, age at ligation and ductal diameter were $28.1 \pm 1.8$ (mean \pm SD) weeks, 981 $\pm 268 \mathrm{gm}, 14.4 \pm 7.5$ days and $2.7 \pm 0.9 \mathrm{~mm}$, respectively.

Infants who had developmental impairment had a significant $\mathrm{CO}$ decline immediately following ligation ( $-18.7 \%$ vs. $3.7 \%, \mathrm{p}=0.041)$, and a trend of generally more decreased $\mathrm{CO}$ was also seen in this group (repeated measured ANOVA between-group $\mathrm{p}=0.103$ ). No difference in demographics, echocardiographic features or blood pressure were observed between the two groups.

Conclusion: An immediate decline of $\mathrm{CO}$ following PDA ligation was associated with developmental impairment at 1-year corrected age in preterm infants. However, future prospective study is warranted to clarify the association between PDA ligation, $\mathrm{CO}$ changes, and afterward neurodevelopment outcome.

341

Neonatology

Congenital Horner and Scimitar Syndrome: Case Report

Mariana Maia ${ }^{1}$, Joana Carvalho ${ }^{1}$, Ágata Mota ${ }^{2}$, Teresa Martins ${ }^{3}$

${ }^{T}$ Paediatrics, Hospital Pedro Hispano, Portugal

${ }^{2}$ Ophthalmology, Hospital Pedro Hispano, Portugal

${ }^{3}$ Neonatology, Hospital Pedro Hispano, Portugal

Introduction: Horner syndrome is a classic neurological syndrome which signs usually include miosis, ptosis and anhidrosis. It can be caused by a lesion anywhere along the sympathetic pathway that supplies the head, eye and neck. A congenital Horner syndrome should be suspected when anisocoria is associated with heterochromia.

Scimitar syndrome is a congenital cardiovascular anomaly in which part or even the entire right lung is drained by right pulmonary veins that connect anomalously to the inferior vena cava.

Case Report: A full-term male newborn presented with left palpebral ptosis, anisocoria and heterochromia. Cleft palate and a heart murmur were also present. During pregnancy, no complications occurred until the third trimester, when a dilated cardiac sinus was detected in a routine fetal ultrasound. Within the first three months of life cleft palate was 
surgically corrected and an echocardiogram was performed, revealing an interauricular communication with left-to-right shunt.

Congenital Horner syndrome was suspected and, in order to rule out neuroblastoma, several exams were performed. Cerebral CT scan, karyotype and urinary levels of vanillylmandelic and homovanillic acids were normal. Thoracoabdominal CT scan revealed an alveolar infiltrate in the upper lobe of the right lung with evidence of anomalous drainage of most of the right lung to the inferior vena cava, suggesting Scimitar syndrome. Also, right cardiac chambers were found prominent, associated with a significant left-to-right shunt. The child is currently 21 months old and has reached every developmental milestone so far, with an adequate growth. A multidisciplinary team is currently following the case.

Conclusion: With this case report the authors aim to alert the medical community to the importance of an early diagnosis, since clinical aspects described may be subtle. Therefore, a high index of suspicion is particularly relevant as well as setting a multidisciplinary team in order to guarantee an adequate follow-up.

\section{1}

\section{Neonatology}

Breast Feeding: One Goal, Many Challenges

$\underline{\text { Inês Rosinha }}^{l}$, Daniela Peixoto ${ }^{1}$, Daniela Ester ${ }^{1}$, Bárbara Leal $^{1}$, Adelaide Bicho

${ }^{1}$ Pediatria, Centro Hospitalar do Baixo Vouga EPE, Portugal

Background: Breast feeding is considered a cornerstone for children's health all over the world. Despite its high prevalence in the immediate post childbirth, high abandonment rates have been registered early in life. World Health Organization (WHO) and The United Nations Children's Fund (UNICEF) recommend six-first months' exclusive breast feeding and have been promoting the Baby Friendly Hospital Initiative (BFHI) since 1992, already counting on fifteen accredited Hospitals.

Objective and Methods: "Centro Hospitalar do Baixo Vouga" (CHBV) has been intending to take part in BFHI since 2012. Therefore, we aim to analyse and compare newborns' feeding practices since birth till maternity leave from 2012 to 2018, using a retrospective study based on surveys completed during maternity stay. Our sample consisted in a variable number of surveys between 1307 and 1474 per year, of approximately 1700 annual live births.

Results: We verified oscillatory exclusive breast feeding rates, maximum in 2015 (80\%). Partial breast feeding rates have been increasing since 2015, maximum of $31 \%$ in 2018 , while exclusive formula feeding rates remained steady around $1 \%$. In what concerns first-hour breast feeding, the rate prowled $85 \%$. Conclusion: Although several efforts in order to fulfil the criteria, CHBV is not an accredited institution yet. We have been facing many difficulties in achieving optimal long-term exclusive breast feeding rates, despite initial high levels of contact with breast milk.

In fact, breast feeding duration depends on several multifactorial determinants, only some of which are modifiable, such as the availability of pre-birth training. Furthermore, it is mandatory some investment in mothers' formation about lactation and its technical difficulties, as well as optimization of health care professionals' training. It is also important to understand the main reasons for breast feeding abandonment and develop strategies to provide support within reach for every mother.

\section{0}

\section{P2: Neonatology 2 (Saturday, September 21, 2019 17:00)}

\section{Neonatology}

Neonatal Alloimmune Thrombocytopenia

Diana Reis Monteiro ${ }^{l}$, Ana Bernardo Ferreira ${ }^{l}$, Teresa Andrade ${ }^{l}$

${ }^{T}$ Pediatrics/Neonatology, Centro Hospitalar Entre Douro e Vouga,

Portugal
Background: Neonatal alloimmune thrombocytopenia (NAIT) occurs when maternal-fetal platelet incompatibility leads to formation of maternal antibodies and results in fetal and neonatal thrombocytopenia. Despite being a rare condition, it is the most common cause of isolated thrombocytopenia in the healthy newborn. Most cases have a mild presentation, but some can present with intracranial hemorrhage (ICH).

Objective: To characterize NAIT cases in a level II hospital.

Methods: Retrospective review of the cases of NAIT between 2014 and 2018. Data analysis was performed using SPSS®.

Results: Ten newborns were included (seven males). All pregnancies were correctly monitored, and all diagnoses were made in the neonatal period. Thirty percent of cases were first pregnancies. Fifty percent were born by eutocic delivery and the rest by dystocic ( $80 \%$ by cesarean). Two patients were preterm. There were six cases of normal birth weight and four cases of low birth weight. The diagnosis of NAIT was suspected in seven cases in the first 24 hours of life, in two cases in the first 48 hours and in one case at 72 hours. Clinically, three cases manifested only with cutaneous hemorrhages, three had $\mathrm{ICH}$, one had both cutaneous lesions and $\mathrm{ICH}$, and three cases were asymptomatic. We recorded minimal platelet values between 4000 and $78000 / \mu \mathrm{L}$. Platelet transfusion was performed in four cases, immunoglobulin in two and corticoid therapy in one case. Human platelet-specific alloantigens (HPA) responsible for causing NAIT were identified in 8 cases, with type HPA-5b being the most frequent. All patients were followed up subsequently in consult.

Conclusion: Most of our cases had a favorable clinical evolution. ICH cases occurred with platelet counts less than $60.000 / \mu \mathrm{L}$ and two of these patients had other comorbidities. Follow-up is essential for all patients, since NAIT can cause severe complications and long-term disabilities, particularly in cases of ICH.

\section{4}

\section{Neonatology}

\section{Infantile Hemangioma}

Joana Morais ${ }^{1}$, Joel Reis $^{2}$, Catarina Viveiros ${ }^{1}$, Cláudia Ferraz ${ }^{3}$, Susana Machado ${ }^{2}$

${ }^{1}$ Serviço de Pediatria, Hospital Pedro Hispano, Portugal

${ }^{2}$ Serviço de Dermatologia, Centro Hospitalar do Porto, Portugal

${ }^{3}$ Serviço de Neonatologia, Hospital Pedro Hispano, Portugal

Background: Infantile hemangiomas (IHs) are skin lesions characterized by abnormal proliferation of endotelial cells and aberrant blood vessel architecture. They represent the most common tumors in childhood, affecting $5 \%$ of general population. Risk factors include low birth weight, caucasian race or female gender. In most cases, IHs are diagnosed based on disease history and physical exam. Manifestations are usually present before 4 weeks of age and growth is completed by $5-8$ months of age (proliferative phase). Then it starts to involute until it disappears, frequently by the age of 4 years (involution phase). It might occur ulceration and pain, and due to localization on or near vital structures may cause functional impairment or permanent disfigurement. Imaging is not usually necessary, but when performed, ultrasound is generally the preferred modality for diagnosis, whereas magnetic resonance imaging (MRI) is requested to assess the extent of lesion. Although the majority of IH's resolves spontaneously, ulcerated IHs or near vital structures require early consultation with a dermatologist.

Case Report: A one-month-old infant, born at $37+5$ weeks and weighted $2540 \mathrm{~g}$, was examined for a progressively increasing right frontoparietal infantile hemangioma. The ultrasound was compatible with the clinical diagnosis of a $3.2 \mathrm{~cm}$ hemangioma reaching the anterior bording of the anterior fontanelle. The MRI confirmed the bone involvement. The patient was referred to Dermatology and started propranolol $2 \mathrm{mg} / \mathrm{kg} /$ day tid. Baseline electrocardiogram and echocardiogram were normal. Monitoring was performed by a pediatric cardiologist. IH significantly reduced after 6 months of therapy, without complications. 
Conclusion: Although most IHs do not require treatment, a minority may develop severe complications, requiring therapeutic intervention. Imaging may be needed when evaluation of extent is necessary. Pediatric providers should remain watchful. When justified, propranolol administered orally at a dose of $2 \mathrm{mg} / \mathrm{kg}$ per day is efficacious in reducing IHs.

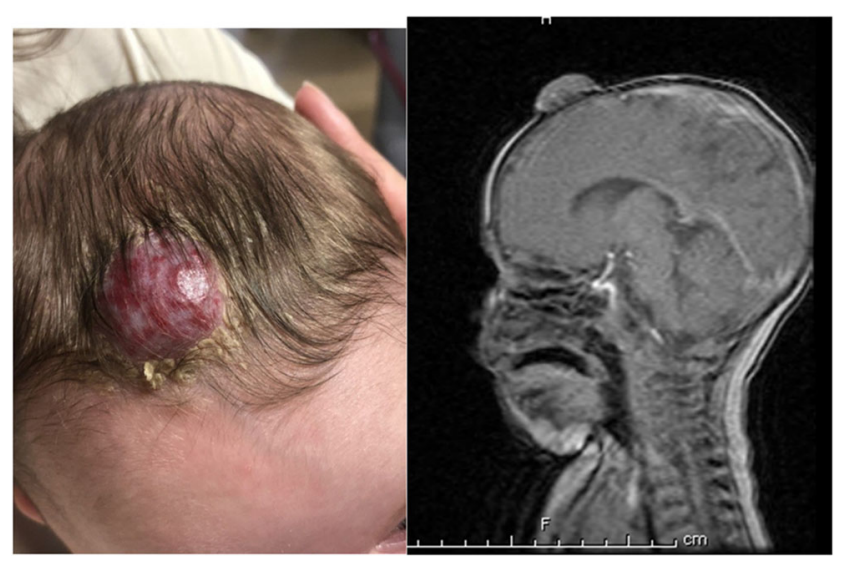

689

Neonatology

Antenatal Magnesium Sulfate: Retrospective Study of Clinically Relevant Outcomes in Preterm Neonates

Ricardo Mota ${ }^{1}$, Vicente Rey y Formoso ${ }^{1}$, Paulo Soares ${ }^{2}$, Hercília $^{2}$ Guimarães $^{2}$

${ }^{1}$ Pediatrics Department, Centro Materno e Pediátrico, Centro Hospitalar e Universitário São João, Portugal

${ }^{2}$ Neonatal Intensive Care Unit, Centro Materno e Pediátrico, Centro Hospitalar e Universitário São João, Portugal

Introduction: Antenatal magnesium sulfate (MgSO4) has been proven to be neuroprotective to the preterm newborn. However, evidence regarding its adverse effects in the newborn is lacking.

Objective: To analyse adverse effects on preterm infants whose mothers have been given $\mathrm{MgSO} 4$ treatment and correlate with serum $\mathrm{Mg}$ levels in the newborn.

Methods: Selected all newborns less than 32 weeks of gestational age born in our hospital between 2016-2018 and admitted to our level 3 Neonatal Intensive Care Unit (NICU) whose mothers have been given antenal $\mathrm{MgSO} 4$. We then conducted a retrospective study with statistical analysis. Results: Fifty infants were included. Serum magnesium was measured in $34 \%$ of the mothers, with a median value of $3.09 \mathrm{mEq} / \mathrm{L}$. The median newborn serum magnesium at approximately 24 hours of life was 2.5 $\mathrm{mEq} / \mathrm{L}$. A strong positive correlation between mother and infant serum magnesium was observed, with a Spearman value of 0.837 . Four infants presented necrotizing enterocoltis (NEC) and its median serum magnesium $(2 \mathrm{mEq} / \mathrm{L})$ was significantly lower than in infants without the condition $(2.54$ $\mathrm{mEq} ; p=0.049)$. When corrected for gestational age, an increase of 0.01 $\mathrm{mEq} / \mathrm{L}$ resulted in a $5.3 \%$ reduction in the odds of developing NEC (Wald $p$ value $=0.023$ ). Twenty-five children were diagnosed with patent ductus arteriosus (PDA). These infants' mothers had a significantly higher serum $\operatorname{Mg}(p=0.035)$. This was not confirmed when corrected to gestational age (Wald $p$ value $=0.783$ ). No difference or correlation in newborn or mother $\mathrm{Mg}$ levels were found when analysing further adverse effects, ventilation days, parenteral nutrition dependency, NICU stay, or other outcomes.

Conclusion: This study suggests that magnesium can be protective against NEC, but not in other morbidities. However, its retrospective nature and the small sample size (only four children developed the condition), determine the need of a larger prospective study.
Table 1 - Antenatal Period

\begin{tabular}{|c|c|c|c|c|}
\hline & & Value & Newborn $\mathrm{Mg}$ (mean) & Mother $\mathrm{Mg}$ (median) \\
\hline \multirow[t]{2}{*}{ Gender } & Male $\mathrm{n}(\%)$ & $24(48)$ & 2.43 & 3.09 \\
\hline & Female $n(\%)$ & $26(52)$ & 2.55 & 1.94 \\
\hline Gestational Age & Weeks (median) & 29,6 & & \\
\hline \multirow{2}{*}{ Multiple Gestation } & Non(\%) & $27(54)$ & 2.56 & 3.61 \\
\hline & Yes $n / \%$ ) & $23(46)$ & 2.42 & 1.65 \\
\hline \multirow[t]{2}{*}{ Antenatal Corticosteroid } & Non(\%) & $3(6.1)$ & 2.32 & 1.42 \\
\hline & Yes $n(\%)$ & $46(93.9)$ & 2.51 & 3.09 \\
\hline \multirow[t]{2}{*}{ Antenatal Ampicilin } & Non(\%) & $24(48)$ & 2.42 & 3.09 \\
\hline & Yes n(\%) & $26(52)$ & 2.56 & 2.20 \\
\hline \multirow[t]{2}{*}{ Maternal Fever } & No n(\%) & $47(94)$ & 2.47 & 3.09 \\
\hline & Yes n(\%) & $3(6)$ & 2.82 & \\
\hline \multirow[t]{2}{*}{ Corioamnionitis } & No $n(\%)$ & $47(94)$ & 2.47 & 3.09 \\
\hline & Yes n(\%) & $3(6)$ & 2.82 & \\
\hline \multirow[t]{2}{*}{ Pre-Eclampsia } & Non(\%) & $37(74)$ & 2.47 & 1.65 \\
\hline & Yesn $n(\%)$ & $13(26)$ & 2.57 & 3.22 \\
\hline \multirow[t]{2}{*}{ HELLP syndrome } & Non(\%) & $46(92)$ & 2.51 & 3.09 \\
\hline & Yes n(\%) & $4(8)$ & 2.29 & 3.04 \\
\hline \multirow[t]{2}{*}{ Gestational Hypertension } & No n(\%) & $45(90)$ & 2.51 & 3.09 \\
\hline & Yes n(\%) & $5(10)$ & 2.35 & 1.69 \\
\hline \multirow[t]{2}{*}{ Gestational Diabetes } & Non(\%) & $48(96)$ & 2.49 & 3.09 \\
\hline & Yesn(\%) & 2(4) & 2.64 & 3.37 \\
\hline \multirow[t]{2}{*}{ Measured Maternal Serum $\mathbf{M g}$} & Non(\%) & $33(66)$ & 2.55 & \\
\hline & Yesn(\%) & $17(34)$ & 2.39 & 3.09 \\
\hline Highest Maternal Serum $\mathrm{Mg}$ & Median, $\mathrm{mEq} / \mathrm{L}$ & 3.09 & & \\
\hline
\end{tabular}

Table 2 - Perinatal Period

\begin{tabular}{|c|c|c|c|c|c|c|}
\hline & & Value & Newborn $\mathrm{Mg}$ (mean) & $p$ Value & Mother $\mathrm{Mg}$ (median) & $p$ Value \\
\hline \multirow[t]{2}{*}{ Delivery } & Eutocic $n(\%)$ & $12(24)$ & 2.69 & & 3.66 & \\
\hline & Dystocic n $(\%)$ & $38(76)$ & 2.43 & & 3.09 & \\
\hline Weight & $\mathrm{g}$, mean & 1253 & & & & \\
\hline Length & $\mathrm{cm}$, median & 37.5 & & & & \\
\hline Head Circumference & $\mathrm{cm}$, mean & 28.12 & & & & \\
\hline \multirow[t]{2}{*}{ Ressusitation } & Non(\%) & $19(38)$ & 2.59 & \multirow{2}{*}{0.311} & 3.66 & \multirow{2}{*}{0.644} \\
\hline & Yes n(\%) & $31(62)$ & 2.43 & & 2.18 & \\
\hline \multirow[t]{2}{*}{$\mathrm{O} 2$} & No n(\%) & $10(20)$ & 2.52 & \multirow{2}{*}{0.876} & 3.09 & \multirow{2}{*}{0.485} \\
\hline & Yes n(\%) & $40(80)$ & 2.49 & & 2.75 & \\
\hline \multirow[t]{2}{*}{ Positive Pressure } & No n(\%) & $15(30)$ & 2.51 & \multirow{2}{*}{0.886} & 3.83 & \multirow[b]{2}{*}{1} \\
\hline & Yes $n(\%)$ & $35(70)$ & 2.49 & & 2.75 & \\
\hline \multirow[t]{2}{*}{ Intubation } & Non(\%) & $33(66)$ & 2.44 & \multirow{2}{*}{0.279} & 2.22 & \multirow{2}{*}{0.101} \\
\hline & Yes n(\%) & $17(34)$ & 2.61 & & 3.88 & \\
\hline
\end{tabular}

Table 3 - Neonatal Period

\begin{tabular}{|c|c|c|c|c|c|c|}
\hline & & Value & Newborn Mg [mean) & $p$ Value & Mother $M_{\mathbb{E}}$ (median) & pValue \\
\hline \multirow[t]{3}{*}{ Non Imasive Ventilation } & Non(96) & $5[10)$ & 2.46 & \multirow{3}{*}{0.930} & 3.09 & \\
\hline & Yesn $n(\%)$ & $45(90)$ & 2.50 & & 2.92 & \\
\hline & Lenggh(medisn dyys) & 14 & & & & \\
\hline \multirow{3}{*}{ Invasive Ventilation } & Non(96) & $24448)$ & 2.45 & \multirow{3}{*}{0.591} & 2.92 & \multirow{3}{*}{0.350} \\
\hline & resn n(s) & 26[52] & 2.53 & & 3.34 & \\
\hline & Lengeth $(m \geq d$ in dayss) & 4.5 & & & & \\
\hline \multirow[t]{2}{*}{ Bronchopulmonary Opplasia } & Non(f6) & 41;82] & 2.52 & \multirow{2}{*}{0.528} & 2.75 & \multirow{2}{*}{0.250} \\
\hline & Yesn n(s) & $9(18)$ & 2.39 & & 4.22 & \\
\hline \multirow{2}{*}{ Infant Respirrtory Distress Syndrome } & Non(\$6) & $20(40)$ & 2.43 & \multirow{2}{*}{0.475} & 2.22 & \multirow{2}{*}{0.304} \\
\hline & Ves n $n(6)$ & $30(60)$ & 2.54 & & 3.34 & \\
\hline \multirow[t]{2}{*}{ Surfactant } & Non(\%) & $27(54)$ & 2.39 & \multirow[b]{2}{*}{0.149} & 2.47 & \multirow[b]{2}{*}{0.058} \\
\hline & Vess n(w) & $23(46)$ & 2.61 & & 3.88 & \\
\hline \multirow[t]{2}{*}{ Patent Ductus Arteriosus } & Non(96) & $25(50)$ & 2.44 & \multirow{2}{*}{0.431} & 2.20 & \multirow{2}{*}{0.035} \\
\hline & Yesn $n(3)$ & $25(50)$ & 2.56 & & 3.88 & \\
\hline \multirow[t]{2}{*}{ Patent Foramen Orale } & Non(56) & 32(64) & 2.51 & \multirow{2}{*}{0.841} & 2.75 & \multirow{2}{*}{0.153} \\
\hline & Yesn $n(s)$ & $18(36)$ & 2.48 & & 3.78 & \\
\hline \multirow[t]{3}{*}{ Aminergic Support } & Non(36) & $44(88)$ & 2.45 & \multirow{3}{*}{0.146} & 2.75 & \multirow{3}{*}{0.154} \\
\hline & resn $n(s)$ & 6(12) & 2.79 & & 4.27 & \\
\hline & Length (median days) & 2 & & & & \\
\hline \multirow[t]{2}{*}{ Fever } & Non(\%) & $48(96)$ & 2.47 & \multirow[b]{2}{*}{0.067} & 3.09 & \\
\hline & Yesn $(3)$ & $2[4]$ & 3.17 & & 2.75 & 1 \\
\hline Parenteric Mutrition & 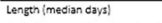 & 15 & & & & \\
\hline & Exclusive Length (median days] & 2 & & & & \\
\hline & \% Exclusive Length & 15.5 & & & & \\
\hline Esophagesal Reflux & Non[96] & 41[22] & 2.45 & & 3.09 & \\
\hline & $\operatorname{Yes}(y)$ & g(18) & 2.70 & 0.199 & 2.77 & 1 \\
\hline Necrotizing Enterocolitis & Non(96) & $46[92)$ & 2.54 & & 3.09 & \\
\hline & Yesn $n(s)$ & $4(8)$ & 2.00 & 0.049 & 1.69 & 1 \\
\hline Introventricular Hemortiage & Non[96] & 41[22] & 2.51 & & 2.75 & \\
\hline & Ves n (s) & $9(18)$ & 2.42 & 0.655 & 3.57 & 1 \\
\hline 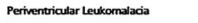 & Non(s) & $47(94)$ & 2.51 & & 3.09 & \\
\hline & $\operatorname{Yess}_{n}(y)$ & $3 / 6)$ & 2.33 & 0.592 & 2.79 & 1 \\
\hline Neonstal Hrpotonia & Non(s) & 49:(98) & 2.50 & & 3.09 & \\
\hline & Yesn $($ (s) & 1[2] & 2.41 & & - & \\
\hline NICU & Length of stary (median days) & 33.5 & & & & \\
\hline Serum $M_{g}$ & First valus & 2.5 & & & & \\
\hline & Second Valuse & 2.32 & & & & \\
\hline Deceased & & $47(94)$ & 2.45 & & 3.09 & \\
\hline & res $n(s)$ & $3(6)$ & & 0.023 & & \\
\hline
\end{tabular}


342

\section{Neonatology}

Less Invasive Surfactant Administration (LISA) using Video Laryngoscopy in Late Preterm Babies in a District General Hospital in The UK

Claudine Ogilvie ${ }^{I}$, Indraneel Adkar ${ }^{I}$, Naomi Robbins ${ }^{I}$, Torsten Hildebrandt ${ }^{I}$

${ }^{1}$ Women and Child Health, Princess of Wales Hospital, UK

Background: Less invasive surfactant administration (LISA) has become an increasingly used intervention in the treatment of respiratory distress syndrome in neonates [1]. Previous reports and meta-reviews document positive outcomes in the avoidance of mechanical ventilation as well as reduction in bronchopulmonary dysplasia [2,3]. During the last few years video laryngoscopes have become available for neonatal practice. The use of video assisted visualisation of the vocal cords makes application of surfactant via LISA easy and safe.

Objective: We report 15 babies who received surfactant using LISA in combination with video laryngoscopy in a District General Hospital in the UK between March and December 2018.

Methods: Babies had a mean gestational age of 34 weeks, with a range of 31 to 39 weeks. Indication for the administration of surfactant was increasing oxygen requirement, clinical impression of respiratory distress and rising capillary $\mathrm{CO} 2$ levels. All babies tolerated the procedure well with improvement in oxygen requirement, clinical assessment of respiratory distress and capillary $\mathrm{CO} 2$. There were no significant adverse effects. 3 babies were given atropine following procedure induced mild bradycardia. None of the babies were sedated.

Conclusion: Our experience with delivering surfactant using LISA assisted by video laryngoscopy has been very positive. The use of a video laryngoscope allows less experienced operators to visualise the vocal cords safely under supervision [4]. This has a positive impact on the team as well as a valuable learning benefit. 6 out of 7 doctors performing the procedure felt that the video laryngoscope had improved their confidence and allowed the procedure to be performed less invasive compared to routine laryngoscopy techniques.

\section{4}

Neonatology

Neonate with Congenital Diaphragmatic Hernia- Case Report Aikaterini Pana ${ }^{l}$, Sofia Vasilakou ${ }^{1}$, Olympia-Panagiota Rozakea ${ }^{1}$, $\overline{\text { Panagiotis Panas }}^{2}$, Ioannis Gripiotis ${ }^{l}$, Ioannis Panagiotou ${ }^{l}$

${ }^{I}$ Pediatrics, General Hospital of Lakonia- Nursing Unit of Sparta, Greece

${ }^{2}$ Obstetrics-Gynaecology, University Hospital Of Patras "Panagia i Voitheia", Greece

Introduction: Congenital diaphragmatic hernia is a defect in the diaphragm that allows the passage of abdominal viscera into the chest cavity. This results to respiratory failure and pulmonary hypertension. It occurs in approximately $1 / 2000$ to $1 / 5000$ live births and it is more common in females, on the posterolateral left side of diaphragm. $30 \%$ of the patients have associated congenital malformations.

Aims: Presentation of a rare congenital malformation.

Material: Neonate with respiratory distress after birth.

Methods: Diagnosis based on clinical examination and chest X-Ray.

Results: Full-term male neonate of Roma origin, without any prenatal screening, presents with respiratory distress in the $1^{\text {st }}$ min of life and bradycardia(HR100/min). During our clinical examination diminished breath sounds were observed on the left side of the neonate's thorax with displacement of the hearth sounds to the opposite side. The diagnosis was confirmed with chest radiography. The newborn was intubated after $15 \mathrm{~min}$ of resuscitation and although he was immediately transported to a neonatal unit, he passed away after a few hours.
Conclusions: Congenital diaphragmatic hernia is a rare congenital malformation and the mortality rate is about $50 \%$. High suspicion must rise in every case where a neonate's respiratory distress deteriorates despite well performed resuscitation. For the treatment it is necessary to avoid ventilation with self-inflating bag and to proceed to early intubation, with PIP

\section{9}

Neonatology

T-Piece Resuscitator or Self Inflating Bag for Positive Pressure Ventilation During Neonatal Resuscitation. A Randomized Controled Trial

Sadbhavna Pandit ${ }^{1}$, Sadbhavna Pandit ${ }^{1}$, Neeraj Dhawan ${ }^{2}$, Suryalata Kookna

${ }^{1}$ Pediatric Department, Government Multi Speciality Hospital Sector 16 Chandigarh India, India

${ }^{2}$ Pediatric Department, Government Multi Speciality Hospital Sector 16 Chandigarh India, India ${ }^{3}$ Pediatric Department, Government Multi Speciality Hospital Sector 16 Chandigarh India, India

Although most newborn infants successfully transit from intrauterine life to extra uterine life without special help but about $10 \%$ of neonates require assistance at birth for smooth transition from intra-uterine to extra uterine life. Positive pressure ventilation in delivery room can be provided with self inflating bag which usually does not provide positive end expiratory pressure (PEEP) and T-Piece resuscitator (TPR) providing PEEP. Methodology: Prospective, randomised controlled, non blinded study was conducted at newborn corners, Government Multi-Speciality Hospital, Sector-16, Chandigarh, India. Fifty eligible newborns more than 28 weeks were randomly allocated in two groups. Group 1 of newborns were resuscitated with T-Piece resuscitator and Group 2 with self inflating bag (SIB). Results: Mean duration of positive pressure ventilation was significantly lesser $(P=0.003)$ in TPR group $(71.48+19.0 \mathrm{sec})$ as compared to SIB $(88.48 \pm 19.28 \mathrm{sec})$. One minute mean $\mathrm{AP} G A R$ score in TPR group $(5.08$ $+1.07)$ was significantly high $(P=0.02)$ as compare to SIB $(4.4+0.91)$ Conclusions: Study suggested that T-Piece resuscitator is a better device as compared to SIB as it reduces the duration of positive pressure ventilation and helps to achieve better APGAR score.

\section{0}

\section{Neonatology}

Avoiding Hypothermia in Preterm Infants

Claire Howarth $^{1}$, Niha Peshimam ${ }^{1}$, Mae Nugent ${ }^{1}$, Cara George ${ }^{l}$, Subhabrata Mitra ${ }^{l}$

${ }^{I}$ Neonatal Unit, University College Hospital London, UK

Background: Preterm neonates are predisposed to develop hypothermia. Admission temperatures below $36^{\circ} \mathrm{C}$ are associated with increased mortality and morbidity.

Objective: To increase compliance of preterm infants' 32 weeks gestation having admission temperatures $36.5 \mathrm{C}-37.5 \mathrm{C}$, as per NNAP standard, by preventing heat loss in the immediate postnatal period. Prior to this project our Unit's compliance was 59\%. Initially we targeted compliance of $75 \%$. Methods: We used the rapid-cycle Plan-Do-Study-Act method. Preterm infants less than 32 weeks gestation who were born at a Regional Neonatal Intensive Care Unit (University College Hospital - UCH, London, England) were enrolled.

Our intervention involved a new education programme and standardising the management at delivery with a thermoregulation guideline (including use of transwarmer, plastic bag and hat). Importantly a major change involved increasing the ambient temperature in theatres to $26 \mathrm{C}$ (previously $23 \mathrm{C}$ ) for those born in obstetric theatres.

Weekly data on admission temperature were collected prospectively. 
Results: Over the 9 weeks that the project has been running $2132 \mathrm{w}$ gestation infants were born at UCH.

$17(81 \%)$ achieved admission temperatures of $36.5 \mathrm{C}-37.5 \mathrm{C}$

Only 1 infant had an admission temperature $37.5 \mathrm{C}(37.7 \mathrm{C})$ and they were septic.

16 of the infants were admitted from obstetric theatres; 13 achieved admission temperatures in range $(81.3 \%)$ by adhering fully to our new guideline. Of the 3 that did not, all were hypothermic (36.5C), and in each case the theatre temperature was not increased to $26 \mathrm{C}$ (only 23$24 \mathrm{C}$ ), although the rest of our guideline was followed.

Conclusion: We reduced hypothermia in $32 \mathrm{w}$ gestation infants using a standardised guideline, and educating and involving the whole multidisciplinary team. Increasing ambient temperature in operating theatres is vital to avoid hypothermia. The PDSA cycles and weekly audits allowed for real time feedback helping to sustain the improvement. Moving forward we aim to target compliance of $90 \%$.

\section{1}

\section{Neonatology}

Congenital Clavicular Pseudarthrosis: A Rare Diagnosis Marta Isabel Pinheiro ${ }^{1}$, Cristina Ferreras ${ }^{1}$, Jorge Coutinho ${ }^{2}$, Maria Gorett Silva ${ }^{I}$, Hercilia Guimarães ${ }^{1,3}$

${ }^{1}$ Department of Neonatology, Regular Nursery, Centro Hospitalar e Universitário de São João, Portugal

${ }^{2}$ Paediatric Orthopaedic Unit. Department of Orthopaedics and Traumatology, Centro Hospitalar e Universitário de São João, Portugal

${ }^{3}$ Faculty of Medicine, University of Porto, Portugal

Background: Congenital clavicular pseudarthrosis is a rare benign anomaly, first reported in 1910. This condition resulting in a failure of coalescence of the two primary ossification centers, during embryogenesis, which produces two portions of the clavicle connected by a fibrous bridge. The exact etiology is unknown. Ninety percent occur on the right side and it's more prevalent in girls. Bilateral occurrence may be associated with genetic syndromes. Generally, simple radiography is sufficient to confirm the diagnosis.

Objective/Methods: The authors aimed to describe a rare clinical case of clavicle pseudarthrosis observed in the maternity ward, in 2018. All clavicular radiographs of newborns from 2018 were reviewed to identify possible non-diagnosed clavicular pseudarthroses.

Case Report: Female newborn with an uneventful pre-natal history. Vaginal delivery at 40 weeks and 3370gr (10-50th percentile) of birth weight. On the first day of life, the paediatrician describes a crackling in the middle third of the right clavicle with symmetrical Moro reflex, no palpable swelling or painful region and normal mobility of the upper limbs. Radiological study confirmed the diagnosis of congenital pseudarthrosis of the clavicle, showed an interruption in the medial region of the clavicle with rounded edges and no evidence of bone callus formation. The patient follow-up is in Children's Orthopaedics outpatient. No more cases of clavicular pseudarthrosis were identified in 2018.

Conclusion: Differential diagnosis, especially in neonates, mainly includes the clavicle fracture due to birth trauma. However, when the clinical findings are contradictory, this condition should be considered, with radiology being an important auxiliary. In most situations, an expectant attitude is adopted. Surgical treatment is recommended when there are functional limitations, progressive pain, onset thoracic outlet syndrome or unacceptable cosmetic deformity.

\section{0}

Effects of Maternal Antiphospholipid Syndrome on Offspring Outcomes: A Literature Review

Vicente Rey y Formoso ${ }^{1}$, Mário Mateus ${ }^{2}$, M.Gorett Silva ${ }^{2}$, Hercília Guimarães $^{2}$

${ }^{1}$ Pediatrics Department, Maternal and Pediatrics Center - São João University and Hospital Center, Portugal
${ }^{2}$ Neonatology Department, Maternal and Pediatrics Center - Sao João University and Hospital Center, Portugal

Background: Antiphospholipid Syndrome (APS) is an autoimmune disorder characterized by pregnancy morbidity and/or thrombosis, associated with positivity for antiphospholipid antibodies (aPL). Despite its relative rarity, because it usually affects women of fertile age, and consequentially their offspring, its knowledge remains essential for any neonatologist or pediatrician.

Objective: To review the latest research and perspectives on the outcomes of children born to mothers suffering from APS.

Methods: A search was conducted in the Pubmed® database using the keyword "antiphospholipid syndrome" in combination with any of the following: "pregnancy", "fetal", "neonatal", "pediatric". Articles were selected considering their pertinence to the subject and relevant references were also reviewed.

Results: Etiology seems multifactorial with multiple genetic and environmental factors likely to play a part. General expert opinion is that these children do not show a significantly increased risk of APS.

A higher frequency of pregnancy complications in APS gravidas such as pre-eclampsia and placental insufficiency (among others) leads to a higher prevalence of prematurity and all its associated complications.

Transplacental passage of aPL is frequent, but its clinical significance remains uncertain and passively transferred aPL levels usually decrease in the first months of life. Early age thrombosis associated with aPL (neonatal/pediatric APS) is extremely rare in these patients - prospective studies found no such episodes in these children's first years of life. Research focused on thrombosis in aPL+ newborns describes that, in most cases, other maternal or neonatal prothrombotic factors can be found.

Several recent prospective studies show a higher prevalence of neurodevelopmental abnormalities - this is compatible with previous experimental studies on animal models.

Conclusion: Despite appropriate treatment, in maternal APS, pregnancy complications still lead to prematurity and higher rates of poor neonatal outcomes. aPL alone are not sufficient for thrombosis development in neonates. Neurodevelopmental screenings should be considered in these patients' follow-up schedule.

\section{4}

\section{Neonatology}

Very Low Birth Weight Neonates: Experience in a Neonatology Intensive Care Unit

Rita Russo Belo ${ }^{l}$, Maria Ventura Nogueira ${ }^{l}$, Carla Sál, Albina Ferreira da Silva', Almerinda Barroso Pereira ${ }^{I}$

${ }^{1}$ Neonatal Special Care Unit, Hospital de Braga, Portugal

Background: The incidence of preterm births is increasing, therefore, so is the incidence of very low birth weight neonates (VLBW), defined as birth weight

Objective: Characterize the population of very low birth weight neonates on a Neonatology Intensive Care Unit (NICU) in a tertiary hospital and compare it with national data.

Methods: Data was collected from the National Registry of Very Low Birth Neonates (

Results: During this period, there were 8623 births in this hospital, 174 (2.0\%) corresponding to VLBW neonates. $31.0 \%$ of these resulted from multiple gestations and $10.9 \%$ occurred in context of medically assisted reproduction. In-utero transfer to our hospital occurred in $33.9 \%$ of the cases and antenatal corticosteroid therapy was performed in $93.7 \%$ of the gestations. $75.3 \%$ of these neonates were born

Conclusion: It is important to have a national registry in order to improve our neonatal care. Our hospital had better results regarding complications and mortality compared to the national total. A correct follow-up of these patients is important. 
365

\section{Neonatology}

The Presence of Nuchal Cord and Need for Resuscitation at Birth in Term Newborns

Zulfiquar Ali Sarani ${ }^{1}$, John Kelleher ${ }^{1}$, Catherine Fitzgerald ${ }^{1}$

${ }^{T}$ Neonatology, Coombe Women and Infants University Hospital., Ireland

Background: The presence of a nuchal umbilical cord is often a normal finding in a fetus. It may be associated with increased risk for adverse neonatal outcomes particularly if tight, coiled or knotted.

Objective: To examine the association between a pathological nuchal cord and need for resuscitation at birth in term newborns.

Methods: This was a retrospective study of an electronic medical record system for an Irish Maternity Hospital examined over twenty years. Inclusion criteria included newborns who delivered at $\geq 37$ weeks gestation, inborn, and without major congenital anomalies. Logistic regression was utilized to model for factors independently associated with need for neonatal resuscitation at birth. The numbers of intrauterine deaths and newborn death within the first seven days of postnatal life were ascertained. A pathological nuchal cord was defined as "tight", "coiled" and/or a "knot" present.

Results: The study included 140,613 newborns. Vaginal delivery occurred in $60.2 \%$ and instrumental/operative delivery in $26.1 \%$ of pregnancies. There were 357 intrauterine and/or neonatal deaths $(0.25 \%)$. Newborn resuscitation, including positive pressure ventilation and/or more advanced resuscitation at birth, was necessitated in 2367 newborns (1.68\%). A pathological nuchal cord was found in 3593 newborns (2.55\%). A non-pathological nuchal cord was described in 36599 newborns (26\%). A pathological cord around the neck was found to be independently associated with need for resuscitation at birth adjusting for mode of delivery, multiple births, and meconium stained amniotic fluid (OR 1.90, 95\% CI $1.56-2.32$ ).

Conclusions: A pathological nuchal umbilical cord including either a knot, tightness or coiling was found to be associated with need for newborn resuscitation at birth in term pregnancies. This study was limited by its retrospective nature and the infrequency of intrauterine death and/or newborn mortality. Its strength is the large sample size.

\section{2}

\section{Neonatology}

Trends in BPD Data Over 9 Years in a Single Tertiary Surgical Neonatal Centre

Sandeep Shetty ${ }^{I}$,Edward Milne ${ }^{I}$, Peter Cornuaud ${ }^{l}$, Anay Kulkarni ${ }^{1}$

${ }^{T}$ Tertiary surgical neonatal unit, St Georges Hospital NHS Foundation Trust, UK

Background: Incidence of significant bronchopulmonary dysplasia (BPD) in babies born

Objective: To observe trend of rates of significant BPD and survivals in babies born at

To determine which gestation category $(23-25+6,26-28+6,29-31+6)$ had the significant change in incidence of significant BPD

To compare rates of significant BPD in babies who were transferred to local hospital before 36 weeks against to babies who were still at the $\mathrm{St}$ Georges hospital at 36 weeks corrected gestation.

Methods: It was retrospective cohort study reviewing babies born at. Babies were transferred Ex-Utero to St. George's Neonatal unit were excluded from the study.

Results: Between 2010-2018 there were 727 live births 32 weeks gestation at St. George's hospital NHS trust. Average incidence of BPD over nine years was observed to be $42 \%$. Incidence of BPD was observed to be increasing from $29 \%$ in 2010 to $51 \%$ in 2018 . We Observed increased BPD rates in gestational group (26 -28+6 (33\% in 2010 to $44 \%$ in 2018) and gestational group $29-31+6$ (19\% in $2010-24 \%$ in 2018).

Conclusions: Rates of significant BPD in babies born
1028

Neonatology

Early Extubation is Associated with a Reduced Risk of Developing Significant Bronchopulmonary Dysplasia - A Retrospective Audit Richard Hutchinson ${ }^{1}$, Anay Kulkarni ${ }^{I}$, Justin Richards ${ }^{I}$, Sandeep Shetty ${ }^{1}$ ${ }^{I}$ Tertiary Surgical Neonatal Unit, St Georges Hospital NHS Foundation Trust, UK

Background: As survival of preterm infants increases, greater attention is being paid to improving the quality of health in survivors. Unfortunately, despite advances in neonatal respiratory care, bronchopulmonary dysplasia (BPD) remains a significant cause of morbidity in the ex-preterm population. Consequently, there remains interest in developing strategies to reduce BPD incidence.

Objective: To identify clinical and demographic features associated with reduced incidence of BPD

Methods: This was a retrospective audit conducted between 2010-18 at a tertiary surgical neonatal unit at St George's Hospital, London, UK. Data were extracted from a database of infants born at 32/40 gestation at $\mathrm{St}$ George`s Hospital. Information was collected on demography, clinical characteristics at birth, and respiratory clinical data. Significant BPD is defined as a need for supplemental oxygen or respiratory support at 36/40 corrected gestational age, in a baby born at 32/40 gestational age (as per National Neonatal Audit Programme definition).

Results: 396 infants were identified as having been born at 32/40 gestational age in the study period. $59 \%$ of the cohort met the definition for significant BPD. Incidence of significant BPD was seen to vary inversely with gestational age and birthweight. An association was seen between delayed extubation, and subsequent development of significant BPD. First extubation attempt beyond 24 hours was associated with an increased risk of developing BPD compared to when extubation was attempted within 24 hours $(61 \%$ incidence vs. $18 \%, \mathrm{OR}=7.1 \mathrm{C} 95 \% \mathrm{CI}$ 4.5-11.4, p0.0001); first extubation attempt beyond one week was associated with a further increased risk of BPD development, compared to extubation attempted within the first week $(96 \%$ vs. $49 \%, \mathrm{OR}=27$ [95\% CI 6.4-114.8, p0.0001].

Conclusion: These data show an association between early extubation, and survival without development of significant BPD. Assessment of strategies to encourage early extubation may demonstrate a causative nature to this relationship, and reduce significant BPD incidence.

301

Neonatology

The Effects of a CXCR2 Antagonist on Postnatal Systemic Inflammation-Disrupted Alveolar Development in a Rat Model of BPD

Jeongmin Shin $^{1}$, Sehyun Kim ${ }^{l}$, Young Hwa Jung ${ }^{l}$, Chang Won Choi ${ }^{1,2}$, $\overline{B e y o n g ~ I l ~ K i m}^{1,2}$

${ }^{I}$ Department of Pediatrics, Seoul National University Bundang Hospital, South Korea

${ }^{2}$ Department of Pediatrics, Seoul National University College of Medicine, South Korea

Background: Bronchopulmonary dysplasia (BPD) is the most common morbidity suffered by surviving preterm infants and is associated with adverse pulmonary and neurodevelopmental outcomes. Inflammation plays a pivotal role in the pathogenesis of BPD. An influx of inflammatory cells and neutrophil-derived chemokines has been implicated in the development of BPD. A competitive CXCR2 receptor antagonist reduces neutrophil activation and influx into tissues.

Objective: To evaluate the effects of a CXCR2 antagonist on reducing postnatal systemic inflammation and consequently developing BPD in rat model. 
Methods: Lipopolysaccharide(LPS) was administered to the amniotic fluid on E20, which corresponds to the late canalicular stage of rat lung development. LPS and a CXCR2 receptor antagonist were administered intraperitoneally on P1, P3, and P5, which correspond to the saccular and early alveolar stages. On P7 and P14, bronchoalveolar lavage fluid (BALF) and peripheral blood (PB) were collected from the rat pups. To assess alveolar development, four random, non-overlapping fields per animal in two distal lung sections were studied at 100X magnification.

Results: Early postnatal LPS administration significantly increased neutrophil counts in BALF and PB and disrupted alveolar development, whereas prenatal LPS alone did not. The CXCR2 antagonist significantly attenuated neutrophil increases in BALF and PB and restored alveolar development in the rat pups exposed to early ponstnatal LPS.

Conclusion: These results suggest that a CXCR2 receptor antagonist restores alveolar development by alleviating pulmonary and systemic inflammation induced by early postnatal LPS.

\section{5}

\section{Neonatology}

Hypoxic-Ischemic Encefalopathy Management in an Ultra Peripheral Hospital

Sarah Stokreef $^{l}$, Joana Fortuna ${ }^{l}$, Catarina Damaso ${ }^{l}$, Ana Lucia Maia ${ }^{l}$, Fernanda Gomes ${ }^{1}$

${ }^{1}$ Serviço de Pediatria, Hospital do Divino Espírito Santo, Portugal

Hypoxic-ischemic encefalopathy (HIE) due to birth asphyxia occurs when there is an acute hypoxic-ischemic event that causes various sings and symptoms. The treatment of choice for this condition is therapeutic hypothermia, accompanied by supportive management. It has been available in Portugal since 2009 and currently there are 4 hypothemia centres.

This restrospective study intends to characterize the patients with this pathology (HIE) in an ultra peripheral hospital. Since this hospital doesn't have the ability to treat with induced hypothermia, our eligible newborns were transferred to a central hypothermia centre. Clinical charts from 2011 to 2018 were analysed.

The first patient submitted to hypothermia was in 2012. Of the newborns admitted to this Neonatology unit, 17 were diagnosed with HIE, $47 \%$ were male. 8 were transferred while 9 remained and received supportive management. From the newborns submitted to hypothermia treatment, 2 developed Cerebral Palsy (CP) while the rest are considered to have a normal psychomotor development. In the group that remained, 3 developed CP. $82 \%$ of patients with HIE suffered acute fetal distress, being late decelerations and sustained bradycardia the most common causes. $82 \%$ had neonatal seizures and were treated with phenobarbital. Head MRI or aEEG are not available, to the pediatric population, at our hospital, making it difficult to assess severity which may lead to unnecessary transfers. Risk factors were present in some patients: 1 uterine rupture; 1 cord prolapse and 1 case of HIE due to Fetomaternal Transfusion. This study intends to demonstrate how important it is to be able to diagnose this condition early to arrange tranportation that isn't always available in the small window we have to start treatment.

\section{2}

\section{Neonatology}

Neonatal Sepsis and Meningitis Caused by Neisseria Oralis: A Case Report

Sangeetha Arjunan ${ }^{1}$, Andrew Eccelston ${ }^{1}$, Sima Svirpliene ${ }^{1}$, Callum McLoughlin ${ }^{1}$

${ }^{1}$ Paediatrics, Dumfries and Galloway hospital, UK

\section{Case:}

Female infant was born by Emergency Caesarian Section for Foetal distress at gestational age of $38+4$ weeks with a birth weight of $2250 \mathrm{~g}$. The delivery was complicated by prolonged rupture of membranes and foul smelling liquor. She was born in poor condition requiring resuscitation .She was admitted to Neonatal unit for IV antibiotics. Her Initial CRP on admission was raised at 46 with a further increase in CRP to 97 on day 2 of life. Her blood culture and CSF culture grew Neisseraia Oralis, sensitive to Cefotaxime. She was treated with IV cefotaxime for 3 weeks. Her infection markers normalised on Day 6 of life. No major morbidity identified at discharge.

Laboratory Investigations:

\begin{tabular}{llll}
\hline & & & Day 6 \\
CRP & 46 & 97 & 5 \\
Bilirubin & - & 88 & 88 \\
Blood Neutrophils & 9.4 & 19 & 2.2 \\
Platelets & 211 & 228 & 426 \\
Blood culture & Neisseria Oralis & & \\
CSF culture & Neisseria Oralis & & \\
CSF PCR & Negative & & \\
\hline
\end{tabular}

\section{Discussion:}

Neisseria Oralis are usually harmless inhabitants of otherwise asymptomatic persons' upper respiratory mucosal surfaces. There have been 7 Serotypes of Nesseria Orlis identified from Healthy Oral mucosa Recently this commensal has been evolving into a pathogen. There has been a previous case report of N.Oralis Cystitis in a Diabetic Patient. Further studies are needed to identify the risk factors that leeds to changes in physiology for these harmless inhabitants to become Pathogens.

\section{9}

Neonatology

Evaluation of Professional Practices Prior to and Subsequent to Training in the use of Phototherapy for Preterm Infants in an Intensive Care Unit

Camille Szcrupak $^{1,2}$, Fatima Chahin-Yassin ${ }^{I}$, Stéphane Delanaud ${ }^{1}$, Estelle Durand $^{\prime}$, Pierre Tourneux ${ }^{1,2}$

${ }^{1}$ PériTox Laboratory UMR-I 01, UFR de Médecine, University of Picardie Jules Verne, France

${ }^{2}$ Pediatric intensive care unit, CHU Amiens-Picardie, France

Background: Newborn jaundice is one of the most common conditions for preterm infants. Phototherapy is the treatment of choice to reduce bilirubin levels. Untreated severe jaundice can cause permanent brain damage but phototherapy has to be carefully used because of its side effects such as melanocytic nevus development.

There are international guidelines regarding the use of phototherapy for preterm infants in order to reduce long-term adverse effects and to improve its efficacy.

Objective: The purpose of this audit was to determine whether the training of staff could improve the compliance with phototherapy good clinical practice guidelines for infants 35 weeks of gestation in incubators in a level III hospital unit.

Methods: The first part of this evaluation was a six-month retrospective study before training, the second part was a two-month prospective study after staff training. Data regarding prescription and indication were gathered and qualified (i.e. compliance with or variation from guidelines), and the two periods were compared.

Results: The study included 70 infants born at 28,6 weeks of gestation on average. 276 prescriptions were analysed. Prior to staff training only $75 \%$ of indications to phototherapy onset complied with guidelines versus $90 \%$ after training (p0,05). No side effect monitoring was prescribed prior to training (i.e $0 \%$ ) versus $78 \%$ after training $(\mathrm{p} 0,0001)$. Treatment 
efficacy monitoring with Transcutaneous Bilirubin Measurements was prescribed in $44,6 \%$ of cases prior to training versus $81 \%$ after training $(p=0,059)$. The prescribing doctor's signature was found in $84 \%$ of cases prior to training versus $92 \%$ after training (p0,0001).

Conclusion: Regarding its side effects, phototherapy must be used only when indicated and with careful monitoring for complications. This clinical audit showed a better compliance with international standards after training with regard to indications and prescription. Training must be considered as an asset to improve clinical practice in the NICU.

\section{7}

\section{Neonatology}

Quality Improvement Through the Introduction of a Standardised Incident and Excellence Reporting Form on a Zambian Neonatal Unit

Rachael Talbot ${ }^{1}$

${ }^{T}$ High Dependency Unit, Alder Hey Children's NHS Foundation Trust, UKNeonatal Unit, University Teaching Hospital, Zambia

Background: It has long been acknowledged that learning from critical incidents is imperative to improving patient safety and the incident report (IR) is now one of the most widely used methods of improving it. The voluntary IR is a standard approach with higher numbers of IRs associated with a generally more effective safety culture. Improvements can be much simpler to action when unit-based IRs are utilized.

On the Neonatal Intensive Care Unit (NICU) in University Teaching Hospital, Lusaka, Zambia, IRs were written but with varying quality as there was no formal structure; they consisted of freehand text on paper. Informal discussions with nursing staff revealed that they had reservations about completing IRs due to concerns of repercussions. These concerns are common in nurses who report incidents and are sometimes well founded. Excellence reporting was not utilised but has been recognised as another tool to aid patient safety.

Methods: All documented IRs on NICU over a two-year period were retrospectively reviewed. Data on the shift and location of incidents was collected when possible. Common themes were identified and data on their occurrence in incidents collected. Based on this, a standardised incident and excellence reporting form was devised and teaching on its use delivered in a series of sessions.

Results: Further data collection awaited. Informal discussions with NICU nursing staff show that they understand and appreciate the importance of IR use but that they still have concerns about potential repercussions they could face. After an initial teaching session on the topic, attendees stated that they felt less reserved about completing an IR but more sessions are planned.

Conclusion: The use of IRs is important and staff do understand and recognise this. They need ongoing support to feel confident using IRs and this should be included as part of a wider culture change.

\section{4}

\section{Neonatology}

Developing an Education Package for a Tertiary Neonatal Unit in a Lower-Middle Income Country

Rachael Talbot ${ }^{1,3}$, Aoife Hurley ${ }^{2,3}$

${ }^{T}$ High Dependency Unit, Alder Hey Children's NHS Foundation Trust, $U K$

${ }^{2}$ Neonatal Unit, Leeds Teaching Hospitals NHS Trust, UK

${ }^{3}$ Neonatal Unit, University Teaching Hospital, Zambia

Background: The Neonatal Intensive Care Unit (NICU) in University Teaching Hospital, Lusaka, is Zambia's only tertiary neonatal unit. It is a busy unit with eighty to a hundred infants. On average, there are twelve infants admitted daily, with the average monthly mortality being $25.1 \%$. In 2015, the Zambian neonatal mortality rate was 21 per 1,000 live births. Currently, there is no formal neonatal nursing training. Discussions with both nursing and medical staff highlighted concerns about inadequate training. This correlated with observed suboptimal clinical practice over a two-month period

Objective: To bridge the knowledge gaps in neonatal nursing through delivering an education package that was established by a UK paediatric nurse with support from a UK paediatric trainee.

Methods: A pilot course of three free separate masterclass sessions were delivered. Topics were based on areas suggested by the senior NICU sisters, neonatal consultants, discussions with NICU nursing staff and the clinical practice observed. Teaching interventions were free or lowcost, aiming to improve short- and long-term outcomes for neonates. These included therapeutic positioning, when to call for help, hypoglycaemia guidelines and neonatal life support. Attendees completed a quiz prior to the study session and afterwards, as well as a post course feedback form.

Results: The course has been attended by nursing and midwifery students and NICU staff. Almost universally, post study session quiz scores were higher than pre study session quiz scores, suggesting an increase in theoretical knowledge on the subject matter. All attendees stated that they would recommend this study session to their peers.

Conclusion: A study day involving practical and theoretical knowledge is welcomed locally, has improved knowledge base and has significant potential for future development. NICU staff will complete the quiz again four weeks after they attended a session. Further sessions are planned, with local staff continuing this work.

\section{1}

Neonatology

Neonatal Effects of Substance Abuse During Pregnancy

Sara Todo Bom Costa ${ }^{1,3}$, David Lito ${ }^{2}$

${ }^{T}$ Pediatric, Pediatric Departament, Santa Maria University Hospital, North Lisbon Hospital Center, Portugal

${ }^{2}$ Pediatric, Pediatric Departament, Vila Franca de Xira Hospital, Portugal

${ }^{3}$ Pediatric, Pediatric Departament, Vila Franca de Xira Hospital, Portugal

Background: Substance use during pregnancy can have harmful perinatal effects. Its impact depends on the drugs used. These women frequently present psychiatric comorbidities, and experience inadequate prenatal care and dysfunctional maternal-infant interactions. Depending on the drug, their children can suffer Neonatal Abstinence Syndrome (NAS) and require pharmacological treatment and extended length of hospital stay.

Objective: The aim of this study was to understand the effects of drug exposure during pregnancy on newborns.

Methods: The authors reviewed the medical records of all newborns exposed to maternal drug use (legal and illicit) during pregnancy over a 6-year period at a second level hospital.

Results: We identified 17 newborns exposed to drugs during pregnancy. Mothers had a median age of 36 years (IQR 13.5), seven (41\%) had correctly monitored pregnancies, $6(35 \%)$ had positive serologic screenings ( $5 \mathrm{HCV}, 1$ syphilis, $1 \mathrm{HIV}, 1 \mathrm{HBV})$. Polysubstance use was observed in 4 women. The psychotropic substances consumed were opioids (7), cannabis (5), cocaine (4), alcohol (1). Three women were treated with benzodiazepines and could not step-down the treatment by the time of birth. Nine women confirmed tobacco consumption. Only 2 women did not present social risk factors. Of the 17 neonates, 8 were male, 3 were preterm, 11 were small for gestational age. Of the 11 neonates at risk for NAS (those exposed to opioids, benzodiazepines and alcohol), 7 (63\%) experienced NAS symptoms, but only $4(57 \%)$ met the criteria for 
treatment -3 were treated with morphine and one with methadone. The duration of treatment was $40,0 \pm 12,2$ days ranging from 27 to 54 days.

Conclusion: The most commonly used drugs were opioids, followed by cannabis and cocaine. Most women didn't seek proper antenatal care. Newborns whose mothers have a history of drug use are at risk and should be closely monitored.

\section{3}

In Vitro Fertilization and Autistic Spectrum Disorder: Clinical Experience- Review of Literature

Efitsek Tsekoura ${ }^{l}$

${ }^{T}$ Developmental Follow Up Clinic, Pediatric Department, Asklepieion General Hospital, Greece

Background: In 1978 the first baby was born in Britain after in vitro fertilization, and it was reported as a healthy female neonate. Since then, according to WHO, there are about 8.000 .000 children born with IVF. In 1984 for the first time it was possible to transfer frozen embryos and in 1991 it was the first successful intracytoplasmic sperm injection (ICSI) to treat mainly male infertility problems. ICSI is now used in more that $80 \%$ of couples treated with IVF. Data for neurodevelopmental outcome of children born with IVF are obscure and inconclusive mainly due to research methodology problems and to factors that can affect outcome such as IVF technique per se, ICSI, culture medium used, transfer of frozen of fresh embryos, the infertility problems and perinatal co factors such as prematurity, multiple birth, parental age. Literature data show a possible link with increased Autistic Spectrum Disorders (ASD) incidence, ADHD, Specific Learning Difficulties (SLDs) and Behavioral problems such as internalizing and externalizing behavior especially with ICSI technique.

Aim: Our aim was to analyze our data from the Developmental and Behavioral Pediatrics Outpatient Clinic and to review current literature.

Material-Methods: We retrospectively analyzed medical records of children referred to our Developmental-Behavioral Pediatrics Outpatient Clinic for evaluation with IVF history and we reviewed recent publications from pubmed library. We excluded extremely and very preterm children, congenital anomalies, syndromes and cerebral lesions such as hemorrhages.

Results: There are no studies or systematic meta analysis to associate increased ASD in relation to IVF. There is a possible link between ICSI and ASD, mental retardation, SLDs. Nevertheless literature studies are of weak methodology mainly due to diagnostic criteria for ASD. In our population we studied medical records of 133 children with IVF history. Boys $61 \%$, girls $39 \%$. The educational level of mother and father was in the middle and upper range in 83 and $86 \%$ respectively. Mean paternal and mean maternal age at conception was 37 and 38 years respectively. ASD of any severity was detected in $9.02 \%$ of cases and of high severity in $1.5 \%$. The relation between boys and girls in ASD cases was 2:1. ADHD detected in $8.2 \%$, internalizing and externalizing behavior in $33.08 \%$ and SLDs in $26.3 \%$

Discussion: In our population we recorded increased ASD incidence amongst children with IVF history $(9.02 \%)$. Nevertheless the incidence of severe cases were close to the general population (1.5\%). In our IVF population there was weaker association between ASD and male sex compared to the general population $(2: 1 \mathrm{vs} 4: 1)$. Literature data need further evaluation regarding entry criteria, methodology and ASD diagnostic criteria in order to come in a clear conclusion between IVF and ASD.

\section{4}

\section{Neonatology}

Rational Use of Antibiotics in Preterm Neonates

Harshal Wagh ${ }^{1}$

${ }^{T}$ Pediatrics, Bharati Vidyapeeth (Deemed to be) University, Medical College \& Hospital, Sangli, India
Background: Neonates are in high risk of developing blood stream infections because of their immature immune system. This is more in case of low birth weight and preterms who are at a higher risk than full term newborns. It is common practice amongst pediatricians to administer antibiotics to at risk neonates without evidence of clinical or microbiologically proven sepsis which has led to resistance to antibiotics.

Objectives: To manage low infection risk preterms without antibiotics and determine their outcome; to find out relation between maternal risk factors, gestational age \& birth weight with regard to need for antibiotics. Methods: Inborn Preterm babies with low risk factors for infection were closely monitored without antibiotics. Babies were started on antibiotics only when sepsis was considered. Detailed maternal history with regard to obstetric risk factors was recorded. Gestational age and birth weight were correlated with need for antibiotics. Babies were followed up till discharge or death.

Results: Of 296 babies admitted in NICU during study period, 74 eligible preterms were enrolled. Sixty two (83\%) out of 74 did not require antibiotics. There was statistically significant difference in mean birth weight $(1.765+0.37 \mathrm{kgs})$ between neonates who required antibiotics and those who did not. (p-value 0.001 ). There was no significant association of gestational age with need for antibiotics. Maternal obstetric risk factors had significant association with need for antibiotics in the babies (p-val$\mathrm{ue}=0.025)$. Three babies $(4 \%)$ who received antibiotics developed NEC as against none in the non-antibiotics group. Only 2(16.3\%) out of the 12 babies in antibiotic group had positive blood cultures. Mortality was $4.1 \%$ Conclusion: Preterms with low risk factors can be managed without antibiotics. Need for antibiotics is strongly associated with maternal obstetric risk factors and low birth weight.

\section{1}

Neonatology

Family Integrated Care in Neonatal Unit: A Service Improvement Project

Muhammad Salman Khurshid ${ }^{1}$, Natalie Webster ${ }^{1}$, Leanne Cumming ${ }^{1}$, Imray Amanda ${ }^{1}$, Nicole Bauwens ${ }^{T}$

${ }^{I}$ Neonatal Intensive Care (NICU), Aberdeen Maternity Hospital, UK

Background: Preterm delivery is associated with an increased risk of anxiety and maternal depression. A recent UK wide survey suggests about one third of parents feel they are not involved in the discussion around the management of their child. A family integrated approach can help overcome some of these challenges.

Objective: This service improvement project was initiated to identify the challenges of involving families in neonatal care and implement changes safely and effectively.

Methods: The nursing colleagues took the initiative and challenged the traditional model of professional led care. A family integrated care working group comprising of professionals from different teams was established. Changes were introduced in a stepwise manner. This included unrestricted access for parents, Parents education sessions, empowering parents to get involved in care, psychological support. Communication among the team was ensured through regular meetings and social media including WhatsApp, twitter. Surveys were done among health professionals and parents to measure outcomes.

Results: Parents now have $24 \mathrm{hr}$ unrestricted access to the neonatal unit. They get a cotside update for their child and present key day to day changes in war round. Regular staff and parent education sessions are being held. Positive impact for parents included a reduction in anxiety, pain, and promotion of breastfeeding. A survey among health professionals identified the need for further research into parental presence during procedures. Further projects include analysis of the impact of preterm delivery on siblings and wider family.

Conclusion: Family integrated care bring positive changes to parental psychological and physical health. This can be a challenge in an intensive 
care environment. Collaborative leadership can be key to bring changes in a multi-professional team.

1017

Neonatology

Home Phototherapy - Audit of Current Practice and Assessment of Family Feedback Caroline Woolley ${ }^{1}$, Richard Mupanemunda ${ }^{l}$

${ }^{T}$ Neonatal Unit, Heartland Hospital, UK

Background: Neonatal jaundice is a common condition in babies. Treatment with phototherapy often means babies remain in hospital or require readmission. The provision of home phototherapy services would reduce hospitalisation. It is a service that Heartlands Hospital is keen to develop and between April and November 2018 they trialled home phototherapy on 14 babies.

Objective: Ensure compliance with our local guideline and gain feedback of family's experiences of the service.

Methods: Performed a retrospective audit of our local guidelines, along with assessment for each baby of required blood tests and days phototherapy. After completing treatment parents completed a feedback questionnaire about their experience and we collated responses.

Results: All 14 babies had the correct paperwork before commencing home phototherapy. All of the babies had their blood group checked along with a full blood count and DCT, but only 4 babies had a conjugated bilirubin tested. The average days spent on home phototherapy was 1.5 days(range 1-3days)and 22 days of home phototherapy were given in total. Each baby required either 2 or 3 bilirubin measurements whilst on home phototherapy. All babies had phototherapy stopped appropriately and rebound levels remained well below treatment threshold. 1 baby required readmission for more intensive phototherapy. We received 12 responses from the questionnaires. 11 families had only good comments about the service. 1 family had mixed comments, however the negatives given related to equipment. Everyone commented on how home phototherapy had allowed them to be together as a family and all families said they would chose to use the service again.

Conclusions: Home phototherapy was a popular alternative to treatment in hospital for all the families who received it. We did not identify any major problems during the trial period. The development of a home phototherapy service would be beneficial for families and the hospital.

759

\section{Neonatology}

Improving Hypothermia in Preterm Infants at Admission to a Tertiary Neonatal Unit- a Quality Improvement Project

Caroline Woolley ${ }^{1}$, Laura Gilbert ${ }^{1}$, Gurpreet Sunsoay ${ }^{1}$, Danika Simkins ${ }^{1}$, Rachel OSullivan $^{1}$, Pinki Surana ${ }^{1}$

${ }^{l}$ Neonatology, Heartlands Hospital, UK

Background: Hypothermia in preterm infants is associated with increased morbidity and mortality. The National Neonatal Audit Programme (NNAP) recommends temperature between $36.5-37.5^{\circ} \mathrm{C}$ at admission to the neonatal unit (NNU) for babies 32weeks gestation. Our tertiary NNU was a national outlier for this NNAP measure for two consecutive years.

Objective: To improve rates of normothermia at admission for infants 32 weeks.

Methods: A baseline audit was conducted on infants born 32 weeks to ascertain areas for improvement. Incomplete documentation of thermalcontrol measures, variable theatre temperature, procedures at delivery and mode of transportation were linked with hypothermia. A quality improvement (QI) initiative was implemented, raising staff awareness about the importance of euthermia. Posters (Fig 1) highlighting good practice were displayed around NNU. A prospective audit was conducted between Dec18-Feb19.

Results: 21 babies included in the audit had a mean gestation of $28+$ 5 weeks $(23-31+3$ weeks) and birth-weight of $1150 \mathrm{~g}(500 \mathrm{~g}-1790 \mathrm{~g})$. All had temperature measured after admission and $62 \%$ were normothermic, $5(24 \%)$ were hypothermic and 3(14\%) were hyperthermic. $18(86 \%)$ had their temperature measured in delivery suite. $16(76 \%)$ used the transport incubator and $15(72 \%)$ transwarmers. Of the 5 hypothermic babies, 3 were hypothermic in delivery-suite. 4 babies were hyperthermic before transfer and 3 remained hyperthermic. Only $37 \%$ of babies born in theatre compared to $75 \%$ of babies born in delivery rooms were normothermic at admission. 4 of the 5 hypothermic babies were born in theatre (mean theatre temperature $23^{\circ} \mathrm{C}$ ). Time of birth and transfer time to NNU did not influence the admission temperature.

Conclusions: Following the QI, rates of normothermia at admission improved from $52 \%$ to $62 \%$. QI highlighted importance of establishing normothermia before transfer to facilitate normothermia at admission and hence the importance of actively measuring the temperature early during stabilisation and adjusting accordingly. Monitoring theatre temperature will be part of our continuous QI project.

\section{9}

\section{Neonatology}

Perinatal Risk Factors in Cochlear Implant Candidates: An Egyptian Sample

Dina Y. Elalfy ${ }^{1}$, Hassnaa Othman Mohammed ${ }^{2}$, Reham Senosy ${ }^{3}$

${ }^{T}$ Lecturer of Pediatric Diseases, Department of Medical Studies for Children, Faculty of Postgraduate Childhood Studies, Ain Shams University, Egypt

${ }^{2}$ Lecturer of Phoniatrics, Department of Medical Studies for Children., Faculty of Postgraduate Childhood Studies, Ain Shams University, Egypt ${ }^{3}$ Lecturer of Audiology, Department of Medical Studies for Children, Faculty of Postgraduate Childhood Studies, Ain Shams University, Egypt

Background: The rate of admission to neonatal intensive care units (NICU) has increased in the past few years. There also has been an improvement regarding the outcome of these NICUs which include a decreased infant mortality rate. However, the impact of these innovative interventions must be reviewed for causality. Several studies have attributed the presence of sensori- neural hearing loss (SNHL) from birth to early childhood to problems related to the birth process. As the burden of hearing impairment falls on the future of the child, it is crucial to investigate the association of adverse perinatal conditions with such a problem.

Objective: To identify the most significant perinatal risk factor among children with severe to profound SNHL who were candidates for cochlear implantation.

Methods: A retrospective study of perinatal risk factors in a group of 129 children ( 71 males \& 58 females) with mean age 61.49 months, who were implemented in the cochlear implant program in one of the Egyptian centers. The assessment protocol included a clinical interview with the child's parent and focused on personal history as well as the history of the perinatal period.

Results: Positive consanguinity was found in $29.5 \%$ of cases in whom $51.3 \%$ also had a positive family history in other siblings. NICU graduates accounted for $11.6 \%$ of cases in which $46.6 \%$ had neonatal jaundice, $26.6 \%$ had +ve consanguinity and $13.3 \%$ had cyanosis.

Conclusion: Among the cochlear implant candidates the most common identified perinatal risk factor was NICU admission, mostly due to neonatal jaundice. In $39.5 \%$ of the sample perinatal risk could not be identified. These findings highlight the cost- effectiveness of the universal neonatal hearing screening (UNHS) that is not yet implemented in our country. 
185

\section{Neonatology}

3D Echocardiographic Evaluation of the Right Ventricle before and after Inhaled Nitric Oxide Therapy for Persistent Pulmonary Hypertension of the Newborn

Yutaka Yamamoto $^{1,2,3}$, Yoshinori Kohno ${ }^{1}$, Masashi Kondo ${ }^{I}$, Toshinari Kohyama $^{I}$, Hisashi Fukutomi ${ }^{I}$, Hiroki Ohtsuka ${ }^{1}$

${ }^{I}$ Department of Neonatology, Gifu Prefectural General Medical Center, Japan

${ }^{2}$ Department of Neonatal Intensive Care Unit, Gifu Prefectural General Medical Center, Japan ${ }^{3}$ Department of Pediatrics, Graduate School of Medicine, Gifu University, Japan

Introduction: In contrast to the near conical shape of the left ventricle $(\mathrm{LV})$, the right ventricle (RV) is more triangular in shape when viewed from the front and it curves over the LV. Therefore, it is difficult to evaluate the RV with 2D Echocardiograpy.

Purpose: The purpose of this study is to examine the RV before and after inhaled nitric oxide therapy (iNO) for persistent pulmonary hypertension of the newborn (PPHN) with Real-time three-dimensional echocardiography (RT3DE) qualitatively and quantitatively.

Methods: Five infants with PPHN were prospectively enrolled, 2 with severe birth asphyxia, 2 with extremely low birth weight and 1 with trisomy 21. RT3DE images were also gathered over using a matrix array transducer for several seconds. The volume analysis was performed on semi-automatic software TomTec RV Function $2.0^{\mathrm{TM}}$ and TomTec LV Analysis 3.1 ${ }^{\mathrm{TM}}$.

Results: In all infants, the RV ejection fraction improved after iNO (median (interquartile range) $28.2 \%$ before $\mathrm{iNO}(21.3 \%-38.4 \%) \rightarrow 40.5 \%$ after iNO (35.0\%-44.6\%)). Furthermore, the ventricular systolic time difference (RV systolic time - LV systolic time) were shortened after iNO(median(interquartile range) $84.7 \mathrm{~ms}$ before iNO (57.1 ms - 100.9 $\mathrm{ms}) \rightarrow 25.4 \mathrm{~ms}$ after iNO $(12.7 \mathrm{~ms}-72.5 \mathrm{~ms})$ ).

Conclusions: RT3DE is easier, faster and more precise method of evaluating RV function in infants than 2D. Furthermore, it needs to be emphasized that it is possible to evaluate the ventricular interdependence only with RT3DE.

\section{4}

\section{Neonatology}

Analysis of Incidence and Risk Factors of Severe Bronchopulmonary Dysplasia (sBPD) among Very Low Birth Weight Infants

Chang-Yo Yang ${ }^{l}$, Shih-Ming Chu ${ }^{l}$, Reyin Lien ${ }^{l}$, Ren-Huei Fu ${ }^{l}$, Jen-Fu Hsu $^{I}$, Ming-Chou Chiang ${ }^{I}$

${ }^{1}$ Division of Neonatology, Department of Pediatrics, Chang Gung Memorial Hospital, Chang Gung University College of Medicine, Taiwan

Background: Although we've made big strides in perinatal care, BPD remains the most common late morbidity of preterm birth, but many controversies persist regarding how to best define BPD, grade its severity, and prevent disease.

Objectives: The aim was to determine the current incidence and analyze possible risk factors of severe $\mathrm{BPD}(\mathrm{sBPD})$ in very-low-birth-weight (VLBW) infants.

Materials and Methods:This was a retrospective study by reviewing medical records of all VLBW infants $(B W \leq 1500 \mathrm{~g})$ admitted to NICU of Chang Gung Children's Hospital over 2 years period. All VLBW infants diagnosed for BPD were included and data were retrieved retrospectively and analyzed for maternal medical and obstetric and neonatal risk factors using logistic regression.

Results: Of the 472 VLBW infants who were enrolled, 412 (87\%) survived. BPD was noted in 247 (59.9\%) of them. There were $14.3 \%, 38.1 \%$ and $7.5 \%$ of mild, moderate, and severe BPD, respectively. Neonates of $\mathrm{GA} \leq 25 \mathrm{wks}$ and 26-28 wks had increased odds ratio of 8.49 and 3.19 for the development of severe BPD, compared to those of 29 wks or more. Pulmonary hypertension complicates the course in up to $25 \%$ of patients with $\mathrm{SBPD}$. By univariant analysis for $\mathrm{SBPD}$, PDA, sepsis, and ROP are strongly associated with the development of sBPD $(P$ 0.05). However, using multiple logistic regression analyses for sBPD, only chorioamnionitis [odds ratio (OR), 2.48; confidence interval (CI), 1.32 to 4.7], birth weight (OR, 0.99; CI, 0.996 to 0.999), and duration of ventilation (OR, 1.06; CI, 1.04 to 1.08) were factors predictive of development of sBPD.

Conclusion: The incidence of severe BPD among VLBW infants was $7.5 \%$. Infants of

673

Neonatology

Swaddling Technique for Alternative to Sedative Administration during Brain Magnetic Resonance Imaging in Very Low Birth Weight Infants

Yeong Myong Yoo ${ }^{1}$, Hye Jung Park ${ }^{1}$, Moon Sung Park ${ }^{1}$, Jang Hoon Lee ${ }^{l}$ ${ }^{T}$ Department of Pediatrics, Ajou University Hospital, South Korea

Background: Magnetic resonance imaging (MRI) of neonates is an essential modality to diagnose brain injury in premature infants and evaluate growth development. Newborns are often sedated during MRI because this modality is required minimal motion to acquire qualified process. However, sedation may cause adverse effects and induces potential neurotoxicity.

Objective: This study investigated the neonate cases that were performed MRI to describe effectiveness of swaddling by comparing with administration of sedative agents.

Methods: This was a retrospective study of premature infants who received swaddling or sedatives (chloral hydrates or benzodiazepines) for brain MRI in the neonatal intensive care unit (NICU). Among the enrolled infants $(\mathrm{N}=79), 40.5 \%(\mathrm{~N}=32)$ were included in the swaddling group and $59.5 \%(\mathrm{~N}=47)$ were in the sedative group.

Results: There were no differences in gestational age (GA) at birth $\left[29^{+3}\right.$ $\pm 4^{+1}$ vs. $\left.29^{+6} \pm 5^{+1}, P=0.439\right]$ and corrected GA at conducting MRI $\left[37^{+4}\right.$ $\pm 2^{+3}$ vs. $\left.37^{+6} \pm 3^{+1}, P=0.649\right]$. In univariate analysis, mean body weight at birth [1188.9 $( \pm 246.4)$ vs. $1167.4( \pm 259.9), P=0.714]$ and at conducting MRI [2439.7 $( \pm 484.4)$ vs. $2250.3( \pm 548.7), P=0.119]$ were similar between groups. There was no significant difference in failure rate of performing MRI [ $12.5 \%(\mathrm{~N}=4)$ vs. $4.3 \%(\mathrm{~N}=2), P=0.174]$. Average of MRI scanning time was longer in swaddling group than sedation group [76.5 ( \pm 20.3$)$ vs. $61.5( \pm 13.6), P=0.001]$. Mean time from end of scan to restart feeding was shorter in swaddling group than sedation group [57.9 $( \pm 48.2)$ vs. $74.0( \pm 70.2), P=0.233]$.

Conclusion: The failure rate of swaddling technique was comparable to sedative administrations during MRI. Furthermore, swaddling may reduces not only adverse events but also neurotoxic effects compare to administration of sedative agents. Therefore, it can be considered prior to an MRI scan, avoiding sedation or anesthesia.

\section{6}

Neonatology

Outcomes of Critical Congenital Heart Disease in Premature Infants at a Single Referral Centre

Young Mi Yoon ${ }^{1}$, Seong Phil Bae ${ }^{2}$, Yoon-Joo Kim ${ }^{3}$, Mi Kyoung Song ${ }^{4}$, Seung Han Shin ${ }^{5}$, Jae Gun Kwak ${ }^{6}$, Woong-Han Kim ${ }^{7}$, Ee-Kyung Kim ${ }^{8}$, Han-Suk Kim ${ }^{9}$

${ }^{I}$ Department of Pediatrics, Jeju National University Hospital, South Korea

${ }^{2}$ Department of Pediatrics, Soonchunhyang University Hospital, South Korea 
${ }^{3}$ Department of Pediatrics, Jeju National University Hospital, South Korea

${ }^{4}$ Department of Pediatrics, Seoul National University, South Korea

${ }^{5}$ Department of Pediatrics, Seoul National University, South Korea

${ }^{6}$ Department of Thoracic and Cardiovascular Surgery, Seoul National University, South Korea

${ }^{7}$ Department of Thoracic and Cardiovascular Surgery, Seoul National University, South Korea

${ }^{8}$ Department of Pediatrics, Seoul National University, South Korea

${ }^{9}$ Department of Pediatrics, Seoul National University, South Korea

Background: Despite advances in neonatal intensive care and surgical procedures, perinatal mortality rates for premature neonates with congenital heart disease (CHD) are relatively high, and survivors are at risk for short-term morbidity and adverse neurological outcomes.

Objective: We aimed to describe the outcomes of premature neonates with critical CHD and to identify the risk factors associated with inhospital mortality at a single referral tertiary centre.

Methods: This was a retrospective cohort study of premature neonates with critical CHD admitted to the neonatal intensive care unit from January 2005 to December 2016.

Results: A total of 78 premature neonates were enrolled (male 38, 48.7\%). The median gestational age (GA) at birth was 34.9 (26.7-36.9) weeks, and the median birth weight was $1.91(0.53-4.38) \mathrm{kg}$. A surgical or percutaneous intervention was performed in 68 patients with a median GA at birth of 34.7 (26.7-36.8) weeks and a median birth weight of 1.92 (0.53-4.38) kg. The in-hospital survival rate was $76.9 \%$ among all enrolled preterm infants and $86.8 \%$ among patients who received an intervention. Very low birth weight (VLBW), persistent pulmonary hypertension of the newborn (PPHN), bronchopulmonary dysplasia (BPD), and CHD complexity were independently associated with in-hospital mortality. For the 68 premature neonates undergoing cardiac interventions, independent risk factors for mortality were VLBW, BPD, and CHD complexity.

Conclusion: For premature neonates with critical CHD, VLBW, PPHN, $\mathrm{BPD}$, and CHD complexity were risk factors for mortality. Careful approaches for surgical intervention and prenatal care should be guaranteed according to the type of CHD and neonatal conditions.

Table 1. Critical congenital heart disease and mortality according to the modified RACHS

\begin{tabular}{|c|c|c|c|c|c|c|}
\hline & $\begin{array}{c}\text { Total } \\
(n=78)\end{array}$ & $\begin{array}{l}\text { Death } \\
(n=18)\end{array}$ & $\begin{array}{l}\text { Mortality } \\
\text { rate (\%) }\end{array}$ & $\begin{array}{c}\text { Intervention } \\
\quad(\mathbf{n}=68)\end{array}$ & $\begin{array}{l}\text { Death } \\
(n=9)\end{array}$ & $\begin{array}{c}\text { Mortalit } \\
\text { y rate } \\
(\%)\end{array}$ \\
\hline Modified RACHS 2 & $29(37.2 \%)$ & 2 & 6.9 & $29(42.6 \%)$ & 2 & 6.9 \\
\hline CoA requiring neonatal surgery & $4(5.1 \%)$ & 1 & 25.0 & $4(5.9 \%)$ & 1 & 25.0 \\
\hline TOF & $15(19.2 \%)$ & 0 & 0 & $15(22.0 \%)$ & 0 & 0 \\
\hline DORV & $2(2.6 \%)$ & 1 & 50.0 & $2(2.9 \%)$ & 1 & 50.0 \\
\hline PS with neonatal intervention & $7(9.0 \%)$ & 0 & 0 & $7(10.3 \%)$ & 0 & 0 \\
\hline Aortopulmonary window & $1(1.3 \%)$ & 0 & 0 & $1(1.5 \%)$ & 0 & 0 \\
\hline Modified RACHS 3 & $24(30.7 \%)$ & 4 & 16.7 & $21(30.9 \%)$ & 2 & 9.5 \\
\hline TGA without VSD & $\hat{3}(3.8 \%)$ & 0 & 0 & $3(4.4 \%)$ & 0 & 0 \\
\hline CAVSD & $3(3.8 \%)$ & 2 & 66.7 & $1(1.5 \%)$ & 0 & 0 \\
\hline DORV with CAVSD & $1(1.3 \%)$ & 0 & 0 & $1(1.5 \%)$ & 0 & 0 \\
\hline CoA with VSD & $5(6.4 \%)$ & 0 & 0 & $4(5.9 \%)$ & 0 & 0 \\
\hline PA with VSD & $12(15.4 \%)$ & 2 & 16.7 & $12(17.6 \%)$ & 2 & 16.7 \\
\hline Modified RACHS 4 & $6(7.7 \%)$ & 1 & 16.7 & $5(7.4 \%)$ & 0 & 0 \\
\hline AS requiring neonatal surgery & $1(1.3 \%)$ & 0 & 0 & $1(1.5 \%)$ & 0 & 0 \\
\hline Internuption of aortic arch & $1(1.3 \%)$ & 1 & 100.0 & - & - & - \\
\hline TAPVR & $3(3.8 \%)$ & 0 & 0 & $3(4.4 \%)$ & 0 & 0 \\
\hline Tnuncus arteriosus & $1(1.3 \%)$ & 0 & 0 & $1(1.5 \%)$ & 0 & 0 \\
\hline Modified RACHS 5 & $13(16.7 \%)$ & 5 & 38.5 & $10(14.7 \%)$ & 2 & 20.0 \\
\hline Single ventricle & $10(12.8 \%)$ & 3 & 30.0 & $8(11.8 \%)$ & 1 & 12.5 \\
\hline Complex CHD with heterotaxy & $\hat{3}(3.8 \%)$ & 2 & 66.7 & $2(2.9 \%)$ & 1 & 50.0 \\
\hline Modified RACHS 6 & $5(6.4 \%)$ & 5 & 100.0 & $3(4.4 \%)$ & 3 & 100.0 \\
\hline HLHS & $5(6.4 \%)$ & 5 & 100.0 & $3(4.4 \%)$ & 3 & 100.0 \\
\hline Cardiomyopathy with PS & $1(1.3 \%)$ & 1 & 100.0 & - & - & - \\
\hline
\end{tabular}

Table 2. Demographics of study population according to the in-hospital mortality

\begin{tabular}{|c|c|c|c|c|}
\hline & $\begin{array}{c}\text { Total } \\
(n=78)\end{array}$ & $\begin{array}{l}\text { Survivors } \\
(n=60)\end{array}$ & $\begin{array}{c}\text { Nonsurvivors } \\
(n=18)\end{array}$ & $P$-value \\
\hline Male gender & $38(48.7)$ & $29(48.3)$ & $9(23.7 \%)$ & 1.000 \\
\hline GA at birth (week) & $34.9(26.7-36.9)$ & $34.7(27.6-36.9)$ & $35.1(26.7-36.9)$ & 0.533 \\
\hline $\mathrm{GA}<32$ at birth (week) & $10(12.8)$ & $3(5.0)$ & $7(70 \%)$ & 0.214 \\
\hline $32 \leq \mathrm{GA}<34$ at birth (week) & $14(17.9)$ & $14(2 \hat{3} .3)$ & $0(0.0)$ & \\
\hline $34 \leq \mathrm{GA}<37$ at birth (week) & $54(69.2)$ & $43(71.7)$ & $11(20.3 \%)$ & \\
\hline Birth weight (kg) & $1.91(0.53-4.38)$ & $1.93(0.53-4.38)$ & $1.80(0.61-3.30)$ & 0.313 \\
\hline Very low birth weight & $11(14.1)$ & $5(8.3)$ & $6(33.3)$ & 0.015 \\
\hline Small for GA & $32(41.0)$ & $24(40.0)$ & $8(44.4)$ & 0.737 \\
\hline foetal hydrops & $3(3.9)$ & $1(1.7)$ & $2(11.1)$ & 0.131 \\
\hline PPHN & $4(5.1)$ & $1(1.7)$ & $3(16.7)$ & 0.036 \\
\hline Noncardiac structural anomaly & $14(17.9)$ & $13(21.7)$ & $1(5.6)$ & 0.169 \\
\hline Chromosome anomaly & $7(9.0)$ & $5(8.3)$ & $2(11.1)$ & 0.659 \\
\hline \multicolumn{5}{|l|}{ Morbidity } \\
\hline Sepsis & $1(1.3)$ & $0(0.0)$ & $1(5.6)$ & 0.231 \\
\hline NEC & $6(7.7)$ & $3(5.0)$ & $3(16.7)$ & 0.132 \\
\hline IVH $\geq$ grade II & $3(3.8)$ & $2(3 . \hat{3})$ & $1(5.6)$ & 0.550 \\
\hline PVL & $4(5.1)$ & $2(3.3)$ & $2(11.1)$ & 0.226 \\
\hline BPD & $12(15.4)$ & $5(8 . \hat{)})$ & $7(38.9)$ & 0.005 \\
\hline ROP & $2(2.6)$ & $2(3.3)$ & $0(0.0)$ & 1.000 \\
\hline$m-\mathrm{RACHS} \geq 5$ & $18(2 \hat{3} .4)$ & $8(13.3)$ & $10(58.8)$ & 0.000 \\
\hline Single-ventricle physiology & $15(19.2)$ & $7(11.7)$ & $8(44.4)$ & 0.002 \\
\hline Preintervention PGE1 & $14(17.9)$ & $7(11.7)$ & $7(38.9)$ & 0.014 \\
\hline Heterotaxy syndrome & $5(6.4)$ & $2(3.3)$ & $3(16.7)$ & 0.078 \\
\hline Intervention & $68(87.2)$ & 59 (98.3) & $9(50.0)$ & 0.000 \\
\hline
\end{tabular}

Data was reported as number of patients(\%) or median(mininum-maxinum).

Table 3. Demographics of patients with cardiac intervention according to the in-hospital mortality.

\begin{tabular}{|c|c|c|c|c|c|}
\hline & $\begin{array}{l}\text { Total } \\
(n=68)\end{array}$ & $\begin{array}{l}\text { Survivors } \\
(n=59)\end{array}$ & $\begin{array}{c}\text { Nonsurvivors } \\
(n=9)\end{array}$ & $P$-value \\
\hline \multicolumn{2}{|c|}{ Male gender } & $34(50.0)$ & $30(50.8)$ & $4(44.4)$ & 1.000 \\
\hline \multicolumn{2}{|c|}{ GA at birth (week) } & $34.7(26.7-36.8)$ & $34.7(27.6-36.9)$ & $31.7(26.7-36.1)$ & 0.043 \\
\hline \multicolumn{2}{|c|}{$\mathrm{GA}<32$ at birth (week) } & $S(11.8)$ & $3(5.1)$ & $5(55.6)$ & 0.039 \\
\hline \multicolumn{2}{|c|}{$32 \leq \mathrm{GA}<34$ at birth (week) } & $14(20.6)$ & $14(23.7)$ & $0(0.0)$ & \\
\hline \multicolumn{2}{|c|}{$34 \leq \mathrm{GA}<37$ at birth (week) } & $46(67.6)$ & $42(71.2)$ & $4(44.4)$ & \\
\hline \multicolumn{2}{|c|}{ Birth weight $(\mathrm{kg})$} & $1.92(0.53-4.38)$ & $1.93(0.53-4.38)$ & $1.73(0.61-3.02)$ & 0.078 \\
\hline \multicolumn{2}{|c|}{ Very low birth weight } & $9(13.2)$ & $5(8.5)$ & $4(44.4)$ & 0.014 \\
\hline \multicolumn{2}{|l|}{ PPHN } & $1(1.5)$ & $1(1.7)$ & $0(0.0)$ & 1.000 \\
\hline \multirow[t]{5}{*}{ Morbidity } & Sepsis & $1(1.5)$ & $0(0.0)$ & $1(11.1)$ & 0.132 \\
\hline & NEC & $6(8.8)$ & $3(5.1)$ & $3(33.3)$ & 0.027 \\
\hline & IVH $\geq$ grade II & $2(2.9)$ & $2(3.4)$ & $0(0.0)$ & 1.000 \\
\hline & PVL & $4(5.9)$ & $2(3.4)$ & $2(22.2)$ & 0.082 \\
\hline & BPD & $11(16.2)$ & $5(8.5)$ & $6(66.7)$ & 0.000 \\
\hline \multicolumn{2}{|c|}{$\mathrm{m}-\mathrm{RACHS} \geq 5$} & $13(19.1)$ & $s(13.6)$ & $5(55.6)$ & 0.010 \\
\hline \multicolumn{2}{|c|}{ Single-ventricle physiology } & $11(16.2)$ & $7(11.9)$ & $4(44.4)$ & 0.032 \\
\hline \multicolumn{2}{|c|}{ Preintervention PGE1 } & $39(57.4)$ & $31(52.5)$ & $8(\$ s .9)$ & 0.068 \\
\hline \multicolumn{2}{|c|}{ Heterotaxy syndrome } & $3(4.4)$ & $2(3.4)$ & $1(11.1)$ & 0.351 \\
\hline \multicolumn{2}{|c|}{ PNA at intervention (days) } & $48.5(1-230)$ & $48.0(1-230)$ & $56.0(2-104)$ & 0.575 \\
\hline \multicolumn{2}{|c|}{ PMA at intervention (week) } & $40.3(31.1-68.6)$ & $40.6(34.7-68.6)$ & $38.0(31.1-46.9)$ & 0.172 \\
\hline \multicolumn{2}{|c|}{ Weight at intervention (Kg) } & $3.10(1.31-7.70)$ & $3.20(1.58-7.70)$ & $2.8 \hat{3}(1.31-\hat{3} .30)$ & 0.066 \\
\hline \multicolumn{2}{|c|}{ Weight $<2.5 \mathrm{~kg}$ at intervention } & $21(30.9)$ & $17(28.8)$ & $4(44.4)$ & 0.442 \\
\hline \multicolumn{2}{|c|}{ Pulmonary artery banding } & $6(8.8)$ & $3(5.1)$ & $\hat{3}(\hat{3} \hat{3} . \hat{3})$ & 0.027 \\
\hline \multicolumn{2}{|c|}{ PS ballooning } & $7(10.3)$ & $7(11.9)$ & $0(0.0)$ & 0.582 \\
\hline \multicolumn{2}{|l|}{ BT shunt } & $15(22.0)$ & $11(18.6)$ & $4(44.4)$ & 0.100 \\
\hline
\end{tabular}

Data was reported as number of pationts $(\%)$ or medion (mininum-maxinum).

Table 4. Multivariate logistic regression analysis for in-hospital mortality in total study population and the intervention group.

\begin{tabular}{|c|c|c|c|c|}
\hline & \multicolumn{2}{|c|}{ Total $(n=78)$} & \multicolumn{2}{|c|}{ Intervention $(\mathrm{n}=68)$} \\
\hline & ${ }_{3} \mathrm{OR}^{*}(95 \% \mathrm{CI})$ & $P$-value & $\mathrm{aOR} \div(95 \% \mathrm{CI})$ & $P$-value \\
\hline VLBW & $7.45(1.11-50.67)$ & 0.039 & $23.63(1.24-451.06)$ & 0.036 \\
\hline PPHN & $31.57(1.64-609.28)$ & 0.022 & & \\
\hline BPD & $9.84(1.71-56.44)$ & 0.010 & $33.07(2.49-438.61)$ & 0.008 \\
\hline $\mathrm{m}-\mathrm{RACHS} \geq 5$ & $18.20(3.51-94.43)$ & 0.001 & $51.45(2.52-1050.04)$ & 0.010 \\
\hline
\end{tabular}


336

\section{Neonatology}

Risk Factors for Retinopathy of Prematurity in Neonates: A Systematic Review

Ruyuan $\mathrm{Zhu}^{l}$, Huijuan $\mathrm{Li}^{2}$, Li Jiang ${ }^{1}$, Lixing Qiao ${ }^{1}$

${ }^{T}$ Pediatrics, Zhongda Hospital Southeast University, China

${ }^{2}$ Neonatal Intensive Care Unit, Nanjing Children's Hospital, China

Background: Retinopathy of prematurity(ROP) is a severe multifactorial disease in preterm neonates associated with childhood blindness. The aim was to systematically review the literature to identify independent risk factors for ROP.
Methods: Pubmed/Medline, Cochrane and Embase were searched systematically for studies reporting risk factors for ROP in neonates using multivariable analysis.

Results: From 951 initial hits, 14 studies were included, with various designs. Significant risk factors for ROP reported in at least two studies were: low birth weight, low gestational age, small for gestational age, multiple gestation, total duration of oxygen supplementation, respiratory distress syndrome, bronchopulmonary dysplasia, sepsis, transfusion.

Conclusion: High quality studies on prognostic factors for ROP are rare. Several prognostic factors, that are not necessarily causal, are associated with ROP. High quality prognostic research is necessary to establish the predictive values of these factors.

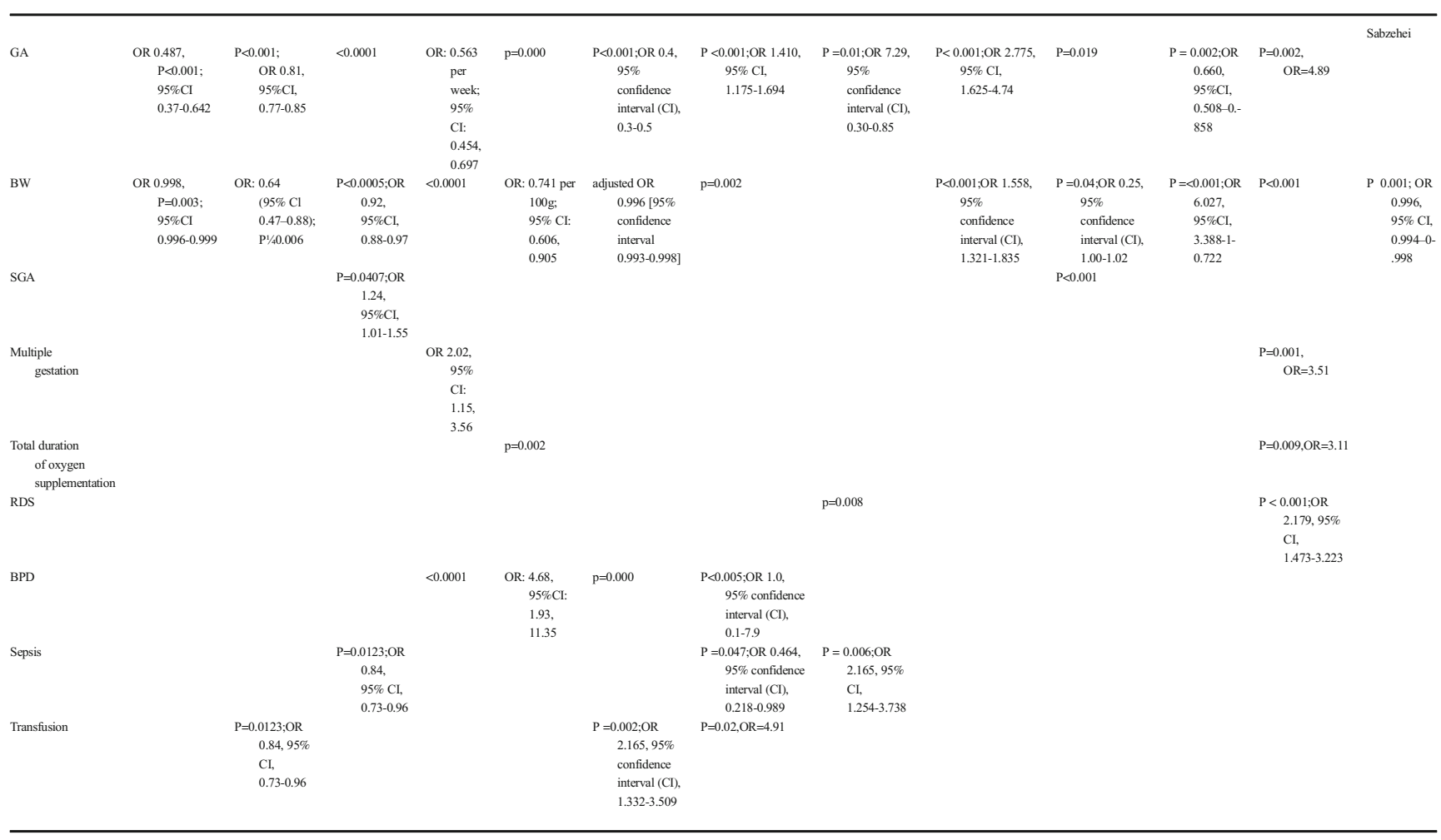

\section{5}

Neonatology

Role of TGF-31/Smad Signaling Pathway in Vascular Injury of Retinopathy of Prematurity

Ruyuan Zhu ${ }^{1}$, Huijuan Li $^{2}$, Li Jiang ${ }^{1}$, Lixing Qiao ${ }^{1}$

${ }^{T}$ Pediatrics, Zhongda Hospital Southeast University, China

${ }^{2}$ Neonatal Intensive Care Unit, Nanjing Children's Hospital, China

Objectives: To explore the changes of TGF- $31 /$ Smad signaling pathway in Phase 1 of retinopathy of prematurity and provide new targets for early intervention of ROP.

Methods: Human umbilical vein endothelial cells were cultured in vitro, and the experiment group was treated with hyperoxiainduced complete medium and hypoxia-induced complete medium intermittently. Wound healing kinetic and transwell migration assay were used to test the proliferation and migration abilities of HUVEC. RT-qPCR was used to detect the mRNA relative expression of TGF- $\beta 1$, and Western blotting was used to detect the expression of $\mathrm{pSmad} 2 / 3$ in cells of two groups.

\section{Results:}

1. After hyperoxia/hypoxia stimulation of HUVEC, the cell's morphology changed, and the ability of proliferation and migration decreased.

2. In RT-qPCR, the relative expression of mRNA in TGF- $\beta 1$ after stimulation was higher than that in the control group.

3. In Western blotting, after stimulation, the expression of $\mathrm{pSmad} 2 / 3$ in experiment group was lower than that in control group, and it was statistically significant.

Conclusions: In the hyperoxia/hypoxia HUVEC model, the expression levels of TGF- $\beta 1$ increased in experiment group. Moreover, the expression of $\mathrm{pSmad} 2 / 3$ was significantly reduced after the stimulation, along with the decrease of cell proliferation and migration ability, which indicated that the decreased of TGF- 31 / Smad2/3 signaling pathway would lead to the decrease of angiogenesis. The pathway may participate in the inhibition of angiogenesis in Phase 1 of ROP. 
Control

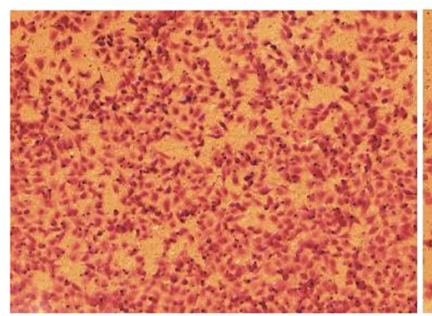

Experiment

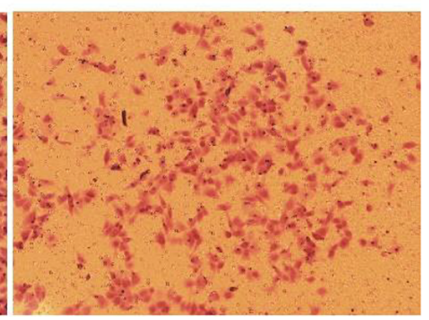

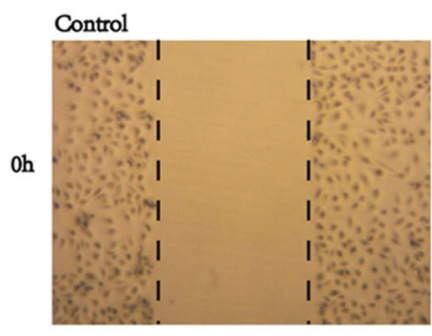
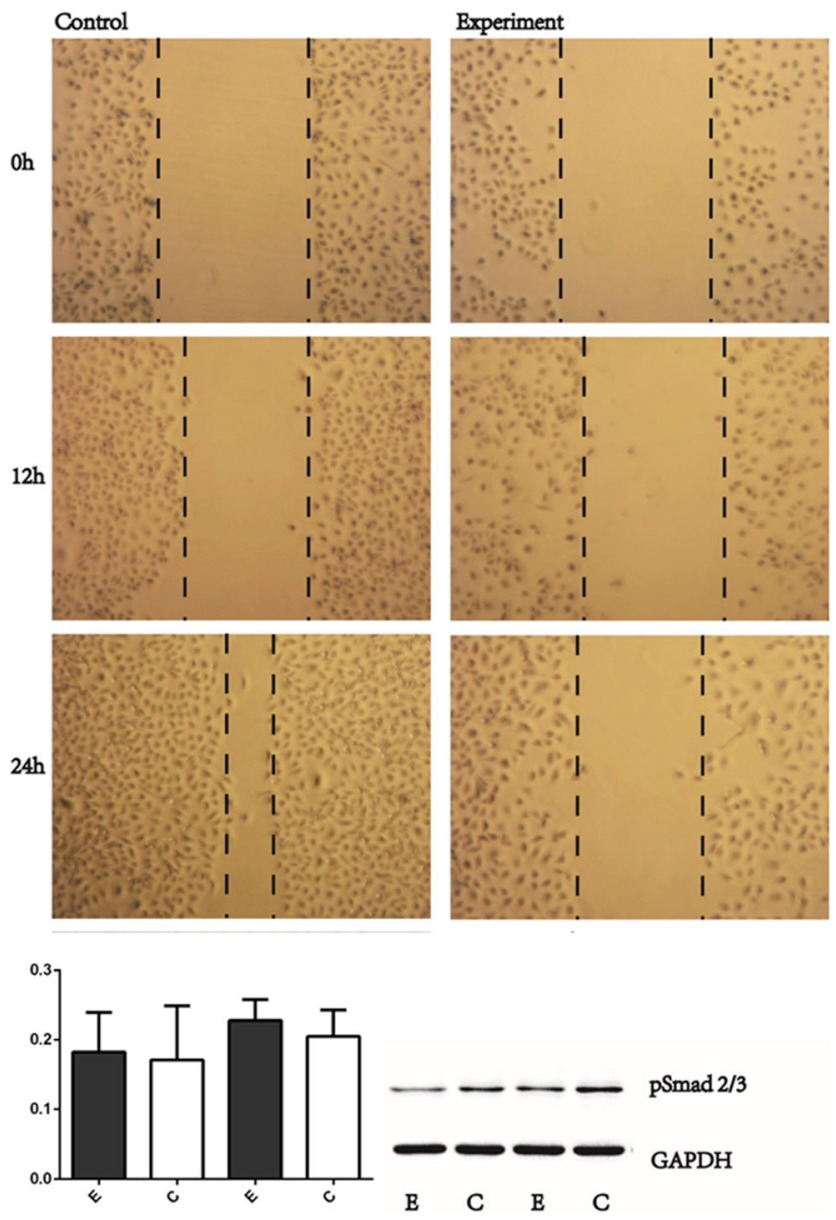

470

\section{Neonatology}

Active and Passive Hepatitis-B Immunization of Newborns in Neonatal Department at Phi Clinical Hospital Shtip Valentina Zlatkovska ${ }^{I}$

${ }^{T}$ Neonatology, PHI Clinical Hospital, Macedonia

Aim: To present the range and results of the active immunization at newborns, as well as the passive immunization from infected mothers. Hepatitis-B is rarely transferred through the placenta. The infection mostly happens during childbirth (except when the birth is performed with S.C.).

Material and method: The subject of this research are data from the Medical documentation and the results from implemented active immunization at the Clinical hospital in Shtip during 2017-2018. Engerix-B has been used for the active immunization process, while the passive immunization is induced by using Hepatitis-B Immunoglobulin. The comparative analysis method and observation by age is used to present the results.

Results: The total number of deliveries in 2017 is 929 , of which 8 newborns are not immunized with active immunization. The total number of deliveries in 2018 is 878 , of which 5 newborns are not immunized with active immunization. The cause for delayed immunization is due to low birth weight and illness of the newborns while being admitted at the Neonatal department. The passive immunization has been conducted at 5 newborns, whose mothers were infected with Hepatitis-B. All 5 children were delivered with natural birth, as it was not indicated to deliver the child with S.C. as a prevention of transmission of Hepatitis-B to the newborn. Once the immunization has been applied, the children were not separated from the mothers and they were fed by breastfeeding. The immunization, both active and passive, caused no side effects.

Conclusion: With complete immunization against Hepatitis-B (active and passive), it is expected to reduce the incidence of Hepatitis-B and to reduce the number of most frequent complications - chronical Hepatitis-B, cirrhosis and Hepatocellular carcinoma.

\section{6}

\section{Nephrology}

Analysis of Markers of Fibrosis Formation in Patients with Pyelonephritis

Tetiana Chekotun ${ }^{1}$, Nadia Tokarchuk ${ }^{1}$, Yulia Vyzhga ${ }^{2}$, Ludmila Starynets $^{3}$ ${ }^{T}$ Department of Pediatrics I, National Pirogov Memorial Medical University, Ukraine

${ }^{2}$ Department of Pediatrics II, National Pirogov Memorial Medical University, Ukraine

${ }^{3}$ Pediatric department, Vinnytsya regional children's hospital, Ukraine

Introduction: Nowadays the scientists are actively studying the role of Galectin-3 in the process of initiation and formation of fibrotic changes. Many studies associate a high level of this marker with disease progression, inflammation processes and fibrosis formation.

Objective: To determine the level of Galectin-3 as a marker of fibrosis formation in children of an early age with pyelonephritis.

Patients and Methods: We examined 100 children of an early age suffering from pyelonephritis. The level of Galectin-3 was determined by immunoenzymatic method (ELIZA) using the kit «Human Galectin-3»

Results: High levels of Galectin-3 were discovered in babies suffering from vesicoureteral reflux $(8,59 \pm 1,03 \mathrm{ng} / \mathrm{ml})$. The concentration of this marker was significantly lower in children of the comparative group $(2,97 \pm 0,94 \mathrm{ng} / \mathrm{ml})$ as well as in almost healthy children who were examined $(1,5 \pm 0,19 \mathrm{ng} / \mathrm{ml}), \mathrm{p} 0,01$. The highest level of Galectin-3 was recorded in children who were $2-3$ years old $(10,3 \pm 1,34 \mathrm{ng} / \mathrm{ml})$ which was reliably different from the level in children aged from 1 month to 1 year $(5,0 \pm 0,76 \mathrm{ng} / \mathrm{ml}),(\mathrm{p} 0,05)$.

It was established that the longer the duration of inflammatory process in kidneys, the higher was the level of pro-fibrotic factor. Thus, the reliably highest level of Galectin-3 $(12,46 \pm 4,21 \mathrm{ng} / \mathrm{ml})$ was in children who were suffering from the inflammatory process in kidneys for 6 months or longer. The children examined after three months of illness showed the level of Galectin-3 which was two times less $(7,03 \pm 3,28 \mathrm{ng} / \mathrm{ml})$, (p0,05). The lowest level of Galectin-3 was found at the beginning of the disease $(5,67$ $\pm 1,64 \mathrm{ng} / \mathrm{ml}),(\mathrm{p} 0,01)$.

Conclusions: Plasmatic concentration of Galectin-3 is reliably higher in children with pyelonephritis accompanied by vesicoureteral reflux than in children with primary pyelonephritis which indicates possible formation of fibrosis in kidneys. It was established that the longer was the duration of pyelonephritis the higher were the levels of pro-fibrotic factors. 
1051

\section{Nephrology}

Infectious Glomerulonephritis, One-Year-Follow-Up After Acute Kidney Injury (Aki) - A Case Report

Claudia Correia $^{1}$, Ana Rita Gomes ${ }^{I}$, Telma Barbosa ${ }^{2}$, Lurdes Morais ${ }^{2}$, Ana Ramos $^{2}$, Ana Teixeira ${ }^{2}$

${ }^{1}$ Department of Pediatrics, Paediatrics Resident, Centro Materno Infantil do Norte, Centro Hospitalar Universitário do Porto, Portugal

${ }^{2}$ Department of Pediatrics, Paediatrics Assistant, Centro Materno Infantil do Norte, Centro Hospitalar Universitário do Porto, Portugal

Background: Post-Infectious Glomerulonephritis (PIG) is the most common cause of acute glomerulonephritis (AGN) in children and manifests clinically as nephritic syndrome. Pneumonia is rarely associated with the occurrence of AGN. PIG prognosis is usually benign, with complete recovery occurring in most cases. Hypocomplementemia is associated with a more severe disease course, namely, azotaemia and nephritic syndrome. Proteinuria persistency, even residual, might be a long-term predictive factor for chronic kidney disease.

Case Report: Four-year-old previously healthy girl with national immunization program up to date, was admitted to our paediatrics unit with right-lobe pneumonia complicated with acute renal injury. The urinalysis revealed haematuria (5-10 erit/uL), proteinuria (protein/creatine ratio 2,4 $\mathrm{mg} / \mathrm{mg}$ ) and leukocyturia. There was no acid-base imbalance. C3 levels were low (11,3 mg/dL, range 81-167), with normal C4 levels. After 24h of standard antibiotic therapy, hypertension, generalized oedema, oliguria and inspiratory bibasal crackles appeared. IV furosemide was initiated, with progressive clinical and analytical improvement, blood pressure and diuresis normalized in 48 hours. Five weeks after discharge, under no medication, she was asymptomatic and normotensive, without other abnormalities on physical examination. Renal function and inflammatory parameters were normal, and C3 levels improved $(58,8 \mathrm{mg} / \mathrm{dL}$, range 81 $167 \mathrm{mg} / \mathrm{dL}$ ). Microscopic haematuria and mild proteinuria (protein/creatinine ratio $0,33 \mathrm{mg} / \mathrm{mg}$ ) were still present. Six months after this episode, there was no haematuria, $\mathrm{C} 3$ levels and the protein/creatinine ratio were normalised, but microalbuminuria, though residual, persisted $(22,7 \mathrm{mg} / \mathrm{g}$ creatinine). One year after the acute episode, microalbuminuria returned to the normal range (10 $\mathrm{mg} / \mathrm{g}$ creatinine).

Conclusions: With this case presentation, the authors highlight the concomitant renal impairment associated with respiratory infection, namely pneumococcal pneumonia, an increasingly diagnosed form of infectious glomerulonephritis. It is important to reinforce the significance of followup to identify possible complications and prevent functional deterioration, even when the PIG has an overall favourable prognosis.

963

Nephrology

Multicystic dysplastic kidney and posterior urethral valve Kristijan Dervishov $^{1}$, Kristijan Bundovski ${ }^{1}$, Doncho Dervishov ${ }^{2}$, Velibor Tasic $^{1}$, Natasha Aluloska ${ }^{1}$

${ }^{1}$ University Children's Hospital, Medical School Skopje, Macedonia

${ }^{2}$ Department of Pediatrics, General Hospital Veles, Macedonia

Introduction: Multicystic dysplastic kidney (MDK) is a rare congenital anomaly of kidney and urinary tract (CAKUT). The disease is characterized with multiple non-communicating cysts without functional renal parenchyma; nowadays the diagnosis is established with prenatal ultrasound screening of the kidneys and urinary tract. The aim of this work is the presentation of rare association of multicystic dysplastic kidney with posterior urethral valves. Case report: A healthy male newborn baby was referred for nephrourological work up due to prenatal diagnosis of multicystic kidney which resolved completely before delivery. The first postnatal ultrasound scan revealed normal morphology of the right kidney and the bladder. At the age of two months the parents complained that the baby had poor urinary stream. Voiding urethrocystography revealed posterior urethral valves and the baby underwent successful valve resection.

Discussion and conclusion: We present a rare association of multicystic dysplastic kidney with posterior urethral valves. This association is very rare and was described in few reports. In the series of Mathiot et al. (J Radiol. 2002) among 54 neonates with diagnosed MDK three had posterior urethral valves. Pooled data from 67 studies including 2159 patients in whom VCUG was performed showed association with posterior urethral valves only in 9 cases $(0.42 \%)$ (Schreuder MF et al, Nephrol Dial Transplant. 2009). Clinician should suspect urethral valves in infants with poor stream even in the case of normal kidney/bladder ultrasound.

Key words: Multicystic dysplastic kidney, posterior urethral valves, resolution, prenatal, ultrasound

\section{6}

\section{Nephrology}

Primary Hyperoxaluria in Macedonian Children Velibor Tasic ${ }^{I}$, Kristijan Dervishov $^{I}$, Zoran Gucev $^{I}$

${ }^{1}$ Pediatric Nephrology, University Children's Hospital Skopje, Macedonia

Background: Primary hyperoxalurias $(\mathrm{PH})$ are hereditary disorders of glyoxylate and oxalate metabolism with consecutive nephrolithiasis, nephrocalcinosis and progressive renal damage resulting in end stage kidney disease and systemic oxalosis.

Objective: The aim of this retrospective study was to analyze the clinical features, biochemical parameters, genetic findings and outcome in Macedonian children with primary hyperroxaluria.

Methods: PH was defined if the urinary oxalate levels were 1.0 $\mathrm{mmol} / 1.73 \mathrm{~m}^{2} / \mathrm{d}$. Secondary causes were excluded on the basis of dietary history, physical examination and laboratory tests. Urinary excretion of glycolate and glycerate was determined within the same samples. 24-hour urine samples were collected for testing pyridoxine sensitivity while in anuric patients plasma oxalate concentration were measured before and after pyridoxine challenge. Mutational analysis of the respective PH1/2/3 genes was performed.

Results: In the period 1995-2018 there were 10 patients with PH (In 6 confirmed with mutational analysis of the $A G X T$ gene). There were no patients with $\mathrm{PH} 2 / 3$. The youngest patient at diagnosis was 4 months old and the oldest 18 years. Four patients progressed to ESKD and one died. Three patients were sensitive to pyridoxine. One patient had nephrolithiasis which was initially considered as a consequence of ureteropelvic junction obstruction, but metabolic investigation revealed hyperoxaluria, hyperlglycolaturia and normalization of urinary oxalate excretion under pyridoxine treatment.

Conclusions: Macedonian PH children were diagnosed late in the course of the disease when progressive kidney damage ensued. There were no cases of $\mathrm{PH} 2$ and $\mathrm{PH} 3$. It seems that yet unidentified genes are responsible for PH in Macedonian children. One should implement mandatory urinary oxalate screening in all children with urolithiasis/nephrocalcinosis in order to initiate early and aggressive conservative management to prevent systemic oxalosis.

\section{8}

Nephrology

Diagnostic Markers of Haematuria in Children with Kidney Disease Anastasiia Konoplitska ${ }^{I}$, Olga Yablon ${ }^{l}$, Yulia Kyslova ${ }^{I}$, Olena Mazur ${ }^{I}$, Nataliia Chornopyshchuk ${ }^{l}$

${ }^{1}$ Paediatric Department №1, National Pirogov Memorial Medical University, Ukraine

Background: A great diagnostic value in the genesis of hematuria in children with nephropathy is the change in cell membranes of the 
nephroteliy, enzymuria and microglobulinuria Objective To determine the level of renospecific enzymes in serum and urine (malonic dialdehydeMDA, cholinesterase-ChE, $\gamma$-glutamyltranspeptidase, $\beta 2$-microglobulin$\beta 2-\mathrm{MG})$ in children with haematuria syndrome as diagnostic markers. Methods We examined 58 children with acute and chronic glomerulonephritis, 50 children with tubulointerstitial nephritis and kristaluriya. Comparison group consisted of 20 children with no signs of kidney damage.

Results: In children with acute and chronic glomerulonephritis observed a significant increase in activity of $\mathrm{ChE}$ in the urine $(20.5 \pm 0.55$ and 9.11 $\pm 0.40 \mathrm{U} / \mathrm{mg}$ creatinine respectively, p0.05, Se $85-92 \%$, Sp $70 \%$, NPV $85-95 \%)$ and MDA in serum $(4.60 \pm 0.20$ and $4.28 \pm 0.25 \mathrm{mmol} / \mathrm{L}$, respectively, p0.05, Se 78-87\%, NPV 85-95\%). Children with kristaluriya and tubulointerstitial nephritis diagnosed by higher values of MDA in serum $(4.22 \pm 0.29$ and $4.41 \pm 0.34 \mathrm{mmol} / \mathrm{L}$ respectively, p0.05, Se $90-93 \%$, NPV $95 \%), \gamma$-glutamyltranspeptidase in urine $(72.9 \pm 3.25$ and $95.5 \pm 3.92 \mathrm{U} / \mathrm{mg}$ creatinine respectively, p0.05, Se 94-100\%, Sp 64-73\%, PPV 72\%, NPV $95-100 \%)$ and $\beta 2-\mathrm{MG}$ in urine $(44.1 \pm 1.37$ and $77.6 \pm 1.64 \mathrm{ng} / \mathrm{ml}$ respectively, p0.05, Se $88-100 \%$, Sp $80 \%$, PPV 78\%, NPV 95-100\%). Correlation analysis revealed specific metabolic markers: the conditions of haematuria glomerular origin - of $\mathrm{ChE}$ activity in urine $(\mathrm{r}=0.693$, p0.05) while for tubular hematuria - the activity of $\gamma$ glutamyltranspeptidase $(\mathrm{r}=0.777, \mathrm{p} 0.05)$ and the level of $\beta 2-\mathrm{MG}$ in urine $(\mathrm{r}=0.669, \mathrm{p} 0.05)$.

Conclusions: Increased urinary excretion of $\gamma$-glutamyltranspeptidase, $\beta 2-\mathrm{MG}$ and increased content of MDA in serum of children with the syndrome of haematuria indicates damage of cell membranes nephrotelial proximal tubules of the kidneys and is an early marker of non-glomerular (tubular) haematuria. Increased urinary excretion of $\mathrm{ChE}$ with normal levels in urine $\beta 2-\mathrm{MG}, \gamma$-glutamyltranspeptidase and elevated levels of MDA in blood serum are markers of glomerular kidney damage.

\section{5}

\section{Nephrology}

Secondary Hypertension: Can it Result from Two Different Causes? Ana Lança ${ }^{l}$, Joana Gonçalves ${ }^{l}$, Paula Nunes ${ }^{l}$

${ }^{T}$ Pediatric department, Hospital de São Francisco Xavier, Centro Hospitalar de Lisboa Ocidental, EPE, Portugal

Background: In Portugal hypertension affects around 13\% of all children. Essential hypertension is the commonest. Renovascular disease is an uncommon, but important cause of hypertension in children, which includes renal artery stenosis (RAS).

Case-Report: A two-year-old girl with a complex cyanotic cardiopathy (type B Aortic branch interruption, subaortic stenosis and a nonrestrictive intraventricular communication) submitted to two correction surgeries is admitted with fever, cough, rhinorrhea and anorexia. At observation she had a good general condition, with cyanotic lips and nails and no signs of respiratory distress and she was hospitalized for surveillance. During the hospitalization serial BP measures revealed hypertensive peaks and she initiated antihypertensive therapy with captopril and furosemide, with poor BP control. An echocardiogram was performed excluding an aortic recoartation and a renal ultrasonography revealed a small right kidney with diminished corticomedullar differentiation. During hospitalization there was no significant decrease in BP raising suspicion of renovascular etiology. A magnetic resonance angiography was performed revealing a stenotic right renal artery and distal aorta in "sack bottom" - consistent with important vascular malformation. Captopril was progressively stopped, a calcium channel blocker was initiated and later a betablocker was added to BP control. After discharge a percutaneous angiography was performed confirming the vascular malformation making it impossible to revascularize. The Tc 99m-MAG3 renal isotope scan was also performed and revealed a right kidney with $36 \%$ function. Currently she has a multidisciplinary follow-up and maintains antihypertensive therapy with propranolol and amlodipine, with therapeutic readjustments periodically needed.

Conclusion: Secondary causes for pediatric hypertension are variable, and should be suspected in children with early-onset hypertension. In a child with a repaired aortic coarctation a recoartation must be excluded as the hypertension etiology. Refractoriness to appropriate medical therapy should raise suspicion for renovascular etiology and CT or MRI angiography reveal the diagnosis.

\section{8}

Nephrology

Clinical Outcomes in Children Admitted with Henoch-Schönlein Purpura

Joana Moscoso ${ }^{1}$, Margarida Serôdio ${ }^{1}$, Liliana Franco ${ }^{1}$, Paula Nunes ${ }^{1}$ ${ }^{T}$ Serviço de Pediatria, Centro Hospitalar Lisboa Central, Hospital São Francisco Xavier, Portugal

Background: Henoch-Schönlein purpura (HSP) is the most common form of systemic vasculitis in small vessels in children. Renal involvement is more prevalent in older children and long-term follow-up of these patients is required.

Objective: To describe a population of children diagnosed with HSP (first onset or relapse) according to demographic data, clinical data, management and outcomes.

Methods: Retrospective analysis from medical files of HSP patients under 18 years who were admitted to paediatric emergency care and paediatric inpatient unit between 2008 and 2018.

Results: A total of thirty-nine children with HSP were analysed. Their median age was 6 years. At onset, purpura was present in all cases, arthritis/arthralgia in $87 \%$, abdominal pain in $38 \%$, renal involvement in $15,4 \%$ and testicular oedema in four patients. $12,8 \%$ presented with hypertension. All the patients were submitted to urine dipstick testing: four had isolated haematuria and two had both macroscopic haematuria and nephrotic proteinuria. $58,9 \%$ of the patients needed hospitalization due to renal, gastrointestinal or severe joint involvement. Treatment with steroids was given to $48,7 \%$ of the patients and about $12,8 \%$ needed antihypertensive medication. During follow-up: three patients had haematuria and nephrotic proteinuria, two had isolated nephrotic proteinuria and two had isolated haematuria; one underwent renal biopsy due to persistent haematuria and nephrotic proteinuria. $69,2 \%$ of the patients experienced no relapse, $17,9 \%$ had one relapse and five patients had $\geq$ 2 relapses. None developed end-stage renal disease until now.

Conclusions: The outcomes of children with HSP are generally excellent and recover spontaneously without medication, however in our cohort most of the children had renal, gastrointestinal or severe joint involvement that required hospitalization. Although the literature describes worse renal outcomes and more relapses in patients with nephrotic-range proteinuria and gross haematuria at the onset, our patients had a good prognosis.

\section{1}

\section{Nephrology}

Resistive Index (RI) in Febrile Urinary Tract Infection: Predictive Value of Renal Outcome

Suzan Naseer ${ }^{1}$, Boshra $N^{1}, N$ Ehsan ${ }^{2}$, Anton Podlubni ${ }^{2}$, Mohammad Sayed $^{2}$, A Z Said , Wael Nasser $^{1}$, Alex Krasnochok ${ }^{2}$

${ }^{1}$ Department of Nephrology Pediatrics, Faculty of Medicine in the Galilee - Bar Ilan University., Israel

${ }^{2}$ Department of Pediatrics, Faculty of Medicine in the Galilee - Bar Ilan University, Israel

Urinary tract infection is the most common bacterial infection in pediatric. 
The Ultrasonography of bladder and kidneys - is a relatively cheap, noninvasive and fast test that enables to find children with anatomical anomalies. Also this test does not pose risk of radiation exposure.

Voiding cystourethrogram - is the test of choice to establish the presence of vesicoureteral reflux, that is a major risk factor for future infections and scaring. This test have a radiation exposure risk, it is invasive and relatively complex to preform .

DMSA scintigraphy test - may be performed to determine acute pyelonephritis or to investigate kidney scarring. This test have greater radiation exposure risk. But it cannot distinguish between dysplasia of a kidney or a scarring

With the development and improvement of the imaging capabilities of Doppler ultrasonography we can measure the Resistive Index, which is a sonographic value to assess the kidney function.

In this work we want to measure if the Resistive Index can predict kidney scarring.

We followed after 71 cases of kids from 0 to 11 years old that was admitted to our hospital - Puria, in the diagnosis of Urinary tract infection.

The diagnosis was made on the base of clinical presentation, laboratory blood analysis, urinary analysis and urinary cultures.

We measured the Resistive index in all of these patients. And they did the ordinary US of kidney parenchyma, the DMSA study and a cystography by the accepted protocols of our department.

In conclusion we found : In the first group under 2 years old we found $\mathrm{e}$ medium correlation between the RI and DMSA study. And there was a strong correlation between RI and cystography. In the second group above 4 years we found a strong correlation between RI both to DMSA and cystography.

966

\section{Nephrology}

Perinephric Urinoma as a Complication of an Ureteropelvic Junction Obstruction- A Case Report

Joana Pereira-Nunes $^{1,2}$, Aida Correia de Azevedo ${ }^{2}$, Ana Sofia Rodrigues $^{2}$, Susana Lopes ${ }^{2}$, Alexandra Sequeira ${ }^{2}$, Clara Vieira $^{2}$, José $^{2}$ Luís Carvalho ${ }^{3}$, Rúben Lamas Pinheiro ${ }^{3}$

${ }^{1}$ Pediatrics Department, Centro Materno Pediátrico, Centro Hospitalar Universitário São João, Portugal

${ }^{2}$ Pediatrics/Neonatology Department, Centro Hospitalar do Médio Ave, Portugal ${ }^{3}$ Pediatric Surgery Department, Hospital de Braga, Portugal

Background: Urinomas are very rare and result from urine extravasation from the urinary tract. They can have an obstructive or a non-obstructive cause. We report a case of a 13 -year-old boy with spontaneous perinephric urinoma secondary to a previously unknown right ureteropelvic junction obstruction (UPJO).

Case Presentation Summary: A 13-year-old boy, with an earlier 6 years' loss of pediatric follow up due to right pielocalicial dilatation, presented with a 1-day history of right flank pain and vomiting. He had no fever, any change in bowel habits, urinary symptoms or change in macroscopic urine characteristics. Previous trauma or surgery were denied. He was hypertensive, prostrated, had sunken eyes and abdominal guarding and pain on abdominal palpation of the right flank. Laboratory evaluation: hemoglobin $15,10 \mathrm{~g} / \mathrm{dL}$, WBC count 15900 cells $/ \mathrm{uL}(88,0 \%$ neutrophils), platelets $281000 / \mathrm{uL}$, creatinine $1,01 \mathrm{mg} / \mathrm{dL}$, BUN $23 \mathrm{mg} / \mathrm{dL}$, normal electrolyte panel, CRP $0,1 \mathrm{mg} / \mathrm{dL}$. He started intravenous fluid therapy and was medicated with ondansetron. Afterwards, he started to complain of lumbar pain and presented a first episode of macroscopic hematuria, maintaining right flank abdominal pain and hypertension. Laboratory revaluation made 6 hours later showed persistance of leukocytosis and neutrophilia and a rise in creatinine's value $(1,23 \mathrm{mg} / \mathrm{dL})$. Urinalyses confirmed the presence of hematuria. Enhanced abdominal CT showed right hydronephrosis with dilatation of the renal pelvis
(5,5 $\mathrm{cm}$ of transverse diameter), which had saccular aspect and presented an abrupt transition to a normal ureter, and significant densification of the perirenal fat attributable to urinary extravasation. Cystoureterography showed contrast extravasation by the right renal lower pole. He was submitted to a bilateral double J stenting and medicated with ceftriaxone, with clinical improvement.

Discussion: Urinomas occur most commonly following renal trauma or surgery. This case has particular interest since it reports perinephric urine extravasation as a complication from UPJO.

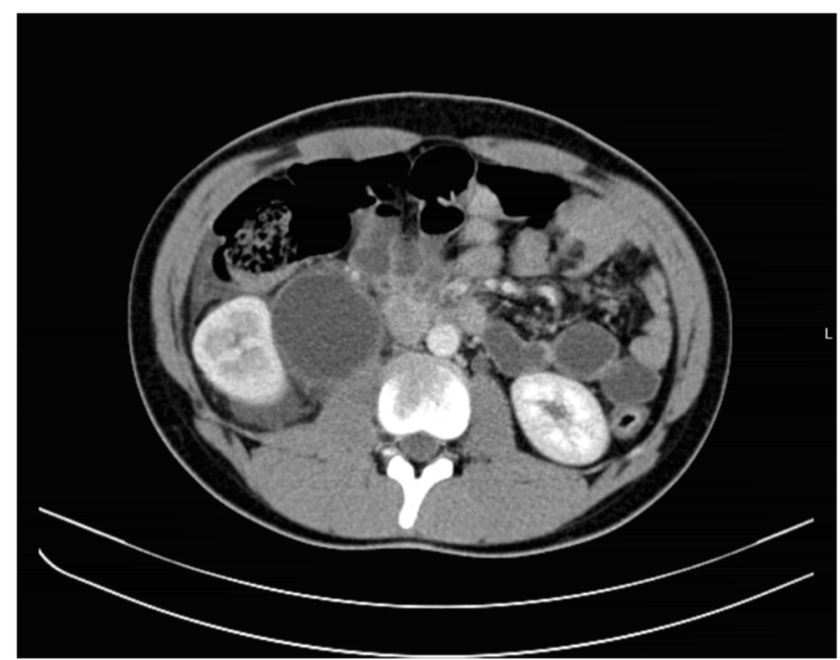

533

Nephrology

Management and Outcome in Childhood Nephrotic Syndrome: A Retrospective Study

Maria Margarida Serôdio ${ }^{1}$, Joana Moscoso ${ }^{1}$, Joana Gonçalves ${ }^{1}$, Liliana Franco', Paula Nunes

${ }^{I}$ Department of Pediatrics, Centro Hospitalar Lisboa Ocidental, Portugal

Background: The Nephrotic Syndrome (NS) is caused by renal diseases that increase the permeability across the glomerular filtration barrier. Despite its low incidence in general population, it is a common cause of chronic kidney disease in childhood. Although corticosteroid response is achieved in the majority of patients at the onset of disease, many patients are resistant or dependent to steroid treatment during follow-up.

Objective: Our aim was to characterize a population of hospitalized children diagnosed with NS according to demographic data, clinical data, management and outcomes.

Methods: A clinical retrospective analysis was performed between 2007 and 2018 .

Results: A total of 22 children were enrolled, with a median age of 5.1 years at the onset of the disease. There was no gender predominance and no secondary causes were identified. We found that $68 \%(\mathrm{n}=15)$ of patients presented with generalized edema and $18 \%(n=4)$ with palpebral edema. Median hospital length of stay was 8 days. All patients were initially treated with corticosteroids, and $82 \%(n=18)$ responded with proteinuria remission during hospital stay. Eighteen percent $(n=4)$ were initially steroid-resistant without remission and subsequently transferred to a tertiary hospital. During follow-up, relapses were found in $68 \%$ $(\mathrm{n}=15)$. Steroid-dependent and steroid-resistant disease was found in $50 \%(n=11)$ and $14 \%(n=3)$ of patients, respectively. Forty-five percent $(\mathrm{n}=10)$ were subsequently treated with additional immunosuppression, mainly cyclophosphamide. Renal biopsies were performed in $32 \%$ $(n=7)$ of patients. 
Conclusions: In contrast to literature, in our cohort there was no gender predominance of NS. We emphasize the initial good corticosteroid response in the majority of our patients, but high incidence of relapses, steroid dependent and resistant disease during follow-up.

290

\section{Nephrology}

Pyelonephritis Xantogranulomatosa: Presentation of Two Cases ${ }_{\text {Magdalena Szymanek-Szwed }}{ }^{l}$, Katarzyna Załeska-Oracka $^{l}$, Joanna Samotyjek ${ }^{\prime}$, Beata Jurkiewicz

${ }^{I}$ Pediatric Surgery and Pediatric Urology Clinic, Centre of Postgraduate Medical Education, Poland

The authors present 2 cases of Pyelonephritis xantogranulomatosa treated in the Clinic. This is a very rare form of chronic kidney inflammation with an unclear cause.

The literature shows the relationship of the disease with disorders in urinary outflow, urolithiasis, infections and prolonged antibiotic therapy. The first patient was found to have doubled kidney and deposits in the kalyxes changed by inflammation. Both patients in the period preceding the diagnosis were subjected to long-term antibiotic therapy due to recurrent respiratory infections and chronic urinary tract infection.

Treatment of the first patient was one-stage due to the intensification of the inflammatory process at the time of diagnosis. In the second girl, the abscess was initially drained, and due to the non-characteristic intraoperative image, the suspicion of xantogranulomatosis was made and confirmed in computed tomography. In the next stage, due to the expansion of the process - nephrectomy was performed.

Proteus mirabillis and Escherichia coli were found in the cultures, which are also reported in the literature as the most common pathogens. It is interesting that despite the generalized inflammatory reaction, only one kidney is involved in the process.

The diagnosis of Pyelonephritis xantogranulomatosa is difficult.

In imaging studies, the kidney is enlarged, with a preserved shape, contains deposits in the calyx and pelvic system and numerous hypoechogenic changes indicating abscesses. Urography and renoscintigraphy may show a weak or inactive organ function. The test of choice is computed tomography, which allows to assess the advancement of the process.

In differential diagnosis in children, the Wilms tumor, neuroblastoma, clear cell carcinoma, inflammatory process like pyelonephritis, kidney tuberculosis, kidney abscess should be considered.

In both described cases, the diagnosis of xantogranulomatosis was confirmed in histopathological examination. Qualification for nephrectomy was based on imaging studies (CT) and persistent high exponents of infection. In the follow up no complications were noted, the remaining kidney in both cases is functioning properly.

\section{7}

\section{Nephrology}

Urinary Tract Infections in Children and Their Early Detection and Treatment

Ilija Timovski ${ }^{1}$, Violeta Angelovska ${ }^{1}$, Maja Timovska ${ }^{2}$, Domnika Rajchanovska ${ }^{3}$, Ana Timovska ${ }^{4}$

${ }^{1}$ Paediatrician, PHI D-r Angelovska and D-r Timovski, Macedonia

${ }^{2}$ Analytics and Research, PRD, Macedonia

${ }^{3}$ Paediatrician, HMS, Macedonia ${ }^{4}$ Student, Medical faculty-Skopje, Macedonia

Introduction: Urinary infections are common problem in pediatric practice. They are more common in female children,except at the young infants with dominant males. The most common causes of infections are
VUR(veziko urethral reflux)congenital anomalies of the urinary tract and concretions. They are commonly caused by gram negative bacteria. In urinary infections nonspecific symptoms occur such as: vomiting, fever and anxiety. After the third year of life as more frequent are the abdominal pain, frequent and painful micturition and difficulties when urinating. Every vague febrile condition should be considered as a possible urinary infection.

Goal: With timely diagnosis and treatment of urinary infections impairment of the renal (kidney) function is prevented.

Materials and methods: Data from 590 children aged 0 to 18 years suspected of urinary infection or febrile for a period of 3 years (20172019) had been processed. Analytical and descriptive method had been used for processing.

Results: Out of 290 children with taken urine - culture, 105 showed positive results $(36,20 \%)$. Escherichia coli had been isolated with 86 children $(81,90 \%)$, Pseudomonas aeruginosa with $(6,4 \%)$, Proteus species $(6,2 \%)$, Klebsiella had been isolated with $(2.6 \%)$ and other agens with $(2,9 \%)$. From the lab findings the most common had been leukocytosis. One child had been diagnosed agenesis of the right kidney, 7 children had been diagnosed VUR, and 1 child ectopic kidney.During the treatment the following drugs had been used: Nitrofurantoin, Amoxicillin + clavulonic acid, Trimetoprim + sulfonamide, Cefuroxim or Cefixime depending on the antibiogram. It resulted in the negative urine-culture in more than $96 \%$ of children, while children with congenital malformations are under regular scrutiny by the pediatrician - nephrologists.

Conclusion: Routine investigation of urine during every obscure febrile condition. Urine- culture method is necessary to determine the specific therapy. Timely diagnosis and treatment leads to successful prevention of renal (kidney) damage.

\section{4}

\section{Neurology}

A Not So Pleasant Fairy Tale

Diana Alba ${ }^{1}$, José Fontoura Matias ${ }^{2}$, Mafalda Moreira ${ }^{1}$, Sara Mosca ${ }^{3}$, $\overline{\text { Rui Chorão }}^{4}$, Maria do Céu Ribeiro ${ }^{1}$

${ }^{I}$ Pediatric Department, Centro Hospitalar Tâmega e Sousa, Portugal

${ }^{2}$ Pediatric Department, Centro Hospitalar Universitário São João, Portugal

${ }^{3}$ Pediatric Department, Centro Materno-Infantil do Norte, Centro Hospitalar Universitário do Porto (CMIN-CHUP), Portugal

${ }^{4}$ Neuropediatric Unit, Pediatric Department, Centro Materno-Infantil do Norte, Centro Hospitalar Universitário do Porto (CMIN-CHUP), Portugal

Background: Alice in Wonderland Syndrome (AiWS) is a neurological condition defined by the presence of perception disorders which translate into an imbalance between the self-representation and/or the perception of the real world. Several conditions have been associated with this syndrome, such as infections, brain injuries, psychoactive drug abuse and epilepsy.

Clinical Case: A 13 year-old male was observed in a general pediatric appointment due to visual distortion. According to his mother, this happened for the first time when he was 5 years old, and it occurred once or twice per year ever since, lasting no longer than 10 minutes. He usually wakes up frightened, claiming he sees everything bigger (macropsia) and sometimes he also refers that the objects seem to run away from him (teleopsia). Physical examination was unremarkable. The following investigation revealed compatible signs of cerebral anoxia at the MRI, probably due to neonatal asphyxia, and a normal initial sleep electroencephalogram (EEG). When repeated 7 months later it showed epileptiform activity. Zonisamide was indicated to be initiated in case of a new seizure.

Discussion: Visual distortions are the hallmark of AiWS, but body image and time distortion can also occur. Patients may have an erroneous 
perception of their body size or a distortion of the size and shape of the objects around them. This condition may be caused by epilepsy which, in turn, and although unusual, may be a later manifestation of neonatal asphyxia. So, it is important to be aware of these associations so that an early and accurate diagnosis can be made, and proper treatment and follow-up be performed.

\section{7}

\section{Neurology}

Are there Differences or Similarities between Girls and Boys with Febrile Seizures?

Ioana Arbanas ${ }^{I}$, Raluca-Ileana Lixandru ${ }^{l}$, Oana Falup-Pecurariu ${ }^{1,2}$ ${ }^{T}$ Paediatrics, Children's Clinical Hospital, Romania ${ }^{2}$ Paediatrics, Faculty of Medicine - Transilvania University, Romania

Background: Febrile convulsions (FC) represent one of the main reasons for presenting at the emergency department.

Objectives: Main objective-to determine the incidence of febrile convulsions for the admitted children in the Childrens Clinic Hospital from Braşov, Romania.As second objectives-to analize possible correlations between gender distribution, clinical and epidemiological aspects, fever, lab exams and antibiotic prescription.

Methods: A retrospective, descriptive observational study between 1st of January 2016 and 31st of December 2018 was designed to include all childern admitted with FC at the Children's Clinic Hospital Brasov.

Results: A total number of 122 children with FC were enrolled with no significant differences between gender distribution, $40 \%$ were the first born, boys being older compared with girls(28months Vs 23 months), FC being most prevalent in March.A positive family history for FC was identified in $14.7 \%$ and $60 \%$ were from rural areas, $28 \%$ of the children received antipyretics at home and $78 \%$ of them presented at the inaugural FC episode.Even though most of the FC are due to viral infections the trend in our patients was to prescribe antibiotics, $19 \%$ of the children received antibiotic although they had no positive laboratory tests for bacterial infection.

Medium time for the seizure was 150 seconds for boys and longer for girls, 180 seconds. The predominant manifestation of the febrile seizure was generalized tonic-clonic seizure for both girls and boys $(63 \%)$, but girls presented more generalized hypotonia and boys presented more generalized hypertonia. $75 \%$ associated loss of consciousness with a predominace of girls(52\% Vs $47 \%)$. Malnutrition was present in $30 \%$ of cases.Laboratory tests showed that more girls had a positive CRP $(21 \%)$ comparative to boys $(14 \%)$. Leucocitosis was presented in $34 \%$ of girls compared with $22 \%$ of boys. The most common underlying condition encountered was respiratory tract infections for both boys and $\operatorname{girls}(36 \% / 43 \%)$.

Conclusions: 1.There were no significant differences between the two studied groups with FC regarding the gender distribution, clinical manifestation, laboratory findings and epidemiological aspects.

2.URTI and LRTI were the most common underlying conditions causing the febrile seizures.

3.Protein-caloric malnutrition was a common risk factor.

4.The administration of antibiotics is high although there is no evident bacterial cause.

\section{0}

\section{Neurology}

\section{A Rare Cause of Development Delay}

$\underline{\text { Sofia Baptista }}^{l}$, Carla Mendonça ${ }^{1}$, Inês Coelho ${ }^{1}$, Teresa Monteiro ${ }^{1}$, Rita Pereira', Daniel Tiago $^{I}$

${ }^{1}$ Serviço de Pediatria, Centro Hospitalar Universitário do Algarve Unidade de Faro, Portugal
Background: Tay-Sachs disease is a neurodegenerative lysosomal disorder with an autosomal recessive inheritance pattern caused by HEXA gene mutations. Children with this disease appear healthy at birth and psychomotor development proceeds normally during the first months of life. Slow neurological and development decline starts around 6 months. Clinical Case: The authors present an eighteen-month old girl, of nonconsanguineous parents, born of a full term bi-chorionic, bi-amniotic twin pregnancy, adequate somatometry at birth, with development delay noticed at the age of 6 months.

We saw her at 18 months at our referral clinic. We identified eating difficulties, motor delay (unable to stand alone), poor visual contact, poor social interaction, no verbal language and an abnormal acoustic-motor response (startle). Mother refers loss of previously acquired skills (hand clapping). On examination we also detected an abnormal posture with neck extension, hypotonia, ataxia, brisk reflexes, optic nerve atrophy and macular cherry red spot bilaterally. No other system anomalies detected. Cranial MRI and EEG were normal. A Gangliosidoses was suspected, so we requested lysosomal biochemical study and deficit of betahexosaminidase A was identified, compatible with GM2-Gangliosidoses - Tay-Sachs Disease. Genetic testing confirmed the diagnosis.

Conclusion: Gangliosidoses are rare diseases, at this moment without specific treatment, but its diagnosis is important for genetic counselling. A high level of suspicion is essential. The presence of a startle response and /or a cherry red spot should alert for these diseases.

233

\section{Neurology}

Cerebrospinal Fluid Hypotension following Fall in a Ten Year Old Child: Case Report

Gabriela Botelho ${ }^{1}$, Ricardo Domingos Grilo ${ }^{1}$, Patrícia Romão ${ }^{1}$, Vera Domingos Almeidal, Susana Gomes ${ }^{1}$, Pedro Soares Pinto ${ }^{2}$, Amets Sagarribay $^{3}$

${ }^{1}$ Pediatria, Hospital do Espírito Santo de Évora, Portugal

${ }^{2}$ Telemedicina, Medwed, Dr. Campos Costa, Portugal

${ }^{3}$ Neurocirurgia, Hospital Dona Estefânia, Centro Hospitalar Lisboa Central, Portugal

Background: Sacral fractures are uncommon injuries resulting from high-energy trauma. They are frequently underdiagnosed and mistreated, and so are their complications. Our goal is to report a case of Cerebrospinal Fluid (CSF) hypotension, under unusual circumstances, and highlight the importance of a careful clinical history and examination. Methods: The authors analyzed the atypical presentation, and diagnostic challenges, of a CSF fistula in a ten-year-old boy following a sacral fracture adjacent to a meningocele.

Results: A previously well ten-year-old boy presented with hip pain, after falling on the previous day, clashing the sacral region against the ground. Later, he reported hip and lower limb pain, while trying to move, that led him to seek medical attention. On examination, he was again able to mobilize both lower limbs. He reported no leg pain nor irradiation, no lack of sphincter control or any sensitive deficits. The neurological examination was normal and there were no cutaneous stigmata of occult spinal dysraphism. However, when asked to stand up, he began biparietal headache, nausea and several vomiting episodes, which improved when laying down and worsened with head elevation.

CT scan showed a sacral fracture of S3 and a meningocele, previously unknown. MRI scan of the head and spine revealed collections of CSF between the posterior arches of C1 and C2, between C7-D1, D1-D11 and along the anterior sacral surface. It confirmed a cystic intracanal lesion between S2-S4: a meningocele with probable spinal dysraphism associated and a recent fracture of S3 with potential laceration of the meningocele and opening of a CSF fistula.

Our diagnosis was CSF hypotension, secondary to the fistula opening. The child was in bedrest for two weeks, under watchful waiting in 
neurosurgical care. Subsequent control MRI showed significant CSF absorption with meningocele refilling and the child started to ambulate gradually.

Conclusion: The final diagnosis was challenging. The child presented with symptoms of CSF hypotension with no evident cause. The discovery of the meningocele led us to hypothesize a possible opening of a fistula, which is a rare diagnosis, later confirmed by MRI.

608

\section{Neurology}

Intracerebral Hemorrhage in an Eight Year Old Girl Marija Dimitrovska-Ivanova ${ }^{1,2}$, Elizabeta Zisovska ${ }^{2}$, Irena Nikolova ${ }^{1,2}$ ${ }^{T}$ Department of Pediatrics, Clinical Hospital Stip, Macedonia

${ }^{2}$ Department of Pediatrics, Faculty of Medical Sciences - University "Goce Delcev" Stip, Macedonia

Background: Intracerebral hemorrhage (ICH) rarely occurs in children. The most common underlying cause for ICH in the pediatric population are arteriovenous malformations, cavernous malformations and aneurysms. The rate of ICH among children is reported to be $0.8 / 100,000$ person-years resulting in mortality up to $33 \%$ and major deficits up to $40 \%$, including seizures, cognitive and motor impairments.

Objective: To present a pediatric case of spontaneous ICH as a result of cerebral vascular malformation.

Methods: Eight year old girl presented with acute onset headache, abdominal pain, nausea, vomiting and hand crumps. On physical examination conscious, afebrile, pale, dehydrated. Blood tests, examinations by ophthalmologist, otorhinolaryngologist and a general surgeon, combined with abdominal ultrasound examination, all with no pathological evidence. Non-contrast computed tomography (CT) scan demonstrated an oval hyperdensed mass with dimensions 27 x $19 \mathrm{~mm}$, on the right temporo-occipital region, subcortically with a local edema and calcifications. CT angiography (CTA), MRI and MRI angiography demonstrated a lesion corresponding to disrupted cavernous angiomas with a possible transformation into venous angiomas and compressive effect of trigonum on the right lateral ventricle. Patient was observed by neurosurgeon and treated symptomatically.

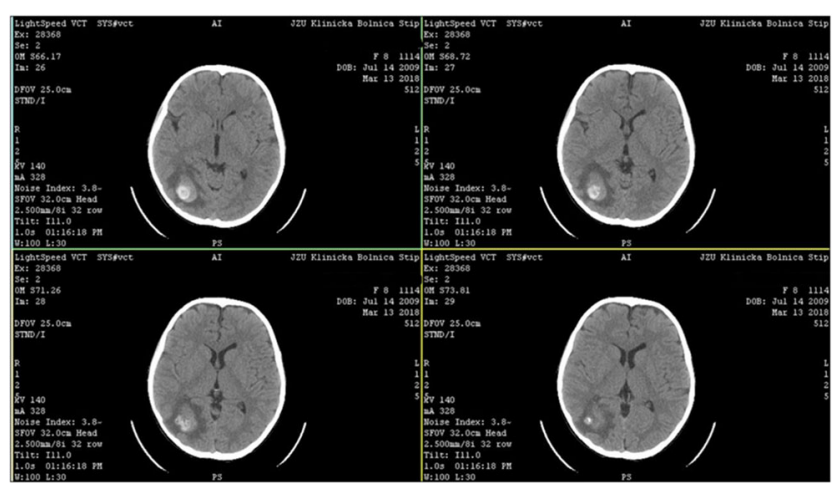

Non-contrast CT

Results: Two days after hospital discharge with epileptic seizure treated outpatient with antiepileptic drugs (Carbamazepin) and Vitamin C. Control MRI demonstrated complete resorption of bleeding with the presence of a small cavernoma in the parieto-occipital right region. Six months after initial ICH, cavernoma was surgically removed. Control MRI of brain in favor of complete excision of the cavernoma. Postoperative without epileptic seizures but still on antiepileptics and Vitamin C.

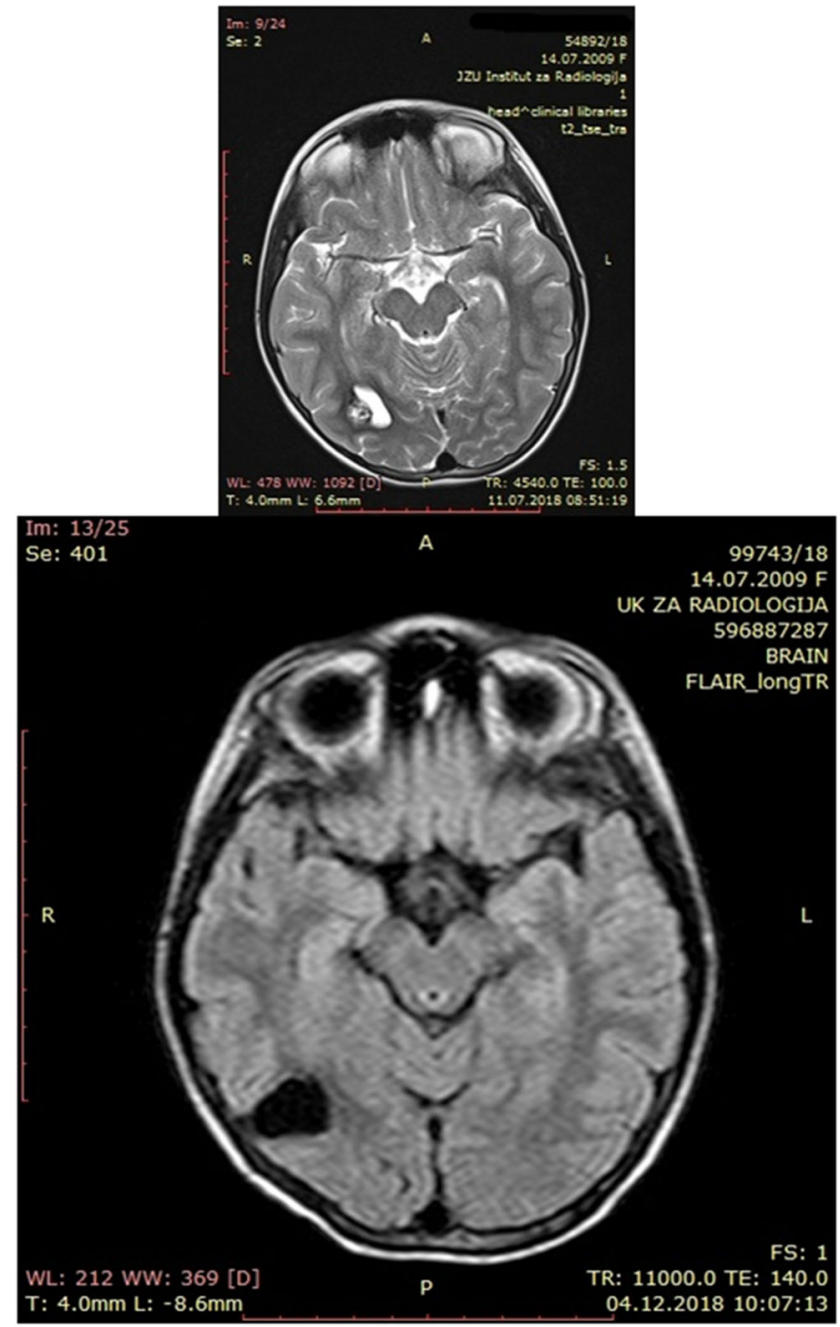

Preoperative MRI and Postoperative MRI

Conclusion: Rupture of brain vascular malformations can cause intracerebral or subarachnoid bleeding. The pressure to the surrounding brain structures, necrosis and focal changes, can develop neurological signs and epileptic seizures as in this case.

1009

Neurology

Epilepsia partialis continua: what lies beneath?

Catarina Amaro ${ }^{1,2}$, Ana Esteireiro ${ }^{1,3}$, Filipe Palavra ${ }^{1,4}$, Sillvia Carvalho ${ }^{5}$, Cristina Pereiral, ${ }^{1}$ Conceição Robalo ${ }^{1}$

${ }^{1}$ Centre for Child Development - Neuropediatrics Unit, Hospital Pediátrico, Centro Hospitalar e Universitário de Coimbra, Portugal

${ }^{2}$ Paediatrics Department, Centro Hospitalar Universitário Cova da Beira, Portugal

${ }^{3}$ Paediatrics Department, Hospital das Caldas da Rainha, Centro Hospitalar do Oeste, Portugal

${ }^{4}$ Faculty of Medicine, Coimbra University, Portugal

${ }^{5}$ Medical Imaging Department - Neurorradiology Unit, Centro Hospitalar e Universitário de Coimbra, Portugal

Background: Epilepsia partialis continua (EPC) is a variant of simple focal motor status epilepticus, in which frequent repetitive muscle jerks continue over prolonged time periods. There are several aetiologies, being Rasmussen's encephalitis (RE) the most common cause of EPC in 
children. RE is a rare and severe progressive disorder of unilateral brain dysfunction, focal seizures and inflammatory histopathology, with an uncertain, but probably immune-mediated etiopathology.

Case report: An 8-year-old girl, with unremarkable past medical and family history, was referred to the Neuropaediatrics clinic because of right hemifacial abnormal movements without loss of consciousness, for the last 3 months. Her physical exam showed repetitive clonic movements affecting the right hemiface, including tongue and soft palate on the right, and orbicularis oculi muscle. Blood tests, lumbar puncture and the initial brain-MRI were normal. The first video-EEG revealed abundant focal paroxysmal activity in the left frontocentral region frequently associated to clonic movements in the right hemiface. She was started on antiepileptic drugs, but there was a progressive clinical and electrophysiolgical worsening, with almost continual paroxysmal activity, despite the several drugs used. Immunosupression was initiated with systemic corticotherapy and intravenous immunoglobulin infusions, with reasonable seizure control. Serial brain-MRI evaluation revealed signal changes in left cortical and sub-cortical areas in the precentral gyrus, as well as cortical atrophy. Clinical, neurophysiologic and brain imaging data suggest the diagnosis of RE.

Conclusion: For the general pediatrician, the early recognition of an EPC is crucial, since it generally has a complex etiological diagnosis, whose functional prognosis can be very difficult. Even so, the early initiation of a targeted therapeutic intervention may help to address the potentially disabling effects of EPC.

\section{3}

\section{Neurology}

Neuroradiological Findings on Pediatric Autoimmune Encephalitis: How can Imageology Contribute to the Diagnosis?

Pedro Barradas ${ }^{1}$, Ana Esteireiro ${ }^{2,3}$, Filipa Rodrigues ${ }^{2}$, Cristina Pereira $^{2,4}$, Silvia Carvalho ${ }^{I}$, Conceição Robalo ${ }^{2,4}$

${ }^{1}$ Neuroradiology Department, Centro Hospitalar e Universitário de Coimbra, Portugal

${ }^{2}$ Centre for Child Development - Neuropediatrics Unit,, Hospital Pediátrico de Coimbra, Portugal

${ }^{3}$ Pediatrics Department, Caldas da Rainha Unit, Centro Hospitalar do Oeste, Portugal

${ }^{4}$ Epilepsy Center - EEG Laboratory, Hospital Pediátrico de Coimbra, Portugal

Background: Autoimmune encephalitis (AE) are a diagnostic challenge and have deserved a focus of increasing attention in pediatric age. The magnetic resonance imaging (MRI) still does not have a well-established role in the diagnosis of this pathology, since the abnormalities reported in the literature are usually nonspecific.

Objective: Characterizing imagiologically the cases hospitalized for $\mathrm{AE}$ in the HP-CHUC.

Methods: Retrospective review of cranioencephalic (CE) MRI images of patients, with ages between 28 days and 18 years, with the diagnosis of probable or definitive AE, between the years 2014 and 2018. Cases with diagnosis of encephalitis of Rasmussen were excluded.

Results: Six patients were identified, 4 males. From the etiological study, anti-GAD65 antibodies were identified in 1 patient and anti-Ma2 antibodies were found in another. The imaging study was performed between the $3 \mathrm{rd}$ and 15 th days of the disease. In the 3 children with imaging abnormalities, the temporal cortex was the most frequently involved, in a unilateral way in 2 cases. CE-MRI revealed slight cortical thickening, without other associated anomalies, in 2 patients. In the other case with imaging abnormalities, in which anti-GAD65 antibodies were identified, hyperintense lesions were observed in T2 FLAIR, involving the parahippocampal gyrus, splenium of the corpus callosum and amygdala, suggesting the diagnostic hypothesis of limbic encephalitis. There was no enhancement of the lesions in any of the cases. One patient presented partial regression of the lesions in the control CE-MRI.

Conclusion: In this study, half of the patients had a normal CEMRI, as reported in the literature. Although in many cases there is no imagiological changes in $\mathrm{AE}$, neuroimaging has a fundamental role in the exclusion of other pathologies involving CNS and the involvement of limbic structures may evoke this diagnosis, although there are still no specific imagiological standards guiding the diagnosis in this pathology.

\section{6}

\section{Neurology}

One Imagiologically Aggressive Presentation of Pediatric Multiple Sclerosis

Pedro Barradas ${ }^{1}$, Ana Esteireiro $^{2,3}$, Sílvia Carvalho ${ }^{1}$, Rui Pedro Pais ${ }^{1}$, Filipe Palavra ${ }^{2,4}$

${ }^{I}$ Neuroradiology Department, Centro Hospitalar e Universitário de Coimbra, Portugal

${ }^{2}$ Centre for Child Development - Neuropediatrics Unit, Centro Hospitalar e Universitário de Coimbra, Portugal

${ }^{3}$ Pediatrics Department, Caldas da Rainha Unit, Centro Hospitalar do Oeste, Portugal

${ }^{4}$ Faculty of Medicine, Coimbra University, Portugal

Background: Multiple sclerosis (MS) is an inflammatory demyelinating disease of the central nervous system (CNS) which, despite affecting mainly young adults, has been diagnosed in the pediatric population, knowing that $2-5 \%$ of all patients display initial symptoms before the age of 16 . Magnetic resonance imaging (MRI) is fundamental in the diagnosis of this pathology.

Clinical case: A 14-year-old teenager was brought to the ER for pain in both thighs and decreased sensitivity in the right leg and foot, 2-3 days after the pain onset. Neurological examination revealed only hypoactive patellar reflexes. Other neurological symptoms were denied. After hospitalization, complaints of low back pain with radiation to the right lower limb arose.

MRI showed countless multifocal injuries in the white matter, including juxtacortical, periventricular, subcortical, callosomarginal, capsular, in the protuberance, cerebellum and cervical spinal cord. To point out, coexisted injuries confluents of the white matter, subcortical and periventricular. Multiple lesions presented enhancement after administration of gadolinium.

Given the distribution in the temporal lobe, MS and CADASIL (cerebral autosomal dominant arteriopathy with subcortical infarcts and leukoencephalopathy) were considered as diagnostic hypotheses. However, the improvement after corticosteroids treatment and the presence of oligoclonal bands CSF helped confirm MS. The imaging findings meet the dissemination criteria in space and time (according to the criteria of McDonald Reviewed from 2017). Was initiated treatment with Natalizumab IV, with good results. Conclusion: Despite the exuberant lesions on MRI, the wearer showed relatively little symptomatology and recovered completely after corticosteroids therapy. This case reminds us that imaging findings do not always reflect the clinical spectrum, highlighting the concept of clinicalradiological dissociation. It is also an atypical case since it is rare for pediatric MS to present itself with confluent white matter lesions in the initial phase of the disease. 


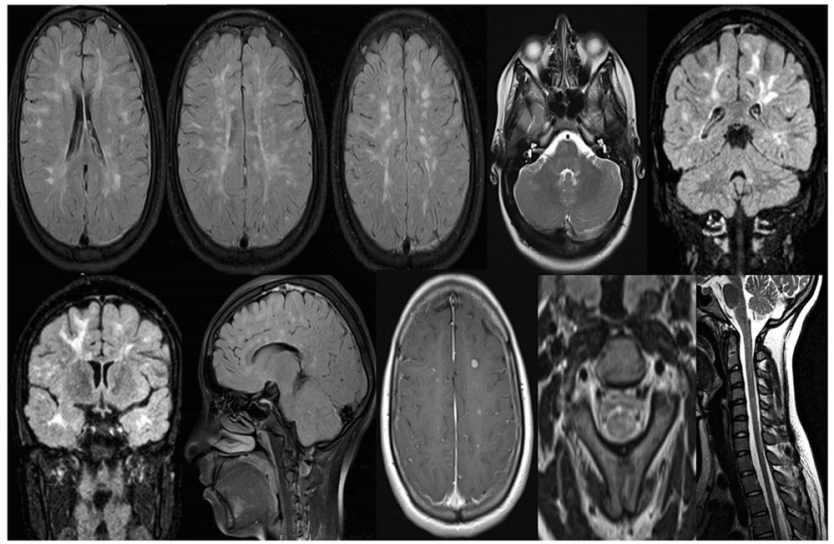

557

\section{Neurology}

Hypotonia: A Clinical Sign, Several Possible Aetiologies

Ana Esteireiro $^{1,2}$, Catarina Abreu Amaro ${ }^{1,3}$, Pedro Barradas ${ }^{1,4}$, Filipe Palavra ${ }^{I, 5}$, Isabel Fineza ${ }^{l}$

${ }^{1}$ Centre for Child Development - Neuropediatrics Unit, Hospital Pediátrico, Centro Hospitalar e Universitário de Coimbra, Portugal

${ }^{2}$ Pediatrics Department, Hospital das Caldas da Rainha, Centro Hospitalar do Oeste, Portugal

${ }^{3}$ Pediatrics Department, Centro Hospitalar da Cova da Beira, Portugal ${ }^{4}$ Neuroradiology Department, Centro Hospitalar e Universitário de Coimbra, Portugal

${ }^{5}$ Faculty of Medicine, Coimbra University, Portugal

Background: Hypotonia is defined as a decrease in muscle resistance to passive movement. It is easily recognized in the observation of the newborn (NB), being a frequent sign of disease in this age group.

Clinical cases:

Case 1: Male NB, childbirth by cesarean section at 35 weeks, Apgar score $2 / 8 / 8$ needing hospitalization in the NICU and ventilatory support. Because of generalized hypotonia, muscular weakness and weak primitive reflexes, a Neuropediatrics evaluation was made at D2 of life. It was observed: batrachian position, facial hypomimia, bilateral eyelid ptosis without ophthalmoparesis and reactive pupils; sialorrhea, compromised swallowing and cough reflexes; narrow chest with predominantly abdominal breathing; poor muscle masses, flaccid quadriparesis, absent reflexes and arthrogryposis. His mother showed a myopathic face and a clear myotonic phenomenon. Genetic studies confirmed the diagnosis of myotonic dystrophy type 1 . The NB left the hospital in D113 of life under non-invasive ventilation, physiotherapy and nasogastric tube feeding. Case 2: Male infant with healthy parents, normal gestational and neonatal periods. He was derived for a Neuropediatrics observation with 1 month of age due to hypotonia, hyporeflexia, and poor active movements and primitive reflexes. On neurological examination, he presented good fixation and eye movements, apparent fasciculations of the tongue, severe axial and peripheral hypotonia, without spontaneous movement observed, bell-shaped chest, inter-bone muscle amyotrophy, areflexia and paradoxical breathing. The genetic study supported the diagnosis of spinal muscular atrophy type 1 . He started on intrathecal Nusinersen and physiotherapy.

Both cases maintain a multidisciplinary follow-up.

Conclusions: Hypotonia is a nonspecific sign with many possible underlying aetiologies (from central nervous system to the muscle itself). The clinical history and neurological examination are vital, providing clues to a methodical and systematic investigation. It is essential to accomplish an early etiological diagnosis to implement adequate therapy (when available), establish prognosis and offer genetic counselling.
468

Neurology

Correlation between Salivary Cortisol Level and Sustained Auditory Attention

Mariza Feniman $^{1,2}$, Maria Renata José ${ }^{1}$, Viviane Fernandes ${ }^{2}$

${ }^{T}$ Fonoaudiologia, Universidade de São Paulo - FOB-USP, Brazil

${ }^{2}$ Fonoaudiologia, Universidade de São Paulo-HRAC-USP, Brazil

Background: Cortisol is a corticosteroid present in body fluids and controlled by the Hypothalamic-Hypophysis-Adrenal (HHA) axis, which under equilibrium conditions maintains the circadian rhythm, and is activated in stressful situations. Thus, measurement of salivary cortisol can provide important information about the functioning of the HHA axis under normal and stress conditions, as well as the involvement of cortisol in the mechanisms that determine activity and adaptation to environmental changes. Stress situations can cause cortisol to be produced in greater amounts than usual, and cortisol levels strongly influence brain function, with reports of decreased attention levels. Thus, it was hypothesized that the increase in salivary cortisol levels is correlated with the low test performance that evaluates the attention, in this study the sustained auditory attention.

Objective: to verify a possible correlation between the level of salivary cortisol and sustained auditory attention in children with cleft lip and palate.

Method: Prospective study with 69 children aged 6 to 11 years, with cleft lip and palate, with normal hearing and no history of inattention. The children were submitted to saliva collection for the measurement of cortisol level and to the Sustained Auditory Attention Ability Test (SAAT) to verify sustained attention.

Results: The results demonstrated the absence of statistical significance between the sexes, both for the cortisol level and for the sustained auditory attention. Seven-year-olds had the worst outcomes in sustained attention and the highest levels of salivary cortisol.

Conclusion: A positive correlation with a statistically significant difference between the salivary cortisol level and the auditory attention ability was observed only in children with cleft lip and palate at the age of seven years.

461

Neurology

The Applicability of Software in the Investigation of Sustained Auditory Attention

Mariza Feniman $^{1,2}$, Maria Renata José ${ }^{1}$, José Roberto Pereira Lauris ${ }^{I}$, Roberta Ribeiro Rosa ${ }^{2}$, Dagma Abramides ${ }^{1}$, Luciana Paula Maximino ${ }^{1,2}$ ${ }^{1}$ Fonoaudiologia, Universidade de São Paulo - FOB-USP, Brazil

${ }^{2}$ Fonoaudiologia, Universidade de São Paulo-HRAC-USP, Brazil

Background: Sustained attention is the ability to focus on a stimulus over an extended period of time. Deficits in this ability cause difficulty in concentration and impairment in development and learning.

Objectives: to examine the performance of children on a software designed to investigate the ability of sustained auditory attention and to compare the scores obtained in this format with those from the original test.

Methods: This prospective study included 52 children of both genders, aged 6 to 11 years with normal hearing and no complaint of inattention and/or hyperactivity. All children used the software. The total error score (inattention and impulsivity) and the vigilance decrement defined the performance in the software. The scores obtained in the two formats (software and original in Compact Disk) were compared. The t-test and correlation coefficient were used in the statistical analysis. The level of significance was $\mathrm{p} 0.05$.

Results: Mean values in the total score ranged from 100.6(46.8) to 70.8(40.6) and from 125.5(50.9) to 58.0(49.1), respectively, gender and 
ages. The vigilance decrement ranged from 3.6(3.3) to 3.0(3.7) and from 3.6(3.5) to 1.9(3.7).

Conclusion: The performance of the male children using the software differed significantly from the female. Male children showed more inattention and impulsivity errors and in the total error score. The number of errors decreases as the age increases, however age did not correlate with vigilance decrement. Inattention errors were more frequent than impulsivity. Higher values were obtained using the software, when compared to the original version.

\section{8}

\section{Neurology}

Miller Fisher Syndrome: A Case Report of an Uncommon Disease Adriana Ferreira ${ }^{1}$, Eulália Sousa ${ }^{1}$, Cristina Garrido ${ }^{2}$, Manuela Santos ${ }^{2}$, Cláudia Monteiro $^{I}, \hat{A}^{\text {ngela Machado }}{ }^{1}$, Ana Garrido ${ }^{1}$

${ }^{I}$ Department of Paediatrics, Centro Hospitalar do Tâmega e Sousa, Portugal

${ }^{2}$ Department of Paediatrics, Centro Materno-Infantil do Norte, CHUP, Portugal

Miller Fisher syndrome (MFS) is considered a variant of Guillian-Barré syndrome (GBS) characterized by the triad of ophthalmoplegia, ataxia and arreflexia. It is an uncommon disorder with an estimated incidence of 1 to $5 \%$ of the total GBS cases in Western countries.

We describe a 15 year-old female who came to the emergency department complaining of dizziness, unsteady gait, diplopia and pain in the hands and feet with a few hours of evolution. The patient reported vomiting and diarrhoea about two weeks before symptom onset. Her vital signs were normal. The neurological assessment revealed bilateral VI nerve palsy, ataxic broad-based gait, arreflexia and limb dysesthesia. Lower limb strength was slightly reduced. Routine blood tests were normal. Cerebrospinal fluid examination didn't show albuminocytologic dissociation (first day of disease) and was negative for the presence of oligoclonal bands, gram stain, culture and polymerase chain reaction for neurotropic viruses. Cranial and medullar magnetic resonance imaging was normal with no cranial nerve enhancement or thickening of the cauda equina. The serologic study revealed evidence of acute infection by Coxsackie virus. The electromyography was normal and serum anti-gangliosides and anti-neuronal antibodies were negative. The patient received intravenous immune globulin with clinical improvement noticed at day two of treatment. Complete recovery was achieved in four months.

MFS is a rare, self-limiting condition and its diagnosis is based on the presence of the typical clinical triad. The albuminocytological dissociation is not present in a majority of cases in the first week of symptoms. Although usually benign, it is important to know this syndrome and how to manage it in order to narrow the differential diagnoses and act properly and promptly.

\section{7}

\section{Neurology}

\section{A Rare Manifestation of Epstein-Barr Virus Carolina Freitas Fernandes ${ }^{I}$, Bernardo Camacho ${ }^{I}$, Conceição Freitas ${ }^{l}$, Paulo Rego Sousa \\ ${ }^{1}$ Paediatrics Department, Hospital Central do Funchal, Portugal}

Background: Epstein-Barr virus (EBV) is a highly disseminated herpesvirus, associated with several manifestations, such as infections of the Central Nervous System. The incidence of EBV encephalitis is less than $0.5 \%$, and commonly presents with confusion, decreased level of consciousness, fever and/or epileptic seizures.

Objectives: Herein, we report a case of 14-year-old female adolescent with confirmed EBV encephalitis being successfully treated with acyclovir.
Methods and Results: Case Report: A 14-year-old female adolescent is brought to the Pediatric Emergency Service with 8 days evolution symptoms, started with general malaise and fatigue, followed by fever and bilateral eyelid edema. On the last day she presented with vomiting, followed by dysarthria, deviation of the labial commissure, sialorrhoea, ocular revulsion and generalized tonic-clonic seizure. Her physical examination showed bilateral palpebral edema (Hoagland Sign), mental confusion and psychomotor agitation. Initial laboratory testing showed $36 \%$ activated lymphocytes and positive Paul-Bunnell test. MRI was performed showing T2 FLAIR hypersignal at the posterior border of the left putamen and laterally of the right putamen, and at the caudates heads bilaterally. Lumbar puncture was done and cerebrospinal fluid was positive for EBV PCR, she started acyclovir therapy, for 14 days (7 days IV). Electroencephalogram was performed at the fourth day of hospitalization, showing generalized background slowing, without paroxysmal activity. She presented gradual clinical improvement being discharged after 10 days, with levetiracetam 500mg twice daily. Patient was followed in pediatric neurology consultation, and after 6 months, was asymptomatic with EEG normalization, starting levetiracetam tapering.

Conclusion: Among infectious causes of encephalitis, viruses are the most frequently identified etiology. A wide variety of viruses can cause encephalitis. Encephalitis caused by EBV does not typically follow an infectious mononucleosis-like illness, so we should be alert for this condition that can be a life-threatening emergency, requiring prompt intervention.

382

\section{Neurology}

Multiple Sclerosis in a Pediatric Point of View: A Case Report

Mariana Gaspar ${ }^{1}$, Ana Catarina Fernandes ${ }^{1}$, Maria Bandeira Duarte ${ }^{1}$, $\overline{\text { Filipa Vilarinho }}^{1}$, Susana Moleiro ${ }^{1}$, Adelaide Palos ${ }^{2}$, João Sequeira ${ }^{3}$, Rita Lopes Da Silva ${ }^{4}$, Aldina Lopes ${ }^{1}$

${ }^{1}$ Serviço de Pediatria, Departamento da Mulher e da Criança, Hospital Distrital de Santarém, Portugal

${ }^{2}$ Serviço de Neurologia, Hospital Distrital de Santarém, Portugal

${ }^{3}$ Serviço de Neurologia, Centro Hospitalar Universitário Lisboa Central, Portugal

${ }^{4}$ Serviço de Neuropediatria, Centro Hospitalar Universitário Lisboa Central, Portugal

Background: Multiple Sclerosis (MS) is an immune-mediated disorder characterized by episodes of demyelination in the Central Nervous System (CNS). Pediatric MS is increasingly recognized, mostly in females, usually presenting as an isolated syndrome (eg. optic neuritis). Diagnosis usually involves Brain Magnetic Ressonance (MRI), Cerebrospinal fluid (CSF) analysis and McDonald criteria for dissemination in space and time. Pulse intravenous methylprednisolone is used in acute attacks. Relapsingremitting MS (RRMS) is the initial form in $97-99 \%$ and the recommended treatment is disease-modifying therapy (injectable or oral).

Objective: Describe a MS case in pediatric age, emphasizing the need for greater awareness of this entity.

Case Report/Results: A 12 year-old girl, previously healthy, complains of sudden onset of paresthesias, right upper limb paresis with inability to write. Symptoms started 5 days before, after her dog death. Examination showed altered fine mobility, right dysmetria and asymmetric gait. Normal Computed Tomography. MRI revealed several areas of hyperintensity in T2-sequences, the major on left pons, suggesting active MS lesions. She was admitted to a Neuropediatric Department and received a methylprednisolone pulse. CSF showed oligoclonal bands and intrathecal synthesis of $\mathrm{IgG}$ and $\mathrm{IgM}$. Two months later, Interferon beta1a was initiated. Meanwhile, she had another relapse with headache, dysarthria, right paretic gait and limb paresthesias correlating with two 
new lesions on MRI. Currently, she shows good clinical and imaging evolution.

Conclusion: This adolescent has a typical pediatric MS presentation, with typical space-time dissemination pattern and two separated clinical attacks involving different areas of CNS. The recent loss of her dog could have led to a misdiagnosed somatization disorder, emphasizing that in this age group some confusion factors can come in the way to a good diagnosis.

\section{0}

\section{Neurology}

\section{ADHD Patients Followed in a Level Two Pediatric Center: Case} Series

Giulia Riggi ${ }^{I}$, Leonor Machado ${ }^{I}$, Joana Gonçalves ${ }^{1}$, Melissa Brigham de Figueiredo ${ }^{I}$, Inês Silval, Ana Lança', Laura Azuraral', Raquel Marta $^{1}$, Constança Gouvêa Pinto ${ }^{1}$, António Macedo ${ }^{1}$

${ }^{1}$ Department of Pediatrics, Hospital de São Francisco Xavier, Portugal

Background: Attention deficit hyperactivity disorder (ADHD) is a childhood-onset neurodevelopmental disorder characterized by inattention, motor hyperactivity, and impulsivity, with difficulties often continuing into adulthood.

Objective: To describe the epidemiological and clinical characteristics of ADHD patients followed in a level two Pediatric Center.

Methods: Retrospective descriptive study of patients diagnosed with ADHD followed in a level two Pediatric Center during a 2.5 year period. Results: 2007 children attended the Neurodevelopment Consultation in the study period and 516 (25.7\%) met ADHD diagnostic criteria. 71\% were male. Median age at first consultation was 7 years. $48 \%$ were sent by their Primary Care Physician and learning disabilities and hyperactivity/ inattention were the main causes for referral. The most frequent associated comorbid disorders were specific learning disorders and intellectual developmental disorder. $52 \%$ of patients required specific school adequacies. At the last appointment, $68 \%$ of patients were under treatment with methylphenidate and $2 \%$ with atomoxetine, with good outcomes.

Conclusion: ADHD was the most frequent diagnosis among pediatric patients attending our Neurodevelopment Consultation, with a significant male predominance, often as a comorbid diagnosis. Intervention with a multidisciplinary and pharmacological approach showed favorable results.

788

\section{Neurology}

Spontaneous Carotid Artery Dissection - A Case Study in Pediatric Aged

Carolina Gonçalves ${ }^{1}$, Alexandra Andrade ${ }^{I}$, Bernardo Camacho ${ }^{1}$, Irina Rosa $^{I}$, Lucília Aveiro ${ }^{I}$, Paulo Sousa ${ }^{I}$

${ }^{1}$ Serviço de Pediatria, Hospital Dr. Nélio Mendonça, Portugal

Background: Craniocervical arterial dissection is rarely recognized in children and usually presents with symptoms of acute ischemic stroke (AIS) or transient ischemic attack. Most cases result from trauma to the oropharynx or neck, but spontaneous dissections have been reported in a variety of systemic conditions (such as connective tissue disorders). In the absence of systemic disease or antecedent trauma, arterial dissection appears to be rare in younger children.

Objective: We present a case report of a child with AIS due to spontaneous carotid artery dissection (CAD).

Methods - Case Report: A healthy five-year-old male, without antecedent illness or history of obvious trauma, presented with holo-cranial headaches, deviation of the right labial commissure, and left hemiparesis twelve hours before admission to the hospital. At admission, computed tomography (CT) was normal, but magnetic resonance imaging (MRI) revealed recent stroke in the cortical region and deep distribution within the right middle cerebral artery. The child was initially treated with subcutaneous low weight heparin and oral acetylsalicylate acid. On the third day, he presented with a severe headache, including vomiting. Repeated CT revealed an increase of intralesional edema in the areas of subacute infarction without hemorrhagic transformation. On the fifth day, neck vessels Doppler ultrasound revealed signs of right internal CAD. After diagnosis of CAD, the patient started therapy with warfarin. During hospitalization, there was improvement in loss-of-strength and regression of the deviation of the labial commissure. There were no additional symptoms, and the patient was discharged after thirteen days of hospitalization. In follow-up control neck and head Angio CT showed total resolution of CAD.

Conclusion: Spontaneous CAD is a potential cause of transient neurological symptoms and AIS and must be considered as an etiology of childhood strokes, especially when symptoms include headache or cervical pain. However, appropriate clinical judgment should be exercised.

\section{4}

\section{Neurology}

Insulin-Like Growth Factor Binding Protein-3 Deficiency Leads to Behavior Impairment through Monoaminergic Dysfunction Masayuki Itoh ${ }^{1}$

${ }^{T}$ Mental Retardation and Birth Defect Research, National Center of Neurology and Psychiatry, Japan

Background: Insulin-like growth factor binding protein-3 (IGFBP3) regulates IGF bioactivity, induces apoptosis and inhibits cell growth independent of IGFs, but the functional role of IGFBP3 in the brain is not clear.

Objective: We revealed the effect of IGFBP3 on the brain by characterizing the phenotype of igfbp3-null mice.

Methodsand Results: Compared to wild-type mice, Igfbp3-null mice showed significantly decreased IGF-1 content in the brain but showed no change in weights of brain and body. In igfbp3-null mice the number of dendritic spines was significantly reduced, and the dendritic diameter was thin. In addition, in igfbp3-null mice, a decrease in phosphorylated Akt and ERK1/2 significantly reduced PSD-95 expression, and GAD65/ 67 expression was significantly decreased.

Conclusion: These results indicate that IGFBP3 deficiency impairs neuronal structure and signaling. In behavioral studies, igfbp 3-null mice were hyperactive, and a Y-maze alternation test revealed impaired spatial working memory, but no anxiety-like behavior. Monoaminergic analysis using HPLC indicated that igfbp3-null mice showed lower levels of dopamine and serotonin compared to wild-type mice, suggesting an abnormal monoaminergic neurotransmission. In conclusion, our studies demonstrated that the deletion of IGFBP3 results in behavioral impairments that are associated with abnormal synaptic function and monoaminergic neurotransmission, which helps to characterize the critical role of IGFBP3 in the brain.

\section{4}

\section{Neurology}

Predictive Factors of Recurrence of Febrile Seizures during the Same Febrile Illness

Jun Kubota $^{1,2}$, Norimichi Higurashi ${ }^{2}$, Daishi Hirano ${ }^{2}$, Hirotaka Isono ${ }^{1,2}$, $\overline{\text { Haruka Numata }}^{1,2}$, Takayuki Suzuki ${ }^{1,2}$, Daisuke Kakegawa ${ }^{1,2}$, Akira Ito $^{1,2}$, Manabu Yoshihashi ${ }^{3}$, Takeru Ito ${ }^{1,2}$

${ }^{1}$ Department of Pediatrics, Atsugi City Hospital, Japan

${ }^{2}$ Department of Pediatrics, The Jikei University School of Medicine, Japan

${ }^{3}$ Department of Pediatrics, Kanagawa rehabilitation center, Japan 
Background: Febrile seizures (FS) are common childhood-onset seizures. The incidence of recurrence of FS during the same febrile illness (RFS) within 24 hours is $15-16 \%$. However, the predictive factors for RFS are unknown.

Objective: To determine the possibility of predicting RFS based on the clinical data at the emergency department.

Methods: This was a cross-sectional, retrospective study in Atsugi City Hospital in Japan, conducted between December 1, 2018 and February 28,2019 . FS are defined as the seizures accompanied by body temperature $\geq 38.0^{\circ} \mathrm{C}$ without central nervous system infection, occurring in children between 6-60 months of age. Exclusion criteria were multiple seizures before visiting the emergency department, diazepam administration before visiting the emergency department or when going home, seizures lasting 15 minutes, underlying diseases (i.e. epilepsy, gastroenteritis, etc.), and no laboratory test at the emergency department. The primary outcome was RFS.

Results: Fifty-one patients fulfilled the inclusion criteria. The incidence of RFS was $17.6 \%(9 / 51)$ and all except one patient reported the recurrence of a seizure within 24 hours from the first seizure. The group with RFS showed significant prevalence in males $(P=0.043)$ and a comparatively lower body temperature $(P=0.013)$. After adjustment for possible confounding factors, RFS showed significant associations with males (odds ratio, 18.13; 95\% confidence interval, 1.18-278.81; $P=0.04$ ) and body temperature (odds ratio, $0.13 ; 95 \%$ confidence interval, $0.02-0.75$; $P=0.02$ ). In this cohort, body temperature cut-off value $\leq 39.4^{\circ} \mathrm{C}$ and males showed specificity and negative predictive values of $78.6 \%$ and $86.8 \%$, respectively.

Conclusion: The incidence of RFS was $17.6 \%$. In this study, RFS was strongly related to a body temperature $\leq 39.4^{\circ} \mathrm{C}$ and a prevalence in males. In future, a prospective and large-sized sample study will be needed to determine the predictive factors for RFS.

892

Neurology

Autism and ADHD: Differential Functioning on a Computerized Performance Test (MOXO-CPT)

Yael Leitner ${ }^{I}$, Moran Hausman - Kedem ${ }^{1,2}$

Institute of Child Development, Dana Children's Hospital, Tel Aviv Sourasky Medical Center, Tel Aviv University, Tel Aviv, Israel, Israel

${ }^{2}$ Pediatric Neurology Unit, Dana Children's Hospital, Tel Aviv Sourasky Medical Center, Tel Aviv University, Tel Aviv, Israel, Israel

Objective: ADHD \& Autism Spectrum Disorder (ASD) have recently been discussed as sharing etiology and symptomatolgy, to such an extent that $60 \%$ of children with ASD show symptoms of ADHD, and some $30 \%$ of ADHD children have difficulties in social communication. The overlap or similarity of these two neurodevelopment disorders requires further studies, to understand their common denominator.

Method: We examined the performance of 25 ASD children (no ADHD) (6years) and $25 \mathrm{ADHD}$ (no ASD) children matched for age, gender and socioeconomic status on a computerized performance test (MOXO-CPT which incorporates visual and auditory stimuli serving as environmental distracters), commonly used as one of the tools in the clinical diagnosis of ADHD.

Results: We found that children with ASD performed better (borderline significance) on $3 \backslash 4$ test items (Attention, Timing, Hyperactivity), while Impulsivity was the same in both groups. Both groups were affected by visual and auditory distracters built-in the MOXO paradigm, but ASD children were significantly less distracted, and showed better performance consistency over time than the ADHD group.

Conclusion: On our computerized test battery MOXO, children with ASD showed better performance than ADHD children on most test items, challenging previous studies on this issue. Computerized Performance Test might provide a "protected" environment for ASD children that does not unravel their attention difficulties under naturalistic conditions.
1048

Neurology

Worn-out Tip Shoes as a Clue for a Family Genetic Diagnosis Sara Mosca ${ }^{1}$, José Fontoura Matias ${ }^{2}$, Diana Alba ${ }^{3}$, Mafalda Moreira ${ }^{3}$, Leonilde Machado ${ }^{3}$, Teresa Temudo ${ }^{4}$, Joana Martins ${ }^{4}$

${ }^{1}$ Pediatric Department, Centro Materno-Infantil do Norte, Centro Hospitalar Universitário do Porto, Portugal

${ }^{2}$ Pediatric Department, Centro Hospitalar Universitário São João, Portugal

${ }^{3}$ Pediatric Department, Centro Hospitalar Tâmega e Sousa, Penafiel, Portugal, Portugal

${ }^{4}$ Neuropediatrics Unit, Pediatric Department, Centro Materno-Infantil do Norte, Centro Hospitalar Universitário do Porto, Portugal

Background: Gait abnormalities and muscle weakness can be the presentation of different conditions, namely inflammatory, infectious, traumatic or degenerative diseases. The etiology is not always easy to define, as the findings of the physical exam can be nonspecific to a general pediatrician, such as lower limb hypertonia and orthopedic deformities. Therefore, it is fundamental to perform a detailed interview and physical exam.

Clinical case: A previously healthy, 11-years old male was observed in a general pediatrics appointment due to pain referred to the feet, muscle weakness, and progressive difficulties to walk associated with worn-out tip shoes. No sphincter complaints. Family history of an uncle and grandfather with similar symptoms, but no definitive diagnosis. General physical examination was normal. He had normal cognitive development, but showed lower limb increased osteotendinous reflexes, and discrete cavus feet. Routine laboratory findings were normal. At this point, the adolescent was referred to a Neuropediatric center. Neurologic evaluation revealed a spastic paraplegia, lower limbs hyperreflexia with right ankle clonus and bilateral Babinski. Brain and medulla magnetic resonance imaging were normal. Further investigation included a hereditary spastic paraplegia (SPG) genetic panel, which allowed the identification of a pathogenic gene mutation, on BSCL2 gene (SPG 17), which is associated with a dominant type of SPG. He is under physiotherapy treatment, and two years after diagnosis, he manages to walk independently.

Discussion: Hereditary spastic paraplegia is a syndromic designation for a clinically and genetically heterogeneous group of inherited neurodegenerative disorders, in which the main neurological symptoms and signs are lower limb spasticity and weakness. In childhood, the most prevalent are SPG 3 and 4. Clinicians should be aware of this diagnosis, especially when combining both clinical symptoms/signs with similar familiar history. Despite the positive family history, this was the first case with a genetic diagnosis, allowing family genetic counseling.

254

Neurology

The Clinical Profile of Childhood Epilepsy in Sudanese Children Attending the Neurology Department in Soba Hospital (Khartoum) Mohamed Osman Omer Dablouk ${ }^{l}$, Amin Alagib Mohamed Musal, Ahlam Abdulrahman Hamed ${ }^{2}$, Mamoun Mohamed Ali Homeida ${ }^{1}$, Rabih Brair Rabih ${ }^{1}$, Khalid Awad Mohamed ${ }^{3}$

${ }^{1}$ Pediatric, University of Medical Sciences and Technology, Sudan

${ }^{2}$ Pediatric, University of Khartoum, Sudan

${ }^{3}$ Pediatric, University of Weil Cornell Medicine, Qatar

Background: Epilepsy is a common neurological disorder affecting around $1 \%$ of children. The incidence in Sudan is likely to be higher given the high rate of genetic and acquired disorders caused by CNS infection and birth injury. Epilepsy can be idiopathic or non-idiopathic, symptomatic epilepsy indicates a known cause while cryptogenic epilepsy implies and unknown cause. 
Objective: To understand the demographic and clinical characteristics of epilepsy in children in the setting of a tertiary epilepsy clinic in Khartoum, Sudan.

Methods: This is a hospital-based cross sectional study recruiting patients with epilepsy attending atertiary pediatric neurology clinic.All patients attending the clinic in the 6 month period were included in the study. Data was collected by medical students and was analyzed on IBM SPSS Version 20.0 in a descriptive fashion.

Results: 284 Children were recruited. The mean age for children with epilepsy was 5.74 (range 0-12 Years). Females constituted 59.9\%, while $94.7 \%$ of patients were of low socioeconomic status. The most common type of seizures found were generalized tonic clinic at 51.8\% (147/284), followed by focal seizures at $21.1 \%$ (60/284). $71 \%$ ofour patients (201/284) had no identifiable cause (idiopathic), while $29 \%$ were non-idiopathic. Of the non-idiopathic group; $41 \%$ were due to congenital anomalies, followed by HIE at $24.1 \%$, infections at $16.9 \%$ trauma at $10.8 \%$ and tumors at $7.2 \%$. Only $18.3 \%(52 / 284)$ had a positive family history of epilepsy.

Conclusions: There appears to be a higher incidence of epilepsy in Children with low socio - economic status with a slight female predominance. The incidence of idiopathic epilepsy appears to be similar to international figures; which may indicate that some children with severe symptomatic epilepsy die at an early age. We acknowledge that this is a hospital based study and recommend broader community based and longterm studies to define the disorder.

\section{2}

\section{Neurology}

Late Diagnosis of MELAS Can Lead to A Serious Brain Edema that Ultimately Requires Decompressive Craniectomy

Oznur Gokce Nizam ${ }^{1}$, Esma Sengenc ${ }^{2}$, Osman Yesilbas ${ }^{3}$, Seyma Sonmez. Sahin $^{2}$, Akin Iscan ${ }^{2}$, Dilara Fusun Icagasioglu ${ }^{2}$

${ }^{1}$ Department of Pediatrics, Besmialem Vakif University, Turkey

${ }^{2}$ Department of Pediatrics, Division of Pediatric Neurology, Bezmialem Vakif Univsersity, Turkey

${ }^{3}$ Department of Pediatrics, Division of Pediatric Intensive Care, Bezmialem Vakif University, Turkey

Background: Mitochondrial myopathy, encephalopathy, lactic acidosis and recurrent stroke-like episodes (MELAS) is a rare, maternally inherited, neurodegenerative disease caused by mutations in the mitochondrial DNA. Early symptoms may include muscle weakness and pain, syncopes and seizures. The main pathophysiology is structural and functional abnormalities of mitochondrial DNA, various mutations affect mainly aerobic cellular metabolism at the level of electron transport chain. The clinical managament of patients is largely supportive.

Case: A 12 year-old-boy who had loss of conciousness one year ago which was treated like encephalitis, was having loss of consciousness spells with accompanying visual symptoms lasting 15-20 minutes since the last year. He presented to our pediatric intensive care unit with worsening episodes described as atonic seizures, inability to walk, vision loss and altered mental states for the last 10 days. He was unconsciousness and had stiff neck. Lumbar puncture was performed, no abnormal findings. His blood gas was normal. (No lactic asidosis.) Results of brain MR and CT imaging showed a lesion in the left occipital lobe with long T1, T2 signals, left shift and edema. On follow up, brain edema treatment was started and was commenced on ceftriaxon and acyclovir. All viral serology that could be cause of encephalitis and autoimmune encephalitis panel came back negative. Although he received antibiotheraphy,IVIG and pulse steroid treatment, his clinical findings and swelling/herniation were worsening so he was undergone decompressive craniectomy. The brain biopsy was determined non-specific encephalitis findings. MR angiography/venography was normal. The blood genetic test suggested a mutation of mt.3243AT, so we got diagnosis of MELAS. After being treated with arginine and coenzyme Q10, the patient dramatically improved.
Conclusion: We should keep in mind that if the diagnosis and treatment was delayed, mitochondrial and metabolic disorders can cause serious brain edema which ultimately may require decompression surgery.

183

\section{Neurology}

Chronic Inflammatory Demyelinating Polyradiculoneuropathy in a 26 Month Old Boy

Sara Teixeira ${ }^{1}$, Marta Vila Real ${ }^{1}$, Fátima Santos ${ }^{1}$

${ }^{T}$ Unit of Neurosciences of Childhood and Adolescence, Pediatric Service, Centro Hospitalar Vila Nova de Gaia/Espinho, Portugal

Introduction: Chronic inflammatory demyelinating polyradiculoneuropathy (CIDP) is an immune mediated treatable disorder of the peripheral nerves, with predominant motor involvement and an insidious onset, or recurrent episodes. Children present slowly progressive or relapsing episodes of gait difficulty, distal symmetric weakness and sometimes paraesthesiae. Reflexes are absent or depressed. Laboratory findings include elevated cerebrospinal fluid (CSF) protein with no increase in cells. Electromyography shows demyelination.

Case Presentation: A previously healthy 26-month-old boy, brought to the emergency department due to imbalanced gait and muscle strength decrease, with two days of evolution. Neurological examination revealed unstable gait, without preferential side, and decrease in distal muscle strength. Reflexes were absent in the lower limbs, and decreased in the uppers. He also presented altered sensitivity.

From the study, we highlight, elevated CSF protein with no increase in cells, and negative microbiological and virological study. Electromyography evidenced severe demyelinating neuropathy. Immunoglobulin and carbamazepine was started. He was oriented to physiotherapy, with partial recovery of limbs strength.

One year later, presented a similar clinical episode, with overlapping study, namely the electromyography. Immunoglobulin was restarted, having performed on a monthly basis for 8 months.

At 9-years-old, there was a new recurrence, in post-infectious context, with affection of upper and lower limbs. Electromyography revealed severe demyelinating neuropathy, without alterations suggestive of acute lesion.

Nowadays, at 12-years-old, he has autonomous gait, with a marked distal motor deficit at lower limbs and slight decrease in upper limbs strength. He has patellar reflexes, but not aquilians.

Conclusion: Authors pretend to emphasize the challenges in the diagnosis and management of paediatric CIDP. Despite being a rare diagnosis, clinical suspicion, early recognition and investigation with prompt management can result in better outcomes of this potentially treatable neuropathy. We also highlight the importance of corticosteroids in this pathology, contrary to it role in Guillain-Barré syndrome.

\section{7}

Neurology

Genotype-phenotype Association In A Boy With Neurofibromatosis Type 1

Sara Teixeira $^{l}$, Helena Santos ${ }^{1}$, Marta Vila Real ${ }^{1}$, Fátima Santos ${ }^{1}$ ${ }^{T}$ Unit of Neurosciences of Childhood and Adolescence, Pediatric Service, Centro Hospitalar Vila Nova de Gaia/Espinho, Portugal

Introduction: Neurofibromatosis type 1 (NF1), is the most common form of Neurofibromatosis. Clinical characteristics include: café-au-lait spots, inguinal and axillary ephelides, multiple neurofibromas and Lisch nodules. Is an autosomal dominant disorder, with extreme clinical variability. The gene complexity and the diversity of mutations in NF1 gene make the genotype-phenotype correlations very difficult. 
Case Presentation: We describe a case of a 9-years-old boy, first child of a healthy and unrelated couple, who had dismorphic facial features, multiple cafe-au-lait spots, and delayed psychomotor development since first year of life. At eight months of age, neurofibroma was detected, and molecular study was requested confirming the presence of mutation in the NF1 gene.

During follow-up, there was an increase in the number of cutaneous neurofibromas and development of numerous neurofibromas in nerve pathways, including intercostal, pelvic nerve plexus, thigh roots, as well as plexiform neurofibroma affecting the upper limb. He also presented intellectual impairment and Lisch nodule in the left eye.

Cerebral magnetic resonance showed small hamartomas in the pale globes, thalamus, peri-aquedutal region, splenius of the corpus callosum on the right side and at the ipsilateral deep slope of the cerebellar hemisphere and protuberance, with no mass effect.

The abdominal study revealed the presence of a retroperitoneal mass, measuring 95x68 mm, involving the large visceral vessels, but without occluding them and exerting mass effect on the adjacent structures. This mass still's under study.

Array-CGH analysis revealed microdeletion of $1.29 \mathrm{Mb}$ in the long arm of chromosome 17 (band 17q11.2).

Conclusion: Although many different mutations associated with neurofibromatosis, have been described, information about genotype-phenotype correlations are still limited. Authors pretend to emphasize the association of deletions involving NF1 gene and a more severe phenotype, as can be seen in our patient.

\section{9}

\section{Neurology}

Extensive Cerebral Venous Thrombosis as a Manifestation of an Uncommon Disease

Mariana Portela ${ }^{1}$, Ana Luisa Carvalho ${ }^{1}$, Andreia Lopes ${ }^{2}$, Teresa Pontes $^{I}$, Ana Paula Barbosa ${ }^{4}$, Carla Ferreira ${ }^{3}$, Arnaldo Cerqueira ${ }^{3}$, Ricardo Maré, ${ }^{5}$ Angela Oliveira ${ }^{3}$

${ }^{1}$ Paediatrics, Hospital de Braga, Portugal

${ }^{2}$ Paediatrics, Hospital Senhora da Oliveira, Portugal

${ }^{3}$ Paediatric Intermediate Care Unit, Hospital de Braga, Portugal

${ }^{4}$ Immunotherapy, Hospital de Braga, Portugal

${ }^{5}$ Neurology, Hospital de Braga, Portugal

Background: Cerebral venous thrombosis (CVT) has a wide range of manifestations and could be challenging to diagnose. Headache is the first and most frequent sign. Intracranial hypertension, encephalopathy, focal neurologic alterations or seizures could be associated. Thus, in presentations concerning CVT, urgent neuroimaging is necessary.

Objective and Methods: A 15 years old female, previously healthy, started a 5 day course of headache and vomiting, associated with left otitis media three days after the onset. Firstly observed in her hospital nearby, she started analgesia and antibiotics. Due the lack of improvement two days later, decreased muscle strength and paresthesias in the left upper limb, as well as left hypovision she resorted again. Normal neurological exam was described, except nuchal rigidity. No fever. A Computed Tomography angiography was performed, showing sagittal, recto and tranverse sinus thrombosis and cerebral edema. She was then transferred to our hospital and admitted in pediatric intermediate care unit. She started intravenous heparin, which was changed to warfarin and enoxaparin at the 13th day. In the Magnetic Resonance Imaging (MRI), extensive venous thrombosis was observed, with findings suggesting acute leukoencephalopathy and optic nerve compromise. Bilateral papilledema was observed. She initiated acetazolamide. The clinical outcome was good, with resolution of the complaints. MRI was repeated 15 days later, with some reduction in the venous congestion. The prothrombotic tests were negative, except elevated levels of homocysteine in urine suggesting classic homocystinuria, which was then confirmed with the mutation in CBS gene. She was discharged, keeping a close multidisciplinary follow up.
Conclusion: Classic homocystinuria is an autossomic recessive disease, related to the metionine metabolism and associated with prothrombotic events, so this patients must be monitored at regular intervals. Further investigations should be made in order to make a correlation with the abnormal extensive alterations in white matter and homocystinuria

\section{2}

\section{Neurology}

Determining the Quality of Life of Children Living with Epilepsy in Kenya: a Cross-Sectional Study Using the CHEQOL-25 Tool

Syeda Ra'ana Hussain ${ }^{1}$, James Orwa ${ }^{1}$, Caroline Mbuba Kathomi ${ }^{1}$, $\overline{D i l r a j}_{\text {Sokhi }}{ }^{I}$, Osman Miyanji ${ }^{1,2}$, Hussein Dossajee ${ }^{3}$, Pauline Samia ${ }^{l}$

${ }^{1}$ The Aga Khan University Hospital, Kenya

${ }^{2}$ Kenya Association for the Welfare of People Living with Epilepsy (KAWE), Kenya

${ }^{3}$ MP Shah Hospital, Kenya

Background: Children with epilepsy (CWE) in low/lower-middle income countries are likely to experience lower health-related quality of life $(\mathrm{HrQoL})$ due to poverty and stigma, especially in sub-Saharan Africa. Few studies have measured this reliably, as most tools capture caregiver rather than child responses; the CHEQOL-25 tool, however, collects both child and caregiver perspectives across five domains: 'interpersonal/social', 'present concerns', 'intrapersonal/emotional', 'secrecy', and 'normality'.

Objective: To measure HrQoL in CWE using CHEQOL-25 at paediatric outpatient settings in Nairobi, Kenya.

Methods: We conducted a prospective cross-sectional study, enrolling CWE aged 7-15 years and their caregivers. Good $\mathrm{HrQoL}$ was defined as CHEQOL-25 score 60 and 50 for CWE and caregiver respectively. CWE-caregiver corroboration was assessed using Kappa statistic; multivariate linear regression analysis elucidated factors affecting $\mathrm{HrQoL}$.

Results: We surveyed 177 CWE-caregiver couples [CWE median age 11 years (range 8-13); 53.1\% (94/177) male]. The commonest seizure type [66.7\% (118/177)] was tonic-clonic [median onset age 5(2-8) years; $41.8 \%$ (74/177) on one anti-epileptic]. $75.3 \%$ (133/177) of CWE visited clinic more than twice in the preceding six months due to uncontrolled seizures. $60.5 \%(107 / 177)$ of CWE and $56.5 \%$ (100/177) of caregivers reported good HrQoL (CHEQOL-25 scores 60.6/100 and 60.4/100 respectively), with significant CWE-caregiver response corroboration in the 'interpersonal/social' and 'intrapersonal/emotional' domains (p0.005). Good HrQoL was significantly less likely for CWE with: (i) male caregivers [odds ratio $(\mathrm{OR})=0.37 ; 95 \%$ confidence interval $(\mathrm{CI}) 0.17-0.81$; $\mathrm{p}=0.001]$; and (ii) caregivers with less than full primary education [OR=0.41(0.17-0.980; $p=0.04]$. 55.3\% (98/177) of CWE had a closeknit $(\mathrm{n} \leq 4$ members) circle of friends, with increasing friend numbers being positively associated with good HrQoL [OR=1.09(1.01-1.17)].

Conclusion: HrQoL was good in the majority of our cohort - as reported by both CWE and their caregivers - especially if the child had a bigger friends circle, but HrQoL was not good if the caregiver was male or uneducated.

280

\section{Neurology}

Profile of Children Admitted with Seizures in a Portuguese Level II Hospital within 8 Years

Diana Simão Raimundo ${ }^{I}$, Catarina Almeida ${ }^{I}$, Carolina Figueiredo ${ }^{l}$, Joana Mendão Carreiral, Joana Fortuna ${ }^{1}$, Sara Dias ${ }^{1}$, Sarah Stokreef ${ }^{1}$, Fernanda Gomes ${ }^{1}$

${ }^{1}$ Department of Paediatrics, Hospital do Divino Espirito Santo, Portugal 
Background: Seizures are a common manifestation of several childhood pathologies, often requiring hospitalization due to associated morbidity and mortality.

Objective: Characterize the population admitted in a pediatric hospital service with seizures, detailed by age groups.

Methods: Retrospective analysis of all admissions from January 1st 2011 to December 31st 2018. Inclusion criteria: seizures as motive of hospital admission and age between 0 and 17 years and 365 days; children with seizures occurring only after hospitalization were excluded. The studied variables were clinical presentation, etiology, gender, age, comorbidities, origin and destination after discharge.

Results: A total of 146 patients were admitted for seizures, with predominance in male gender $(61,4 \%)$ and in children under 4 years old $(53,4 \%)$. It was the first episode in $46,6 \%$, of which $26,5 \%$ had comorbidities. Presence of fever was commoner among 1-4 years old $(48,3 \%)$. Generalized tonicclonic were the most common type of crisis in every age group $(41,1 \%)$; $7,5 \%$ presented in status epilepticus. The most common causes were epilepsy $(33,6 \%)$ (prevailing in those older than 4 years old); complex febrile seizures $(20,6 \%)$ (uppermost in those under 4 years old) and structural brain injury associated epilepsy $(11,6 \%)$. Only $5(3,4 \%)$ were due to infection of the central nervous system (CNS). There was 1 death $(0,7 \%)$. There were abnormalities in $65,7 \%$ of the children who performed EEG $(73,9 \%)$ and in $55.2 \%$ of those who underwent neuroimaging $(19,9 \%)$.

Conclusion: The causes of seizures requiring hospitalization varies by age: complex febrile seizures in younger and epilepsy in older children. In this study, there were few episodes of CNS infection manifesting with seizures. Comorbidities may be an important predictor in first episode. A detailed medical history is crucial. Seizures require long-term surveillance: they can occur even when underlying pathology is identified and medicated and may require hospitalization for clinical stabilization.

\section{5}

\section{Neurology}

Regional Demographic Analysis of Status Epilepticus in Children Diana Raimundo ${ }^{1}$, Patrícia Torcato Ferrão ${ }^{2}$, Catarina Almeida ${ }^{1}$, Paula Pires $^{3}$, Fernanda Gomes ${ }^{1}$

${ }^{I}$ Department of Pediatrics, Hospital do Divino Espirito Santo, Portugal ${ }^{2}$ Department of Family Medicine, Villa Longa Family Health Unit, Portugal ${ }^{3}$ Department of Neurology, Hospital de Santo Espirito da Ilha Terceira, Portugal

Background: Status epilepticus is the most frequent neurologic emergency in children. Mortality is estimated as 3-15\%, with a much higher morbility. Etiology depends on age and most episodes may be triggered by infectious processes and previous lesions / congenit malformations of central nervous system.

Objective: To described the demographics of children admitted in a regional pediatric service with status epilepticus, detailed by age groups.

Methods: Retrospective analysis of department's records from January 1st 2011 to December 31st 2018. Inclusion criteria: all children with status epilepticus as motive of hospital admission and age from 29 days to 17 years and 365 days. Exclusion criteria: age under 29 days; children with status epilepticus occurring only after hospitalization. Studied variables: gender, age, comorbidities, clinical presentation, etiology, origin and destination after discharge. Results: A total of 14 episodes from 11 children (71,4\% male) were enrolled, representing $9,4 \%$ of all children admitted with seizures. Age range was 1 month to $13 \mathrm{y} / \mathrm{o}(1 \mathrm{y} / \mathrm{o}: \mathrm{n}=2,1-3 \mathrm{y} / \mathrm{o} ; \mathrm{n}=5,4-9 \mathrm{y} / \mathrm{o}: \mathrm{n}=4 ; 10-13 \mathrm{y} /$ o:n=3). Four presented with fever, all aged 1 to 3 years old. It was first seizure episode in 2 children, both with no fever. Most comon comorbidities were epilepsy due to structural malformation $(n=3)$, genetic syndrome with epilepsy $(n=3)$, epileptic encephalopathy $(n=3)$ and perinatal hypoxic-ischemic cerebral palsy $(n=2)$. The majority of crisis where related to those comordibities. Generalized tonic-clonic were the most common unset. One patient died during hospitalization and 1 was transfered to Pediatric Intensive Care.
Conclusions: Being the only hospital in a regional island area, it receives all children needing hospital care, so those data are representative of the region's demography. There was male but no age predominance. Most of etiology were according to literature, but there was no case of infectious processes. Comorbidities was the most important factor in this study and may be an important predictor.

\section{0}

\section{Neurology}

Cerebral Venous Thrombosis in a Patient with Nephrotic Syndrome Irina Rosa ${ }^{l}$, Carolina Gonçalves ${ }^{l}$, Francisco Silva $^{l}$, Maria João Borges ${ }^{l}$, Paulo Sousa

${ }^{1}$ Pediatric department, Hospital Dr Nélio Mendonça, Portugal

Introduction: Nephrotic syndrome (NS) is the most frequent glomerular disease of childhood, reaching about 2-7: 100,000 children. This may be associated with thromboembolic complications, mostly with venous origin. The diagnosis of cerebral venous thrombosis is difficult, since the clinic may be non-specific. In the acute phase recommended treatment is low molecular weight heparin for 5 to 7 days.

Case report: A 3-year-old female child, previously healthy presented to the pediatric emergency department with two days of irritability, prostration and periorbital edema. Physical examination showed peri-orbital edema and blood preassure was normal.

Laboratory workup revealed nephrotic proteinuria, megaloblastic anemia, hypercholesterolemia, hypertriglyceridemia and hypoalbuminemia $(15.3 \mathrm{mg} / \mathrm{dL})$, and elevated sedimentation rate. Under the diagnosis of Nephrotic Syndrome, she was admitted on steroid therapy.

Remission occurred on the 8th day of therapy, being discharged on the 12th under steroid therapy with Pediatric Nephrology follow-up.

Five days after discharge she started headache with photophobia, nocturnal awakening and morning vomiting. On general observation she was complaining, slightly dehydrated, normotensive, with normal cardiopulmonary evaluation. Neurologic examination was normal without fundoscopic alterations. Due to this the clinical manifestations and concomitant risk factors angiotomography was performed, showing an extensive venous thrombosis of the superior sagittal sinus with an apparent extension to the transverse sinus and started antithrombotic therapy.

Progressive improvement of headache episodes was observed, being asymptomatic after 3 days of antithrombotic therapy (Enoxaparine).

She remained clinically stable throughout the hospitalization period and was discharged on the 15th day taking prednisolone, enoxaparin and enalapril, with pediatric nephrology and neuropediatrics follow-up. Hereditary thrombophilia and dysfibrinogenemias were studied. She remains clinically well without neurologic symptons.

Conclusion: Cerebral venous thrombosis is a rare yet a important complication of NS. The hypercoagulant state contributes to this phenomenon due to its multifactorial etiology.

The high clinical suspicion with contrast-enhanced CT allowed the early institution of therapy, thus preventing the occurrence of post-thrombotic complications.

In the absence of major intracranial hemorrhage anticoagulation is the gold standard of treatment. There is no consensus for prophylactic treatment.

\section{1}

\section{Neurology}

Vomit as an Alert in a Rare Pathology

Mariana Santos ${ }^{1}$, Ricardo Maré2, Maria João Nogueira ${ }^{1}$, Margarida

Reis Morais $^{I}$, Susana Carvalho ${ }^{1}$, Sónia Figueiroa ${ }^{3}$, Teresa Pontes ${ }^{1}$

${ }^{1}$ Paediatrics, Hospital de Braga, Portugal

${ }^{2}$ Neurology, Hospital de Braga, Portugal

${ }^{3}$ Paediatric Neurology, Centro Materno Infantil do Norte, Portugal 
Background: Vomits are a frequent symptom in paediatric ages and can be related to severe diseases, like neuromyelitis optica (NMO), characterized by imuno-mediated demyelination and axonal destruction, involving particularly the optic nerve and spinal cord. Anti-aquaporin antibodies (AbAQ4) are involved in the pathogenesis and distinguishes it from multiple sclerosis.

Case Description: Ten year-old boy with irrelevant medical history, with 10 days of recurrent vomiting, food refusal and belching, not responsive to symptomatic treatment. The blood tests and abdominal ultrasound were normal. The upper digestive endoscopy revealed esophagitis, and he started domperidone and pantoprazole, with slight improvement. Seven days after admission he started feeling less active, with frontal headache. The brain MRI showed a lesion at the posterior side of rachidian bulb (postrema area), probably with an inflammatory origin. The CSF revealed pleocytosis ( 8 cells) with negative virologic, microbiologic and oligoclonal bands. The immunologic study was normal. Vomiting ceased after 5 days of methylprednisolone. Once he stopped the treatment, the vomiting and headache re-emerged, associated with somnolence, dysarthria, blurred vision, vertical nystagmus, unbalance and right hypoesthesia. He restarted corthycoterapy at growing doses. The neuroaxis MRI revealed a new lesion at C3, of inflammatory nature and anti-MOG and AbAQ4 were requested. The patient was transferred to a clinic center with Neuropediatrics, where he initiated plasmapheresis and a course of IV immunoglobulins after the positive result of AbAQ4, with significant improvement. He was readmitted one month later with relapse suspicion, medicated with rituximab. Currently he is under corthycoterapy, asymptomatic and with negative AbAQ4.

Conclusion: The postrema area syndrome is relativity common within NMO and precedes the involvement of the optic nerve or spinal cord, which can be considered an important warning sign. Early recognition and treatment can prevent the occurrence of outbreaks of optic nevritis or transverse myelitis.

\section{7}

Neurology

Significant Laboratory Findings in Children with Simple Febrile Seizures

Emilija Shpritova ${ }^{l}$, Jadranka Breslieva ${ }^{l}$

${ }^{T}$ Pediatric, PHI Clinical hospital, Macedonia

Background: Simple febrile seizures (SFS) are generalized tonic clonic seizures, with a duration less than 15 minutes, no recurrence within the next 24 hours, no underlying neurological illness, no focal features during or after and presented during fever in children between 6 months and 5 years of age.

Sodium, has a well established role in the function of the neurons, by being a part of the sodium potassium ATPase complex and transitions trough voltage gated channels.

Iron deficiency anemia (IDA) is the most common nutritional deficiency in the world.Available data suggest significant association between SFS and IDA.

Objective: 78 hospitalized children with SFS in a 3 year period (20162018) were included. Sodium blood levels were measured in the first hour after the seizure and hemoglobin and iron values were evaluated after appropriate hydration.

Methods: Sodium and iron blood levels were measured with the assay method of Architect 4000 device and hemoglobin values with the Humacount device.

Reference ranges :sodium 136-145 mmol/L, iron 10-30 $\mu \mathrm{mol} / \mathrm{L}$,

Hemoglobin $95-150 \mathrm{~g} / \mathrm{L}$ ( ranges by age considered)

Results:

Sodium levels

- 49 children $(62,8 \%)$ lower than $136 \mathrm{mmo} / \mathrm{L}$.

- 20 children $(25,6 \%)$ higher than $136 \mathrm{mmol} / \mathrm{L}$.
- 9 children $(11,5 \%)$ equal to $136 \mathrm{mmol} / \mathrm{L}$.

- Iron levels

- 72 children $(92,3 \%)$ lower than $10 \mu \mathrm{mol} / \mathrm{L}$

- 6 children $(7,6 \%)$ higher than $10 \mu \mathrm{mol} / \mathrm{L}$

- Hemoglobin values

- 39 children (50\%)- age appropriate values

- 39 children (50\%)- below range

Conclusion: There is a significant finding of a low sodium blood levels in the first hour after the SFS.We strongly recommend oral electrolyte fluids during fever in children who experienced SFS. There is a significant result that the IDA is very common in children with SFS as preexisting condition. Consequently, iron is a better marker than hemoglobin.We strongly recommend iron+ vitamin supplementation and parental nutrition education.Further investigations are necessary to explain the possible role of the sodium blood levels in the etiology of SFS in predisposed children.

1024

\section{Neurology}

Vascular Anomaly as a Probable Cause of Acute Ischemical Stroke in a 13-yr Old Girl

Daniel Martins Tiago ${ }^{I}$, Rita Justo Pereira ${ }^{I}$, Teresa Monteiro ${ }^{I}$, Inês Coelho $^{I}$, Sofia Baptista ${ }^{1}$, Pablo Grande ${ }^{2}$, Carlos Amaral ${ }^{3}$, Carla Mendonça $^{I}$

${ }^{1}$ Serviço de Pediatria, Centro Hospitalar Universitário do AlgarveHospital de Faro, Portugal

${ }^{2}$ Serviço de Radiologia, Centro Hospitalar Universitário do Algarve Hospital de Faro, Portugal

${ }^{3}$ Serviço de Angiologia e Cirurgia Vascular, Centro Hospitalar Universitário de Lisboa Central - Hospital de Santa Marta, Portugal

Background: Acute ischemic stroke (AIS) is a rare condition in children (0.6-7.9 / 100.000 year), more common in boys $(\sim 60 \%)$, associated with heart conditions, hematologic changes, vasculopathies, metabolic disorders and drugs. In infants, AIS typically presents with seizures and altered mental status, while older children present with focal deficits, hemiparesis, visual disturbance or cerebellar signs. Anamnesis and neuroimaging, CT-angiography or MRI, are essential for timely diagnosis and intervention. Further investigation includes blood tests, ECG, and echocardiography.

Objective: We report a case of AIS in a 13-yr old girl previously diagnosed with arteriovenous malformation (AVM). We discuss etiologies for AIS including its association with AVM.

Case Study: M.D.G., complained of ocular pain 2 days prior to admission and presented with headache and vomiting, followed by facial asymmetry and slowed speech. Past medical history of an AVM in left sub-scapular region. On examination a right hemiparesis with homolateral facial involvement, past pointing on the right, and ataxia. Full blood count, biochemistry screen and CSF results were normal. CT scan was normal but cranial MRI revealed a left anterolateral pons lesion, confirming early stage AIS. Echocardiogram was normal. Prothrombotic factors were not identified. LMW-heparin and acetylsalicylic acid were initiated promptly as well as a rehabilitation program. Cranial MRA revealed occlusion of the distal $1 / 3$ of the basilar artery and no other evidence of a vasculopathy. On cervical MRA the vertebrobasilar circulation was not directly related to the AVM. Clinical and angiographic course were favorable.

Discussion and Conclusion: Diagnosis of AIS in children is difficult and requires a high level of suspicion. In this age group etiological investigation is essential to identify treatable causes. In our patient we could not identify usual causes, nor syndromes associating AIS with AVM. We question if the AVM could be responsible for a "steal" syndrome resulting in AIS. 
582

Neurology

The Forgotten Children With Disabilities: A Study on the State of Children with Disabilities from a Base Hospital in the Central Province of Sri Lanka

Kamanee Menik Upatissa ${ }^{l}, T R M L$ Bandara $^{l}, D M P W K$

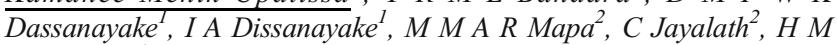
Weeraratne

${ }^{1}$ Paediatrics, District Base Hospital Rikillagaskada, Sri Lanka

${ }^{2}$ Paediatrics, Teaching Hospital Kandy, Sri Lanka

Background: According to the State of the Worlds' Children 2013 report by UNICEF only a few countries have reliable information on children with disabilities. These children encounter different forms of exclusion and are affected by them to varying degrees. Local data on these children are not available and therefore we undertook this descriptive study with the following objectives.

Objectives: To identify children with disabilities in a rural area, categorise the disability according to the type and to assess the current services they received.

Design, setting and method: A cross sectional study was undertaken in the area of Rikillagaskada. The Public Health Midwives were given a basic training to detect such children in the community and invited them to a special clinic conducted at the hospital. All children underwent a physical examination including a neurological assessment to arrive at a diagnosis by a medical officer. Data were collected on pre tested questionnaires.

Results: Fifty five ( $\mathrm{n}=55)(\mathrm{M}: \mathrm{F}=28: 27)$ children were identified. Their age ranged from 10 months-12 years. Their disabilities were categorized as: physical $(60 \%)$, sensory (visual and hearing) (12\%), intellectual (20\%), and mixed (7\%). $49 \%$ were confirmed to have cerebral palsy on neurological examination. A cause for the disability could be identified in $72 \%$. Only $31 \%$ were attending rehabilitation. None of the children with Down Syndrome received follow up. 64\% of 6-12 years children received education. Majority (69\%) of children did not attend rehabilitation due to the distance to travel. $96 \%$ of families did not receive any funds $(53 / 55)$. Conclusions: $50 \%$ disabilities were due to Cerebral Palsy. A significant proportion of children were not getting followed up mainly due to the lack of local rehabilitation facilities. Community support and provision of funds for the families were negligible.

\section{4}

Neurology

Cases of Neurological Impairment in PICU

Elpis Chochliourou ${ }^{I}$, Eleni Volakli ${ }^{1}$, Asimina Violaki ${ }^{l}$, Peristera-Elena Matzafleri $^{l}$, Menelaos Svirkos ${ }^{l}$, Anastasia Anastasiou ${ }^{l}$, Maria Sdougka ${ }^{l}$

${ }^{1}$ Pediatric Intensive Care Unit, Hippokration General Hospital of Thessaloniki, Greece

Background: Cases with neurological impairment, due to a variety of pathology seem to be frequent symptomatology of patients in a PICU. The above incidences were studied in a period of 3 years, while sex, age, clinical status in entrance, total stay in PICU and final outcome were retrospectively reviewed.

Objectives: The properties of the pathology determine the extent of the damage with sometimes mild symptoms and sometimes a rapid development that may end up in death. Hospital stay maybe long.

Methods: 56 cases were described with affected neurological image. 16 cases in 2016, 15 cases in 2017 and 25 cases in 2018. The neurological image varied with coma, convulsions, status epilepticus, or symptoms due to encephalitis or meningitis. 1 incident with known Dravet syndrome, 1 with metabolic edema and 1 case with surgical hematoma.

Results: The incidences concerned status epilepticus were 18/56 (32\%), with coma were $8 / 56(14 \%)$, with febrile convulsions $1 / 56((1,7 \%)$, meningitis 4/56 (7\%), encephalitis 6/56 (11\%). From them, 38/56 were boys (68\%), while 18 were girls (32\%). 5/56 (9\%) were died (3/5 boys) while $3 / 5$ had brain death (2/3 boys). Mean average of hospital stay14,4 days. The ages of the hospitalized children variety from 42 days to 13 years old.

Conclusions: A lot of incidences need the helpful treatment of PICUmechanical or non mechanical ventilation, hemodynamic stabilization, combination of anticonvulsant drugs, antibiotic therapy- while a percentage of these are very serious with a long hospital stay. The population of boys is significantly higher, also the outcome.

\section{8}

Neurology

Epidemiological Features of Autism Spectrum Disorder (ASD): An Egyptian Sample Dina Y. Elalfy, ${ }^{1}$, Hassnaa Othman Mohammed ${ }^{2}$

${ }^{T}$ Lecturer of Pediatric Diseases, Department of Medical Studies for Children, Faculty of Postgraduate Childhood Studies, Ain Shams University, Egypt

${ }^{2}$ Lecturer of Phoniatrics, Department of Medical Studies for Children, Faculty of Postgraduate Childhood Studies, Ain Shams University, Egypt

Background: ASD is a neurodevelopmental disorder. The epidemiological features of ASD and the variability of communicative abilities among both genders were poorly studied among Egyptian children. Moreover, the relation between intellectual disability (ID) and severity of symptoms among ASD children in both genders was an area of contradiction among literature. Objective: Determine some epidemiological features of ASD. Determine the variability of Intelligent Quotient (IQ) and Childhood Autism Rating Scale (CARS) and total language age. Compare these variables for both genders.

Methods: This retrospective study was done in the period between January 2017 and May 2018 at the special needs center of Ain-Shams University, Cairo, Egypt. The study was carried on 705 children with neuro- developmental disorders (510 males and 195 females) attending the pediatric and phoniatric clinics. Assessment was done utilizing standardized Arabic language test, DMS- 5, severity of ASD symptoms using CARS and assessment of IQ using Wechsler non-verbal test (WNV).

Results: The ratio of ASD among the studied sample was 1/ 20 (36 in 705) $(5.1 \%)$ (27 males and 9 females). The male/ female ratio was 3:1. After application of CARS, twenty four children (67\%) were mildly autistic, 5 children (14\%) were moderately autistic and 7 children (19\%) were severely autistic. After application of WNV, 60\% had mild ID, $20 \%$ had moderate ID and 20\% had a borderline IQ. Males with ASD received significantly higher IQ scores than females. The relationship between IQ and CARS went in the reverse direction. After application of standardized Arabic language test, males were significantly higher in their verbal communicative abilities than females. Conclusions: The severity of ID and severity of ASD symptoms went in direct proportional relationship. ASD in females is accompanied by marked reduction in IQ than in males. The verbal communicative abilities are higher among male children.

989

Obesity

Leptin Resistance and Hypertension in Children with Metabolic Syndrome

Gnyloskurenko Ganna ${ }^{l}$, Aliusef Maya ${ }^{l}$

${ }^{1}$ Pediatric Department \#4, O.O.Bogomolets National Medical University, Ukraine

Background. Metabolic syndrome (Mets) is a group of risk factors which include abdominal obesity, diabetes mellitus, dyslipidemia, glucose 
intolerance and hypertension. Mets is diagnosed from the age of 10 years old according to IDF criteria if there are three and more criteria listed above are present. The high waist-to-height ratio (WtHR) $(\geq 0.5)$ is associated with the increased risk of Mets. Leptin, like other neuropeptides, is a possible link between obesity and the development of hypertension.

Objective: The aim of this work is to compare the leptin resistance in children with Mets associated with hypertension and without one.

Methods. A cross-sectional study was accomplished in a group of 78 children (10-17 years old) with Mets. Patients were classified into main group (38 patients with Mets and hypertension) and comparison group (40 children with Mets without hypertension). In addition to the criteria for metabolic syndrome for the patients the waist-to-height ratio (WtHR), serum leptin level were determined and leptin resistance was calculated. The statistical analysis was done using the SPSS 22.0.

Results: According to the results of the examination at the main group average values of serum leptin level were significantly higher $(p 0.05)$ than in the comparison group (29.9 \pm 0.74 and $20.0 \pm 0.52)$. Leptin resistance were determined in $57.9 \%$ of patients with arterial hypertension and only $25 \%$ in the group without it ( $p 0.05)$. There was a positive correlation between leptin and WtHR $\geq 0.5 \mathrm{r}=0.575$.

Conclusion. Leptin resistance and $\mathrm{WtHR} \geq 0.5$ are associated with the increase of cardiovascular risks of Mets in children.

170

Obesity

Nutritional Profile of Children of Public Schools of Southern Brazil Jane Cardoso ${ }^{I}$, Mayara Maria De Jesus Rozante ${ }^{I}$

${ }^{T}$ Medicine, Center of University of Maringa, Brazil

Objective: Describe the nutritional and epidemiologic profile of children in public schools in an urban area in Southern Brazil.

Methods: cross-section study using convenience sample of 283 children ages 7 to 8 , from day care facilities and public schools in Southern Brazil. Data regarding weight, height, waist circumference and Body Mass Index (BMI) was collected, according to Health Ministry Technical Normative. The nutritional status was defined and weight, height and BMI's Z score averages were obtained, by age and sex. Height by age (H/A) and BMI/A of pre-schoolers and schoolchildren were compared.

Results: The mean age was 7.8 years, $42 \%$ were female and $58 \%$ were male. Regarding waist circumference, $9.54 \%$ were elevated, considering $55.55 \%$ men and $44.44 \%$ women. Comparing the waist-to-height ratio, $31.50 \%$ of the students had this high rate, being $66.29 \%$ female and $33.70 \%$ male.

Conclusions: A high proportion of severe obesity is observed, 3.7 times higher in males. The waist circumference is slightly higher in females, but the waist-to-height ratio is almost double in females. Although males have severe obesity, females seem to also present cardiovascular risk because they have the highest waist-to-height ratio. There was also a statistically and highly significant linear correlation between the waistto-height ratio and waist circumference with $\mathrm{p}$

\section{8}

Correlation of Nutritional Status and Growth in Adolescents Jane Cardoso ${ }^{1}$, Claudio Leone ${ }^{2}$

${ }^{T}$ Medicine, Center of University of Maringa, Brazil

${ }^{2}$ Public Healthy, University of Sao Paulo, Brazil

Aim: The objective of the study is to analyse the growth achieved and its relation with nutritional status among school adolescents attending public schools in Southern Brazil.

Subjects and Methods: A cross-sectional, analytical study with a convenience sample of 7,225 schoolchildren, aged 10 to 16 years, was selected from the anthropometric evaluation of the Health Program of School. Classification was defined according to groups of height-forage: Group of High with z-scores height-for-age (zE) greater than +1 , Average Group with $\mathrm{zE}$ between +1 and -1 and Group of Low with $\mathrm{zE}$ below -1 , for both sexes. Body Mass Index z-score values (zBMI) of Group High and Group Low were compared, and zBMI correlation and sex were found.

Results: There is a statistically significant difference between BMI of Male High versus BMI of Male Low (p 0.0001), BMI of Female High versus BMI of Female Low (p 0.0001) and BMI of Male Hersus BMI of Female High $(\mathrm{p}=0.0005)$. There is a correlation between $z B M I$ and $z E$ of Male High $(p=0.0023)$ and $z B M I$ with Male Low zE (p 0.001).

Conclusion: Fast growth is associated with body weight gain and a tendency towards overweight and obesity.

\section{7}

Obesity

Increased Fibroblast Growth Factor 21 Serum Levels and Impaired Endothelial Function in Children With Obesity and Metabolic Disorder

${ }_{\text {Eleni Domouzoglou }}{ }^{l}$, Antonios Vlahos $^{I}$, Anna Challa ${ }^{1}$, Michail Papafaklis $^{2}$, Agathocles Tsatsoulis ${ }^{3}$, Lampros Michalis ${ }^{2}$, Nikolaos Chaliasos $^{1}$, Katerina Naka ${ }^{2}$

${ }^{1}$ Department of Paediatrics, University of Ioannina, Greece

${ }^{2}$ 2nd Department of Cardiology, University of Ioannina, Greece

${ }^{3}$ Department of Endocrinology, University of Ioannina, Greece

Background: Obesity is linked to higher risk for endothelial dysfunction which is critical in the progression of cardiovascular disease. Obesity is constantly increasing in childhood. Early detection of metabolic disorders and cardiovascular risk is of utmost importance. Fibroblast growth factor 21 (FGF21) is known to influence metabolic pathways and is shown to be increased in the serum of obese adults. However, there are limited data regarding the potential role of FGF21 in metabolic and cardiovascular disorders in children.

Objective: Our aim was to investigate FGF21 serum levels and endothelial function in children with obesity and signs of metabolic disorder.

Methods: Seventy-eight children (8-16 years old) were classified as obese/overweight $(n=41 ; 53 \%$ of total) and normal weight defined by age- and sex-specific body mass index percentiles. We defined metabolically unhealthy children as those with any classical feature of the metabolic syndrome (International Diabetes Federation criteria). Endothelial function was assessed by the brachial artery flow-mediated dilation (FMD) technique and normalized to the shear stimulus [i.e. peak\%FMD normalized to shear rate (normalized FMD)]. The Mann-Whitney U test was used to investigate the differences between groups.

Results: In obese/overweight children, FGF21 serum levels were significantly increased [median 73.6 (interquartile range 41.6$127.8)$ vs. $30.4(13.9-92.6) \mathrm{pg} / \mathrm{ml}, \mathrm{p}=0.017]$ and normalized FMD was significantly lower $[0.06(0.04-0.09)$ vs. $0.09(0.04-$ $0.12), \mathrm{p}=0.044]$ compared to normal BMI children. Metabolically unhealthy obese/overweight children $(n=23 ; 29 \%$ of total $)$ had significantly higher FGF21 serum levels [87.4 (49.1-185.5) vs. $29.3(11.9-91.3) \mathrm{pg} / \mathrm{ml}, \mathrm{p}=0.003$ ] and a tendency for lower normalized FMD [0.06 (0.04-0.07) vs. 0.07 (0.04-0.12), p=0.082] compared to metabolically healthy normal weight children $(n=32)$. Conclusion: Obese/overweight and metabolically unhealthy obese/ overweight children had increased FGF21 serum levels and impaired endothelial response to FMD. FGF21 may serve as a new biomarker that could be associated to obesity, metabolic disorders and endothelial dysfunction in childhood. 
214

Obesity

Ghrelin/Obestatin Ratio as a Potential Mediator for Food Intake and Fat Distribution among a Sample of Obese Egyptian Children

Nayera Elmorsi Hassan ${ }^{1}$, Gamal Samy Aly ${ }^{2}$, Reham Sabry Abdelaal ${ }^{2}$, Sahar Abd-El-Rauf El-Masry, Hanaa Hamdy Ahmed', Nehad Hassan Ahmed $^{4}$, Ayat Nageeb Kamal Abd-Elaziz ${ }^{l}$

${ }^{1}$ Biological Anthropology Department, National Research Centre, Egypt ${ }^{2}$ Child Heath Department, Faculty of Postgraduate Childhood Studies, Ain Shams University, Egypt

${ }^{3}$ Hormones Department, National Research Centre, Egypt

${ }^{4}$ Nutrition and food science Department, National Research Centre, Egypt

Background: Ghrelin and Obestatin are two hormonal peptides convey to central nervous system information concerning nutritional status and energy stores.

Objective: Assessment of Ghrelin/Obestatin ratio relation to food intake and fat distribution in obese children.

Methods: Study was cross-sectional case-control, comprised 60 Egyptian obese children aged (6-12y) of both sexes with body mass index(BMI) $\geq 95^{\text {th }}$ percentile, and 31 healthy (age-sex matched)as controls, collected from "Visceral Obesity and Growth Disturbances Unit" National Research Centre. All children were subjected to Food Intake questionnaire for 24hours with average dietary recall of 3 days, food frequency items and any snacks taken between meals. Anthropometric assessment (Weight, Height; Waist, Hip circumferences; Calculated BMI, Waist/ hip, Waist/ height; Triceps, Bicepes, Subscapular, Suprailiac and Abdominal skin fold thickness and Laboratory investigations (Serum Ghrelin, Obestatin, fasting Glucose, fasting Insulin, Ghrelin/Obestatin ratio, HOMA; and Lipid profile).

Results: Obese children had tendency for central obesity.In obese group: Ghrelin showed significant positive correlation with serum level of Glucose, total Energy, protein, Fat, Saturated and Monounsaturated Fatty acids intake. In control group: Obestatin had significant positive correlations with total Energy, total Fat and Saturated Fatty acids intake; while, Ghrelin/Obestatin ratio had significant negative correlation with total Energy intake. Ghrelin did not show any statistically significant correlations with fat distribution among the studied groups but on the other hand, Obestatin and Ghrelin/Obestatin ratio showed significant association with Subscapular skin fold thickness (central subcutaneous fat).Step wise linear regression analysis showed that total energy intake is a highly significant predictor for $19 \%$, Fat energy intake for $14 \%$ of changes occurring in Ghrelin, while total energy intake responsible for $4 \%$ of changes occurring in Ghrelin/Obestatin ratio.

Conclusion: Ghrelin /Obestatin ratio was negatively correlated with total energy intake. It showed significant association with central subcutaneous fat. Ghrelin/Obestatin ratio may have a role in the etiology and pathophysiology of obesity.

\section{1}

Obesity

Physical Exercise among University Students and The Role of the University in its Promotion

Luisa Macieira $^{2}$, Manuel Almeida ${ }^{1}$, Lelita Conceição ${ }^{3}$

${ }^{T}$ Faculty of Medicine of the University of Coimbra, Portugal

${ }^{2}$ Faculty of Medicine of the University of Coimbra, Pediatric Clinc of the Faculty of Medicine, Hospital and University Center of Coimbra, Portugal

${ }^{3}$ Faculty of Medicine of the University of Coimbra, Hospital and University Center of Coimbra, Portugal

Introduction: Going to university leads to a series of changes in the life of a student, especially with regards to physical exercise.
Objectives: the comparison between the physical exercise habits of students of University of Coimbra, Portugal and students of Yasar University in Ismir, Turkey. Evaluation of the roles of universities in practicing physical exercise.

Material and Methods: Observational analytical study, using data collected by means of a questionnaire on physical exercise. 818 students participated - 428 from Yasar University in Ismir, Turkey, and 390 from University of Coimbra, Portugal. The data were collected during the $1^{\text {st }}$ Semester of the 2018/2019 academic year. The statistical analysis was carried out using SPSS, version 22 (p0.5).

Results: 818 students were included - 428 from Yasar University in Ismir, Turkey (162 males and 266 females) and 390 from University of Coimbra, Portugal (136 males and 254 females). The average age of the students was 21-22 years old.

Half of the students practiced physical exercise (Yasar 46.3\% / Coimbra $50 \%$ ). Half practiced physical exercise less than 3 times a year (Yasar $47.6 \%$ and $52 \%$ in Coimbra).

Most of the students had practiced physical exercise prior to going to university. The university was considered a decisive factor for the practice of physical exercise by $56.7 \%$ at Coimbra vs. $39.7 \%$ at Izmir (p0.001). A minority recognised the role of the universities in practicing physical exercise $-2.1 \%$ at Coimbra and $0.9 \%$ at Izmir.

Only $3.1 \%$ of the Coimbra students who practice physical exercise do so at the university, in comparison to $28.5 \%$ of the students at Izmir (p0.001). The reasons cited were: lack of knowledge by $23.9 \%$ of the Coimbra students and $7.8 \%$ of the students at Yasar (p0.001) and lack of time by $54.8 \%$ of the students at Yasar and $46.9 \%$ of the Coimbra students (p0.001). Most of the students who practice physical activity have a normal BMI.

\section{Conclusions/ Discussion}

Half of the students practice physical exercise. The role of physical activity for having a healthy life is recognised by students. Most university students have an adequate body mass index. The majority of the students who practice physical exercise do so out of the university. Students at Yasar recognise the positive role of the university for practicing exercise. Universities will have to reflect and take measures to encourage physical activity.

167

Obesity

The Role of Neutrophil to Lymphocyte Ratio and Platelet to Lymphocyte Ration in Children's Obesity

Cristina Oana Mărginean ${ }^{1}$, Lorena Elena Meliț ${ }^{1}$, Dana Valentina

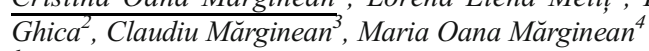

${ }^{I}$ Pediatrics, University of Medicine, Pharmacy, Sciences and Technology, Romania

${ }^{2}$ Medical Informatics and Biostatistics, University of Medicine, Pharmacy, Sciences and Technology, Romania

${ }^{3}$ Obstetrics \& Gynecology, University of Medicine, Pharmacy, Sciences and Technology, Romania

${ }^{4}$ Pediatric Cardiology, University of Medicine, Pharmacy, Sciences and Technology, Romania

Overweight and obesity is a current global health problem carrying multiple burdens in both children and adults. In obesity we have a chronic inflammatory status due to an alteration of immune activity in adipose tissue consisting in a transiently infiltration of neutrophils within the abdominal fat and their binding to adipocytes. Neutrophil to lymphocyte ratio (NLR) and Platelet to lymphocyte ration (PLR) are cost-effective markers for the detection of subclinical inflammation.

Material and Method: We performed a prospective study on 132 children aged from 5 to 18 years, admitted in a Pediatric Tertiary Hospital during the year 2018, divided according to the value of body mass index (BMI) into 2 groups: group 1, study group - 55 obese children (BMI ${ }^{3}$ 
P95), and group 2, control group - including 77 children with normal $\mathrm{BMI}$ in order to evaluate the correlation of BMI with laboratory parameters (CBC, ESR, transaminases, total proteins, albumin), inflammatory biomarkers, as NLR and PLR and abdominal ultrasound. changes

Results: We found that leukocytes, lymphocytes, erythrocytes, platelets and transaminases were significantly higher in overweight/obese group $(\mathrm{p}=0.033 / \mathrm{p}=0.0003 / \mathrm{p}=0.0005 / \mathrm{p}=0.0003 / \mathrm{p}=0.034 / \mathrm{p} 0.0001)$. We did not encounter any significant statistical differences between the 2 groups in terms of neutrophils, hemoglobin, albumin, total proteins and glycaemia (p0,05). Also, NLR and PLR did not differ significantly between the 2 groups.

Conclusions: Obesity is associated with systemic low-grade inflammation reaching alarming rates worldwide among both children and adults. We emphasize that leukocyte, lymphocyte, erythrocyte and platelet count are significantly higher in obese children related to inflammatory status in this condition.

\section{3}

\section{Obesity}

Childhood Obesity and Sleep: Friends or Foes?

Raluca Maria Vlad ${ }^{1,2}$, Livia Brezeanu ${ }^{1}$, Oana Elena Iaru ${ }^{3}$, Daniela Pacurar $^{1,2}$

${ }^{T}$ Pediatrics, "Grigore Alexandrescu” Emergency Hospital for Children, Bucharest, Romania, Romania

${ }^{2}$ Pediatrics, "Carol Davila" University of Medicine and Pharmacy, Romania

${ }^{3}$ Oncopediatrics, “Alexandru Trestioreanu” Oncologic Institute, Romania

Background: Obesity is one of the leading causes of morbidity in children worldwide. Research in the past two decades has focused on determining correlations between obesity and sleeping disorders. Many authors suggest that sleep duration and multiple aspects of sleep patterns, including sleep related breathing disorders (SRBD), are in close association with weight problems in children of all ages.

Objective: The authors aimed to evaluate how overweight/obese children sleep.

Methods: We conducted a prospective study from November 2018 until January 2019 that included overweight (BMI $85^{\text {th }}-95^{\text {th }}$ percentile) or obese (BMI $\geq 95^{\text {th }}$ percentile) $3-18$ years old children. The patients were enrolled in the Pediatrics Department of "Grigore Alexandrescu" Children's Hospital, where they were admitted for unrelated pathology. Parents were asked to fill in a SRBD scale. The obese/overweight children were scored on this scale; scores $\geq 0.33$ were considered positive and suggestive of high risk for pediatric sleep-related breathing disorder. The length of the children's sleep during the day and overnight were also recorded.

Results: Forty five patients were enrolled (53.3\% obese). Mean age was 11 years 4 months, sex ratio $\mathrm{M} / \mathrm{F}=1.25 / 1,62.2 \%$ came from urban areas. The parents declared an average 8.3 hours of night sleep. $60 \%$ of the children did not take naps during the day. $6.66 \%$ of parents declared their children to have difficulty breathing during sleep, $15.5 \%$ perceived periods of apnea during their child's sleep and $26.6 \%$ felt the child was tired in the morning. A significant proportion of patients $(26.6 \%)$ were scored positive for SRBD, percentages being slightly higher for obese vs overweight children (29 vs.23.8\%).

Conclusions: A quarter of the obese/overweight children evaluated were at high risk of sleep related breathing disorder. Addressing obesity from a young age should be expected to improve the quality of life on many levels, including sleep quality and consequently daytime activity.
163

Obesity

What Children Eat and what Parents Say or Think they Eat Teresa Pinheiro ${ }^{I}$, Teresa Caldeira ${ }^{I}$

${ }^{7}$ Neonatology/Pediatric Department, Centro Hospitalar de Entre-oDouro e Vouga, Portugal

Child obesity is a public health problem. Children learn about nutrition at school and parents are constantly reminded of the importance of healthy eating but doubt remains whether they are aware of what their children eat, as they spend most of their days at school, some of them with pocket money to spend on food.

Our goal was to compare what children say about their meals and what parents know about it. We applied a food survey to children in three schools in the region of Porto and asked their parents to answer the same inquiry. It involved questions about frequency of meals, consumption of various foods and use of the cafeteria. The results were analyzed using SPSS Statistics®.

The survey was applied to 201 children, mean age of 8,4 years ( $\min 6$, max 12 , SD+-1,5) and $53 \%$ male. $48 \%$ reported having 5 meals/day. $42 \%$ reported eating 3 or more fruits/day. $80 \%$ reported eating meat 3 or more times/week and $52 \%$ reported the same frequency of fish. 50\% consumed dairy 3 or more times/week. 14\% reported drinking soft drinks and $12 \%$ eating candy every day. The parents' survey reported a $4 \%$ and $5 \%$ consumption of soft drinks and candy every day, but the results were not statistically different between groups. $71 \%$ of the children reported using the cafeteria, $41 \%$ to buy candy, $66 \%$ bread and pastry and $19 \%$ soft drinks. Food surveys may not be the best test to evaluate food consumption. Children' memory may not be accurate and parents' answers may be biased by what they know is healthy. Our sample shows high frequency of soft drinks and candy consumption, that was not reported by the parents, although with no statistical significance. These results and the low frequency of fish consumption demonstrates the need for more educational programs regarding healthy nutrition, aimed at children and their parents. Also, schools should be aware and accountable for foods they have available.

\section{8}

Obesity

Emotional Feeding Model (EFM) in Early Childhood to Prevent Obesity, Eating Disorders, Child Neglect and Abuse

Shlomith Samish ${ }^{1}$, Pnina Hertz ${ }^{2}$, Nurit Samish ${ }^{3}$

${ }^{1}$ School of Nutritional Scinces, The Hebrew University of Jerusalem, Israel

${ }^{2}$ Feeding Clinic, Hadassah Medical Center, Jerusalem, Israel ${ }^{3}$ Education, Educational Psychology Services, Israel

The increasing prevalence of childhood obesity in the world leads to more and more health problems throughout life.

"Interventions to prevent childhood obesity by changing dietary, physical activity and sedentary behaviors have not proved to be very effective, and did not significantly improve BMI" (Anderson, 2010)

"There is increasing evidence linking the stress response to obesity and the metabolic syndrome.... A novel approach to preventing obesity is to help children develop healthy emotion regulation, controlling their physiologic and behavioral responses to emotions that arise from psychological stress" (Anderson, 2011)

The Emotional Feeding Model (EFM) is suggested as a novel approach to nurture the emotional well-being in early childhood and to prevent obesity and its outcomes, eating disorders, child neglect and abuse.

Feeding and family mealtime's experiences in early childhood nurture the emotional, social, as well as cognitive and motor development. The common daily situation of feeding and mealtimes serve as learning and 
training laboratory, where parent-child relationships are experienced and shaped. Positive transactions and experiences during feeding, like:

- listening and responding appropriately to the baby's hunger and satiation signals

- placing full trust in his ability to adjust the amount of food he needs respecting and encouraging his initiatives and experiences to feed himself

- Create the basic elements for building positive well-being, emotionally and socially.

- When caregivers feed the child mechanically or force feed him, it creates the toxic stress and unhealthy environment that might damage his brain development. This has far-reaching effects of his psychological, social and physical development.

It is of great significance and in our responsibility to guide and train young parents, as well as professionals working with young children, in how to create an environment that nurtures the emotional well-being and prevents the toxic stress and its damaging outcomes.

300

Obesity

Statistical Relationship of Overweight, Obesity and High Blood Pressure in Children Based on the Analysis of Russian Big Data Svetlana Shchelykalina ${ }^{1}$, Dmitry Nikolaev ${ }^{2}$, Natalia Bulanova ${ }^{3}$

${ }^{T}$ Department of Medical Cybernetics and Computer Science, Pirogov Russian National Research Medical University (RNRMU), Russia

${ }^{2}$ Department of Development, CRC Medass, Russia

${ }^{3}$ Cardiology Research Department, Federal State Autonomous Educational Institution of Higher Education I.M. Sechenov First Moscow State Medical University of the Ministry of Health of the Russian Federation (Sechenov University), Russia

It is considered, overweight and obesity are a risk factor for hypertension and high blood pressure (HBP). In 2014, big data of health exam in Russian health centers are available for analysis under grant of the Russian Science Foundation.

Aim of the Work: Estimate statistical relationship of overweight, obesity, and HBP in children based on the analysis of Russian big data.

Materials and Methods: The present study used data from 107,015 children aged 6-18 who visited Russian health centers in 2009-2015. The survey results are accumulated in the Federal Information Resource of Health Centers.

The analysis included the calculation of the Spearman correlation coefficient between blood pressure (SBP, DBP) and BMI and body fat mass (\%FM) for each month of life and an estimate of the prevalence of HBP (SBP or DBP the height-age standard) for children with normal weight (NW) and overweight or obesity (Ov/Ob, by BMI).

Results and Discussion: The correlation coefficient for BMI and SBP had pronounced fluctuations in 0.15-0.40. The general age trend (polynomial approximation) has a maximum at 12.5 years $(\mathrm{r}=0.30)$. The general age trend of correlation of \%FM and SBP has a maximum at 8 years and 4 months $(\mathrm{r}=0.25)$. Thus, SBP is $9 \%$ determined by changes in BMI and $6 \%-$ in $\%$ FM. The correlation values with DBP are about 0.1 less than with SBP.

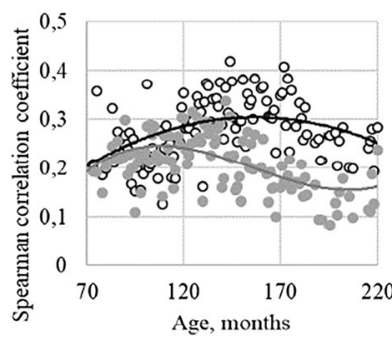

$\circ \mathrm{BMI} \times \mathrm{SBP} \bullet \% \mathrm{FM} \times \mathrm{SBP}$

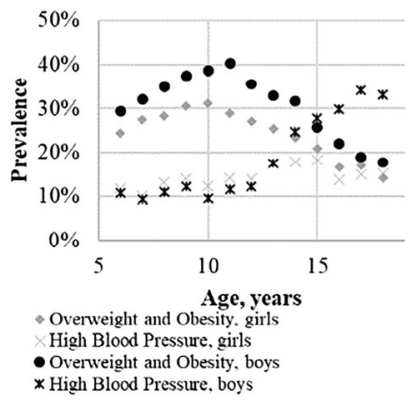

The presence of $\mathrm{Ov} / \mathrm{Ob}$ increased the prevalence HBP: $16.6 \%$ for boys and $11.5 \%$ for girls with NW and $26.3 \%$ and $24.4 \%$ for boys and girls with $\mathrm{Ov} / \mathrm{Ob}$.
Conclusions: The analysis of Russian big data showed the presence of a correlation of BMI and \%FM with SBP and DBP. The presence of $\mathrm{Ov} / \mathrm{Ob}$ increased the prevalence of HBP by 2 times.

Acknowledgment:

This work was supported by grant No. 14-15-01085 of the Russian Science Foundation (Hwad: V. Starodubov)

\section{7}

\section{Paediatric Cardiology}

Risk Factors in the First 1000 Days of Life and Carotid Intima-Media Thickness in Children: A Systematic Review and Meta-Analysis Adina Mihaela Epure ${ }^{1}$, Magali Leyvraz ${ }^{1}$, Daniela Anker ${ }^{2}$, Yvan Mivelaz ${ }^{3}$, Stefano Di Bernardo ${ }^{3}$, Bruno R da Costa ${ }^{2,5}$, Arnaud Chiolero ${ }^{1,2,4}$, Nicole Sekarski ${ }^{3}$

${ }^{I}$ Department of Epidemiology and Health Services, Center for Primary Care and Public Health (UNISANTE), University of Lausanne, Switzerland

${ }^{2}$ Institute of Primary Health Care (BIHAM), University of Bern, Switzerland

${ }^{3}$ Pediatric Cardiology Unit, Woman-Mother-Child Department, Lausanne University Hospital (CHUV), Switzerland

${ }^{4}$ Department of Epidemiology, Biostatistics and Occupational Health, McGill University, Canada

${ }^{5}$ Institute of Health Policy, Management and Evaluation, University of Toronto, Canada

Background: The first 1000 days of life, i.e. from conception to age 2 years, could be a critical period in the development of cardiovascular disease (CVD) risk. Carotid-intima media thickness (CIMT) is a surrogate marker of CVD. Although early-life risk factors, such as low birth weight or premature birth, are associated with increased CIMT in adults, it is unclear whether the relationship is present at younger ages.

Objective: Our objective was to assess the relationship between exposures or interventions in the first 1000 days of life and CIMT in children through a systematic review and meta-analysis.

Methods: Systematic searches of MEDLINE, EMBASE and CENTRAL databases were performed. Experimental and observational studies investigating the association between ultrasound CIMT and exposures or interventions at the child, familial, or environmental level, which occurred in the first 1000 days of life, were included. Data will be synthesized descriptively and, when appropriate, random-effects meta-analyses will be conducted. Funding: SNSF 32003B-163240.

Results: A total of 5,561 publications were screened and 38 full-text publications, from 26 observational and 2 experimental studies, were retained. Observational studies comprised a total of 5,610 participants $(0$ to 18 years) and reported a wide range of exposures, from abnormal fetal growth to maternal dysglycemia or air pollution. Experimental studies comprised a total of 788 participants (5 to 8 years) and focused on nutritional interventions. Most of the studies were conducted in high-income countries, with 18 in Europe and only 2 in North America. Data analysis is ongoing and further results will be available by September 2019 .

Conclusion: Preliminary results indicate that the relationship between multiple early-life exposures or interventions and CIMT was evaluated across various populations and in different pediatric age groups. This systematic review will improve the knowledge on the mechanisms underpinning the developmental origins of CVD.

\section{3}

\section{Paediatric Cardiology}

Epstein-Barr Virus (EBV) Infection as a Cause of Myopericarditis Ana Luísa de Carvalho ${ }^{1}$, Mariana Santos ${ }^{1}$, Maria Ventura Nogueira ${ }^{l}$, Joana Pimenta ${ }^{2}$, Teresa Pontes ${ }^{1}$, Susana Carvalho ${ }^{1}$, Margarida Morais ${ }^{1}$, Ana Antunes ${ }^{1}$, Sofia Martins ${ }^{1}$

${ }^{1}$ Pediatrics, Hospital de Braga, Portugal

${ }^{2}$ Pediatric Cardiology, Hospital de Braga, Portugal 
Introduction: Myopericarditis is a rare complication of Epstein-Barr infection, with nonspecific symptoms.

Case description: A 9-year-old boy, with no relevant past medical history, was admitted to ER with a two week history of intermittent pleuritic chest pain, vomiting, nausea, anorexia and periumbilical pain. In the last ten days he also had productive cough and unilateral knee pain, but no inflammatory signs and in the last month he had high fever and nasal symptons for eight days.

At the admission he was afebrile, with a normal physical examination. Blood tests revealed elevated biochemical markers of liver injury (AST 361U/L, ALT 604U/L, FA 214U/L and GGT 105U/L) with normal liver function, elevated serum creatine kinase (998U/L) and cardiac biomarkers (troponin I 19.1ng/ $\mathrm{mL}, \mathrm{CK}-\mathrm{MB} 122 \mathrm{U} / \mathrm{L}$ ). Chest radiography was normal. The electrocardiogram showed ST-elevation in leads II, III, aVF, V5 and V6. Myopericarditis was the initial diagnosis and after Pediatric Cardiology evaluation AINE therapy was initiated. Transthoracic echocardiogram was normal. Investigation revealed no active infection for hepatitis A, B and C, cytomegalovirus, herpes I and II and adenovirus. IgM and IgG antibodies to viral capsid Epstein Barr Virus (EBV) antigen and to nuclear EBV antigen were positive. Early antigen antibodies to EBV were negative. The positive EBV DNA pointed out a recent EBV infection with hepatitis and myopericarditis.

Chest pain resolution was observed on day three and improvement in transaminases and cardiac biomarkers on day five; electrocardiogram showed flattened $\mathrm{T}$ waves on aVF, V5 and V6.

He was discharged to Pediatric Cardiology follow-up consultation and he remains asymptomatic with normal ECG.

Discussion: Myopericarditis is a rare disease, often caused by viral infections but exact etiology remains undetermined in most patients. This report highlights the importance of considering this diagnosis as a rare presentation of a common infection.

\section{0}

\section{Paediatric Cardiology}

Kawasaki Disease: An Overview of 16 Years Experience At University Hospital of Algarve

$\underline{\text { Rita Justo Pereira }}^{l}$, Daniel Martins Tiago ${ }^{I}$, Inês Coelho ${ }^{l}$, Sofia Baptista $^{I}$, Teresa Monteiro ${ }^{I}$, Filipa Mestre Dias ${ }^{1}$, Maria João Virtuoso ${ }^{I}$ ${ }^{1}$ Pediatria, Centro Hospitalar Universitário do Algarve, Portugal

Introduction: Kawasaki disease (KD) is one of the most common childhood vasculitis although its aetiology still remains unknown. It is a systemic inflammatory illness that particularly affects medium-sized arteries, especially the coronary arteries. The most feared complications include aneurysms and ischemic disease. Most cases occur in children younger than five years and rarely in those younger than six months.

The aim of this study was to evaluate the experience of this clinical entity at the main University Hospital of Algarve.

Methods: A retrospective study was performed through the analysis of epidemiological, clinical, laboratory and echocardiographic data of hospitalized KD cases between January 2004 and June 2019.

Results: A total of 22 cases were obtained. The ages ranged from 3 months to 8 years, with a median of 22 months, and subjects were predominantly male. There was a higher incidence in the months of June and December. At presentation, the majority had exanthema, ocular and mucosal involvement. The time of fever prior to diagnosis ranged from 1 to 15 days, with an average of 6 days. All had an echocardiogram, 8 had echographic alterations, of which 6 had aneurysms. All patients started treatment with immunoglobulin until the 10th day of illness, except for three patients who had a late diagnosis. They also all started acetylsalicylic acid therapy. The length of hospitalization ranged from 2 to 21 days, with an average of 9 days. Half of the patients will continue to be followed by pediatric cardiologist, and there were no deaths. Three of the six cases of aneurisms still have some degree of coronary dilatation and one patient underwent cardiac catheterization.
Conclusion: $\mathrm{KD}$ is known for its cardiovascular complications and is the leading cause of acquired heart disease in developed countries. An early diagnosis is critical to achieve optimal treatment results and a better outcome.

867

\section{Pediatric Cardiology}

A Case of Congenital Annular Pericardial Fibrous Band Causing Ventricular Strangulation in a Neonate Hyo Ju Yang ${ }^{1}$, Juae Shin ${ }^{1}$, Ji Hong Yoon ${ }^{1}$, Jae Young Lee ${ }^{1}$

${ }^{T}$ Department of Pediatrics, Seoul St. Mary's Hospital, College of Medicine, the Catholic University of Korea, South Korea

Congenital pericardial anomalies are very rare, and manifested as pericardial cyst, diverticulum and absence of pericardium. As an acquired and localized pericarditis manifested as a constrictive pericardial band causing strangulation and hourglass shaping of ventricles has been rarely reported in adolescents and adults, but this type of congenital pericardial anomaly has not been reported in neonates. We report a case of congenital pericardial fibrous band causing strangulation of both ventricles, diagnosed at fetal life and confirmed after birth. At 22 weeks of gestation, a fetal echocardiography showed an unusual ring-like constriction of both mid-ventricular cavities and multiple mass-like lesions in the both ventricular cavities, suggesting cardiac tumor. Both ventricular systolic and diastolic functions were within normal limits. After an uneventful pregnancy, the baby was born at $38^{+3}$ weeks of gestation with a birth weight of $3.3 \mathrm{~kg}$. Echocardiography showed a pericardial fibrous band causing strangulation of both ventricles, extending from mid left ventricular (LV) lateral wall, diaphragmatic surface of both ventricles, lateral wall of right ventricle (RV) and into the RV outflow track. A three-dimensional cardiac computed tomography also showed the same findings. There was no definite cardiac tumor. The baby was asymptomatic and discharged home 4 days after birth. At 6 months of age, the baby was still asymptomatic, but echocardiography showed a merged pattern of mitral inflow Doppler and mitral annular tissue Doppler imaging, along with mild dilatation of both atria, suggesting an early stage of ventricular diastolic dysfunction. Close follow up is needed to assess ventricular functions and to decide necessity of any surgical intervention timely.

\section{2}

\section{Paediatric Cardiology}

\section{Echocardiographic Diagnosis of Foramen Ovale at Child Age ${ }_{\text {Jadranka Breslieva }}{ }^{1}$,Emilija Shpritova ${ }^{I}$ \\ ${ }^{T}$ Pediatric, PHI Clinical Hospital, Macedonia}

Background: Foramen ovale ( FO) is a hole in the fetal heart through which in the embryo phase, the blood flows free between the left and the right atriums. Scientists agree that the hole closes lately to the first year of life.

Objective: Data analyses of children with FO, examined in the echocardiography room at the Pediatric Ward, Clinical hospital Shtip in the period from 2015 to 2018 year.

Material and Methods: The data is classified according to the age, heart auscultatory finding, echocardiography, pregnancy risk factors, gestational age .

Results: 250 children were examined with diagnosis FO. According to heart auscultatory finding 225 (90\%) had systolic murmor 1-2/ 6 degree precordial, 25 ( $10 \%)$ did not have any murmors. By their age, the infants of 0-6 months were mostly affected, $150(60 \%)$, then those of 6-12 months- 80 babies (32\%) and $20(8 \%)$ were at the age of 12-14 months. Acording to the anamnestic data from the mothers, only 45 (18\%) were with complications during the pregnancy and child complications after 
the birth. By gestational age, $177(70,8 \%)$ children were delivered in term in $40^{\text {th }}$ gestational week $(\mathrm{GW})$, and only $73(29,2 \%)$ were preterm births in 33-36 GW.Echocardiographic examination showed the folowing results: $174(69,6 \%)$ had only FO, $76(30,4 \%)$, the rest had more complex congenital heart defects, such as: $30(12 \%)$ with Ductus arteriosus persistens, $18(7,2 \%)$ with mild degree of pulmonary stenosis, $12(4,8$ $\%)$ with Defectus septi ventriculorum perimembranous , $10(4 \%)$ with Defectus septi ventriculorum muskulrum , 5 (2\%) with Tetralogy of Fallot and one child $(0,4 \%)$ with dextrocardia.

Conclusion: Parents of children with $F O$ should know that their children are healthy without any physical disabilities. In $20 \%$ of the population with FO it persists in older age and can result in migraine and paradoxical embolism with a stroke and it is the reason for dual attitudes should the hole be closed in school age.

\section{0}

\section{Pediatric Cardiology}

Effects of Hematopoietic Stem Cell Transplantation on Cardiac Function

Kyung Min Kim ${ }^{1}$, Ju Ae Shin ${ }^{1}$, Ji Hong Yoon ${ }^{1}$, Jae Young Lee ${ }^{1}$

${ }^{T}$ Department of Pediatrics, Seoul St. Mary's hospital, College of Medicine, The Catholic University of Korea, South Korea

Background: The acute effects of chemotherapy on cardiac function have been researched, but the effects of hematopoietic stem cell transplantation (HSCT), on cardiac function are studied less frequently.

Objective: We aimed to evaluate serial cardiac functional changes during on year, from pre- to 1-year post-HSCT by echocardiography in pediatric acute myeloid leukemia (AML) patients.

Methods: From July 2009 to December 2014, 49 (33 males, 16 females) AML patients underwent HSCT at The Catholic University of Korea, Seoul St. Mary's Hospital, survived up till 1-year post-HSCT, and had completed echocardiographic studies according to our institutional protocol. Median (range) age at diagnosis and HSCT were 7.9 (1.1-16.0) years and 9.1 (1.5-16.5) years, respectively. Median (range) cumulative Anthracycline dosage administered prior to HSCT was $318 \mathrm{mg} / \mathrm{m}^{2}(135-$ $604 \mathrm{mg} / \mathrm{m}^{2}$ ). None had radiation therapy before HSCT. Echocardiographic systolic and diastolic functional parameters, especially using tissue Doppler imaging (TDI), within 1 month before, 6-month and 1-year after HSCT were analyzed.

Results: Left ventricular ejection fraction, shortening fraction, mass and end-diastolic wall thickness did not change significantly after HSCT, but left ventricular end-diastolic dimension decreased significantly after HSCT. Mitral inflow Doppler indices did not change after HSCT. TDI S' velocity showed transient, but significant decreased at 6 month in mitral septal annulus. Mitral septal and lateral annulus TDI E' velocity decreased significantly at 6 month and 1 year after HSCT, but tricuspid TDI E' velocity did not change significantly. TDI myocardial performance index in the mitral septal and tricuspid lateral annulus increased at 6 month and 1 year after HSCT, and mitral lateral annulus TDI myocardial performance index showed transient increase at 6 months after HSCT.

Conclusion: This study showed that significant changes, although subclinical, in left ventricular systolic and diastolic functions occur after hematopoietic stem cell transplantation in pediatric acute myeloid leukemia patients. Close follow-up study is mandatory in this population to elucidate the long term effects of hematopoietic stem cell transplantation on cardiac functions.

\section{7}

\section{Paediatric Cardiology}

Epidemiological Features of Kawasaki Disease in Pediatric Population in The State of Qatar
Manasik Hassan $^{1,2}$, lolwa Anaimi ${ }^{I}$, Mohamed Sobhy ${ }^{I}$, Noura Almarri ${ }^{I}$, Amr Daia', Hibaq Jama $^{1}$, Ahmed Alhammadi ${ }^{2}$

${ }^{1}$ Peditaric, Hamad Medcial Corporation, Qatar

${ }^{2}$ Peditaric, SIDRA medicine, Qatar

Background and aim: Kawasaki disease (KD) is a systemic vasculitis with an acute and self-limited course. The incidence of $\mathrm{KD}$ is differs widely among ethnic groups and countries which may reflect both a genuine increase in disease burden and increased disease recognition . Limited patient series have been reported from countries in the Middle East our aim of this study is to describe the demographic ,clinical presentation, echocardiographic data, treatment options and complication of children's diagnosed with Kawasaki diseases over two years duration from( 2013-2015) in state of Qatar

Methods and material: A retrospective and descriptive study was conducted at main tertiary hospital in Qatar, Children hospitalized in our pediatric department with KD from January 2013- December 2015 were included. it include details of demographic, clinical presentation of Kawasaki disease, echocardiogram finding and treatment option offered for the patients

Result: There were $133 \mathrm{KD}$ cases, with typical KD in $111(84 \%)$ and Atypical in 21(16\%).Male to female ratio was 1.3:1 and median age was 30 months. presenting symptoms frequency were as follow :Fever were in $100 \%$ of the patients from which $32 \%$ were diagnosed on day 5 of fever and $65 \%$ on day 7 and by 2 weeks $94 \%$ were diagnosed .Conjunctivitis and lymphadenopathy were reported as common as $66 \%$ of the total patient followed by skin rash in $63 \%$ and Strawberry tongue $59 \%$ .Irritability was common also in $57 \%$ of the patients. Desquamation was the least frequent symptoms in $33 \%$ of the total patients. Echocardiographic (ECHO) done for $51 \%$ of the patients in the first week of the illness and $96 \%$ by the end on 2 nd week, reported normal in $75 \%$ and abnormal in $25 \%$. Nearly $98 \%$ of patients received intravenous immunoglobulin and aspirin.

Conclusions: The epidemiology and cardiovascular outcomes in our study are similar to other predominantly international EuropeanCaucasian populations. Management decisions in our center is following the standard evidence based medicine treatment . our study showed a good numbers of cases diagnosed with Atypical KD , further description looking into possible risk factor and cardiac outcome is required

\section{4}

\section{Paediatric Cardiology}

Condition of Vegetative Regulation of Cardiac Activity in Newborns with Heart Rhythm Disorders

$\underline{\text { Yevheniia Ivanova }}^{I}$, Margaryta Gonchar $^{I}$, Tetiana Teslenko ${ }^{1}$, Maryna Strelkova

${ }^{1}$ Department of Pediatrics №1 and Neonatology, Kharkiv National Medical University, Ukraine

Evaluation of heart rate variability is a method of assessing the state of the mechanisms of regulation of physiological functions in the body. Neonatal arrhythmias can causes life-threatening conditions.

Objective: To clarify the mechanisms of development of heart rhythm disorders in newborns by assessing the state of autonomic regulation of cardiac activity.

Materials and Methods: Holter ECG, analysis of heart rate variability (SDNN, SDANN, SDNN indices, rMSSD, pNN50), statistical analysis. Results: The study involved 187 newborns with cardiac rhythm disturbances at auscultation of the heart. All the newborns were administered Holter ECG, following which they were divided into 2 groups. Group 1 $(n=126)$ - newborns with heart rate and conduction impairment, Group 2 ( $\mathrm{n}=61)$ - newborns with no rhythm and conduction disorders. Taking into account the gestational period, Group 1 and 2 newborns were divided into subgroups of full-term and pre-term infants: Group 1a - full-term 
with arrhythmia $(n=34)$, Group $1 \mathrm{~b}$ - pre-term with arrhythmia $(n=92)$, Group $2 \mathrm{a}$ - full-term without arrhythmia $(\mathrm{n}=24)$, Group $2 \mathrm{~b}$ - pre-term without arrhythmia $(\mathrm{n}=37)$.

SDNN and SDANN factors, SDNN and pNN50 indices were higher in the subgroups of full-term newborns $(46,5[33,0 ; 64,0]$ and $46,0[31,0$; $54,0] ; 28,5[19,0 ; 35,0]$ and $27,0[20,5 ; 36,0] ; 34,0[21,0 ; 53,0]$ and 34,5 $[21,5 ; 39,5] ; 1,01[0,33 ; 5,66]$ and $0,8[0,31 ; 2,73])$. The rMSSD values were higher in the la subgroup $(16,5[11,0 ; 40,0])$, which indicated a higher activity of the parasympathetic link of the autonomic nervous system in full-term infants with arrhythmias.

Conclusions: Assessment of heart rate variability in newborns is an important component of the characteristics of the heart rate; gestational immaturity of the vegetative centers and imbalance of the vegetative regulation are arrhythmogenic factors.

\section{5}

\section{Pediatric Cardiology}

Undiagnosed Giant Coronary Aneurysms Leading to Sudden Cardiac Arrest in a 15 Year Old Male- A Case Report

Michaela Nikolaou, Letitia Cooper ${ }^{1}$, Michael Bowes ${ }^{2}$, Krasimir Atanasov

${ }^{1}$ Paediatric Cardiology, Royal Manchester Children's Hospital, Manchester University NHS Foundation Trust, UK

${ }^{2}$ Paediatric Cardiology, Alder Hey Children's Hospital NHS Foundation Trust, UK

Background: The most common causes of sudden cardiac arrest (SCA) are congenital abnormalities in individuals 0 to 13 years of age, presumed primary arrhythmia in those 14 to 24 years of age, and coronary artery disease in individuals 25 to 35 years of age. Coronary artery aneurysms are extremely rare in paediatric population and mainly due to the sequelae of Kawasaki Disease.

Objective: We herein report a case of undiagnosed giant coronary aneurysms leading to SCA in 15 -year-old previously well male patient.

Methods: A 15-year-old Caucasian male presented with sudden out of hospital cardiac arrest during a moderate exercise activity. He was successfully resuscitated and received one direct cardioversion by AED. AED recorded ventricular fibrillation. His past medical and family history were negative for cardiovascular disease as well as Kawasaki disease.

Results: His physical examination and the 15-lead ECG were unremarkable. Initial echocardiogram demonstrated dilated left ventricle with reduced systolic function. 24-hour ECG recording showed frequent monomorphic ventricular ectopics. Exercise testing demonstrated ST depression in the inferior leads. The CMRI study was diagnostic showing several small subendocardial areas of infarction and giant aneurisms with thrombi involving proximal left anterior descending and mid left circumflex coronary arteries. The patient was commenced on anticoagulation therapy and he had an S-ICD implanted. He was discharged home on beta blockers, ACEI and Warfarin. He remains under follow up.

Conclusion: This case demonstrates the importance of comprehensive evaluation and diagnostic workup in survivors of SCA. Coronary pathology although rare should be considered as possible aetiology of SCA in childhood, even in case of no clear history of Kawasaki disease. Our case illustrates the usefulness of advanced imaging modalities such as CMRI to identify a rare cause of cardiac arrest. In patients not previously identified as high risk, secondary prevention is as important as primary prevention.

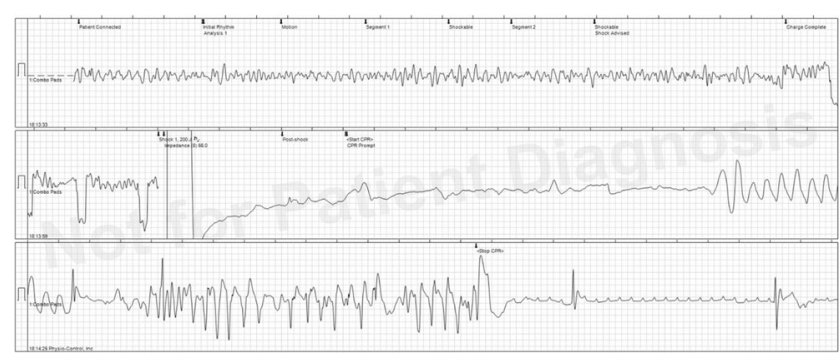

568

Pediatric Cardiology

Supraventricular Tachycardia as a First Sign of Inborn Metabolic Disease

Simona Cainap ${ }^{1}$, Gabriel Cismaru ${ }^{2}$, Gheorghe Popa ${ }^{1}$, Ioana Nascu ${ }^{3}$, Daniela Dreghiciu ${ }^{3}$, Romana Vulturar ${ }^{4}$, Alexandra Maris ${ }^{3}$, Tudor Lucian Pop

${ }^{I}$ Second Departament of Pediatrics, University of Medicine and Pharmacy "Iuliu Hatieganu", Romania

${ }^{2}$ Departament of Cardiology, Rehabilitation Hospital, University of Medicine and Pharmacy "Iuliu Hatieganu", Romania

${ }^{3}$ Emergency Departament, Clinical Emergency Hospital for Children, Romania

${ }^{4}$ Biochemistry, University of Medicine and Pharmacy "Iuliu Hatieganu", Romania

Introduction: Supraventricular tachycardia (SVT) is the most common arrhythmia in children. SVT may manifest as self-limited paroxysm, or on the contrary as a prolonged dysrhythmia with the subsequent development of cardiac dysfunction, heart failure and multiple organ failure. Cardiac disease associated with an inborn metabolic disorder (IEM) can present as an immediate life-threatening pathology with symptoms affecting growth and development, or as a "silent" disease with an increased risk of sudden cardiac death.

Case Presentation: We report two cases of children who were initially admitted for supraventricular tachycardia, but who have been later diagnosed with an IEM.

Our first patient, a four months old infant, presented in the emergency department with irritability, inappetence and tachypnea. He was diagnosed with SVT and found to be hemodinamically unstable, so he required electrical conversion to sinus rhythm. The initial clinical and biological evaluation pointed towards cardiogenic shock and multiple organ failure. Echocardiography revealed the presence of eccentric hypertrophic cardiomyopathy. Acylcarnitine profile was also evaluated, indicating a low free carnitine level. Treatment with oral carnitine led to normal carnitine values at following visits and a good cardiologic outcome, without other SVT episodes. Our second patient, of one year and two months, presented two episodes of SVT, at two months interval, both occurring during an infectious episode. Both episodes responded promptly to adenosine therapy. Echocardiography indicated a dilated left ventricle, with preserved systolic and diastolic functions. Shortly after the second episode, the patient presented another infectious episode with fever, vomiting and many loose stools, without cardiac rhythm disturbances associated. He presented in the emergency room, where the biological evaluation uncovered severe metabolic acidosis with an increased anion gap, which does not respond to classical rehydration therapy, revealing also ketonemia and increased ammoniemia. A spectroscopic MRI examination of his urine sample indicated high levels oh methyl-malonic acid, establishing the diagnosis of methyl-malonic acidemia, However, the patient died 44 hours after admission. 
Discussion: The diagnosis of an IEM as a cause of cardiomyopathy is extremely important as there is often a specific treatment available for this disorders, highlighting also the importance of genetic counseling in this cases.

541

\section{Paediatric Cardiology}

A Rare Case of Acute Myocarditis

Teresa Brito $^{1}$, Hugo Teles ${ }^{1}$, Graça Nogueira ${ }^{2}$, Fracisco Abecasis ${ }^{3}$, Cláudio D'Elial, Sara Carmo ${ }^{4}$, Susana Parente ${ }^{1}$

${ }^{1}$ Paediatric Department, Centro Hospitalar de Setúbal, Portugal

${ }^{2}$ Paediatric Cardiology Department, Hospital Santa Cruz, Portugal

${ }^{3}$ Paediatric Intensive Care Unit, Centro Hospitalar Lisboa Norte, Portugal

${ }^{4}$ Paediatric Surgery Departement, Centro Hospitalar de Setúbal, Portugal

Background: Syntomatic Esptein-Barr virus (EBV) acute infection is more common in adolescents and young adults, classically manifesting as infectious mononucleosis. Although this is a common disease, complications are rare.

Objective: to report rare complications of EBV infection in one child.

Methods: We present the case of a 10 years old child admitted for persisting fever (medicated with amoxicillin PO for a tonsillitis a week earlier), associated with vomiting and abdominal pain in the right hypochondrium. The blood count revealed: WBC 13700 (N 68\%), CRP 5,3mg/dL, ALT 31U/L and AST 240U/L and the abdominal ultrassound showed an acute acalculous cholecystitis. She was medicated with cefuroxime and metronidazol ev. After 2 days, she suddenly began chest pain, hemoptysis, respiratory distress, hypoxemia, paleness, tachycardia, hypotension, pulmonary crackles and hepatomegaly. Biochemistry revealed WBC $16900(\mathrm{~N}$ 78,4\%), ESR 45mm/h, AST 224U/L, ALT 79U/L, CK 1361U/L, CK-MB 211U/L, LDH 1377U/L, CRP 18.38mg/dL, D-dimers 6536ng/ml, Troponin 8184ng/L, NT-proBNP 33530pg/mL. EBV serology was positive. Cardiac monitorization showed ST segment elevation. Thoracic-CT revealed ARDS and right pleural effusion and the showed biventricular dysfunction and pericardiac effusion, compatible with cardiogenic shock. Inotropic therapy was started and she was transferred to a Pediatric Intensive Care Unit, under mechanical ventilation. On admission, the patient was hemodynamically unstable with an episode of ventricular tachycardia and was treated with amiodarone, adrenaline and dobutamine. She was discharged under treatment by carvedilol, linisopril, ivabradine, espironolactone and furosemide, but kept a moderate left ventricular dysfunction.

Conclusion: The initial clinical presentation suggesting mononucleosis and the positive serology for EBV were essential to establish the diagnosis of acute myocarditis, a rare complication of EBV infection.

868

Pediatric Cardiology

A Case of Refractory Atypical Kawasaki Disease with Pericardial and Hemorrhagic Pleural Effusion

YeJi Kim ${ }^{l}$, Kyung Min Kim ${ }^{1}$, Jae Young Lee ${ }^{I}$, Ji Hong Yoon ${ }^{1}$, Gi Beom $\mathrm{Kim}^{2}$

${ }^{1}$ Department of Pediatrics, Seoul St. Mary's Hospital, College of Medicine, the Catholic University of Korea, South Korea

${ }^{2}$ Department of Pediatrics, Seoul National University Children's Hospital, South Korea

Kawasaki disease (KD) combined with pericardial and hemorrhagic pleural effusion is very rare. We report the case of refractory atypical KD in 10months-old infant who was initially manifested in fever, nonsuppurative conjunctival injection, red fissured lip, BCG site injection, respiratory failure and gastrointestinal symptoms. She was noted pericardial and pleural effusion in echocardiogram, so we did pericardiocentesis and thoracentesis, and exudate pericardial and hemorrhagic pleural effusion were revealed. Treatment with infliximab after the failure of high dose methylprednisolone and repeated intravenous immunoglobulin therapy, resolved KD symptoms and pleural effusion. Echocardiogram showed normal coronary arteries during and after the entire treatment period. This case would help clinicians recognize initial presentation of atypical $\mathrm{KD}$ with pericardial and hemorrhagic pleural effusion.

535

Paediatric Tropical Medicine

Typhoid Fever in the Returning Traveler: Similar Presentation, Different Clinical Course

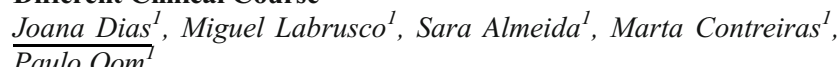

${ }^{1}$ Paediatric Department, Beatriz Ângelo Hospital, Portugal

Background: In recent years the number of children travelling abroad has substantially increased. The possibility of enteric fever should be considered if a patient presents with fever after visiting an endemic area. Case Description: We present a case of an eight-year-old girl and her tenyear-old brother, both previously healthy, whom were admitted to the emergency department with a febrile disease six weeks after returning from Malawi. They had not taken a pre-travel appointment and therefore didn't receive any additional vaccinations or malaria chemoprophylaxis. After being initially diagnosed with a respiratory infection and treated with Azithromycin they returned, after four days, due to persistent fever, nausea, intermittent vomiting, abdominal pain and constipation. Routine laboratory tests showed an elevated C-reactive protein and elevated transaminase enzymes. Malaria tests results, viral serologies and stool cultures were negative. Our female patient had a positive Widal test which motivated a presumptive diagnosis of enteric fever. Salmonella typhi was isolated from a blood culture obtained from her brother. Both patients were treated with intravenous Ceftriaxone for 14 days with complete resolution of symptoms. Approximately four weeks after discharge our female patient was readmitted with high fever and anorexia. Widal test was positive and Salmonella typhi was identified in a new blood culture. The patient was initially treated at home with azithromycin but given the persistence of fever she was readmitted to the hospital and started intravenous Ceftriaxone. After eight days of Cetriaxone she reinitiated fever and knee pain. An ultrasound was performed and identified bursitis. She completed 14 days of PiperacillinTazobactam and Amikacin and was discharged from the hospital.

Conclusion: This case draws attention to a disease that remains a major health problem in many developing countries. Early recognition of the syndrome and possible relapses is critical to the institution of appropriate therapy.

838

Paediatric Tropical Medicine

Prevalence and Associated Factors of Diarrhoea During Peak Season at a Tertiary Hospital in Southeast Nigeria

Chikosolu Okiche $^{1,2}$, Chineze Okorowo ${ }^{1,2}$, Lilian Nwobashi ${ }^{1}$, Lilian Nwobashi ${ }^{2}$, Theresa Nnaji ${ }^{2}$, Theresa Nnaji ${ }^{1}$

${ }^{1}$ Paediatrics, Federal Teaching Hospital Abakaliki, Nigeria

${ }^{2}$ Paediatrics, Federal Teaching Hospital Abakaliki, Nigeria

Introduction: Diarrhoeal disease is the second leading cause of death in children under five years old especially in developing countries, and this is responsible for killing around 525, 000 children every year. The commonest cause of diarrhoea in children is rotavirus. In the tropics, more cases of diarrhoea occur during the dry season (between Oct- April) and this also corresponds to the peak seasonality period of the rota virus 
infection. Rotavirus vaccine is yet to become one of the free vaccines in Nigeria hence, children here still suffer from severe form of the rota virus infection.

Objectives: The study's aim was to determine the prevalence and other associated factors of diarrhoea during the disease peak period at a tertiary hospital in South East Nigeria.

Method: Data of children who had diarrhoea and presented to the Children Emergency Room between October 2018- March 2019 was extracted from their records. Data was analysed using the SPSS version 22.

Results: A total of 1,167 children were seen at the Children emergency room during the period under study and of this 377 children had diarrhoea giving a prevalence of $32.3 \%$. Males accounted for $56.6 \%$ of these cases. $82.8 \%$ of the children had severe to moderate dehydration with $1.6 \%$ of them having hypovolemic shock. The commonest age affected was the 12 month old (13.3\%) however the mean age of affected children was 20months. A total of $8(2.4 \%)$ children died and the average duration of hospital stay was 2 days. Conclusion: The study shows that diarrhoea is still here with us and more work needs to be done in making sure that our children don't die from the disease. More health education to care givers and more advocacy is needed so as to ensure that the rotavirus vaccine is free.

\section{2}

\section{Primary Care}

Risk, Complexity and Special Health Care Needs Scoring for Hospitalized Children with Medical Complexities(CMC) in an Academic Hospital of Univ California San Francisco, California Alya Ahmad $^{l}$, Luella Guzman ${ }^{I}$

${ }^{T}$ Pediatric, University of California San Francisco at Fresno, USA

High acuity, complexity, \& special needs in hospitalized pediatric patients have been shown to have measurable economic costs \& effect on quality of care.

This study examines criteria for Children with Medical Complexities (CMC) $\&$ scoring tools to stratify risk using Exeter HOMES(Hospitalization, Outpatient care, Medical chronic disorders, Extra services, \& Social determinants) complexity index, \& a validated Children with Special Health Care Needs (CSHCN) Screener. By measuring both complexity \& special needs an effect on higher health care utilization, Length of Stay, (LOS) \& readmission rates (RR) are predictive of economic burden.

A 6-month retrospective observational study was conducted from MayNov, 2018, for a(n=204) pediatric patients, admitted to the general pediatric unit. Demographics, ICD10, PCP's, total number of admission (TA), Gestational age, LOS, \#RR, occurring in last $180 \& 90$ days of the study period. Criteria fitting CMC \& (Cystic Fibrosis, asthma, cardiac, \& mental health), Gestational Age (GA), clinical encounters, medications, medical devices, \& social determinants were reviewed for CMC patients. The total number of RR, LOS \& Risk scores were determined. The scoring was compared for CMC \& an equal number of non-CMC pediatric patients admitted during the same study period using Excel Statistical program.

Pediatric Patients Admitted Age<1yr old,

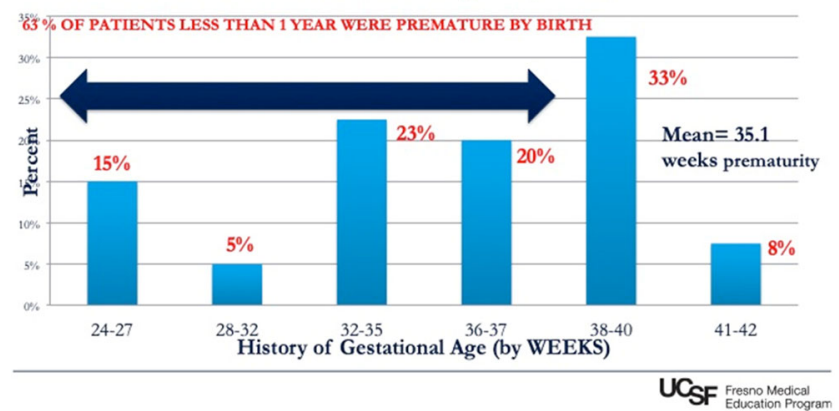

High Utilization of Hospital Resources for Pediatric Patients (admitted May 2018-Nov 2018)
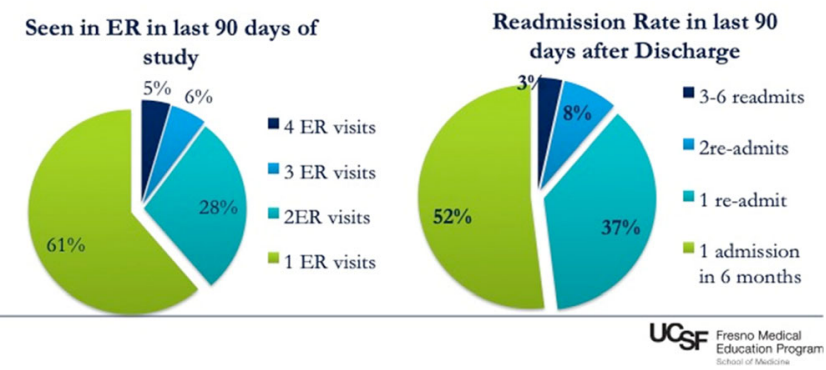

DISEASE REGISTRY

Patient admitted (6month)(May 2018-Nov 2018) UCSF-Fresno Hospital

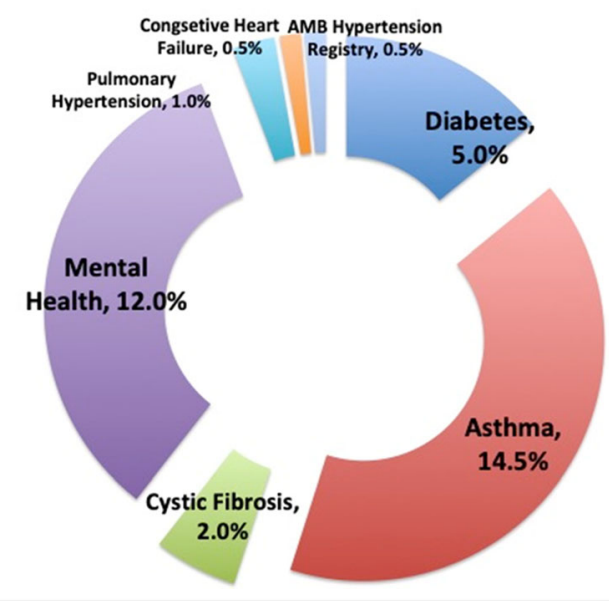

Age distribution of patient admitted (6month)(May 2018-Nov 2018) UCSF-Fresno Hospital

60

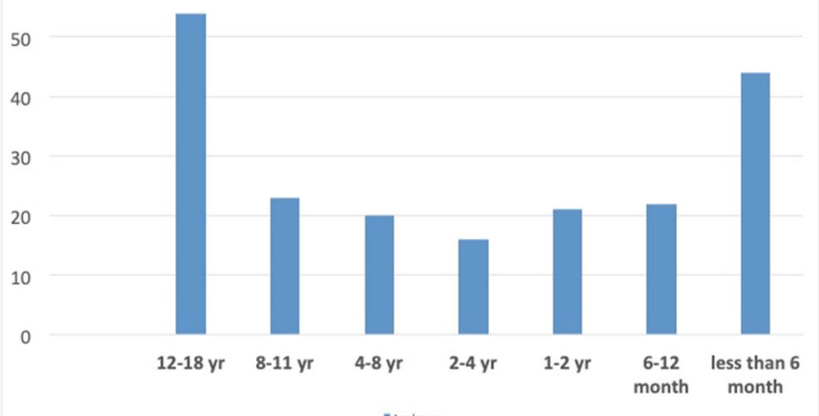

EXETER Complexity Index scores

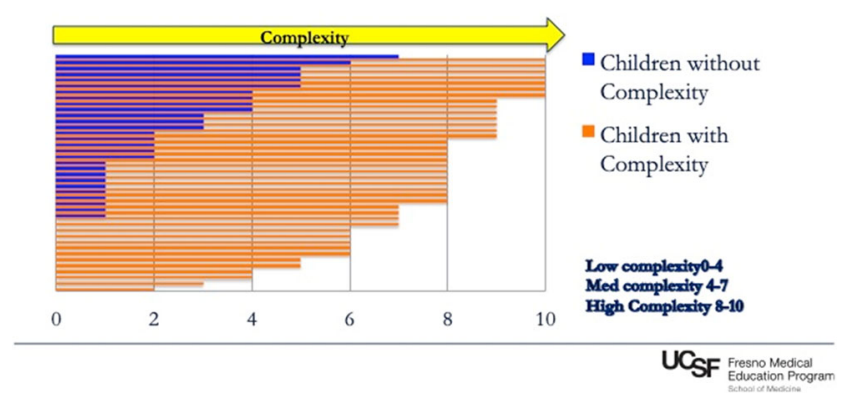


Risk Based Tools: Degree of Complexity \& Effect on outcomes

\begin{tabular}{|c|c|c|c|}
\hline SCORES (Mean reaulto) & $\begin{array}{l}\text { Children with Complex } \\
\mathrm{n}=40\end{array}$ & $\begin{array}{l}\text { Non Complex } \\
\text { Children(random) } n=40\end{array}$ & \\
\hline $\begin{array}{l}\text { Exeter H.O.M.E.S. } \\
\text { (10=Most complex and high } \\
\text { utilization) }\end{array}$ & $\begin{array}{l}7.55 \text { out of } 10 \\
\text { Standard Deviation } 2.04 \\
\text { Variance } 4.15\end{array}$ & $\begin{array}{l}2.10 \text { out of } 10 \\
\text { Srandard Deviation } 2.18 \\
\text { Variance } 4.7\end{array}$ & \begin{tabular}{|l} 
McanDifferencec $=5.45 / 10$ \\
P-Value <0.00001 \\
(t-test) $99 \%$ CI \\
Difference is significant
\end{tabular} \\
\hline $\begin{array}{l}\text { Special Needs } \\
\text { Score( } 5=\text { high disability) }\end{array}$ & $\begin{array}{l}3.63 \text { out of } 5 \\
\text { Standard Deviation }=1.15 \\
\text { Variance }=1.32\end{array}$ & $\begin{array}{l}\mathbf{2 . 0} \text { out of } 5 \\
\text { Standard Deviation } 1.31 \\
\text { Variance }=1.73\end{array}$ & \begin{tabular}{|l} 
Mean Difference $=2.9$ \\
P-Valuc < 0.000001 \\
$($ t-t-test) $99 \%$ CI \\
Difference is significant
\end{tabular} \\
\hline $\begin{array}{l}\text { Number of Readmission } \\
\text { (in } 6 \text { months) after discharge }\end{array}$ & $\begin{array}{l}2.1 \\
\text { Standard deviation }=1.77\end{array}$ & $\begin{array}{l}1.3 \\
\text { Standard Deviation }=0.47\end{array}$ & $\begin{array}{l}p \text { value }<0.0001 \\
\text { Difference is significant }\end{array}$ \\
\hline $\begin{array}{l}\text { Number of } \\
\text { Readmission(90days) after } \\
\text { discharge }\end{array}$ & $\begin{array}{l}1.0 \\
\text { Stardard Deviation }=1.29\end{array}$ & $\begin{array}{l}1.4 \\
\text { Standard Deviation }=0.59\end{array}$ & $\begin{array}{l}\text { P value }=108 \\
\text { C1 } 199 \% \text { Difference is not } \\
\text { siggificant }\end{array}$ \\
\hline 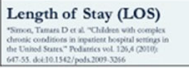 & $\begin{array}{l}7.5 \text { days } \\
\text { National Average (2006)= } \\
5.2 \text { days* }\end{array}$ & 2.1 days & P. valuc 0.048 Statistically different \\
\hline
\end{tabular}

For CMC patients, the complexity index scores are 2.5- 2.6 times higher \& LOS also increases by 2.1. Twenty percent of patients of admitted are of high complexity. Validated risk stratification tools applied to CMC patients demonstrate increased LOS \& RR that are more than twice that of the non-CMC patients. A highrisk index can lead to inefficient health care utilization, unaccountable patient/family cost, \& poor outcomes.

\section{7}

\section{Primary Care}

Factors Contributing to Patient No-Show in General Pediatrics Ambulatory Clinics In Qatar

Ahmed AlMohannadi $^{1}$, Amina Alansari ${ }^{1}$, Manasik Hassan ${ }^{1}$, Ahmed Alhammadi ${ }^{I}$

${ }^{1}$ Sidra Medicine, Qatar

Introduction and aim: Patients no-show in the clinics represent a critical and continous problem in the healthcare system. It creates negative impact in many aspects: disruption in continuity of patient care, long waiting times for new patients, waste of clinic and hospital resources.

Our aim was to explore why patients may not show for their appointments and to make potential recommendations to decrease the non-attendance rate

Methods: Prospective study of patients referred to general pediatrics out patients' clinics at Sidra Medicine- the newly opening tertiary children hospital in Qatar from June 2018-sept 2018, phone interview-based survey conducted and were performed by the unit clerk of clinics. Survey included details from patients exploring different reasons of not attending clinics although previously patients' families confirmed attendance by phone call reminders.

Result: 2036 patients were schedules to general pediatric clinics. 883 they attended the clinics, 899 they canceled /re-schedules the appointments and 254 were no-show. The phone interview included 254 out of 1140 of all patients confirmed initially attending clinics in phone call reminder.

The main reasons identified of no show up were: no answer of the call back $143 / 256(55 \%)$, the time of clinics is not suitable with parents $44(17 \%)$,parents forget the appointment $26(10 \%)$, family work issues $(5 \%)$ patients/parents were sick $(4 \%)$, patients clinically improved $(3 \%)$, no available transportation $(3 \%)$.

Discussion and conclusion: The international benchmark for primary care clinics of no-show is $10 \%$. In our observation the no show rate were $20 \%$. Identified multiple reasons of no show it is similar to the international ones. Initiating new strategies to decrease no-show in the clinics like revisiting cancelation policy, modifying working hours, appointment reminders via sending telephone massage with option of choice of appointments will decrease patients waiting, reduce health care cost and maintain full capacity scheduling of the clinics.
390

\section{Primary Care}

Sports Related Concussion in Paediatric Age: The Importance of Gradual Return to School and Sport Strategy

Tiago Almendra ${ }^{1}$, Sofia Miranda ${ }^{2}$

${ }^{T}$ Resident at Family Medicine Department, USF d'Terras de Lanhoso/ Braga Hospital, Portugal

${ }^{2}$ Resident at Paediatric Department, Braga Hospital, Portugal

Background: Sports Related Concussion (SRC) is a traumatic brain injury that has been defined as a complex pathophysiological process affecting the brain, induced by biomechanical forces with several common features that help define its nature. The diagnosis of concussion remains a clinical diagnosis despite the availability of auxiliary tests.

A 7 year's old child, five hours later a history of head trauma when was playing football, started to experience in his football training dizziness, headache, nausea and vomiting and was referred for urgent assessment. A physical examination was performed and a CT was requested.

Serious intracranial injury was excluded by neuroimaging, the diagnosis of concussion has been established and the child was discharged, with surveillance of the alarm signals.

A brief period of physical and cognitive rest was advised followed by a gradual program, which included 10 steps with 3 medical evaluations. A return to play was achieved with no complications.

Objective: Encourage paediatric care providers to follow-up after an SRC to monitor recovery and help with return to school. Ensure the importance of spreading the SRC prevention and management for teachers, staff, students and parents.

Methods: The literature on paediatric concussion was reviewed. A case report of a 7 years old child was detailed. A graduated return to school and sport was designed.

Conclusion; Although most concussions solve without complications, they may occur, especially when concussions are not recognized or are sustained multiple times, which include post concussion symptoms, second impact syndrome, and chronic traumatic encephalopathy. The applicability of implemented tools like Child SCAT5 baseline testing can be a key for better interpreting post-injury test scores. Continued efforts to train and educate practicing and future physicians about concussion diagnosis and management should be promoted to better serve patients.

\section{7}

\section{Primary Care}

Pediatric Cutaneous Horn of the Eyelid

Inês F. Viegas ${ }^{1}$, Maria Filomena Cardosa ${ }^{3}$, Helena Costa Pinto ${ }^{2}$

${ }^{1}$ Serviço de Pediatria Médica, Departamento de Pediatria, Hospital de Santa Maria, Centro Hospitalar Universitário Lisboa Norte, Portugal

${ }^{2}$ USF São João do Pragal, ACES Almada Seixal, Portugal

${ }^{3}$ Serviço de Pediatria, Hospital Garcia de Orta, Portugal

Background: A cutaneous horn consists of a keratotic projection that resembles a spicule or cone. Cutaneous horns of the eyelid are uncommon and even rarer in paediatric age. Typically, they grow in elder people with light skins and on sun-exposed areas. A variety of histopathological findings can be associated with this lesion.

Clinical Case: A 4-year old girl presented to the urgency room with an upper eyelid lesion of the right eye. This lesion had 4 months of evolution. At examination she had no complains but pain with localized pressure. A minor haemorrhage happened the day before and motivated her consultation. This lesion had already been seen both at primary care center and general paediatric urgency before. Topical antibiotics and corticosteroids have been applied with no improvement. At this point, a referral was made to Ophthalmology. The clinical diagnosis of a cutaneous horn was made and she was sent to the ambulatory clinic for further investigation and excision biopsy. 
Discussion: We present this case to highlight that, although most paediatric patients in Portugal are first seen by paediatricians or family doctors, there should be a low threshold for Ophthalmology referral in face of eyelid pathologies. Even though most of these lesions in children are benign, a careful management should be followed due to the possibility of a premalignant or malignant transformation at the lesion's base.

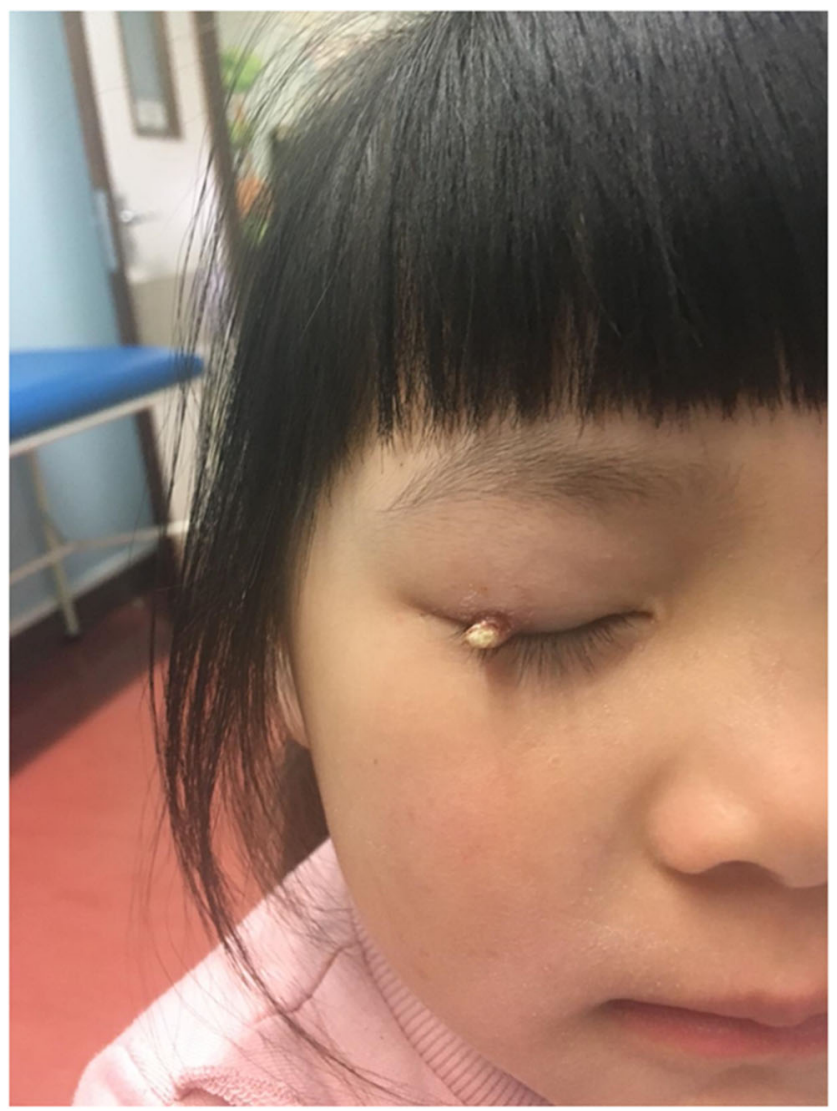

169

\section{Primary Care}

Body Composition in Children at the Beginning of School Age Jane Cardoso ${ }^{I}$, Luana Dalla Costa ${ }^{I}$

${ }^{T}$ Medicine, Center of University of Maringa, Brazil

Objective: the present study aims to analyze the body composition, through the children's arm anthropometry at the beginning of school age. Method: this is a cross-sectional study using a database of a cohort of elementary school students from municipal and state schools in Florianopolis, aged between 7 and 8 years. Measurements were obtained from the arm circumference and triceps skinfold thickness (TSF) and Arm Muscle Area (AMA), Arm Fat Area (AFA) e Arm Total Area (ATA) were calculated. There were obtained mean, median and standard deviation and rated the percentiles of the indices according to Frisancho's referential. Correlations of AMA, AFA, ATA e TSF between boys and girls were analyzed.

Results: there was no statistically significant linear correlation of the Arm Muscle Area (AMA) between boys and girls, there was a statistically significant correlation of the Arm Fat Area (AFA) between boys and girls, and there was a strong linear correlation of Arm Total Area (ATA) between boys and girls. There was a statistically significant negative linear correlation of Triceps Skinfold Thicknesses (TSF) between boys and girls.
Conclusion: in the group the mean and medians of AFA are higher and the mean and medians of AMA and TSF are lower in relation to the referential. We can say that especially girls are overweight at the beginning of school age.

172

\section{Primary Care}

Diagnosis of Hypertension in Children and Adolescents Jane Cardoso $^{I}$, Bruna Domingues ${ }^{I}$, Claudia Pellegrini ${ }^{I}$ ${ }^{\mathrm{T}}$ Medicine, Center of University of Maringa, Brazil

Objective: To carry out an integrative review on the topic of hypertension diagnosis in children and adolescents and their recommendations.

Methodology: This is a study with data collection performed from secondary sources, through a bibliographic survey to identify studies on the diagnosis of hypertension in children and adolescents. The integrative literature review was adopted. Fifteen studies were analyzed, of which 7 were in the English language and 8 in Portuguese. Among the studies, the guidelines and guidelines for hypertension in Portugal, Brazil, the United States, Canada and Europe were analyzed. It was identified that the predominant type of study was the transversal one, besides the literature review. Regarding the year of publication, articles from 2005 to 2018 were used. Results: The results showed that for an adequate measure of BP in children, a quiet environment, an appropriate cuff, and technical knowledge are important. In addition, guidelines have shown that for diagnosis of target organ damage and concomitant clinical diseases, a BP measurement, medical history, including family history, physical examination, laboratory investigations, and other diagnostic tests is required.

Conclusion: It is difficult to use screening to predict which children and adolescents will have high blood pressure as adults, there is limited information on blood pressure cuff accuracy in the diagnosis of hypertension in children and adolescents, and evidence of inappropriate use, incorrect calibration, or lack of this material in outpatient clinics.

999

\section{Primary Care}

A Study of Adverse Events Following Immunization (AEFI) in Children, in Primary Care $\underline{\text { Emiliana Costiug }}^{1,5}$, Valeria Herdea ${ }^{2,5}$, Claudia-Felicia Pop ${ }^{3,5}$, Laura Comnea $^{4,5}$

${ }^{I}$ M.D, General Practitioner, Family Medicine, Iuliu Hațieganu University of Medicine and Pharmacy, Romania

${ }^{2}$ M.D. General Practitioner, PhD student, University of Medicine and Pharmacy of Târgu Mureș, Romania

${ }^{3}$ MD, PhD, General Practitioner, Family Medicine, Iuliu Hațieganu University of Medicine and Pharmacy, Romania

${ }^{4}$ MD, General Practitioner, Family Medicine Office, Romania The Romanian Association for Pediatric Education in Family Medicine AREPMF, Romania

Background: The surveillance of AEFI is important to improve the vaccine safety and also to build public confidence in immunization's effects. The lack of confidence in immunization's benefits and the fear of possible side effects are the main reasons why there is a refusal to vaccination.

Objective: To analyze the adverse events following immunization (AEFI) in 0-16 year old children and to quantify the rates and severity of these events.

Methods: This prospective study was conducted in 3 family medicine offices between the 1st of January 2017 and the 31st of December 2017 (2445 vaccine doses administered).During appointments for vaccination, parents were given information concerning possible AEFI and were advised on how to act, should these reactions appear, including reaching out 
to the family doctor by telephone or e-mail. There were records of adverse reactions being noticed by parents and by medical personnel and recorded following the methodology of the National Center of Control for Infectious Diseases.

Results: There were records of 283 minor AEFI in the first 2 to 10 days following vaccine administration, which consisted of $11,57 \%$ of the total vaccine doses administered. The adverse reactions were: moderate or low grade fever, food intake behavior disorder, sleep behavior disorder and mild local reactions. There was no need of emergency care treatment or clinical admission.

Conclusion: The evaluation and analysis of AEFI confirms the low rates of occurrence and the lack of severity compared to risk of the vaccine preventable disease. The active involvement and counseling of parents in reporting possible AEFI gives them responsibility, lowers the unease about possible reactions and heightens vaccination acceptance rates

284

\section{Primary Care}

Utilization of Medical Facilities by Children in Japan at Night and During Holidays

Akira Ehara

${ }^{T}$ Faculty of Health Services Management, Hiroshima International University, Japan

Background: Population of children under 6 years in Japan is decreasing from 6,761 thousands in 2005 to 6,031 thousands in 2015. Compared with the past, today's parents are raising fewer children. It is probable that they do not have an enough experience with treating children's illness and that they bring their children even with a mild illness to medical facilities not only in daytime but also at night and holidays.

Objective: In this analysis, I tried to clarify the number of children`s first visit to medical facilities in Japan in day- and night-time and their hospitalization rate between 2007 and 2017.

Methods: I used data from Statistics of Medical Care Activities in Public Health Insurance, 2007, 2012, and 2017 published by the Ministry of Health, Labour and Welfare of Japan. I calculated the number of the fee charged for a first visit of children under 6 years old in and out of medical hours.

Results: The number of the fee charged for a patient's first visit increased 576.36 (/1000 person-months) in 2007 to 606.17 (/1000 person - months) in 2017. The increment was highest in daytime (Table 1).

Table 1. The number of the fee charged for a patient's first visit. (First visit/1000 person-months)

\begin{tabular}{lrrrr}
\hline $\begin{array}{l}\text { First Vist/ } \\
1000 \text { person-months }\end{array}$ & $2007 \mathrm{Yr}$ & $2012 \mathrm{Yr}$ & $2017 \mathrm{Yr}$ & $2017 / 2007$ \\
\hline Daytime & 487.50 & 514.87 & 530.79 & $108.9 \%$ \\
$\begin{array}{l}\text { 6 p.m. to 10 p.m. \& } \\
\begin{array}{l}\text { a.m. to 8 a.m. } \\
\text { of Weekdays }\end{array}\end{array}$ & 35.47 & 36.96 & 33.98 & $95.8 \%$ \\
$\begin{array}{l}\text { Holidays } \\
10 \text { p.m. to 6 a.m. }\end{array}$ & 46.49 & 47.72 & 34.86 & $75.0 \%$ \\
\hline Total & 6.90 & 7.40 & 6.54 & $94.7 \%$ \\
\hline & 576.36 & 606.95 & 606.17 & $105.2 \%$ \\
\hline
\end{tabular}

Hospitalization of infants and toddlers was also growing from $0.48 \%$ in 2007 to $0.86 \%$ in 2017 , and the rate was highest at midnight (from $3.55 \%$ in 2007 to $7.59 \%$ in 2017, Table 2).

Table 2. Hospitalization rate of infants and toddler at first visit

Hospitalization Rate 2007 Yr 2012 Yr 2017 Yr $\quad 2017 / 2007$

\begin{tabular}{|c|c|c|c|c|}
\hline Daytime & $0.33 \%$ & $0.58 \%$ & $0.60 \%$ & $182.1 \%$ \\
\hline 6 p.m. to 10 p.m. 8 & & & & \\
\hline $\begin{array}{l}\text { a.m. to } 8 \text { a.m. } \\
\text { of Weekdays }\end{array}$ & $1.51 \%$ & $1.88 \%$ & $2.79 \%$ & $184.5 \%$ \\
\hline Holidays & $0.78 \%$ & $1.01 \%$ & $1.68 \%$ & $215.0 \%$ \\
\hline 10 p.m. to 6 a.m. & $3.55 \%$ & $4.95 \%$ & $7.59 \%$ & $213.6 \%$ \\
\hline Total & $0.48 \%$ & $0.75 \%$ & $0.86 \%$ & $180.2 \%$ \\
\hline
\end{tabular}

Conclusion: Most of parents bring their children to medical facilities in daytime, and they tend to refrain from utilizing medical facilities at night and during holidays when their children have a mild illness.

255

\section{Primary Care}

Role of Lactobacillus Rhamnosus 19070-2 and Lactobacillus Reuteri DSM 12246 in Infant Colic: A Multi-Center Double-Blind PlaceboControlled Randomized Dietary Study

Sergei Gerasimov ${ }^{1}$, Jesper Ganzel ${ }^{2}$

${ }^{T}$ Department of Pediatrics \#2, Lviv National Medical University, Ukraine

${ }^{2}$ Life Sciences, Biocare Copenhagen, Denmark

Introduction: Infant colic is a common condition of unknown pathogenesis that brings frustration to families seeking for effective management. Accumulating evidence suggests that some single strains of lactobacilli may play a positive dietary role in attenuation of colic in exclusively breastfed infants. The objective of this study was to evaluate mixture of 2 lactobacillus strains in decreasing of infant cry and fuss in this population.

Methods: Infants aged 1-3 months received L. rhamnosus 19070-2 and L. reuteri DSM 12246 in a daily dose of $250 \times 10^{6} \mathrm{CFU}$ and 200 IU of vitamin $\mathrm{D}_{3}$ (84 infants, probiotic group) or just vitamin $\mathrm{D}_{3}$ (84 infants, control group) for 28 days. Cry and fuss time were measured with validated Baby`s Day Diary on days 0 and 28.

Results: At baseline, mean (SD) duration of cry and fuss time was comparable in the probiotic and control groups: 305 (81) vs. 315 (90) min., respectively $(\mathrm{P}=0.450)$. On day 28 , mean cry and fuss time became statistically different: 142 (89) vs. 199 (72), respectively (P0.05). Mean change in cry and fuss time from day 0 through day 28 was -163 (99) minutes in the probiotic and -116 (94) minutes in the control group $(\mathrm{P}=0.019)$. Infants in the probiotic group recovered faster from colic in Cox`s F-test (P0.001) (Figure 1). 


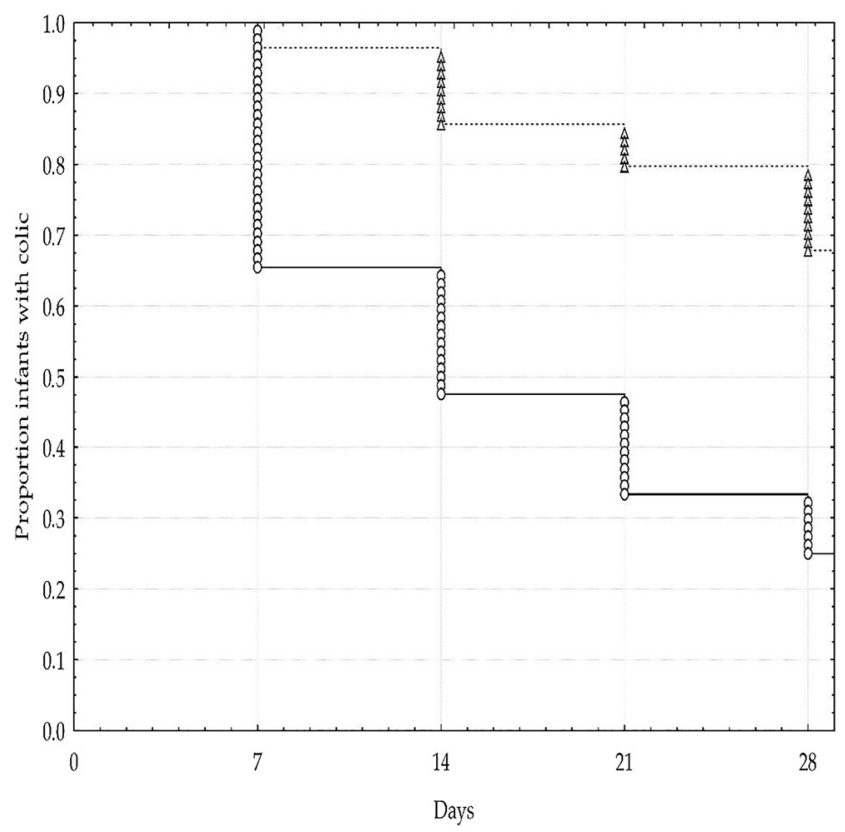

Conclusion: Our findings confirm that lactobacilli decrease cry and fuss time and provide meaningful dietary support in exclusively breastfed infants with colic.

\section{2}

\section{Primary Care}

Evaluation of the Knowledge and Photoprotection Habits of Children and their Caregivers

Cátia Granja ${ }^{I}$, Filipa Cunha ${ }^{1}$

${ }^{T}$ Pediatrics, Hospital Distrital da Figueira da Foz, Portugal

Introduction: Spending time outdoors is an essential part of an active and healthy lifestyle. On average, the first 18 years of age account for $25 \%$ of an individual's total sun exposure. However, the time in the sun without adequate sun protection is responsible for $50-80 \%$ of the deleterious effects of accumulated exposure to ultraviolet radiation (UVR), particularly for its carcinogenic action.

Objectives: To assess the level of knowledge and to understand the children/adolescents and their caregiver's habits regarding sun protection, in outpatient, our Pediatric emergency department, Hospital level II, and in the child health consultation of a local USF.

Methods: Cross-sectional study in which was applied a questionnaire about knowledge and habits concerning sun protection. Frequency tables and chi-square tests were applied, statistically significant p 0.05 .

Results: Sample totaled 295, 63.7\% caregivers and 36.3\% young people under 18 years. The period of the day of greatest exposure to sunlight was after 4:00pm (78.3\%). They use sunscreen, $93 \%$ of children/adolescents and $90.6 \%$ of caregivers, however, the majority of the population only use it on the beach $(61 \%)$ (p0.05). As for the frequency of renewal of sunscreen, $40.2 \%$ of the children/adolescents renewed two times, and $52.6 \%$ of the caregivers only once ( $\mathrm{p} 0.05)$. It is the female who responds to use more umbrella (76\%) (p0.05) and more girls use SPF 30+ (59\%) and 50+ (73.7\%) while more boys use SPF

Conclusion: The present study's data point to the need for a wider dissemination of adequate knowledge, both by the media and by physicians, to nurture healthy practices regarding exposure to the sun. The multidisciplinarity between primary health care and Pediatrician becomes crucial.
958

\section{Primary Care}

Changing Atittudes Towards Patients in Family MedicineIntrafamilial Predictive Medicine

Valeria Herdea $^{I}$, Petruta Tarciuc ${ }^{1}$, Carmen Raluca Ghionaru ${ }^{2}$, Simona Magdalena Cioc ${ }^{2}$, Oana Maria Rosu ${ }^{I}$, Andra Carmina Elsayed ${ }^{I}$, Smaranda Diaconescu ${ }^{1}$

${ }^{1}$ Institute for Doctoral Studies Targu Mures, University of Medicine, Pharmacy, Science and Technology of Targu Mures, Romania

${ }^{2}$ Family Medicine, Romanian Association for Pediatric Education in Family Medicine (AREPMF), Romania

Background: According to WHO 2018, 41 million children are obese in the world (1)

In Romania, $24.5 \%$ of children aged 5 to 19 years are obese (2016, NIS) Aims: Introduction of Intrafamilial Predictive Model (MIP) - Preventive interventional mechanism applicable to the child with the role of reducing the risk of major chronic diseases in the future adult

Method: Between 10.2017- 04.2018, we developed a prospective observational interventional cohort study, including two groups of 36 children (study group- control group) aged 6-18 years old, sex ratio girls: boys 2: 1 , average BMI(body mass index) 27 , coming from families struggling with chronic diseases: HBP(high blood pressure), dyslipidemia, diabetes. Clinical parameters were recorded: 3 visits for every patient over a period of 6 months: Weight, Height, BP(blood pressure), PA(abdominal perimeter), BMI $(2,3,4)$. Based on these data, a MIP has been prepared and a schedule of recommendations for the child and the his /her family has been developed and applied (5).

Results: After 6 months:

- $10 \%$ weight loss in children

- Reduction of Systolic BP values averaged by $15 \mathrm{mmHg}$ in girls and $10 \mathrm{mmHg}$ in boys

- Daily sports activity - average $60 \mathrm{~min}$

- Professional orientation for children with static vertebral disorders - 30 min every day- spending quality time with family (sport, dinner) Conclusions: Obesity remains the main common, identified major risk factor (MRF) in the studied patient. MPI allows the early identification and evaluation of MRF in childhood with a possible role in the etiology of chronic diseases in adulthood. MPI allows the development and implementation of a personalized, cost-effective program, based on education for health. Future study is needed for a larger group of children to allow correlations between RF(risk factors) and chronical diseases occurrence.

729

\section{Primary Care}

Morbidity During the First Year of Life in Children Conceived by In Vitro Fertilization in Clinic "ISIDA-IVF" Oksana Honcharuk $^{1}$, Mariia Krepak ${ }^{1}$, Tatiana Petreman ${ }^{l}$ ${ }^{T}$ Pediatric department, LCC"ISIDA-IVF", Ukraine

Background and aims: As in vitro fertilization (IVF) are increasingly used to overcome infertility, there is concern about the health of the children conceived via IVF. It is important to follow up these children and evaluate whether they have elevated health risks compared to naturally conceived children. Inspite of big amount of researches, it is still not enough information to make an accurate assessment of health of children, born via IVF technologies.

Methods: We monitored 81 children born in 2016 - 2018 years via IVF and 102 naturally conceived children. We assessed the morbidity during the first year of life in children conceived naturally or via IVF.

Results: Children conceived with use of IVF were more likely to have anemia (OR 1.21 95\%CI 0.47 to 3.13), diseases of gastrointestinal (OR $1.8495 \% \mathrm{CI} 0.70$ to 4.83 ) and genitourinary systems (OR $2.5595 \% \mathrm{CI}$ 
0.22 to 28.71 ), also they are at higher risk of the skin and subcutaneous tissue diseases (OR 1.17 95\%CI 0.57 to 2.39). We observed that children in research group have significantly lower risk of acute respiratory infection (OR $0.8895 \%$ CI 0.49 to 1.58 ), neonatal diseases (OR $0.8195 \% \mathrm{CI}$ 0.41 to 1.83 ), eye (OR $0.3095 \% \mathrm{CI} 0.03$ to 2.79 ) and ear diseases(OR $0.4195 \%$ CI 0.01 to 10.32 ).

Conclusion: Children conceived by IVF were at significantly increased risk of anemia, diseases of gastrointestinal and genitourinary systems, also they are at higher risk of the skin and subcutaneous tissue diseases, when compared to those conceived by spontaneous conception. We think that further large scale research should be undertaken with long-term follow-up to assess the morbidity of IVF conceived children.

\section{3}

\section{Primary Care}

The Health Status of Primary-School Aged Children in Adjara Region

Liana Jorjoliani ${ }^{1}$, Rusudan Karseladze $e^{1}$, Lali Saginadze $e^{l}$

${ }^{1}$ Faculty of Medicine, Department of Pediatrics, Iv. Javakhishvili Tbilisi State University, Georgia

Background: According to the data of numerous researches, in the recent years the situation, with respect of children's health is unfavorable: number of absolutely healthy children has reduced, frequency of functional disorders has increased, $68 \%$ of the pre-school children has multiple functional disorders, $13.5 \%$ has physical development anomalies and $17 \%$ of them has chronic diseases. Structure of chronic pathologies has changed; share of the digestive organs, locomotion and nervous systems has increased. There are many cases of body posture anomalies, myopia, impairment of the functional capabilities of cardio-respiratory system and this plays significant role in organism resistance reduction and high morbidity.

Objective: Goal of the research was complex evaluation of the health status of the primary-school aged children residing in various regions (urban, rural) of Adjara.

Methods: Cross-section, one-stage research was conducted in the City of Batumi and village Tsikhisdziri. In the process of survey health status of children of 4 public schools, from 6 to 9 years old was studied. Public schools' selection was provided by cluster randomization method. Observations covered up to 800 school children in total. Screening included consultations of the multidisciplinary group of specialists, additional laboratory and instrumental studies intended for the purpose of accurate diagnostics.

Results: Performed studies showed that $28.3 \%$ of the studied population was actually healthy, $55 \%$ had functional disorders and $16.7 \%$ - chronic diseases. Prevalence of both, functional disorders and chronic diseases was tow times greater in the 6-7 years' group, compared with the group of 8-9 years-old children.

Conclusion: Negative trends in health status of primary school aged children, given the regional characteristics, evidences necessity of timely identification of the key causes of morbidity and adequate diseases' management and this is of great importance, with respect of reduction of the children's morbidity burden.

\section{6}

\section{Primary Care}

Loss of Cerebral Volume on MRI in a Child with Severe Iron Deficiency Anaemia: Case Repot and Systematic Review of the literature. ${ }_{\text {Helen Leveret }}{ }^{l}$, Tamsin Woodbridge ${ }^{l}$, Amabetare Biu ${ }^{l}$, Fiona Finlay ${ }^{2}$, Richard Lee-Kelland ${ }^{l}$

${ }^{1}$ Community Paedaitrics, Sirona Healthcare, UK

${ }^{2}$ Community Paedaitrics, Virgincare, UK
Background: A 2 year old girl presented to hospital with severe lethargy, pallor and increased work of breathing on a background of regression of motor and cognitive skills; having previously been able to walk she was now unable to weight bear. The girl was drinking up to 2 litres of cow's milk daily and eating little solid food.

Blood tests revealed a haemoglobin of $11.7 \mathrm{~g} / \mathrm{L}$ (94-130) MCV 63f/L (87$103)$ and ferritin of $12 \mathrm{ug} / \mathrm{L}$ (15-150). She was stabilised with a blood transfusion. Echocardiogram showed mild left ventricular dysfunction alongside a pericardial effusion. MRI brain revealed generalised volume loss with prominent sulci, subarachnoid spaces and ventricles (see figure 1). After investigations into potential haematological, metabolic or malabsorptive causes, a diagnosis of severe iron deficiency anaemia was made.

Objective: Iron deficiency anaemia is the most common mineral deficiency of childhood (WHO 2017) and is known to be associated with cognitive impairment and developmental delay (Lozoff 2006). Iron is essential for brain development and iron deficiency has been demonstrated to produce structural brain changes on MRI in animal models (Mudd 2018). We wondered if there were any previous studies finding structural brain changes in children as a result of iron deficiency anaemia.

Methods: Systematic literature review

Results: We found no evidence of any previous studies reporting structural MRI changes. One case series (Munot 2011) reported on 4 children aged 14 months to 4 years with ischemic stroke as a result of IDA.

Conclusion: While it has been shown that iron deficiency produces developmental impairment, it has yet to be elucidated how the deficiency directly affects the brain in children. This case shows a severe case of Iron deficiency anaemia with associated widespread volume loss. Further neuroimaging studies are required to characterize the effect of iron deficiency anaemia on the brain in childhood.

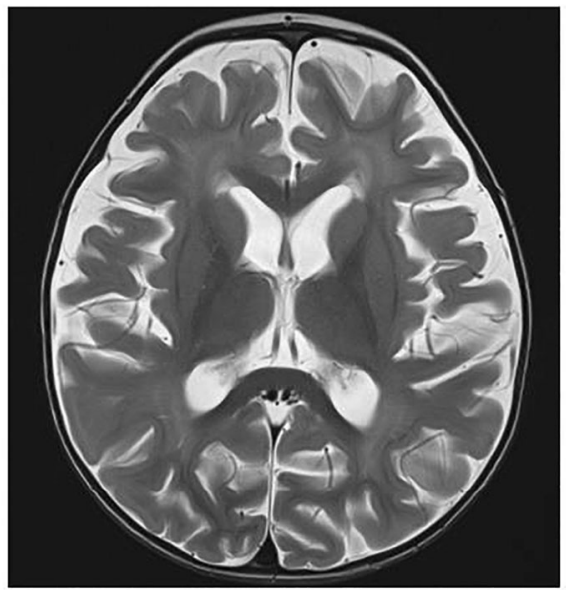

Figure 1: T2 Axial slice showing MRI brain Generalised lack of cerebral volume with prominent sulci, subarachnoid spaces and ventricles. There is subtle abnormal high $\mathrm{T} 2$ intensity in cerebral white matter.

\section{1}

\section{Primary Care}

Socio-Demographic Profile of Bullying among Grade 6 Pupils in a Public Elementary School in Manila Pamela Losa ${ }^{1}$, Edwina Losa ${ }^{2}$, Kris Ian Mendoza ${ }^{1}$, Jennie Wong ${ }^{1}$, Rose $\overline{\text { Lyn Bernardo }}^{1}$, Sittie Noraisa Ussam Arimao ${ }^{l}$

${ }^{I}$ Pediatrics, Ospital ng Maynila Medical Center, Philippines

${ }^{2}$ Pediatrics, Presidential Security Group Station Hospital, Philippines

In the Philippines a law that ensure that cases and instances of abuse are closely monitored at school level. However in 2015 Department of Education reports an increasing trend in bullying. This study aims to 
determine the socio-demographic profile of bullying among grade 6 students enrolled in a Public school in Manila. This is a Cross-sectional Study. Grade 6 students were chosen by cluster sampling and 3 classes were included. Participants were asked to answer a questionnaire. Data were arranged in tables expressed percentages. Chi-square test was done to determine the association of variables.Results showed that $84 \%$ said they were bullied by other children, $77.2 \%$ bully other children. Verbal bullying was the most commonly experienced type by victims and done by perpetrators. Frequency of bullying for both bullies and victims happened less than once a week (61\%). Retaliation (53\%) is the most common reason of bullies and for the victims they are bullied just for fun(43\%).Majority of the bullies felt guilty after they have bullied other kids(40\%), while most of the victims felt nothing after they were bul$\operatorname{lied}(23 \%)$. Majority of the students $(81 \%)$ reported that the most common reaction of teachers to bullying incidence they have witnessed was stopping the act immediately and resolving the matter right there and then. Those who are bullied others have higher incidence of being bullies $(83.9 \%)$ than those who were not bullied $(47.1 \%)$. There is no significant association between sex and being bullies or being bullied by other students. In conclusion, there is a high percentage of bullying in a public elementary school in Manila despite an existing law against bullying. Programs which may increase bullying awareness must be done in schools. The Department of Education together with the national and local government leaders and the whole community should work hand in hand to be able to lessen if not eradicate bullying in our country.

\section{6}

\section{Primary Care}

\section{Measles Outbreak in Macedonia: A 4 Year Old Girl with Varicella and Measles Infection One after Another Elena Manoleva Nikolovska ${ }^{1}$ \\ ${ }^{T}$ Pediatrics, PHO Polyclinic MANOLEVI, Macedonia}

Backgrounds: Varicella is a highly contagious disease caused by varicella-zoster virus. Most commonly, virus is affecting children, teens and young adults. Incubation period is usually 14 days. Varicella-zoster virus enters in the body through the respiratory system.

Measles is a highly contagious infectious disease caused by measles virus. Symptoms usually develop 10-12 days after exposure to an infected person and last 7-10 days. Measles virus spreads easily through the coughs and sneezes of infected people.

Varicella and measles are important infectious diseases because they can cause severe complication even death in young children under one year. Methods: A 4 year old girl came on exam four weeks after the onset of the varicella rash. She came with high fever over 40C, caught, vomitus and decreased appetite. Physical examination shown signs of pharyngitis. Blood test showed normal leucocytes and high elevated CRP. After 5 days of antibiotic therapy, she came on exam, still febrile over 39-40C, with drowsiness and decreased appetite. Second laboratory findings revealed normal leucocytes and reduced value of CRP. Two days after, she was hospitalised, still febrile, dehydrated, with drowsiness and presence of red maculopapular rash in the body, starting from the head through the corps. Girl wasn`t immunised with MMR vaccine.

Results: First laboratory findings revealed leucocytes 5,4x109/1 (Ne69\%,Ly24\%), CRP 96mg/d (6). Second laboratory findings revealed leucocytes 4,0x109/1 (Ne58\%,Ly35\%), CRP 24mg/dl (6). Test for Measles (IgM positive) confirmed the disease.

Conclusions: Varicella and measles are highly contagious infectious diseases. They are diseases that cause a big decline in immune system and can cause severe complications. Also, they are diseases that can be prevented by a vaccine. Therefore, it is recommended regular immunisation per calendar to prevent the occurrence of the diseases as well as to reduce the complications and mortality from these diseases, especially from the measles.
933

\section{Primary Care}

Parental Attitudes toward the Human Papilloma Virus (HPV) Vaccine: A New Concept in the State of Qatar

Tasabeh Mohamed $^{1}$, Manasik Hassan ${ }^{1}$, Reem Mohamed ${ }^{1}$, Moza Al $\overline{\text { Sulaiti }}^{I}$, Hadeel Mahjoob ${ }^{I}$, Dure Yasrab ${ }^{l}$, Ahmed Al Hammadi ${ }^{l}$, Mohamed Hendaus-Rahal ${ }^{1}$

${ }^{I}$ Department of Pediatrics, SIDRA Medicine, Qatar

Background: Human papillomavirus (HPV) is one of the leading causes of genital cancer in both genders. The HPV vaccine is recommended by the CDC for children 14 years and above. Still, many countries do not include the HPV vaccine in their immunization schedule. Awareness of the families about the virus itself in addition to the availability of the vaccine is essential to prevent cancer-related viruses in the future.

Objective: To explore the HPV vaccine in Qatar, a rapidly developing country that does not include the HPV vaccine in its immunization schedule.

Methods: A cross-sectional study using a questionnaire conducted in SIDRA medicine, the only tertiary pediatric hospital in the state of Qatar. Results: 232 questioners were completed (response rate from parents is $94 \%$ ). Almost $60 \%$ of participating parents were between 30 and 39 years of age. $60 \%$ of the parents were not aware that human papillomavirus could cause cancer. More than $75 \%$ of the parents felt confident about the idea of giving their children the HPV vaccine to prevent genital cancer. Unfortunately, $90 \%$ of the parents stated that their primary care physician has never mentioned HPV vaccine. When asked about their idea for the suitable time for HPV vaccination, $41 \%$ of the parents stated that it should be before a child is mature, while $22 \%$ stated it should be given before marriage. $12 \%$ of the parents stated that the decision about the HPV vaccine should be left to the children when they become adults. $25 \%$ of parents do not have an opinion.

Conclusion: A large proportion of parents residing in Qatar have a positive perception regarding the HPV vaccine. We will share the result of our study with the ministry of public health in Qatar with a goal to incorporate the HPV vaccine in the national immunization schedule.

\section{9}

\section{Primary Care}

Mind Your Child's Screen Time: A Word of Warning! Alexandra Coroleuca ${ }^{1}$, Raluca Maria Vlad ${ }^{1,2}$, Livia Brezeanu ${ }^{1}, \underline{\text { Daniela }}$ Pacurar $^{1,2}$

${ }^{T}$ Pediatrics, “Grigore Alexandrescu” Emergency Hospital for Children, Bucharest, Romania, Romania

${ }^{2}$ Pediatrics, "Carol Davila" University of Medicine and Pharmacy, Romania

Background: Today's children grow up immersed in digital media, which has both positive and negative effects on healthy development. The experts' recommendations are: no screen time below 18 months, for children 2-5 years limited screen time to one hour/day, for children $\geq 6$ years there should be limits on the time spent using media and the types of media.

Objective: The authors aimed to evaluate how much screen time the children are exposed to during the day.

Methods: We conducted a prospective, observational study in February 2019 assessing the time children spend in front of a screen. This is a pilot study. The patients were enrolled in the Pediatrics Department of "Grigore Alexandrescu" Children's Hospital. Parents were asked to fill in a questionnaire containing screen time, type of screen, behavior related to screens.

Results: Fifty one patients were enrolled in one week. Mean age was 7 years 3 months, sex ratio $\mathrm{M} / \mathrm{F}=1.04 / 1,78.4 \%$ came from urban areas. The 
parents declared the children started using screens at an average age of 3 years (minimum 6 months). 9.8\% of children were exposed to screens below the age of one. The devices used were smartphones, tablets, TV, laptop, gameboard in $74.5 \%, 41.1 \%, 35.2 \%, 27.4 \%$ and $19.6 \%$ respectively. Children used screens alone on average 3 hours/day, 5.4 days/week and alongside their parents on average 2.2 hours/day, 5.2 days/week. In half the cases, a device was used during meal time. $25.4 \%$ of parents declared they use screen time as reward and $47 \%$ felt taking the child's device to be an effective punishment. $11.7 \%$ of children were falling asleep with the TV on.

Conclusions: Screen time for children is progressively increasing as the age of exposure decreases. The most frequently used device is by far the smartphone. The screen related behavior is "educated" in the family.

\section{7}

\section{Primary Care}

Lipschütz Ulcer: A Rare Cause of Genital Ulcer

Eva Cristina Pedrero Segura ${ }^{I}$, Anabel Valdivielso Martínez ${ }^{I}$, Silvia Gallego Gutiérrez

${ }^{1}$ Pediatrics, Primary Care Attention Málaga-Guadalhorce District, Spain

Background: ulcer (LU) is an uncommon disease characterized by vulvar ulcerations with sudden onset in nonsexually active girls or young women. It has a diagnosis of exclusion, discarding sexually transmitted ulcers, autoimmune diseases or trauma lesions. Most cases are idiopathic but several viral and bacterial infections have been envolved.

Objective: We report a case of acute onset of vulvodynia presented in emergency department (ED).

Methods: Case report

Results: A 13 years old female presented to pediatric ED reporting acute vulvar pain and dysuria for the last 24 hours. Fever, mialgias, rhinorrhea, astenia and odynophagia had begun three days before. Genital examination showed two ulcers with red border and a central gray-black eschar one in left labia minor and the other in vulvar haipin. Blood test showed discrete leucocitosis and C-reactive protein $30 \mathrm{mg} / \mathrm{L}$. Influenza A/B test, urianalysis and throat swab test for Group A Streptococcus were negative. The ulcer swab culture was also negative. We started oral and topic analgesia (lidocaine 2\%) and warm stiz baths. Two days later, she reconsulted at primary care associating cough and otalgia with diseminated wheezings and right acute otitis media at physical examination. She started inhaled bronchodilators and based on the suspicion of Mycoplasma Pneumoniae (MP) infection, oral macrolide was prescribed. Two days after she was afebrile with better general conditions. Serological tests showed EBV, CMV, HSV negatives and IgM MP positive in $1 / 256$. The ulcer complete healed in about two weeks with no scarring.

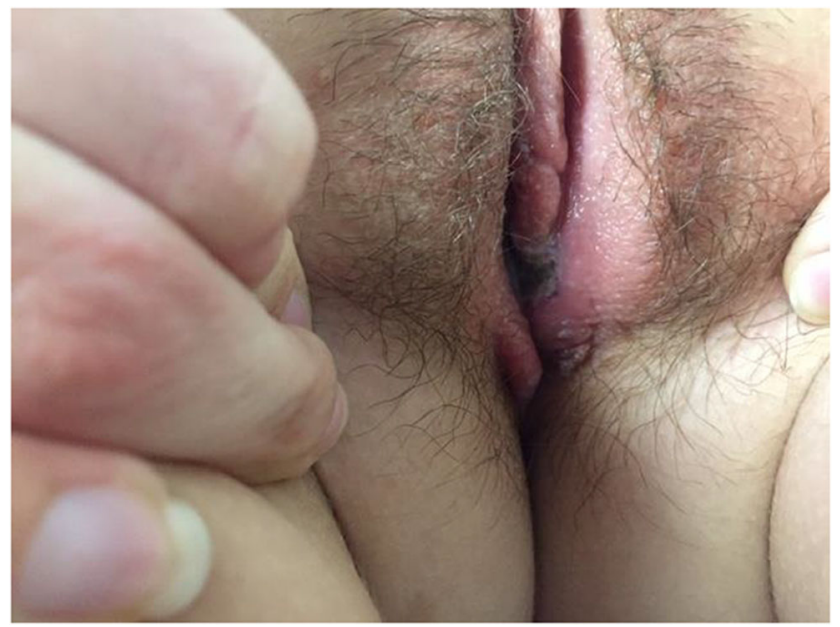

Conclusion: LU is an infrecuent disease that typically present in sexually inactive girls. It is probably misdiagnosed by pediatrician who are unaware of them. The diagnosis is based on clinical features and exclusion of other causes of genital ulcers. Although most of the cases are idiopathic, $E B V$ is the most identified cause but MP is also reported.

\section{5}

\section{Primary Care}

Motivators and Barriers of Primary Healthcare to Vaccines not included in National Immunization Schedule

Joana Rosa ${ }^{1}$, Beatriz Amaral ${ }^{2}$, Filipa Rebelo ${ }^{2}$, Ana Ventura ${ }^{2}$, Ana Raposo

${ }^{1}$ Pediatric Department, Divino Espirito Santo Hospital, Portugal

${ }^{2}$ Primary Care, Ponta Delgada Health Care Center, São Miguel Island Health Unit, Portugal

Background and Objectives: Vaccination has made an enormous contribution to the population growth and mortality rate decrease. The Portuguese National Vaccination Program includes 8 vaccines against 12 target diseases. Although some vaccines are not yet present in the program, they are considered safe and effective. The aim of this study was to evaluate opinion amongst primary healthcare workers about vaccines not included in the National Immunization Schedule (NIS).

Methods: An observational and descriptive study using a questionnaire to primary health care professionals about vaccines not include in NIS. The study had two phases (2017 and 2018), separated with clinical sessions and pamphlets distribution.

Results: 58 health professionals participated in the survey in 2017, of which $66 \%$ were nurses and $34 \%$ doctors. In the second questionnaire (2018), 83 health professionals were interviewed (53\% nurses and $47 \%$ doctors). An average of 12 and 15 years of professional activity was observed in 2017 and 2018, respectively. Of the study participants, 54 (91\%) in 2017 and $82(97.6 \%)$ in 2018 addressed the topic in primary health care appointment. The most recommended vaccines were against rotavirus and Neisseria meningitidis B. Approximately 19\% in 2017 refer that they did not feel comfortable discussing these issues with parents, a value that decreases to $2.4 \%$ after clinical sessions. The main reasons for the lack of initiative to recommend were monetary value and lack of knowledge. Considering the professional category, it was observed a difference in the recommendation of rotavirus (p-value 0.004$)$, hepatitis A ( $p$-value 0.036 ) and human papillomavirus virus ( $p$-value 0.035 ) vaccines in 2017 questionnaire. Most participants agreed with human papillomavirus virus in the male genre, but only $22.4 \%$ and $51.2 \%$ recommend it.

Conclusion: This study allows to recognize motivators and barriers that are important for identifying intervention points for improving the approach of this topic with parents.

447

\section{Primary Care}

Immunization Coverage and the Reasons for Unvaccination in Indjija, Srem District, Republic of Serbia

Vesna Petrovic ${ }^{1}$, Danilo Visnjevac Visnjevac ${ }^{l}$, Tanja Rozek Mitrovic ${ }^{1}$

${ }^{1}$ Health Center Indjija, Serbia

Background: Vaccination is one of the most successful public health measures. It is administration of pathogens or their parts in order to improve an individual's immunity to a particular disease. The aim of mandatory National immunization programme is to achieve and maintain 95\% immunization coverage of the entire population. Children should be vaccinated according to the National immunization schedule.

Objective: The aim of the study was to evaluate the immunization coverage of children born from 1997. till 2008. year according to the National 
immunization programme in Indjija, Srem District, Republic of Serbia and to determine the reasons for their incomplete vaccination at the time of conducting the cross-section.

Methods: The descriptive epidemiological method was performed. The data were gathered in July 2016. at Pediatric Department in Primary Health Center Indjija, from immunization records of children born between 1997. and 2008.

Results: There have been 3366 medical records of schoolchildren, born from 1997. till 2008. year. Vaccination coverage was above $95 \%$ in all volumes, except in one (1998. year of birth), it was $94.8 \%$. The total immunization coverage was $96.6 \%$. Parents refused vaccination for $35 /$ 3366 children, $1.03 \%$. Incompletely immunized were $77 / 3366$ children, $2.28 \%$. Medical reasons for incomplete vaccination had 11/77 children, $14.3 \%$. Between nonmedical reasons for incomplete vaccination vaccine deficiency 11/77 (14.3\%) and family removal 26/77 (33.7\%) were the most common

Conclusion: Immunization coverage according to the National immunization program in the schoolchildren in Indjija was in accordance with the recommendations $(95 \%)$. It is necessary to point out the importance of organizational measures and parental education about the necessity of vaccines to reduce the percentage of refused and incomplete vaccination.

\section{5}

\section{Primary Care}

Preventive Child Health, the Key to Ensure Healthy Lives and Wellbeing for All

Liesbeth Siderius $^{1}$, Sanath Lamabadusuriya ${ }^{2}$, Petra Poulissen ${ }^{3}$, Marc de Graauw

${ }^{1}$ Youth Health Care, GGD IJsselland, Netherlands

${ }^{2}$ Emeritus Professor of Paediatrics, University of Colombo, Sri Lanka

${ }^{3}$ Patient Representative, Rare Care World, Netherlands ${ }^{4}$ Healthcare ICT specialist, magrit. Nl, Netherlands

Introduction: The United Nations Sustainable Development Goals \#3 "Ensure healthy lives and promote well-being for all ages". Noncommunicable diseases are recognized as a major cause of illness and death globally. In this context, chronic and disabling (rare) conditions are receiving increased attention.

To meet the challenge of sustainable healthcare, cooperation between all involved in child health should be supported. tools to improve health for all. Preventive child health, as well as management of chronic diseases, follow schemes that can be aligned with international semantic terminologies. How can person-centred care, contribute to healthy lives?

Methods: Patient representatives of people with a rare condition designed a Unified Modelling Language (UML), as a graphical language software, to support personal health management: https://rarecare.world. A checklist based on preventive child health schemes was designed to identify recognizable features and diagnostic tests of rare conditions at primary care level. Characteristic symptoms were linked to international terminologies applicable in primary care; the International Classification of Primary Care, Human Phenotype Ontology and Standard for identifying health measurements, observations, and documents. Each symptom was associated with a disease using the International Classification of Diseases.

Results: Rare and disabling conditions present in preventive child health with recognizable features where schemes include data on birth, family history, physical examination, development and growth. For each rare condition, an application programming could be provided to link the features to preventive schemes.

Conclusions: Regardless of the chronic condition, the development of a generic set of skills and tools will allow individuals to manage their own illnesses and improve health outcomes. Different rare conditions have served as examples of a promising model for early recognition and better care of the disabled child.
736

\section{Primary Care}

Pigmented Pururic Dermatosis, a Case in Primary Care Silvia Gallego Gutierrez ${ }^{I}$, Ana Isabel Valdivielso Martinez ${ }^{I}$, Eva Pedrero Segural

${ }^{1}$ Malaga-GuadalHorce District, Primary Care Attention, Spain

Background: Pigmented purpuric dermatoses (PPD) are an heterogeneous group of dermatoses characterized by petechia and hyperpigmented macules and histologically by capillaritis, erythrocyte extravasation and hemosiderin deposition. Vasculitis is not present. Although it is most commonly seen in adults, this disease can also affect children.

Objective: We report on a male infant with progressive purpura who underwent laboratory tests to rule out a systemic disease. Based on the laboratory findings and clinical course, the diagnosis of Schamberg`s purpura was established.

Methods: Case reports

Results: A 7 years-old male consulted to us at primary care because of a purpuric rash that had started on the extremities 5 months prior to presentation. On physical examination the patient appeared as a well-developed healthy infant. He had a patchy, poorly demarcated light-purple to reddish hyperpigmentation over the trunk and the extremities.

No associated disease, significant drug exposure, or contact allergens were found.

Routine laboratory test including coagulation were all normal. Punch skin biopsy revealed perivascular mononuclear infiltrates with extravasated erythrocytes and few hemosiderin deposits in the upper dermis without apparent damage to vessel walls.

The clinical and laboratory findings in our patient suggested the diagnosis of chronic pigmented purpuric dermatosis (Schamberg`s purpura).

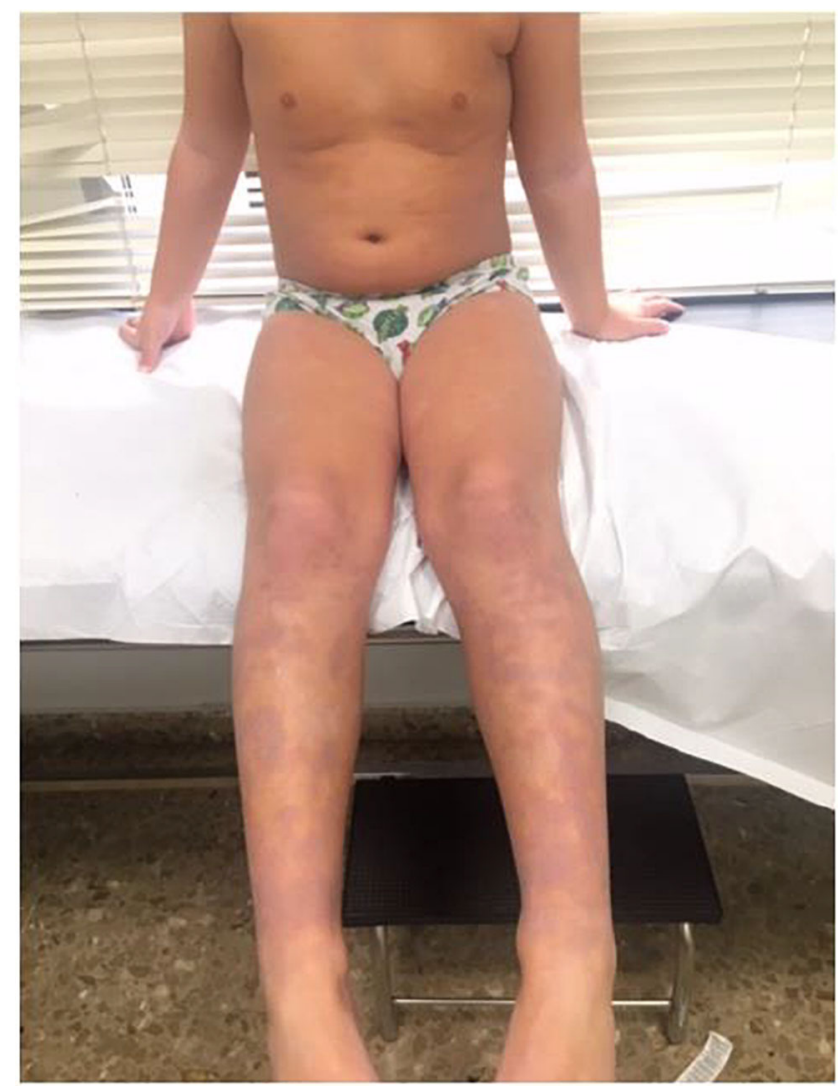

Conclustion: The presented case demonstrates a rare cause of purpura in infancy. PPD in children are idiopathic and chronic eruptions. The patient's history and routine laboratory tests allow to make a differential 
diagnosis with thrombocytopenia, coagulopathy, vasculitis, drugs or traumatic purpura. Recently, successful treatment with griseofulvin, pentoxifylline, and with PUVA have been reported in few patients. In view of the benign nature of the pigmented purpuras, the patients can benefit from watchful waiting.

\section{7}

\section{Primary Care}

Terra Firma-Forme Dermatosis: It's Easy When You Know It Nuno Vilas Boas ${ }^{1}$, Jacinta Mendes ${ }^{I}$, Anabela Bicho Nunes ${ }^{I}$, Rute Machado ${ }^{I}$

${ }^{1}$ Paediatrics, Centro Hospitalar do Oeste - Caldas da Rainha, Portugal

Background: Terra firma-forme dermatosis, also called "Duncan's dirty dermatosis" is an acquired, benign and asymptomatic condition characterized by a disorder of keratinization. It's usually presented as a brownishblack, dirt-like plaques, in patients with normal hygiene. The lesions often affect the neck, ankles, trunk and face. This condition is most frequently seen in children and adolescents but doesn't have a predilection for any gender and is also unrelated to any known genetic condition or familial inheritance. Objective: Raise awareness amongst pediatricians for an early diagnosis of this condition, as it could save children from unnecessary investigations and treatments.

Case Presentation: A 16-year-old adolescent girl with a personal history of type I tuberous sclerosis and epilepsy presented at her regular pediatric consultation for her annual follow-up with an asymptomatic brownish dirt-like lesion at the anterior region of the thorax with some weeks of evolution. Both the patient and her mother thought the lesion was dirt and tried to clean it by rubbing it with water and soap without success. There was no history of an inflammatory dermatosis in the area or other dermatologic conditions before this. There was also no history of bad hygiene habits. At the consultation, rubbing the lesion with an $70 \%$ isopropyl alcohol-soaked gauze pad and applying pressure resulted in complete disappearance of the lesion, therefore, the diagnosis of terra firma-forme dermatosis was confirmed. After this consultation the adolescent girl manifested frequent recurrences of the lesions, mostly in both her arms, which always disappear after she applies an alcohol-like solution.

Conclusion: Both diagnosis and treatment of Terra firma-forme dermatosis consists in the application of $70 \%$ isopropyl alcohol. It's important to recognize this condition and not confuse this benign and easily treatable condition with other dermatologic disorders in order to avoid unnecessary exams and medications.

\section{8}

\section{Primary Care}

Bi-acromial Dimples: A sign of beauty?

Nuno Vilas Boas ${ }^{I}$, Jacinta Mendes ${ }^{I}$, Rute Machado ${ }^{I}$, Anabela Bicho Nunes

${ }^{1}$ Paediatrics, Centro Hospitalar do Oeste - Caldas da Rainha, Portugal

Background: Bi-acromial dimples, also known as "supraspinous fossae" or shoulder dimples are an anatomic peculiarity that should be considered an anatomic variation. They seem to have an autosomal dominant inheritance pattern. These dimples are thought to result from the entrapment of skin between the shoulder bones and the wall of the uterus. They can also appear in other parts of the body and coexist in the same individual. These dimples are found infrequently, and are solitary findings in the majority of the cases. However, bi-acromial dimples have been reported as part of malformation syndromes such as $18 \mathrm{q}$ deletion syndrome, and skeletal dysplasias such as Apert's syndrome.

Objective: Raise awareness amongst pediatricians for anatomic variations in the skin and its benign nature.
Case Presentation: A 2-year-old boy with no previous medical history presented at his regular pediatric consultation for his annual follow-up. The parents have noticed he had a dimple on both his shoulders which made them worried that was pathologic. There was no family history of these kind of dimples that was known to them. On physical examination deep dimples were present in the acromial region bilaterally and symmetrically, $10 \mathrm{~mm}$ in diameter, about 5 to $10 \mathrm{~mm}$ deep and without dermal or hypodermic atrophy. No other somatic abnormalities were found. There was also no restriction in range of movement of the shoulder joints.

Conclusion: Bi-acromial dimples may be considered an anatomic curiosity. However, it is important that pediatricians are aware of this entity in order to reassure parents of its benign nature. It is of special interest to look for this abnormality in several generations, but familial observations are not necessarily associated with a genetic syndrome. In this case biacromial dimples were an isolated anatomical peculiarity, with no known familial occurrences. Skin dimples were discovered incidentally and are most probably underdiagnosed.

\section{5}

\section{Primary Care}

A Need to Provide Safety and Quality in The Health System Elpis Chochliourou ${ }^{1}$, Elena Volakli ${ }^{1}$, Asimina Violaki ${ }^{1}$, Anna Kalaitzi ${ }^{1}$, Eleni Karakeke ${ }^{I}$, Vasiliki Papadopoulou ${ }^{I}$, Maria Sdougkal

${ }^{1}$ Pediatric Intensive Care Unit, Hippokration General Hospital of Thessaloniki, Greece

Background: The non-qualitative provision of health services is a certainty in Greece and concerning the difficult economic situation in the country, the problems are constantly increasing. Despite advances in science, the successive meetings of responsible in Health Care Services, specialization in the management of Health Care, it is clear the inability of a concerted effort to improve quality. At international level, a comprehensive reorganization effort is being undertaken to identify weaknesses, find appropriate solutions and establish quality assessment systems.

Objectives: To highlight the need to create a National Agency for Quality in Health and to develop quality assessment systems. We identify shortcomings in terms of both quality and security and present possible proposals at the level of structures, processes and outcomes for the efficient management of the above weaknesses.

Methods: A bibliographic research was made using papers and online literature to identify and review relevant articles.

Results: Electronic prescribing, automation of regular outpatient appointments, adopting international treatment protocols on daily and serious medical issues, the "thirst" of younger doctors to further specialize in their subject of interest, the plethora of research work at university departments and the attempt to gradually renew more and more departments in Public Institutions, show change.

Conclusions: With the proper management, specialized administration in the field of Health Units, rehabilitation and prioritization, a new road opens up with the ultimate goal of the sustainable development of Quality and Safety Systems in the field of Health.

970

\section{Primary Care}

Dental Home for Children with Special Needs in Qatar: Analyzing Their Dental Health Issues and Challenges faced by Families to Acquire Dental Care

Dure Yasrab ${ }^{l}$, Mohamed Hendaus Rahal ${ }^{I}$, Sara Hamad ${ }^{l}$, Deena Assem ${ }^{I}$, Lina Salah $^{1}$, Aya Al Naubani ${ }^{2}$, Ahmed Alhammadi ${ }^{I}$

${ }^{1}$ Pediatrics, Hamad Medical Corporation, Qatar ${ }^{2}$ 
Methods: A cross-sectional study using a questionnaire was conducted at Hamad Medical Corporation in Qatar.

Results: 302 questionnaires were completed by caregivers of children with special needs (response rate 92\%). 70\% of children had developmental delay, $20 \%$ had musculoskeletal disabilities, remaining had respiratory compromise(on non- invasive ventilation), learning,visual and hearing disabilities. $92 \%$ of the children had multiple comorbidities Regarding their children's dental problems,45\% stated multiple problems(pain, cavities, halitosis, broken teeth, and teeth discoloration), whereas $10 \%$ complained only of cavities. Upon inquiring about oral hygiene habits care of their children, around $70 \%$ reported daily use of toothbrush, toothpaste and mouthwash. However $8 \%$ of them performed no oral hygiene routine.

$70 \%$ of children didn't have a routine dentist visit in last year due to reasons such as long appointment time(25\%), difficulty in child's mobility(17\%), perception that dental care is expensive(9\%), lack of dentist's experience in dealing with children with special needs $(5 \%)$, and combination of the above factors $(25 \%)$. When asked of what factors will encourage caregivers to acquire a dental home for their children, $63 \%$ mentioned quick appointments, followed by dentists specialized in children with special needs $(51 \%)$, child friendly atmosphere(21\%), low $\operatorname{cost}(26.6 \%)$, close to home $(20 \%)$, and others $(6 \%)$. Majority of parents $(75 \%)$ believed that the primary pediatrician of their child should initiate the dental home process.

Conclusion: Children with special needs lack proper oral health care, and it could be attributed to the lack of a dental home. The primary pediatrician's role is crucial in initiating the process of acquiring a dental home for this special population. We will share our results with the ministry of public health of Qatar with a goal to provide awareness among primary care physicians to consider oral health as an important aspect of the overall wellbeing of such children and to create an easy referral system to obtain a dental home.

\section{9}

\section{Primary Care}

The Association of Atopic Dermatitis and Food Allergies among Infants in our Primary Care Setting

Helena Zivic ${ }^{1}$

${ }^{T}$ Pediatrics, Health facility Zagreb Center, Croatia

Background: Food allergens are most relevant in infants with moderate to severe atopic dermatitis (AD) and when there is a history of reactions to food. Skin testing and specific IgE levels are useful diagnostic tools but predict probability, not necessarily the severity of allergic reactions. Children with early-onset severe $\mathrm{AD}$ have the greatest risk for positive $\mathrm{IgE}$ testing to foods.

Objective: Aim was to analyze the association of $\mathrm{AD}$ and food allergies among infants with $\mathrm{AD}$ in our primary care setting.

Methods: Retrospective analysis of our patients 1-12 month of age with AD and food allergy, in a period of 3 years (2015-2018). Descriptive statistical analysis was used.

Results: Overall 70 children age 1-12 months had AD. 43 boys (61.4\%) and 27 girls (38.6\%). Median age for AD was 5 months. Associated food allergy was found in 23 children $(32.8 \%)$, of which $19(82.6 \%)$ had moderate to severe $\mathrm{AD}$ and $4(17.4 \%)$ had mild AD. Most patients $17(73.9 \%)$ had strong positive skin testing for egg and peanut, with elevated specific IgE levels (mostly class 4$), 4$ patients $(17.4 \%)$ had positive skin testing for egg, cow's milk and soy, with specific IgE levels mostly class 3 , and 2 patients $(8.7 \%)$ had positive skin testing for wheat and soy, with specific IgE levels mostly class 3 . All children were referred to pediatric dermatologist, elimination diet was introduced only to infants with confirmed food allergy. Most infants 20 (86.9\%) with $\mathrm{AD}$ and food allergy were breastfed.

Conclusion: Among foods that have been implicated in refractory, moderate to severe $\mathrm{AD}$, egg and peanut are the most common triggers, only $5 \%$ of all our infants with AD had a clinically relevant milk allergy. Testing should be considered when infants have severe AD refractory to standard treatments, and a history of a reaction to food.

\section{3}

\section{Respiratory}

Pneumonia Complicated with Recurrent Pneumothorax: A Case Report

Alexandra Andrade ${ }^{l}$, Carolina Gonçalves ${ }^{l}$, Teresa Jacinto $^{l}$, Filomeno Paulo $^{2}$, Conceição Freitas ${ }^{l}$, Cristina Freitas ${ }^{1}$

${ }^{I}$ Serviço de Pediatria, Hospital Central do Funchal, Portugal

${ }^{2}$ Serviço de Cirurgia Pediátrica, Hospital Central do Funchal, Portugal

Background: Pneumonia is an infection of the lung parenchyma. It is occasionally associated with pleural effusion and empyema, but rarely is complicated with pneumothorax. We present a case of a previously healthy child with a pneumonia complicated with pneumothorax.

Objective: To report a rare complication of pneumonia and our approach.

Case Report: A previously healthy 2-year-old child was admitted due to a four day history of cough and high fever. On the day of admission he presented with difficulty breathing and pleuritic pain. He had completed pneumococcal vaccination.

On admission, the child was afebrile, with no signs of respiratory distress, oxygen saturation of $95 \%$ on room air. Pulmonary auscultation revealed decreased breath sounds on the right side.

Chest radiograph presented with a condensation of the right medial lobe. He was admitted with the diagnosis of pneumonia and started treatment with ampicillin. After eighteen hours his clinical state deteriorated with worsening tachypnea and desaturation. Chest radiograph (Figure 1) revealed right pneumothorax and a chest drain was placed. He developed subcutaneous emphysema, which extended to the neck, thorax and abdomen. There was a good clinical evolution until the $4^{\text {th }}$ day of hospitalization when he presented with respiratory distress, worsening of the emphysema and increased oxygen needs. A second right hypertensive pneumothorax was diagnosed (Figure 2) and repositioning of the chest drain was accomplished. At this time we changed the antibiotics to vancomycin and ceftriaxone. A thoracic CT revealed evidence of necrotizing pneumonia. The patient had an uneventful recovery, being discharged after fifteen days of iv antibiotics. Blood cultures were negative and further investigations showed no evidence of other underlying pulmonary disease.

Conclusions: Pneumonia is a frequent diagnosis in the ED. This case demonstrates a rare and potentially life-threatening complication if not approached quickly and efficiently. It can happen on healthy children, but further investigation should be considered. 

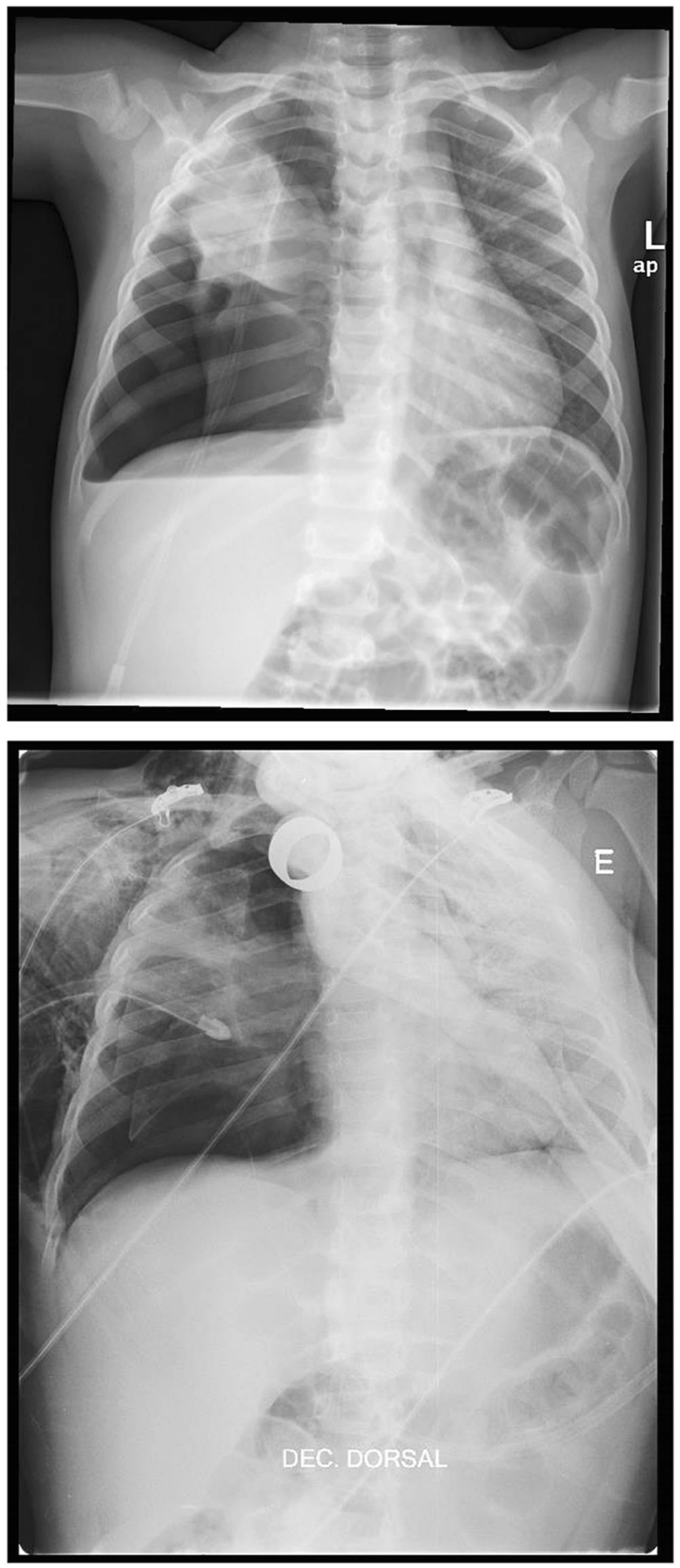

425

Respiratory

Prevalence of Indoor Air Pollution by Cooking Fuel and its Association with Respiratory Infections in Children in Peru: An Analysis of a Population Based, Cross-Sectional Survey Antonio Cunha ${ }^{1}$, César Gutiérrez ${ }^{2}$, Edith Alarcón-Matutti ${ }^{2}$, Jorge Alarcón ${ }^{2}$, Manuel Sobrino-Toro ${ }^{3}$
${ }^{1}$ Pediatrics, Universidade Federal do Rio de Janeiro (UFRJ), Brazil ${ }^{2}$ Epidemiology, Universidad Nacional Mayor San Marcos, Peru ${ }^{3}$ Farmacología, Pediatría y Radiología, Universidad de Sevilla, Spain

Background: Household air pollution accounts for about 3 $\cdot 5-4$ million deaths every year. Interventions should target priority geographic areas where the problem is more prevalent.

Objective: To estimate the overall prevalence, the prevalence by region and area of residence, of indoor air pollution by cooking fuel in children in Peru; and to study the association of indoor air pollution by cooking fuel with of acute respiratory infection (ARI) in children.

Methodology: We used data from the 2013-Demographic and Health Survey, with nationwide representativeness. We included children under 5 years of age. Air pollution was present if the child lived in a house that used coal, charcoal, wood, straw, bush, grass, crops or livestock dung as a fuel for cooking. We used logistic regression models to study the independent association of air pollution by cooking fuel and ARI, controlling for potential confounders.

Results: We studied 8,616 children; overall prevalence of indoor air pollution was $37.4 \%$ (95\%CI: $36.4-38.4 \%$ ). Among those exposed to cooking fuel, in $24.4 \%$ (95\% CI: $23.5-25.3 \%$ ) of the houses there were no chimney. The ARI prevalence was $13.0 \%$ (95\%CI:12.3-13.7\%). ARI was associated to indoor air pollution in urban and rural areas, and a dose response pattern was observed (p0.05). By region the association remained but was statistically significant only in the Forest region.

Conclusion: Prevalence of indoor air pollution caused by cooking fuel in children was high in Peru and ARI was independently associated to it. Interventions to reduce this source of pollution need to be implemented in Peru, and the Forest should be a priority region for any implementation.

\section{Respiratory}

Just another Case of Pneumonia?

Sara Almeida ${ }^{l}$, Joana Dias $^{1}$, Miguel Labrusco ${ }^{1}$, Ana Sofia Nicolau ${ }^{l}$, Joana Rios ${ }^{l}$, Paulo Oom ${ }^{I}$

${ }^{1}$ Pediatric Department, Hospital Beatriz Ângelo, Portugal

Background: Pneumonia is a common infection but it can be the first presentation of an underlying disease, such as tumors.

Case Summary Presentation: An 11 year-old boy, with atopic dermatitis, allergic rhinitis and asthma, presents to the emergency department with a 2 day history of thoracic pain accompanied with shortness of breath. Physical exam reveals fever and normal oxyhemoglobin saturation $(95 \%-100 \%)$ on room air. On auscultation he has absent sounds in the right hemithorax but normal breath sounds on the left. A chest radiograph reveals a round area of consolidative opacity in the right hemithorax and a pleural effusion, confirmed by chest ultrasonography, with $14 \mathrm{~mm}$. Blood tests show a leucocyte count of $16.600 / \mathrm{mL}$ with $78.3 \%$ of neutrophils, and an elevated C-reactive protein level of 17.59 $\mathrm{mg} / \mathrm{dL}$. He is admitted to the hospital and starts intravenous ampicillin. At day 2 after admission, the chest ultrasonography reveals a pleural effusion of $21.6 \mathrm{~mm}$ at the right hemithorax and images compatible with an empyema. He is submitted to a Video Assisted Thoracic Surgery (VATS) and drains about $100 \mathrm{~mL}$ of non-pure pleural fluid. The antibiotic therapy is changed to intravenous ceftriaxone. All cultural exams were negative. After 8 days, clinically improved, he is discharged and continues ceftriaxone until completing 14 days. One month later he repeats the chest radiograph which continues to reveal the same round area of opacity. The CT scan and the MRI show a round, encapsulated and cystic mass located in the anterior mediastinum compatible with a teratoma. He was submitted to excision of the tumor and pathologic evaluation revealed a mature teratoma. Subsequently, he is clinically improved and asymptomatic. 
Conclusion: This case demonstrates that complicated pneumonia should always be followed up until complete resolution of symptoms and radiologic findings, and that a round consolidation should always be suspicious.

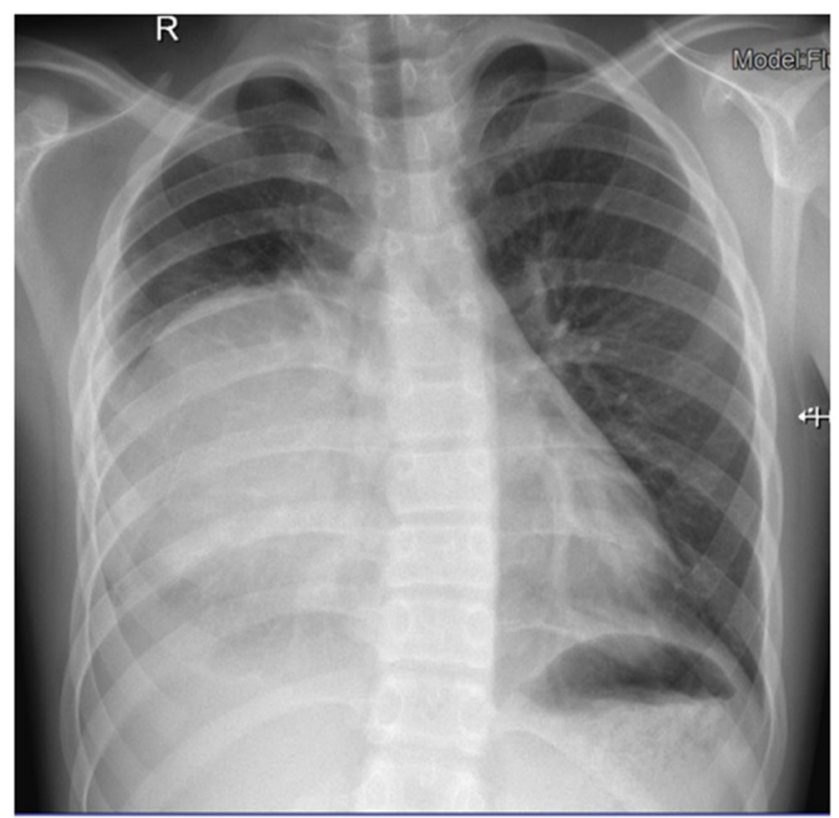

849

Respiratory

Acute Bronchiolitis - What Changed in 10 Years?

${ }_{\text {Joana Figueirinha }}{ }^{2}$, Diana Bordalo ${ }^{1}$, Filipa Pinto ${ }^{l}$, Ana Sofia Gomes ${ }^{1}$, Fernanda Carvalho

${ }^{1}$ Pediatrics, Centro Hospitalar do Médio Ave, Portugal

Background: Acute Bronchiolitis (AB) is a fairly common cause of hospitalization in children. In 2012, Portuguese national guidelines regarding the diagnosis and treatment of $\mathrm{AB}$ were issued, aiming to prevent doctors from requesting unnecessary diagnostic tests or using unhelpful drugs. The aim of this study is to determine if the guidelines were useful in changing the medical approach to children hospitalized with $\mathrm{AB}$, in a second-level hospital.

Methods: Inclusion criteria: every child with a first episode of AB below the age of 23 months that was hospitalized in Centro Hospitalar do Médio Ave between October 2005 and March 2006 (Group A) and between October 2015 and March 2016 (Group B). Chi-square tests were performed, with a confidence interval of $95 \%$.

Results: A total of 84 children were included, 45 in group A ( $51.1 \%$ male) and 39 in group B (56.4\% male). Median age was 4 months and 2 months, respectively. $100 \%$ of the children in group A got a chest X-ray and $93.3 \%$ got their blood tested, whereas in group B the frequency of chest $\mathrm{X}$-rays and blood tests was $74.4 \%$ in both categories. Comparing both groups, we have a significant reduction regarding the use of both tests ( $p$

Conclusion: After the guidelines were published, there was a significant shift both in the use of diagnostic tests and therapeutic measures, however, there's still room for improvement.

924

\section{Respiratory}

The Investigation of Mediastinal Opacity in A 10 Years Old Boy Presenting with Asthma Exacerbation

Hani Gowai ${ }^{1}$, Kelechi Ugonnal

${ }^{T}$ Paediatric Respiratory, Sheffield Children's Hospital, UK
Case: A 10 years old boy, known to have asthma, house dust-mite allergy and unilateral dysplastic kidney, presented to $\mathrm{A} \& \mathrm{E}$ with an exacerbation. His chest radiograph showed bulky mediastinum so was referred to respiratory team. He was treated for atypical infection and echocardiography and abdominal USS were done and were unremarkable. CT chest showed mediastinal lymph node enlargement. The case was discussed with oncology/ radiology teams and agreed on treating as atypical infection. Serial chest radiographs revealed gradual improvement, then was lost for follow up for 10 months. When he was seen in clinic, a chest X-ray showed that the hilar prominence is still there, but was asymptomatic, and later on, in 2 months the changes were persistent, so he went on to have a thoracic MRI that revealed a mediastinal mass likely lymphadenopathy with a splenic lesion and an abnormal marrow signal along the spine. Splenic and bone marrow biopsies both showed no malignant cells. A PET scan revealed activity consistent with lymphoma that was later confirmed on axillary lymph node biopsy and the boy was subsequently started on chemotherapy.
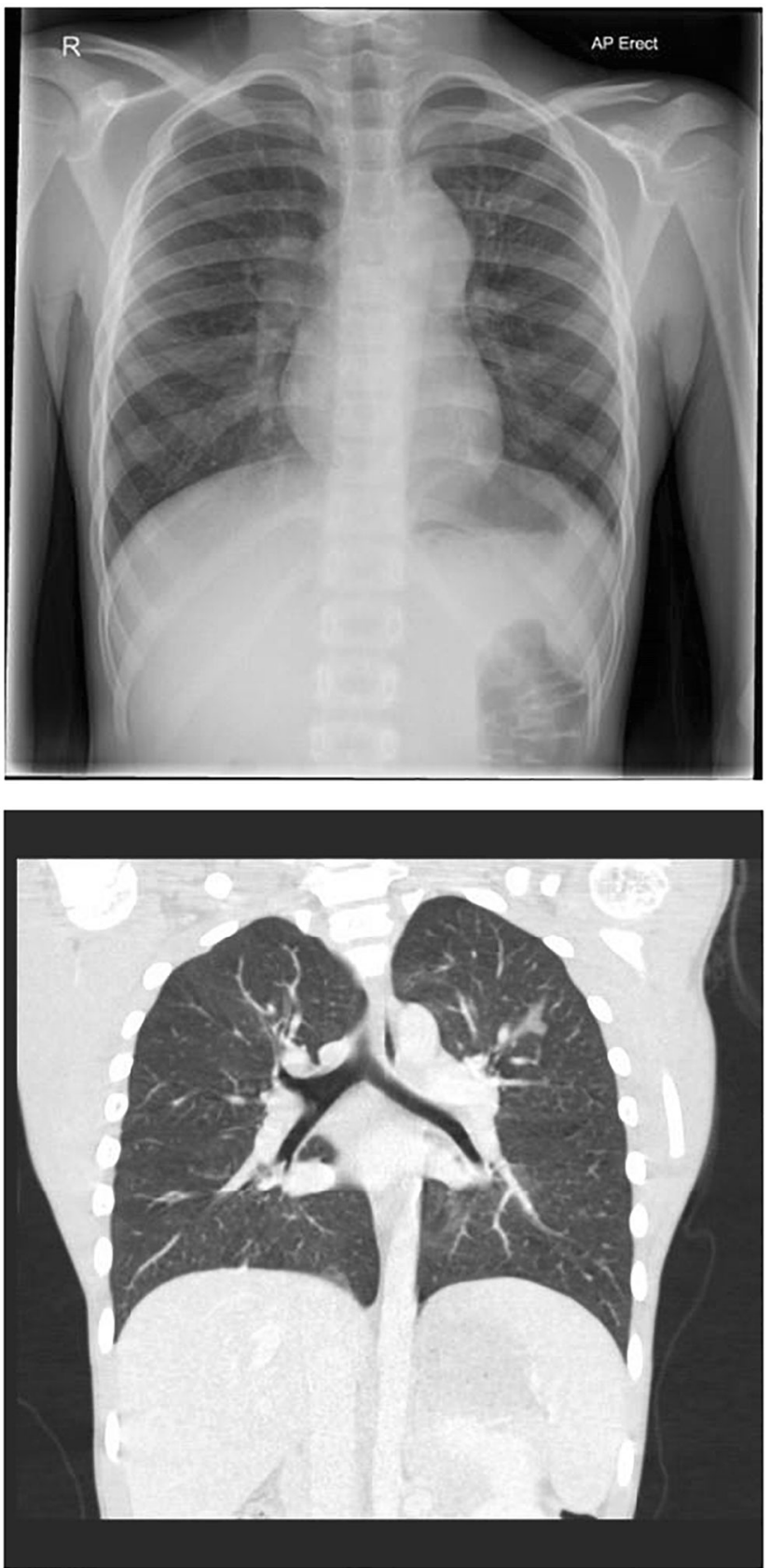
Conclusions and learning points: This case shows that it can be difficult to interpret mediastinal opacities on plain radiographs and in this case the CT scan has not changed the management significantly. PET scan helped guide further investigations. Biopsy of an appropriate site is the gold standard (as two biopsies from spleen and bone marrow were negative), and in this particular case, would have saved much effort and time.

\section{1}

Respiratory

Mediastinal Mass Presenting as Suspected Dysfunctional Breathing in A 14 Years Old Girl

Hani Gowai ${ }^{1}$, Kelechi Ugonna ${ }^{1}$

${ }^{T}$ Paediatric Respiratory, Sheffield Children's Hospital, UK

A 14 years old girl, known to have autism and eczema, was referred to general paediatrics by her GP with history of right lower chest pain for 2 years. Pain has worsened recently, became more central, was aggravated by exercise and with no relieving factors. Her clinical examination was normal. Chest X-ray was unremarkable, the pain was thought to be of musculoskeletal origin. A trial of salbutamol inhaler and antacid did not help her symptoms and she was referred to physiotherapy. She was assessed by the physiotherapist who referred her to the respiratory clinic to rule out exercise induced laryngeal obstruction. She was seen in clinic 2 months later, where the chest radiograph was repeated and revealed mediastinal enlargement. Her echocardiography and spirometry were normal. She went on to have a CT chest that confirmed mediastinal lymphadenopathy. A thoracoscopic biopsy confirmed the diagnosis of Hodgkin's lymphoma. She completed the treatment protocol and subsequent work up showed no residual disease; she is currently asymptomatic under regular follow up.

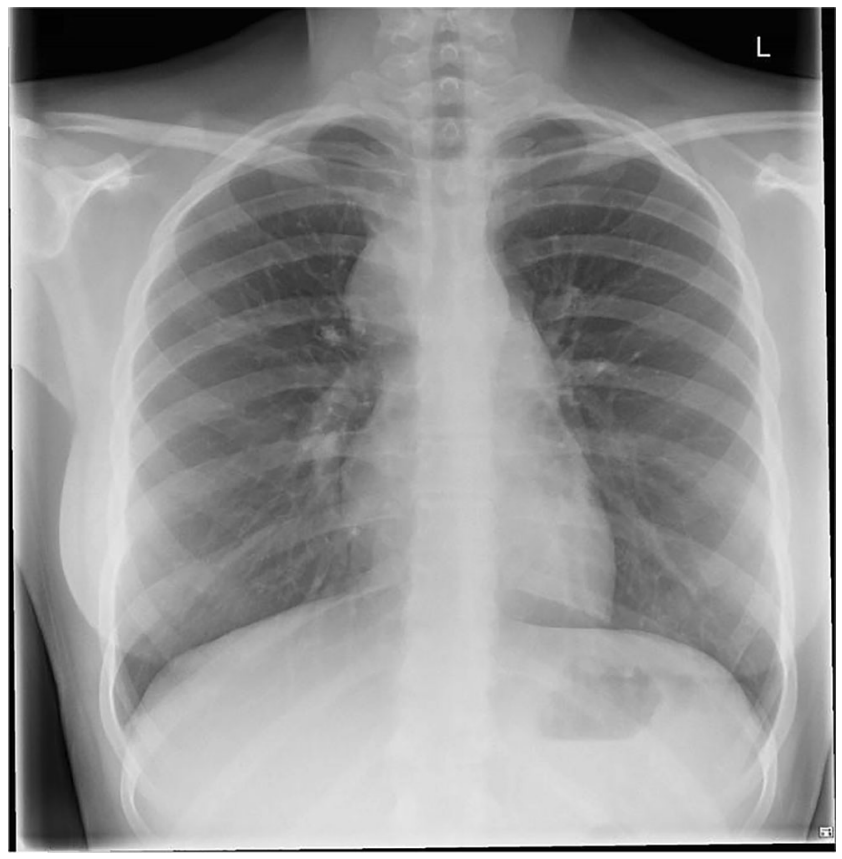

Conclusions and learning points This case presented with shortness of breath on exertion which was thought to be due to dysfunctional breathing, which is an unusual presentation of a mediastinal mass. A normal radiograph at the first presentation should not cause hesitation in repeating it especially with persistent symptoms. Tissue biopsy is the gold standard investigation in such cases.
337

\section{Respiratory}

Bronchial Obstruction Caused by Pulmonary Tuberculosis in $\mathbf{3}$ Months Baby

Oana Maria Guta ${ }^{1}$, Laura Carasava ${ }^{1,2}$

${ }^{T}$ Department of Pediatrics, "Grigore Alexandrescu” Emergency Hospital for Children, Romania

${ }^{2}$ Department of Pediatrics, "Carol Davila" University of Medicine and Pharmacy, Romania

Introduction: Tuberculosis remains an important cause of morbidity and mortality in children worldwide. Diagnosing the pulmonary tuberculosis can be challenging in infants and young children since clinical and radiological signs can be non-specific.

Purpose: The main purpose of this case report is to highlight the challenge of diagnosing tuberculosis in young children.

Materials and Methods: We present the case of a 3 months baby, admitted in Pediatrics Department of "Grigore Alexandrescu" Hospital in October 2018 for rhinorrhea and fever.

Case Description: A previously healthy 3-months-old male was brought to the emergency department due to rhinorrhea in the previous 10 days and fever in the admission day. At the clinical examination, he had a good general appearance, rhinorrhea, spastic cough and symmetric breathing sounds in the pulmonary auscultation. The blood analysis revealed elevated platelets count and C-reactive protein with anemia. The chest radiography showed a density in the left upper lobe. Given this workup, the patient was diagnosed with Pneumonia, admitted and treated with Ceftriaxone. Fever persisted during the first 10 days of hospital stay. At the insistent resumption of the anamnesis, we found out that the father was hospitalized about 2 years ago for Tuberculous Pleurisy. A bronchoscopy was performed, revealing stricture in the area of left upper bronchus. The lung computer tomography confirmed the upper left atelectasis and several hilar and mediastinal adenopathies that amputated the left main bronchus. The baby and his mother started anti-tuberculosis treatment with good clinical evolution. Conclusion: Primary pulmonary tuberculosis in childhood is a difficult diagnose to make. Radiographic features, even if it may compress the trachea or main bronchus causing external stenosis, can be misleading. Rethinking the case and inclusion of the anamnestic data can lead to early diagnosis and appropriate treatment, therefore avoiding disastrous consequences.

\section{3}

\section{Respiratory}

Relationship of Indoor Aerosol Pollution and Morbidity of Children in Primary Schools of Vilnius

Nina Prokopciuk ${ }^{1}$, Ulrich Franck ${ }^{2}$, Arunas Valiulis ${ }^{3}$, Izabele Juskiene ${ }^{3}$, Vadimas Dudoitis ${ }^{1}$, Nikolaj Tarasiuk ${ }^{1}$, Vidmantas Ulevicius ${ }^{1}$

${ }^{I}$ Center for Physical Sciences and Technology, SRI, Lithuania

${ }^{2}$ Helmholtz Centre for Environmental Research, UFZ, Germany

${ }^{3}$ Institute of Clinical Medicine, M.K.Ciurlionio St. 21/27,, Vilnius University Faculty of Medicine, Lithuania

Objective. Identify relationship of indoor aerosol pollution and morbidity in primary school children.

Materials and methods. Indoor aerosol pollution was measured in 7 schools in Vilnius (Lithuania) in 2017-2018. Retrospective analysis of 6-11 years old patients health history (number of upper and lower respiratory infections) from records of outpatient clinics was performed. 953 pupils from primary schools were involved. Total aerosol particle number concentration (PNC) in the size range of $0.01-1.00 \mu \mathrm{m}$ was estimated using a Condensation Particle Counter (CPC, TSI model 3007). Particle number and mass concentrations in the size range of $0.3-10 \mu \mathrm{m}$ were measured using an Optical Particle Seizer (OPS, TSI model 3330). 
Results. The median values of PNC (CPC) and (OPS) in schools varied in the range of $1965-10194 \mathrm{part} / \mathrm{cm}^{3}$ and $16-210$ part $/ \mathrm{cm}^{3}$, respectively. The maximum values of PNC (CPC) and (OPS) in schools varied in the range of $12379-30999$ part $/ \mathrm{cm}^{3}$ and $215-1334$ part $/ \mathrm{cm}^{3}$, respectively. Morbidity positive correlation with the indoor aerosol pollution was obtained with the PNC (OPS) in the size range of $0.3-1.00 \mu \mathrm{m}(95 \%)$ ). Correlation coefficients determined for the seasonal morbidity and PNC data were equal to $0.45,0.51$ and 0.6 for autumn, winter and spring, respectively. Seasonal dependence of the morbidity on the PNC can be expressed by linear function with a constant term responsible only for morbidity due to the outdoor exposure and a term proportional to $\mathrm{PNC}$ in the size range of $0.3-10 \mu \mathrm{m}$. A value of the proportionality coefficient was estimated to be equal to 0.02 , 0.12 and 0.03 for autumn, winter and spring, respectively.

Conclusion. Relationship between indoor aerosol pollution in schools and seasonal morbidity of primary school children was found. This study shall be continued involving a larger number of schools.

\section{5}

\section{Respiratory}

Improving Prescription Process for Cystic Fibrosis Patients Attending a Tertiary Referral Centre

Evodia Ludusan $^{I}$, Aoibhinn Walsh ${ }^{I}$, Emma O'Grady $^{I}$, Mary Morgan ${ }^{I}$, $\overline{S h a r o n}$ Deignan $^{1}$, Joan Maye ${ }^{1}$, Michael Williamson ${ }^{I}$, Fiona Healy ${ }^{I}$

${ }^{1}$ Respiratory Department, Children's University Hospital Temple Street, Dublin, Ireland

Background: There was no standardised process for providing repeat prescriptions for patients with cystic fibrosis (CF) attending our department. Risks of poor patient compliance with medications and medication error were identified.

Aim: Using the SMART acronym (Specific, Measurable, Applicable, Realistic, Timely) the aim was to increase the number of repeat prescriptions written after review appointment, from a baseline of $0 \%$ to $100 \%$, for patients with CF.

Methods: Current practice in our department is that parents contact the $\mathrm{CF}$ team as needed for repeat prescriptions. Using the fishbone diagram and process map, we identified variation within the system.

After meeting with the CF multidisciplinary team (consultants, registrars, nurses and pharmacist) we identified change ideas. The first Plan/Do/ Study/Act (PDSA) cycle was to write classic repeat prescriptions during each clinic visit. Then we applied PDSA for both classic and electronic high-tech prescriptions. A final cycle then included preparing and processing all prescriptions after clinic.

Results: There are 100 patients with $\mathrm{CF}$ attending our tertiary referral CF centre. Number of repeat prescriptions written at the end of the clinic (primary outcome measure) increased to $100 \%$ from $0 \%$. There are $4-$ 12 patients per clinic and every patient requires prescription medication. All prescriptions are checked by the CF pharmacist.

Previously we received approximately 5 parental requests per day, each taking typically 20 minutes to process (balancing measures). By improving the primary outcome, we improved balancing measures and reduced the time spent daily on repeat prescriptions.

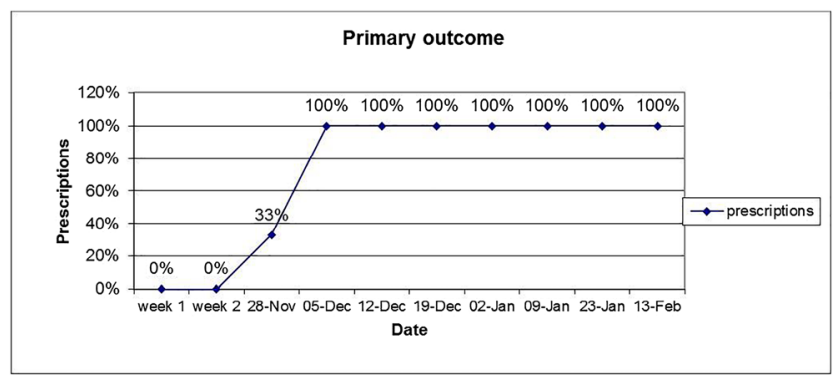

Conclusion: By improving the prescribing process, we minimised the waste within the department and for the patients. We transferred the responsibility for the repeat prescriptions from the patients to medical team, we improved medication safety and reduced patient risk.

937

\section{Respiratory}

Long QT Syndrome In Neonate - The Management Challenges In Case Of Bronchiolitis

Cristiana Maximiano $^{1}$, Ivo Neves ${ }^{1}$, Graça Sousa ${ }^{2}$, Albina Silva ${ }^{3}$, Helena Silva $^{1}$

${ }^{1}$ Pediatric Department, Hospital of Braga, Portugal

${ }^{2}$ Pediatric Cardiology Department, Hospital of Braga, Portugal

${ }^{3}$ Intensive Neonatal Care Unit, Hospital of Braga, Portugal

Background: Long QT syndrome (LQTS) is a disorder of ventricular myocardial repolarization characterized by a prolonged QT interval on electrocardiogram (ECG) that can lead to ventricular arrythmias and an increased risk of sudden cardiac death. LQTS may be congenital or acquired. The gold standard therapy is b-blockers, usually propranolol or nadolol. In patients with LQTS, $b_{2}$ agonist therapy is associated with an increased risk of cardiac events.

Case presentation summary: An 1-month old boy, with personal history of congenital LQTS, family history of congenital LQTS type 2 and pending genetic study, with prescribed propranolol but failure to adherence (his mother decided no to follow the prescription), was admitted to the pediatric department due to acute bronchiolitis. On his second day of hospitalization there was a clinical worsening, with respiratory effort, prostration and feeding difficulties. He presented with type 1 respiratory failure and signs of dehydration. Promptly initiate oxygen therapy but he maintained signs of respiratory distress and hypoxemia. Due LQTS, it was not possible to prescribe nebulized salbutamol or adrenaline, so it was decided transfer to the Intensive Neonatal Unit Care to high flow nasal cannula (HFNC) therapy. There was favourable clinical outcome: he required HFNC during four days, maximum flow of $7 \mathrm{~L} / \mathrm{min}$ and humidified $\mathrm{O}_{2}$ concentration of $30 \%$, general condition improved and appetite increased. $\mathrm{He}$ remained hemodynamically stable, without any cardiac event. There was a positive rapid test for respiratory syncytial virus.

Learning Points/Conclusion: Bronchiolitis is one of the most common infection during the first two years of life. The general management include, in mild to severe forms, bronchodilators as salbutamol or adrenaline. In this case, due to LQTS, those drugs are not recommended and there are very limited therapeutic options.

In resume, we would like to highlight the challenging in the management of a mild bronchiolitis in case of infants with LQTS.

992

Respiratory

Crunching Heartbeat

Jacinta Mendes $^{1}$, Nuno Vilas Boas ${ }^{1}$, Catarina Gomes $^{I}$, Sara Santos ${ }^{1}$

${ }^{T}$ Centro Hospitalar do Oeste - Caldas da Rainha, Serviço de Pediatria de Caldas da Rainha, Portugal

Pneumomediastinum is an uncommon entity in pediatric practice, with a peak of incidence during adolescence, particularly affecting tall, thin males. It is defined as free air within the mediastinum and its etiology is multifactorial, spontaneous or idiopathic. The most common symptom of spontaneous pneumomediastinum (SPM) is chest pain; however, most patients are asymptomatic. The pain is characteristically pleuritic, retrosternal and intensified by movement, breathing, and position 
changes. Other common symptoms are dyspnea, cough, neck pain, odynophagia and dysphagia.

We report a case of a previously healthy, 15-year-old male adolescent, high performance athlete, who was admitted to the hospital with three days evolution chest pain, which began a few hours after an intensive sports practice. The pain irradiated to the neck and was intensified by deep breathing. No shortness of breath, weakness, heart palpitations or other heart symptoms were associated. There was no history of respiratory infections, invasive procedures or trauma in the previous days.

On physical examination he had palpable subcutaneous neck emphysema and a crunching sound in cardiac auscultation, synchronous with the heartbeat. Chest and neck radiography confirmed ectopic air in the mediastinum and subcutaneous neck emphysema. The treatment consisted in rest and avoidance of maneuvers that could increase pulmonary pressure. Clinical symptoms resolved after 5 days and there were no radiologic findings at a 7 day follow up.

Hamman`s sign, Hammond 's sign or Hammond's crunch is a rare but pathognomonic sign characterized by a crunching, rasping sound, synchronous with the heartbeat, heard over the precordium. This sound is produced by the heart beating against air-filled tissues. SPM diagnosis can be made by physical examination and chest radiography or computed tomography may be helpful to document the diagnosis.

This case highlights the importance of physical examination in SPM diagnosis, especially in the presence of the pathognomonic Hamman's sign.

904

\section{Respiratory}

Pleuropneumonia In a 7 Year Old Boy Irena Nikolova $^{1,2}$, Marija Dimitrovska-Ivanova ${ }^{1,2}$, Luiza Doneva ${ }^{l}$ ${ }^{T}$ Department of Pediatrics, Clinical hospital Shtip, Macedonia ${ }^{2}$ Department of Pediatrics, University Goce Delcev Shtip, Macedonia

Background: Pleuropneumonia takes a prominent place in childhood morbidity. Very often in children it can be manifested with atypical clinical presentation.

Objective: To present atypical case of Pleuropneumonia in a seven year old boy.

Methods: Seven year old boy with acute onset headache, abdominal pain, vomiting and febrile. On physical examination conscious, febrile (39 C), pale, dehydrated, pharynx hyperemic, abdominal distension and pain on abdominal palpation. Blood test with leucocytosis $(\mathrm{Le}=31)$, elevated infalamotory markers $(\mathrm{CRP}=221 \mathrm{mg} / \mathrm{l})$, abdominal ultrasound with normal findings. Abdominal $\mathrm{x}$ ray - Mesohypogastric righ with distended intestinal viscera and with hydroaeric levels in formation. Due to suspicion of an acute abdomen, the child was transferred to the surgical department. After 12 hours of obsrevation still febrile, dehydrated, but with acute appearance of tachidispnea and chest pain on the left hemitorax. On examination febrile $40 \mathrm{C}$, tachidispnoic. Lung auscultation - vesicular breathing weakened to the left in the middle and basal parts. Chest $x$ ray Paracardiac basal left with larger zone of non-homogeneous shading in addition to inflammatory consolidation, right with peribronchitic infiltrates and sings of pleural effussion - Left dome of the diaphragm isn't followed and left frenicocostal sinus is shaded. Pleural ultrasound showed pleural effusion in left frenicocostal sinus.

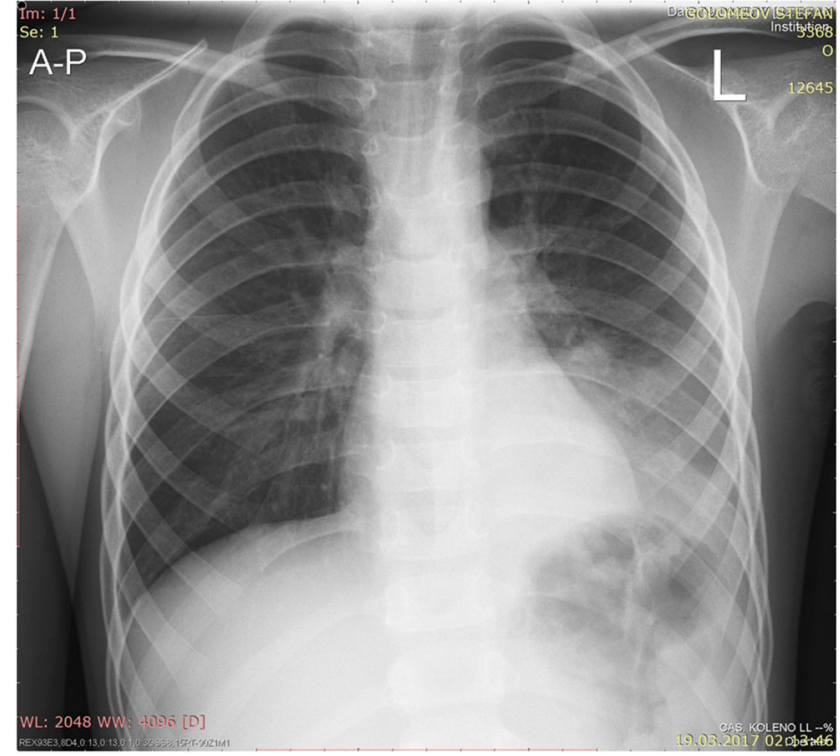

Chest X ray

Results: After appropriate antibiotic treatment, oxygen therapy, corticotherapy and inhalation therapy with a complete regression of the auscultatory finding. Control chest $\mathrm{x}$ ray and pleural ultrasound with complete regression of inflammatory consolidation and pleural effusion. Conclusion: Very often pleuropneumonia in children can be manifested with atypical clinical picture and it's a diagnostic and therapeutic problem because it's often not recognized and not thought of.

1012

Respiratory

Refining Clinical Guidelines for Bronchiolitis: Factors Contributing to the Use of Antibiotics. A Single Center Review in Cyprus Antigoni Papaioannou ${ }^{l}$, Spyridon Bekas ${ }^{l}$, Athina Lazaridou ${ }^{l}$, Elena Sotiriou'

${ }^{1}$ Medical School, University of Nicosia, Cyprus

${ }^{2}$ Pediatric, Limassol General Hospital, Cyprus

Background: Several evidence-based clinical guidelines for bronchiolitis discourage the use of antibiotics. It was identified that our center was using non-recommended evidence-based care for the treatment of bronchiolitis.

Objective: We aimed to gather and analyze data from our center on the trends of bronchiolitis management and on the factors that prompts physicians to prescribed antibiotics.

Methods: Retrospective observational study accomplished by reviewing charts of children less than 2 years with a discharge diagnosis of bronchiolitis from September 2018 to March 2019. The study included 76 infants and children between the age 0-24 months with discharge diagnosis of bronchiolitis. Patients were divided into two groups. Both groups were treated with inhaled bronchodilators. Group A did not receive any antibiotics and patients in Group B received antibiotics. Group A included 34 patients with mean age of 5.5 months. Group B included 42 patients, 8 patients were excluded due to documented diagnosis of bacterial pneumonia. Mean age of patients in group B 11.8 months.

Results: No statistical significant difference was observed in the length of hospital stay in two groups. (Group A, mean 3.18 Group B mean 3.68, p= 0.38 ). There was no significant difference between the two groups on the 
percentage of RSV/flu positive cases (Group A 14\% vs Group B 12\%) and percentage of CXR abnormalities (Group A $62.5 \%$ vs Group A $61.1 \%$ ). There was a statistically significant difference between CRP values in two Groups (Group A CRP mean 8.39, Group B CRP mean $19.63 \mathrm{p}=0.012$ ).

Conclusions: Antibiotics do not change the clinical course in bronchiolitis.We plan to implement new evidence-based clinical practice guidelines for our center for the upcoming RSV season.CPR levels need to be interpreted with caution when treating children with bronchiolitis and not to be used alone as a predictor for a concomitant bacterial infection.

\section{2}

\section{Respiratory}

Spontaneus Pneumomediastinum in a Pediatric Patient Cristina Rodrigues ${ }^{I}$, Ana Ribeiro ${ }^{1}$, Sofia Martins ${ }^{1}$, Susana Carvalho ${ }^{l}$, Teresa Pontes ${ }^{I}, A n a$ Antunes ${ }^{l}$

${ }^{I}$ Department of Pediatrics, Hospital de Braga, Portugal

Background: Spontaneous pneumomediastinum is the presence of air in the mediastinum in the absence of traumatic or iatrogenic causes. It is rare in pediatric age and several precipitating factors are described. It is usually benign and self-limited, and treatment is supportive.

Case Report: A 17-year-old male, with a previous right spontaneous pneumothorax submitted to thoracentesis 14 months before, immunizations updated, presented to our emergency department with sudden suprasternal thoracic pain throughout a handball training, which worsened with inspiration and cervical mobilization, in the last 24 hours. He also had an history of odynophagia and sporadic coughing and an upper respiratory infection two weeks ago. He denied fever, trauma, medications or drug use. He was hemodynamically stable, oxygen saturation $100 \%$. His weight was $55 \mathrm{~kg}$ (10th centile) and height $170 \mathrm{~cm}(15-50$ th centile). On physical examination he had suprasternal subcutaneous crepitations and tonsilar erythema. The chest radiograph revealed linear air leaks along the mediastinal borders, without pneumothorax, and the lateral radiograph showed paratracheal air and suprasternal subcutaneous emphysema. Pharingeal Group A Streptococcus test and C-reactive protein were negative and blood count was normal. IgM antibody Mycoplasma pneumoniae was positive. He presented sinus bradycardia in the electrocardiogram. Treatment included supplemental oxygen and azithromycin $10 \mathrm{mg} / \mathrm{kg} /$ day for five days. He had no need of analgesia. At the second day of hospitalization he was asymptomatic. The chest and lateral radiograph 4 days after admission showed almost complete resolution of the pneumomediastinum and subcutaneous emphysema. IgM antibody Mycoplasma pneumoniae remained positive one month after episode.

Discussion: In this case, physical activity, Mycoplasma pneumoniae infection with cough and longilineus habit could be the triggering factors. The autors pretend to alert to the occurrence of this pathology with nonspecific symptoms, requiring a high level of suspicion for a correct diagnosis.

\section{5}

\section{Respiratory}

Idiopathic Pulmonary Hemosiderosis: Diagnostic and Therapeutic Challenge

Rita Russo Belo ${ }^{l}$, Cristiana Martins ${ }^{1}$, Cristina Cândido ${ }^{1}$, Natalina Miguel $^{\prime}$, Vânia Martins ${ }^{1}$

${ }^{1}$ Department of Pediatrics, Centro Hospitalar de Trás-os-Montes e Alto Douro, Portugal

Background: Idiopathic pulmonary hemosiderosis is a rare cause of alveolar hemorrhage characterized by iron deficiency anemia, hemoptysis and pulmonary infiltrates. The diagnosis implicates the exclusion of other disorders causing diffuse alveolar hemorrhage. Treatment is not well established and consists on glucocorticoids and other immunosuppressant agents.

Case Report: Nine-year-old child with type 1 diabetes mellitus diagnosed by the age of three and idiopathic pulmonary hemosiderosis diagnosed at five. At diagnosis, he presented with severe anemia ( $\mathrm{Hb} 4.9$ $\mathrm{g} / \mathrm{dL}$ ) and after exclusion of infectious, gastrointestinal and hemolytic causes, the study revealed diffuse pulmonary infiltrates. Bronchoalveolar lavage and lung biopsy revealed hemosiderin-laden macrophages. Other causes of pulmonary hemosiderosis were excluded. Autoimmune study was negative. Initially treated with intravenous corticosteroids (methylprednisolone, monthly pulses), prednisolone and hydroxychloroquine. After three months, a pulmonary computerized scan revealed no evidence of pulmonary hemorrhage. He completed six methylprednisolone pulses, eight months of prednisolone and 13 months of hydroxychloroquine. Two months later, he had a relapse of pulmonary hemorrhage and reinitiated corticosteroids and hydroxychloroquine. $\mathrm{He}$ repeated methylprednisolone pulses, maintained prednisolone for 20 months and maintained hydroxychloroquine until nowadays. Eight months after suspending prednisolone, and while on treatment with hydroxychloroquine, he had a new relapse of pulmonary hemorrhage, reinitiating prednisolone. While on corticosteroids, glycemic control required insulin adjustment.

Conclusions: The treatment of this condition on a child with type 1 diabetes mellitus is challenging, since glycemic control is very difficult and demands high insulin doses. The coexistence of type 1 diabetes mellitus rises questions on the idiopathic etiology, since autoimmunity is one of the etiologies proposed for this disorder. During follow-up, we understood that the suspension of corticosteroids, even maintaining hydroxychloroquine, lead to the relapse of pulmonary hemorrhage. It is important to manage the balance between pulmonary hemorrhage control and glycemic control in order to minimize the sequels from both disorders.

\section{8}

\section{Respiratory}

Polymorphism of EPOX1 Gene in Children with an Acute Bronchitis with Wheezing Syndrome

Maryna Strelkova ${ }^{I}$, Ganna Senatorova ${ }^{I}$, Yevheniia Ivanova ${ }^{I}$, Nelli Bashkirova

${ }^{I}$ Department of Pediatrics №1 and Neonatology, Kharkiv National Medical University, Ukraine

${ }^{2}$ Department of pulmonology, Kharkiv Regional Children's Clinical Hospital, Ukraine

Background: The development of wheezing during acute bronchitis is recorded in $20-25 \%$ of cases in children. The formation of wheezing is determined by the anatomical organism's features, as well as the nature and severity of the inflammatory process of the bronchial tree which is the result of the interaction of genetic, medical, biological, social factors.

Objective: To determine the informativity of the study of polymorphism of the gene of microsomal epidergylysis (EPOX1) to predict the development of wheezing in acute bronchitis in children.

Methods. The checkup was conducted on 54 children aged from 3 to 5 years with acute bronchitis. The group of 28 children $(51.9 \%)$ with the acute form of bronchitis with wheezing manifestations was the most observed. The comparison group 26 children $(48.1 \%)$ with acute bronchitis without wheezing.Determination of the genetic polymorphism of Tyr113His in the EPOX1 gene was carried out by the polymerase chain reaction.

Results. The diffusion of the single-nucleotide replacement genetic polymorphism of Tyr113His in the EPOX1 gene in children with acute bronchitis has been studied. It was established that the homozygous Tyr/Tyr 
genotype was determined in $53.8 \%$ of the examined children with acute bronchitis, the heterozygous genotype Tyr/His was determined in $42.6 \%$, and the homozygous genotype His/His was determined in $3.7 \%$. The gene polymorphism analysis revealed that children with wheezing syndrome 2.2 times more often had the genotype Tyr/Tyr, in the children of the comparison group $(p=0.023)$. It has been established that children with a "weakened" genotype Tyr/His and His/His disease have more favorable course.

Conclusion. The EPOX1 gene may be a candidate for the detection of a predisposition to the development of wheezing in children. The obtained data confirm the facts of the genetic determination of the propensity to character of the course of bronchopulmonary diseases in children.

451

\section{Respiratory}

Epidemiological Evaluation of Lower Respiratory Infections in Admitted Children in a Public Hospital in Southeastern Brazil Eneida Veiga ${ }^{l}$, Alvaro Veiga ${ }^{1}$, Patrícia Lopes Silva ${ }^{2}$, Maria Mariana Muniz Jorge ${ }^{2}$, Luana Fonseca Vita Souza ${ }^{2}$, Felipe Moliterno ${ }^{1}$, Nathalia Veiga Moliterno ${ }^{I}$, Solimar Stumpf Cordeiro ${ }^{I}$

${ }^{I}$ Pediatrics, Faculty of Medicine of Petrópolis, Brazil

${ }^{2}$ Medicine student, Faculty of Medicine of Petrópolis, Brazil

Background: Respiratory infections are among the most common causes of mortality in children throughout the world. Pneumonias are responsible for $20 \%$ of causes of mortality in children below five years of age. Bronquiolitis is the most common infection of the inferior respiratory tract and main cause of hospitalization in infants throughout the world, especially due to syncytial respiratory virus.

Objective: To identify and compare the prevalence of pediatric hospitalizations due to inferior respiratory tract infections, through the analysis of the clinical and epidemiological characteristics of admitted patients from March $20^{\text {th }}, 2017$ through September $21^{\text {st }}$, 2018.

Methods: Retrospective, transversal and descriptive study of the pediatric hospitalizations in the period of March $20^{\text {th }}, 2017$ to September $21^{\text {st }}$, 2018 due to pneumonia and bronquiolitis in a public hospital in the Serrana region of Rio de Janeiro.

Results: 495 hospitalizations due to respiratory causes were evaluated, totalizing $30,3 \%$ of the total of 1.632 general hospitalizations. The majority occurred in patients younger than 1 year old. The most prevalent diagnosis was pneumonia, $67 \%$, followed by bronquiolitis, $19,5 \%$, and the existence of both, $13,5 \%$. Males were the most affected with $63 \%$. There were more hospitalizations in the fall of 2018. 4,4\% of patients were in the intensive care unit due to these respiratory infections and $8,4 \%$ were readmissions.

Conclusion: There was an increase in the number of hospitalizations for respiratory infections in the fall and winter periods. It is empirical that the means of prevention of lower respiratory infections be improved in order to reduce the number of cases observed.

\section{4}

\section{Respiratory}

Conditions of Vulnerability to Inadequate Treatment of Bronchiolitis Kattia Naves ${ }^{2}$, Sandra Vieira

${ }^{I}$ Pediatrics, Faculdade de Medicina da Universidade de São Paulo, Brazil

${ }^{2}$ Pediatrics, Hospital do Servidor Publico do Estado de São Paulo, Brazil

Goals: To analyze clinical and demographic variables possibly associated with the prescriptions of non-recommended but routinely used therapies for infants with acute viral bronchiolitis.
Methods: A cross-sectional study included hospitalized infants with bronchiolitis caused by respiratory syncytial virus. Those with other associated infections and/or morbidities were excluded. The data were collected from medical records.

Results: Among 120 cases, $90 \%$ used inhaled beta agonists, $72.5 \%$ corticosteroids, $40 \%$ antibiotics and $66.7 \%$ inhaled hypertonic saline solution. The use of bronchodilators did not present an independent association with another variable. More frequent use of corticosteroids was associated with low oximetry, longer hospitalization time and age 3 months. Antibiotic therapy was associated with the presence of fever, longer hospitalization and age3 months. Inhaled hypertonic saline solution was associated with longer hospitalization time.

Conclusions: Non-recommended prescriptions were frequent. Corticosteroid and antibiotic therapy were associated with signs of severity, as expected, but interestingly, they were more frequently used in infants above $3 \mathrm{~m}$, which suggested less safety in the diagnosis of viral bronchiolitis in these patients. The use of bronchodilators was even more worrying since they were indiscriminately used, without association with another variable related to the severity or characteristics of the host. The use of inhaled hypertonic solution, although not associated with severity, seems to have implied a longer hospitalization time. The identification of these conditions of greater vulnerability to the prescription of inappropriate therapies contributes to the implantation of protocols for the bronchiolitis treatment, for continuing education and for analysis of the effectiveness of the strategies employed.

\section{5}

\section{Respiratory}

The Role of Respiratory Virus Infection in Suspected Pertussis: A Prospective Study

Sandra Vieira ${ }^{1}$, Angela Ferronato ${ }^{2}$

${ }^{T}$ Pediatrics, Faculdade de Medicina da Universidade de São Paulo, Brazil

${ }^{2}$ Pediatrics, Hospital Universitário da Universidade de São Paulo, Brazil

Background: Pertussis is a disease caused by Bordetella pertussis (BP), being more frequent and severe in infants less than 1 year old. Respiratory viruses (RV) may mimic pertussis in infants.

Methods: A prospective cohort study with children under 1 year of age hospitalized with suspected clinical pertussis between 2014 and 2016 and submitted to etiological research to identify BP (nasopharynx swab for culture and/or PCR) and RV (nasopharyngeal aspirate for indirect immunofluorescence). Clinical, demographic and follow up data were collected.

Results: During the study period, 59 infants were analyzed. In $30.5 \%$ there was identification of BP, in $39 \%$ of some respiratory virus. In four (7\%), there was BP detection and some RV. In the bivariate analysis they presented a greater chance of BP infection: lower age, no fever, not being vaccinated, leukocytosis $20,000 / \mathrm{mm}^{3}$, lymphocytosis $10,000 / \mathrm{mm}^{3}$ and RV infection: wheezing. After adjustment for confounders, the largest predictors for $\mathrm{BP}$ independently were: no wheezing $(\mathrm{OR}=5.7)$ and leukocytosis $20,000 / \mathrm{mm}^{3}(\mathrm{OR}=5.38)$. The number of patients with codetection did not allow the comparative analysis of severity with those with single agent.

Conclusion: Clinical/laboratory characteristics suggest, but are not pathognomonic, of the etiologies, which corroborates the need for VR and $\mathrm{BP}$ research in this clinical situation.

753

Respiratory

Home Mechanical Ventilation: An Emerging Necessity in the Pediatric Patient with Complex Chronic Conditions 
Eleni Volakli ${ }^{1}$, Asimina Violaki ${ }^{1}$, Elpis Chochliourou ${ }^{l}$, Menelaos Svirkos $^{I}$, Serafeia Kalamitsou ${ }^{l}$, Peristera-Eleni Mantzafleri ${ }^{1}$, Eleni Karakeke $^{I}$, Anna Kalaitzi ${ }^{1}$, Anastasia Anastasiou ${ }^{1}$, Maria Sdougka ${ }^{1}$

${ }^{I}$ Pediatric Intensive Care Unit, Hippokration General Hospital, Greece

Introduction: Home mechanical ventilation (HMV) is an emerging necessity for children with complex chronic conditions (CCC). The aim of our study was to record all PICU patients that were discharged with HMV. Methods: Retrospective study of PICU patients discharged with HMV from 5/2011 to 3/2019. Data recorded: Demographics, interfaces, ventilator components, $\mathrm{O}_{2}$ needs, mode and settings of $\mathrm{HMV}$, and the outcome.

Results: 28 children (11M/17F), median (IQR) age of 18(8.25-72)mo, BW 9(5.6-13)kg, ideal BW 11(8.6-20)kg, were discharged on HMV due to epileptic encephalopathy (11), neuromuscular diseases (13), and congenital syndrome diseases (4). The majority (23) was put on Invasive pressure/volume MV (IMV) through tracheostomy, while 5 were on mask NIV (BIPAP). All IMV patients were connected through a bellow connector and all but one used single circuit with active hydration. Main IMV mode: SIMV-PS (19), assist (2), and PS (2). Main IMV settings: Vt as ml/ ideal BW 9.16(7.5-10.43), RR 20(11-22), PEEP 6(5-6), Ti 0.8(0.8-1.2), PS 12(10-14), Trigger 1.25(0.5-1.87)L/min, Flow squared(11)/decelerating(12), aimed at satisfactory lung expansion $\left(9^{\text {th }} \mathrm{rib}\right)$, and normal/proper ABG. Alarms were set appropriately. All patients were discarded on oxygen and at a follow up time of 31.5(12-47)mo, they remained in a stable condition. All families were supported by a national Home Care system.

Conclusions: Pressure/volume compressors with single circuit and hydration were overwhelmingly used, in accordance to recent guidelines on HMV for children. Pediatric patients with $\mathrm{CCC}$ were underweighted, and the use of ideal BW coupling with the increased VD due to connector should be taken into account for the appropriate setting of VT. Proper alarm settings contribute to better patient safety and family satisfaction.

483

\section{Rheumatology}

Incomplete Kawasaki Disease: A Diagnostic Challenge

Ana Abreu ${ }^{l}$, Ivo Neves ${ }^{l}$, Maria João Nogueira ${ }^{l}$, Augusta Gonçalves ${ }^{1}$, Manuela Alves ${ }^{1}$, Edite Gonçalves ${ }^{2}$, Liliana Branco ${ }^{3}$, Arnaldo Cerqueira ${ }^{3}$, Liliana Abreu ${ }^{3}$

${ }^{1}$ Paediatrics, Hospital de Braga, Portugal

${ }^{2}$ Paediatric Cardiology, Hospital de Braga, Portugal

${ }^{3}$ Paediatric Intermediate Care Unit, Hospital de Braga, Portugal

Background: Kawasaki disease (KD) is an acute inflammatory vasculitis defined by clinical criteria. Coronary artery (CA) aneurysms may occur, leading to significant morbidity and mortality. Young infants are at increased risk, as prevalent incomplete forms pose diagnostic difficulties that often delay timely treatment.

Objective: This work aims to raise awareness to incomplete KD trough a clinical case, highlighting its complexities.

Clinical Case: Previously healthy 6 month-old female admitted with high fever, rhinorrhea, cough and irritability for 4 days. Besides bilateral ocular hyperaemia, the remaining physical examination was unremarkable. Laboratory tests showed leukocytosis with neutrophilia, C-reative protein $128 \mathrm{mg} / \mathrm{L}$, mild normocytic normochromic anaemia and significant leukocyturia. Lumbar puncture revealed limpid liquor with pleocytosis (414/uL) and normal biochemistry. Ceftriaxone was initiated. During hospitalization, intermittent polymorphous exanthema, hands and feet oedema were observed. Persistent fever, fluctuant after day 5, and irritability accounted for progressive therapeutic escalation with vancomycin and meropenem whilst awainting for a positive result of liquor bacterial DNA; after confirmed contamination, anphotericin B was started. Repeated and extensive blood, urine, liquor and respiratory secretions studies showed no evidence of causative infectious agent. Electroencephalogram was normal. Other analytic findings: thrombocytosis, hypoalbuminemia and sedimentation rate $111 \mathrm{~mm} / \mathrm{h}$. At day 16 after admission, an echocardiogram revealed left and right coronary ectasia, and KD was confirmed. She received intravenous immunoglobulin, methylprednisolone, high-dose oral acetylsalicylic acid (ASA) $60 \mathrm{mg} / \mathrm{kg} / \mathrm{day}$, with further favourable clinical and analytic evolution. Periungual desquamation was noticed during $3^{\text {rd }}$ week. She was discharged for ambulatory follow-up after 28 days, with oral ASA (low dose).

Conclusion: Incomplete KD is a clinical challenge, especially in infants. Heterogeneous laboratory findings and unspecific clinical features may be misleading. A high index suspicion is fundamental, as correct diagnosis might rest upon detection of CA abnormalities. Prompt adequate treatment has a potential prognosis impact, besides limiting further futile therapeutic interventions.

\section{8}

\section{Rheumatology}

Saudi Female with Successful Arrest of Progressive Juvenile Localized Scleroderma Using Interleuken-6 Antagonist Logain Alahmadi ${ }^{1}$, Mohammed A. Muzuffer ${ }^{2}$

${ }^{7}$ Department of Pediatric, MBBS , SSC-PED, King Abdulaziz Universty Hospital, Saudi Arabia

${ }^{2}$ Department of Pediatric, MD, FRCPC ,King Abdulaziz University Hospital, Saudi Arabia

Juvenile localized Scleroderma is one of the pediatric rheumatological disorders that primarily affect the skin and subcutaneous tissue beside the other type which is systemic sclerosis as it is rare in pediatric population. Management of such diseases is really crucial as its significantly effects the health and the life quality of the children. Data shows the effectiveness of systemic corticosteroids in association with methotrexate (MTX) in patients with active JLS especially in progressive linear scleroderma and generalized or pansclerotic morphoea. If MTX is ineffective or the disease relapses after a period of clinical remission or in the case of MTX-intolerance mycophenolate mofetil (MMF) may be used despite the lack of good evidence in the literature. Few studies shows that using of tocilizumab is safe, well tolerated and may have therapeutic benefit in the cases with severe and refractory JLS. In this case which is first time reported in Saudi Arabia describing the response and the efficacy of Intereuken-6 Antagonist in JLS .

\section{2}

\section{Rheumatology}

\section{Two Siblings with Recurrent Fevers}

Cristina Ferreras $^{1}$, Teresa Campos $^{2}$, Esmeralda Rodrigues ${ }^{2}$, Francisca Aguiar $^{3}$, Mariana Rodrigues ${ }^{3}$, Iva Brito ${ }^{3}$

${ }^{1}$ Department of Pediatrics, Centro Hospitalar São João, Portugal ${ }^{2}$ Inborn Errors of Metabolism Unit, Centro Hospitalar São João, Portugal ${ }^{3}$ Pediatric Rhematology Unit, Centro Hospitalar São João, Portugal

Recurrent fevers are a diagnostic challenge in Pediatrics which requires a systematic structured approach since differential diagnosis is vast. The authors present the case of a 14 year-old caucasian girl, with no relevant family history. She presented with recurrent fevers since the first year of life, with a duration of 3-7 days at variable intervals (1-6 months). Accompanying symptoms included oral ulcers, cervical lymphadenopathies and arthralgia. She was assyntompatic between attacks, with normal growth and development. After excluding infectious, neoplastic and autoimmune causes, a PFAPA diagnosis was assumed. The patient was treated with a 1-2 day course of prednisolone with favorable response. 
At age 12 she presented with genital ulcers during a febrile episode and was referred to our Pediatric Rheumatology Unit. During our evaluation, she complained also of macular rash and abdominal pain with ocasional diarrhea during the episodes.

Investigations during attacks revealed increased inflammatory markers (IM), IgA, IgD and urinary mevalonic acid. Between attacks IM are normal, with mild anemia.

Genetics identified MVK variants c.79-2AG and c.1129GA in heterozygosity, which confirmed HyperIgD syndrome (HIDS) diagnosis. She's receiving NSAIDs and rare short courses of steroids during attacks with adequate control. Her 9 year-old brother was then brought to our attention with a history of recurrent fevers and rash since the first year of life. Currently, he presents with 6-7 episodes/year, 5-6 days duration, associated with painful cervical lymphadenopathies and occasional diarrhea and artralgia. His genetic study identified the same MVK variants, confirming a HIDS diagnosis. HIDS is an autosomal recessive autoinflamatory syndrome caused by mutations in the MVK gene. Its presentation is very variable but usually early onset, and can be confused with PFAPA.

Diagnosis is paramount for its therapeutic implications, monitoring for secondary amyloidosis and to detect other cases within the same family, such as in this case.

\section{0}

\section{Rheumatology}

Ehlers-Danlos Syndrome: An Unusual Etiology of Enuresis Ines Ganhao ${ }^{1}$, Maria São Pedro ${ }^{1}$, Cristina Esteves ${ }^{1}$, Inês Marques ${ }^{1}$ ${ }^{T}$ Serviço de Pediatria, Centro Hospitalar Barreiro-Montijo, Portugal

Background: Enuresis is a common problem that affects approximately $15 \%$ of children and adolescents. Although it can resolve spontaneously, in most cases it can lead to low self-esteem and dysfunctional family and social relationships, especially among pairs. Meticulous approach, identification of comorbidities and behavioral/pharmacologic treatment are for that reason fundamental.

Objective: To alert pediatric community regarding association of enuresis and hypermobility syndromes.

Methods: Case report from suburban hospital in Portugal.

Results: We report the case of a ten year old boy, with family history of spina bifida with enuresis (mother) and primary enuresis (brother) but no relevant personal history. He was first referred to Pediatric consultation for primary nonmonosymptomatic enuresis. No previous response to behavioral measures or desmopressin. Physical examination revealed skin hyperextensibility, joint hypermobility of the shoulders and the $1^{\text {st }}$ phalangeal-metacarpal joint of both hands, with subluxation. The score of Beighton was 4 , foot/height relation 0,15 and upper segment/lower segment relation 0,89 , with no other marfanoid habitus characteristics. Behavioral measures were reinforced and it was associsted oxybutynin to the ongoing treatment with a decrease of $50 \%$ of enuresis episodes. Genetic study confirmed Ehlers-Danlos Syndrome.

Conclusion: Enuresis is a multifactorial dysfunction with known association with hypermobility syndromes. Dysautonomia had probably a central role in the etiology of enuresis in this case report, do to scarcity of autonomic nerves associated with mutation of collagen, which leads to structural changes of bladder.

\section{6}

\section{Rheumatology}

PENTRAXIN 3 is the Best Biomarker of Kawasaki Disease: Predictor of IVIG Non-Responder and Coronary Artery Lesion and Sequellae Toshiyuki Kitoh $^{2}$, Tsuyoshi Ohara ${ }^{2}$, Taichiro Muto ${ }^{I}$, Akihisa Okumura ${ }^{1}$, Yusuke Koizumi ${ }^{3}$, Yuka Yamagishi, Hiroshige Mikamo ${ }^{3}$, Reizo Baba ${ }^{4}$, Kenji Daigo ${ }^{5}$, Takao Hamakubo
${ }^{I}$ Department of Pediatrics, Aichi Medical University, Japan

${ }^{2}$ Department of Pediatric Pharmacology, Aichi Gakuin University, School of Pharmacy, Japan

${ }^{3}$ Department of Clinical Infectious Diseases, Aichi Medical University, Japan

${ }^{4}$ College of Life and Health Sciences, Chubu University, Japan

${ }^{5}$ Laboratory for protein-protein interaction research, The Institute for Advanced Medical Sciences,, Nippon Medical School, Japan

Kawasaki disease $(\mathrm{KD})$ is a disease of unknown cause whose main symptom is systemic vasculitis due to immune reaction frequently seen in children. The patients rarely develop fatal episodes, coronary artery aneurysm even after established IVIg treatment. Useful biomarkers are required for accurate treatment. From January 2013 to March 2015, all patients with Kawasaki Disease admitted to Pediatrics at Aichi Medical University Hospital were requested consents and obtained plasma was saved before IVIG administration. We examined the correlation between plasma values of PTX3, sCD24-ST (Presepsin) and NT-proBNP and acute coronary artery lesion (CAL) and coronary sequelae in $\mathrm{KD}$. We also examined the IVIG dose number correlated with the severity to measure the predictive ability of the test value. Total 97 cases were registered. 22 case of incomplete KD were excluded from PTX3 analysis. 75 case of complete KD were concluded for statistical analyses. Of the 75 patients with $\mathrm{KD}$, three had CAL and the rest 72 cases did not have (nonCAL group). These were confirmed by echocardiography. Statistically significant difference was seen only in PTX3 among the correlation with coronary lesion acute phase disorder, coronary artery sequelae. 1) When the cutoff value was set at $19.1 \mathrm{ng} / \mathrm{ml}$ of PTX3, the specificity was 63.03 , and the sensitivity was 72.73 , and the likelihood ratio was 19.1 in acute phase CAL. When the cutoff value was set at $68.85 \mathrm{ng} / \mathrm{ml}$ of PTX3, specificity 98.21 , sensitivity 100 , and its likelihood ratio was 56 in coronary artery sequelae. 2) The statistical correlation with PTX only IVIg doses was observed. The $r$ values (correlation coefficients) of PTX3, Presepsin, and NT-proBNP were 0.5398, 0.01893, and 0.05664 ,respectively.In this cohort, Kobayashi's Score (KS) was also checked. ROC of IVIG response and KS AUC $0.518895 \%$, confidence interval [0.3288 to 0.7088 ], P value 0.8366 IVIG response and PTX3 AUC $0.907995 \%$, confidence interval [0.8275 to 0.9883 ], $P$ value

\section{0}

\section{Rheumatology}

An Adolescent with Deforming Osteoarthropathy of the Fingers Pedro Marinho $^{1}$, Soraia Azevedo ${ }^{2}$, Ana de Carvalho Vaz ${ }^{1}$, Carolina Germana Silva $^{1}$, Mariana Costa ${ }^{1}$, Daniela Peixoto ${ }^{2}$

${ }^{1}$ Pediatrics Department, Unidade Local de Saúde do Alto Minho, Portugal ${ }^{2}$ Rheumatology Department, Unidade Local de Saúde do Alto Minho, Portugal

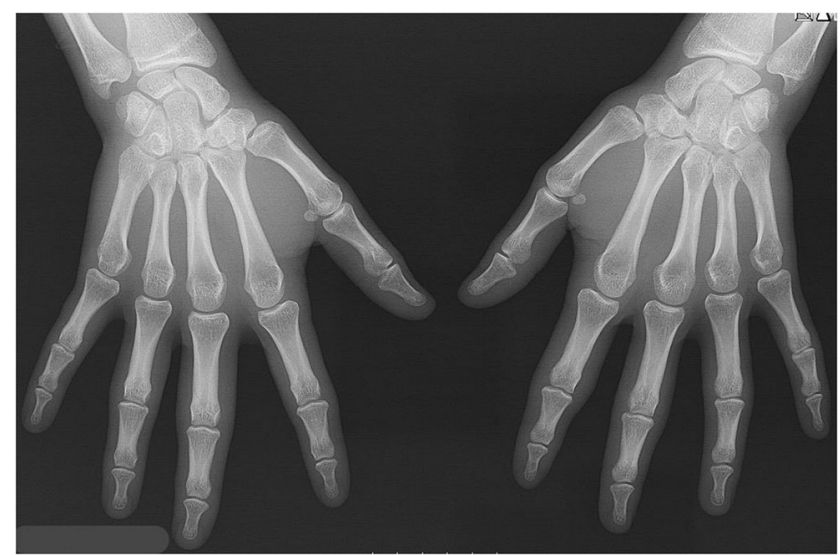


The authors report a 15-year-old Caucasian female, presenting with a oneyear history of bilateral fusiform swelling of the proximal interphalangeal (PIP) joints. No other joints were involved, no other clinical symptoms described. The swelling was initially painless, but after two months she started to develop transient episodes of increased swelling of the PIP joints, with limitation of finger mobility, tenderness and pain associated with finger movements, without an identifiable trigger. During these exacerbation episodes, symptomatic therapy using NSAID's had little effect.

The past medical history of the patient included a traumatic fracture of the proximal phalanx base of the right hand, three years prior to the initial symptoms. The patient has four siblings, one of which has been described with similar PIP joint swelling, but because our patient is adopted, further details are absent. Blood tests were normal (including markers of acute inflammation C-reactive protein and erythrocyte sedimentation rate, rheumatoid factor, antinuclear antibodies and anti-neutrophil cytoplasmic antibody); radiography revealed soft-tissue enlargement of the PIP joints, without irregularities of the phalangeal epiphyses.

The clinical features and positive family history point to the diagnosis of Thiemann's disease - a rare familial juvenile osteochondrosis of the phalangeal epiphyses, a form of idiopathic avascular necrosis, transmitted in an autosomal dominant pattern. Diagnostic features include onset before the age of twenty-five and swelling of PIP joints, with normal laboratory tests; radiographs aid diagnosis if typical changes are present. Role of trauma is undetermined.

Clinicians should be aware of this rare disorder which might be evocative of other forms of arthritis, not only for greater clinical recognition but also avoidance of unnecessary treatments - this disease has a benign course, most patients are asymptomatic after growth-plate closure.

415

\section{Rheumatology}

Systemic Lupus Erythematosus with Prolidase Deficiency in a 16 Year Old Boy

$\underline{\text { Satoshi Sato }}^{1}$, Yoji Uejima ${ }^{1}$, Mihoko Furuichi ${ }^{l}$, Eisuke Suganuma ${ }^{1}$, Yutaka Kawano
${ }^{1}$ Division of Infectious Diseases and Immunology, Saitama Children's Medical Center, Japan

Introduction: Prolidase deficiency (PD) is a rare autosomal recessive disorder which may have a wide spectrum of clinical features. The disorder is caused by a mutation in the PEPD gene.

Objective: $\mathrm{PD}$ is considered to be monogenic cause that are suspected in onset of SLE.

Methods: Two siblings had cognitive impairment, rash or skin ulceration, hyper IgG-emia, hyper IgE-emia. Younger sister had severe interstitial pneumonia. They were diagnosed as PD by performed whole-exome sequencing and measurement of prolidase enzyme activity.

Case1: At the age of 16 years, older brother had gross hematuria. Laboratory findings included hemoglobin $(10.6 \mathrm{gm} / \mathrm{dl})$, white blood cell $(2,500 / \mu \mathrm{l})$, platelet $\left(87 \times 10^{3} / \mu \mathrm{l}\right)$, elevated erythrocyte sedimentation rate (ESR). Immunologic evaluation revealed high immunoglobulins: IgG 4,015 mg/dl, IgA $662 \mathrm{mg} / \mathrm{dl}, \operatorname{IgM} 87 \mathrm{mg} / \mathrm{dl}$, and very high IgE 18,563 $\mathrm{IU} / \mathrm{ml}$. Antinuclear antibodies (ANA) were positive in a titer of 1:80 (homogenous pattern). Antibodies to double-stranded DNA IgG and SS-A were positive. Complement $\mathrm{C}_{3}, \mathrm{C}_{4}$, were low. He underwent a kidney biopsy which confirmed the LN diagnosis with class IV-G (A/C) with mesangial diffuse proliferative changes, with crescent.

$\mathrm{He}$ was started on Intravenous pulses of methylprednisolone and MMF. Later, four infusions of rituximab were given due to incomplete response. He is currently stable in complete remission. His levels of anti-dsDNA and $\mathrm{C} 3$ normalised.

Case2: A younger sister was diagnosed as interstitial pneumonia at six months old. She had recurrent pyoderma, clubbing. ANA and SS-A were positive. Antibodies to DNA IgG was negative. Complement were low. Her urinary tests showed normal. She has been treated with low dose of prednisolone and cyclosporine A for interstitial pneumonia.

Conclusion: $\mathrm{PD}$ was diagnosed in siblings with skin lesions, cognitive impairment, hyper IgG-emia, hyper IgE-emia. And they have ANA and low levels of complement. PD might be a risk factor for the development of SLE. 